ANL-7165 $352-66$

ANL-7165

Argonne Rational Laboratom

TABLES FOR THE CALCULATION

OF OPERATING PARAMETERS FOR

COMPOUND SOLVENT-EXTRACTION COLUMNS

by

Terry R. Johnson and Nelda K. Clark 


\section{DISCLAIMER}

This report was prepared as an account of work sponsored by an agency of the United States Government. Neither the United States Government nor any agency Thereof, nor any of their employees, makes any warranty, express or implied, or assumes any legal liability or responsibility for the accuracy, completeness, or usefulness of any information, apparatus, product, or process disclosed, or represents that its use would not infringe privately owned rights. Reference herein to any specific commercial product, process, or service by trade name, trademark, manufacturer, or otherwise does not necessarily constitute or imply its endorsement, recommendation, or favoring by the United States Government or any agency thereof. The views and opinions of authors expressed herein do not necessarily state or reflect those of the United States Government or any agency thereof. 


\section{DISCLAIMER}

Portions of this document may be illegible in electronic image products. Images are produced from the best available original document. 


\section{LEGAL NOTICE}

This report was prepared as an account of Government sponsored work. Neither the United States, nor the Commission, nor any person acting on behalf of the Commission:

A. Makes any warranty or representation, expressed or implied, with respect to the accuracy, completeness, or usefulness of the information contained in this report, or that the use of any information, apparatus, method, or process disclosed in this report may not infringe privately owned rights; or

B. Assumes any liabilities with respect to the use of, or for damages resulting from the use of any information, apparatus, method, or process disclosed in this report.

As used in the above, "person acting on behalf of the Commission" includes any employee or contractor of the Commission, or employee of such contractor, to the extent that such employee or contractor of the Commission, or employee of such contractor prepares, disseminates, or provides access to, any information pursuant to his employment or contract with the Commission, or his employment with such contractor.

Printed in USA. Price $\$ 5,00$. Available from the Clearinghouse for Federal Scientific and Technical Information, National Bureau of Standards,

U. S. Department of Commerce, Springfield, Virginia 22151 
RELEASED FOR ANNOUINCEMENT

II NUCTEAR SCTENCE ABSTRACTS
ANL- 7165

Mathematics and Computers (TID-4500)

AEC Research and

Development Report
ARGONNE NATIONAL LABORATORY 9700 South Cass Avenue Argonne, Illinois 60439

\section{TABLES FOR THE CALCULATION \\ OF OPERATING PARAMETERS FOR \\ COMPOUND SOLVENT-EXTRACTION COLUMNS}

by

Terry R. Johnson

Chemical Engineering Division

and

Nelda K. Clark

Applied Mathematics Division

\section{LEGAL NOTICE}

This report was prepared as an zooount of Guvejument sponsored work. Nelther the United States, nor the Commission, nor any person acting on behalf of the Commission:

A. Makes any warranty or representation, expressed or implled, with respect to the accuracy, completeness, or usefulness of the information contained in this report, or that the use of any Information, apparatus, method, or process disclosed in this report may not Infringe privately owned rights; or

B. Assumes any liabllttes with respect to the use of, or for damages resulting from the use of any Information, apparatus, method, or process disclosed in this report.

As used in the above, "person acting on behalf of the Commission" includes any employee or contractor of the Commiseion, or employee of such contractor, to the extent that such employee or contractor of the Commlssion, or employee of euch contractor prepares, disseminates, or provides access to, any Information pursuant to his employment or contract with the Commiasinn, or hls omployment with such contractor.

February 1966

Operated by The University of Chicago

under

Contract W-31-109-eng-38

with the

U. S, Atomic Energy Commission 
THIS PAGE

\section{WAS INTENTIONALLY LEFT BLANK}


TABLE OF CONTENTS

$\underline{\text { Page }}$

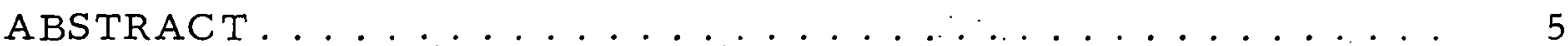

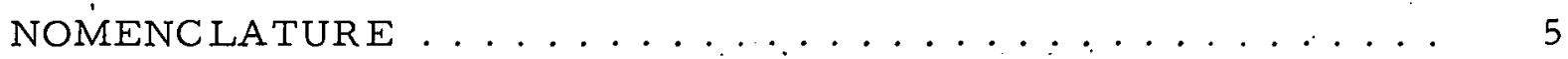

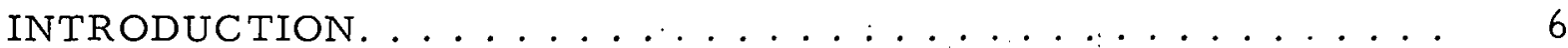

KREMSER - BROWN EQUATION FOR COMPOUND COLUMNS $\therefore . . .8$

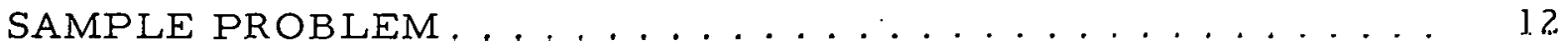

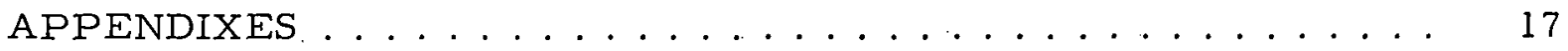

A. Parameter $f$ as a Function of Extraction Factor $\alpha$, Scrub Factor $\beta$, and Numbers of Stages $n$ and $n^{\prime} \ldots \ldots$

B. Extraction Factor $\alpha$ as a function of $f$, Scrub Factor $\beta$, and Numbers of Stages $n$ and $n ! \ldots \ldots \ldots$

C. Parameter $g$ as a Function of Extraction Factor $\alpha$, Scrub Factor $\beta$, and Numbers of Stages $n$ and $n^{\prime} \ldots \ldots 120$

D. FORTRAN Program Statement............. 171

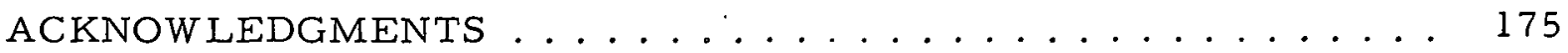

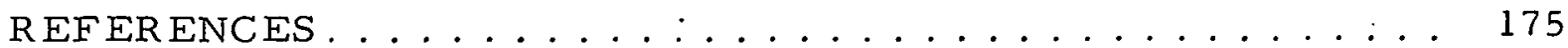




\section{LIST OF FIGURES}

No.

1. Simple Extraction Column . . . ............... 7

2. Compound Extraction Column ............... 8

3. Recoveries and Flow Ratios for Plutonium and Rare-earth

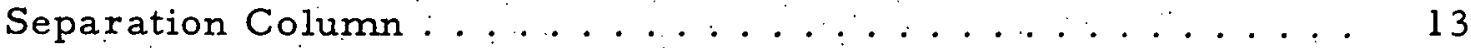




\title{
TABLES FOR THE CALCULATION \\ OF OPERATING PARAMETERS FOR \\ COMPOUND SOLVENT - EXTRACTION COLUMNS
}

by

Terry R. Johnson and Nelda.K. Clark

\begin{abstract}
A set of tables is presented to assist in the calculation of the solvent flow rates and the number of theoretical stages that will effect a specified separation between two distributing solutes in a countercurrent, fractional-extraction column in which the distributing components are introduced on an intermediate plate. The procedure that is outlined is an extension of the Kremser-Brown equation, which applies to extraction columns that have feeds only at the top and bottom. Similar assumptions are required; distribution coefficients and solvent flow rates are constant above and below the feed. Additional tables are presented for use with equations that relate column parameters when distributing components are also fed to the top or bottom of a compound extraction column. A sample problem is described which is applicable to a high-temperature liquid metal-liquid salt extraction process for separating rare-earth fission products from uranium and plutonium. However, the computational procedure is applicable to any system in which the simplifying assumptions are reasonably accurate. The procedure is quite rapid and straightforward.
\end{abstract}

NOMENCLATURE
A. $\quad(\alpha-1) /\left(\alpha^{n+1}-1\right)$
B $\left.\left(\beta^{n^{\prime}+1}-1\right) / \beta-1\right)$
$\mathrm{K} y / \mathrm{x}=$ distribution cocfficient in extraction section
$K^{\prime} y^{\prime} / x^{\prime}=$ distribution coefficient in scrub section
$\mathrm{R}$ Flow rate of raffinate solvent in extraction section
$R^{\prime}$ Flow rate of scrub solvent in scrub section
$\overline{\mathrm{R}}$ : Flow rate of feed solvent in principal feed stream 


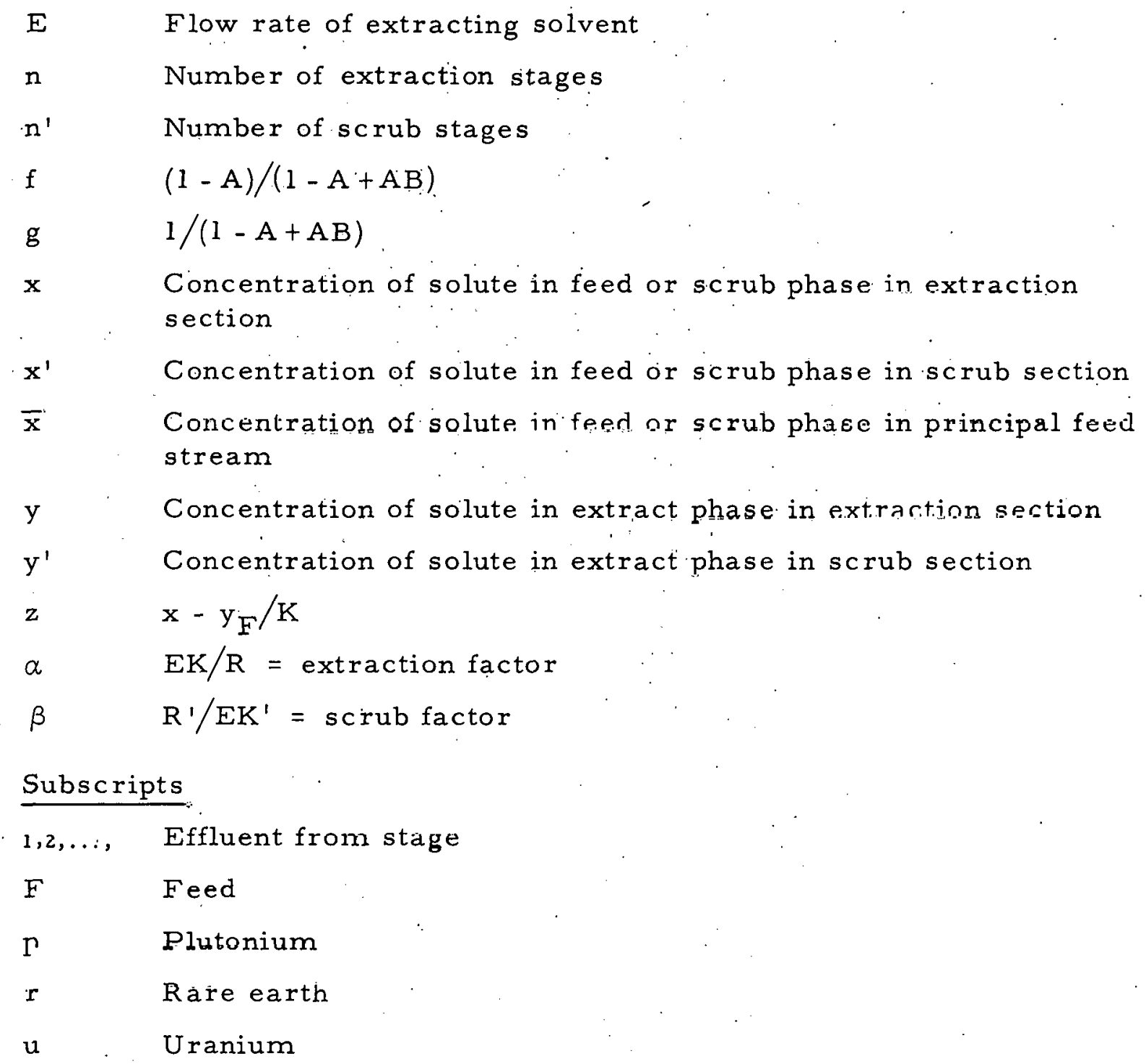

\section{INTRODUCTION}

A common type of solvent-extraction problem can be expressed as follows: In a particular two-phase system of immiscible solvents, estimate the number of equilibrium extraction and scrub stages, and the solvent flow rates above and below the feed stage that are required to achieve a given separation between two distributing solutes.

If it is assumed that the necessary equilibrium constants are known, several methods are available for obtaining sets of operating parameters that will effect the required recovery of one component in countercurrent extraction columns. However, if the recoveries of two distributing elements in the product are specified, time-consuming trial-and-error calculations are often required. With electronic computers, the tediousness 
of the computational methods may appear to be immaterial. However, many problems that are sufficiently difficult to discourage hand calculations, hardly warrant the expense necessary to write the initial computer program. Quick and straightforward methods are still needed for reaching solutions to problems similar to that stated above.

If the equilibrium and operating lines can be assumed to be straight, exact solutions can be derived that provide rapid means of solving the above problem. The familiar Kremser-Brown ${ }^{1}$ equation is applicable to extraction columns that have no central feed (simple columns). This equation can be applied also to problems concerning extraction columns having a central feed (compound columns) by considering the compound column to be two simple columns. However, the stream compositions at the feed stage must be matched by trial and error. If two distributing components are involved, this procedure can be difficult. Smith ${ }^{2,3}$ has derived a solution to a relatively general, countercurrent, equilibrium-stage process, i.e., one having a central feed and product reflux at both the top and bottom of the columin. An expression is described below that can be considered either an extension of the Kremser-Brown equation to compound columns or a special case of Smith's general equation. This expression can be adapted readily to hand calculations and can be used to solve the above problem in which two component recoveries are specified and a compound column is employed.

These exact solutions only approximately describe real systems because of the simplifying assumptions used in their derivations. However, the answers obtained can be used as first estimates for more rigorous computational procedures, such as plate-to-plate or graphical methods, that can better describe the real situation.

The Kremser-Brown equation relates the amount of a distributing solute in a product stream to the extraction factor and the number of equilibrium stages. For the simple column shown in Fig. 1, one form of this equation is

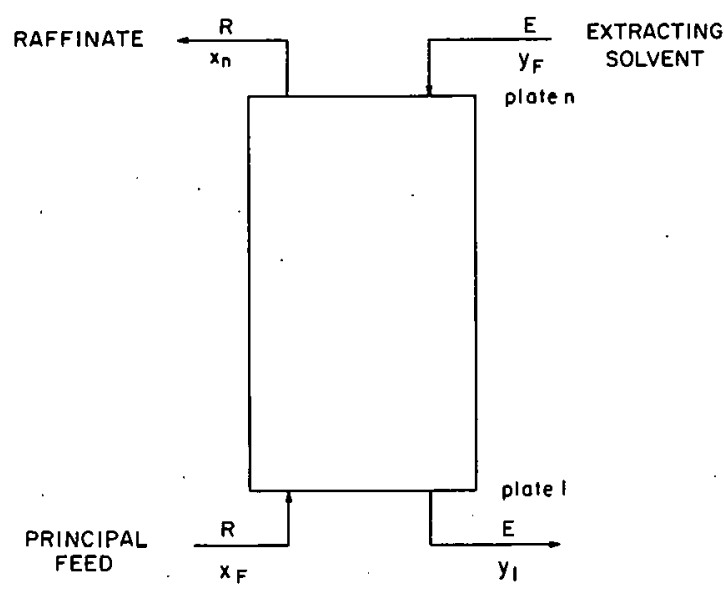

Fig. 1. Simple Extraction Column

$$
\frac{x_{F}-x_{n}}{x_{F}-\left(y_{F} / K\right)}=\frac{\alpha^{n+1}-\alpha}{\alpha^{n+1}-1}
$$

where $\alpha=$ extraction factor $=\mathrm{EK} / \mathrm{R}$.

In the derivation of Eq. (1), a straight equilibrium line (constant dis tribution coefficient) and a straight operating line (immiscible solvents) are assumed. For dilute systems, these are reasonable assumptions. Graphs of Eq. (1) are available to aid calculations. If the recovery of only 
one component is specified, more than one set of operating conditions satisfy the recovery specification, i.e., a number of plates and a corresponding extraction factor. If the recoveries of two components are fixed, there is at most only one solution (a certain number of stages and a related extraction factor).

\section{KREMSER - BROWN EQUATION FOR COMPOUND COLUMNS}

Expressions similar to Eq. (1) have been presented by others $\mathrm{s}^{5,6}$ for compound solvent-extraction columns for the case in which:

a. The distribution coefficients are constant throughout the column,

b. The distributing components fed to the column contain no solvents, and/or

c. 'L holvents fed at the top and bottom of the column contain no distributing components.

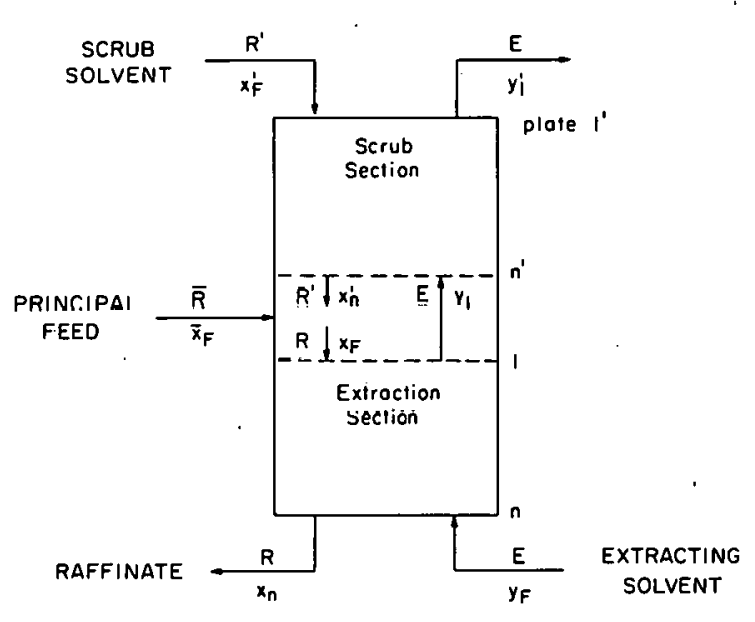

Fig. 2. Compound Extraction Column

These three restrictions are relaxed to some degree in the expressions derived below. The derivation pertains to the column shown in Fig. 2 in which the principal feed dissolved in the heavy solvent is introduced on an intermediate plate. The plates below the feed plate constitute the extraction secliun, and those above the teen plater. constitute the scrub scction. In the extraction section, components are transferred from the feed solvent to the extracting solvent, and in the scrub section, components are transferred in the oppusile direction.

The solvent flow rates ( $R, R^{\prime}$, and $E$ ) and the distribution coefficients ( $K$ and $K^{\prime}$ ) are assumed to be constant through each section of the column. Extracting solvent containing some distributing solute. is fed to the bottom of the column, and pure scrub solvent is fed to the top of the column; i.e., $\mathrm{y}_{\mathrm{F}} \neq 0$ and $\mathrm{x}_{\mathrm{F}}^{\prime}=0$.

For a compound column, the Kremser-Brown equation can be used lu relate the compositions of the streams entering and leaving the section below the feed and also to relate these stream compositions for the section above the feed. Material balances can then be used to combine the two relationships. The treatment presented here is similar to that used by Treybal. ${ }^{6}$ However, the results are somewhat more general and are in a form that can be solved relatively easily with the use of the tables of functions in Appendixes A, B, and C. 
The Kremser-Brown equation below the feed is

$$
\frac{x_{F}-x_{n}}{x_{F}-\frac{y_{F}}{K}}=1-A=\frac{\alpha^{n+1}-\alpha}{\alpha^{n+1}-1}
$$

Define $z=x-\frac{y_{F}}{K}:$ Then

$$
\frac{\left(x_{F}-\frac{y_{F}}{K}\right)-\left(x_{n}-\frac{y_{F}}{K}\right)}{x_{F}-\frac{y_{F}}{K}}=1-\frac{z_{n}}{z_{F}}=1-A .
$$

The Kremser-Brown equation above the feed is

$$
\frac{y_{1}-y_{1}^{\prime}}{y_{1}-x_{F}^{\prime} K^{\prime}}=1-\frac{y_{1}^{\prime}}{y_{1}}=1-\frac{1}{B}=\frac{\beta^{n^{\prime}+1}-\beta}{\beta^{n^{\prime}+1}-1} \text {. }
$$

Solving for $A$ and $B$, one obtains

$$
\mathrm{B}=\frac{\mathrm{y}_{\mathrm{1}}}{\mathrm{y}_{1}^{\prime}} ; \quad \mathrm{A}=\frac{\mathrm{z}_{\mathrm{n}}}{\mathrm{z}_{\mathrm{F}}} .
$$

The material-balance equation above the feed is

$$
E_{y_{1}}=E B_{1}^{\prime}=E_{y_{1}^{\prime}}^{\prime}+R^{\prime} x_{n}^{\prime} .
$$

Then,

$$
R^{\prime} x_{n}^{\prime}=E_{y 1}^{\prime}(B-1) \text {. }
$$

The material-balance equation below the feed is

$E y_{F \cdot}+R x_{F}=E y_{1}+R x_{n}=E B y_{1}^{\prime}+R x_{n}$.

Rearranging Eq. (8) results in

$E\left(y_{F}-B y_{1}^{\prime}\right)=R\left(x_{n}-x_{F}\right)$.

Substituting Eq. (3) into Eq. (9) results in

$$
E\left(y_{F}-B y i\right)=R(A-1) z_{F}
$$


or

$$
R z_{F}=\frac{E\left(y_{F}-B y_{1}^{i}\right)}{A-1}
$$

The material-balance equation around the feed is

$$
R x_{F}=\vec{R} x_{F}+R^{\prime} \dot{x}_{n}^{\prime}
$$

or

$$
R x_{F}=R\left(z_{F}+\frac{y_{F}}{\dot{K}}\right)=\frac{E\left(y_{F}-B y_{1}^{\prime}\right)}{A-1}+R \frac{y_{F}}{\bar{K}}
$$

Substituting Eqs. (7) and (13) into Eq. (12) produces

$$
\frac{E Y_{F}-E B y_{1}^{\prime}}{A-1}+R \frac{Y_{F}}{K}=\bar{R} \bar{x}_{F}+E y_{1}^{\prime}(B-1)
$$

Rearranging Eq. (14) results in

$$
\frac{E y_{1}^{\prime}}{\bar{R} \bar{x}_{F}}=f \cdot\left[1-\left(\frac{R}{\bar{R}}\right)\left(\frac{y_{F}}{\bar{x}_{F}^{K}}\right)\right]+g\left(\frac{E_{y_{F}}}{\bar{R} \bar{x}_{F}}\right) \text {. }
$$

where

$$
\begin{aligned}
& f=\frac{1-A}{1-A+A B}, \\
& g=\frac{1}{1-A+A B}, \\
& 1-A=\frac{\alpha^{n+1}-\alpha}{\alpha^{n+1}-1}, \\
& 1-\frac{1}{B}=\frac{\beta^{n^{\prime}+1}-\beta}{\beta^{n^{1}+1}-1}, \\
& \alpha=E K / R=\text { extraction factor, }
\end{aligned}
$$

and

$$
\beta=R^{\prime} / E K^{\prime}=\text { scrub factor. }
$$


In a similar fashion, an analogous expression.can be derived for the case of an:impure scrub solvent and a. pure extracting. solvent, i.e., $\mathrm{y}_{\mathrm{F}}=0, \mathrm{x}_{\mathrm{F}}^{\prime} \neq 0$. This result is

$$
\frac{E y_{1}^{\prime}}{\bar{R}_{\bar{x}} \bar{x}_{F}+R^{\prime} x_{F}^{\prime}}=f+(1-g) \frac{E K^{\prime} x_{F}^{\prime}}{\bar{R} \bar{x}_{F}+R^{\prime} x_{F}^{\prime}} \text {. }
$$

The general case, in which distributing components are fed to the top and bottom of the column as well as. to an. intermediate stage, is essentially the equation derived by Smith. The above equations are special cases of this general equation and.can be deduced from it. The derivation used here shows the relationship to the more familiar Kremser-Brown: equation.

If both scrub and extracting solvents are pure, Eqs. (15) and (22) reduce to

$$
\frac{E_{y_{1}^{\prime}}^{\prime}}{\bar{R} \bar{x}_{F}}=f=\frac{1-A}{1-A+A B} .
$$

The above relationships are valid also if the principal feed is dissolved in a solvent lighter than the extracting solvent. In: this case, the extraction section is above the feed and the scrub section is below.

The terms $\alpha$ and $\beta$ are analogous to the extraction factor in the Kremser-Brown. equation for a simple column. Since $\alpha$ includes the solvent flow rates and distribution factor for the extraction section, $\alpha$ is the extraction factor, and for a similar reason $\beta$. is the scrub factor. For the special case in which the distribution. coefficients of one component in the extraction and scrub sections of the column are the same, $\alpha \beta \leq 1$, since

$$
\alpha \beta=\left(\frac{E K}{R}\right)\left(\frac{R^{\prime}}{E K}\right)=\frac{R^{\prime}}{R}
$$

which is $\leq l$ for $\bar{R} \geq 0$ (a nonnegative feed). In the derivation, however, the distribution coefficients were not assumed to be equal in each section; they: were only. assumed to be constant through each section. In.this more general case, $\alpha \beta$ can be greater than one.

The use of these equations in hand calculations is greatly facilitated by tabulations of $f$ and $g$ as functions of the extraction factor, the scrub factor, and the numbers of extraction and.scrub stages. Because recoveries are usually specified, tables of the extraction factor as a function of $f, \beta$, $n,$. and $n$ ' are also useful. Tables of $f, \alpha$, and $g$ for all integer combinations of $n$ up to 10 and. $n^{\prime}$ up to 5 have been prepared on a digital computer, and are presented. in Appendixes $A, B$, and $C$ of this report. The program statement from which these tables were prepared is included in Appendix D. 


\section{SAMPLE PROBLEM}

A sample calculation is presented here to illustrate the use of the expressions derived. The example refers to a nuclear-fuel recovery process which uses a solvent-extraction step to separate uranium and plutonium from rare-earth fission products. The solvents in this process are a liquid-metal alloy $(65 \mathrm{a} / \mathrm{o} \mathrm{Cd}, 15 \mathrm{a} / \mathrm{o} \mathrm{Zn}, 20 \mathrm{a} / \mathrm{o} \mathrm{Mg})$ and a liquid salt $\left(50 \mathrm{~m} / \mathrm{o} \mathrm{MgCl}_{2}, 30 \mathrm{~m} / \mathrm{o} \mathrm{NaCl}, 20 \mathrm{~m} / \mathrm{o} \mathrm{KCl}\right.$ ). The rare earths are preferentially dissolved (as chlorides) in the salt and the actinides (as the elements) in the metal. The following distribution coefficients apply throughout the column:

for uranium, $\mathrm{K}_{\mathrm{u}}=0.015 \frac{(\mathrm{kg} \text { of } \mathrm{U} \text { in salt }) /(\mathrm{kg} \text { of solute-free salt solvent })}{(\mathrm{kg} \text { of } U \text { in metal }) /(\mathrm{kg} \text { of solute-free metal solvent })}$

for plutonium, $\mathrm{K}_{\mathrm{p}}=0.035$ (similar units);

for rare earths, $K_{r}=1.2$ (similar units)

(an average value for all rare earths).

The distribution coefficients of several rare earths and actinides have been determined by Knighto ${ }^{7}$ in a variety. of liquid metal-liquid salt systems..

For this illustration, it is required that spent nuclear fuel be recovered which contains the following, important constitutents:

$\begin{array}{lc}\text { Uranium } & 40 \mathrm{~kg} / \text { day } \\ \text { Plutonium } & 8 \mathrm{~kg} / \mathrm{day} \\ \text { Rare earths } & 0.8 \mathrm{~kg} / \text { day. }\end{array}$

This fuel is roughly equivalent to the fuel discharged from a fast breeder reactor that has a thermal power of $2,400 \mathrm{MW}$ and discharges fuel having an average burnup of $5 \%$ of the heavy atoms. It is required that greater than $99.2 \%$ of the plutonium be recovered and that $20 \%$ or less of the rare earths be present in the metal solution product. The fuel, dissolved in:the metal solvent, is fed to the center of the column, and the rare earths are extracted into the salt phase. In this case the extracting solvent is the salt, and the scrub solvent is the liquid metal.

The number of stages in the column:can be selected arbitrarily within. limits. Unlike the simple column, the specification of two component recoveries in a compound column does. not limit ${ }^{8}$ the possible operating conditions to a single set of values of the flow ratio and number of stages. There is a range of values of the extraction and scrub factors corresponding to a number of stages above and below the feed that will achieve the. required separation of plutonium from rare earths. For this example, the 
numbers of stages above and below the feed were chosen as three. Values of $\alpha$ and $\beta$ corresponding to the specified recovery can be found in the table for $n=3$ and $n^{\prime}=3$ in Appendix $B$ for each distributing element. $A$ line of constant plutonium recovery can be plotted on coordinates of the solvent flow ratios above and below the feed (Fig. 3) since

$$
\frac{\mathrm{R}}{\mathrm{E}}=\frac{\mathrm{K}_{\mathrm{p}}}{\alpha_{\mathrm{p}}} \text { and } \frac{\mathrm{R}^{\prime}}{\mathrm{E}}=\beta_{\mathrm{p}} \mathrm{K}_{\mathrm{p}}
$$

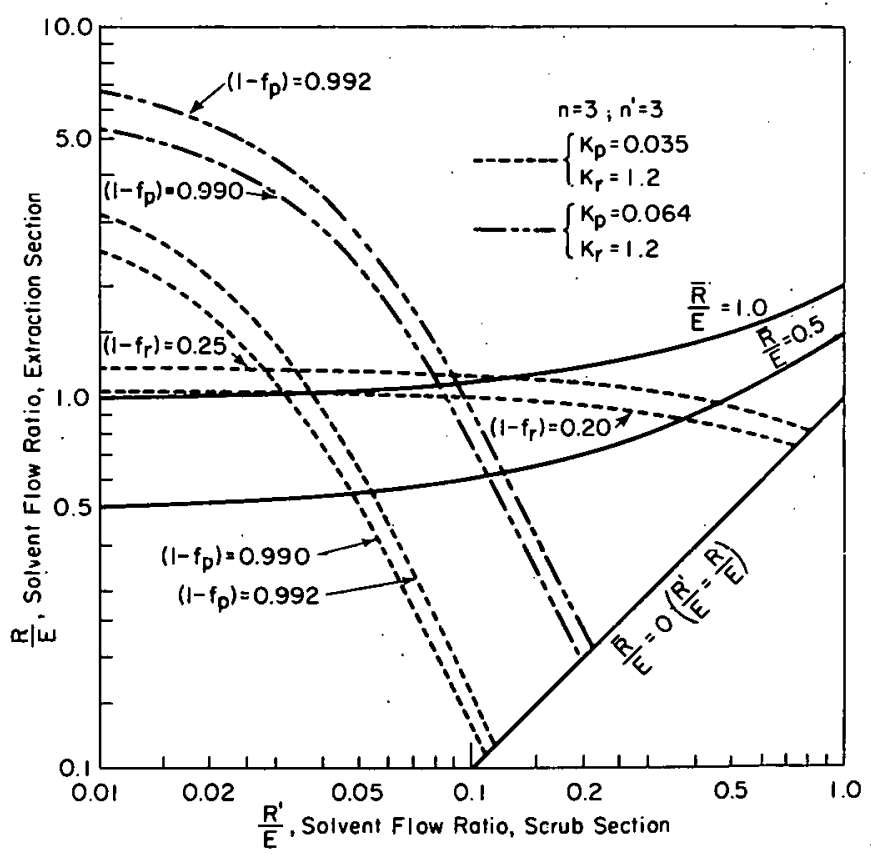

Fig. 3

Recoveries and Flow Ratios for Plutonium and Rare-earth Separation Column

In a similar manner, a line of constant rare-earth recovery can be drawn. The intersection of the lines $1-f_{r}=0.20$ and $1-f_{p}=0.992$ represents the flow ratios that will yield the required recoveries in a countercurrent column having three extraction and three scrub stages. The distribution coefficient of each component is assumed to be independent of the other solute. The area bounded by the lines $l-f_{p}=0.992,1-f_{r}=$ 0.20 , and $R / E=R 1 / E$ represents possible flow conditions that will yield $1-f_{p}>0.992$ and $l-f_{r}<0.20$. If the required separation were not possible with this number of stages, the recovery lines would not intersect or would intersect to the right of the line $R / E=R^{\prime} / E$.

The solution to the sample problem is

$$
R / E=1.030 ; R^{\prime} / E=0.0376 ; \bar{R} / E=0.992 \text {. }
$$


The amount of feed solvent $\bar{R}$ is fixed in this case by: the uranium solubility in the feed. The uranium solubility is $0.0267 \mathrm{~kg}$ of $\mathrm{U} / \mathrm{kg}$. of solute-free solvent metal.* Thus,

$$
\begin{aligned}
& \bar{R}=\frac{\mathrm{kg} \text { of uranium per day }}{0.0267}=40 / 0.0267=1500 \mathrm{~kg} / \mathrm{day}, \\
& E=R / 0.992=1512 \mathrm{~kg} / \text { day }, \\
& \mathrm{R}^{\prime}=(1512)(0.0376)=56.8 \mathrm{~kg} / \text { day, }
\end{aligned}
$$

and

$$
R_{u}=1.030 \mathrm{E}=15.57 \mathrm{~kg} / \mathrm{day} .
$$

For solvent flow rates above, the recovery of uranium: in the metal product can be fonind as follows:

$$
\begin{aligned}
& \mathrm{K}_{\mathrm{u}}=0.015 \\
& \alpha_{\mathrm{u}}=\mathrm{EK}_{\mathrm{u}} / \mathrm{R}=(1512)(0.015) /(1557)=0.01458 \\
& \beta_{\mathrm{u}}=\mathrm{R} ! / \mathrm{EK}_{\mathrm{u}}=(56.8) /(1512)(0.015)=2.50 .
\end{aligned}
$$

From Appendix A $\left(n=3, n^{\prime}=3\right), f_{u}=0.0009$

The effect of changing parameters can be visualized on the coordinates of Fig. 3. The change of plutonium and rare-earth recoveries caused by changing solvent flow ratios can be shown by plotting additional lines of constant recovery; for example, lines for $1-f_{p}=0.990$ and $1-f_{r}=0.25$ are plotted on Fig. 3. Feed dilutions can be shown on this graph since

$$
\frac{R}{E}=\frac{\bar{R}}{E}+\frac{R^{\prime}}{E}
$$

If the distribution coefficient for a component increases, the com: ponent recovery lines shift upwards. Lines of constant plutonium recovery are plotted in Fig. 3 for $K_{p}=0.064$. If the specified recoveries are to be met, the feed solvent ratio $(\bar{R} / E)$ is lower. Since the amount of feed.solvent (metal) used in this example is fixed by solubility, larger amounts of light solvent (salt) and scrub metal must be used.

If the extraction or scrub solvents are not free of the distributing solutes, Eq. (15) or (22) must be used to calculate flow ratios. These equations appear formidable for hand calculations, but the estimation of column

*The maximum solute concentration may not necessarily occur in the feed stream, but may occur on the feed plate. If a solubility criterion is used to determine absolute solvent flow rates, this possibility should not be ignored. 6 
operating parameters can be made quickly by a few trial-and-error steps. For example, if the extracting solvent is impure, $y_{F}$ is assumed to be zero as a first approximation, and operating conditions are computed as outlined in the sample problem. When these solvent flow rates and the assumed number of plates are used, a new value of $\mathrm{Ey}_{1}^{\prime}$ is calculated from Eq. (15). If the new computed recovery. is not as required, the values of $R^{\prime}$ and/or. $E$ are adjusted and a new value of Eyilfound. If EyF is relatively small compared to $\overline{\mathrm{R}} \overline{\mathrm{x}}_{\mathrm{F}}$, the calculated recoveries will converge to the desired value in one or two trials.

In the sample problem, let the rare-earth concentration in the extracting salt be $8 \times 10^{-5} \mathrm{~kg}$ of rare earth $/ \mathrm{kg}$ of solvent salt. The amount of rare earth in the product metal is not to exceed $0.16 \mathrm{~kg} /$ day, and the same number of equilibrium stages is to be employed. First,calculate the rare-earth recovery in the extractant when the flow rates are as found above. From the table for $\mathrm{n}=3$ and $\mathrm{n}^{\prime}=3$. in Appendix $\mathrm{A}$,

$$
f_{r}\left(\alpha_{r}=1.17, \beta_{r}=0.0312, n=3, n^{i}=3\right)=0.796 .
$$

From the proper table in Appendix C,

$$
g_{r}\left(\alpha_{r}=1.17, \beta_{r}=0.0312, n=3, n^{\prime}=3\right)=0.91
$$

Equation (15) can be rearranged into the following more convenient form for the calculation of the amount of rare earth that leaves the column in the extractant:

$$
\begin{aligned}
E_{y_{1}^{\prime}} & =f_{r}\left(\bar{R} \bar{x}_{F}-\frac{R y_{F}}{K_{r}}\right)+g_{r} E_{F} \\
& =0.796\left[0.80-\frac{(1557)\left(8 \times 10^{-5}\right)}{1.2}\right]+(0.91)(1512)\left(8 \times 10^{-5}\right) \\
& =0.664 \mathrm{~kg} / \text { day. }
\end{aligned}
$$

Rare earths in metal product $=0.800+(1512)\left(8 \times 10^{-5}\right)-0.664=$ $0.257 \mathrm{~kg} /$ day.

To increase the amount of rare earth recovered in the extractant and to keep the plutonium recovery constant, a new set of flow ratios is selected by moving to the right in Fig. 3. along a line of constant plutonium recovery $\left(1-f_{p}=0.992\right)$ and the above series of calculations is repeated. Select $R^{\prime} / E=0.060$. As a consequence, $\bar{R} / E=0.46, R / E=0.40, \alpha_{r}=$ 2.61 , and $\beta_{r}=0.050$. From the table for $n=3$ and $n^{\prime}=3$ in Appendix $A$,

$$
f_{r}(2.61,0.050,3,3)=0.962 \text {. }
$$


From the proper table in Appendix $C$

$$
g_{r}(2.61,0.050 ; 3,3)=0.998
$$

Then $E_{y}^{\prime}$ is found to be $0.791 \mathrm{~kg} /$ day. The amount of rare earth in the product metal is $0.130 \mathrm{~kg} /$ day. One more trial at $R^{\prime} / E=0.050$ and $R / E=$ 0.645 yields a rare-earth content of the product metal of 0.158 , which meets the specification reasonably well. The new operating flow rates are

$$
\begin{aligned}
& \bar{R}=1500 \mathrm{~kg} / \mathrm{day}, \\
& E=2520 \mathrm{~kg} / \mathrm{day}, \\
& \mathrm{R}=1626 \mathrm{~kg} / \mathrm{day},
\end{aligned}
$$

and

$$
R^{\prime}=126 \mathrm{~kg} / \text { day. }
$$




\section{APPENDIXES}

Because of limitations in the format of the computer print-out (only upper-case letters are available), the nomenclature used in these appendixes differs somewhat from that used in the body of this report. The differences are as follows:

$\begin{array}{cc}\begin{array}{c}\text { Nomenclature in } \\ \text { Body of Report }\end{array} & \frac{\begin{array}{c}\text { Nomenclature in } \\ \text { Tables }\end{array}}{\mathrm{F} f(\alpha)} \mathrm{N} \text { or } \mathrm{F}(\mathrm{A}) \\ \mathrm{n} & \mathrm{NP} \\ \mathrm{n} & \mathrm{G}\end{array}$

In Appendixes $A$ and $C$, values of beta, listed in the left-hand vertical column, are given as an integer and a power of ten; e.g.,

$$
1.0-003=1.0 \times 10^{-3} \text {. }
$$


APPENDIX A :.. !

Parameter $f$ as a Function of Extraction Factor $\alpha$, Scrub Factor $\beta$, and Numbers of Stages $n$. and $n^{\prime}$ 


\begin{abstract}
VALUES OF F FOH N $=1$ APSO NPE 1
BETA

$\begin{array}{llllllllllllll}1.0-003 & 0.0010 & 0.0020 & 0.0130 & 0.0050 & 0.0069 & 0.0009 & 0.0196 & 0.0291 & 0.0476 & 0.0654 & 0.0908 \quad 0.1665 & 0.2306 \quad 0,3331 & 0.4115\end{array}$

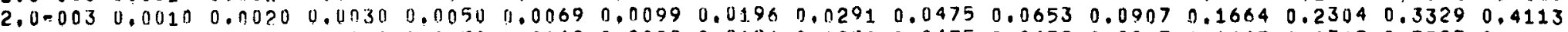

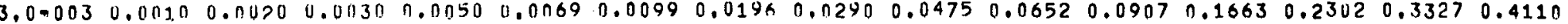

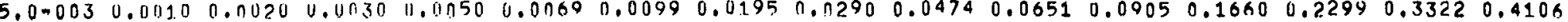

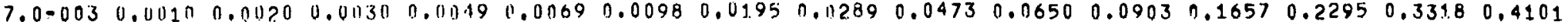
$1,0-0020.00140 .00200 .0130 \quad 0.00490 .00690 .0098 \quad 0.01940 .02880 .04720 .06480 .09010 .16530 .2290 \quad 0.33110 .4094$

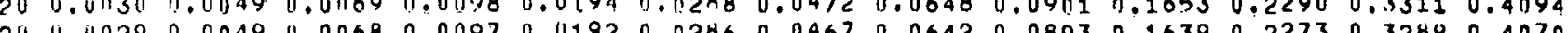

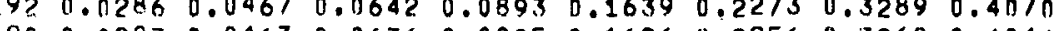

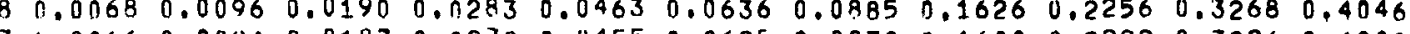

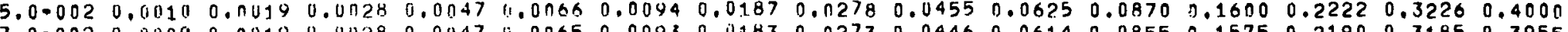

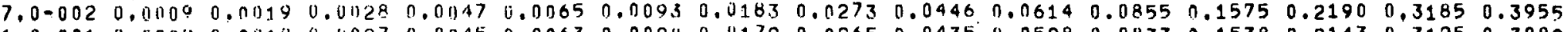

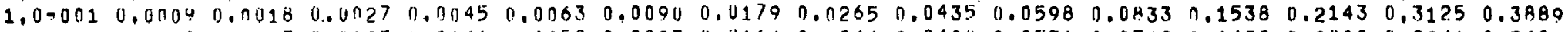

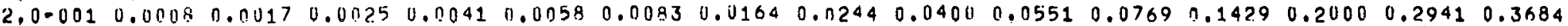
$3.0=0010.00090 .0015 \quad 0.0 n 23 \quad 0.0038 \quad 0.0054 \quad 0.0076 \quad 0.015 ? \quad 0.02260 .03700 .05110 .07140 .13330 .18750 .2778 \quad 0.3500$ $5.0=0010.010070 .00130 .0020 \quad 0.0033 \quad 0.0046 \quad 0.0066 \quad 0.0132 \quad 0.0196 \quad 0.03230 .0446 \quad 0.06250 .1176 \quad 0.1667 \quad 0.2500 \quad 0.3182$ $7.0=0010.00060 .00120 .0018 \quad 0.00390 .0041 \quad 0.0058 \quad 0.0116 \quad 0.0173 \quad 0.0286 \quad 0.03950 .0556 \quad 0.1 .053 \quad 0.1500 \quad 0.2273 \quad 0.2917$ $1.0+000 \quad 0.00050 .00100 .0 n 150.0025 \quad 0.00350 .0050 \quad 0.00990 .01480 .02440 .03380 .04760 .0909 \quad 0.1304 \quad 0.2000 \quad 0.2593$

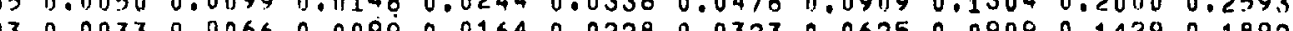

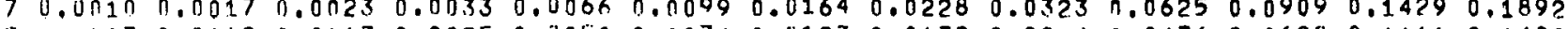
$3.0+0000.0 n 12.0 .01550 .00070 .0 n 120.00170 .00250 .005 n 0.00740 .01230 .01720 .02440 .04760 .06980 .11110 .1489$ $5.0+000 \quad 0.00080 .0030 .00050 .00080 .00120 .00170 .0 n 330.00500 .00830 .01150 .01640 .03230 .04760 .07690 .1045$ $7.0+000 \quad 0.00010 .0002 \quad 0.013040 .00060 .00090 .00120 .00250 .00370 .00620 .00870 .01230 .02440 .03610 .05880 .0805$

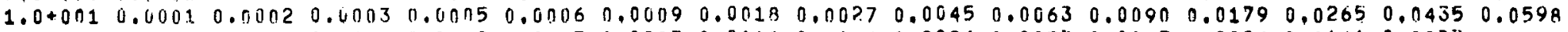
$2.0+001$ v.000n 0.013010 .00010 .001120 .00030 .00050 .00100 .00140 .00240 .00330 .00470 .00940 .01410 .0233

$3,0+001$ u.0nol $0.00010 .0 n 010.001120 .00020 .00030 .00060 .00100 .00160 .00230 .0 n 320.00640 .0096$
\end{abstract}

$5.0+0010.000010 .0000 \quad 10.010010 .001110 .00010 .00020 .00040 .00960 .00100 .00140 .07200 .0039$

$7,0+0010.00000 .00000 .00000 .0001 .0 .00010 .00010 .00030 .00340 .00070 .00100 .0014$

$1.0+0020.000010 .0100 \quad 0.00000 .00000 .0010 .00010 .00020 .001330 .00050 .00070 .0010$

$2.0+002 \quad 0.0000: 0.0000$ U. 0n00 $0.0000 \quad 0.0000 \quad 0.0000 \quad 0.00010 .00310 .0002$

$3.0+0020.0000 .0 .0000 \quad 0.011000 .00000 .001000 .00000 .00010 .0001$

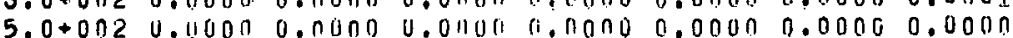

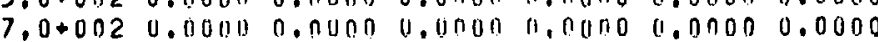

$1.0+0030.0000 .00000 .00000 .0000 \quad 0.00000 .0000$

ALPHA $1.000 \quad 2.000 \quad 3,000 \quad 5,000 \quad 7.000 \quad 10.00 \quad 20.00 \quad 30.00 \quad 50.00 \quad 70,00 \quad 100.0 \quad 200.0 \quad 300,0 \quad 500.0 \quad 700.0 \quad 1000$ BETA

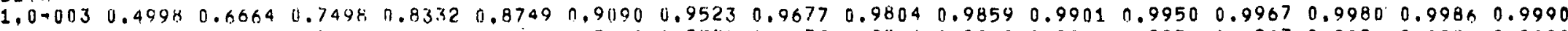

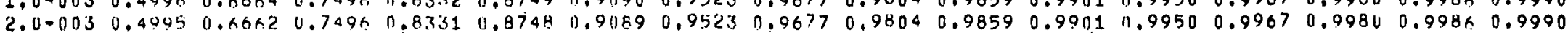
$3.0-0030.49930 .6660 \quad 4.74940 .8320 .87470 .90880 .95220 .96760 .98030 .98590 .99010 .99500 .99670 .99800 .99860 .9990$

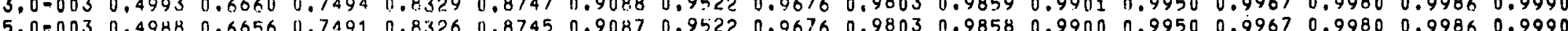

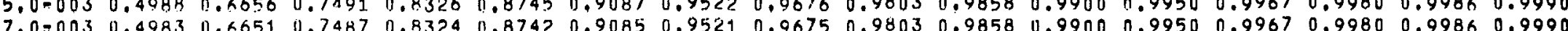

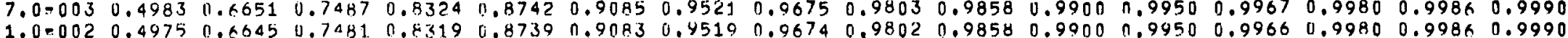

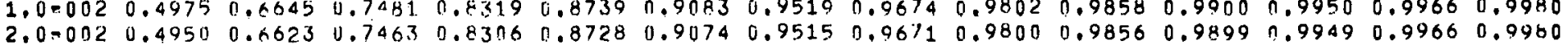
$\begin{array}{llllllllllllllll}3.0=002 & 0.4926 & 0.6601 & 0.7444 & 0.8292 & 0.8717 & 0.9066 & 0.9510 & 0.9668 & 0.9798 & 0.9855 .0 .9898 & 0.9949 & 0.9966\end{array}$

$\begin{array}{lllllllllllllllllll}5.0-002 & 0.4874 & 0.6557 & 0.7407 & 0.8264 & 0.8696 & 0.9050 & 0.9501 & 0.9662 & 0.9794 & 0.9852 & 0.9896 \quad 0.9948\end{array}$

$\begin{array}{llllllllllllllll}7.0=002 & 0.4831 & 0.6515 & 0.7371 & 0.8237 & 0.8674 & 0.9033 & 0.949 ? & 0.9656 & 0.9790 & 0.9849 & 0.9894\end{array}$

$1.0=001 \quad 0.4762 \quad 0.6452 \quad 0.7317 \quad 0.8197 \quad 0.8642 \quad 0.90090 .94790 .9646 \quad 0.97850 .9845 \quad 0.9891$

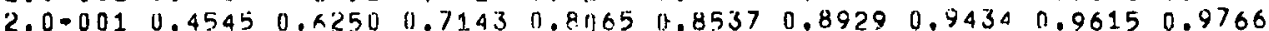

$3.0-0010.4344 \quad 0.6061 \quad 11.0977 \quad 0.7937 \quad 0.8434 \quad 0.8850 \quad 0.9390 \quad 0.0555$

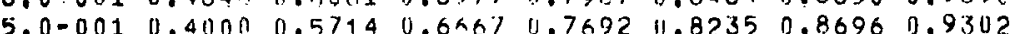

7.0 .001
0.3704 $0.54050 .63830 .7463 \quad 0.8046 \quad 0.8547$

$1.0+000 \quad 0.3333 \quad 0.5000 \quad 0.01000 .7143 \quad 0.7778 \quad 0.8333$

$2.0+000 \quad 0.25010 .41100 \quad 0.50110 \quad 0.6250$

$3.0+000 \quad 0.200 ! 10.3333 \quad 4.4286$

$5.0+000 \quad 0.1429 \quad 0.2500$

$7.0+000 \quad 0.1111$

$1.0+0010.0833$ 
VALUES OF F FUN ALPHA BETA

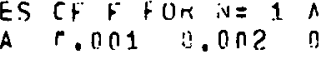

$1.0=003 \quad 0.0010 \quad 0.0020 \quad 0.003 n \quad 0.0050 \quad 0.00690 .0999 \quad 0.0194 \quad 0.02910 .04760 .06540 .09080 .016650 .2306 \quad 0.33310 .4115$ 2.0-003 0.001n 0.0020 0.003110 .0050 .00690 .01790 .01960 .02910 .04750 .06530 .09070 .16640 .23040 .33290 .4113

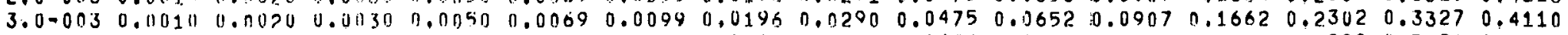

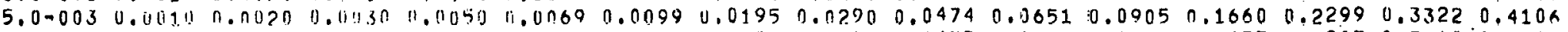

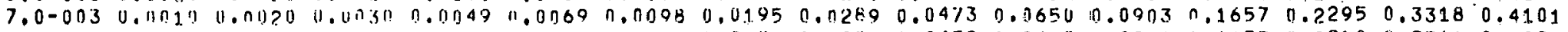

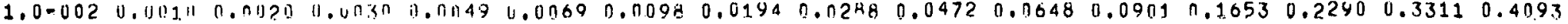

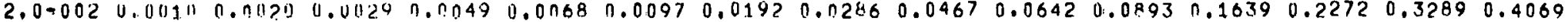

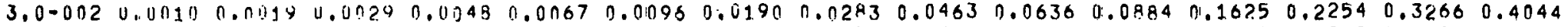

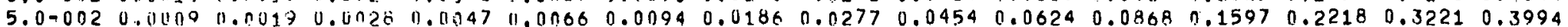
7.0-002 0.0114 U.0119 11.4n28 $0.0046 \quad 0.0650 .00920 .01830 .02720 .04440 .06110 .08510 .15690 .21820 .31750 .3944$

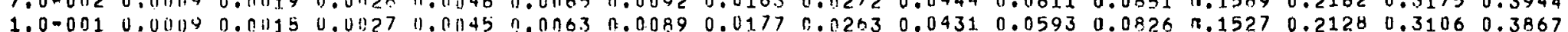

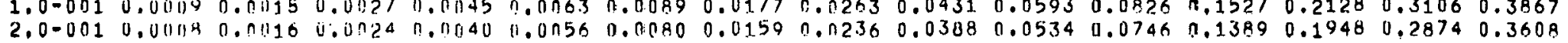

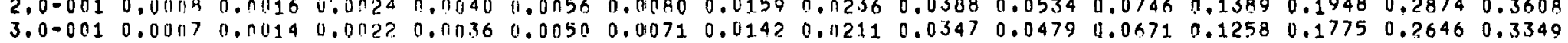
5.0-001 $0.0006 \quad 0.0011 \quad 1.00170 .0028 \quad 0.0040 \quad 0.0057 \quad 0.01130 .01690 .02780 .0385 \quad 1.0541 \quad 0.1026 \quad 0.1463 \quad 0.2222 \quad 0.2857$

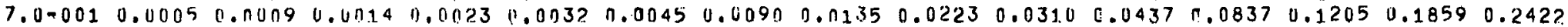

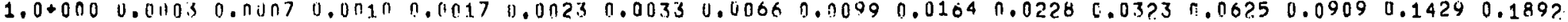

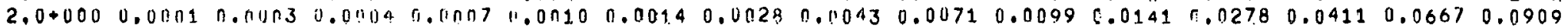

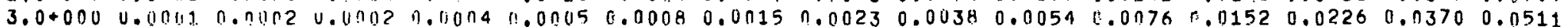
$5.0+0000.00010 .01010 .00010 .00 n 20.00020 .00030 .00060 .00100 .00160 .00230 .0032000340 .00960 .01590 .0221$ $7.0+000$ 0.000

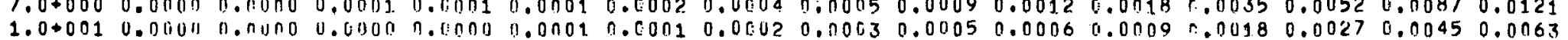
$2.0+0010.00000 .00 n 0$ u. $10040.0 n 0018.00000 .00000 .00000 .00010 .0001000020 .00020 .00050 .00070 .0012$

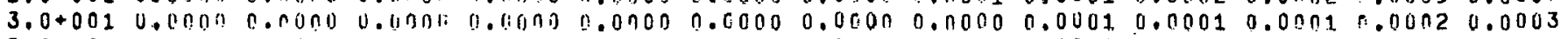

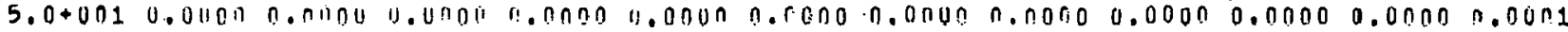

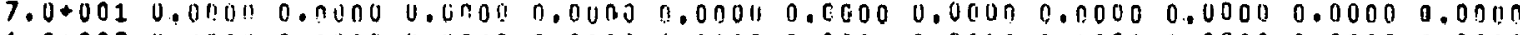

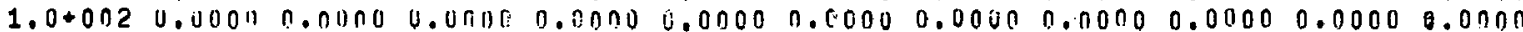

$2.0+0020.0000$ o. 0uno $0.4000,0.00000 .00000 .00000 .00000 .00000 .0000$

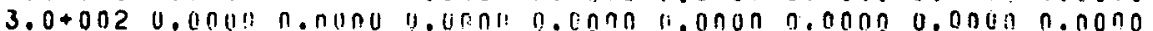

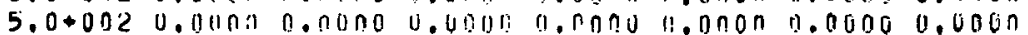

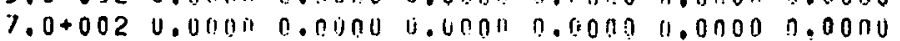

$1.0+0030.00000 .01300$ 0.0non 0.000010 .00000 .0000 BETA

ALPHA $1.000 \quad 2.000 \quad 3.000 \quad 5.000 \quad 7.000 \quad 10.00 \quad 20.00 \quad 30.00 \quad 50.00 \quad 70.00 \quad 100.0 \quad 200.0 \quad 300.0 \quad 500.0 \quad 700.0 \quad 1000$

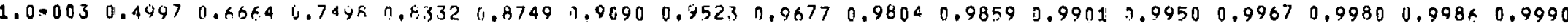

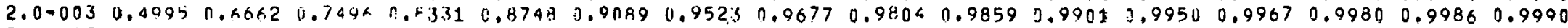

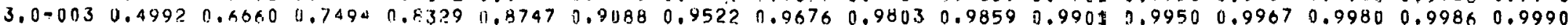

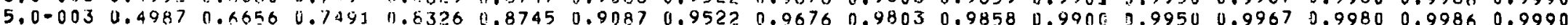

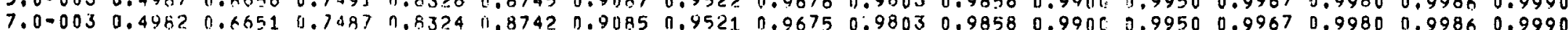

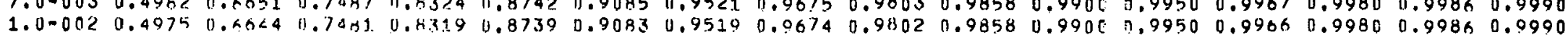
$2.0-0020.49500 .00220 .74670 .43950 .87280 .90740 .95150 .96710 .98000 .98560 .99960 .99490 .9960 \quad 0.9980$

$3.0-0020.492411 .4509$ 0.7143 0.42910 .87160 .90650 .95100 .96680 .97980 .98550 .98980 .99490 .9906

$5.0 .0020 .4872 .0 .65520 .14030 .8261 \quad 0.86930 .70480 .950100 .96610 .97940 .98520 .98960 .9948$

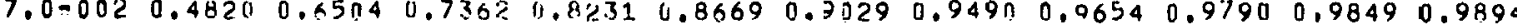

$\begin{array}{llllllllllllllll}1.00001 & 1.4739 & 0.6431 & 0.7299 & 0.8193 & 0.8631 & 0.7001 & 0.9474 & 0.9643 & 0.9783 & 0.9844 & 0.9890\end{array}$

$2.0 .001 \quad 0.4464 \quad 0.0173 \quad 1.797 \% 0.801 .3 \quad 0.8495 \quad 0.3397 \quad 0.94160 .96030 .9758$

$3.0=001 \quad 0.4164 \quad 0.5900 \quad 11.0334 \quad 0.7325 \quad 11.8343 \quad 10.3780 \quad 0.9350 \quad 0.9557$

$5,0-001 \quad 0.3636 \quad 0.5333 \quad 0.03160 .741570 .801000 .3511 \quad 1.9195$

$7,0=001 \quad[.3135 \quad 0.4773 \quad 0.57610 .0954 \quad 0.76170 .3203$

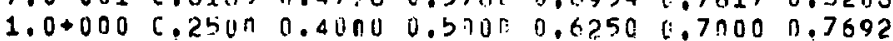

$2.0+000 \quad 0.1250 \quad 0 . ? 2220.30000 .41 .57$

$3.0+000 \quad 1.07140 .1333 \quad 0.1275$

$5,0+000 \quad 5.0313 \quad 1.0606$

$7,0+000 \quad 6.0172$

$1,0+001 \quad 0,0089$ 


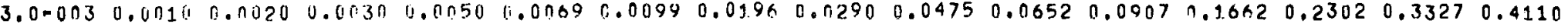

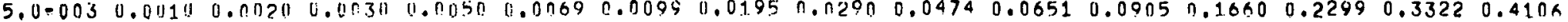

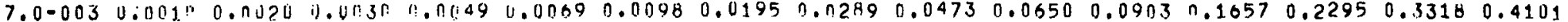

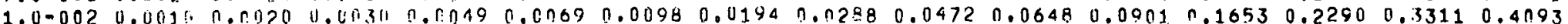
$1.0-002$ 0.0016

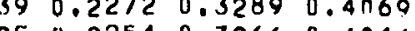
1.

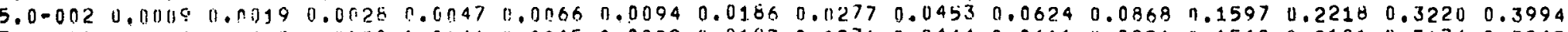

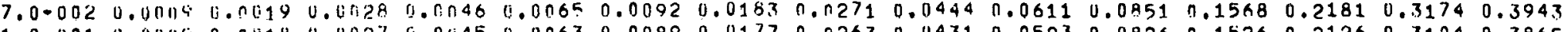

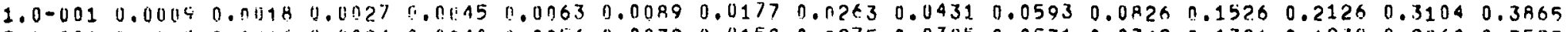

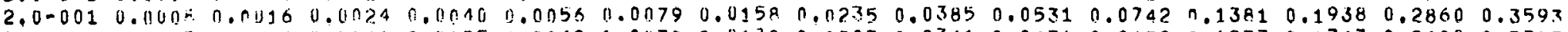

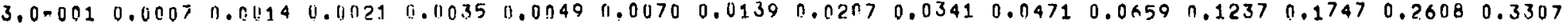

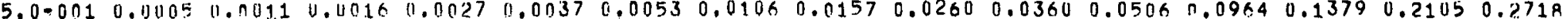

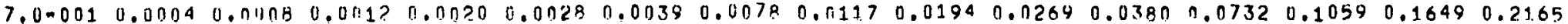

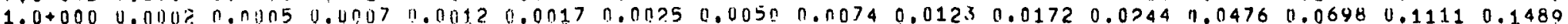

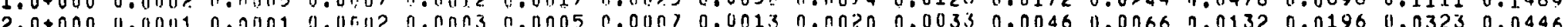
.

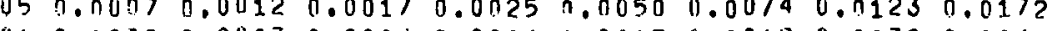

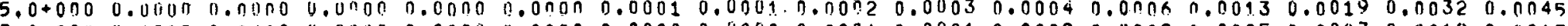
$7.0+0000.000 n$ n. $10000.0 n \pi$. $1.0+001 \quad 0.000 ! 0.00000 .00000 .00000 .00000 .00000 .00000 .00000 .00000 .00010 .00010 .00020 .00030 .001040 .0006$

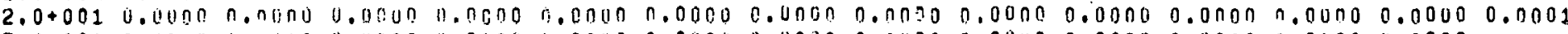

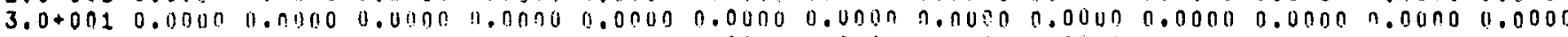

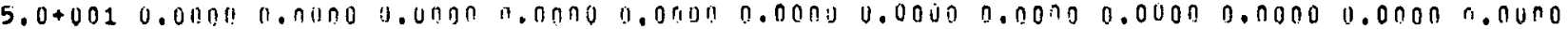

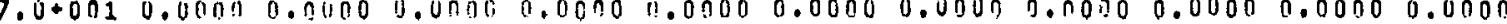

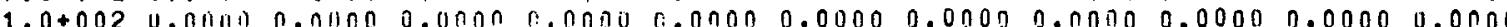

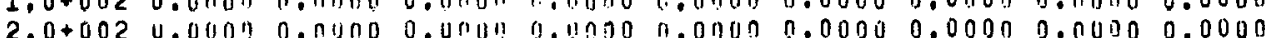

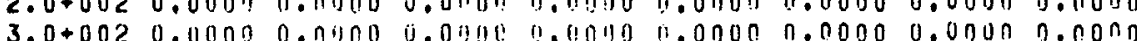

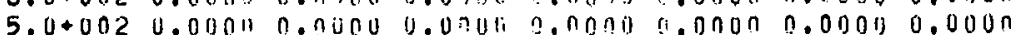

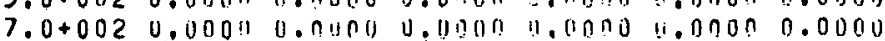

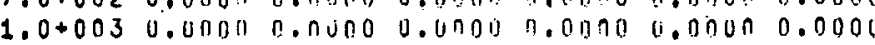

ALPHA $1.000 \quad 2.0003 .005$ 5.000 7.000 $10.00 \quad 20.00 \quad 30.00 \quad 50.00 \quad 70.00 \quad 1.00 .0 \quad 200.0 \quad 300.0 \quad 500.0 \quad 700.0 \quad 1000$ BETA

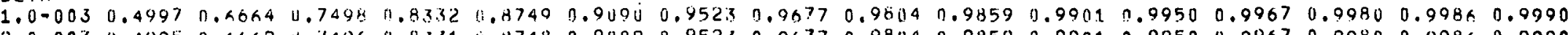

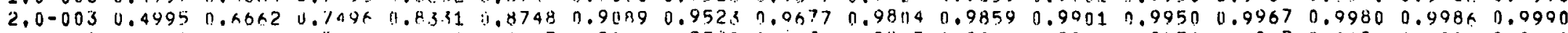
$3.0-0030.49920 .66600 .74940 .83290 .87470 .90880 .95220 .06760 .98030 .98590 .99010 .99500 .99670 .99800 .09840 .9990$

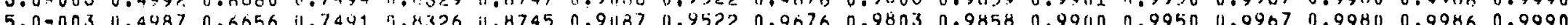
$9.0-0030.49870 .5656$ 0.7491.9.4325

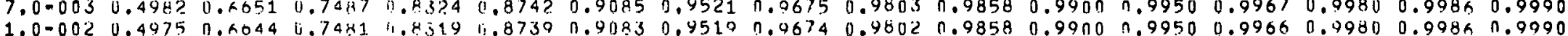

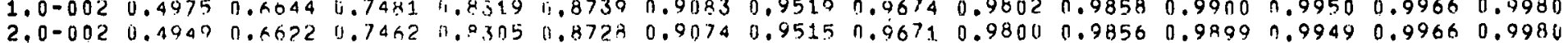

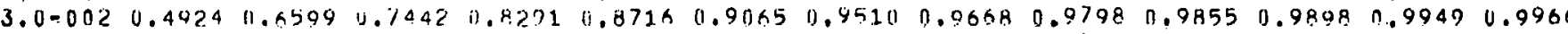

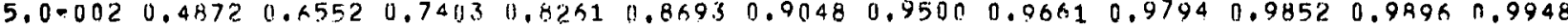

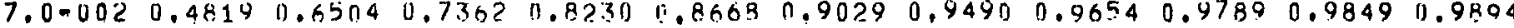

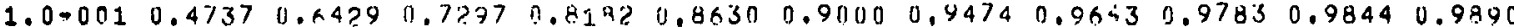

2.0.001 $0.4448 \quad 0.0158 \quad 0.7062 \quad 0.4073 \quad 0.8487 \quad 0.8890 \quad 0.9413 \quad 0.9601 \quad 0.9756$

$3.0-0010.4137 \quad 0.5553 \quad 0.079 ? \quad 0.7792 \quad 11.8317 \quad 0.87590 .9338 \quad 0.0549$

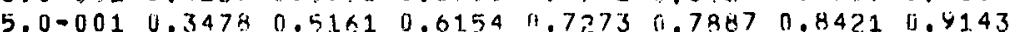

$\begin{array}{llllllll}7.0-001 & 0.2830 & 0.4412 & 0.5422 & 0.6637 & 0.7343 & 0.7979\end{array}$

$1.0+000 \quad 0.2000 \quad 0.33330 .4286 \quad 0.5556 \quad 0.6364 \quad 0.7143$

$2.0+000 \quad 0.0625 \quad 0.1176 \quad 0.1667 \quad 0.25110$

$3.0+000 \quad 0.02440 .0476$ U. 1698

$5.0+000 \quad 0.0164 \quad 0.0127$

$7.0+000 \quad 0.01025$

$1.0+0010.00019$ 


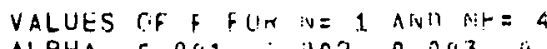
AFTA

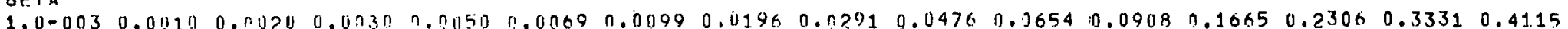

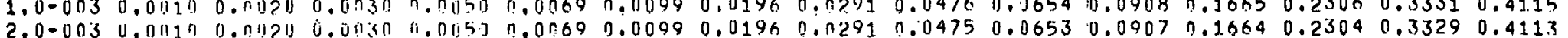

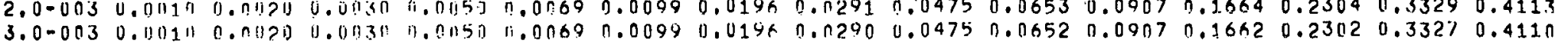

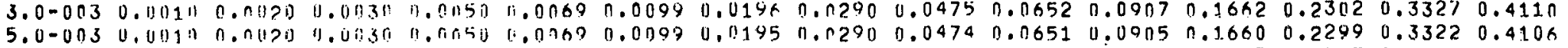

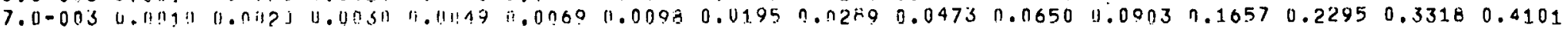

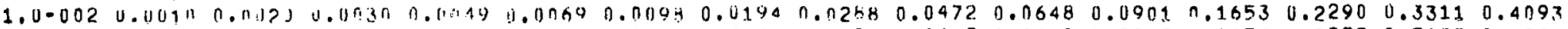

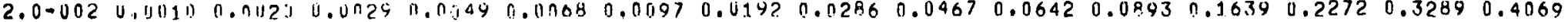

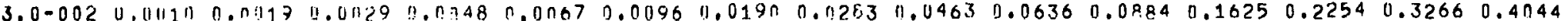
$5,0-0020.110090 .00174 .0411 .10470 .00650 .00940 .01860 .02770 .04530 .06240 .08680 .15970 .22980 .32200 .3994$ 5.0-002 v.

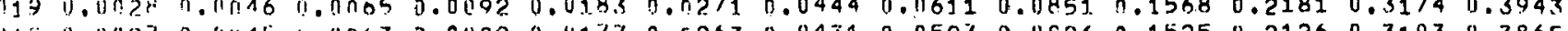
(1)

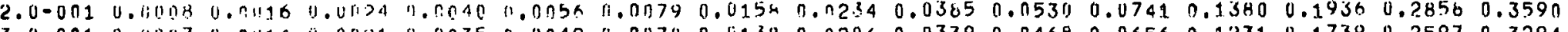

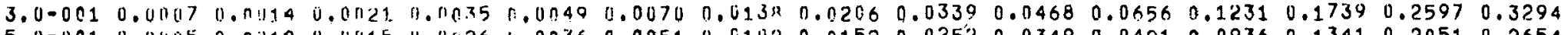
5.0-001 0.0uns n.0110 0.0115 J.1126 1.0036 0.0051 0.010? 0.01520 .02520 .03490 .04910 .09360 .13410 .20510 .2654

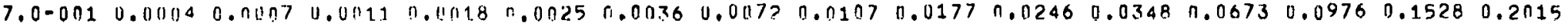

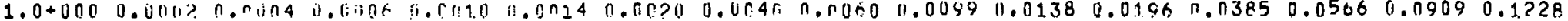

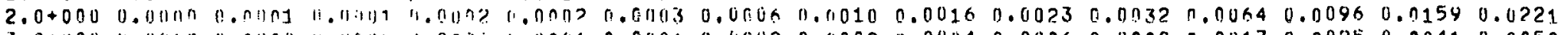

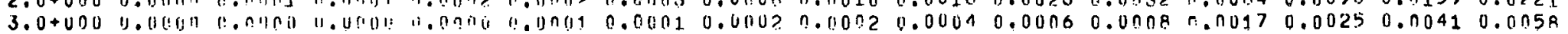

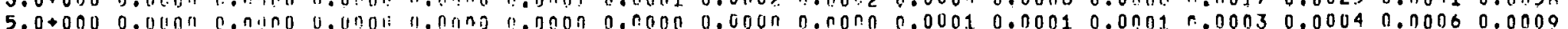

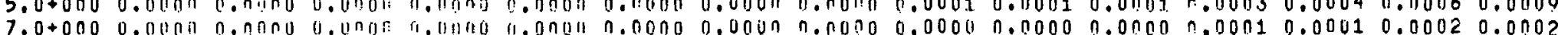

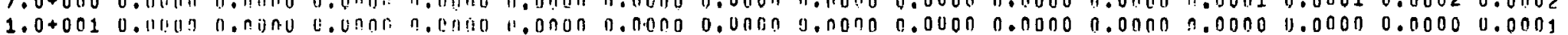

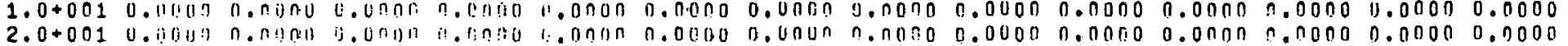

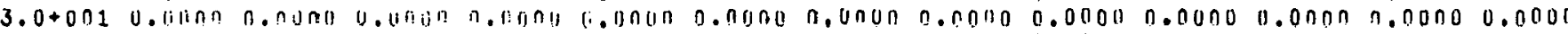

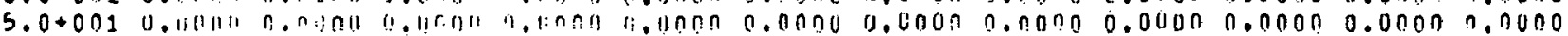

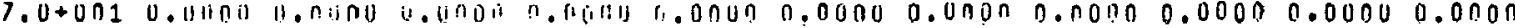

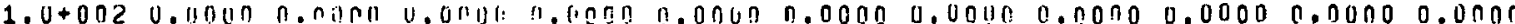

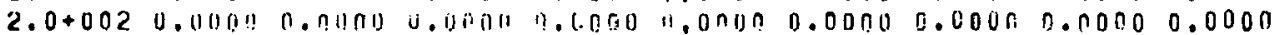

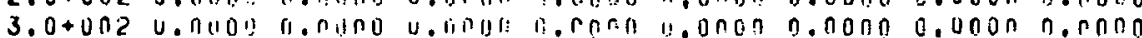

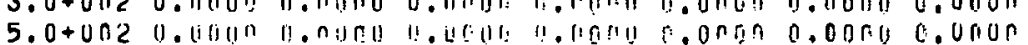

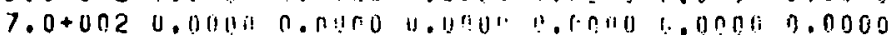

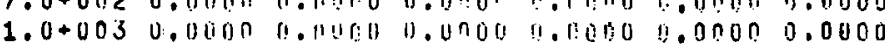

ALPH

BETA

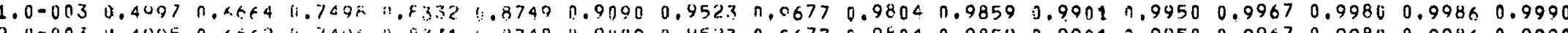

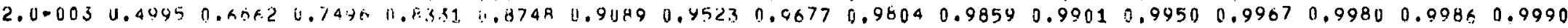

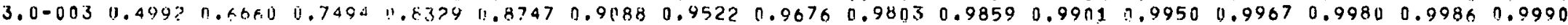

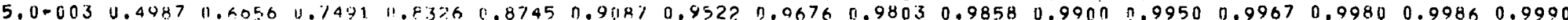

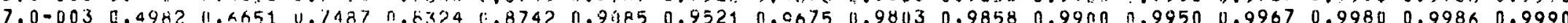

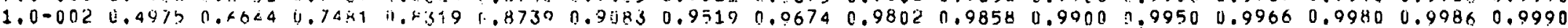

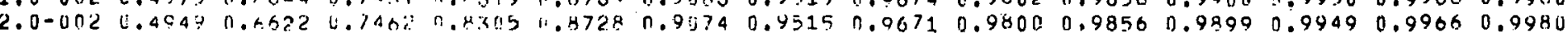

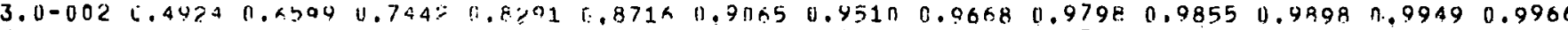

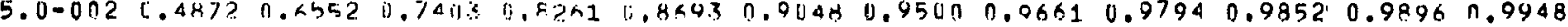

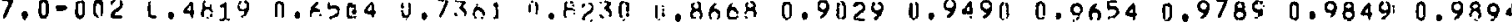

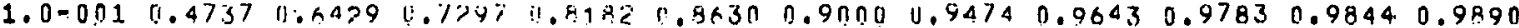

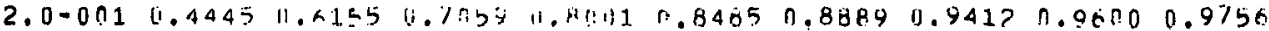

$3.0-001 \quad 0.4124 \quad 0.5879$ 11.0/4: $11.7 \% 420.8309 \quad 0.87530 .93350 .0547$

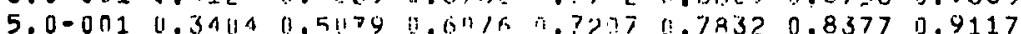

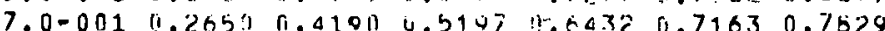

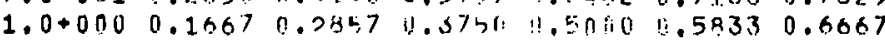

$2.0+0000.0313$ 1..1!b36 0.04de 1.9339

$3.0+0000.408$ ? $11.01 \% 3$ ij. 1.24?

$5.0+000 \quad 0.0101 \mathrm{~s} \mathrm{fi.n11?6}$

$7.0+000 \quad 0.001004$

$1.0+0010.000$ 


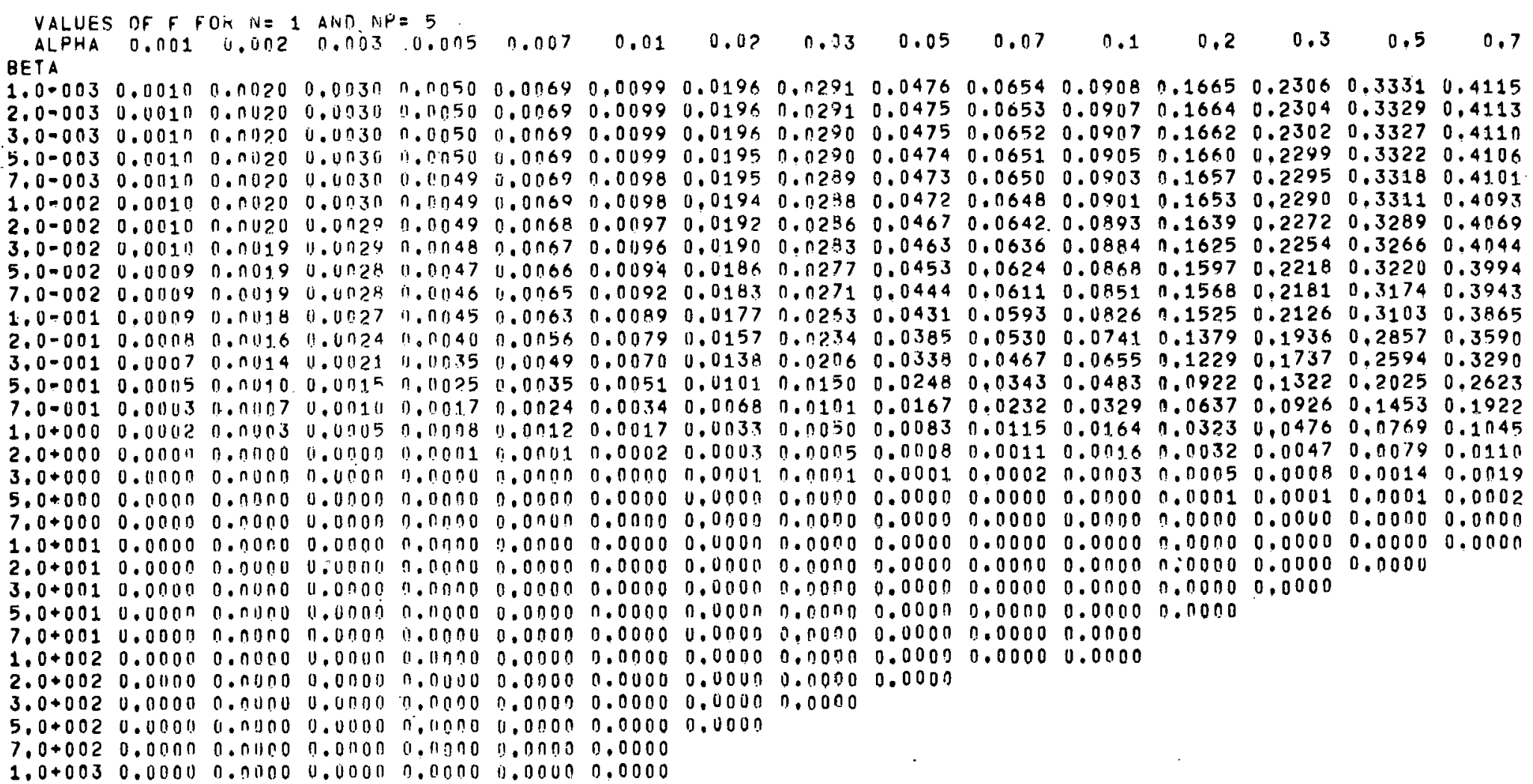


VALUES OF F FOR $N=2$ ANIS NP= 1

ALPHA 0.001 H. 002 0.003 U.0

BETA

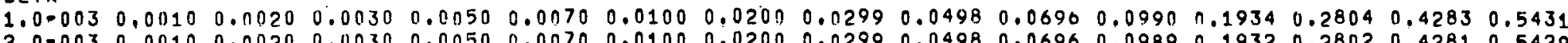
$2,0=0030.0010 \quad 0.00200 .0030 \quad 0.0050 \quad 0.0070 \quad 0.0100 \quad 0.0200 \quad 0.02990 .04980 .0696 \quad 0.09890 .1932 \quad 0.28020 .4281 \quad 0.5429$

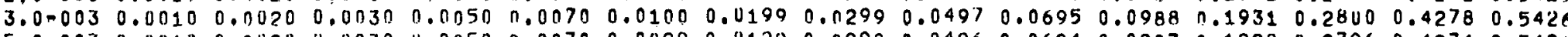

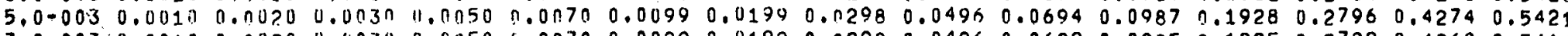
$7,0=0030.00100 .01200 .00300 .0350 \quad 0.0070 \quad 0.00990 .01990 .02980 .04960 .06920 .09850 .19250 .27920 .42690 .5414$ $\begin{array}{lllllllllllllllll}1,0-002 & 0.0010 & 0.0020 & 0.11030 & 0.0050 & 0.0069 & 0.0099 & 0.0198 & 0.0297 & 0.0494 & 0.0690 & 0.0982 & 0.1920 & 0.2786 & 0.4261 & 0.5409\end{array}$

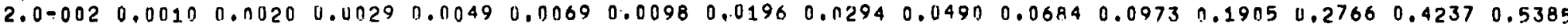
$3.0-0020.00100 .00190 .00290 .00490 .00680 .00970 .01940 .02910 .04850 .06780 .09650 .18900 .27460 .42130 .5360$

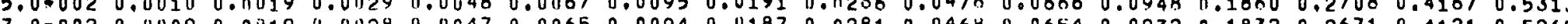

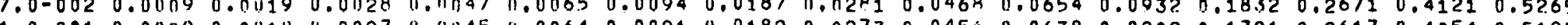

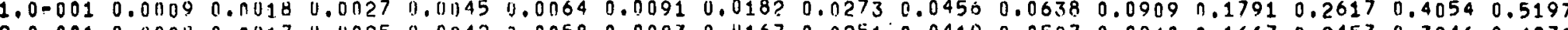
2,0-001 $0.0004 \quad 0.0017 \quad 1.0025 \quad 0.0042 \quad 0.0058 \quad 0.0083 \quad 0.0167 \quad 0.02510 .04190 .0587 \quad 0.0840 \quad 0.1647 \quad 0.2453 \quad 0.3846 \quad 0.4979$

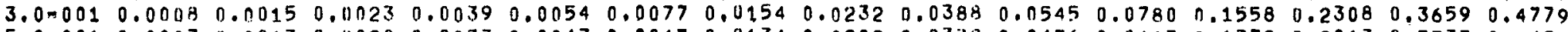
$5.09001 \quad 0.0007 \quad 0.00130 .0020 \quad 0.0033 \quad 0.0047 \quad 0.00670 .01340 .02020 .03380 .04760 .06830 .13790 .2063 \quad 0.3333 \quad 0.4424$ $7.0=001 \quad 0.0006 \quad 0.0012 \quad 0.0018 \quad 0.00290 .0041 \quad 0.00590 .01190 .01790 .03000 .0422 \quad 0.04080 .1237 \quad 0.1866 \quad 0.3061 \quad 0.4118$

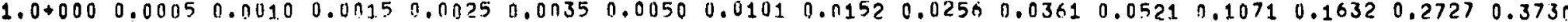
$2.0+000 \quad 0.0003 \quad 0.0007 \quad 0.00100 .00170 .00230 .00340 .00680 .01020 .01720 .02440 .03540 .07410 .1150 \quad 0.20000 .2840$

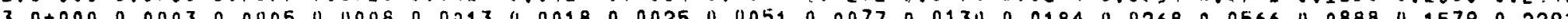
$3.0+0000.00020 .00030 .00050 .00080 .00180 .00170 .00340 .00510 .00070 .01230 .01800 .03850 .06100 .11110 .2295$

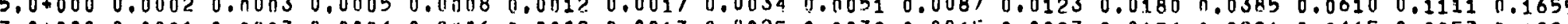

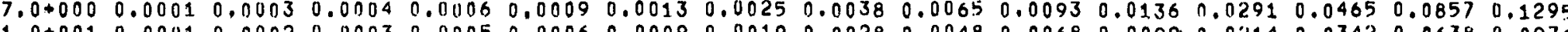

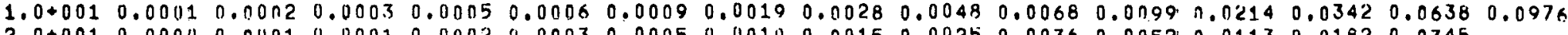
$2.0+0010.00000 .00010 .00010 .00020 .0003 \quad 0.00050 .001) 0.00150 .00250 .00360 .0052 \quad 0.01130 .01820 .0345$ $3.0+0010.00000 .00010 .00010 .0002 \quad 1.00020 .00030 .00070 .00100 .00170 .00240 .00350 .00770 .0124$

$5.0+0010.00000 .00000 .00010 .00010 .00010 .00020 .00040 .00060 .00100 .00150 .00220 .0047$

$7.0+0010.00000 .00000 .00000 .00010 .00010 .00010 .00030 .00040 .00070 .00110 .0015$

$1.0+0020.00000 .00000 .00000 .00000 .00010 .00010 .000 ? 0.00030 .00050 .00070 .0011$

$2.0+002 \quad 0.0000 \quad 0.0000 \quad 0.0000 \quad 0.0000 \quad 0.0000 \quad 0.00010 .0001 \quad 0.00320 .0003$

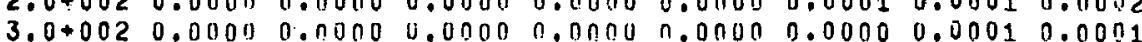

$5.0+0020.00000 .00000 .0000,1.00000 .00000 .00000 .0000$

$7.0+0020.00000 .0000 \quad 0.00090 .0000 \quad 10.00000,0000$

$1.0+0030.00000 .00000 .00000 .0000 \quad 0.00000 .0000$ BETA

ALPHA $1.000 \quad 2.000 \quad 3.000 \quad 5.000 \quad 7.000 \quad 10.00 \quad 20.00 \quad 30.00 \quad 50.00 \quad 70.00 \quad 100.0 \quad 200.0 \quad 300.0 \quad 500.0 \quad 700.0 \quad 1000$

$1.0-0030.66640 .8570 \quad 0.9230 \quad 0.9677 \quad 0.98240 .9910 \quad 0.9976 \quad 0.99890 .9996 \quad 0.99980 .99991 .00001 .00001 .00001 .00001 .0000$ $2.0=003 \quad 0.666 ? 0.95690 .92290 .9677 \quad 0.9824 \quad 0.9910 \quad 0.9976 \quad 0.99890 .9996 \quad 0.99980 .99991 .00001 .00001 .00001 .00001 .00 n 0$

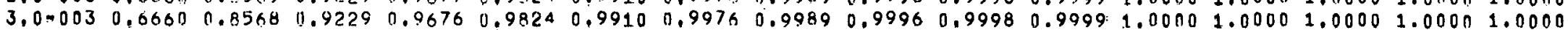

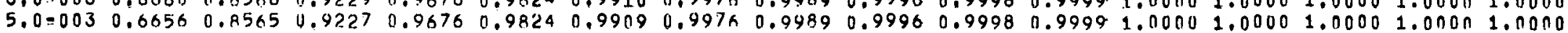

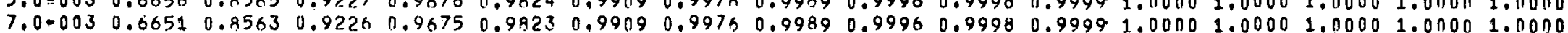

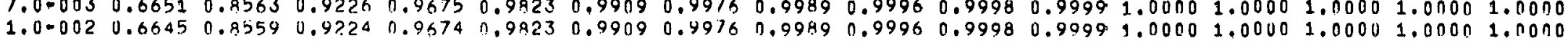

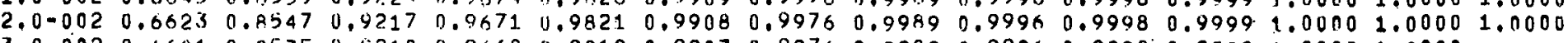
$3.0-0020.6601 \quad 0.8535 \quad 10.92100 .06680 .98190 .99070 .99760 .99890 .99960 .9998 .0 .99991 .00001 .0000$

$5.0=002 \quad 0.65570 .8511 \quad 0.91 .95 \quad 0.96620 .9816 \quad 0.99050 .99750 .99890 .9990 \quad 0.99980 .99991 .0000$

$\begin{array}{llllllllllllllll}7.0-002 & 0.6515 & 0.8487 & 0.9181 & 0.9556 & 0.9813 & 0.9904 & 0.9975 & 0.9989 & 0.9990 & 0.9998 & 0.9999 .\end{array}$

$\begin{array}{llllllllllllll}1.0-001 & 0.6452 & 0.8451 & 0.9160 & 0.9646 & 0.9807 & 0.9901 & 0.9974 & 0.9988 & 0.9996 & 0.9998 & 0.9999\end{array}$

$\begin{array}{llllllllllll}2.0-001 & 0.6251] & 0.8333 & 0.4091 & 0.0615 & 0.9790 & 0.9892 & 0.997 ? & 0.0987 & 0.9995\end{array}$

$\begin{array}{llllllllll}3.0 .001 & 0.0061 & 0.4219 & 0.91023 & 0.9585 & 0.9773 & 0.9883 & 0.9969 & 0.9986\end{array}$

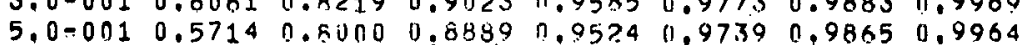

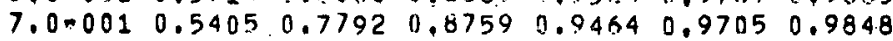

$1.0+000 \quad 0.5000 \quad 0.7500 \quad 0.8571 \quad 0.937 .50 .96595 \quad 0.9821$

$2.0+000 \quad 0.4000 \quad 0.4667 \quad 0.8000 \quad 0.9091$

$3.0+000 \quad 0.33330 .6000 \quad 0.7500$

$5,0+000 \quad 0.25000 .5000$

$7.0+000 \quad 0.2000$

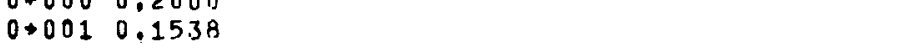

$1.0 \rightarrow 0010.1538$ 


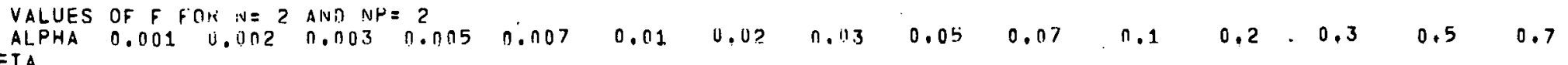

BETA

$1.0=003 \quad 0.0010 \quad 0.0020 \quad 0.0030 \quad 0.0050 \quad 0.0070 \quad 0.0100 \quad 0.0200 \quad 0.02990 .0498 \quad 0.0696 \quad 0.0990 \quad 0.19340 .2804 \quad 0.4283 \quad 0.5431$ 2.0-003 $0.00100 .0020 \quad 0.0030 \quad 0.0050 \quad 0.0070 \quad 0.0100 \quad 0.0200 \quad 0.02990 .04980 .06960 .09890 .19320 .28020 .42810 .5429$

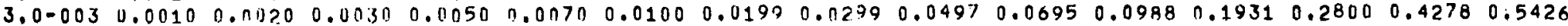
$\begin{array}{llllllllllllllll}5.0=003 & 0.0010 & 0.01120 & 0.0030 & 0.0050 & 0.007 n & 0.0099 & 0.0199 & 0.0298 & 0.0496 & 0.0694 & 0.0987 & 0.1928 & 0.2796 & 0.4273 & 0.5421\end{array}$ $7.0-0.30 .00100 .00200 .0030 \quad 0.0050 \quad 0.0070 \quad 0.00990 .0190 \quad 0.02980 .04950 .06920 .09850 .1925 \quad 11.2792 \quad 0.4269 \quad 0.5416$ $1.0-0020.00190 .011000 .00300 .00500 .00690 .00990 .01790 .02970 .04940 .06900 .09820 .19200 .27860 .42610 .5409$ 0.09730 .19040 .27650 .42360 .5384 $2.0-0020.00100 .00200 .01290 .14490 .01690 .00980 .01960 .02940 .04890 .068$

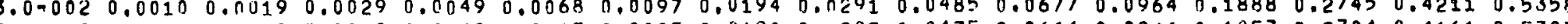

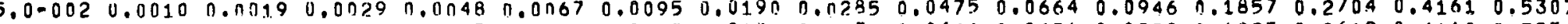

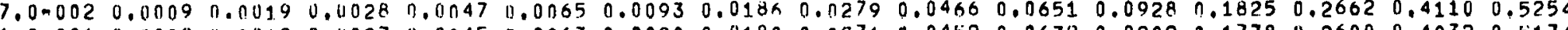

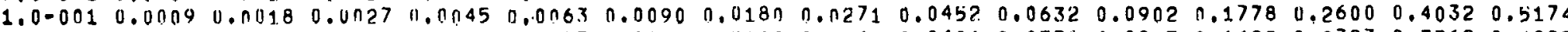

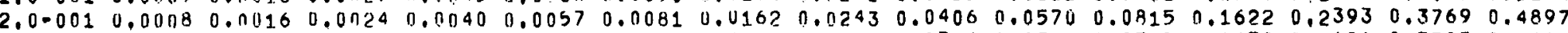
$3.0-001 \quad 0.0007 \quad 0.00140 .0022 \quad 0.0036 \quad 0.0050 \quad 0.0072 \quad 0.0145 \quad 0.02170 .03640 .05110 .0733 \quad 0.1472 \quad 0.21910 .3505 \quad 0.4612$ $5.0-0010.0006 \quad 0.00110 .0 n 17 \quad 0.00290 .0040 \quad 0.0057 \quad 0.01150 .01740 .02910 .04100 .05910 .1206 \quad 0.18220 .3000 \quad 0.4048$ $7.0 .0010 .00050 .00090 .00140 .00230 .0032 \quad 0.00460 .0092 \quad 0.01390 .02340 .03310 .04780 .09880 .1512 \quad 0.25510 .3521$ $1.0+0000.00030 .00070 .0010 .00170 .00230 .00340 .40680 .01020 .01720 .02440 .03540 .07410 .11500 .20000 .2840$

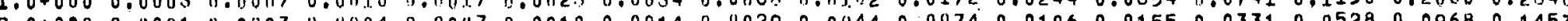
.

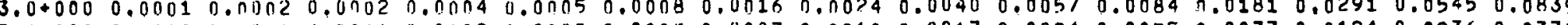

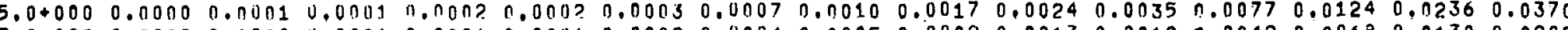
$7.0+000 \quad 0.0000 \quad 0.00000 .00010 .00 n 10.00010 .00020 .00040 .00050 .00090 .00130 .00190 .00420 .00680 .01300 .0205$ $1.0+0010.0000 \quad 0.00000 .011000 .00000 .00010 .00010 .00020 .00130 .00050 .00070 .00100 .00220 .00350 .00670 .0106$ $2.0+0010.00000 .00000 .0000 \quad 1.00 n 00.00000 .00000 .000010 .0 n 010.00010 .00020 .00030 .00060 .00090 .0018$ $3.0+0010.000 n \pi .00000 .0 n 000.00000 .00000 .00000 .000 n 10.00000 .00010 .00010 .00010 .00030 .0004$
\end{abstract}

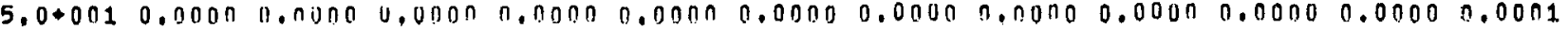

$7.0+0010.000 n \quad 0.00000 .00000 .00000 .0000 \quad 0.0000 \quad 0.00000 .00 n 00.00000 .00000 .0000$

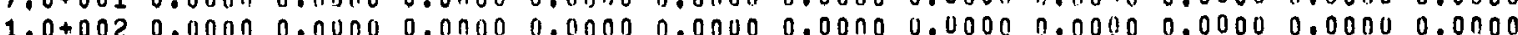

$1.0+0020.000 n$.

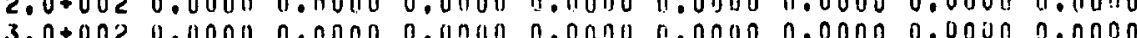

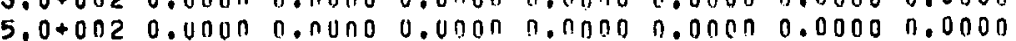

$7.0+0020.00030 .00000 .00000 .00000 .00000 .0000$
$1.0+003$

$1.0+0030.00000 .00000 .00000 .00000,00000.0000$

ALPHA $1.000 \quad 2.000 \quad 3.000 \quad 5.000 \quad 7.000 \quad 10.00 \quad 20.00 \quad 30.00 \quad 50.00 \quad 70.00 \quad 100.0 \quad 200.0 \quad 300.0 \quad 500.0 \quad 700.0 \quad 1000$ BETA

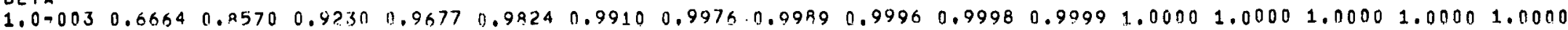

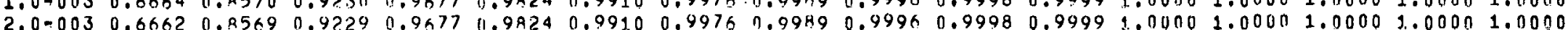
$2.0 .0030 .66520 .05690 .92290 .95770 .90^{2}$

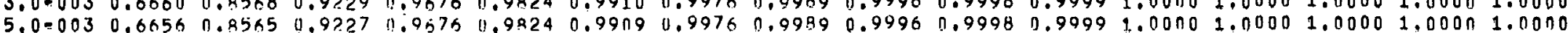

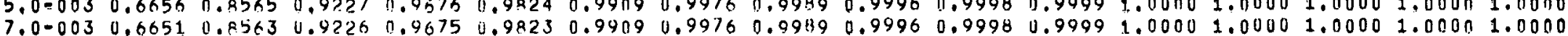

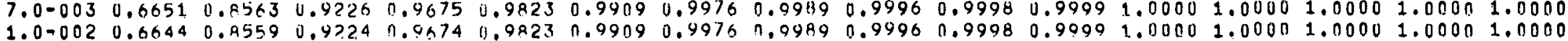
2.0-002 U.6622 $0.85470 .9216 \quad 0.0671 \quad 1.9821 \quad 0.99080 .9976 \quad 0.99890 .9996 \quad 0.99980 .99991 .00001 .00081 .0000$

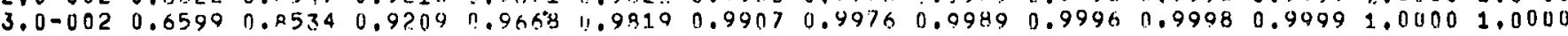

$\begin{array}{lllllllllllll}5.0-002 & 0.6552 & 0.8508 & 0.9194 & 0.9641 & 0.9916 & 0.9905 & 0.9975 & 0.9989 & 0.9996 & 0.9998 & 0.9999 & 1.0400\end{array}$

$\begin{array}{llllllllllllll}7.0 & 0002 & 0.6504 & 0.9481 & 0.9178 & 0.9654 & \text { ü.9812 } & 0.9903 & 0.9974 & 0.9988 & 0.9996 & 0.9998 & 0.9099\end{array}$

$\begin{array}{llllllllllllll}1.0-001 & 0.0431 & 0.2439 & 0.9153 & 0.9643 & 0.9806 & 0.9900 & 0.9974 & 0.9988 & 0.9996 & 0.9998 & 0.9999\end{array}$

$2,0-0010.01730 .42870 .906311 .0603 \quad 0.97830 .98890 .99790 .99870 .9995$

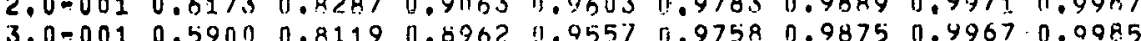

$5.0-001 \quad 0.53330 .71420 .67270 .9444 \quad 1.96970 .98430 .9950$

$\begin{array}{lllllll}7.0 .001 & 0.4773 & 0.7326 & 0.8457 & 0.9320 & 0.9624 & 0.9805\end{array}$

$\begin{array}{llllllll}1.0+000 & 0.4000 & 0.6667 & 0.6700 & 0.9091 & 0.9492 & 0.9735\end{array}$

$2.0+000 \quad 0.22220 .46150 .6316 \quad 0.8103$

$3.0+000 \quad 0.1333 \quad 0.3158 \quad 0.4300$

$5.0+000 \quad 0.0606 \quad 0.1622$

$7.0+000 \quad 0.0339$

$1.0+001 \quad 0.0177$ 
VALUES OF F FOH $N=2$ ANII $N P=3$ ALPH

ALPHA 0.001 U. $002 \quad 0.003 \quad 0.005$ BETA

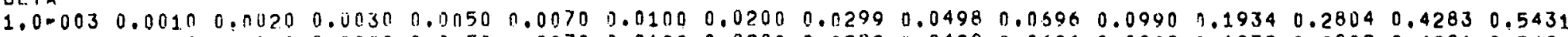
$2.0=0030.0010 \quad 0.00200 .00300 .0050 \quad 0.0070 \quad 0.01000 .02000 .02990 .04980 .06960 .09890 .19320 .28020 .42810 .5429$ $3.0=0030.00100 .00200 .0030 \quad 0.0050 \quad 0.0070 \quad 3.0100 \quad 0.01990 .02990 .0497 \quad 0.0695 \quad 0.09880 .19310 .2800 \quad 0.4278 \quad 0.5426$

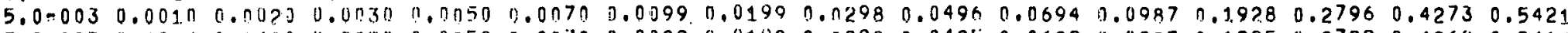
7.0-003 $0.0010 \quad 0.011200 .0030 \quad 0.0050 \quad 0.0070 \quad 0.0099 \quad 0.01990 .0298 \quad 0.0495 \quad 0.0692 \quad 0.0985 \quad 0.19250 .2792 \quad 0.4269 \quad 0.5416$

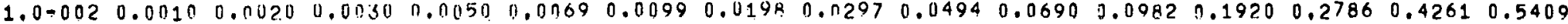
2,0-002 $0.0010 \quad 0.00 ? 0 \quad 0.00290 .011490 .00690 .0098 \quad 0.0196 \quad 0.02940 .04890 .06840 .0973 \quad 0.1904 \quad 0.2765 \quad 0.4236 \quad 0.5384$ $\begin{array}{llllllllllllllllllll}3.0 .002 & 0.0010 & 0.0019 & 0.0029 & 0.01149 & 0.0008 & 0.0097 & 0.0194 & 0.0291 & 0.0485 & 0.0677 & 0.0964 & 0.1888 & 0.2745 & 0.4211 & 0.5358\end{array}$ 5.0 .0020 .00100 .00190 .00290 .00480 .00670 .00950 .01900 .02850 .04750 .06640 .09460 .18570 .27030 .41610 .5306

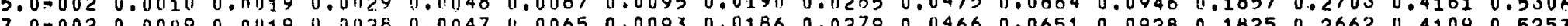

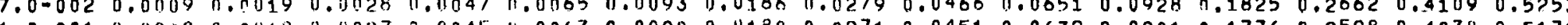

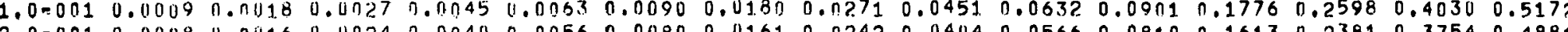

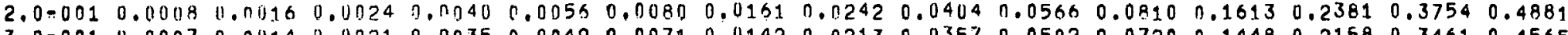
$3.0=001 \quad 0.0007 \quad 0.00140 .0021 \quad 0.0035 \quad 0.00490 .0071 \quad 0.0142 \quad 0.0213 \quad 0.0357 \quad 0.0502 \quad 0.0720 \quad 0.1448 \quad 0.2158 \quad 0.3461 \quad 0.4565$ $\begin{array}{llllllllllllllll}5,0-001 & 0.0005 & 0.0011 & 0.0016 & 0.0027 & 0.0037 & 0,0054 & 0.0108 & 0.0162 & 0.0272 & 0.0384 & 0.0554 & 0.1135 & 0.1722 & 0.2857 & 0.3883\end{array}$

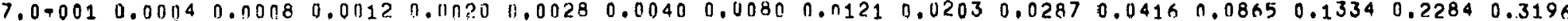

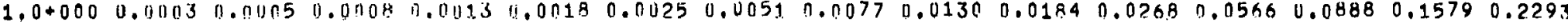

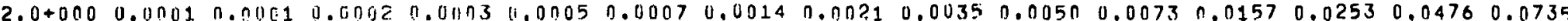

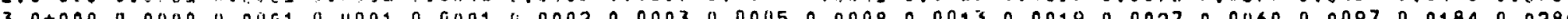
$5.0+000$ a. 0000 n.0.00 5.0+000 a.0.000 $7.0+000 \quad 0.00000 .00000 .0 n 00,0.00001 .00000 .00000 .00010 .00010 .00010 .00020 .00030 .00060 .00100 .00190 .0030$ $1.0+001 \quad 0.00000 .00000 .00000 .00 n 00.00000 .00000 .00000 .10000 .00000 .00010 .00010 .00020 .00040 .00070 .0011$

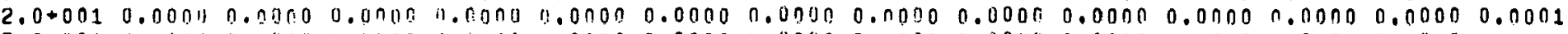
$3.0+0010.00000 .01000 .00000 .00000 .00000 .00000 .00000 .00000 .00000 .00000 .00000 .00000 .0000$

$5.0+0010.00000 .0000 \quad 0.00000 .00000 .00000 .00000 .0 n 000.00000 .00000 .00000 .00000 .0000$

$7.0+0010.00000 .00000 .0000 .00000 .00000 .00000 .00000 .00000 .00000 .00000 .0000$

$1.0+0020.0000 .0 .00000 .0000,0.000 \pi 0.0000 \quad 0.00000 .00 .000 .00000 .00000 .00000 .0000$

$2.0+0020.00000 .00000 .0000 \quad 0.00000 .0000 \quad 0.0000 \quad 0.0000 \quad 0.00000 .0008$

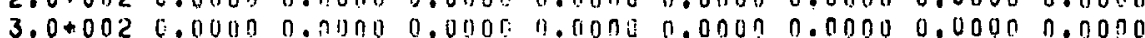

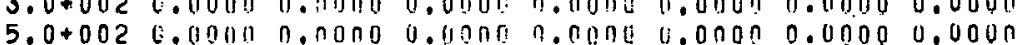

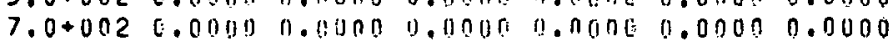

$1,0+0030.000011 .00000 .00000 .00100000000000$ GETA

ALPHA $1.000 \quad 2.000 \quad 3.000 \quad 5.000 \quad 7.000 \quad 10.00 \quad 20.00 \quad 30.00 \quad 50.00 \quad 70.00 \quad 100.0 \quad 200.0 \quad 300.0 \quad 500.0 \quad 700.0 \quad 1.000$

$1.0=0030.66640 .8570 \quad 0.9230 \quad 0.9677 \quad 0.9824 \quad 0.9910 \quad 0.9976 \quad 0.99890 .9996 \quad 0.99980 .90991 .00001 .00001 .00001 .00001 .0000$ $2.0-003 \quad 0.06620 .85890 .92290 .06770 .98240 .99100 .9976 \quad 0.39890 .99960 .99980 .99991 .00001 .00001 .00001 .00001 .0000$

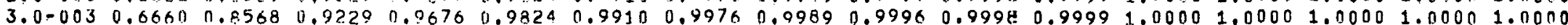

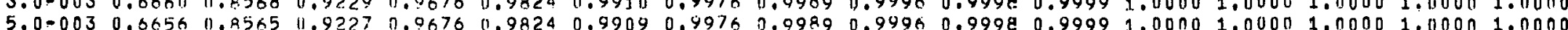

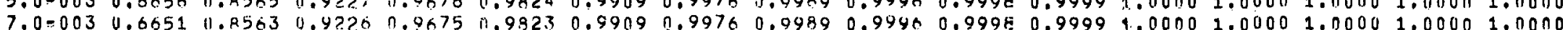

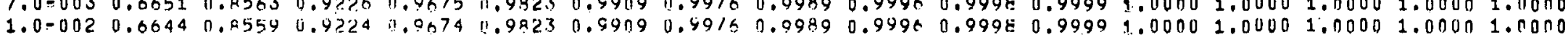

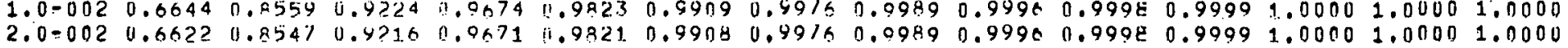
$3,0=002 \quad 0.65990 .8534 \quad 1.07090 .9658 \quad 1.99190 .9907 \quad 0.9976 \quad 0.99490 .9996 \quad 0.9996 \quad 0.90991 .00001 .0000$

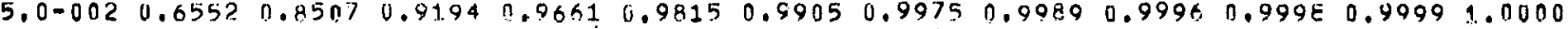

$\begin{array}{lllllllllllll}7.0=002 & 0.65 n 4 & 0.8 .480 & 0.9178 & 0.9654 & 0.9812 & 0.5903 & 0.9974 & 0.0988 & 0.9996 & 0.999 \varepsilon & 0.9999\end{array}$

$\begin{array}{llllllllllll}1.0=001 & 0.6429 & 0.8438 & 0.9153 & 0.9043 & 0.9905 & 0.9900 & 0.9974 & 0.9988 & 0.9996 & 0.999 \varepsilon & 0.9999\end{array}$

$\begin{array}{lllllllllll}2.0-001 & 0.3158 & 0.12278 & 0.9058 & 0.0611: & 0.9782 & 0.9888 & 0.9970 & 0.0987 & 0.9995\end{array}$

$3.0=0010.5853 \quad 0.81190 \quad 11.8944$ i1.9549 0.97530 .98730 .99660 .0985

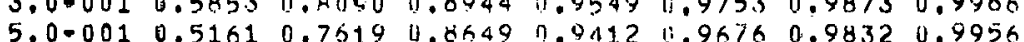

$\begin{array}{lllllll}7.0=001 & 0.4412 & 0.7032 & 0.8257 & 0.9223 & 0.9567 & 0.9775\end{array}$

$1.0+000 \quad 0.33330 .60100 .7500 \quad 0.8924 \quad 0.93330 .9649$

$2.0+000 \quad 0.11760 .2857 \quad 0.44440 .6667$

$3.0+000 \quad 0.04750 .13540 .2308$

$5.0+000 \quad 0.0127 \quad 0.0370$

$7.0+000 \quad 0.0050$

$1.0+0010.0018$ 


\begin{abstract}
VALUES OF F FOR $N=2$ AND $N P=4$
BETA

BETA

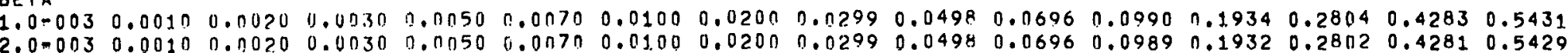
$\begin{array}{llllllllllll} & \end{array}$

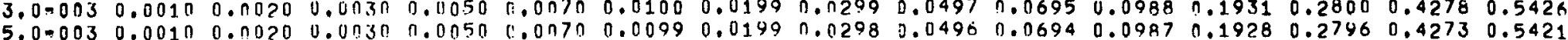
7.0-003 $0.0010 \quad 0.0020 \quad 0.0030 \quad 0.0 n 50 \quad 0.0070 \quad 0.0099 \quad 0.01990 .02980 .0495 \quad 0.0692 \quad 0.0985 \quad 0.1925 \quad 0.2792 \quad 0.4269 \quad 0.5414$ $1.0 .0020 .00100 .00200 .01300 .0050 \quad 0.00690 .0094 \quad 0.0198 \quad 0.02970 .0494 \quad 0.06900 .0982 \quad 0.1920 \quad 0.2786 \quad 0.4261 \quad 0.5400$

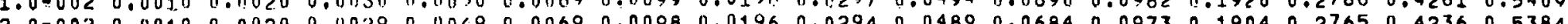
2.0 .0020 .00100 .00200 .01290 .00490 .00690 .00970 .01960 .02940 .04890 .06840 .09730 .19040 .27650 .42360 .5384 (1)

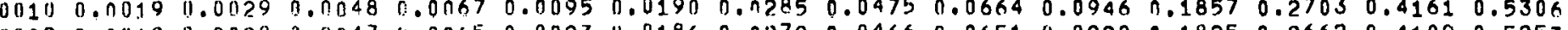
$7.0 .0020 .00090 .00190 .0 .2280 .01470 .0 n 650.0093 \quad 0.0186 \quad 0.02790 .04660 .06510 .09280 .18250 .26620 .41090 .5253$ $\begin{array}{lllllllllllllll}1.0-001 & 0.0009 & 0.0018 & 0.0027 & 0.0045 & 0.0003 & 0.0090 & 0.0180 & 0.0271 & 0.0451 & 0.0632 & 0.0901 & 0.1776 & 0.2598 .0 .4030 & 0.5171\end{array}$ 2.0-001 $0.0008 \quad 0.0016 \quad 0.0024 \quad 0.0040 \quad 0.0056 \quad 0.0080 \quad 0.0161 \quad 0.02410 .04030 .05650 .08090 .16910 .23790 .3751 \quad 0.4878$ $3.0-0010.0007 \quad 0.0014 \quad 0.0021 \quad 0.00350 .00490 .0070 \quad 0.0141 \quad 0.02120 .0355 \quad 0.0499 \quad 0.0717 \quad 0.1441 \quad 0.2149 \quad 0.3448 \quad 0.4550$ $\begin{array}{lllllllllllllllllll}5.0-001 & 0.0005 & 0.0010 & 0.0016 & 0.00 ? 6 & 0.0036 & 0.0052 & 0.01 .04 & 0.0157 & 0.0264 & 0.0372 & 0.0537 & 0.1102 & 0.1676 & 0.2791 & 0.3805\end{array}$ $\begin{array}{llllllllllllllllll}7.0-001 & 0.0004 & 0.0007 & 0.0011 & 0.0018 & 0.0025 & 0.0036 & 0.0073 & 0.0110 & 0.0186 & 0.0263 & 0.0382 & 0.0797 & 0.1233 & 0.2129 & 0.3003\end{array}$ $1.0+000 \quad 0.00020 .0004 \quad 0.0006 \quad 0.001 .0 \quad 0.0014 \quad 0.0020 \quad 0.00410 .00610 .0104 \quad 0.0148 \quad 0.02150 .0458 \quad 0.0724 \quad 0.1304 \quad 0.1922$ ?

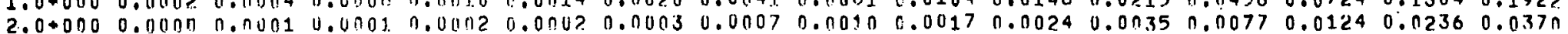

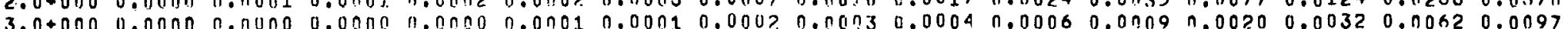
$3.0+000.000 n$.

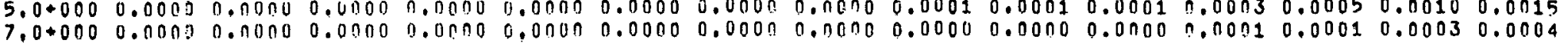

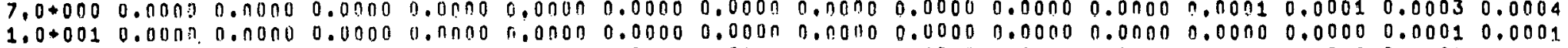
$2.0+0010.0000,0.00000 .0000,0.110000 .00000 .00000 .00000 .00000 .00000 .00000 .00000 .00000 .00000 .0000$

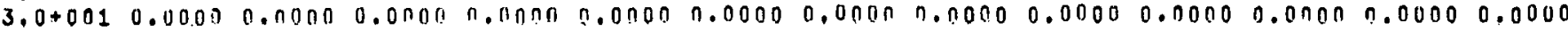

$5.0+0010.00000 .00000 .000 n \pi .00000 .00000 .00000 .00000 .00000 .00000 .00000 .00000 .0000$

$7.0+0010.00000 .00000 .00000 .0000 \quad 1.00000 .00000 .00000 .00000 .00000 .00000 .0000$

$1.0+0020.00000 .00000 .00000 .00000 .00000 .00000 .00000 .00000 .00000 .00000 .0000$

$1,0+0020.0000$.

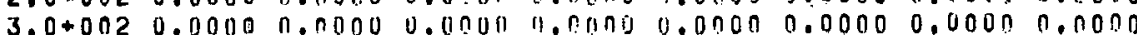

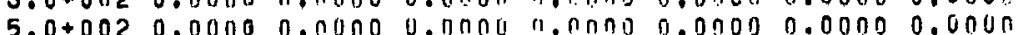

$7,0+0020.00000 .0000,0000,0.001000,00000,0000$

$1.0+0030.00000 .00000 .00000 .00000 .00000 .0000$

ALPHA $1.006 \quad 2.000 \quad 3.000 \quad 5.000 \quad 7.000 \quad 10.00 \quad 20,0.0 \quad 30.00 \quad 50.00 \quad 70.00 \quad 100.0 \quad 200.0 \quad 300.0 \quad 500.0 \% 700.0 \quad 1000$ BETA

$1.0=0030.66640 .8570 \quad 0.9730 \quad 0.9677 \quad 0.9824 \quad 0.9910 \quad 0.9976 \quad 0.99590 .9996 \quad 0.9998 \quad 0.99991 .00001 .00001 .00001 .00001 .0000$ $2.0=0030.60620 .85690 .92290 .96770 .9924 \quad 0.99100 .99760 .09990 .99960 .99980 .99991 .00001 .00001 .00001 .000 n 1.0000$ $3,0-0030.66600 .85680 .92290 .90760 .92240 .99100 .99760 .09290 .99960 .99980 .99991 .00001 .00001 .00001 .00001 .0000$

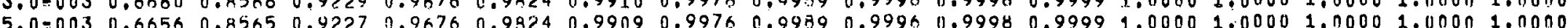

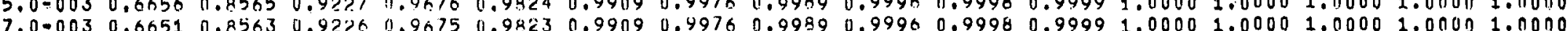

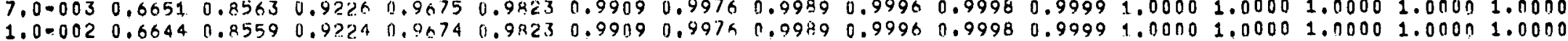
$2.0-0020.66220 .85470 .92160 .06710 .99210 .99060 .99760 .99290 .99960 .99980 .99991 .00001 .00001 .0000$

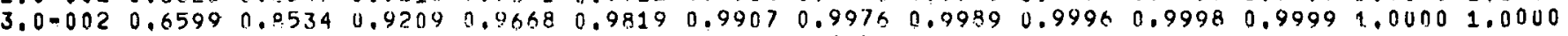

$\begin{array}{llllllllllllll}5.0-002 & 0.6552 & 0.8507 & 0.9194 & 0.9661 & 0.9815 & 0.9905 & 0.9975 & 0.9989 & 0.9996 & 0.9998 & 0.9999 & 4.0000\end{array}$

$\begin{array}{llllllllllllll}7.0=002 & 0.6504 & 0.8480 & 0.9178 & 0.9654 & 0.9812 & 0.9903 & 0.9974 & 0.0998 & 0.9996 & 0.9998 & 0.9999\end{array}$

$100=0010.64290 .84380 .915301 .96430 .98050 .99000 .99740 .99880 .99960 .99980 .9999$

$2.0=001 \quad 0.6155 \quad 0.8276 \quad 1.9057 \quad 0.9600 \quad 0.9782 \quad 0.98880 .9970 \quad 0.09870 .9995$

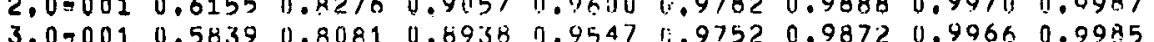

$5.000010 .50700 .75590 .86100 .0393 \quad 0.96660 .98270 .9954$

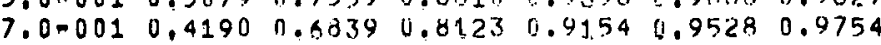

$1.0+000$
$0.2857 \quad 0.5455 \quad 0 . \% 059$ $0.8571 \quad 0.9180 \quad 0.9565$

$2.0+000 \quad 0.0606 \quad 0.1622 \quad 0.27910 .4918$

$3.0+000 \quad 0.01630 .104720 .090 ?$

$5,0+000 \quad 0.0026 \quad 0.0076$

$7.0+000 \quad 0.0007$

$1,0+0010,0002$ 

BETA

$1.000030 .00100 .00200 .0030 \quad 0.05500 .0070 \quad 0.0100 \quad 0.0200 \quad 0.02990 .04980 .06960 .09900 .19340 .28040 .4283 \quad 0.5431$ $2.0-0030.00100 .00200 .00300 .0050 \quad 1.00700 .01000 .02000 .02990 .04980 .06960 .09890 .19320 .28020 .42810 .5429$

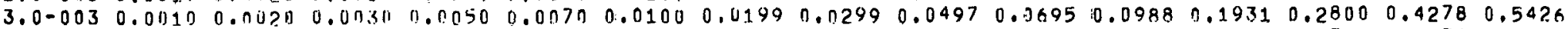

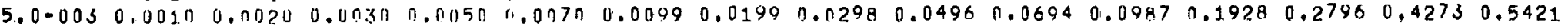

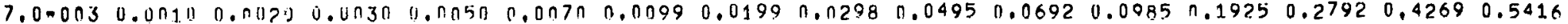

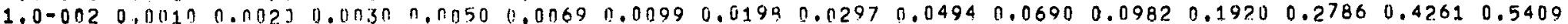
2.0-002 $0.00100 .00270 .00290 .04490 .00690 .00980 .0196 \quad 0.02940 .04890 .06840 .09730 .19040 .27650 .42360 .5384$ 3.0-002 $0.00100 .00170 .00290 .00490 .0068=06970.01940 .02910 .04850 .0677 \quad 8.09640 .18880 .27450 .42110 .5358$ $5.0-0020.00100 .00170 .00290 .00480 .00670 .0050 .01900 .02850 .04750 .06645 .09460 .18570 .27030 .41610 .5306$

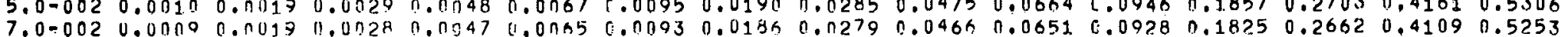

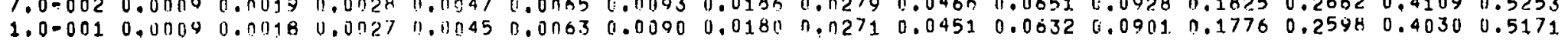

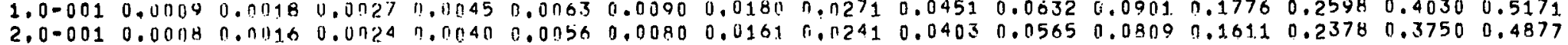

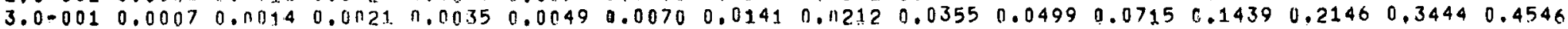

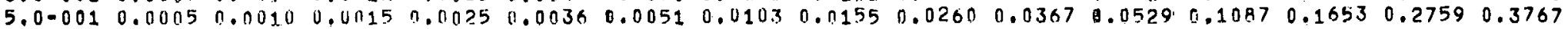

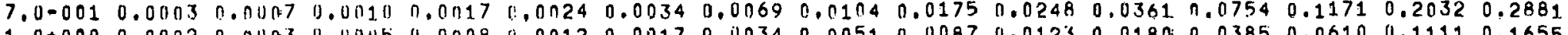

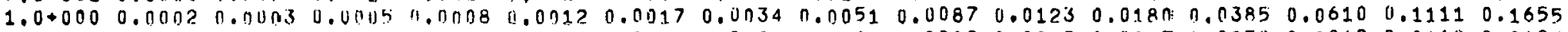

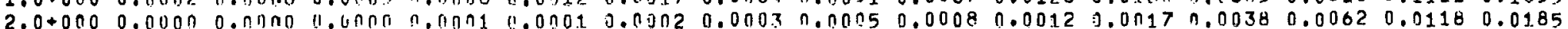

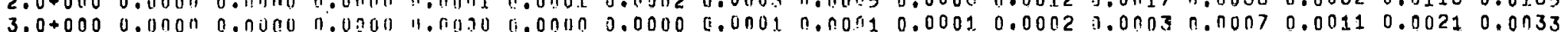

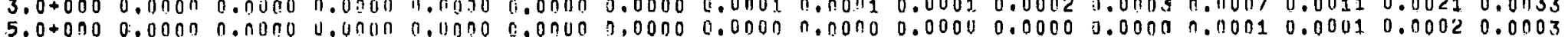

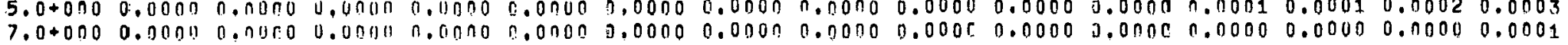

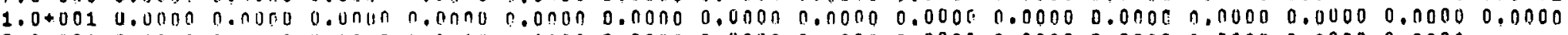

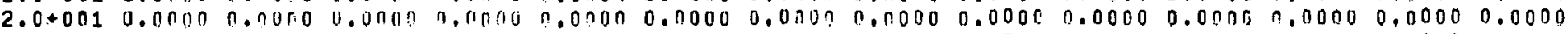

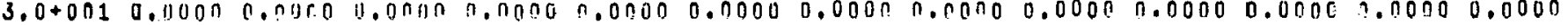

$5.0+0010.00000 .00000 .01000 .00000 .00000 .00000 .00000 .00000 .00000 .00000 .00000 .0000$

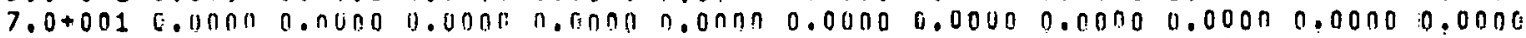

$1.0+0020.00000 .00000 .00050 .110000 .00000 .00000 .00000 .00000 .00000 .00000 .0000$

$1.0+002$.

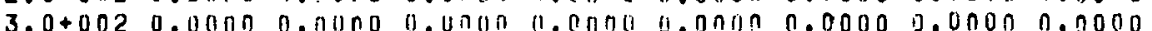

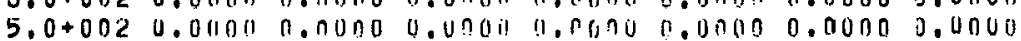

$7.0+002 \quad 0.0000 \quad 0.010 .000000110 .00000 .00000 .00000$

$1.0+0030.00000 .0030$ ij.0000 0.0000 0.0000 0.0000

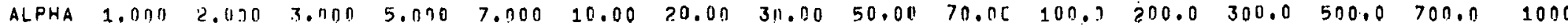
BETA

$1.0-003 \quad 3.6664 \quad 0.8570 \quad 0.9250 \quad 9.9677 \quad 0.9924 \quad 0.99100 .5976 \quad 0.99890 .9996 \quad 0.9998 \quad 0.9997 \quad 1.00001 .00001 .00001 .00001 .0000$ 2.0-003 J.6662 0.85690 .92200 .967711 .98240 .99100 .99750 .99890 .99960 .99980 .99991 .00001 .00001 .00001 .00001 .0000 $3.0-0030.66600 .85 \times 80.92200 .36760 .98240 .99100 .99760 .09890 .99960 .99980 .99901 .00001 .00001 .00001 .00001 .0000$

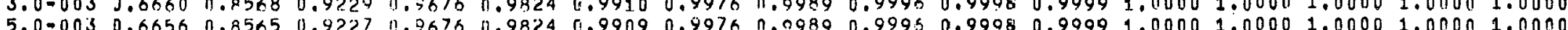

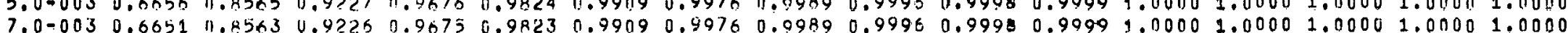

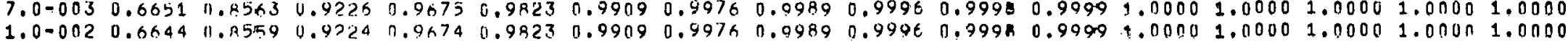

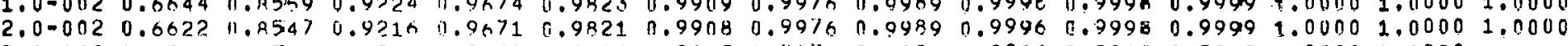
$3.0 .002 \quad 0.65990 .8534 \quad 0.97090 .0668 \quad 0.98190 .9907 \quad 0.9976 \quad 0.99890 .9996 \quad 0.999 .0 .9999 \quad 1.00001 .0000$

$\begin{array}{lllllllllllll}5.0 .002 & 0.655 ? & 0.9507 & 0.9194 & 0.9661 & 0.9815 & 0.9905 & 0.9975 & 0.9989 & 0.9996 & 0.9993 & 0.99991 .0000\end{array}$

$\begin{array}{lllllllllllllll}7.0-002 & 0.6503 & 0.9480 & 0.9178 & 0.9654 & 0.9812 & 0.7903 & 0.9974 & 0.9988 & 0.9956 & 0.9993 & 0.99 .59\end{array}$

$\begin{array}{llllllllllll}1.0-001 & 0.6429 & 0.8438 & 0.9153 & 1.9643 & 0.9805 & 0.9900 & 0.9974 & 0.0988 & 0.9996 & 0.9993 & 0.9959\end{array}$

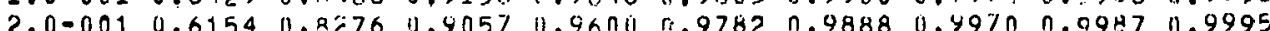

$3.0=001 \quad 0.5835 \quad 0.8178 \quad 0.8937 \quad 0.9546 \quad 0.9751 \quad 0.9872 \quad 0.9966 \quad 0.9985$

$5.0-0010.50390 .7529 \quad$ li. $05910.0384 \quad 0.9600 \quad 0.98240 .9953$

$7.000010 .40480 .0,1110.803111 .91070 .95010 .9740$

$1.0+000 \quad 0.2500 \quad 0.5100 \quad 0.666 .7 \quad 0.8333 \quad 0.9 n 32 \quad 0.9483$

$2.0+000 \quad 0.0308 \quad 0.0870 \quad 0.160100 .3726$

$3.0+000 \quad 0.0055 \quad 0.0 .62 \quad 0.0331$ ?

$5.0+000 \quad 0.00050 .0115$

$7.0+000 \quad 0.0001$

$1.0+0010.0000$ 


\begin{abstract}
VALUES UF F FOR $N=3$ AND $N P=1$
BETA

$\begin{array}{lllllllllllllllll}\text { ALPHA } & 0.001 & 0.012 & 0.003 & 0.005 & 0.007 & 0.01 & 0.0 \% & 0.03 & 0.05 & 0.07 & 0.1 & 0.2 & 0.3 & 0.5 & 0.7\end{array}$

$1.0=0030.00110 .00200 .0030 \quad 0.0050 \quad 0.0070 \quad 0.0100 \quad 0.0200 \quad 0.0300 \quad 0.04990 .06990 .09980 .1986 \quad 0.29410 .46640 .6050$

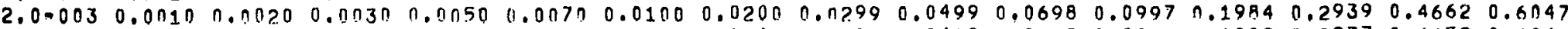

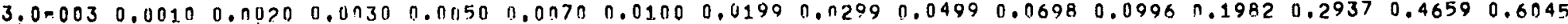

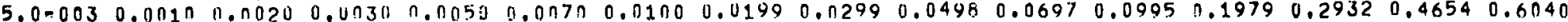
7.0-003 $0.0010 \quad 0.00200 .4 n 300.00570 .0 n 7 n \quad 0.00990 .01990 .02980 .04970 .06950 .09930 .19760 .2928 \quad 0.46490 .6035$ $10-0020.00100 .00200 .07300 .00500 .00690 .00990 .01980 .12970 .04950 .06930 .09900 .19710 .29220 .46420 .6028$ 2.0-002 $0.00100 .10200 .01290 .01490 .01690 .00980 .01960 .02940 .04910 .06870 .09310 .195610 .29020 .46170 .6 n 05$

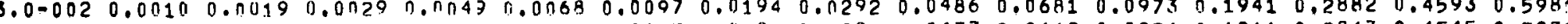
5.0.002 $0.00100 .00190 .03290 .00430 .0067 \quad 0.00950 .0191 .002860 .04770 .06690 .0956 \quad 0.191 .10 .28430 .45450 .5935$

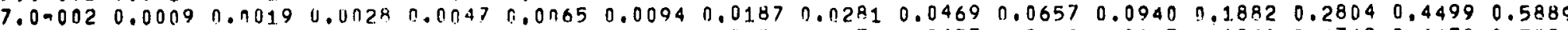

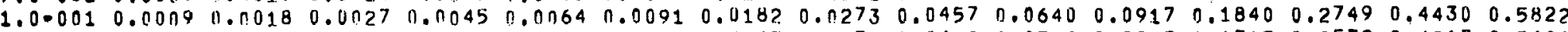

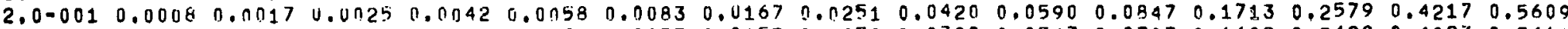
$\begin{array}{lllllllllllllllllll}3.0-001 & 0.0008 & 0.0015 & 0.0023 & 0.0 n 39 & 0.0054 & 0.0077 & 0.0155 & 0.0232 & 0.0389 & 0.0547 & 0.0787 & 0.1602 & 0.2429 & 0.4023 & 0.5411\end{array}$

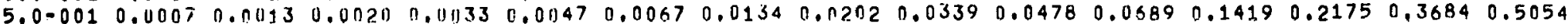
7.0-001 $0.00060 .00120 .00180 .00790 .00410 .00590 .01190 .01790 .03000 .04240 .0613 \quad 0.1273 \quad 0.1970 \quad 0.3398 \quad 0.4742$ 1.00000 .00050 .0100 .00150 .00250 .00350 .00500 .01010 .01520 .02560 .03630 .05260 .11030 .17250 .30430 .4330

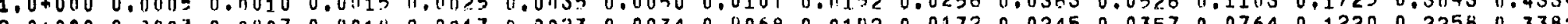

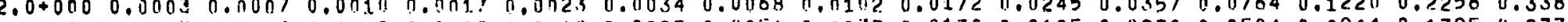

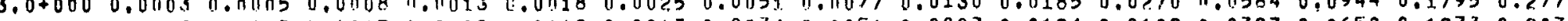

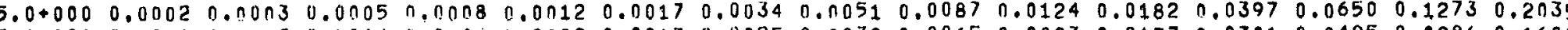
$7.0+000 \quad 0.00010 .0003 \quad 0.00040 .0006 \quad 1.00090 .00130 .00250 .00390 .00650 .00930 .01370 .03010 .04950 .09860 .1608$ $1.0 .0010 .00010 .00020 .0003 \quad 0.00050 .00060 .00090 .00190 .0028 \quad 0.00480 .00680 .01000 .02200 .03650 .07370 .1223$ $2.0+0010.00010 .00010 .40010 .0 n n 20.00030 .00050 .00100 .00150 .00250 .00360 .00530 .01170 .01950 .0400$

$3.0+0010.00000 .00010 .0 n 010.00020 .00020 .00030 .00070 .011 .00 .00170 .00240 .0036 \quad 0.00790 .0133$
\end{abstract}

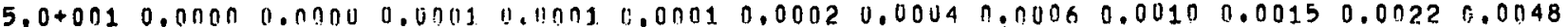

$7.0+0010.00000 .00000 .40000 .00010 .00010 .00010 .00030 .00040 .00070 .00110 .0016$

$1,0+0020.00000 .00000 .00000 .00000 .00010 .00010 .00020 .00030 .00050 .00070 .0011$

$30+0020.00000 .01000 .40000 .00050 .00000 .00010 .0041 .0 .00020 .0003$

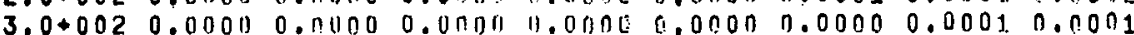

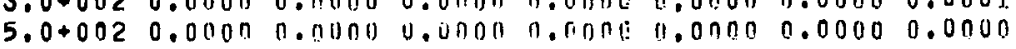

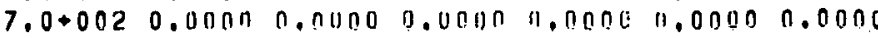

$1,0+0030.000110 .0000 \quad 3,00000.00000 .00000 .0000$

ALPHA $1.000 \quad 2.0003 .000 \quad 5.000 \quad 7.000 \quad 10.00 \quad 20.00 \quad 30.00 \quad 50.00 \quad 70.00 \quad 100.0 \quad 200.0 \quad 300.0 \quad 500.0 \quad 700.0 \quad 1000$ BETA

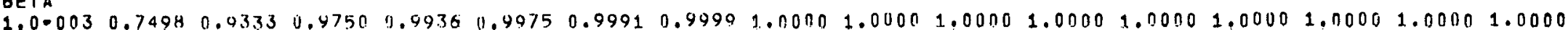

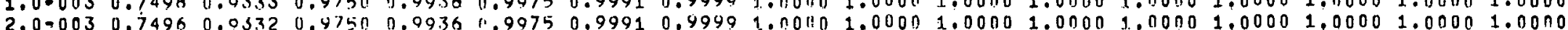

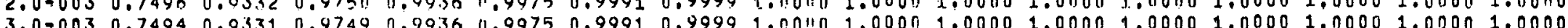

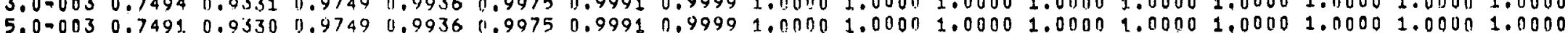

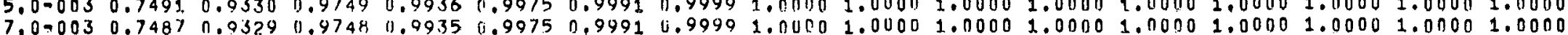

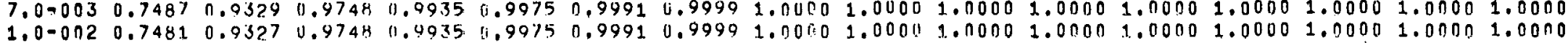
$2,0=0020.74630 .93210 .97450 .99350 .99750 .99910 .99991 .00501 .00001 .00001 .00001 .00001 .00001 .0000$ 3.0 .0020 .74440 .93150 .97430 .99340 .99740 .99910 .99991 .00701 .00001 .00001 .00001 .00001 .0000

$5.0=002 \quad 0.74070 .93020 .97380 .99330 .99740 .99910 .99991 .00001 .00001 .00001 .00001 .0000$

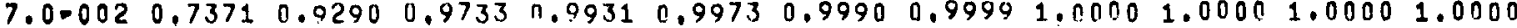

$1.0-0010.73170 .02720 .97260 .99300 .99730 .99900 .99991 .00001 .00051 .00001 .0000$

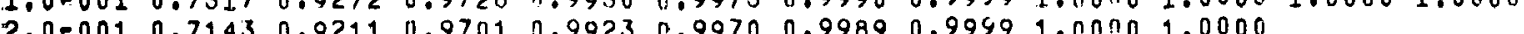

$3.0-0010.69770 .01500 .9677099170 .90640 .99880 .99981 .0000$

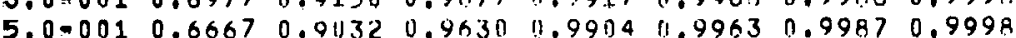

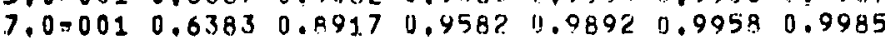

$1.0+000 \quad 0.6000 \quad 0.8750 \quad 0.9512 \quad 9.9873 \quad 0.9950 \quad 0.9982$

$2.0+000 \quad 0.5000 \quad 0.82350 .9266 \quad 1.9810$

$3.0+000 \quad 0.4286 \quad 0.7778 \quad 0.4070$

$5.0+000 \quad 0.33330 .7000$

$7,0+000 \quad 0,2727$

1. $0+0010.2143$ 


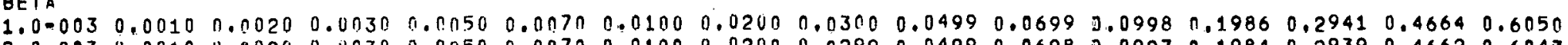

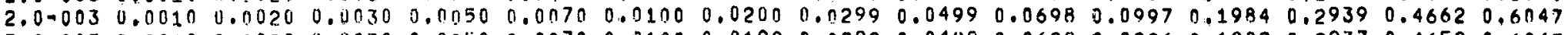

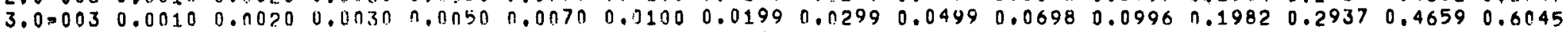

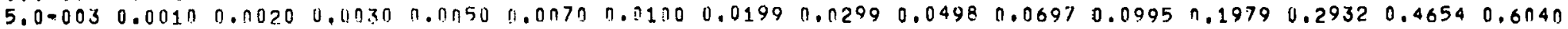

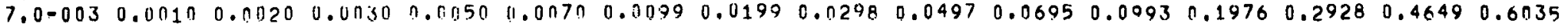
1,0-002 $0.0010 \quad 0.01120 \quad 0.0030 \quad 0.0050 \quad 0.00690 .0099 \quad 0.0198 \quad 0.02970 .04950 .196930 .0990 \quad 0.1971 \quad 0.29220 .4642 \quad 0.6028$ $2.0=0020.00100 .0020 \quad 0.00290 .00490 .00690 .0098 \quad 0.01960 .02940 .0490 \quad 0.06870 .09810 .19550 .29010 .46160 .6004$

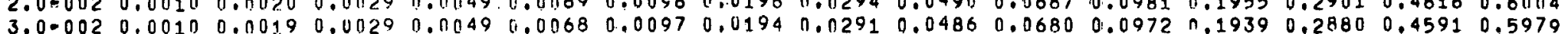

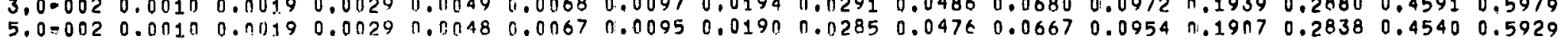

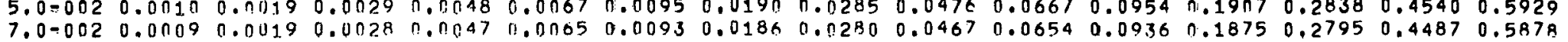

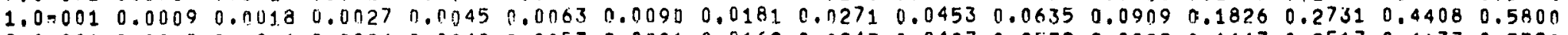
$2,0=001 \quad 0.0008 \quad 0.0015 \quad 0.0024 \quad 0.0010 \quad 0.0057 \quad 0.0081 \quad 0.01620 .0243 \quad 0.0407 \quad 0.0572 \quad 0.08220 .1667 \quad 0.25170 .4137 \quad 0.5528$ $\begin{array}{llllllllllllllll}3.0=001 & 0.0007 & 0.0014 & 0.0022 & 11.0036 & 0.0050 & 0.0072 & 0.0145 & 0.0218 & 0.0365 & 0.0514 & 0.0740 & 0.1514 & 0.2308 & 0.3863 & 0.5245\end{array}$

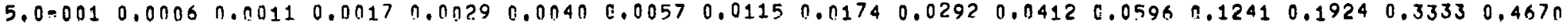
7.0-001 $0.0005 \quad 0.00090 .00140 .0023 \quad 0.0032 \quad 0.0046 \quad 0.0092 \quad 0.01390 .02350 .0332 \quad 0.0482 \quad 0.10170 .1600 \quad 0.2855 \quad 0.4118$ $1.0+000 \quad 0.00030 .00 n 7 \quad 0.00100 .0097 \quad 0.0 n 23 \quad 0.00340 .00680 .01020 .01720 .02450 .0357 \quad 0.07640 .1220 \quad 0.2256 \quad 0.338 ?$ $2.0+0000.00010 .00030 .00040 .00070 .00100 .00140 .00290 .00440 .00750 .0106 \quad 0.0156 \quad 0.03420 .05620 .11110 .9797$

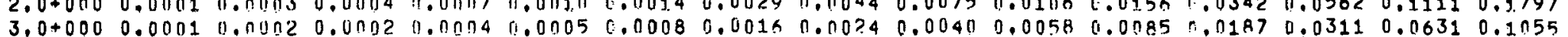

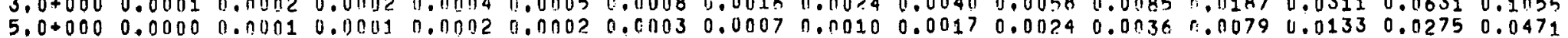

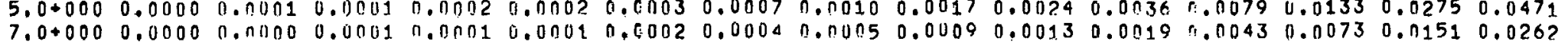
$1.0+0010.00000 .00000 .00000 .00000 .00018 .00010 .00020 .00030 .00050 .00070 .00100 .00220 .00370 .00780 .0136$ $2.0+0010.0000$ 0.nuño $0.00000 .00000 .00000 .00000 .000 n 0.00010 .0001 .0 .00028 .00030 .00060 .00100 .0021$ $3.0+0010.00000 .00090 .00000 .00000 .0000 .00000 .00000 .00000 .00010 .00010 .00010 .00030 .0004$ $5.0+0010.00000 .00000 .0000 .0 .00000 .00000 .00000 .00400 .00000 .00000 .00000 .00000 .0001$ $7.0+0010.00000 .00000 .00000 .00000 .00000 .00000 .00000 .00000 .00000 .00000 .0000$ $1.0+0020.00000 .00000 .00000 .00000 .00000 .00000 .00000 .00000 .00000 .00000 .0000$

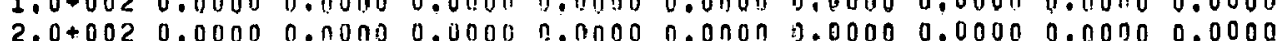

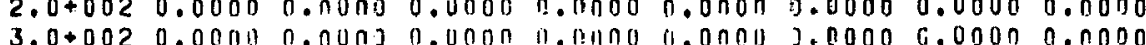

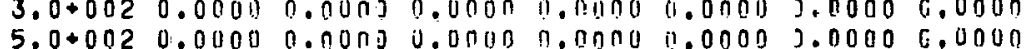

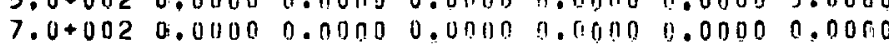

$1,0+0.030 .00000 .00000 .00000 .00000 .00000,0000$ BETA

$1.0-003 \quad 0.7498 \quad 0.0333 \quad 0.9750 \quad 0.0936 \quad 0.9075 \quad 0.7991 \quad 0.99991 .00001 .00001 .00001 .00001 .00001 .00001 .00001 .00001 .0000$

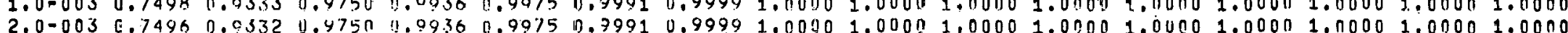

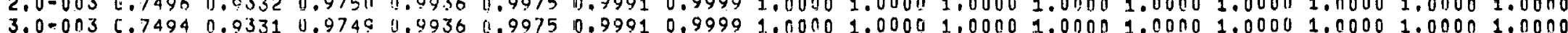

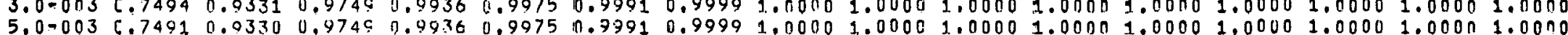

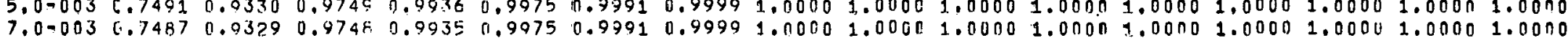

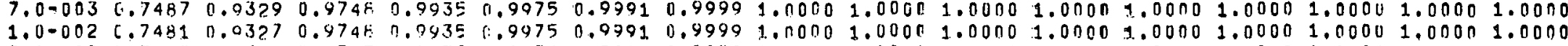
$2,0.0020 .74620 .93210 .97450 .99350 .99740 .99910 .99991 .00001 .00001 .00001 .00001 .00001 .00001 .0000$ $3.0-0020.74430 .93140 .97420 .9934 \quad 0.99740 .99910 .99901 .00001 .00001 .00001 .00001 .00001 .0000$

$5.0-002 \quad 0.7403 \quad 0.03010 .97370 .99330 .99740 .99910 .99991 .00001 .00001 .00001 .00001 .0000$

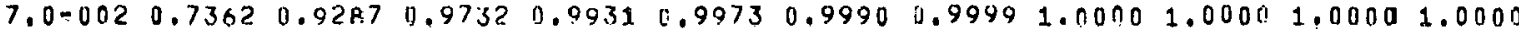

$\begin{array}{llllllllll}1.0-001 & 0.7299 & 0.0265 & 0.9723 & 0.9979 & 0.9972 & 0.9990 & 0.9999 & 1.00001 .0001 & 1.00001 .0000\end{array}$

$2.09001 \quad 0.7075 \quad 0.0186 \quad 1.9592 \quad 0.9921 \quad 0.9069 \quad 0.99 .89 \quad 0.9999 \quad 1.001001 .0000$

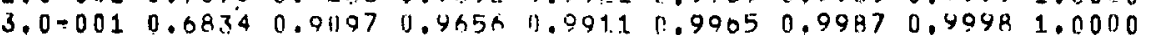

5.0-001 $0.03160 .88890 .957: 0.78880 .99560 .99840 .9998$

$7.0=001 \quad 0.5780 \quad 0.86470 .9468 \quad 0.9861 \quad 0.99450 .9980$

$1.0+000 \quad 0.5000 \quad 0.82350 .92860 .9810 \quad 0.90250 .9973$

$2.0+000 \quad 0.3000 \quad 0.6667 \quad 0.8478 \quad 0.9568$

$3.0+000 \quad J .18750 .5155 \quad 0.7500$

$5.0+000 \quad 3.0882 \quad 0.3111$

$7.0+000 \quad 3.0500$

$1,0+0013.0263$

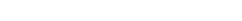




\begin{abstract}
VALUES OF F FOR $N=3$ AIN $N P=3$ BETA

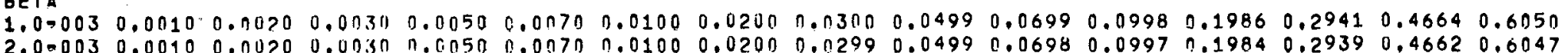

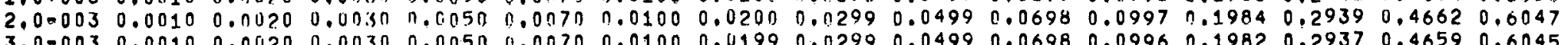
$\begin{array}{llllllllllllllll}3.0 .003 & 0.0010 & 0.0020 & 0.0130 & 0.0 .050 & 0.007 n & 0.0100 & 0.0199 & 0.0299 & 0.0499 & 0.0698 & 0.0996 & 0.1982 & 0.2937 & 0.4659 & 0.6045\end{array}$

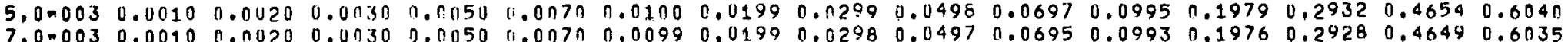

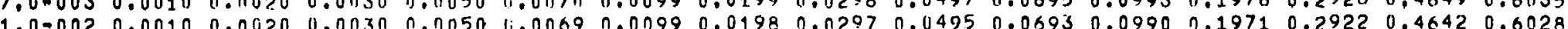
$1.0=0020.0010 \quad 0.00200 .0030 .0050 \quad 1.00690 .00990 .01980 .02970 .04950 .06930 .0990 \quad 0.19710 .29220 .46420 .6028$

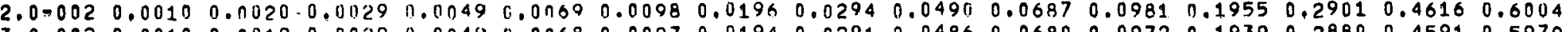
$\begin{array}{llllllllllllllll}3,0=002 & 0.0010 & 0.0019 & 0.0029 & 0.0049 & 0.0068 & 0.0097 & 0.0194 & 0.0291 & 0.0486 & 0.0680 & 0.0972 & 0.1939 & 0.2880 & 0.4591 & 0.5979\end{array}$ $\begin{array}{llllllllllllllll}5.0-002 & 0.0010 & 0.0019 & 0.0029 & 11.0048 & 0.0167 & 0.0095 & 0.0190 & 0.0285 & 0.0476 & 0.0667 & 0.0954 & 0.1907 & 0.2837 & 0.4539 & 0.5929\end{array}$

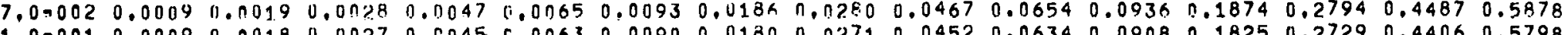

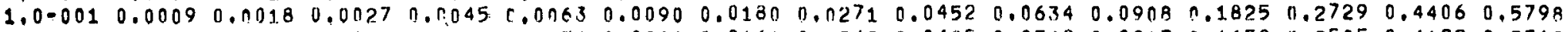
2.0-001 $0.00080 .0016 \quad 0.07240 .0040 \quad 0.0056 \quad 0.0080 \quad 0.0161 \quad 0.02420 .04050 .05690 .0917 \quad 0.1658 \quad 0.25050 .4122 \quad 0.5512$ $\begin{array}{lllllllllllllllllll}3.0001 & 0.0007 & 0.0014 & 0.0021 & 0.0035 & 0.005 n & 0.0071 & 0.014 ? & 0.0214 & 0.0358 & 0.0504 & 0.0726 & 0.1489 & 0.2274 & 0.3818 & 0.5197\end{array}$ $5.0=001 \quad 0.0005 \quad 0.00110 .0016 \quad 0.0027 \quad 0.0037 \quad 0.0054 \quad 0.0108 \quad 0.01620 .0273 \quad 0.0386 \quad 0.0559 \quad 0.11680 .18190 .3182 \quad 0.4498$

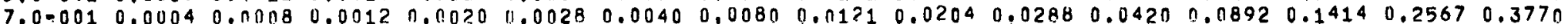
1.0 .0010 .00030 .00050 .00080 .00130 .00180 .00250 .00510 .00770 .01300 .01850 .02700 .05840 .09440 .17950 .2771

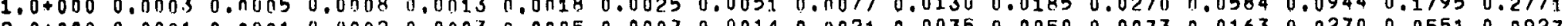

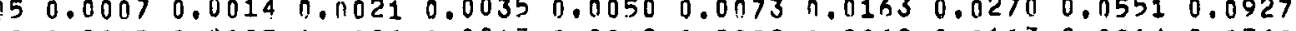
$3.0+0000.00000 .10110 .0 n 01.0 .00110 .0 .020 .00030 .0005 n .001080 .00130 .00190 .00280 .00620 .01030 .02140 .0369$ $5.0+000 \quad 0.0000 \quad 0.00000 .00000 .00000 .00000 .00010 .00010 .00020 .00030 .00050 .00070 .00160 .00270 .00560 .0097$ $7.0+000 \quad 0.0000 \quad 0.00000 .0000 \quad 0.00000 .0000 \quad 0.0000 \quad 0.00010 .00010 .00010 .00020 .00030 .00060 .00100 .00220 .0038$ $1.0+0010.00000 .00000 .00000 .00000 .00000 .00000 .00000 .00000 .00000 .00010 .00010 .00020 .00040 .00080 .0014$

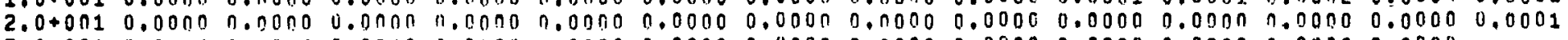

$3.0+0010.00000 .00000 .00000 .00000 .00000 .00000 .00000 .00000 .00000 .00000 .00000 .00000 .0000$
\end{abstract}

$5.0+0010.00000 .00000 .0000,0.00000 .00000 .00000 .00000 .00000 .00000 .00000 .00000 .0000$

$7.0+0010.00000 .00000 .00000 .00000 .00000 .00000 .00000 .00000 .00000 .00000 .0000$

$1,0+0020.00000 .00000 .00000 .00000 .00000 .00000 .00000 .00000 .00000 .00000 .0000$

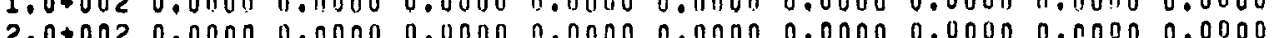

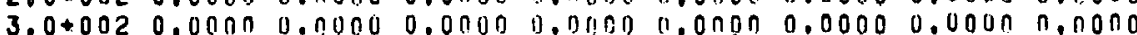

$5,0+0020.00000 .00000 .0000,0,0000 \%, 00000.00000 .0000$

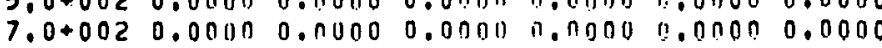

$1.0+0030,00000.00000 .00000 .00000,00600.0000$

ALPHA $1.000 \quad 2.000 \quad 3.000 \quad 5.000 \quad 7.000 \quad 10.00 \quad 20.00 \quad 30.00 \quad 50.00 \quad 70.00 \quad 100.0 \quad 200.0 \quad 300.0 \quad 500.0 \quad 700.0 \quad 1000$ BETA

$1.0-0030.7498 \quad 0.03330 .9750 \quad 0.9936 \quad 0.9975 \quad 0.9991 \quad 0.99991 .00001 .00001 .00001 .00001 .00001 .00001 .00001 .00001 .0000$ $2.0=0030.74960 .03320 .9750 \quad 0.99360 .97750 .99990 .99991 .001409 .00001 .00001 .00001 .00001 .00001 .00001 .00001 .0000$

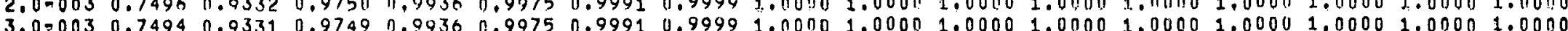

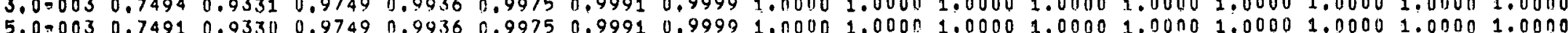

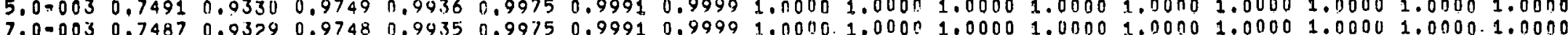

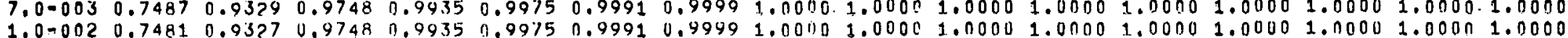
$2.0-002 \quad 0.7462 \quad 0.93210 .97450 .99350 .99 / 40.99910 .99991 .00011 .00001 .00001 .00001 .00001 .00001 .0000$

$3.0=0020.74420 .93140 .97420 .99340 .99740 .99910 .99991 .00101 .00001 .110001 .00001 .00001 .0000$

$5.0-0020.7403 \quad 0.9301 \quad 0.97370 .99330 .99740 .99910 .99991 .00001 .00001 .00001 .00001 .0000$

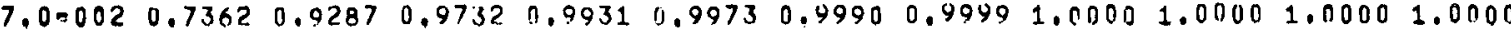

$1.0 .0010 .72970 .9265 \quad 0.97230 .9929 \mathrm{j}, 9972 \quad 0.9990 \quad 0.99991 .00001 .00001 .00001 .0000$

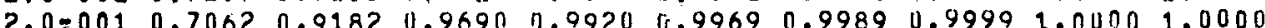

$3,0=001 \quad 0.6792 \quad 0.9081 \quad 0.96490 .90090 .99650 .9987 \quad 0.99980 .9999$

5.070010 .61540 .88190 .95410 .94800 .90530 .99830 .9998

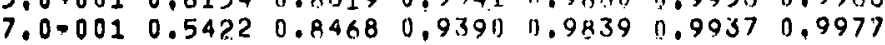

$1.0+000 \quad 0.4286 \quad 0.7778 \quad 0.9070 \quad 0.9748 \quad 0.9001 \quad 0.9964$

$2.0+000 \quad 0.1667 \quad 0.4828 \quad 0.7222 \quad 0.9118$

$3.0 .000 \quad 0.0698 \quad 0.2503 \quad 0.4937$

$5.0+000 \quad 0.01890 .0 .824$

$7.0+000 \quad 0.0074$

$1.0+001 \quad 0,0027$ 

BETA

$1.0=003 \quad 0.0010 \quad 0.0020 \quad 0.0030 \quad 0.0050 \quad 0.0070 \quad 0.0100 \quad 0.0200 \quad 0.031000 .0499 \quad 0.0599 \quad 0.0998 \quad 0.1986 \quad 0.2941 \quad 0.46640 .6050$ $2.0=0030.00100 .00200 .00300 .00500 .00700 .0100 \quad 0.0200 \quad 0.02990 .04990 .0598 .0 .0997 \quad 0.19840 .29390 .46620 .6047$ $3,0=003 \quad 0.00100 .0020 \quad 0.0030 \quad 0.0050 \quad 0.0170 \quad 0.0100 \quad 0.01990 .02990 .04990 .06980 .0996 \quad 0.1982 \quad 0.2937 \quad 0.4659 \quad 0.6045$

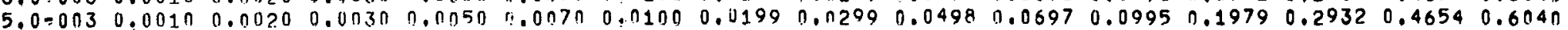
7.0-003 0.0010 $0.0020 \quad 0.0030 \quad 11.0050 \quad 0.007 n \quad 0.00990 .01990 .02980 .04970 .06950 .0993 \quad 0.19760 .2928 \quad 0.4649 \quad 0.6035$

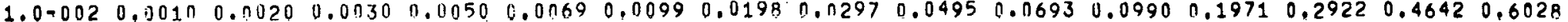
$2,0-0020.10100 .00200 .0029 \quad 0.00490 .00690 .00980 .0196 \quad 0.02940 .0490 \quad 0.0687 \quad 0.0981 \quad 0.1955 \quad 0.2901 \quad 0.4616 \quad 0.6004$ 2.0-002 0.0100 .00190 .00290 .00490 .00680 .00970 .01940 .02910 .04860 .06800 .09720 .19390 .28800 .45910 .5979 5.0 .002 0.001 0.00190 .00290 .00480 .00670 .00950 .01900 .02850 .04760 .06670 .09540 .191070 .28370 .45390 .5929 .0009 n.0018 0.0027 $1.0 .0010 .00090 .0180 .0027 \quad 1.10450 .05630 .00900 .01800 .02710 .04520 .06340 .09080 .18250 .27290 .44060 .5798$

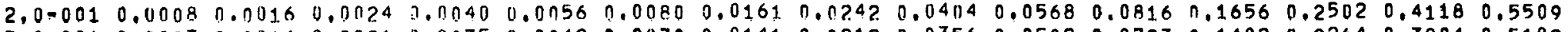
$3.0=001 \quad 0.0007 \quad 0.0014 \quad 0.0021 \quad 0.0035 \quad 0.00490 .0070 \quad 0.0141 \quad 0.02120 .0356 \quad 0.0502 \quad 0.0723 \quad 0.1482 \quad 0.2264 \quad 0.3804 \quad 0.5182$ $5.0=0010.0005 \quad 0.0010 \quad 0.0016 \quad 0.0036 \quad 0.0036 \quad 0.0052 \quad 0.0104 \quad 0.0157 \quad 0.0264 \quad 0.03740 .0542 \quad 0.11350 .1771 \quad 0.3111 \quad 0.4417$

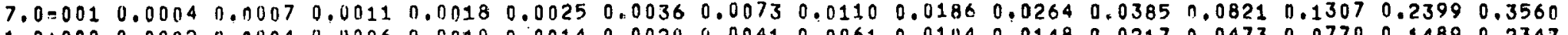
$1.0+000 \quad 0.0002 \quad 0.0004 \quad 0.0006 \quad 0.00100 .00140 .0020 \quad 0.00410 .00610 .0104 \quad 0.01480 .0 ? 17 \quad 0.0473 \quad 0.0770 \quad 0.1489 \quad 0.2347$

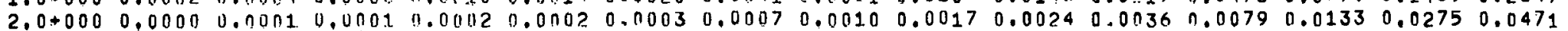

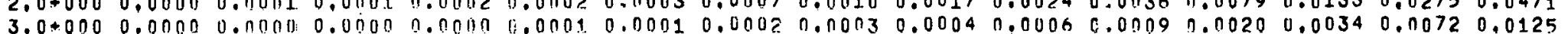

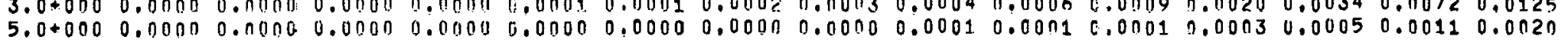

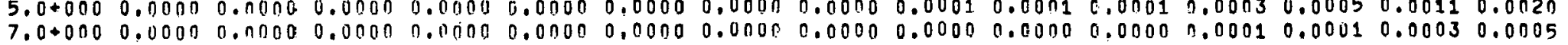
$1.0+0010.00000 .00000 .00000 .0000 \quad 0.00000 .00000 .00000 .00000 .00000 .00000 .00000 .00000 .00000 .00010 .0001$ $2.0+0010.00000 .00000 .00007 .00005 .00000 .00000 .00000 .00000 .00000 .00000 .00000 .00000 .00000 .0000$

$3.0+0010.0000 \quad 0.00000 .00000 .0000$ lj.0000 0.00000 .00000 .00000 .00000 .00000 .00000 .00000 .0000

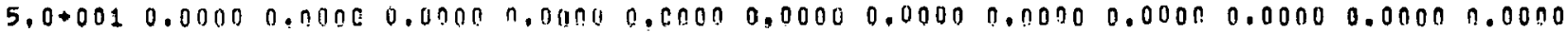

$7.0+0010.00000 .00000 .00000 .00000 .00000 .00000 .00000 .00000 .00000 .00000 .0000$

$1.0+0020.00000 .00000 .00000 .00000 .00000 .00000 .00000 .00000 .00000 .00000 .0000$

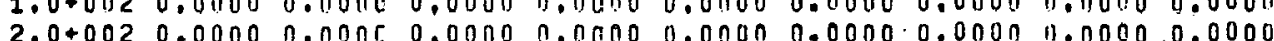

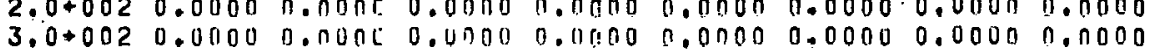

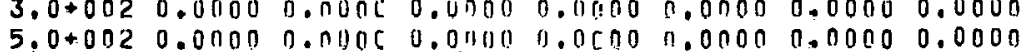

$7,0+0020.00000 .000 \mathrm{C} 0.00000 .00000 .00000 .0000$

$1,0+0030.00000 .00060 .0000 \quad 0.05000 .0000 \quad 0.0000$ BETA

$1.0-003 \quad 0.7498 \quad 0.033 j 0.975110 .09360 .9075 \quad 0.99910 .99991 .00001 .00001090001 .00001 .00001 .00001 .00001 .00001 .0000$ $2.0=0030.74960 .03320 .9750 \quad 1.9936 \quad 1.99750 .99910,99991.00001 .00001 .00001 .00001 .00001 .00001 .00001 .00001 .0000$ $3.0=0030.74940 .933 .0 .97490 .99360 .99750 .99910 .99991 .00001 .00001 .00001 .00001 .00001 .00001 .00001 .00001 .0000$

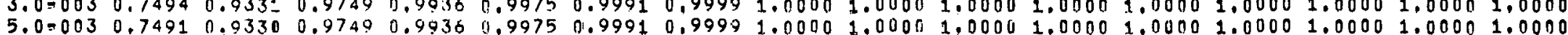

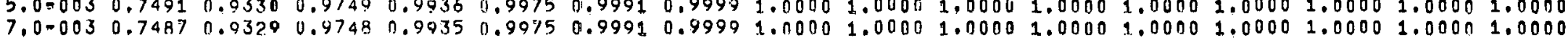
$1.0=002 \quad 0.74810 .03270 .97490 .99350 .99750 .99910 .99991 .00001 .00001 .00001 .00001 .00001 .00001 .00001 .00001 .0000$ $2,0.0020 .74620 .03210 .97450 .00331099740 .99910 .99901 .00001 .00001 .00001 .00001 .00001 .00001 .0000$

$3.0-0020.74420 .93170 .9742 \quad 0.9934 \quad 1.99740 .99910 .99991 .00001 .00001 .00001 .00001 .00001 .0000$

$5.0 .002 \quad 0.7403 \quad 0.93010 .97370 .9933 \quad 0.9974 \quad 0,99910.99991 .00001 .00001 .00001 .00001 .0000$

$7.000020 .73610 .92870 .97320 .99310 .9973 \quad 0.99900 .99991 .00001 .00001 .00001 .0000$

$1.0 .0010 .72970 .02650 .97230 .99290 .9972 \quad 0.99900 .99991 .00001 .00001 .00001 .0000$

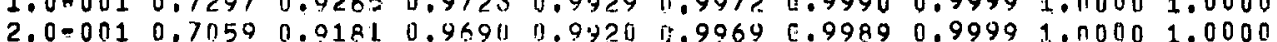

$\begin{array}{lllllllll}2.0=001 & 0.7058 & 0.9181 & 0.9691 & 0.9920 & .9969 & 0.9989 & 0.9999 & 1.0000 \\ 3.0 .001 & 0.6780 & 0.9075 & 0.9647 & 0.9309 & 11.9964 & 0.9987 & 0.9998 & 0.9999\end{array}$

$5.0=001 \quad 0.6076 \quad 0.878=0.9527 \quad 0.937 \% \quad 0.9952 \quad 0.9983 \quad 0.9998$

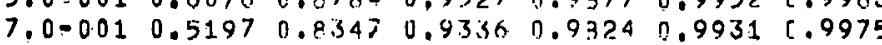

$1.0+000 \quad 0.3750 \quad 0.7363 \quad 0.8864 \quad 0.9337 \quad 0.9876 \quad 0.9955$

$2.0+000 \quad 0.08820 .31110 .55710 .8333$

$3.0+000 \quad 0.024 ? \quad 0.103 .70 .2438$

$5,0+000 \quad 0.0038 \quad 0.0176$

$7.0+000 \quad 0.0011$

$1.0+001 \quad 0.00103$ 


\begin{abstract}
VALUES OF F FOR $N=3$ AND $N P=5$
BETA

$\begin{array}{llllllllllllllll}1.0-003 & 0.0010 & 0.0020 & 0.0030 & 0.0050 & 0.0070 & 0.0100 & 0.0200 & 0.0300 & 0.0499 & 0.0699 & 0.0998 & 0.1986 & 0.2941 & 0.4664 & 0.6050 \\ 2.0-003 & 0.0010 & 0.0020 & 0.0030 & 0.0050 & 0.0070 & 0.0100 & 0.0200 & 0.0299 & 0.0499 & 0.0698 & 0.0997 & 0.1984 & 0.2939 & 0.4662 & 0.6047\end{array}$ $3.0 .003 \quad 0.0010 \quad 0.0020 \quad 0.0030 \quad 0.0050 \quad 0.0170 \quad 0.0100 \quad 0.0199 \quad 0.02990 .04990 .06980 .09960 .19820 .29370 .46590 .6045$

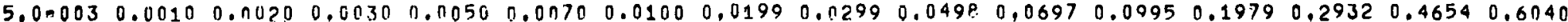

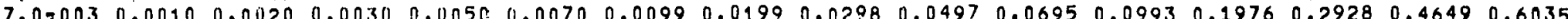
$1.0=0020.00100 .00200 .0030400500 .00690 .00990 .01980 .02970 .04950 .06930 .09900 .19710 .29220 .46420 .6028$ 0.01960 .02970 .04950 .06930 .09900 .19710 .29220 .46420 .6028 2.0 .0020 .00100 .0020 .0029 .10049 .00690 .00980 .01960 .02940 .0490 .06870 .09810 .19530 .29010 .46160 .6004 3.0 .0020 .00100 .001 .90 .00290 .00490 .00680 .00970 .01940 .12910 .04860 .06800 .09720 .19390 .28800 .45910 .5979 $5.0 .0020 .0010 \quad 0.00190 .00290 .00480 .01670 .0095 \quad 0.0190 \quad 0.02850 .04760 .06670 .0954 \quad 0.1907 \quad 0.28370 .45390 .5929$

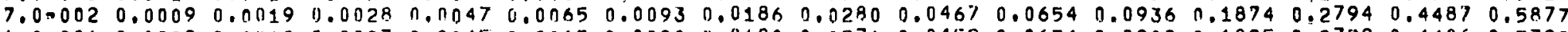
1.0-001 $0.00090 .00180 .0027 \quad 0.0045 \quad 0.0 n 63 \quad 0.0090 \quad 0.0180 \quad 0.02710 .04520 .06340 .09080 .18250 .27290 .4406 \quad 0.5798$ $2.0-001 \quad 0.0008 \quad 0.0016 \quad 0.0024 \quad 0.0040 \quad 0.0056 \quad 0.0080 \quad 0.0161 \quad 0.02410 .0404 \quad 0.0568 \quad 0.0816 \quad 0.1656 \quad 0.2502 \quad 0.4118 \quad 0.5509$

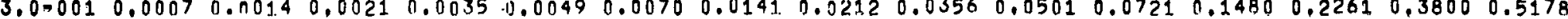
$\begin{array}{lllllllllllllllllll}5.0=001 & 0.0005 & 0.0010 & 0.0015 & 0.0025 & 0.0036 & 0.0051 & 0.0103 & 0.0155 & 0.0260 & 0.0368 & 0.0534 & 0.1119 & 0.1748 & 0.3077 & 0.4378\end{array}$ $7.0-001.0 .00030 .00070 .00100 .00170 .00240 .00340 .00690 .01040 .01760 .02490 .03640 .07780 .12420 .22930 .3426$ 1.000000 .00020 .00030 .00050 .00000 .00120 .00170 .00340 .00510 .00870 .01240 .01820 .03970 .06500 .12730 .3426

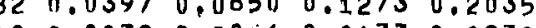
0.0238

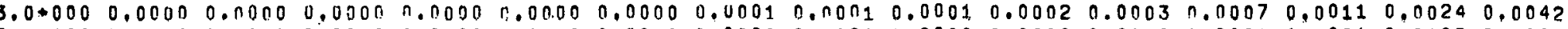
$5.0+000 \quad 0.00000 .00000 .00000 .00000 .00000 .00000 .00000 .00000 .00000 .00000 .00000 .00010 .00010 .00020 .0004$ $7.000000 .00000 .00000 .00000 .0000 \quad 0.00000 .0 .0000 .00000 .00000 .00000 .00000 .00000 .00000 .00000 .00000 .0001$ $1.000010 .00000 .00000 .00000 .0000 \quad 0.0000 \quad 0.00000 .00000 .00000 .00000 .00000 .00000 .00000 .0000 \quad 0.00060 .0000$ $2.0+0010.00000 .00000 .00000 .00000 .00000 .00000 .00000 .00000 .00000 .00000 .00000 .00000 .00000 .0000$
\end{abstract}

$3.0+0010.0000 \quad 0.00000 .00000 .00000 .00000 .00000 .00000 .00000 .00000 .00000 .00000 .00000 .0000$

$5.0+0010.00000 .00000 .00000 .0000 \quad 1.00000 .00000 .0000,0.00000 .000 c 0.00000 .00000 .0000$

$7.0+001 \quad 0.0000 \quad 0.00000 .0000,0.00000 .0000 \quad 0.00000 .00000 .00000 .00000 .00000 .0000$

$1.0+0020.00000 .00000 .00000 .00900 .00000 .00000 .00000 .00000 .00000 .00000 .0000$

$2.0+0020.00000 .00000 .00000 .00000 .00000 .00000 .00000 .00000 .0000$

$3.0+0020.0000 \quad 0.00000 .00000 .00000 .00000 .00000 .00000 .0000$

$5,0+0020.00000 .00000 .00000 .00000 .00000 .00000,0000$

$7.0+0020.000 n \quad 0.00000 .00000 .0070 \quad 1.00000 .0000$

$1,0+0030.00000 .00000 .00000 .00000 .00000 .0000$

ALPHA $1.000 \quad 2.000 \quad 3.000 \quad 5.000 \quad 7.000 \quad 10.00 \quad 20.00 \quad 30.00 \quad 50.00 \quad 70.00 \quad 100.0 \quad 200.0 \quad 300.0 \quad 500.0 \quad 700.0 \quad 1000$ BETA

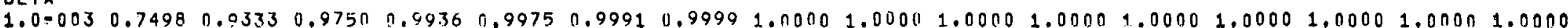

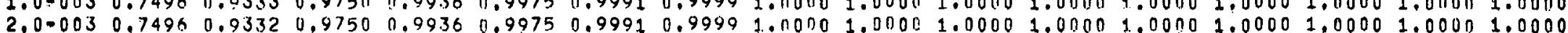

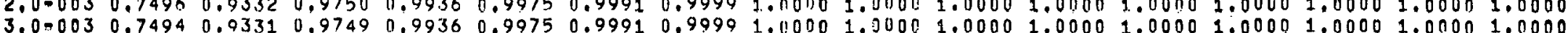

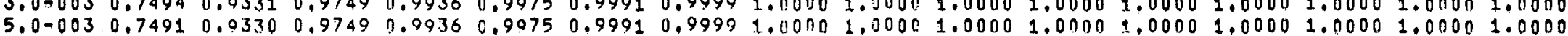

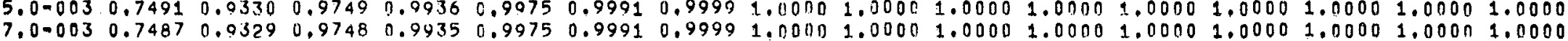
$1.0-0020.7481 \quad 0.9327 \quad 0.9748 \quad 0.9935 \quad 0.9975 \quad 0.99910 .99991 .00001 .00001 .00001 .00001 .00001 .00001 .00001 .00011 .0000$ $2.0-0020.74620 .93210 .97450 .99350 .90740 .99910 .99991 .00001 .00001 .00001 .00001 .00001 .00001 .0000$

$3,000020.74420 .93140 .9742 \quad 0.9934 \quad 0.99740 .9991 \quad 0.99991 .00 n 01.00001 .00001 .00001 .00001 .0000$

$5.0=0020.7403 \quad 0.03 n 10.97370 .99330 .99740 .99910 .99991 .00001 .00001 .00001 .00001 .0000$

$7,0=0020.7361 \quad 0.9287 \quad 0.97320 .99310 .99730 .99900 .99991 .00001 .00001 .00001 .0000$

$1.0=0010.72970 .02650 .97230 .99290 .99720 .99900 .99991 .00001000011 .00001 .0000$

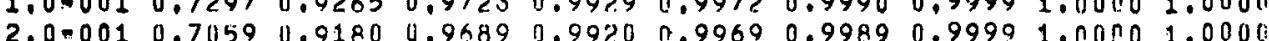

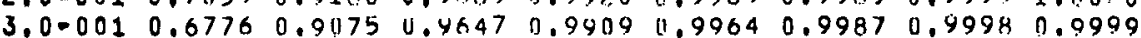

$\begin{array}{lllllllll}5.0 .001 & 0.6038 & 0.8767 & 0.9519 & 0.9575 & 0.9951 & 0.9982 & 0.9998\end{array}$

$7.0 .001 \quad 0.5050 \quad 0.8264 \quad 0.9299 \quad 0.9814 \quad 0.9927 \quad 0.9974$

$1.0+000 \quad 0.3333 \quad 0.7000 \quad 0.8667 \quad 0.9627 \quad 0.9852 \quad 0.9946$

$2.0+000 \quad 0.0455 \quad 0.1818 \quad 0.3824 \quad 0.7110$

$3,0+000 \quad 0.0082: 0.0370 \quad 0.0968$

$5,0+000 \quad 0.0008 \quad 0.0036$

$7,0+000 \quad 0.0002$

$1.0+0010.0000$ 


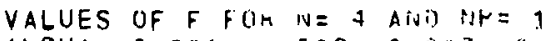

ALPHA 0.001 y.0त2 0.003 0.0105

ก. ก 07

0.01

(i. 02

0.03

0.0

0.07

0.1

0,2

0.3

0.5

$n, 7$ BETA

$1.0=0030.0010 \quad 0.00200 .0113 n \quad 0.0050 \quad 0.0070 \quad 0.0100 \quad 0.0200 \quad 0.0300 \quad 0.05010 .06990 .0999 \quad 0.1996 \quad 0.2981 \quad 0.4836 \quad 0.6392$

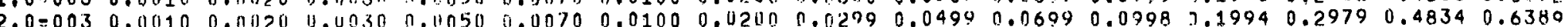

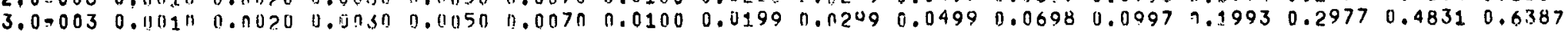

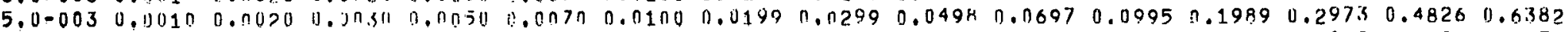

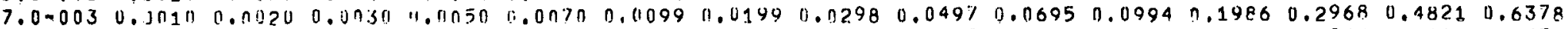

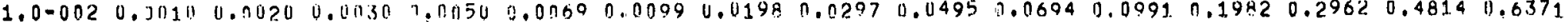

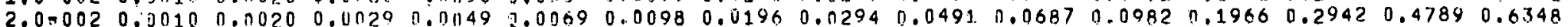
$3,0=0020.00100 .00190 .00290 .0049 \quad 0.0068 \quad 0.0097 \quad 0.0194 \quad 0.02920 .04860 .06810 .09740 .1951 \quad 0.29210 .4765 \quad 0.6325$ $5,0=0020.00100 .0090 .00290 .00480 .00670 .00950 .01910 .02860 .04770 .06690 .09570 .19210 .28820 .47170 .6281$.

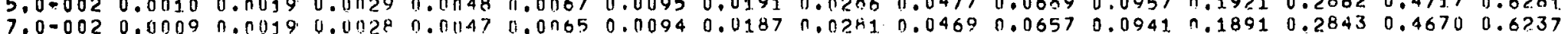

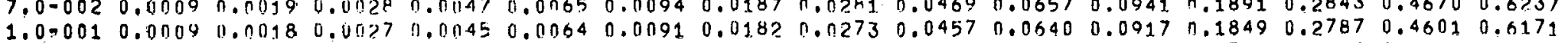

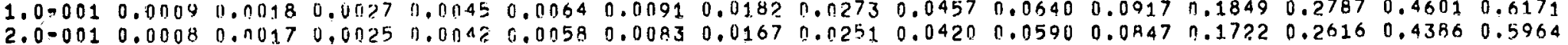

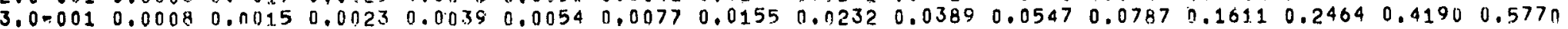

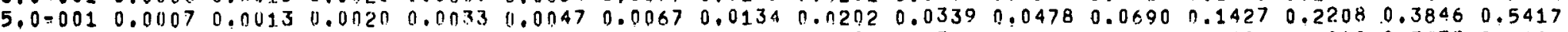
$\begin{array}{llllllllllllllll}7.05001 & 0.0006 & 0.001 \varepsilon & 0.0018 & 0.01129 & 0.0041 & 0.0059 & 0.0119 & 0.0179 & 0.0300 & 0.0424 & 0.061 .3 & 0.1280 & 0.2000 & 0.3555 & 0.5105\end{array}$

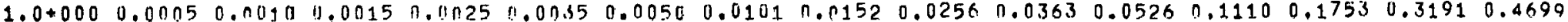
2.00000 .00030 .00070 .00100 .00170 .00230 .00340 .00680 .01020 .01720 .02450 .03570 .07680 .12410 .23810 .3715

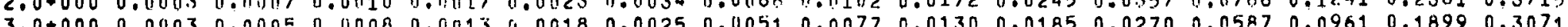
$3.0+000$ 0.0003 0.000

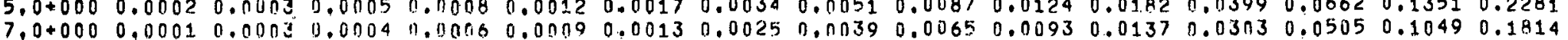

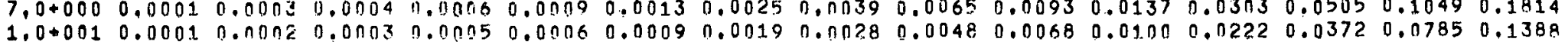
$2.0+0010.000 \pi 0.00010 .0 n 01.0 .00020 .00030 .00050 .00100 .00150 .00250 .00360 .0 n 530.01170 .01980 .0427$

$3.0+0010.00000 .00030 .0001 .0 .00020 .00020 .00030 .00070 .00100 .00170 .00240 .00360 .00800 .0135$

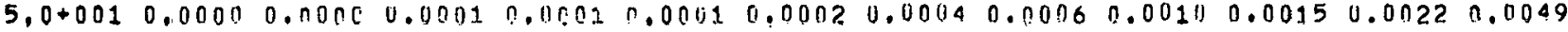

$7.0+0010.0000 \quad 0.0000 \quad 0.00000 .0001 \quad 0.00010 .00010 .00030 .00040 .00070 .00110 .0016$

$1.0+002 \quad 0.0000 \quad 0.00000 .0000 \quad 0.0000 \quad 0.00010 .00010 .00020 .00030 .00050 .00070 .0011$

$2.0+0020.00000 .00000 .00000 .00000 .00000 .00010 .00010 .00020 .0003$

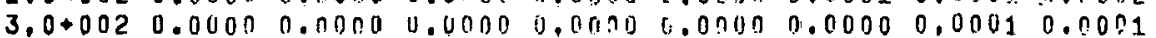

$5.0+0020.00000 .00000 .00000 .00 ! 100,00000.00000,0000$

$7.0+0020.00000 .00090 .00000 .00000 .0000 \quad 0.0000$

$1,0+0030.00000 .00000 .00000 .001300 .0000 \quad 0.0000$ ETA

$1.0 .003 \quad 0.7998 \quad 0.0677 \quad 0.9917 \quad 0.9987 \quad 0.9096 \quad 0.9999 \quad 1.00001 .00001 .00001 .00001 .00001 .00001 .00001 .00001 .000 n 1.0000$ $2.0=0030.7997 \quad 0.06770 .99170 .9987 \quad 0.99960 .99991 .00001 .00001 .00001 .00001 .00001 .00001 .00001 .00001 .00001 .0000$

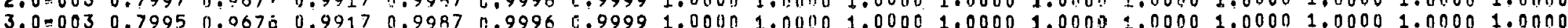

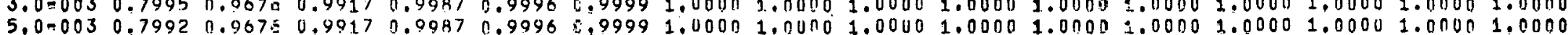

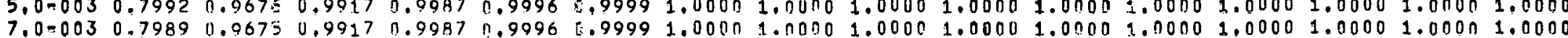
$1.0-0020.79840 .06740 .9917 \quad 0.99470 .9996 \quad 5.99991 .00001 .00501 .00001 .00001 .00001 .00001 .00001 .00001 .00001 .0000$ 2.070020 .79680 .96710 .99160 .99470 .99960 .99991 .00001 .00001 .00001 .00001 .00001 .00001 .00001 .0000

$3.0 .0020 .7952 \quad 0.06630 .99150 .99870 .99960 .99991 .00001 .00001 .000111 .0000=00001.00001 .0000$

$5.0=002 \quad 0.79210 .9662 \quad 0.99130 .97870 .99960 .99991 .00001 .00001 .00001 .0000 \div .00001 .0000$

$7.0=0020.7890 \quad 0.9655 \quad 0.99120 .97860 .9996 \quad 0.99991 .00001 .00001 .00001 .0000=.0000$

$1.0=001 \quad 0.7843 \quad 0.06460 .99090 .9786 \quad 0.9996 \quad 0.99991 .00001 .00001 .00001 .0000 \div .0000$

$2.0 .001 \quad 0.7692 \quad 0.00150 .9901 \quad 0.9985 \quad 0.9996 \quad 0.9999 \quad 1.00001 .00001 .0000$

$3.0 .0010 .7547 \quad 0.95850 .949 z \quad 0.9433 \quad 0.9995 \quad 0.9999 \quad 1.00001 .0000$

$5.0=0010.72730 .95240 .9877 \quad 0.99131 \quad 0.9995 \quad 0.99991 .0000$

$7,0.0010,70180.94640,9860 \quad 0.9978 \quad 0.9994 \quad 0.9998$

$1.0+000 \quad 0.6667 \quad 0.9375 \quad 0.9836 \quad 0.9974 \quad 0.9993 \quad 0.9998$

$2,0+000 \quad 0.5714 \quad 0.9091 \quad 0.9756 \quad 0.9962$

$3.0+000 \quad 0.5000 \quad 0.8824 \quad 0.9677$

$5.0+000 \quad 0.4000 \quad 0.8333$

$7.0+000 \quad 0.3333$

$1.0+0010.2667$ 
$\begin{array}{llllllllllllllll}\text { VALUES OF F FOH N }=4 & \text { AND NP }=\text { ? } \\ \text { ALPHA } & 0,001 & 0.002 & 0.003 & 0.005 & 0.007 & 0.01 & 0.02 & 0.03 & 0.05 & 0.07 & 0.1 & 0.2 & 0.3 & 0.5 & 0.7\end{array}$ BET

$1.0-0030.0010 \quad 0.0020 \quad 0.0030 \quad 0.0050 \quad 11.0070 \quad 0.0100 \quad 0.0200 \quad 0.03000 .0500 \quad 0.06990 .09990 .19960 .29810 .48360 .6392$ $2,0.0030 .00100 .0020 \quad 0,00300.0 n 50 \quad 0.0070 \quad 0.0100 \quad 0.02000 .02990 .04990 .06990 .09980 .19940 .2979 \quad 0.48340 .6389$ $3.0=003 \quad 0.0010 \quad 0.0020 \quad 0.0030 \quad 0.01150 \quad 0.0 n 70 \quad 0.0100 \quad 0.0199 \quad 0.112990 .04990 .06980 .0997 \quad 0.1993 \quad 0.2977 \quad 0.4831 \quad 0.6387$ $5.000030 .0010 \quad 0.0020 \quad 0.0030 \quad 0.01150 \quad 0.0070 \quad 0.0100 \quad 0.0199 \quad 0.02990 .04980 .0697 \quad 0.0995 \quad 0.1989 \quad 0.2972 \quad 0.4826 \quad 0.6382$ 7.0-003 $0.0010 \quad 0.0020 \quad 0.0030 \quad 0.00500 .0 n 7 n \quad 0.0099 \quad 0.01990 .02980 .0497 \quad 0.06950 .0094 \quad 0.1986 \quad 0.2968 \quad 0.4821 \quad 0.6378$

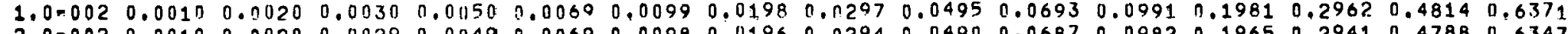

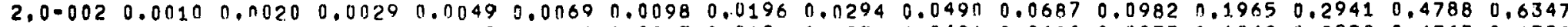
$3.0-002 \quad 0.001 n \quad 0.00190 .00290 .00490 .0068 \quad 0.0097 \quad 0.0194 \quad 0.02910 .0486 \quad 0.06800 .0973 \quad 0.1949 \quad 0.2920 \quad 0.4763 \quad 0.6323$ $5.0=0020.00100 .00190 .00290 .00480 .0 n 67 \quad 0.00950 .0190 \quad 0.02850 .04760 .06670 .09550 .19170 .28770 .47110 .6275$ 7.0 .0020 .00090 .00190 .00280 .00470 .07650 .00930 .01860 .02500 .04670 .06540 .09370 .18840 .28340 .46590 .6226

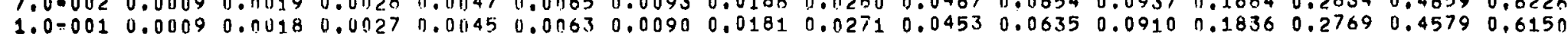

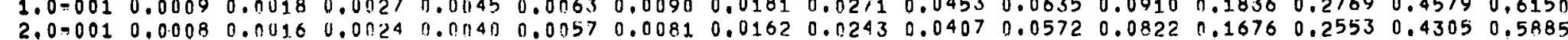
$\begin{array}{llllllllllllllllll}3.0 .001 & 0.0007 & 0.0014 & 0.0022 & 0.0036 & 0.0050 & 0.0072 & 0.0145 & 0.0218 & 0.0365 & 0.051 .4 & 0.0740 & 0.1522 & 0.2342 & 0.4028 & 0.5606\end{array}$

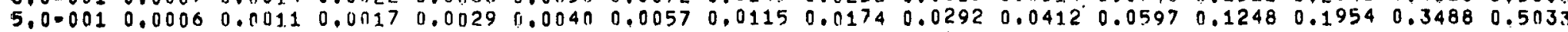
$\begin{array}{lllllllllllllllllll}7.0 .001 & 0.0005 & 0.0039 & 0.0014 & 0.0023 & 0.0032 & 0.0046 & 0.0092 & 0.0139 & 0.0235 & 0.0332 & 0.0483 & 0.1023 & 0.1626 & 0.2998 & 0.4474\end{array}$ $1.0+000 \quad 0.0003 \quad 0.0007 \quad 0.0010 \quad 0.00170 .0023 \quad 0.0034 \quad 0.0068 \quad 0.01020 .01720 .02450 .0357 \quad 0.0768 \quad 0.12410 .23810 .3715$ $2.0+000 \quad 0.00010 .0003 \quad 0.0 n 04 \quad 0.0007 \quad 0.0 n 10 \quad 0.00140 .00290 .00440 .00750 .0106 \quad 0.0156 \quad 0.03440 .0573 \quad 0.11810 .2021$

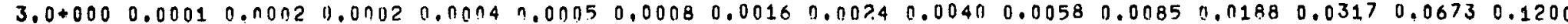
$5.0+0000.00000 .00010 .00010 .00020 .00020 .00030 .00070 .00100 .00170 .00240 .00360 .00800 .01350 .02940 .0541$ $3.0+0000.00000 .0000 .00010 .10010 .00010 .00020 .00040 .00050 .00090 .00130 .00190 .00440 .00740 .01620 .0302$ $1.0+0010.00000 .0000 .00000 .00000 .00010 .00010 .0002 .000030 .00050 .00070 .00100 .00220 .00380 .00840 .0157$

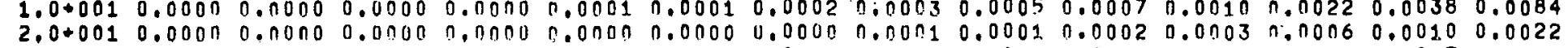
$3.0+0010.00000 .00000 .0 n 000.0 n 000.00000 .00000 .00000 .00000 .00010 .00010 .00010 .00030 .0005$ $5,0+0010,00000.00000 .00000 .00000 .00000 .00000 .00000 .00000 .00000 .00000 .00000 .0001$

$7.0+0010.0000 \quad 0.0000 \quad 0.0000 \quad 0.00000 .00000 .00000 .00000 .00000 .00000 .00000 .0000$ $1.0+0020.0000 \quad 0.0000 \quad 0.0000 \quad 0.00000 .0000 \quad 0.00000 .00000 .00000 .00000 .00000 .0000$

$2.0+0020.00000 .00000 .00000 .00000 .00000 .00000 .00000 .00000 .0000$

$3.0+0020.00000 .0000 \quad 0.00000 .00000 .00000 .00000 .00000 .0000$

$5,0+0020.000 n 0.00000 .0000 \quad 0.00000 .00000 .00000 .0000$

$7,0+0020.00000 .00000,00000.00000 .00000 .0000$

$1.0+0030.00000 .0000 \quad 0.00000 .0000 \quad 0.00000 .0000$

$$
\text { AETA }
$$

$1.090030 .79980 .0677 \quad 0.99170 .99870 .9996 \quad 0.99991 .00001 .00001 .00001 .00001 .00001 .00001 .00001 .00001 .00001 .0000$ $2,0=0030.7997 \quad 0.0677 \quad 0.9917 \quad 0.9987 \quad 0.99960 .99991 .00001 .00001 .00001 .00001 .00001 .00001 .00001 .00001 .00001 .0000$ $3.0=0030.7995 \quad 0.96760 .99170 .9987 \quad 0.9996 \quad 0.99991 .00001 .00001 .00001 .00001 .00001 .00001 .00001 .00001 .00001 .0000$ 5.0-003 $0.79920 .96760 .99170 .9987 \quad 0.99960 .99991 .00001 .00031 .00001 .00001 .00001 .00001 .00001 .00001 .00001 .0000$ 7.0-003 $0.79890 .06750 .99170 .9997 \quad 0.99960 .99991 .00001 .00001 .00001 .00001 .00001 .00001 .00001 .00001 .00001 .0000$ $1.0-0020.79840 .06740 .99170 .99870 .99960 .99991 .00001 .00001 .00001 .00001 .00001 .00001 .00001 .00001 .00001 .0000$ 2.0-002 0.79670 .96710 .99160 .99471 .99960 .99991 .00001 .001101 .00001 .00001 .00001 .00001 .00001 .0000 3.0 .0020 .79510 .96680 .99150 .99700 .99960 .99991 .00001 .00001 .00001 .00001 .00001 .00001 .0000 $5.000020 .79170 .96610 .9913 \quad 9.9987 \quad 1.99960 .99991 .00001 .00701 .00001 .00001 .00001 .0000$

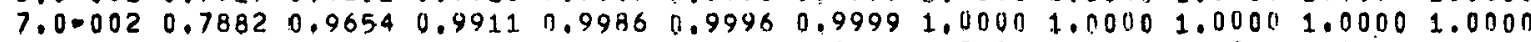
$1.0=0010.7828 \quad 0.06430 .99080 .9986 \quad 0.9996 \quad 0.99991 .00001 .00001 .00001 .00001 .0000$

$2.0=0010.76340 .9603 \quad 0.9894 \quad 0.9994 \quad 0.9996 \quad 0.99991 .00001 .00001 .0000$

$\begin{array}{llllllllll}3.0 .001 & 0.7421 & 0.9557 & 0.9485 & 0.9982 & 11.9995 & 0.9999 & 1.0000 & 1.0000\end{array}$

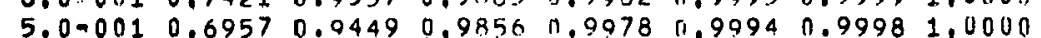

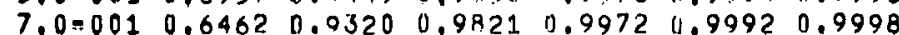

$\begin{array}{lllllllll}1.0+000 & 0.5714 & 0.9091 & 0.9756 & 0.9962 & 0.9989 & 0.9997\end{array}$

$2.0+000 \quad 0.3636 \quad 0.8108 \quad 0.9449 \quad 0.9911$

$3,0+000 \quad 0.2353 \quad 0.6977 \quad 0.9023$

$5,0+000 \quad 0.1143 \quad 0.491 .8$

$7.0+000 \quad 0.0656$

$1.0+0010.0348$ 
VALUES OF F FOR $N=4$ AND $N P=3$ BETA

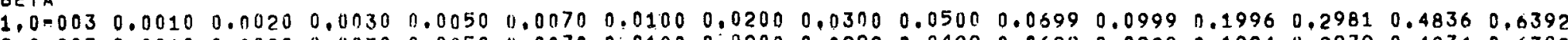

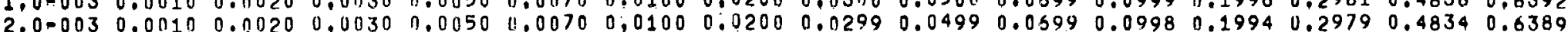
$3.0 .0030 .0010 \quad 0.01200 .0030 \quad 0.00500 .00700 .0100 \quad 0.01990 .02990 .04990 .06980 .09970 .19930 .29770 .48310 .6387$

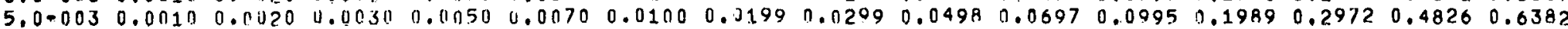

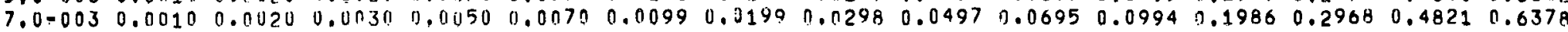

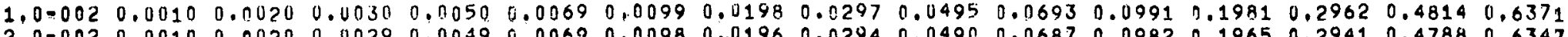
$2,0=002 \quad 0.0010 \quad 0.0020 \quad 0.0029 \quad 0.0049 \quad 0.00690 .0098 \quad 0.0196 \quad 0.0294 \quad 0.0490 \quad 0.0687 \quad 0.0982 \quad 0.1965 \quad 0.2941 \quad 0.4788 \quad 0.6347$ $\begin{array}{llllllllllllllll}3.0-002 & 0.0010 & 0.0019 & 0.0029 & 0,0049 & 0.0068 & 0.0097 & 0.0194 & 0.0291 & 0.0486 & 0.0680 & 0.0973 \quad 0.1949 & 0.2920 & 0.4763 \quad 0.6323\end{array}$

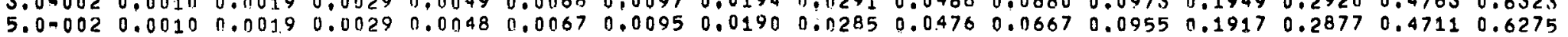

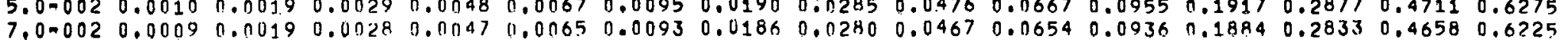

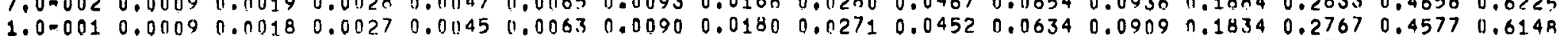

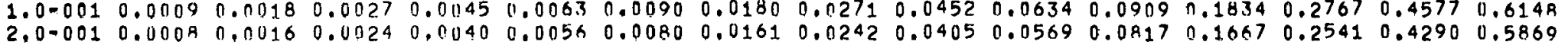
$3.0 .0010 .00070 .00140 .0021 \quad 0.00350 .0050 \quad 0.0071 \quad 0.0142 \quad 0.02140 .03580 .0504 \quad 0.0727 \quad 0.1498 \quad 0.2308 \quad 0.3982 \quad 0.5558$

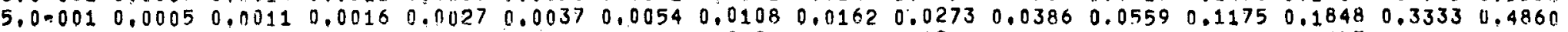
$\begin{array}{lllllllllllllllllll}7.0 .001 & 0.0004 & 0.0008 & 0.001 ? & 0.0020 & 0.0028 & 0.0040 & 0.0080 & 0.0121 & 0.0204 & 0.0289 & 0.0420 & 0.0897 & 0.1437 & 0.2701 & 0.4118\end{array}$

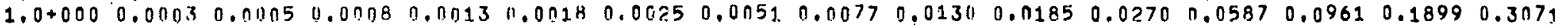
$2.0+000 \quad 0.00010 .0001 \quad 0.00820 .00030 .0005 \quad 0.0007 \quad 0.00140 .00210 .00350 .0050 \quad 0.0074 \quad 0.01640 .0270 \quad 0.05880 .1057$ $3.0+0000.00000 .00010,00010.00010 .00020 .00030 .00050 .00080 .00130 .00190 .00280 .00620 .01050 .02290 .0424$ $5.0+000 \quad 0.00000 .00000 .00000 .00000 .00000 .00010 .00010 .00020 .00030 .00050 .00070 .00160 .00270 .00600 .0112$

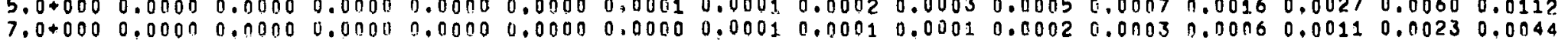

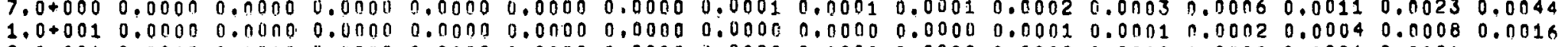

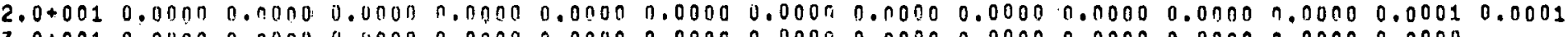
$3.0+0010.00000 .00000 .07000 .00000 .00000 .00000 .00000 .00000 .00000 .00000 .00000 .00000 .0000$

$5.0+0010.00000 .00000 .00000 .00000 .00000 .0000 \quad 0.00000 .00000 .00000 .00000 .00000 .0000$

$7.0+0010.0000 \quad 0.00000 .0000 \quad 0.0000 \quad 0.0000 \quad 0.0000 \quad 0.00000 .00000 .00000 .00000 .0000$

$1.0+0020.00000 .00000 .00000 .00000 .00000 .00000 .00000 .00000 .00000 .00000 .0000$

2.0+002 $0.00000 .0000 \quad 0.00000 .0 n 000.0000 \quad 0.00000 .0000 \quad 0.00000 .0000$

$3,0+0020.0000 \quad 0.00000 .0000 \quad 0.0090 \quad 0.0000 \quad 0.0000 \quad 0.0000 \quad 0.0000$

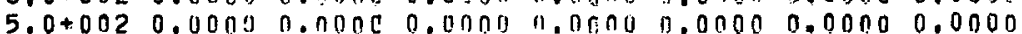

$7.0+0020.00000 .0000 .00000 .00000 .00000 .0000$

$1,0+0030.00000 .00060 .000011 .00000 .00000,0900$ BETA

ALPHA $1.000 \quad 2.005 \quad 3.000 \quad 5.000 \quad 7.000 \quad 10.00 \quad 20.00 \quad 30.00 \quad 50.00 \quad 70.00 \quad 100.0 \quad 200.0 \quad 300.0 \quad 500.0 \quad 700.0 \quad 1000$

$1.0-0030.7998 \quad 0.96770 .9917 \quad 0.99870 .9996 \quad 0.99991 .00001 .000001 .00001 .00001 .00001 .00001 .00001 .00001 .00001 .0000$ 2.0-003 0.79970 .06770 .9917 1.9997 0.99960 .99991 .00091 .00001 .00001 .00001 .00001 .00001 .00001 .00001 .00001 .0000 $3.0-0030.79950 .9670 \quad 0.99170 .99870 .99960 .99991 .00001 .00001 .00001 .00001 .00001 .00001 .00001 .00001 .00001 .0000$ $5,0=0030.79920 .06760 .99170 .9987 \quad 0.99960 .99991 .00001 .00001 .00001900001 .00009 .00001 .00001 .00001 .00001 .0000$

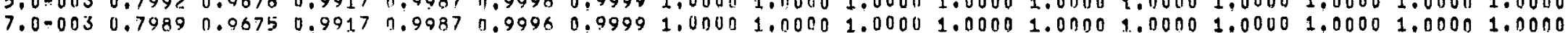

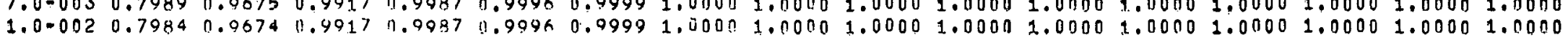

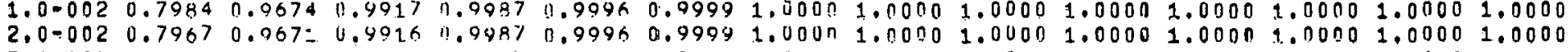
$3,0-0020.79510 .96680 .99158 .99970 .9996 \quad 0.99971 .00001 .00001 .00001 .00001 .00001 .00001 .0000$ $5.0=0020.79170 .966=0.99130 .9987 \quad 1.9996 \quad 0.99971 .00001 .00001 .00001 .00001 .00001 .0000$

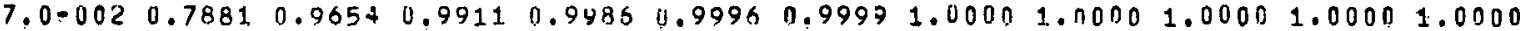

$1.0=001 \quad 0.7826 \quad 0.9643 \quad 0.9908 \quad 11.9986 \quad 0.9996 \quad 0.9999 \quad 1.00001 .00001 .00001 .00001 .0000$

$2.0=0010.7622 \quad 11.9601 \quad 0.9897 \quad 0.99840 .9996 \quad 0.9999 \quad 1.0000 \quad 1.00001 .0000$

$3.0=001 \quad 0.7384 \quad 0.95490 .9883 \quad 0.9942 \quad 0.9995 \quad 0.9999 \quad 1.00001 .0000$

$5.0=001 \quad 0.6809 \quad 0.9412 \quad 0.9846 \quad 0.9976 \quad 0.9943=.99981 .0000$

$\begin{array}{lllllll}7.0 .001 & 0.6123 & 0.0221 & 0.9793 & 0.9368 & 0.9091 & 0.9998\end{array}$

$1.0+000 \quad 0.5000 \quad 0.98240 .9677 \quad 0.9349 \quad 0.9986 \quad[.9996$

$2.0 .000 \quad 0.2105 \quad 0.6667 \quad 0.89890 .9811$

$3.0+000 \quad 0.0909 \quad 0.4285 \quad 0.750 n$

$5.0+000 \quad 0.0250 \quad 0.16: 13$

$7.0+000 \quad 0.0099$

$1,0+0010,003$ 


\begin{abstract}
VALUES OF F FOR $N=4$ AND $N P=4$ BETA $\begin{array}{llllllllllllllll}B E T A & \\ 1.0=003 & 0.0010 & 0.0020 & 0.0030 & 0.0050 & 0.0070 & 0.0100 & 0.0200 & 0.0300 & 0.0500 & 0.0699 & 0.0999 & 0.1996 & 0.2981 & 0.4836 & 0.6392 \\ 2.0-003 & 0.0010 & 0.0020 & 0.0030 & 0.0050 & 10.0070 & 0.0100 & 0.0200 & 0.0299 & 0.0499 & 0.0699 & 0.0998 & 0.1994 & 0.2979 & 0.4834 & 0.0389\end{array}$ $\begin{array}{llllllllllllllllll}3.0=003 & 0.0010 & 0.01020 & 0.0030 & 0.0050 & 0.007 n & 0.0100 & 0.0199 & 0.0299 & 0.0499 & 0.0698 & 0.0997 & 0.1993 & 0.2977 & 0.4831 & 0.6387\end{array}$

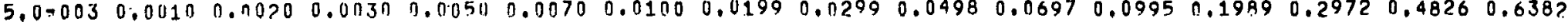
$7.0=003 \quad 0.0010 \quad 0.0020 \quad 0.0030 \quad 0.0050 \quad 0.0070 \quad 0.00990 .0199 \quad 0.02980 .0497 \quad 0.0695 \quad 0.0994 \quad 0.1986 \quad 0.2968 \quad 0.4821 \quad 0.6378$ $10.0020 .00100 .00200 .003110 .00510 .00690 .00990 .01980 .02970 .04950 .06930 .09910 .19810 .2962 \quad 0.48140 .6371$

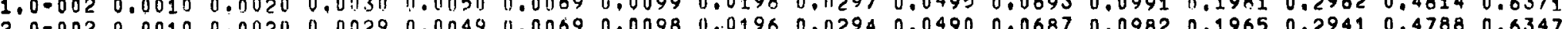

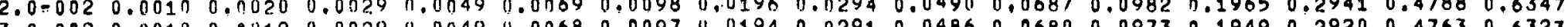
(1048 0.00670 .00950 .01900 .02850 .04760 .06670 .09550 .19170 $5,0=0020.00100 .0190 .01290 .01480 .01670 .00950 .01900 .12850 .04760 .06670 .09550 .19170 .28770 .47110 .6275$

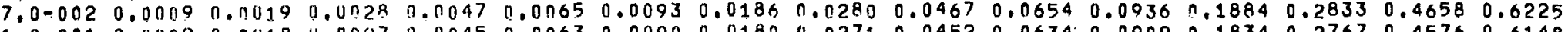
$1.0=0010.00090 .0018 \quad 0.0027 \quad 0.0045 \quad 0.0 n 63 \quad 0.0090 \quad 0.0180 \quad 0.02710 .04520 .06340 .09090 .18340 .27670 .45760 .6148$

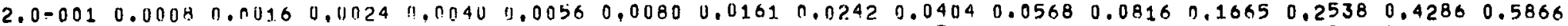

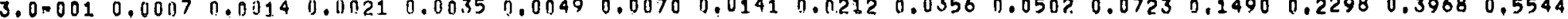

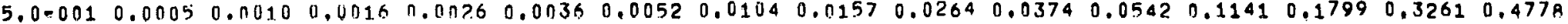
$\begin{array}{lllllllllllllllll}7.0-001 & 0.0004 & 0.00 n 7 & 0.0011 & 0.0118 & 0.0 n 25 & 0.0036 & 0.0073 & 0.0110 & 0.0186 & 0.0264 & 0.0385 & 0.0826 & 0.1329 & 0.2527 & 0.390 n\end{array}$ $1.0+000 \quad 0.00020 .0004 \quad 0.0006 \quad 0.0010 \quad 0.0014 \quad 0.00200 .00410 .00610 .01040 .0148 \quad 0.02170 .0475 \quad 0.0784 \quad 0.1579 \quad 0.2618$ $1.0+0000.0000 .0 .0010 .00010 .00020 .00020 .00030 .0007 .0 .00100 .00170 .00240 .00360 .00800 .01350 .02940 .0541$

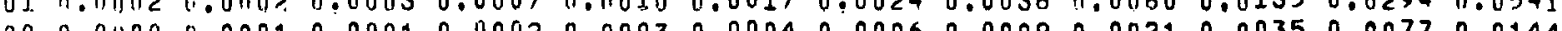
$3.0+0.0$ 0.00n 0.000 0.000

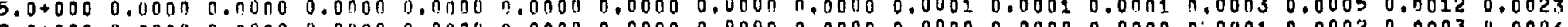

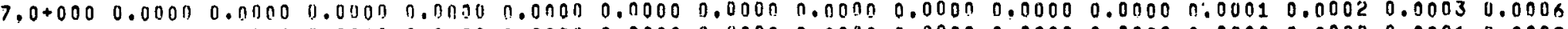
$1.0+0010.000 \pi 0.00000 .0000 n .0 n \pi 00.00000 .00000 .00000 .00000 .00000 .00000 .00000 .00000 .00000 .00010 .0002$ $2.0+0010.00000 .00000 .07000 .00000 .00000 .00000 .00000 .00000 .00000 .00000 .00000 .00000 .00000 .0000$

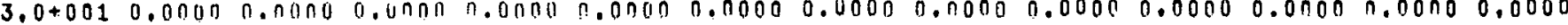

$5.0+0010.00000 .00000 .00000 .00000 .00000 .00000 .0000 \quad 0.00000 .00000 .00000 .00000 .0000$

$7.0+0010.00000 .00000 .030 n \quad 1.0000 \quad 0.0000 \quad 0.00000 .00000 .00000 .00000 .00000 .0000$

$1.0+0020.00000 .00000 .0000 \quad 1.00100 .0000 \quad 0.0000 \quad 0.0000 \quad 0.00000 .00000 .00000 .0000$

$2.0+0020.0000 \quad 0.0000 \quad 0.430 \pi 0.0030 \quad 0.0000 \quad 0.0000 \quad 0.0000 \quad 0.00000 .0000$

$3,0+0020.00000 .011000 .0000,0.00000 .0000 \quad 0.0000 \quad 0.00000 .0000$

$5.0+0020.00000 .00000 .0000,0.00000 .00000 .00000 .0000$

$7.0+0020.0000 \quad 1.0000 \quad 0.0000 \quad 0.0000 \quad 0.0000 \quad 0.0000$

$1.0+0030.00000 .00000 .00000 .00000 .0000 \quad 0.0000$

ALPHA $1.000 \quad 2.000 \quad 3.000 \quad 5.000 \quad 7.000 \quad 10.00 \quad 20.00 \quad 30.00 \quad 50.00 \quad 70.00 \quad 100.0 \quad 200.0 \quad 300.0 \quad 500.0 \quad 700.0 \quad 1000$ BETA

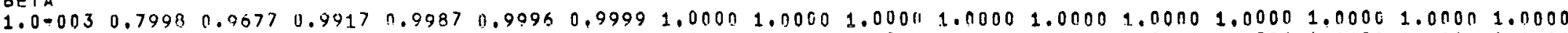
$2.0-0030.79970 .06770 .97170 .99770 .99960 .99991 .00001 .00001 .00001 .00001 .00001 .00001 .00001 .00001 .00001 .0000$ $2.0-0030.79050 .06760 .99170 .99870 .99960 .99991 .00001 .00001 .00001 .00001 .00001 .00001 .00001 .00001 .00001 .0000$ $5.0-0030.79920 .06760 .99170 .99970 .99960 .99991 .00001 .00001 .000 n 1.00001 .00001 .00001 .00001 .00001 .00001 .0000$ $5.0-0030.99920 .9676$.

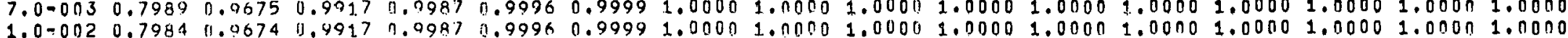

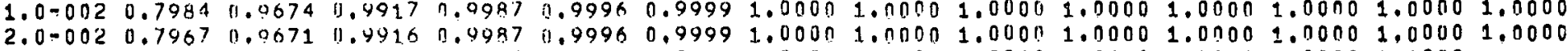
$3,0=0020.79510 .966810 .99150 .99870 .99950 .99991 .00001 .00001 .00001 .00001 .00001 .00001 .0000$

$5.0-002 \quad 0.79170 .06610 .9913 \quad 0.0997 \quad 0.9996 \quad 0.99991 .00001 .00001 .00001 .00001 .00001 .0000$

$7,0=002 \quad 0.7881 \quad 0.0654 \quad 0.9911 \quad 0.9986 \quad 10.9996 \quad 0.99991 .00001 .00001 .00001 .00001 .0000$

$1.0-0010.7826 \quad 0.9643 \quad 0.9908 \quad 3.9986 \quad 0.9996 \quad 0.99991 .00001 .00001 .00001 .00001 .0000$

$2.0-0010.76290 .0600 \quad 11.98970 .94840 .99960 .99991 .00001 .00001 .0000$

$\begin{array}{lllllllll}3.0001 & 0.7373 & 0.0547 & 0.9883 & 0.9982 & 0.9995 & 0.9999 & 1.0000 & 1.0000\end{array}$

$5.0=001 \quad 0.07370 .9393 \quad 0.9341 \quad 0.9975 \quad 1.9993 \quad 0.9998 \quad 1.0000$

$\begin{array}{lllllll}7.0001 & 0.5906 & 0.0154 & 0.9774 & 0.9965 & 0.9990 & 0.9998\end{array}$

$1.0+000 \quad 0.4444 \quad 0.9571 \quad 0.9600 \quad 0.9936 \quad 0.9982 \quad 0.9996$

$2.0+000 \quad 0.1143 \quad 0.4918 \quad 0.7947 \quad 0.961 \mathrm{H}$

$3.0+000 \quad 0.03290 .1987 \quad 10.4979$

$5.0+000 \quad 0.00510 .0370$

$7.0+000 \quad 0.0014$

$1.0+0010.0004$ 

$B E T A$

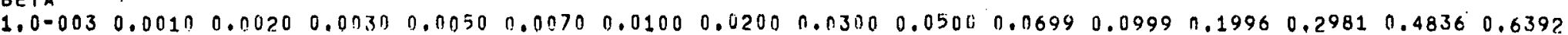
$2,0=0030.0010 \quad 0.0020 \quad 0.003011 .0050 \quad 0.0070 \quad 0.0100 \quad 0.0200 \quad 0.02990 .04990 .06990 .0998 \quad 0.19940 .29790 .48340 .6389$

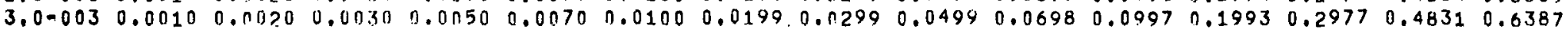

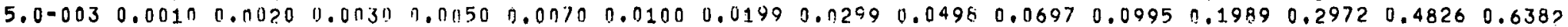

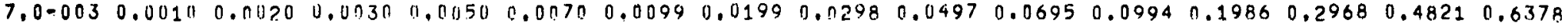

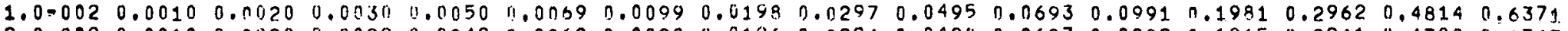
$2.0-0020.00100 .00200 .00290 .00490 .00690 .00980 .0196 \quad 0.02940 .04900 .06870 .09820 .1965 \quad 0.29410 .47860 .0347$ $3.0-0020.00100 .00190 .00290 .00490 .00680 .00970 .01940 .02910 .04660 .06800 .09730 .19490 .29200 .47630 .6323$

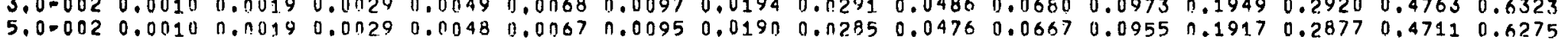

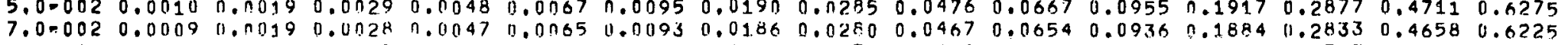
$1.000010 .00090 .0018 \quad 0.0027 \quad 0.0045 \quad 1.0003 \quad 0.0090 \quad 0.01800 .02710 .0452 \quad 0.06340 .0909 \quad 0.1834 \quad 0.2767 \quad 0.4576 \quad 0.0148$

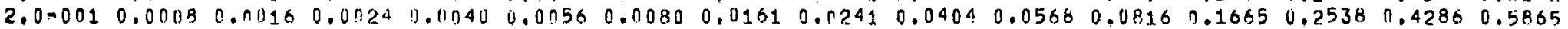
$\begin{array}{lllllllllllllllllll}3.0 .001 & 0.0007 & 0.011 .4 & 0.0121 & 0.0035 & 0.0049 & 0.0070 & 0.0141 & 0.0212 & 0.0356 & 0.0501 & 0.0722 & 0.1488 & 0.2295 & 0.3964 & 0.5540\end{array}$ 5.0-001 $0.0005 \quad 0.00100 .0015 \quad 0.0025 \quad 0.0036 \quad 0.0051 \quad 0.0103 \quad 0.01550 .02600 .03680 .05340 .11250 .1776 \quad 0.3226 \quad 0.4739$ $\begin{array}{llllllllllllllllllll}7.0 .001 & 0.0003 & 0.0007 & 0.0010 & 0.0017 .0 .0024 & 0.0034 & 0.0069 & 0.0104 & 0.0176 & 0.0250 & 0.0364 & 0.0782 & 0.1263 & 0.2 .417 & 0.3761\end{array}$ $1.0+000 \quad 0.0002 \quad 0.00 n 3 \quad 0.0705 \quad \pi .00 n 8 \quad 1.00120 .00170 .00340 .00510 .00870 .01240 .01820 .03090 .06620 .13510 .2281$ $2,0+0000.0000 .0100 .0000 n .00010 .00010 .00020 .00030 .00050 .00000 .00120 .00180 .00390 .00670 .01470 .0274$

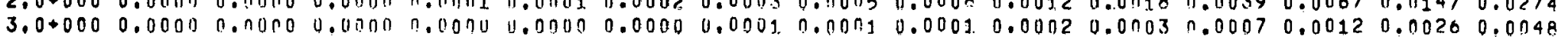

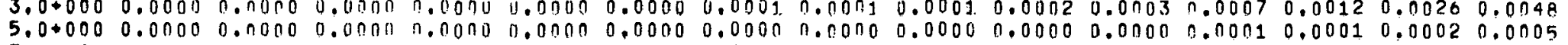
$7.0+000.0 .00000 .011000 .00000 .00000 .00000 .00000 .00000 .00000 .00000 .00000 .000010 .00000 .00000 .00000 .0001$

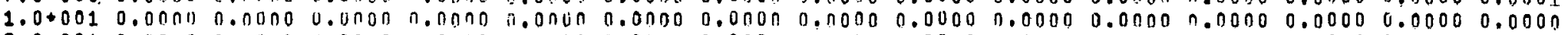
$2,0+0010.00000 .00000 .00001 .00000 .00000 .00000 .00000 .00000 .00000 .00000 .00000 .00000 .00000 .0000$

$3.0+0010.00000 .010000 .00110,1.00011 .00000 .00000 .00000 .00000 .00000 .00000 .00000 .00000 .0000$

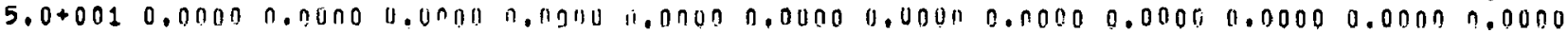

$7,0+0010.00000 .00000 .00000 .00 \pi 00.00000 .00000 .00000 .00000 .00000 .00000 .0000$

$1.0+0020.00000 .00000 .0000 .01100 .00000 .00000 .00000 .00000 .00000 .00000 .0000$

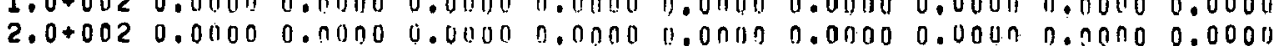

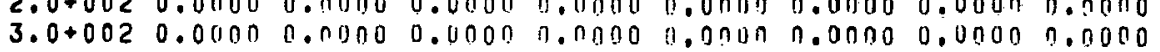

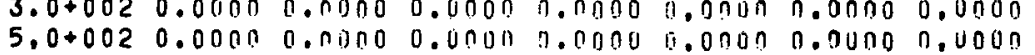

$7.0+0020.00000 .0000$ u. unon 1.00000 .00000 .0000

$1.0+0030.0000 \quad 0.0000 \quad 0.0000 \quad 0.0000 \% .0000 \quad 0.0000$ BETA

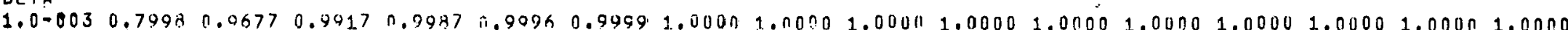

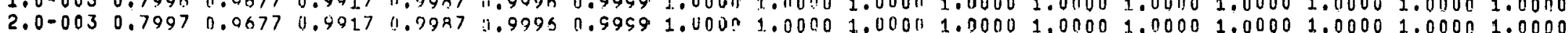

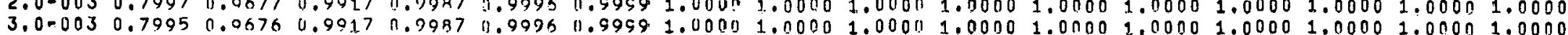

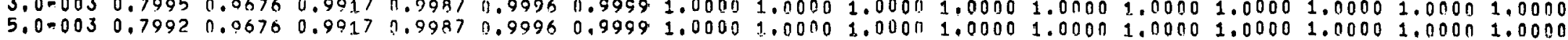

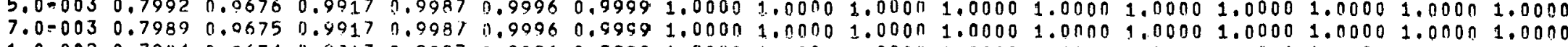

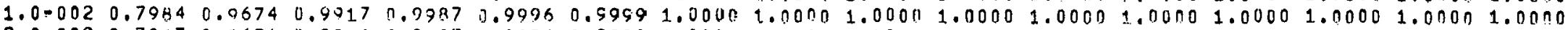
$2.0-0020.79670 .96710 .9916 \quad 0.9997 \quad 0.9996 \quad 0.99991 .00001 .00001 .00001 .00001 .00001 .00001 .00001 .0000$

$3.0-002 \quad 0.7951 \quad 0.96680 .99150 .9987 \quad 0.9996 \quad 0.99991 .00001 .000001 .00001 .00001 .00001 .00001 .0000$

$5.0-0020.79170 .96610 .99130 .9987 \quad 0.99960 .99991 .00001 .00001 .00001 .00001 .00001 .0000$

$7.0 .0020 .78810 .96540 .99110 .9986 \quad 1.9996 \quad 0.99991 .00001 .00001 .00001 .00001 .0000$

$1.0=001 \quad 0.7826 \quad 0.96430 .9908 \quad 0.9986 \quad 0.9996 \quad 0.99991 .00001 .00001 .000111 .00001 .0000$

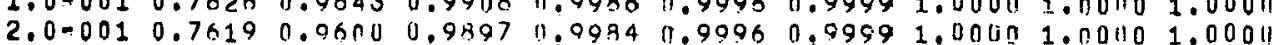

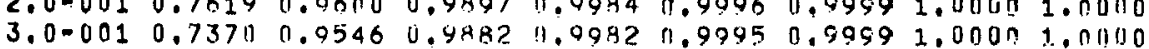

5.0-001 $0.6702 \quad 0.03840 .9959 \quad 0.9975 \quad 0.9993 \quad 0.39981 .0000$

$\begin{array}{lllllll}7.0-001 & 0.5763 & 0.9107 & 0.9761 & 0.9962,11.9990 & 0.9997\end{array}$

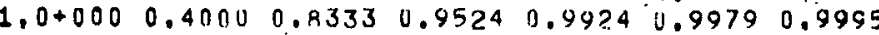

$2.0+000 \quad 0.0597 \quad 0.32260 .6557 \quad 0.9253$

$3.0+000 \quad 0.01090 .0761 \quad 0.2479$

$5.0+000 \quad 0.0010 \quad 0.0076$

$7.0+000 \quad 0.0002$

$1.0+001 \quad 0.0000$ 


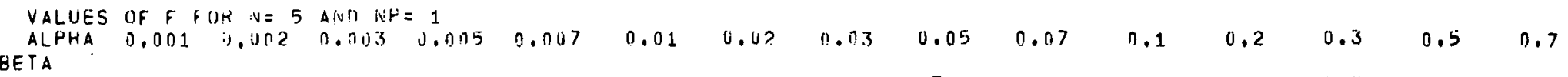

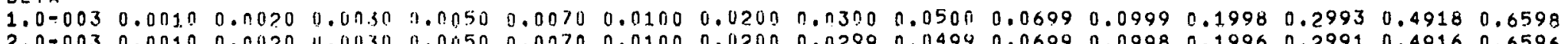

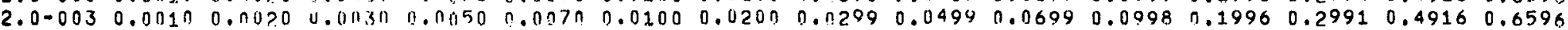

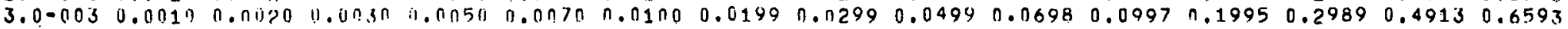

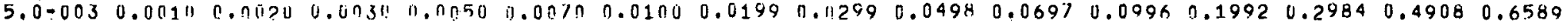

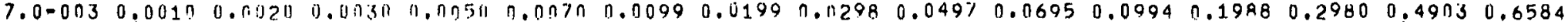

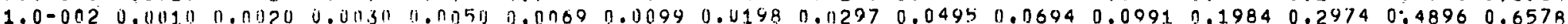

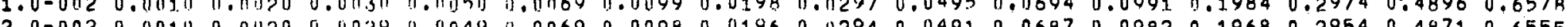

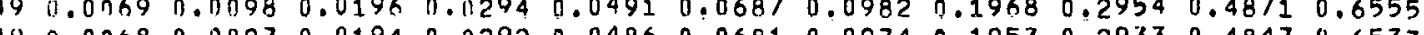
.

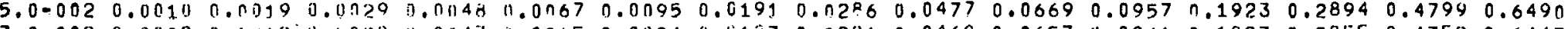

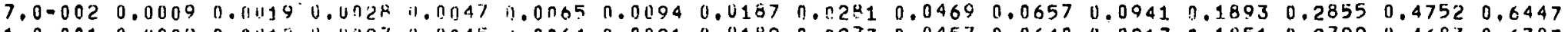
$1.0-0010.00090 .01318 \quad 0.01127 \quad 1.0045 \quad 1.0064 \quad 0.00910 .018 ? 0.0273 \quad 0.0457 \quad 0.0640 \quad 0.0917 \quad 0.1851 \quad 0.2799 \quad 0.4683 \quad 0.6383$

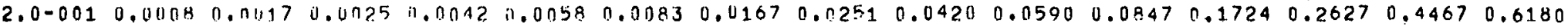

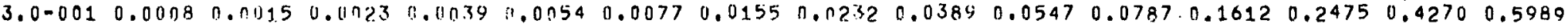

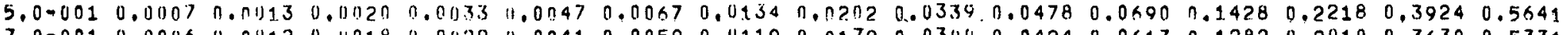

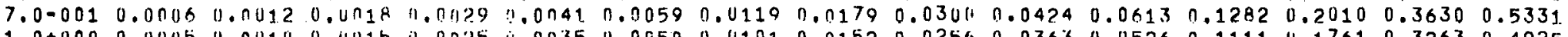

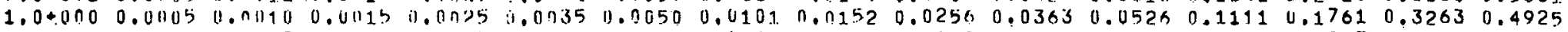
$2.0+000 \quad 0.0003$ u.non? 0.00110 .0017 i. 00230.00340 .00680 .01020 .01720 .02450 .03570 .07690 .12470 .24410 .3929

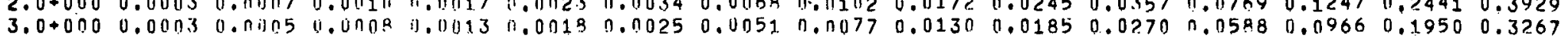

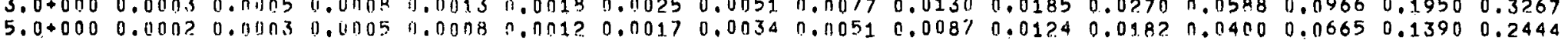

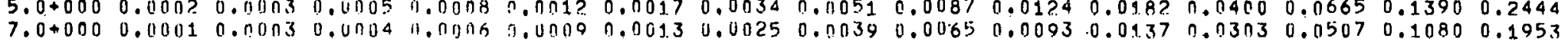
$1.0+0010.00010 .010 n 20.011330 .0011,00050.00090 .00190 .00280 .00460 .00680 .01 .000 .02220 .03740 .08090 .1500$

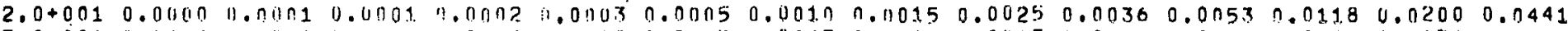

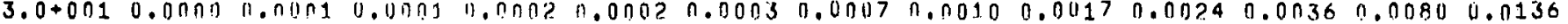

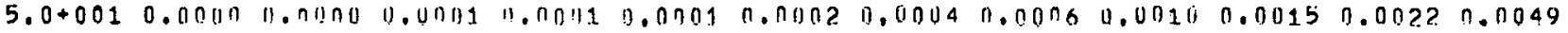

$7.0+0010.00000 .013000 .000(10.00010 .0002 \quad 0.00010 .00030 .00040 .000 \% 0.00110 .0016$

$1.0+0020.000 \pi 0.00000 .0 n n 0,0.000 j 0.0 n 020.00010 .00020 .00030 .00050 .00070 .0011$

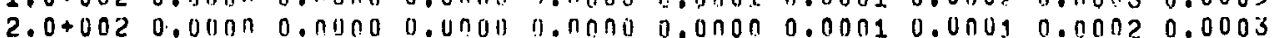

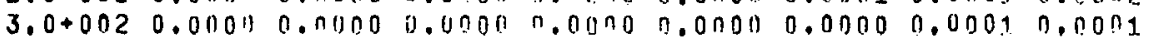

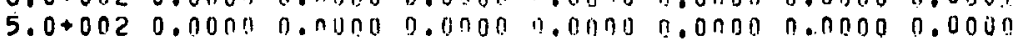

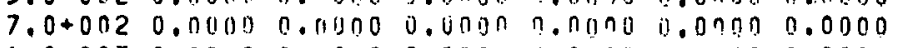

$1.0+0030.00000 .00000 .000011,00000.00000 .0000$

ALPHA $1.000 \quad 2.000 \quad 3.000 \quad 5.000 \quad 7.000 \quad 10.00 \quad 20.00 \quad 30.01050 .00 \quad 70.00 \quad 100.0 \quad 200.0 \quad 300.0 \quad 500.0 \quad 700.0 \quad 1000$ BETA

$1.0-0030.83320 .04410 .99730 .9407 \quad 0.99901 .00001 .00001 .001101 .00001 .00001 .00009 .00001 .00001 .00001 .00001 .0000$ $2.0-0030.83310 .08410 .99720 .090711 .99901 .00001 .00001 .00110900001 .00001 .00001 .00001 .00001 .00001 .00001 .0000$

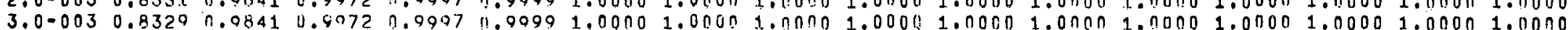

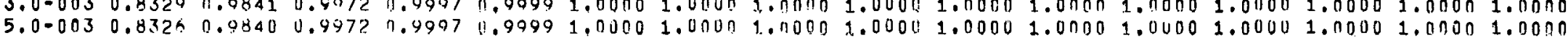

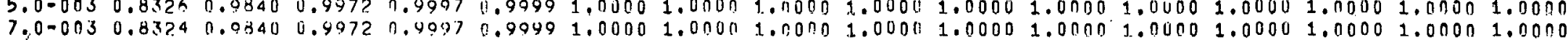
$1.0=0020.83190 .98400 .99720 .99970 .90901 .00001 .00601 .00001 .00001 .00001 .00001 .00001 .00001 .00001 .00001 .0000$ $2.0-0020.83050 .03380 .90170 .9997 \quad 1999991.00001 .00001 .001101 .00001 .00001 .00009 .00001 .00001 .0000$

$3.0 .0020 .82920 .93370 .997 ? 1.0997 \quad 1.99991 .00001 .00001 .00001 .00041 .00001 .00001 .00001 .0000$

$5,0-0020.82640 .98330 .9971 .0 .9997 \quad 1.9999 \quad 1.00001 .00001 .00001 .00001 .00001 .00001 .0000$

7.070020 .82370 .93300 .99710 .99970 .99991 .00001 .00003 .00001 .000111 .00001 .0000

$1.0-0010.8197 \quad 0.9876 \quad 0.9971) 0.9997 \quad 11.9999 \quad 1.00001 .00001 .0 .0901 .00001 .00001 .0000$

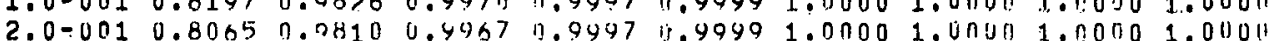

$3,0=0010.7937 \quad 0.91950 .49040 .99370 .97991 .00001 .00101 .00000$

$5.0-0010.769$ ? $0.9764 \quad 0.94590 .90960 .99991 .00001 .0000$

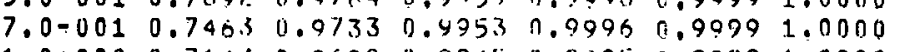

$1.0+000 \quad 0.714 .50 .0688 \quad 0.9945 \quad 0.9995 \quad 0.9999 \quad 1.0000$

$2.0+000 \quad 0.6250 \quad 0.9538 \quad 0.9918 \quad 11.9992$

$3.0+000 \quad 0.5550 \quad 0.0394 \quad 0.4591$

$5,0+000 \quad 0.4545 \quad 0.9118$

$7,0+000 \quad 0.3846$ 
VALUES OF F FOK $N=5$ ANU NPB $=2$ $B E T A$

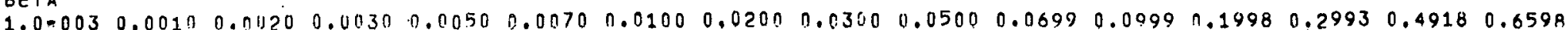

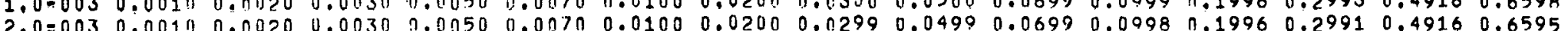
$2.0=003$
$3,0-0030.0010$

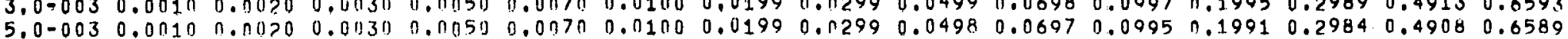

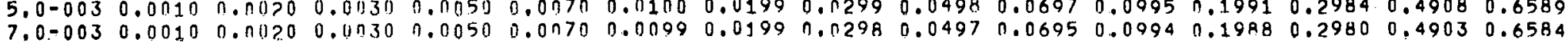

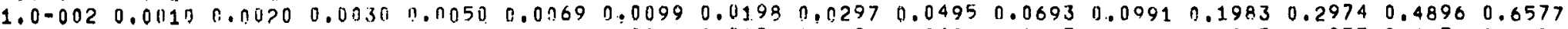

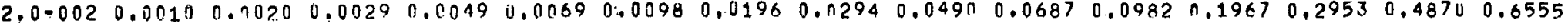
$\begin{array}{lllllllllllllllllll}3.0-002 & 0.0010 & 0.0019 & 0.0029 & 0.0049 & 0.0068 & 0.0097 & 0.0194 & 0.0291 & 0.0486 & 0.0680 & 0.0973 & 0.1951 & 0.2931 & 0.4845 & 0.0531\end{array}$

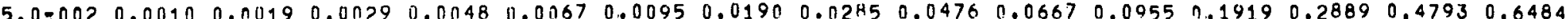
$7.0-0020.00090 .00190 .00280 .00470 .00650 .00930 .01860 .02300 .04670 .06540 .09370 .18860 .28460 .47400 .6436$ 0.01810 .07710 .04530 .06 0.610 ?

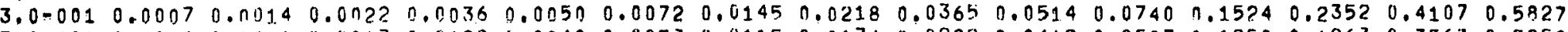
$\begin{array}{lllllllllllllllll}5.0-001 & 0.0006 & 0.0011 & 0.0017 & 0.0029 & 0.0040 & 0.0057 & 0.0115 & 0.0174 & 0.029 ? & 0.0412 & 0.0597 & 0.1250 & 0.1963 & 0.3563 & 0.5259\end{array}$ $\begin{array}{llllllllllllllll}7.0-001 & 0.0005 & 0.0009 & 0.0014 & 0.0033 & 0.0032 & 0.0046 & 0.0092 & 0.0139 & 0.0235 & 0.0332 & 0.0483 & 0.1024 & 0.1633 & 0.3067 & 0.4699\end{array}$

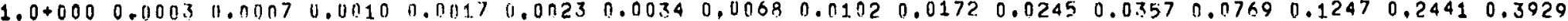
$2.0+000 \quad 0.00010 .0003 \quad 0.00040 .00170 .00100 .00140 .00290 .00440 .00750 .0106 \quad 0.0156 \quad 0.03450 .0576 \quad 0.12160 .2171$ $3,0+0000.00011100020 .40020 .00040 .00050 .00080 .00160 .04240 .00400 .00540 .00850 .01890 .03180 .06940 .1290$ $5.0+0000.00000 .0010 .00010 .00020 .00020 .00030 .00070 .00100 .00170 .00240 .00360 .00800 .01360 .03030 .0589$

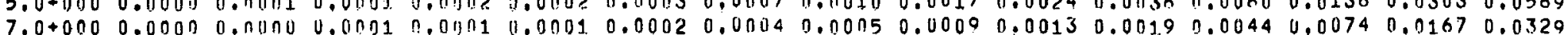

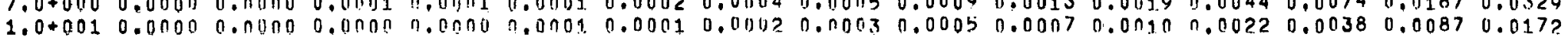

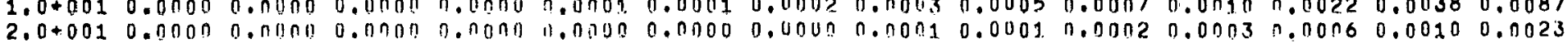

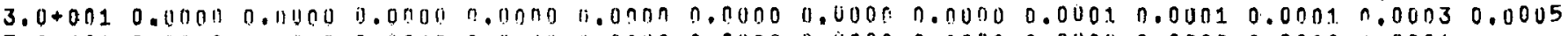
$5.0+0.010 .00000 .00000 .00000 .00100500000 .00000 .00000 .00000 .000110 .00000 .00000 .0001$

$7.0+0010.00000 .00000 .0000 \quad 0.00000 .00000 .00000 .00000 .00000 .00000 .00000 .0000$

$1.0+0020.00000 .00000 .011000 .00000 .00000 .00000 .00000 .00000 .00000 .00000 .0000$

$2.0+0020.0000 \quad 0.00000 .00000 .00000 .00000 .00000 .00000 .00000 .0000$

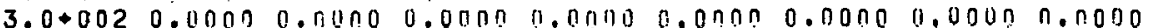

$5.0+002 \quad 0.0000 \quad 0.0000 \quad 0.0000 \quad 0.0000 \quad 0.0000 \quad 0.0000 \quad 0.0000$

$7,0+0020.00000 .0000 \quad 0.0000 \quad 0.00000 .0000 \quad 0.0000$

$1,0+003 \quad 0.00000 .00000,00000,00000.00000,0000$

$$
\text { AL }
$$

$1.0-0030.63320 .95410 .49730 .9497 \quad 0.99991 .00001 .00001 .00001 .000 n 1.00001 .00001 .04001 .00001 .00001 .00001 .0000$ $2.0 .0030 .83310 .98410 .49720 .9907 \quad 0.99991 .00001 .00001 .00001 .00001 .00001 .00001 .00001 .00001 .00001 .00001 .0000$ $3.0-0030.83290 .98410 .997 ? 0.99970 .99991 .00001 .00001 .00001 .00001 .00001 .00001 .04001 .00001 .00001 .00001 .0000$ $5.0=0030.83260 .9840 \quad 0.99720 .99970 .99901 .00001 .00001 .00001 .00001 .00001 .00001 .00001 .00001 .00001 .00001 .0000$ 5.0 .003
$7.0-0030.8324$

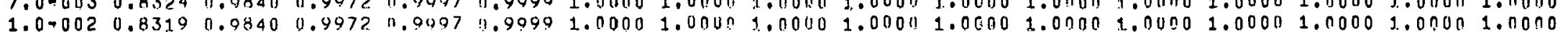

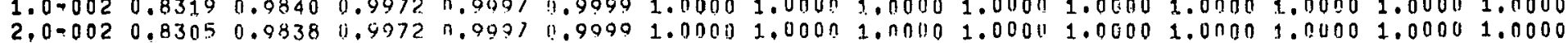

$3.0=0020.82910 .98360 .99720 .999780 .99491 .00001 .00001 .00001 .000111 .00001 .00001 .00001 .0000$

$5.0=0020.82610 .98330 .9971 .0 .99970 .99991 .00001 .00001 .00001 .000111 .00001 .00001 .0000$

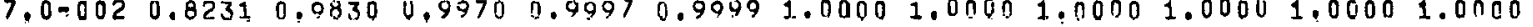

$1.0=0010.8183 \quad 0.9824 \quad 0.99700 .9997 \quad 0.9099 \quad 1.00001 .00001 .00001 .00001 .00001 .0000$

$2.0-6010.8013 \quad 0.98040 .9966 \quad 7.9997 \quad 0.9949 \quad 1.00001 .00001 .00001 .0000$

$3.0-0.010 .7825 \quad 0.0781 \quad 0.4962 \quad 11.9096 \quad 0.9099 \quad 1.00001 .00001 .0000$

$5.0=0010.7407 \quad 0.9725 \quad 1.9952 \quad 0.9996 \quad 11.9099 \quad 1.00001 .0000$

$7.0-0010.69540 .06590 .9940 \quad 0.9994 \quad 0.99991 .0000$

$1.0+000 \quad 0.6250 \quad 0.0538 \quad 0.9918 \quad 0.9992 \quad 0.99981 .0000$

$2.0+000 \quad 0.4167 \quad 0.8946 \quad 0.9819 .119982$

$3,0+0.00 \quad 0.27780 .8267 \quad 0.9654$

$5,0+000 \quad 0.13890 .566 .7$

$7,0+0000.0800$

$1.0+001 \quad 0.0431$ 

GETA

1.0-003 0.0010 0.0020 0.0n30 n.0050 1.0070 $0.0100 \quad 0.0200 \quad 0.0300 \quad 0.0500 \quad 0.0699 \quad 0.0999 \quad 0.1998 \quad 0.2993 \quad 0.4918 \quad 0.6598$ $2.0-003 \quad 0.001 \pi \quad 0.00200 .0030 \quad 1.005010 .0070 \quad 0.0100 \quad 0.0200 \quad 0.02990 .04990 .0699000998 \quad 0.19960 .29910 .4916 \quad 0.6595$

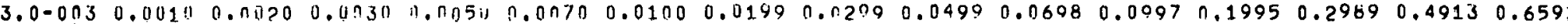

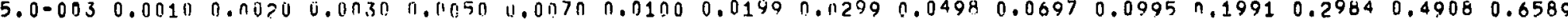

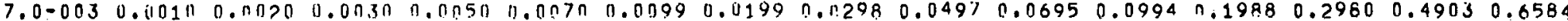

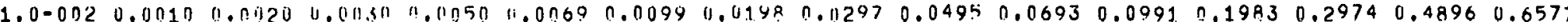
$1.0-0020.00100 .00200 .00290 .00490 .00690 .00980 .01960 .02940 .04900 .068 \% 0.09820119670 .29530 .48700 .6555$

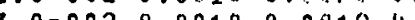

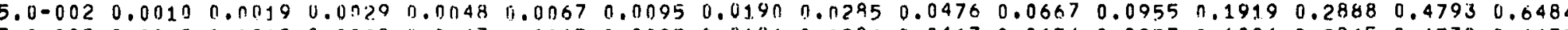

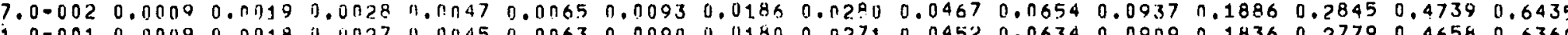

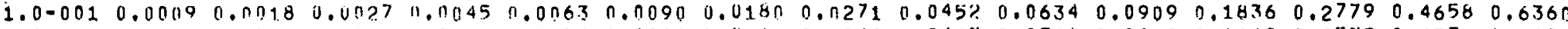

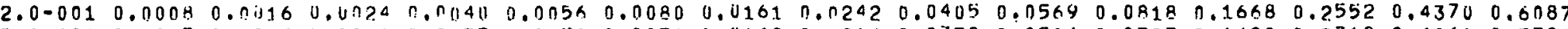

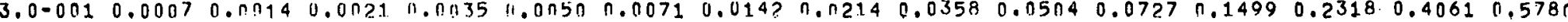

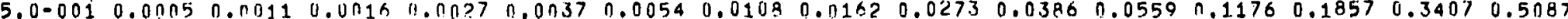

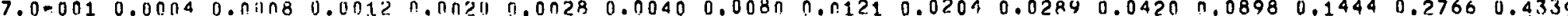

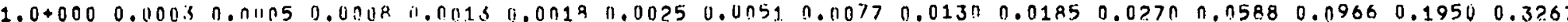
2.04000 0.1011 0.0U1 U.0002 0.0103 11.00050 .00070 .00140 .00210 .00350 .00500 .00740 .01640 .02770 .06070 .1146 3.0+000 0.0000 , $5.0+000$. $000 n$. n.

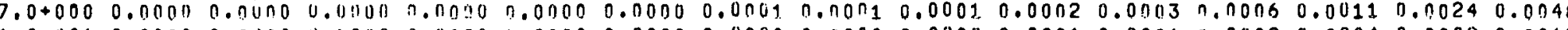

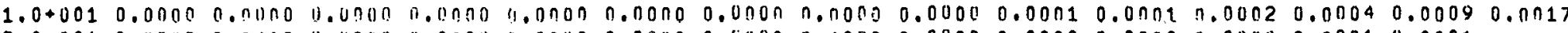

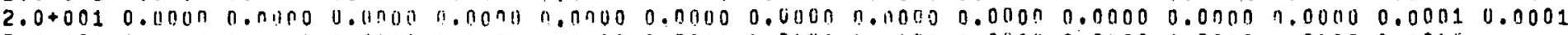

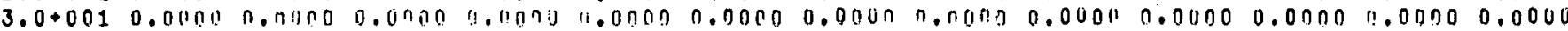

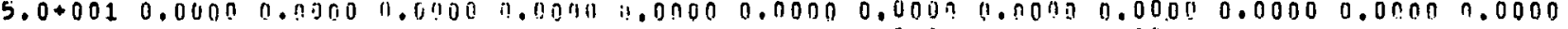

$7.0+0010.00000 .00000 .00000 .00000 .00000 .00000 .00000 .00000 .00000 .00000 .0000$

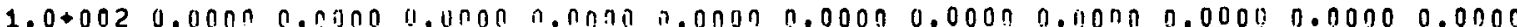

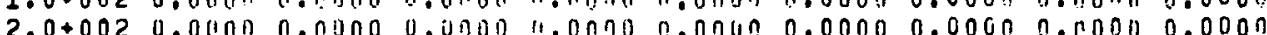

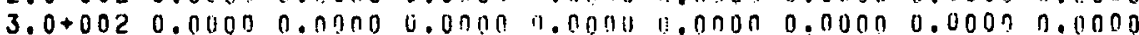

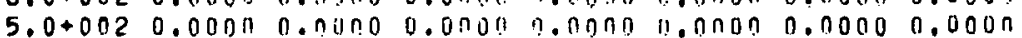

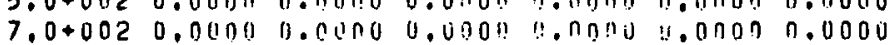

$1,0+0030.00000 .00000 .0000 \pi, 00000.00000 .0000$

$A L P H$

EFTA

$1.0=0030.83320 .98410 .9073 \quad 0.99970 .90991 .00001 .000 .79 .00001 .00001 .00001 .00001 .00001 .00001 .00001 .00001 .0000$ $2.0-0030.83310 .08410 .44720 .0997 \quad 0.99991 .00001 .00001 .00101 .00001 .00001 .00001 .00001 .00001 .00001 .00001 .0 .000$ $3.0-0030.83290 .05414 .9972 \pi .99970 .99991 .00001 .00401 .00 n 01.000 n 1.00001 .00001 .00 n 01.00001 .00001 .00001 .0000$ $5.0-0030.83260 .03400 .90730 .99970 .90991 .00001 .00011 .00001 .0001 .900001 .00001 .00001 .00001 .00001 .00001 .0000$ $7.0-0030.83240 .0840 \quad 10.99720 .99471 .99991 .00001 .00001 .00001 .00001 .00001 .00001 .00001 .00001 .00001 .00001 .0000$ $1.0-0020.83190 .0840 \quad 0.99720 .99970 .90991 .00001 .00001 .00001 .000111 .00001 .00001 .00001 .00001 .00001 .00001 .0000$ $2.0-0020.83050 .08380 .997211 .0997: 1.90941 .00001 .00001 .1701001 .00001 .00001 .00001 .00001 .00001 .0000$ $3.0-0020.82910 .93360 .997 ? 0.9907 \quad 1.99991 .00001 .00001 .00001 .000111 .00001 .00001 .00001 .0000$

$5.0-0020.82610 .03330 .99710 .94970 .99991 .00001 .001101 .00001 .000011 .00001 .00001 .0000$

$7.0-0020.823110 .9530 \quad 0.997110 .9997 \quad 0.90991 .00001 .00001 .00001 .00011 .00001 .0000$

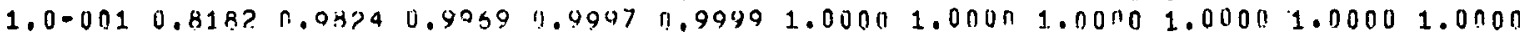

$2,0=0010.8003 \quad 0.0403 \quad 4.996 n 14.9097 \quad 0.90991 .00001 .00001 .0001101 .0000$

$3,0=0010.77920 .97770 .97510 .9006$ i. 90991.00001 .00001 .0000

$5.0=0010.72730 .0706 \quad 0.90490 .49050 .901391 .00001 .0000$

$\begin{array}{lllllll}7.0-001 & 0.6637 & 0.0507 & 0.9731 & 0.9994 & 9.9999 & 1.0000\end{array}$

$1.0+000 \quad 0.5556 \quad 0.9594 \quad 0.9841 \quad 1.9990 \quad 0.9998 \quad 1.0000$

$2.0+000 \quad 0.2500 \quad 0.91152 \quad 0.960 \% 11.9952$

$3,0+000 \quad 0.11110 .41) 78 \quad 4.9007$

$5.0+000 \quad 0.0311 .0 .2544$

$7.0+000 \quad 0.012 .5$

$1.0+001 \quad 0.0) \div 4$ 
VALUES OF F FOF $N=5$ ANO NP $=4$

$=4$

BETA

$1.0-0030.0010 \quad 0.00200 .00300 .0050 \quad 0.0070 \quad 0.0100 \cdot 0.0200 \quad 0.03000 .0500 \quad 0.06990 .09990 .19980 .2993 \quad 0.4918 \quad 11.6598$

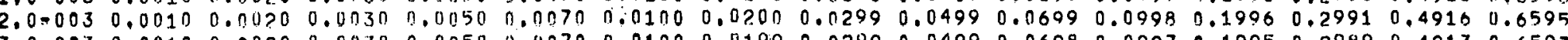

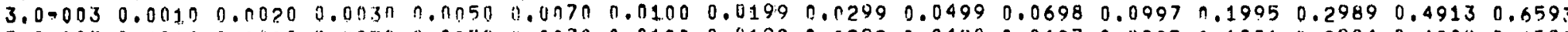

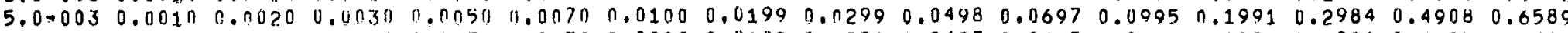

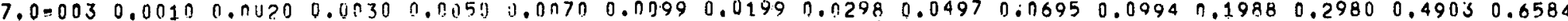

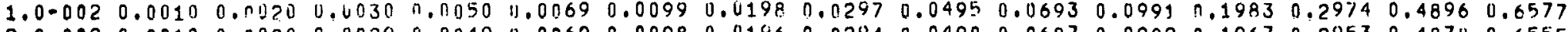

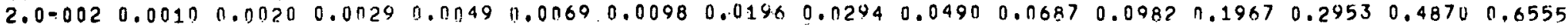
$3.0-0020.00100 .0190 .0020 \quad 0.00490 .00680 .00970 .0194 \quad 0.02910 .04860 .06800 .0973 \quad 0.19510 .29310 .48450 .6531$ $5.0-0020.0010 .01190 .00290 .00480 .00670 .00950 .019 r_{1} 0.02850 .04760 .06670 .09550 .19190 .28880 .47930 .0484$ 7.0.002 0.001

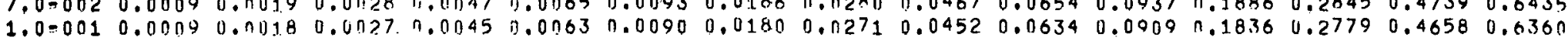

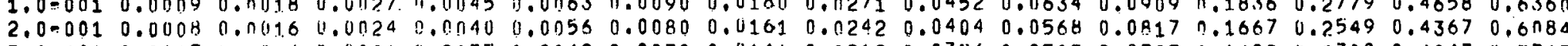
3.0-001 $0.0007 \quad 0.0014 \quad 0.0021 \quad 0.0035 \quad 0.0049 \quad 0.0070 \quad 0.01410 .02120 .0356 \quad 0.05020 .0723 \quad 0.1492 \quad 0.2308 \quad 0.4047 \quad 0.5767$

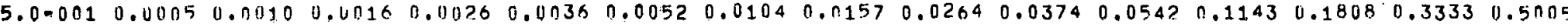
$\begin{array}{llllllllllllllllll}7.0 .001 & 0.0004 & 0.01107 & 0.0011 & 0.0018 & 0.0125 & 0.0036 & 0.00 \% 3 & 0.0110 & 0.0186 & 0.0264 & 0.0385 & 0.08 ? 7 & 0.1336 & 0.2589 & 0.4118\end{array}$ $1.0+000 \quad 0.0002 \quad 0.0004 \quad 0.0006 \quad 0.0010 \quad 0.0014 \quad 0.0020 \quad 0.0041 \quad 0.00610 .01040 .0148 \quad 0.02170 .0476 \quad 0.0788 \quad 0.1623 \quad 0.2797$

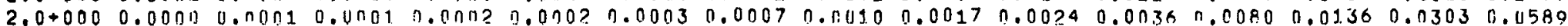

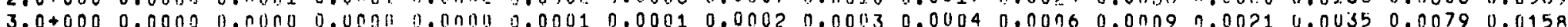

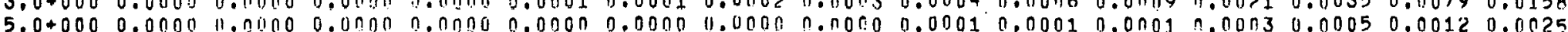

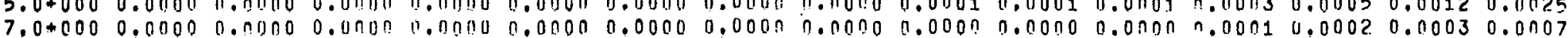

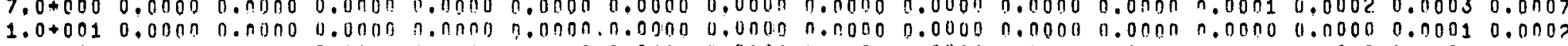
$2.0+0010.00000 .00000 .0000,0.00000 .00000 .00000 .00000 .00000 .00000 .00000 .00000 .00000 .00000 .0000$

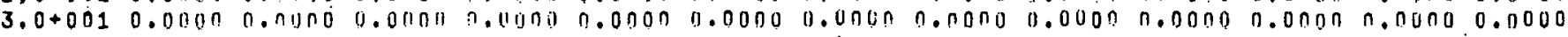

$5.0+0010.00000 .00000 .00000 .00000 .00000 .00000 .00000 .00000 .00000 .00000 .00000 .0000$

$7.0+0010.00000 .00000 .00000 .00000 .00000 .00000 .00000 .00000 .000110 .00000 .0000$

$1.0+002 \quad 0.0000 \quad 0.0000 \quad 0.0000,0.00000 .0000 \quad 0.0000 \quad 0.0000 \quad 0.00000 .00000 .00000 .0000$

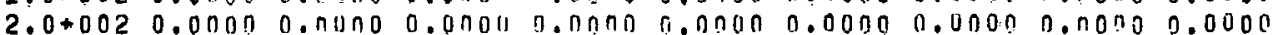

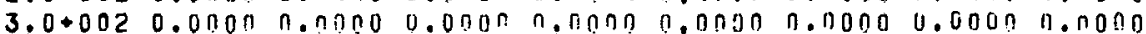

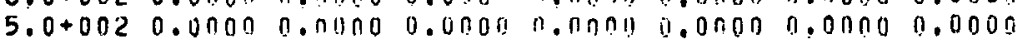

$7.0+0020.00000 .0000 \quad 0.00000 .0000 \quad 0.00000 .0000$

$1.0+0030,00000.00000 .00000 .00000 .00000 .0000$

$$
\text { BETA }
$$
BETA

ALPHA $1.000 \quad 2.000 \quad 3.000 \quad 5.000 \quad 7.000 \quad 10.00 \quad 20.00 \quad 30.00 \quad 50.00 \quad 70.00 \quad 100.0 \quad 200.0 \quad 300.0 \quad 500.0 \quad 700.0 \quad 1000$

$1.0-0030.833 ? 0.90410 .90730 .99970 .99901 .00001 .40001 .00001 .000191 .00001 .00001 .00001 .00001 .00001 .00001 .0000$

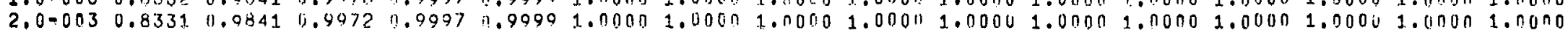
$3.0 .0030 .83290 .04410 .99720 .99970 .90991 .00001 .00001 .00 n 01.000 n 1.00001 .00001 .00001 .00001 .00001 .00001 .0000$

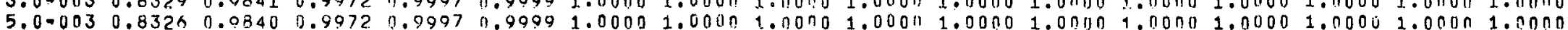

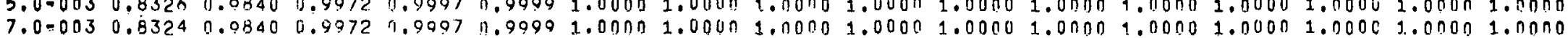

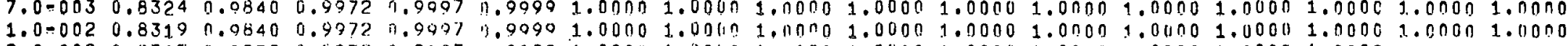
$2,0-0020.83050 .98380 .49720 .99979 .99901 .00001 .00001 .010001 .00001 .00001 .0000900001 .00001 .0000$ $3.0-0020.8291,0.96360 .9772$ 1.999\% 0.99901 .00001 .00001 .00001 .00001 .00001 .00001 .00001 .0000

5.0-002 $0.82610 .05330 .9971 .0 .9997 \quad 0.99991 .00001 .00001 .00001 .00001 .00001 .00001 .0000$

$7.0-0020.8230 \quad 0.9630 \quad 0.9970 \quad 0.99970 .99991 .00001 .00601 .00001 .00001 .00001 .0000$

$1.0-0010.8182 \quad 0.98240 .99690 .999 \% \quad 0.99991 .00001 .00001 .00001 .00011 .00001 .0000$

$2.0-0010.80010 .08020 .9060 \quad 1.590 \%, 1.99901 .00 n 01.00001 .000001 .0000$

$3.0-0010.778$ ? 0.07750 .9961 i. 99760.99991 .07001 .00001 .0000

$5.0=0010.72070 .05070 .94470 .99750 .90991 .00001 .0000$

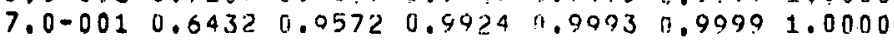

$1.0+000 \quad 0.5000 \quad 0.0254 \quad 0.9564 \quad 0.9937 \quad 11.9997 \quad 1.0000$

$2.0+000 \quad 0.13890 .6667 \quad 0.9 ? 13 \quad 0.99 ? 1$

$3.0+000 \quad 0.0397 \quad 0.33880 .710700$

$5.0+000 \quad 0.0064 \quad 0.0735$

$7.0+000 \quad 0.001 .8$

$1.0+0010.0004$ 
VALUES OF F FOH $N=\{$ ANII NF= 1 ALPHA

$1.0=003 \quad 0.0010 \quad 0.0020 \quad 0.0730 \quad 0.0050 \quad 0.0070 \quad 0.0100 \quad 0.0260 \quad 0.0300 \quad 0.05000 .06990 .09090 .1998 \quad 0.2956 \quad 0.4958 \quad 0.6729$ 2. $0=0030.0010 \quad 0.0020 \quad 0.0030 \quad 0.0050 \quad 0.0070 \quad 0.0160 \quad 0.0200 \quad 0.02990 .04990 .06990 .0998 \quad 0.19970 .29540 .4956 \quad 0.6726$ $3.0=0030.00100 .0020 \quad 0.00300 .01500 .0070 \quad 0.0160 \quad 0.01590 .02990 .04990 .06980 .09970 .19950 .29920 .49530 .6724$

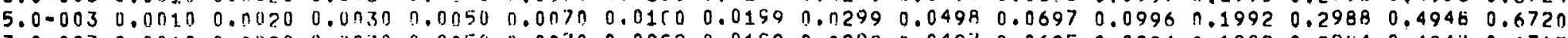

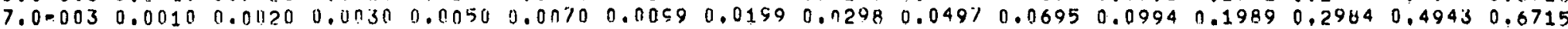

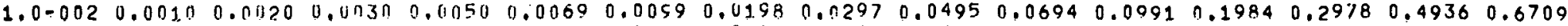

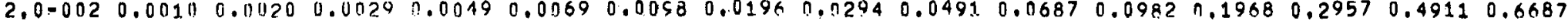
$\begin{array}{llllllllllllllll}3.0-002 & 0.0010 & 0.0019 & 0.01029 & 0.0049 & 0.0068 & 0.0057 & 0.0194 & 0.0292 & 0.0486 & 0.0681 & 0.0974 & 0.1953 & 0.2937 & 0.4887 & 0.6065\end{array}$ $5,0-0020.00100 .00190 .00290 .00480 .0007 \quad 0.0 .0550 .01910 .02860 .04790 .06690 .09570 .19230 .28970 .48390 .6623$ $7.0-0020.00040 .00190 .00280 .011470 .00650 .00940 .01870 .02810 .04690 .06570 .09410 .18940 .28580 .47920 .6580$

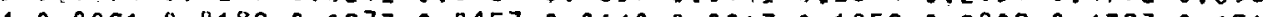
$1.0 .0010 .00 n$. $010180.0 n 27 n .0 n 450.0 n 640.00910 .01820 .02730 .04570 .06400 .09170 .18520 .28020 .47230 .6518$ $2.0-0010.0009$ n. $\begin{array}{llllllllllllllll}3.0 .001 & 0.0000 & 0.0015 & 0.0023 & 0.0039 & 0.0054 & 0.0077 & 0.0155 & 0.0232 & 0.0389 & 0.0547 & 0.0787 & 0.1613 & 0.2478 & 0.4309 & 0.6130\end{array}$ $\begin{array}{lllllllllllllllll}5.0-001 & 0.0007 & 0.0113 & 0.0020 & 0.0033 & 0.0047 & 0.0067 & 0.0134 & 0.0202 & 0.0339 & 0.0478 & 0.0690 & 0.1428 & 0.2221 & 0.3962 & 0.5785\end{array}$ $\begin{array}{llllllllllllllll}7.0-001 & 0.0006 & 0.0012 & 0.0018 & 0.0079 & 0.0041 & 0.0059 & 0.0119 & 0.0179 & 0.03011 & 0.0424 & 0.0613 & 0.1282 & 0.2012 & 0.3667 & 0.5477\end{array}$

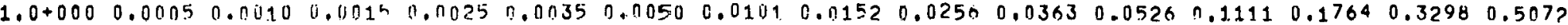
$2.0+000 \quad 0.0003 \quad 0.00070 .0010 \quad 0.0017 \quad 0.0023 \quad 0.00340 .0068 \quad 0.01 C 20.01720 .02450 .0357 \quad 0.0769 \quad 0.1249 \quad 0.2471 \quad 0.4070$ $3.0+000 \quad 0.00030 .0005 \quad 4.00080 .00130 .07180 .00250 .0051 \quad 0.00770 .01300 .01850 .02700 .05880 .09670 .19750 .3398$ $5.0+0000.00020 .00034 .00050 .00080 .00120 .00170 .00340 .00510 .00870 .01240 .01820 .04000 .06660 .14090 .2555$

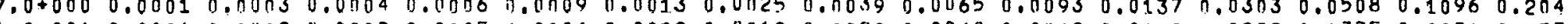

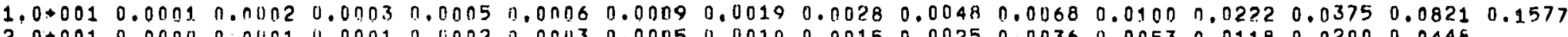

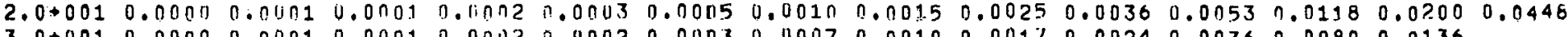
$3.0+0010.00000 .00010 .0 n 010.001320 .000 ? 0.00030 .00070 .00100 .001 \% 0.00240 .00360 .00800 .0136$ $5.0+0010.00000 .00000 .00010 .00113,00010.000210 .00040 .00060 .00100 .00150 .00220 .0049$

$7.0+0010.0000 \pi .00000 .00000 .00010 .00010 .00010 .0003 \quad 11.00040 .00070 .00110 .0016$

$1.0+0020.00000 .00000 .0000300010 .00010 .00010 .00020 .00030 .00050 .00070 .0011$

$2.0+0020.0000 \quad 0.01000 \quad 0.0000 \quad 0.0000 \quad 0.0000 \quad 0.00310 .0001 \quad 0.00020 .0003$

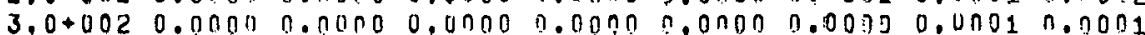

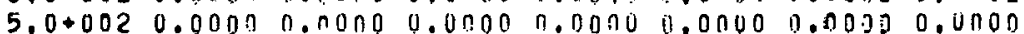

$7.0+0020,00000.011000 .00000 .00000 .00000 .0000$

$1,0+0030.00000 .00000 .0000 \%, 0000,00000.0000$

$$
\text { BETA }
$$
BETA

ALPHA $1.000 \quad 2.000 \quad 3.000 \quad 5.000 \quad 7.000 \quad 10.00 \quad 20.00 \quad 30.00 \quad 50.00 .70 .00 \quad 1.00 .0 \quad 200.0 \quad 300.0 \quad 500.0 \quad 700.0 \quad 1000$

$1.0-0030.857110 .09210 .9991 .0 .99091 .00001 .00031 .00001 .00001 .00001 .00001 .00001 .000001 .00001 .00001 .00001 .0000$ 2.0-003 0.85690 .99210 .9991 .0 .99991 .00001 .00031 .00001 .00001 .000101 .00001 .00001 .00001 .00001 .00001 .00001 .0000 3.0-003 $0.85690 .99210 .99910 .99991 .00001 .50091 .000 n 1.00001 .000131 .00001 .00001 .00001 .00001 .00001 .00001 .0000$

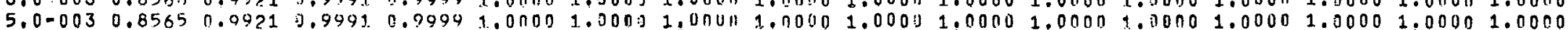

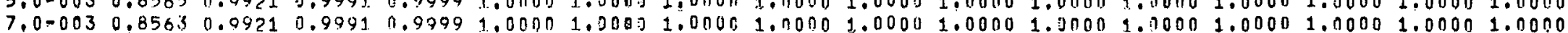

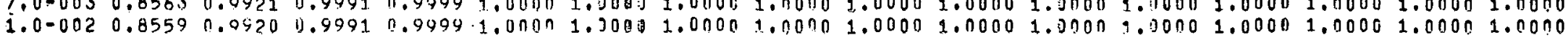

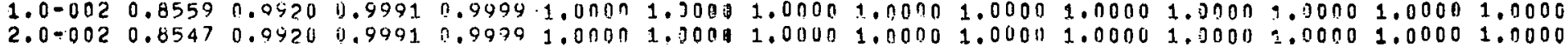
$3.0-0020.85350 .99190 .90910 .99991 .00001 .30001 .00001 .00001 .0001) 1.00001 .3000 \quad 0.00001 .0000$ 5.0-002 $0.8511 .0 .09170 .9990 \quad 0.99991 .00001 .90011 .00031 .00001 .00001 .0000 .1,00001.0000$

$7,0-0020.84870 .0916 \quad 11.9990 \quad 0.99991 .00001 .0001 .00001 .00001 .00001 .00001 .0000$ $1.0-0010.84510 .09130 .9990 \quad 0.99991 .000101 .00041 .00001 .00001 .00001 .00001 .0000$ $2,0=0010.83330 .9906 \quad 4.99890 .09991 .000111 .00601 .00001 .00001 .0000$

$3.0-0010.82190 .0998 \quad 0.5988$ ri.9499 $1.00001 .00 C 01.00001 .0000$

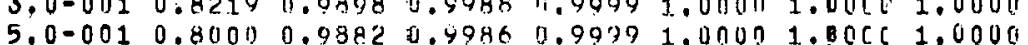

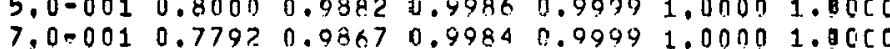

$1.0+000 \quad 0.7500 \quad 0.94440 .99820 .99991 .001001 .000 \mathrm{C}$

$2.0+000 \quad 0.6667 \quad 0.9767 \quad 0.9973 \quad 0.9998$

$3.0+000 \quad 0.6000 \quad 0.9692 \quad 11.9964$

$5.0+000 \quad 0.5000 \quad 0.8545$

$7.0+000 \quad 0.4286$

$1.0+0010.3529$ 
$\begin{array}{llllllllllllllll}\text { VALUES OF F FOR N }=6 & \text { AND NP= } 2 \\ \text { ALPHA } & 0.001 & 0.002 & 0.003 & 0.005 & 0.007 & 0.01 & 0.02 & 0.03 & 0.05 & 0.07 & 0.1 & 0.2 & 0.3 & 0.5 & 0.7\end{array}$ BETA

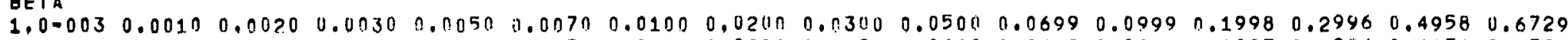
$2,0.0030 .0010 \quad 0.0020 \quad 0.0030 \quad 0.0050$ ij.0n7n $0.0100 \quad 0.0200 \quad 0.02990 .04990 .06990 .09980 .19970 .29940 .4956 \quad 0.6726$ $3.0-0030.0010 \quad 0.0020 \quad 0.0030 \quad 0.0050 \quad 0.0 n 7 n \quad 0.0100 \quad 0.0 .199 \quad 0.02990 .0499 \quad 0.0698 \quad 0.0997 \quad 0.1995 \quad 0.2992 \quad 0.4953 \quad 0.6724$

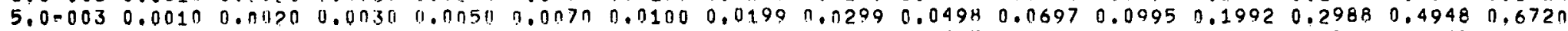

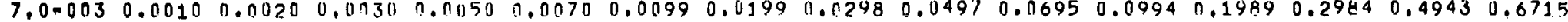
$\begin{array}{llllllllllllllll}1.0-002 & 0.0010 & 0.0020 & 0.0030 & 0.0050 & 0.0069 & 0.0099 & 0.0198 & 0.0297 & 0.0495 & 0.0693 & 0.0991 .00 .1984 & 0.2977 & 0.4936 & 0.6709\end{array}$ $2.0-0020.00100 .00200 .00290 .00490 .0000 \quad 0.0098 \quad 0.0196 \quad 0.02940 .049100 .0687 \quad 0.0982 \quad 0.1968 \quad 0.2956 \quad 0.4910 \quad 0.6686$ $3.0-0020.00100 .00190 .00290 .01490 .00080 .00970 .01940 .02910 .04860 .06800 .09730 .19520 .29350 .4885 \quad 1.6663$

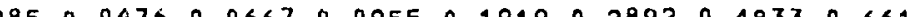

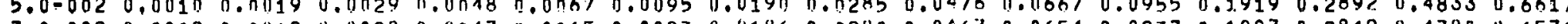

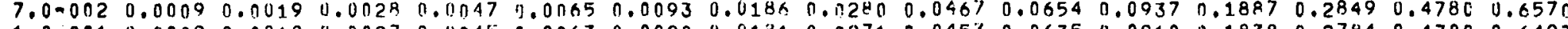
$1.0=0010.00090 .0018 \quad 1.0027$ n.11045 $1.00630 .00900 .01810 .02710 .04530 .06350 .09100 .18380 .27840 .4700 \quad 0.6497$

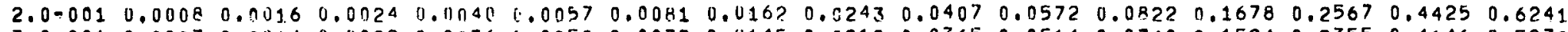
$3,0=0010.0007 \quad 0.0014 \quad 0.0022 \quad 0.0036 \quad 1.0050 \quad 0.0072 \quad 0.0145 \quad 0.02180 .0365 \quad 0.05140 .0740 \quad 0.15240 .2355 \quad 0.4146 \quad 0.5970$

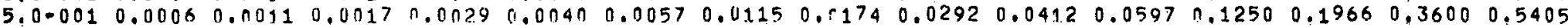

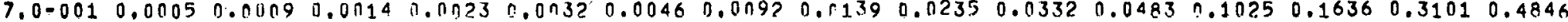
$\begin{array}{llllllllllllllll}1.0+000 & 0.0003 & 0.0007 & 0.0010 & 0.001 .7 & 1.0023 & 0.0034 & 0.0008 & 0.0 .102 & 0.0172 & 0.0245 & 0.0357 & 0.0769 & 0.1249 & 0.2471 & 0.4070\end{array}$ $2.0+000 \quad 0.00010 .00030 .0 n 040.00 n 70.001 n 0.00140 .0 n 290.00440 .00750 .01060 .01560 .03450 .05770 .12330 .2273$ 3.040000 .00010 .00020 .00020 .00040 .00050 .00080 .00160 .00240 .00400 .00580 .00850 .01890 .03190 .07040 .1367

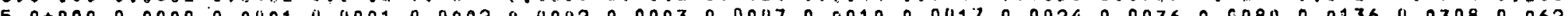

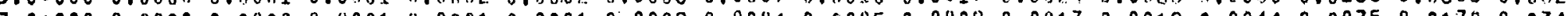
1.00010 .0000 .0000 .00000 .00000 .00010 .00010 .00020 .00030 .00050 .00070 .01100 .00220 .00380 .00880 .0182

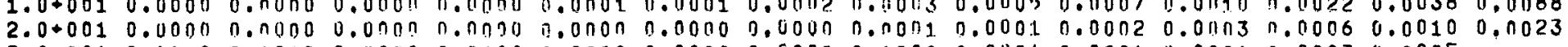
$3,0+0010.00000 .00000 .00000 .00000 .00000 .00000 .00000 .00000 .00010 .00010 .00010 .00030 .0005$ $5,0+0010.00000 .0 u 000.0000,1.00000 .00000 .00000 .00000 .00000 .00000 .00000 .00000 .0001$

$7.0+0010.00000 .00000 .00000 .00700 .00000 .00000 .00000 .00000 .00000 .00000 .0000$

$1.0+0020.00000 .04000 .00000 .0000,0.00000 .0000 .0 .00000 .00000 .00000 .00000 .0000$

$2.0+002 \quad 0.0000 \quad 0.01100 \quad 0.0000 \quad 0.00 n 00.0000 \quad 0.0000 \quad 0.0000 \quad 0.000 \pi 0.000 ! 3$

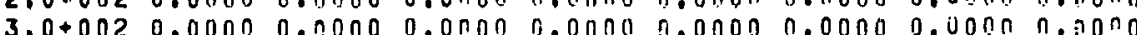

$5.0+002 \quad 0.00000 .0000 \quad 0.0000,0.00000 .0000 \quad 0.00000 .0000$

$7.0+0020.00000 .00000 .00000 .00000 .00000 .0000$

$1,0.0030 .00000 .00000 .00000 .00000 .00000 .0000$

$$
A L P H
$$
$B E T A$

ALPHA $1.000 \quad 2.000 \quad 3.000 \quad 5.000 \quad 7.000 \quad 10.00 \quad 211.00 \quad 30.00 \quad 50.00 \quad 70.00 \quad 100.0 \quad 200.0 \quad 300.0 \quad 500.0 \quad 700.0 \quad 1000$

1.000030 .85700 .09210 .99910 .99091 .00001 .00001 .00001 .00001 .00001 .00001 .00001 .00001 .00001 .00001 .00001 .0000 2.0-003 0.85690 .99210 .90910 .99991 .09001 .00001 .00001 .00001 .00001 .00001 .00001 .00001 .00001 .00001 .00001 .0000 $3.0-0030.85680 .99210 .99910 .99991 .00001 .00001 .00001 .00001 .00001 .00001 .00001 .00001 .00001 .00001 .00001 .0000$ $5.0-0030.85650 .09210 .99910 .991991 .00001 .00001 .00001 .00001 .00001 .00001 .00001 .00001 .00001 .00001 .00001 .0000$

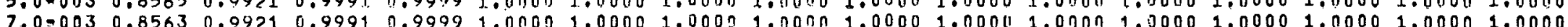

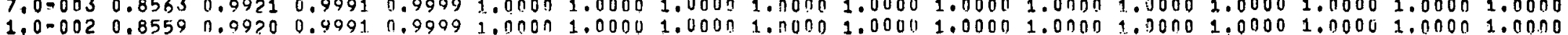

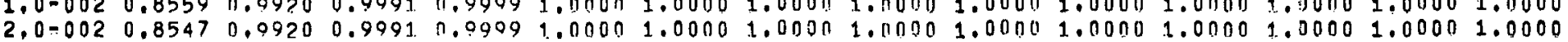
$3.0=0020.85340 .09190 .9991 \quad 0.99991 .00001 .00001 .00001 .00001 .00001 .00001 .00001 .00001 .0000$ $5.0 .0020 .85 n 80.99170 .9990 \quad 0.99991 .00001 .00001 .00001 .00301 .00 n 01.00001 .00001 .0000$

7.0 .0020 .84810 .09150 .99900 .99991 .00001 .00001 .00001 .00001 .00001 .00001 .0000

$1.090010 .84390 .99130 .9990 \quad 0.99991 .00001 .00001 .00001 .00001 .00001 .00001 .0000$

$2.000010 .8287 \quad 0.0903 \quad 0.99890 .9999 \quad 1.0000 \quad 1.00001 .00001 .00001 .0000$

$3.0 .0010 .81190 .9891 \quad 0.9987 \quad 0.99991 .0000 \quad 1.00001 .00001 .0000$

$5,09001 \quad 0.7742 \quad 0.9863 \quad 0.99340 .99991 .00001 .00001 .0010$

$7,0=0010.7326 \quad 0.98290 .9980 \quad 0.99991 .00001 .0000$

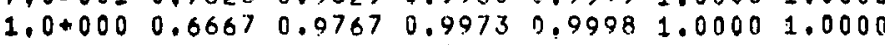

$2.0+000 \quad 0.4615 \quad 0.9474 \quad 0.9936 \quad 0.9996$

$3.0+000 \quad 0.3158 \quad 0.9065 \quad 0.9882$

$5.0+000 \quad 0.16220 .9025$

$7.0+000 \quad 0.0952$

$1.0+0010.0513$ 
VALUES OF F FOK $H=$ E AND NP= 3
ALPHA 0.001 (1.002 $0.003 \quad 0.005$

VALUES OF F FOK $M=$ E AND NP= 3
ALPHA $0.001 \quad 0.002$ O.0.03 0.005 BETA

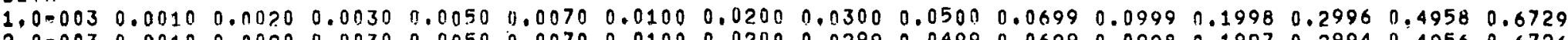
$2.0=003 \quad 0.0010 \quad 0.0020 \quad 0.0030 \quad 0.0050 \quad 0.0070 \quad 0.0100 \quad 0.0200 \quad 0.02990 .04990 .06990 .09980 .1997 \quad 0.2994 \quad 0.4956 \quad 0.6726$

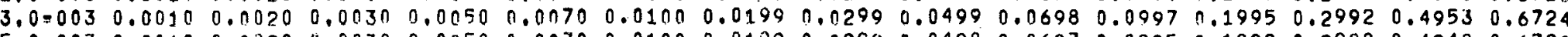

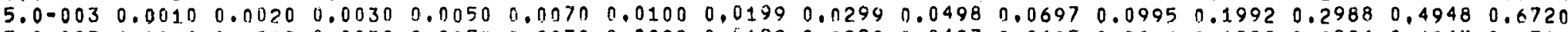

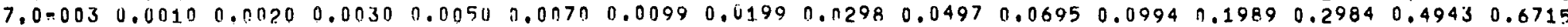

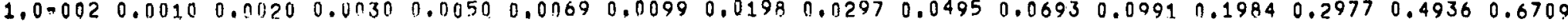

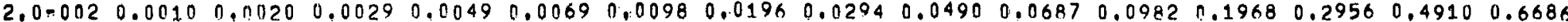

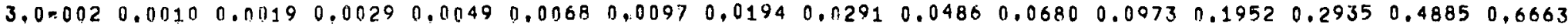

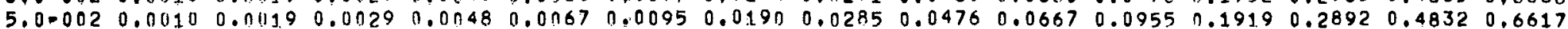
$3.0-0020.0000 .0190 .00280 .00470 .00650 .00930 .01860 .02800 .04670 .06540 .09370 .18860 .28480 .47790 .6569$

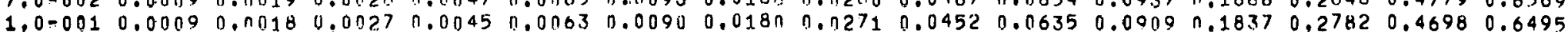

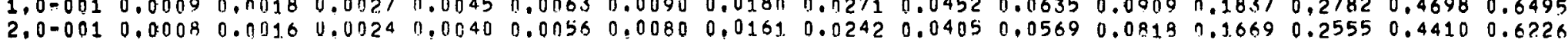

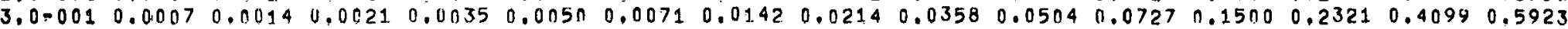

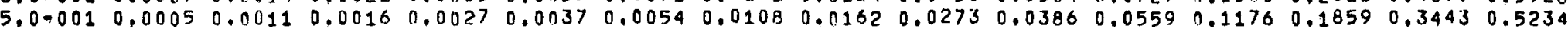
$7,0=0010.00040 .0008 \quad 0.00120 .0020 \quad 0.0 n 28 \quad 0.0040 \quad 0.0080 \quad 0.01210 .02040 .02890 .0420 \quad 0.08980 .1446 \quad 0.2799 \quad 0.4484$ $\begin{array}{lllllllllllllllll}1.0+000 & 0.0003 & 0.0005 & 0.0008 & 0.0013 & 0.0018 & 0.0025 & 0.0051 & 0.0077 & 0.0130 & 0.0185 & 0.0270 & 0.0588 & 0.0967 & 0.1975 & 0.3398\end{array}$ $2.0+000 \quad 0.00010 .0001 \quad 0.0002 \quad 0.0003 \quad 0.00050 .00070 .00140 .00210 .00350 .0050 \quad 0.00740 .01640 .02780 .06160 .1207$ $3.0+0000.00000 .00010 .000110 .00010 .00020 .00030 .00050 .00080 .00130 .00100 .00280 .00620 .01060 .02400 .04918$ $5,0+0000.00000 .00000 .00000 .00000 .00000 .00010 .00010 .00020 .00030 .00050 .00070 .00160 .00270 .00630 .0130$ $7,0+000 \quad 0.0000 \quad 0.0000 \quad 0.00000 .00000 .00000 .00000 .00010 .00010 .00010 .00020 .00030 .00060 .00110 .00250 .0051$

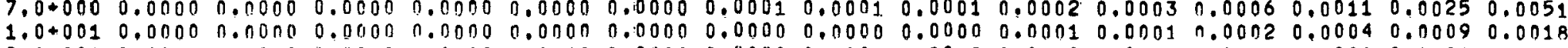

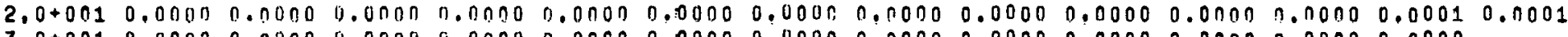
$3,0+0010.00000 .01000 .000 \pi 0.00000 .00000 .00000 .00000 .00000 .00000 .00000 .00000 .00000 .0000$

$5.0+0010.00000 .0000 \quad 0.0000 \quad 0.00000 .00000 .00000 .00000 .00000 .00000 .00000 .00000 .0000$

$7.0+0010.00000 .00000 .00000 .00000 .00000 .00000 .00000 .00000 .00000 .00000 .0000$

$1.0+0020.00000 .04000 .0000 \quad 0.00000 .00000,00000.00000 .00000 .00000,00000.0000$

$2,0+0020.0000 \quad 0.00000,0000,0,0000,0,0000.00000 .00000 .00000 .0000$

$3,0+002 \quad 0.0 .000 \quad 0.0000 \quad 0.0000 \quad 0.0000 \quad 0.0000 \quad 0.0000 \quad 0.0000 \quad 0.0000$

$5.0+0020.00000 .01) 00.0000 \quad 0.00000 .00000 .00000 .0000$

$7,0+0020.00000 .00000 .00000 .00000 .00000 .0000$

$1,0+0030,00000.00000,00000.00000,00000.0000$

$$
\text { ALPH }
$$

BETA

$1.0=0030.8570 \quad 0.99210 .99910 .99091 .00001 .30001 .00001 .00001 .00001 .00001 .00001 .00001 .00001 .00001 .00001 .0000$ $2.0-0030.85690 .99210 .4091 \quad 0.99991 .00001 .00001 .00001 .00001 .00001 .00001 .00001 .00001 .00001 .00001 .00001 .0000$ 3.0 .0030 .85680 .09210 .99910 .99991 .00001 .30001 .00001 .00001 .00001 .000111 .00001 .00001 .00001 .00001 .00001 .0000 $5.0-0030.85650 .99210 .59910 .99991 .00001 .90001 .00001 .00001 .00001 .00001 .00001 .00001 .00001 .00001 .00001 .0000$

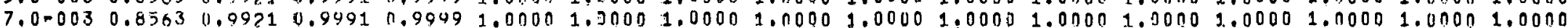
$1.0-0020.85590 .99 ? 0 \quad 1.40910 .99991 .00001 .00001 .00001 .00001 .00001 .00001 .00001 .00001 .00001 .00001 .00001 .0000$ $2,0=0020.85470 .99201 .99910 .99971 .00001 .00001 .00001 .00001 .00001 .00001 .00001 .00001 .00001 .0000$

$3.0=0020.85340 .99190 .99910 .99991 .00001,30001.00001 .00001 .00001 .00001 .00001 .00001 .0000$

$5.0 .0020 .8507 \quad 0.99170 .9990 \quad 0.99991 .00001 .30001 .00001 .00001 .00001 .00001 .00001 .0000$

$7.0 \div 002 \quad 0.84800 .99150 .9990 \quad 0.99991 .00001,30001.00001 .00001 .00001 .00001 .0000$

$1,0=0010.84380 .99130 .9990 \quad 0.99991 .00001 .30001 .00001 .00001 .00001 .00001 .0000$

2. $0=001 \quad 0.8278 \quad 0.99 n 2 \quad 0.9469 \quad 0.9999 \quad 1.0000 \quad 1.30001 .000 n \quad 1.00001 .0000$

$3,0=001 \quad 0.819100 .0889 \quad 1.9987 \quad 0.9998 \quad 1.00001 .30001 .00001 .0000$

$5.0-001 \quad 0.75190 .9853 \quad 0.9983 \quad 0.99991 .00001 .30001 .0000$

$7.0-001 \quad 0.79320 .9803,9,99770.99991 .00001,3000$

$1.0+000 \quad 0.61000 .9692 \quad 3.99640 .99981 .00001 .3000$

$2.0+000 \quad 0.2857 \quad 0.8936 \quad 3.9864 \quad 0.9992$

$\begin{array}{lllll}3.0+000 & 0.1304 & 0.7500 & 3.5647\end{array}$

$5,0+000 \quad 0,0370 \quad 0.4468$

$7,0+000 \quad 0,0 \leq 48$

$1.0+0010.0054$ 
VALUES OF F FOK IN= 6 ANI! Nr= 4 BETA

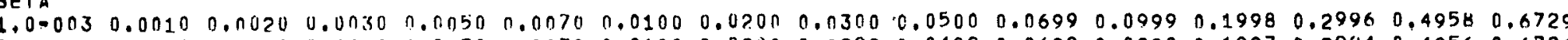

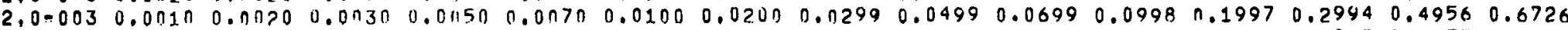

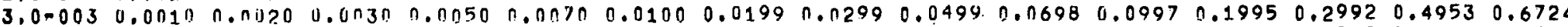

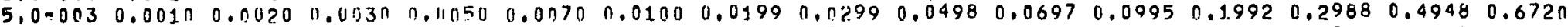

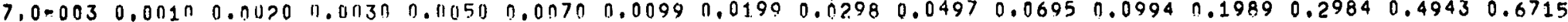

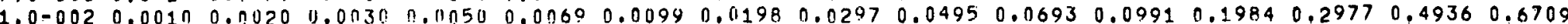
$2.0-0020.00100 .00200 .00290 .00490 .00690 .00980 .01960 .02940 .04900 .06870 .09820 .19680 .29560 .49100 .6686$

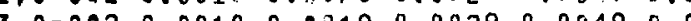

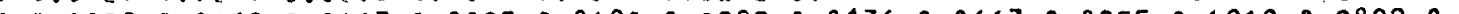

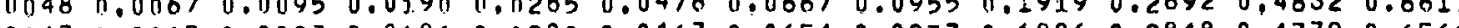
7.0-002 0.00090 .00190 .012811 .00470 .00650 .00930 .01860 .02800 .04670 .06540 .09370 .18860 .28480 .47790 .6569 $1.0 .0010 .00090 .01180 .018270 .00450 .0063 \quad 0.0090 \quad 0.01800 .02710 .04520 .06340 .09090 .18370 .27820 .46980 .649 .5$

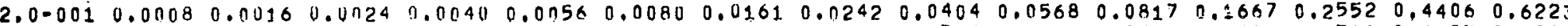
$3,0.0010 .0007 \quad 0.001 .40 .0021 \quad 0.00350 .00490 .0070 \quad 0.01410 .02120 .0356 \quad 0.05020 .07230 .1492 \quad 0.23110 .40850 .5909$ $\begin{array}{llllllllllllllll}5.0=001 & 0.0005 & 0.0010 & 0.0116 & 0.002 .6 & 0.0036 & 0.0052 & 0.0104 & 0.0157 & 0.0264 & 0.0374 & 0.0542 & 0.1143 & 0.1810 & 0.3369 & 0.5152\end{array}$

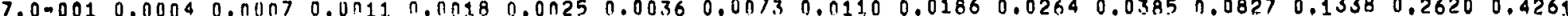
$1,0+000 \quad 0.00020 .010040 .0006 \quad 1.0010 \quad 0.00140 .00200 .0 n 490.00610 .01040 .01480 .02170 .04760 .07890 .16450 .2917$ $10+0000.00000 .00010 .00010 .00020 .00020 .00030 .00070 .00100 .00170 .00240 .00360 .00800 .01360 .03080 .0623$ 00010.00020 .00030 .00040 .0006 0.0009 0.00210 .00350 .00810 .0167 $5.0+000$ 0.000 n.

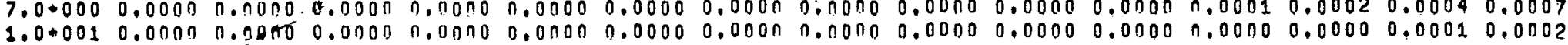
$2.0+0010.00000 .00000 .00000 .00 n 00.0000 \pi .00000 .00000 .00000 .00000 .00000 .00000 .00000 .00000 .0000$ $3.0+0010.00000 .00000 .00030 .00000 .00000 .00000 .00000 .00000 .00000 .00000 .00000 .00000 .0000$

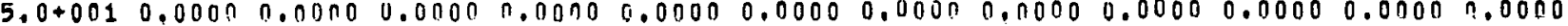

$7.0+0010.00000 .00000 .0000 \quad 0.0000 \quad 0.00000 .0000 \quad 0.0000 \quad 0.00000 .00000 .00000 .0000$

$3.0+0020.0000 \quad 0.00000 .00000 .00000 .0000 \quad 0.0000 \quad 0.00000 .0000 \quad 0.0000 \quad 0.00000 .0000$

$2,0+0020.00000 .00000 .00000 .00000 .00000 .00000 .00000 .00000 .0000$

$3,0+0020.0000 \quad 0.0000 \quad 0.00000,00000.0000 \quad 0.00000 .00000 .0000$

$5,0+0020.00000 .00000 .01000 .0000 \quad 0.00000 .00000 .0000$

$7.0+0020.00000 .0000 .0000 .0000,00000,0000$

$1.0+0030,00000.00000,00000,00000.00000 .0000$

ALPHA $1.000 \quad 2.000 \quad 3.000 \quad 5.000 \quad 7.000 \quad 10.00 \quad 20.00 \quad 30.00 \quad 50.00 \quad 70.00 \quad 100.0 \quad 200.0 \quad 300.0 \quad 500.0 \quad 700.0 \quad 1000$ BETA

1.000030 .85700 .99210 .99910 .99991 .00001 .00001 .00011 .00001 .00001 .00001 .00001 .00001 .00001 .00001 .00001 .0000 $2.0=0030.85690 .99210 .99910 .99991 .00001 .00001 .00001 .00001 .00001 .00001 .00001 .00001 .00001 .00001 .00001 .0000$ $3,0=0030.85680 .09210 .99910 .99991 .00001 .00001 .00001 .00001 .00001 .00001 .00001 .00001 .00001 .00001 .00001 .0000$ $5,0=0030.85650 .99210 .90910 .90091 .00001 .00001 .00001 .00001 .00001 .00001 .00001 .00001 .00001 .00001 .00001 .0000$

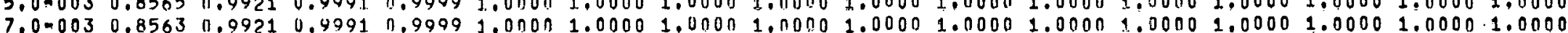

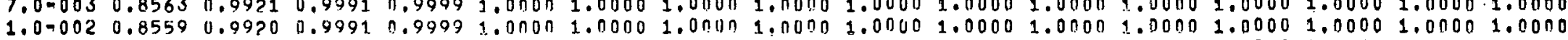
2.000020 .85470 .99200 .9791 .0 .99991 .00001 .00001 .00001 .00001 .00001 .00001 .00001 .00001 .00001 .0000 $3,0=0020.85340 .99190 .99910 .99991 .00001 .00001 .00001 .00001 .00001 .00001 .00001 .00001 .0000$

5.0 .0020 .85070 .99170 .99900 .99991 .00001 .00001 .00001 .00001 .00001 .00001 .00001 .0000

$7.0 .0020 .84800 .99150 .9990 \quad 0.99991 .00001 .00001 .00001 .00001 .00001 .00001 .0000$

$1.0-0010.8438 \quad 0.09130 .9990 \quad 0.90991 .00001 .00001 .00001 .00001 .00001 .00001 .0000$

$2.0=001 \quad 0.82760 .99020 .99890 .99991 .00001 .00001 .00001 .00001 .0000$

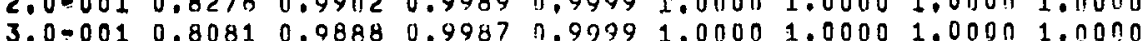

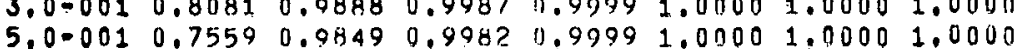

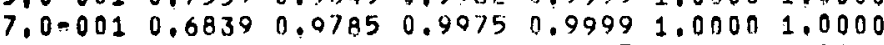

$1.0 .000 \quad 0.5455 \quad 0.9618 \quad 0.9954 \quad 0.9997 \quad 1.00001 .0000$

$2.0+000 \quad 0.1622 \quad 0.8025 \quad 0.9724 \quad 0.9984$

$3.0+000 \quad 0.0472 \quad 0.5101 \quad 0.9002$

$5.0+000 \quad 0.0076 \quad 0.1389$

$7.0+000 \quad 0.0021$ 
VALUES OF F FOK $N=6$ AND NIP $=5$

VALUES OF $F$ FOR $N=6$ AND NIP $=5$

BETA

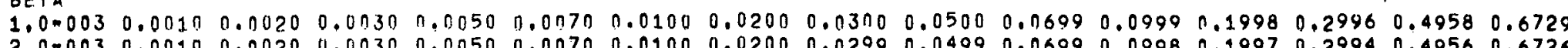
$2,0=003 \quad 0.00190 .0020 \quad 1.0030 \quad 0.0050 \quad 0.0070 \quad 0.0100 \quad 0.02000 .02990 .04990 .06990 .0998 \quad 0.1997 \quad 0.2994 \quad 0.4956 \quad 0.6726$

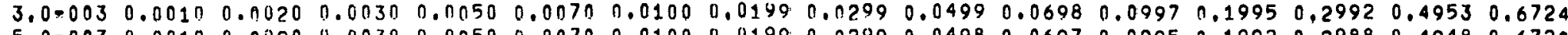
$5.0-0030.001 n \quad 0.0020 \quad 0.0030 \quad 0.0 .050 \quad 0.0070 \quad 0.0100 \quad 0.01990 .02990 .0498 \quad 0.0697 \quad 0.0995 \quad 0.1992 \quad 0.2988 \quad 0.4948 \quad 0.6720$

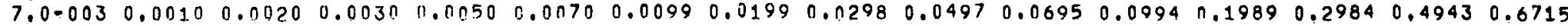

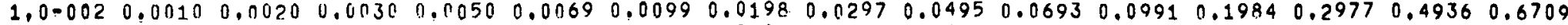

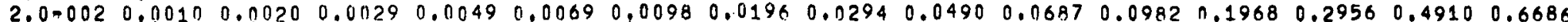
$3,0=0020.00100 .00190 .40290 .00490 .00680,00970.01940 .02910 .04860 .06800 .09730 .19520 .29350 .48850 .6663$

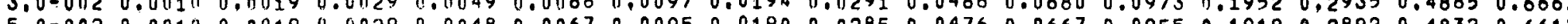

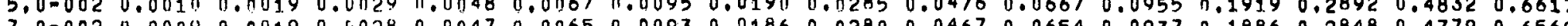

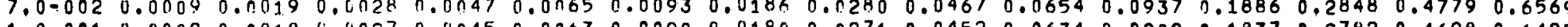

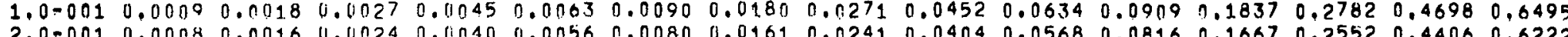

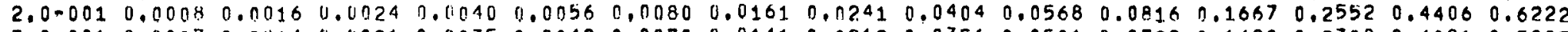
$3.0-001 \quad 0.0007 \quad 0.01140 .0021 \quad 0.0 n 35 \quad 0.00490 .0070 \quad 0.01410 .02120 .0356 \quad 0.05010 .07220 .1490 \quad 0.2308 \quad 0.40810 .5905$ $\begin{array}{lllllllllllllllllllllll}5.0=001 & 0.0005 & 0.0010 & 0.0015 & 0.0025 & 0.0036 & 0.0051 & 0.0103 & 0.0155 & 0.0260 & 0.0368 & 0.0534 & 0.1127 & 0.1787 & 0.3333 & 0.5112\end{array}$ $\begin{array}{llllllllllllllllll}7.0=001 & 0.0003 & 0.0007 & 0.0010 & 0.0017 & 0.0024 & 0.0034 & 0.0069 & 0.0104 & 0.0176 & 0.0250 & 0.0364 & 0.0783 & 0.1271 & 0.2508 & 0.4118\end{array}$

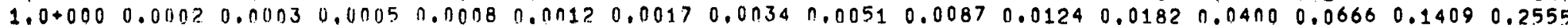
$2.0+0000.00000 .00000 .00000 .00010 .00010 .00020 .00030 .00050 .00080 .00120 .00180 .00400 .00680 .01540 .0316$ $3.0+0000.00000 .00000 .00000 .00000 .00000 .00000 .00010 .00010 .00010 .00020 .00030 .00070 .00120 .00270 .0056$ $3.0+0000.0000 .000 .0056$

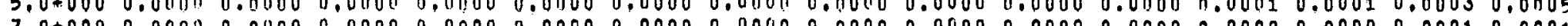
$7.0+000 \quad 0.00000 .00000 .00000 .00000 .0000 \quad 0.00000 .00000 .00000 .00000 .00000 .00000 .00000 .00000 .00010 .0001$ $1.0+0010.00000 .00000 .00000 .00 n 00.00000 .00000 .00000 .00000 .00000 .00000 .00000 .00000 .00000 .00000 .0000$ $2,0+0010.011000 .00000 .00000 .00000 .00000 .00000 .00000 .00000 .00000 .00000 .00000 .00000 .00000,0000$ $3.0+0010.00000 .00000 .00000 .00000 .00000 .00000 .00000 .00000 .00000 .00000 .00000 .00000 .0000$

$5.0+0010.00000 .00000 .00000 .00000 .00000 .00000 .00000 .000000 .00000 .00000 .00000 .0000$

$7.0+0010.0000 \quad 0.0000 \quad 0.0000 \quad 0.00000 .00000 .00000 .00000 .00000 .00000 .00000 .0000$ $1.0+0020.00000 .00000 .00000 .00000 .00000 .00000 .00000 .00000 .00000 .00000 .0000$ $2.0+0020.00000 .00000 .00000 .00000 .00000 .00000 .00000 .00000 .0000$

$3,0+0020.00000 .0000$ i. $0000,0.00000 .00000 .00000 .001100 .0000$

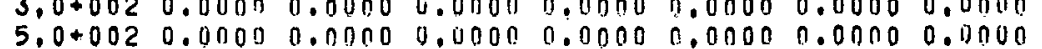

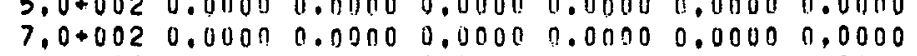

$1,0+0030,00000,00000,00000.00000,00000,0000$

ALPHA $1.000 \quad 2.000 \quad 3.000 \quad 5.000 \quad 7.000 \quad 10.00 \quad 20.00 \quad 30.00 \quad 50.00 \quad 70.00 \quad 100.0 \quad 200.0 \quad 300.0 \quad 500.0 \quad 700.0 \quad 1000$ BETA

$1.0=0030.85700 .99210 .99910 .99991 .00001 .00001 .00001 .00001 .00001 .00001 .00004 .00001 .00001 .00001 .00001 .0000$ $2.0=0030.85690 .99210 .99910 .99991 .00001 .00001 .00001 .00001 .00001 .00001 .00001 .04001 .00001 .00001 .00001 .0000$ $3.0=0030.85680 .09210 .99910 .99991 .00001 .00001 .00001 .00001 .00001 .00001 .00001 .00001 .00001 .00001 .00001 .0000$

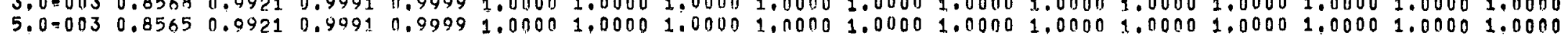

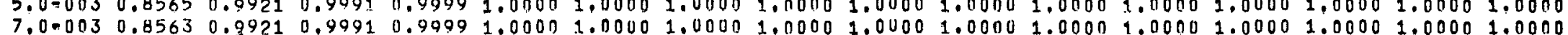

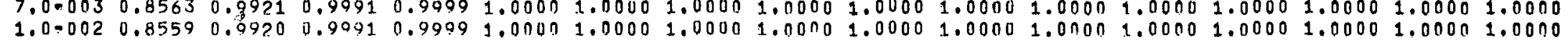
$2.0-0020.85470 .09200 .99910 .99991 .00001 .00001 .00001 .00001 .00001 .00001 .00001 .00001 .00001 .0000$ $3.0=0020.85340 .99190 .9991 \quad 1.99991 .00001 .00001 .00001 .00001 .00001 .00001 .00001 .00001 .0000$

$5,0=0020.85170 .99170,9990 \quad 0.99991 .00001 .00001 .00001 .00001 .00001 .00001 .00001 .0000$

$7,0=0020.84800 .991 .5 \quad 0.9990 \quad 0.99991 .00001 .00001 .00001 .00001 .00001 .00001 .0000$

$1.0 .0010 .84380 .99130 .99900 .9999 \quad 1.00001 .00001 .00001 .00001 .00001 .00001 .0000$

$2.0 .0010 .8276 \quad 0.9902 \quad 0.99890 .9999 \quad 1.00001 .00001 .00001 .00001 .0000$

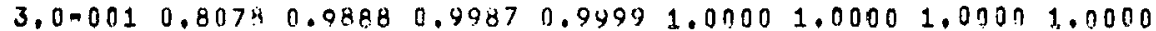

$\begin{array}{llllll}5.0-001 & 0.7529 & 0.9846 & 0.9982 & 0.9999 & 1.00001 .00001 .0000\end{array}$

$\begin{array}{lllllll}7,0-001 & 0.6711 & 0.9772 & 0.9973 & 0.9998 & 1.00001 .0000\end{array}$

$1.0+000 \quad 0.5000 \quad 0.0545 \quad 0.9945 \quad 0.9997 \quad 1.0000 \quad 1.0000$

$2.0 \$ 000 \quad 0.0870 \quad 0.6667 \quad 0.9455 \quad 0.9968$

$3.0+000 \quad 0.016 ? \quad 0.2571 \quad 0.7500$

$5.0+000 \quad 0.00150 .0313$

$7.0+000 \quad 0.0003$

$1.0+001 \quad 0.0001$ 


\begin{abstract}
VALUES UF F FCiN N= 7 AND $N: F=1$ BETA

$\begin{array}{llllllllllllllll}\text { ALPHA } & 0.001 & 10.0102 & 0.003 & 0.005 & 0.007 & 0.01 & 0.02 & 0.03 & 0.05 & 0.07 & 0.1 & 0.2 & 0.3 & 0.5 & 0.7\end{array}$

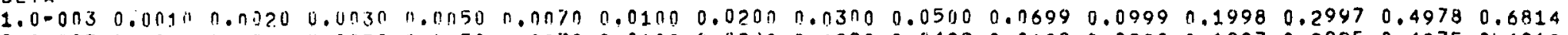

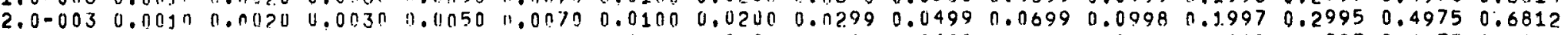

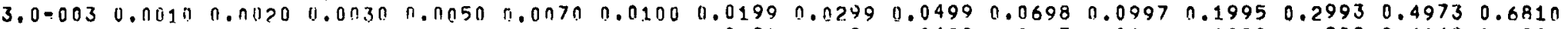

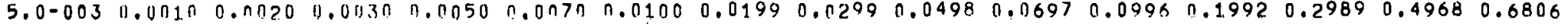

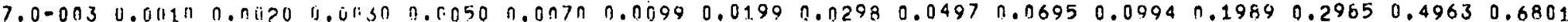
$1.0-00200113) 0.01720 .00300 .00501100690 .00990 .01980 .02970 .04950 .06940 .09910 .19840 .29790 .49560 .6795$ $1.0-002$ v.010

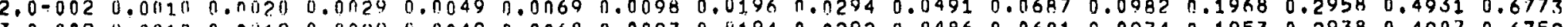
, 0.002 v.

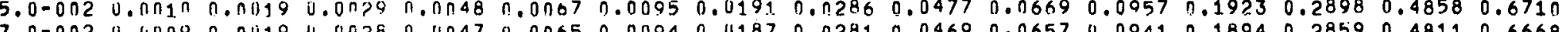

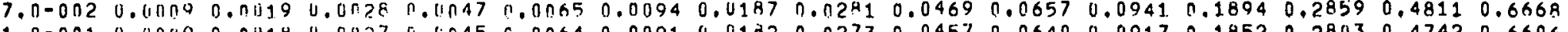

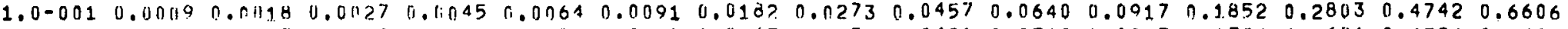

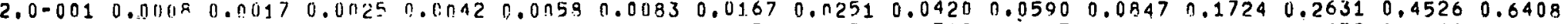

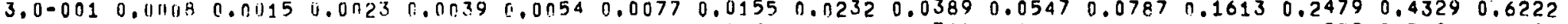
5.0-001 0.unn\% $0.01133 \quad 0.0 n 2 n \quad 0.0033 \quad 0.0 n 47 \quad 0.00670 .01340 .02020 .03390 .04780 .0690 \quad 0.14290 .2222 \quad 0.39810 .5880$

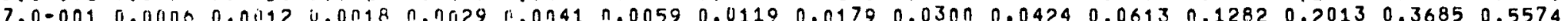
$1.0+0000.00150 .01100 .05150 .025 \quad 1.00350 .00500 .01010 .01520 .02560 .03630 .05260 .11110 .17640 .33160 .5170$

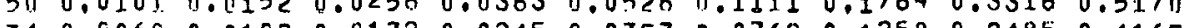
.

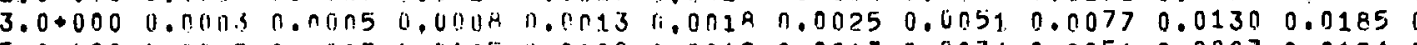

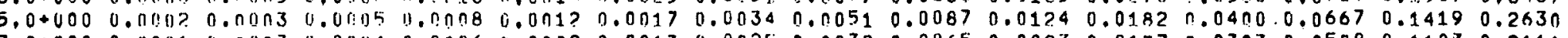

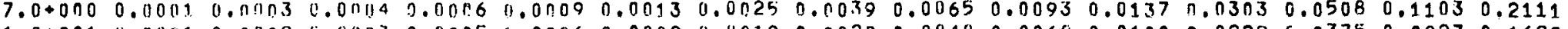

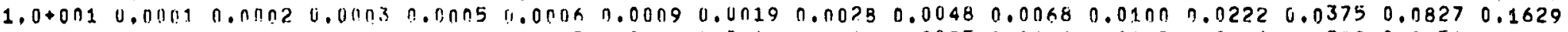

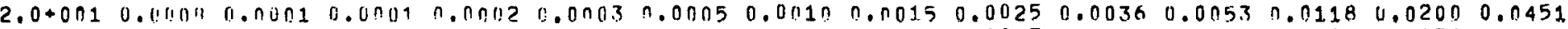

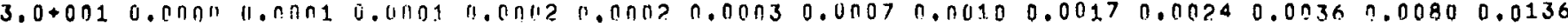

$5.0+0 n 10.00000 .010000 .010010 .00 n 10.0001 .0 .00020 .00040 .00050 .00100 .001 .50 .00220 .0049$

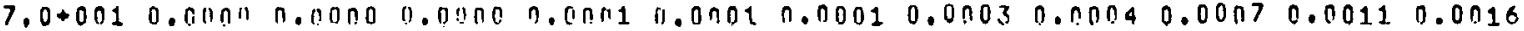

$1.0+0.20 .040 n$ n.01100 0.001180 .0011000010 .00010 .00020 .00030 .00050 .00070 .0011

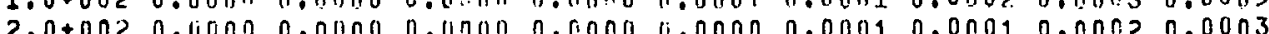

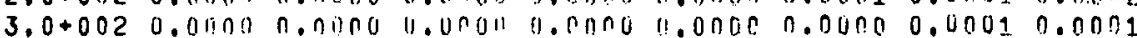

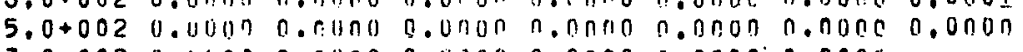

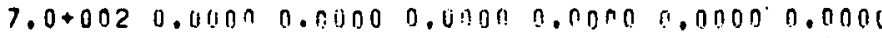

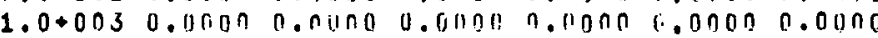

ALPHA $1.0002 .000 \quad 3.0005 .000 \quad 7.000 \quad 10.00 \quad 20.00 \quad 30.00 \quad 50.00 \quad 70.00 \quad 100.0 \quad 200.0 \quad 300.0 \quad 500.0 \quad 700.0 \quad 1000$ BETA

$1.0-003 \quad 0.87440 .0961 \quad 0.9097 \quad 1.00009 .00001 .00001 .00009 .00001 .00001 .00001 .00001 .00001 .00001 .00001 .00001 .0000$ $2.0-0030.87490 .096110 .40071 .001101 .09001 .00001 .00001 .00001 .00001 .00001 .00001 .00001 .00001 .00001 .00001 .0000$ $3.0-0 n 30.07470 .09610 .97971 .00001 .000 n 1,00001.000 n 1.00001 .00001 .00001 .00009 .00001 .00001 .00001 .00001 .0000$

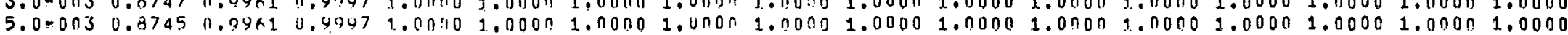

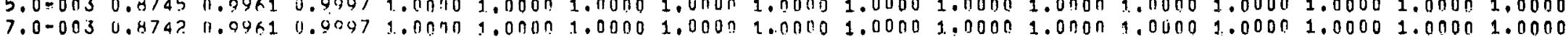
$1.0-0020.87390 .09600 .98971 .00001 .00001 .00001 .0001) 1.00001 .00001 .00001 .00001 .00001 .00001 .00001 .00001 .0000$ $2.0-0020.67200 .0960 \quad 1.9097 \quad 1.0 n 1101.00001 .00001 .00001 .00001 .00001 .00001 .00001 .00001 .00001 .0000$

$3,0-0020.87170 .04600 .40971 .001101 .00001 .001101 .011011 .001101 .00001 .00001 .00001 .00001 .0000$

5.0-002 0.4ngs 0.9959 u.9097 1.00100 1.0n00 1.0000 1.000n 1.0000 1.0000 1.0000 1.00001.0000

$7.0-0020.80740 .99580 .9097 \quad 1.00001 .00001 .00001 .000 n 1.00001 .00001 .00001 .0000$

1.0-001 0.864? $0.04570 .9097,1.00001 .00001 .00001 .00001 .00001 .00001 .00001 .0000$

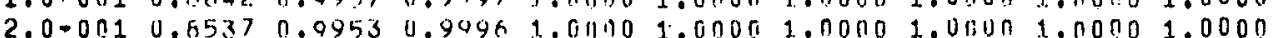

$3.000010 .44340 .09490 .9096 \quad 1.001101 .00001 .000101 .00001 .0000$

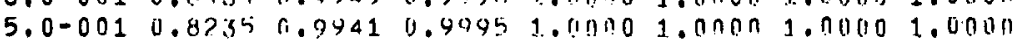

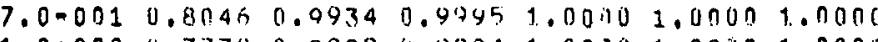

$1.0+000 \quad 0.77790 .0922 \quad 0.9094 \quad 1.001009 .00001 .0000$

$2.0+000 \quad 0.7010010 .9383 \quad 0.9791 \quad 1.01190$

$3.0+000 \quad 0.6304 \quad 11.0845 \quad 0.9988$

$5.0+000 \quad 0.534 h$ h $0.0 \% 69$

$7.0+000 \quad 0.466 \%$

$1.0+0010.3880$ 
VALUES OF F FOR $N=$ ? $\triangle N !) \quad N P=2$

ALPH

BETA

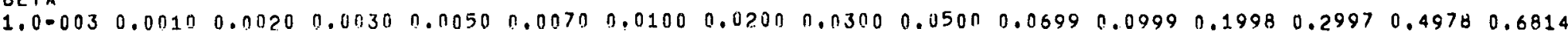

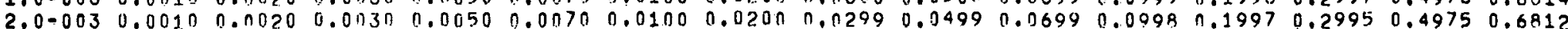
$3.0-0030.0010 \quad 0.01320 \quad 0.00300 .0050 \quad 0.0 n 70 \quad 0.01000 .0199 \quad 0.02990 .04990 .06980 .0997 \quad 0.19950 .2993 \quad 0.4973 \quad 0.6810$

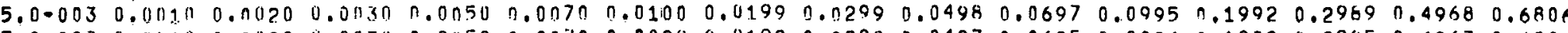

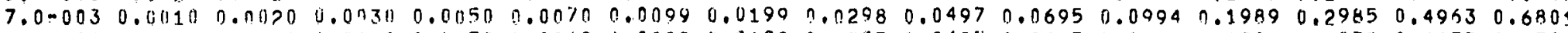

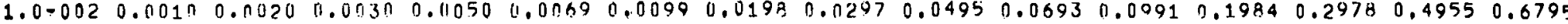

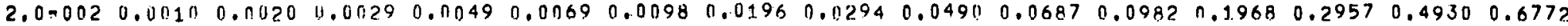

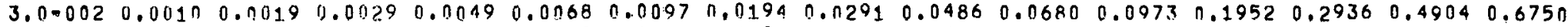
$5.0-0020.00100 .00190 .00290 .00480 .00670 .00950 .01900 .02850 .04760 .06670 .00550 .19190 .28930 .48530 .6704$

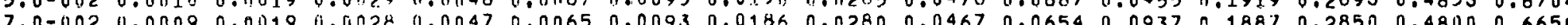
$1.0-0010.00090 .000 .0027$. 00450.00630 .00900 .01810 .02710 .04530 .06350 .00100 .18300 .27050 .47200 .6586

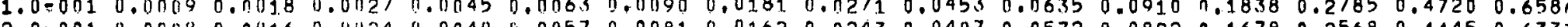

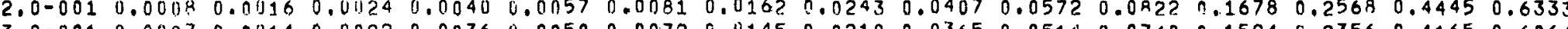

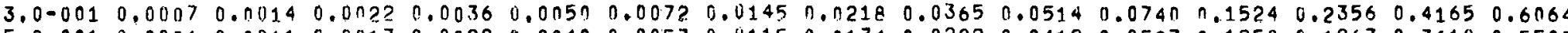

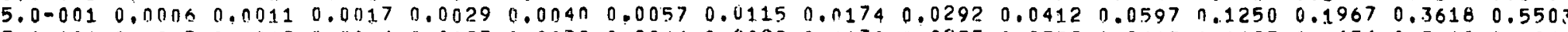
7.0-001 $0.0005 \quad 0.00090 .00140 .0023 \quad 0.0 n 320.00460 .009 ? \quad 0.01390 .02350 .03320 .04830 .10250 .16360 .31180 .4944$

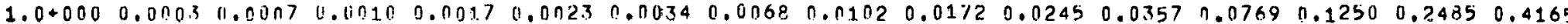

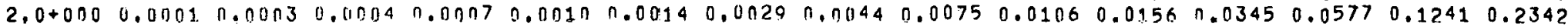
$3.0+000 \quad 0.01010 .00020 .0002 \quad 0.00040 .00050 .0008 \quad 0.00160 .00240 .0040 \quad 0.0058 \quad 0.0085 \quad 0.01890 .03190 .07090 .1414$ $5.0+0000.00000 .00010 .0 n 010.00 n 20.00020 .00030 .00070 .00100 .00170 .00240 .00360 .00800 .01360 .03100 .0646$ $7.0+0000.00000 .00000 .00010 .00010 .00010 .00020 .00040 .00050 .00090 .00130 .00190 .00440 .00750 .01710 .0362$

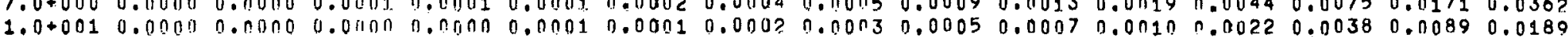

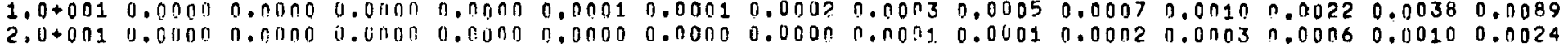

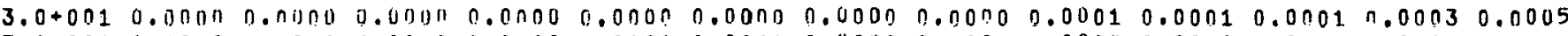
$5.0+0010.00000 .0000 \quad 0.00000 .00000 .00000 .00000 .00000 .00000 .00000 .00000 .00000 .0001$

$7.0+0010.010000 .0000 \quad 0,00000.00000 .00000 .00000 .0000,0.00000 .00000 .00000 .0000$

$1.0+0020.00000 .00000 .00000 .00000 .00000 .0000000000 .00000 .00000 .00000 .0000$

$2.0+0020.00000 .00000 .00000 .00000 .00000 .00000 .00000 .00000 .0000$

$3.0+0020.0000 \quad 0.00000 .0 n \pi 00.00100 .00000 .00000 .0000,0.0000$

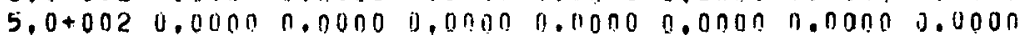

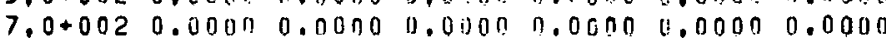

$1.0+0030.00000 .00000 .00000 .00000 .00000 .0000$

$$
A L P H
$$

BETA

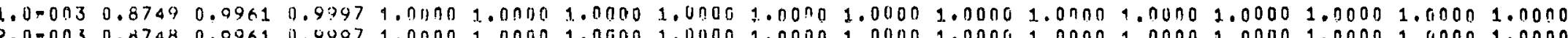
$2,0=0030.07480 .09610 .49971 .00001 .00001 .00001 .0000 \quad 2.00001 .00001 .00001 .00001 .00001 .00001 .00001 .00001 .0000$ $3.0-0030.87470 .0961 \quad 0.9097 \quad 1.001101 .00001 .00001 .00001 .001001 .00001 .00001 .00001 .00001 .00001 .00001 .00001 .0000$ $5.0-0030.87450 .09610 .99971 .00001 .00001 .05001 .00001 .00001 .00001 .00001 .000 n 1.00001 .00001 .00001 .00001 .0000$

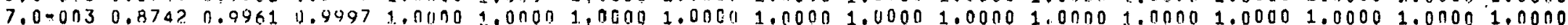

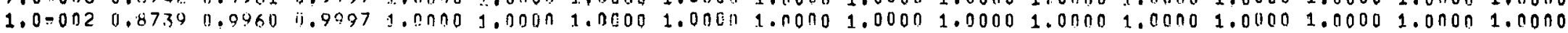
$2.0-0020.87200 .9960 \quad 3.9997 \quad 1.00001 .00001 .00001 .00001 .00001 .00001 .00001 .00001 .00001 .00001 .0000$ $3.0-0020.87150 .99600 .40971 .00001 .00001 .00001 .00001 .00001 .00001 .00001 .00001 .00001 .0000$ $5.0-0020.86930 .99590 .99971 .00001 .00001 .06001 .00601 .00001 .00001 .00001 .00001 .0000$

$7.0-002 \quad 0.86690 .9958 \quad 0.9997 \quad 1.00901 .00001 .00001 .00001 .00001 .00001 .00001 .0000$

$1.0=0010.8631,0.9950 \quad 0.9997 \quad 1.00001 .00001 .00001 .00601 .00001 .00001 .00001 .0000$

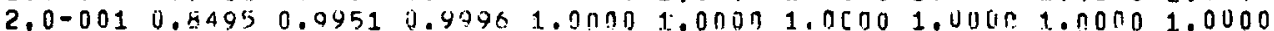

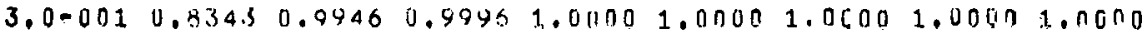

$5.0=0010.80000 .99320 .9095 \quad 1.01001 .00001 .06001 .0000$

7.0-001 0.76170 .99150 .99931 .00001 .00001 .0600

$1.0+000 \quad 0.711000 .0883 \quad 0.9991 .00001 .00001 .0600$

$2.0+000 \quad 0.5000 \quad 0.9732 \quad 0.99790 .9999$

$3.0+000 \quad 0.35000 .9593 \quad 0.9061$

$5.0+000 \quad 0.144 ? \quad 0.8912$

$7.0+000 \quad 0.1094$

$1.0+0010.0593$ 


\begin{abstract}
VALUES OF F FON $N=7$ AND NPP= 3
BETA

$\begin{array}{llllllllllllllllllll}\text { ALPHA } & 0.001 & 0.012 & 0.003 & 0.005 & 0.007 & 0.01 & 0.02 & 0.03 & 0.05 & 0.07 & 0.1 & 0.2 & 0.3 & 0.5 & 0.7\end{array}$

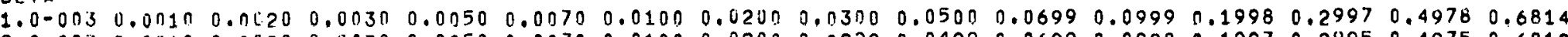
2.0-003 $0.0010 \quad 0.0120 \quad 0.0030 \quad 0.0050 \quad 0.0070 \quad 0.0100 \quad 0.0200 \quad 0.02990 .04990 .06990 .0998 \quad 0.19970 .2995 \quad 0.49750 .6812$

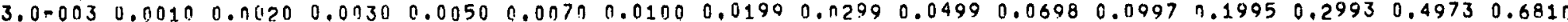

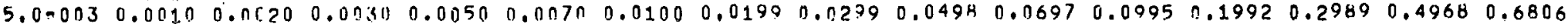

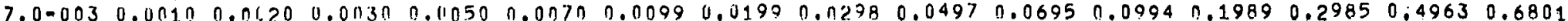
$1.0-0020.00100 .00200 .00300 .00500 .00690 .00990 .01980 .02770 .04950 .06930 .09910 .19840 .29780 .49550 .6795$

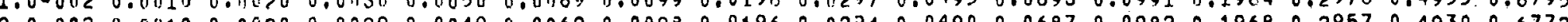
$2,0-0020.00100 .06200 .00290 .00490 .00690 .00980 .01964 .02740 .04900 .06870 .09820 .19680 .29570 .49300 .6772$ $3.0=002$ 0.1n1 0.00190 .00290 .00490 .00680 .00970 .01940 .12910 .04860 .06800 .09730 .19520 .29360 .49040 .6750

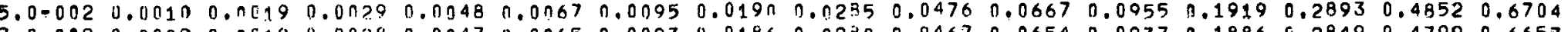

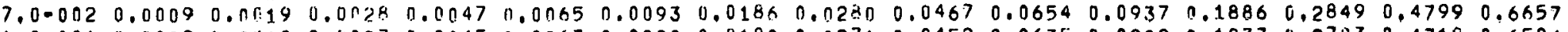

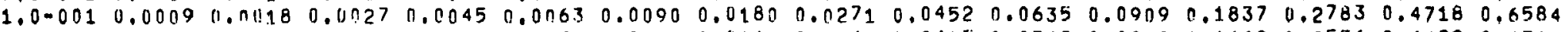
$2.0-0010.0008 \quad 0.0016 \quad 0.0024 \quad 0.0040 \quad 0.0056 \quad 0.00800 .01610 .02420 .04050 .0569 \quad 0.08180 .1669 \quad 0.2556 \quad 0.44290 .6318$

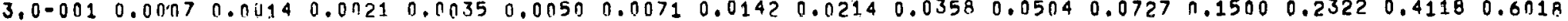
$\begin{array}{lllllllllllllllllll}5.0001 & 0.0005 & 0.0011 & 0.0016 & 0.0027 & 0.0037 & 0.0054 & 0.0109 & 0.0162 & 0.0273 & 0.0386 & 0.0559 & 0.1176 & 0.1860 & 0.3460 & 0.5331\end{array}$ 7.0-001 $0.00040 .00080 .00120 .0020 \quad 0.00280 .0040 \quad 0.008 n \quad 0.01210 .02040 .02890 .04200 .0898 \quad 0.1447 \quad 0.28150 .4581$ $1.0+0000.00030 .00050 .00080 .00130 .00180 .00250 .00510 .00770 .01300 .01850 .02700 .05880 .09680 .19870 .3487$ (0.0740.010 $0.00350 .00500 .00740 .01640 .02780 .0620 \quad 0.1249$

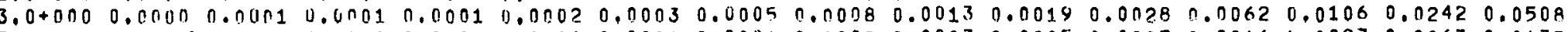
$5.0+\cos 0.000 .00 .00 .00 \quad 0.0000 \quad 0.0000 \quad 0.0000 \quad 0.00010 .00010 .00720 .00030 .00050 .00070 .00160 .00270 .00630 .0135$

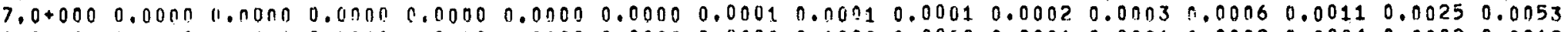
$1.0+0010.000 n$ n. n000 $0.1000 .1100 n 00.00000 .00000 .00000 .00000 .00000 .00010 .00010 .00020 .00040 .00090 .0019$ $2.0+0010.00000 .00000 .00000 .00000 .00000 .00000 .00000 .00000 .00000 .00000 .00000 .00000 .00010 .0001$

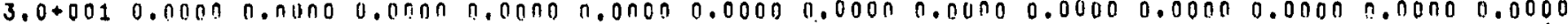

$5.0+0010.000 n \quad 0.00000 .011000 .00000 .00000 .00000 .00000 .00000 .00000 .00000 .00000 .0000$

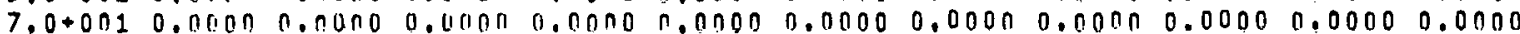

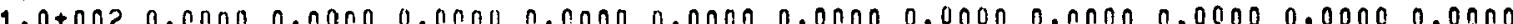

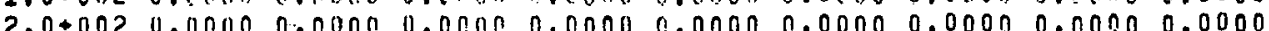

$3.0+0020.010000 .00000 .00000 .00000 .00000 .00000,00000.0000$

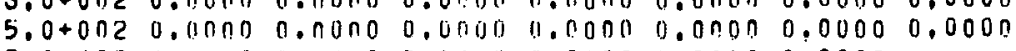

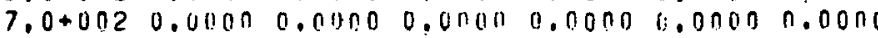

$1,0+0030.00000 .00 n 00.000 n 0.00000 .00000 .0000$

BETA

$1.0-0030.87490 .04610 .90971 .00001 .00001 .00001 .00001 .00001 .00001 .00001 .00001 .00001 .00001 .00001 .00001 .0000$ $2.0-0030.87480 .09610 .40971 .00001 .00001 .00001 .00001 .00001 .00001 .00001 .00001 .00001 .00001 .00001 .00001 .0000$ $3,0=0030.87470 .09610 .40971 .00001 .00001 .00001 .00001 .00001 .00001 .00001 .00001 .00001 .00001 .00001 .00001 .0000$ $5.0-0030.87450 .09610 .49971 .00001 .00001 .00001 .00001 .00001 .00001 .00001 .00001 .00001 .00001 .00001 .00001 .0000$ 7.0-003 0.874? 0.9961.0.4497 1.00001.00001.00001.00001.00001.00001.00001.00001.00001.00001.00001.00001.0000

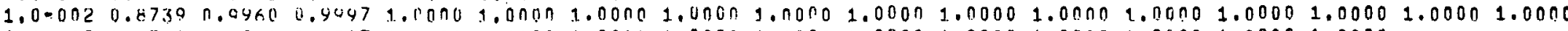
2.0-002 $0.87280 .0960 \quad 0.49971 .00001 .00001 .00001 .00001 .00001 .00001 .00001 .00001 .00001 .00001 .0000$

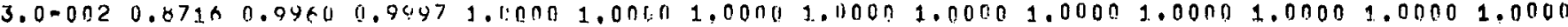

$5.0-0020.86930 .99590 .9097 \quad 1.00001 .00001 .00001 .00001 .00001 .00001 .00001 .00001 .0000$

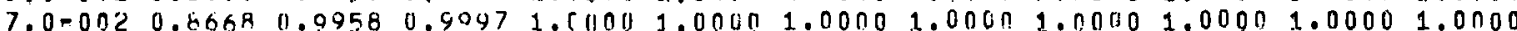

$1.0-0010.8631) 0.09560 .409 \% 1.00001 .00001 .00001 .00001 .00001 .00001 .00001 .0000$

2.0-001 0.84870 .0951 0.4ngh 1.0000 1.0nen 9.0000 1.000n 1.0n110 1.0000

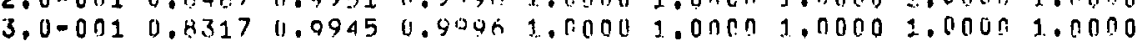

$5.0-0010.78870 .99770 .40941 .010001 .00001 .00001 .0000$

$7.0-0010.7343 \quad 0.99110 .994 ? \quad 1.010001 .00001 .0000$

$1,0+000 \quad 0.63640 .0545 \quad 1.90841,00001.00001 .0000$

$2.0+000 \quad 0.31820 .0442 \quad 0.9034 \quad 0.9998$

$3.0+000 \quad 0.14490 .46390 .4479$

$5.0+000 \quad 0.014290 .6195$

$7.0+0000.1177$

$1,0+0010.0063$ 
$1.0-003.0 .0010 \quad 0.0020 \quad 0.00300 .0050 \quad 0.0070 \quad 0.0100 \quad 0.0200 \quad 0.0300 \quad 0.0500 \quad 0.06990 .0999 \quad 0.1998 \quad 0.2997 \quad 0.4978 \quad 0.6814$

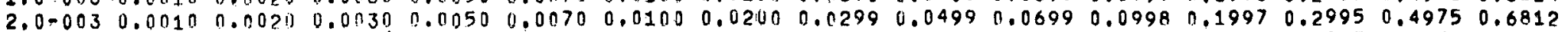

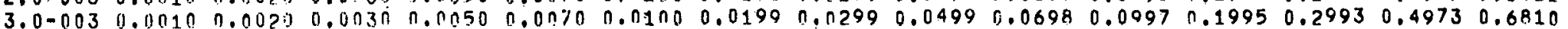

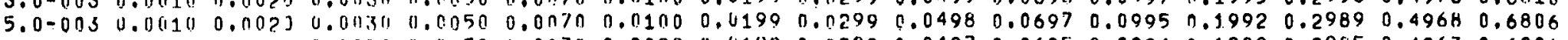

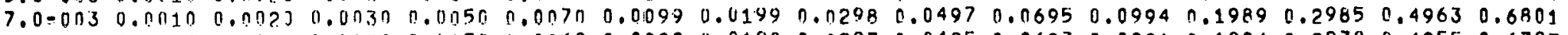

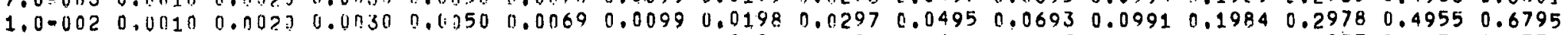
2.0-002 0.01010 0.0020 $0.00290 .00490 .00690,00980.0196 \quad 0.02940 .04900 .06870 .0982 \quad 0.19680 .2957 \quad 0.4930 \quad 0.677 ?$ $3.0=002 \quad 0.00100 .04170 .0 n 290.00490 .00680 .0097 \quad 0.01940 .02910 .04860 .06800 .0973 \quad 0.1952 \quad 0.2936 \quad 0.49040 .6750$ 5.0-002 $0.00100 .0018 \quad 0.0 n 29 \quad 0.0048 \quad 0.0167 \quad 0.0095 \quad 0.019 n \quad 0.02850 .0476 \quad 0.06670 .0055 \quad 0.19190 .2893 \quad 0.4852 \quad 0.6704$ 7.0-002 0.0009 0.0017 0.0128 0.00470 .00650 .00930 .01860 .02800 .04670 .06540 .09370 .18860 .28490 .47990 .6657 $1.0-0.010 .00090 .01180 .00270 .00450 .00630 .00900 .01800 .02710 .04520 .06340 .09090 .18370 .27830 .47170 .6584$

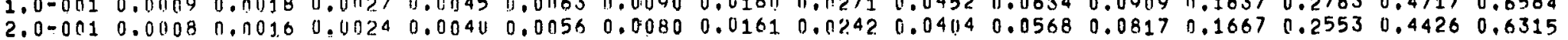

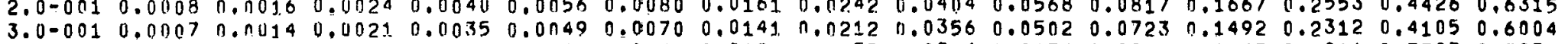

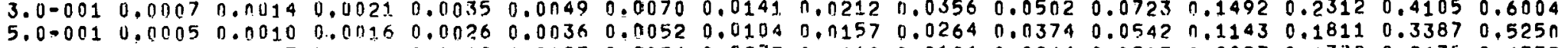

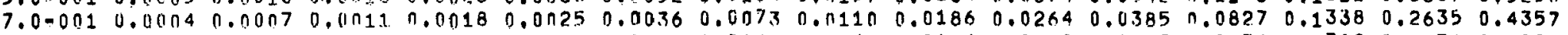
$1.0+0000.0110 ? 0.00040 .00060 .00100 .00140 .0020 \quad 0.00410 .011610 .01040 .01480 .03170 .04760 .07890 .16560 .2998$

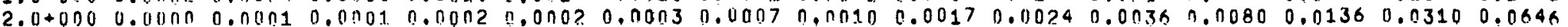
$3.0+0000.00000 .00000 .00000 .00000 .00410,00010.00020 .00030 .00040 .00000 .00090 .00210 .00350 .00810 .0174$ $5.0+0000.00000 .00000 .00000 .00000 .00000 .00000,00110.00000 .0009,0.00010 .00010 .00030 .00050 .00130 .0027$

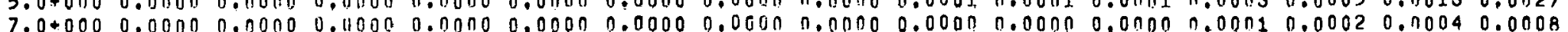
$7.0+000$
$1.0+001$

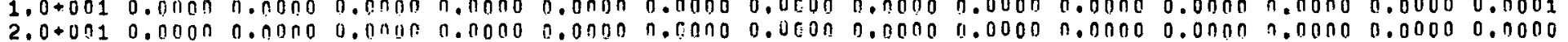

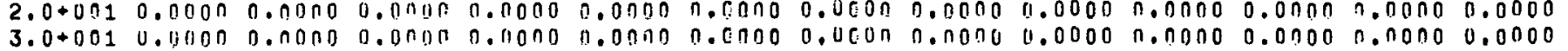
$5.0+0010.00000 .00000 .00000 .00000 .00000 .60000 .00000 .00000 .00000 .00000 .00000 .0000$ $7.0+0010.00000 .00000 .01000 .00000 .0000 \quad 0,00000.00000 .00000 .00000 .00000 .0000$ $1,0+0020.00000 .00000 .00000 .00000 .00100 .00000 .00000 .00000 .00000 .00000 .0000$

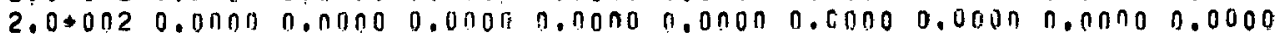

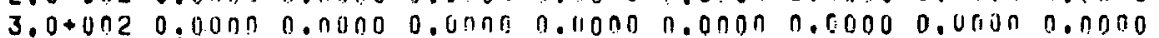

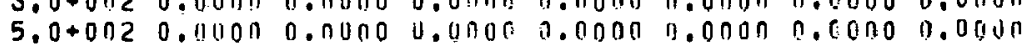

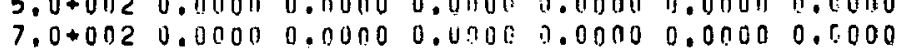
$1.0+0030.00000 .00000 .00000 .00000 .0000 .0 .0000$

$$
\text { BETA }
$$

$1.0-0030.87490 .0961 \quad 0.99971 .00001 .00001 .00001 .00001 .00001 .00001 .00001 .00001 .00001 .00001 .00001 .00001 .0000$ $2.0-0030.87480 .9961 \quad 0.49971 .00001 .00001 .00001 .000 n 1.00001 .00001 .00001 .00001 .00001 .00001 .00001 .00001 .0000$ $3.0-0030.87470 .99,610.99971 .00101 .00001 .00001 .000 n 1.00001 .00001 .00001 .00001 .00001 .00001 .00001 .00001 .0000$ $5.0-0030.87450 .99610 .99971 .00001 .00001 .00001 .00001 .00001 .00001 .00001 .00001 .00001 .00001 .00001 .00001 .0000$

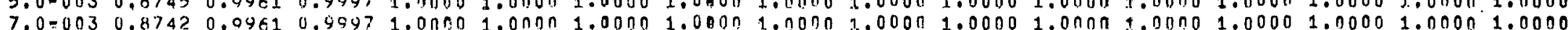

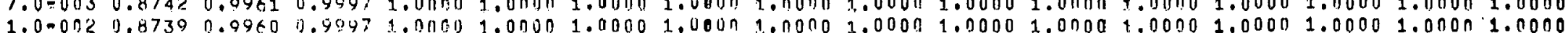

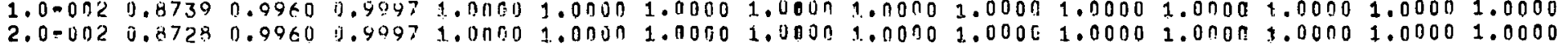
$3.0-0020.87160 .0960 \quad 0.90971 .00001 .00001 .00001 .00001 .00001 .00001 .00001 .00001 .00001 .0000$ $5.0-0020.86930 .99590 .99971 .00001 .00001 .00001 .00001 .00001 .00001 .00001 .00001 .0000$ 7.0.002 $0.86680 .99580 .99971 .00001 .00001 .00001 .000 n 1.00001 .00001 .00001 .0005$ $1.0-0010.36300 .09560 .99971 .00001 .00001 .00001 .00001 .00001 .00001 .00001 .0000$

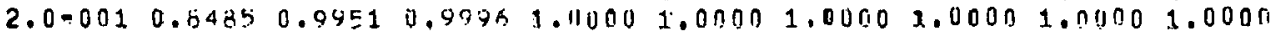
$3.0-0010.83090 .09440 .9096 \quad 1.00001 .00001 .0000 \quad 1.00001 .0000$ $5.0-0010.79320 .99240 .99941 .00001 .00001 .00001 .0000$

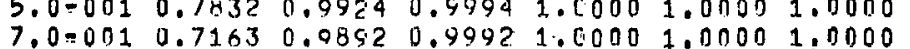

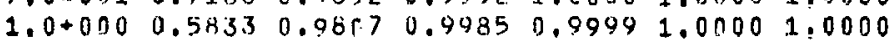
$2.040000 .184 \% 0.89120 .99000 .9997$

$3.0+0 \cap 0 \quad 0.115470 .4773 \quad 0.9644$

$5.0+0000.00890 .2454$

$7.0+000 \quad 0.0025$

$1.0+001 \quad 0.0006$ 


\begin{abstract}
VALUES OF $F$ FOH
BET A

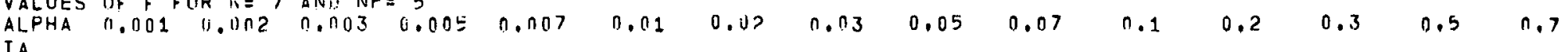

$1.0-0030.7 n 100.0020 \quad 10.0030 \quad 0.005 \pi \quad 0.0 n 70 \quad 0.0100 \quad 0.0200 \quad 0.0 .300 \quad 11.0500 \quad 0.0699 \quad 0.09990 .1998 \quad 0.2997 \quad 0.4978 \quad 0.6814$ $2.0-0030.0010 \quad 0.0020 \quad 0.003 \pi \quad 0.10050 \quad 0.007 n \quad 0.0100 \quad 0.0200 \quad 0.02990 .0499 \quad 0.0699 \quad 0.0998 \quad 0.1997 \quad 0.2995 \quad 0.4975 \quad 0.6812$

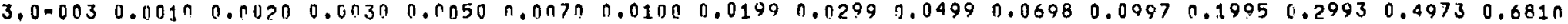

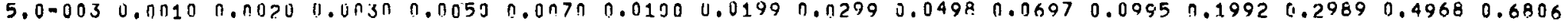
$7.0-003 \quad 0.001 n \quad 0.0020 \quad 0.0030 \quad 0.0050 \quad 0.007 n \quad 0.00990 .0190 \quad 0.02980 .04970 .06950 .00940 .19890 .09850 .49630 .6801$

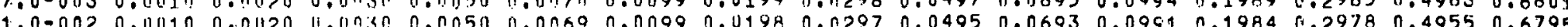

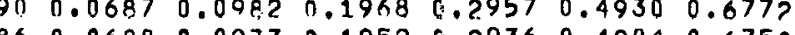

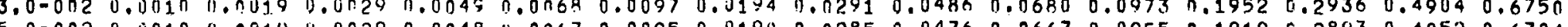

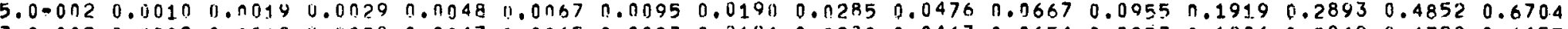

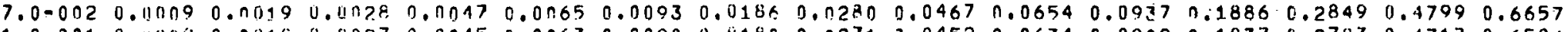
1.0-001 $0.10090 .0018 \quad 0.0027 \quad 0.00450 .0063 \quad 0.0090 \quad 0.018 n \quad 0.0271 \quad 0.0452 \quad 0.06340 .09090 .1837 \quad 0.2783 \quad 0.47170 .6584$

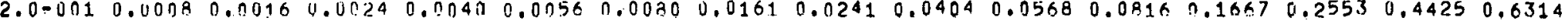

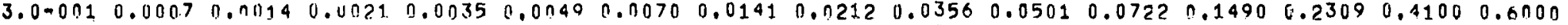
$\begin{array}{llllllllllllllllll}5.0-001 & 0.0005 & 0.0010 & 0.1015 & 0.0025 & 0.0 n 36 & 0.0051 & 0.0103 & 0.0155 & 0.0260 & 0.0368 & 0.0534 & 0.112 .7 & 0.1787 & 0.3351 & 0.5210\end{array}$

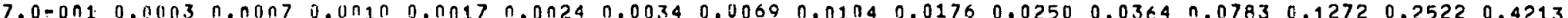
$1.0+0000.11020 .00030 .40050 .00080 .00120 .00170 .00340 .00510 .00870 .01240 .09820 .04000 .06670 .14190 .2630$

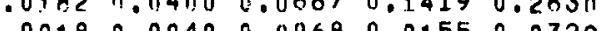

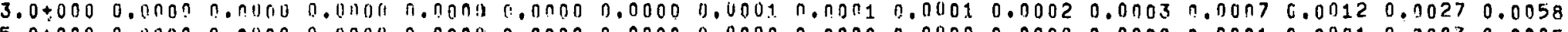

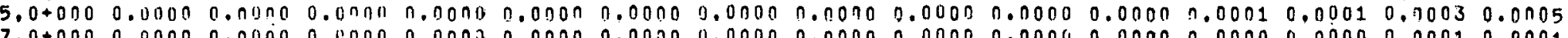
$7.0+000 \quad 0.0000 \quad 0.00000 .00000 .00000 .00000 .00000 .00000 .00000 .00000 .00000 .00000 .00000 .00000 .00010 .0001$

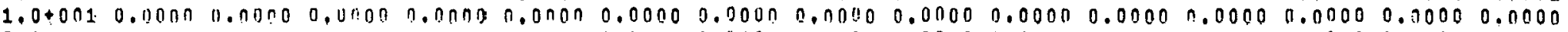

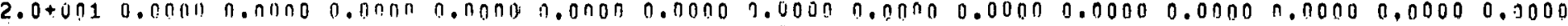

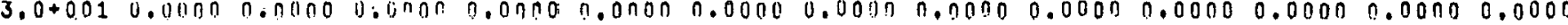

$5.0+0010.00000 .00000 .00000 .00000 .00000 .00000 .00000 .00000 .00000 .00000 .00000 .0000$

$7.0+0010.0 n 000.00000 .0 n n 0,0.00000 .00000 .00000 .000 n 0.00000 .00000 .00000 .0000$

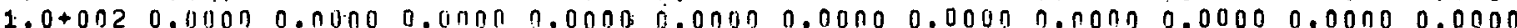

$2.0+0020.00000 .00000 .00000 .00000 .00000 .00000 .00000 .00000 .0000$

$3.0+0 n 2$ o.

$5.0+0 n 20.00000 .013000 .00000 .000000 .00000 .00000 .0000$

$7.0+0.020 .00000 .00000 .60009 .00000 .00000 .0000$

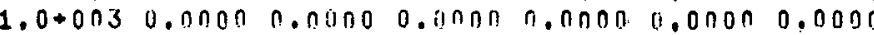
HETA

$1.0-003 \quad 0.87490 .9961 .4 .4997 \quad 1.00001 .00001 .00001 .00001 .00001 .00001 .00001 .00001 .00001 .00001 .00001 .00001 .0000$ 2.0-00S 0.47490 .99610 .49971 .00011 .00001 .00001 .00001 .00001 .00001 .00001 .00001 .00001 .00001 .00001 .00001 .0000 $3.0-0030.67470 .09610 .99971 .00301 .00001 .00001 .00001 .00601 .00001 .00001 .00001 .00 n 01.00001 .00001 .00001 .0000$ $5.0-0030.87450 .9961 \quad 0.59471 .00001 .00001 .00001 .00001 .00001 .00001 .00001 .00001 .00001 .00001 .00001 .00001 .0000$ $7.0-0030.87420 .99610 .40971 .00001 .00001 .00001 .00001 .00001 .00001 .00001 .00001 .00001 .00001 .00001 .00001 .0000$ $1.0-0020.87390 .994000 .44971 .00001 .00001 .00001 .000 n+1.00001 .00001 .00001 .00001 .00001 .00001 .00001 .00001 .0000$ $2.0-0020.47280 .09600 .4097 \quad 1.00001 .00001 .00001 .00001 .00001 .00001 .00001 .00001 .00001 .00001 .0000$

$3.0-0020.87160 .9960 .0 .90971 .00001 .00001 .00001 .00001 .00001 .00001 .00001 .00001 .00001 .0000$

$5.0-002 \quad 0.86930 .99590 .9997 \quad 1.00001 .00001 .00003 .00001 .00001 .00001 .00001 .00001 .0000$

7.0-002 0.8658 $0.09580 .9997 \quad 1.00001 .00 n 01.00001 .00001 .00001 .00001 .00001 .0000$

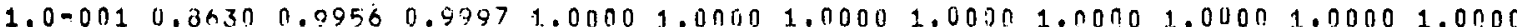

$2.0-0010.84850 .0451 \quad 0.99441 .00001 .00001 .00001 .00041 .00001 .0005$

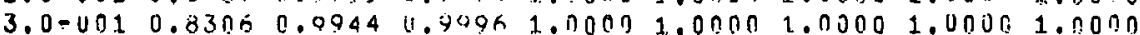

$5.0-001 \quad 0.7805 \quad 0.0923 \quad 0.90941 .00001 .00001 .00001 .0000$

$7.0-0010.70410 .98$ B $0.9991 \quad 1.00101 .00001 .0000$

$1.0+000 \quad 0.5385 \quad 0.9169 \quad 1.978 ? \quad 0.99991 .00001 .0000$

$2.0+000 \quad 0.1100 \quad 0.0013$ ij. 9.8110 .9994

$3.0+000 \quad 0.01690 .11160 .41101$

$5.0+0000.0018 \quad 0.0611$

$7.0+000 \quad 0.0004$

$1.0+001 \quad 0.0001$ 
VALUES OF F FOR $N=8$ AIN! $N P=1$

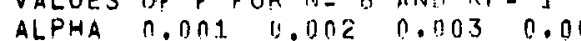
BETA

$1.0-0030.011100 .00200 .0030 \quad 0.0050 \quad 0.0070 \quad 0.01000 .0200 \quad 0.03000 .05000 .06990 .09990 .19980 .2998 \quad 0.49880 .6872$ $2.0=0030.01100 .0020 \quad 0.00300 .00500 .00700 .01000 .02000 .02990 .04990 .06990 .09980 .19970 .29960 .49850 .6870$

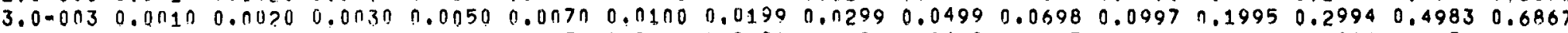
$5.0-003 \quad 0.0010 \quad 0.0020 \quad 0.4030 \quad 0.0050 \quad 0.007 n \quad 0.01 .00 \quad 0.0199 \quad 0.02990 .0498 \quad 0.0697 \quad 0.0996 \quad n .1992 \quad 0.29890 .4978 \quad 0.6863$

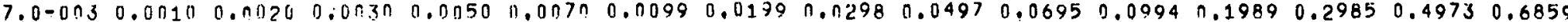
$\begin{array}{llllllllllllllll}1.0-002 & 0.0010 & 0.0020 & 0.0030 & 0.0050 & 0.0069 & 0.0099 & 0.0198 & 0.0297 & 0.0495 & 0.0694 & 0.0091 & 0.1984 & 0.2979 & 0.4965 & 0.6852\end{array}$ $2.0-0020.00100 .00200 .00290 .00490 .00690 .00980 .01960 .02940 .04910 .06870 .09820 .1968 \quad 0.29580 .49410 .6831$ $3.0-0020.00100 .00190 .00290 .00490 .00680 .00970 .01940 .02920 .04860 .06810 .09740 .19530 .29380 .49160 .6810$ $3.0-0020.1010 .0019 .00290 .00490 .00680 .00970 .01940 .02920 .04860 .06810 .0974 n 19530.29380 .49160 .6810$ $5.0-0020.0010 .00190 .00290 .0048 n .00670 .00950 .01910 .02860 .04770 .06690 .0957 n .192 .30 .28980 .48680 .6768$ $7.0-0020.00090 . n 1.90 .0128 .0 .00470 .05650 .00940 .0187 n .02810 .04690 .06570 .09410 .18940 .28600 .48210 .6727$ $1.0=0010.00090 .00180 .01270 .0 n 450.09640 .00910 .01820 .02730 .04570 .06400 .0917 n .18520 .28040 .47520 .6665$

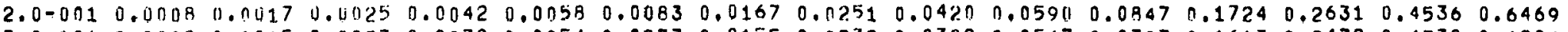
$\begin{array}{lllllllllllllllll}3.0 .001 & 0.0008 & 0.0015 & 0.0 n 23 & 0.0039 & 0.0 n 54 & 0.0077 & 0.0155 & 0.0232 & 0.0389 & 0.0547 & 0.0787 & 0.1613 & 0.2479 & 0.4338 & 0.6284\end{array}$ $\begin{array}{llllllllllllllllll}5.0=001 & 0.0007 & 0.0013 & 0.0020 & 0.0033 & 0.0047 & 0.0067 & 0.0134 & 0.0202 & 0.0339 & 0.0478 & 0.0690 & 0.1429 & 0.2222 & 0.3991 & 0.5945\end{array}$ $\begin{array}{llllllllllllllll}7.0-001 & 0.0006 & 0.0012 & 0.0 n 18 & 0.0129 & 0.0041 & 0.0059 & 0.0119 & 0.0179 & 0.0300 & 0.0424 & 0.0513 & 0.1282 & 0.2013 & 0.3695 & 0.5640\end{array}$ $\begin{array}{lllllllllllllllll}1.0+000 & 0.0005 & 0.0010 & 0.0115 & 0.0025 & 0.0035 & 0.0050 & 0.0101 & 0.0152 & 0.0256 & 0.0363 & 0.0526 & 0.1111 & 0.1765 & 0.3325 & 0.5237\end{array}$ $2.0+000 \quad 0.00 n 3 \quad 1.0007 \quad 0.0 n 10 \quad 0.0 n 17 \quad 0.0 n 23 \quad 0.00340 .00680 .01020 .01 \% 20.02450 .0357 \quad 0.0769 \quad 0.1250 \quad 0.24930 .4229$ $3.0+0000.00030 .0050 .01080 .00130 .00180 .00250,00510.01170 .01300 .01850 .02700 .05880 .09680 .19940 .3547$ $5.0+0000.00020 .0030 .00050 .00080 .00120 .00170 .00340 .00590 .00870 .01240 .01820 .04000 .06670 .14240 .2682$

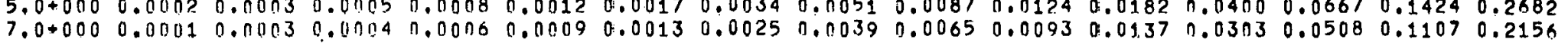

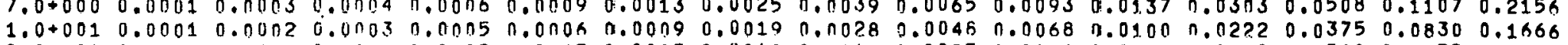
$2.0+0010.01000 .00010 .00010 .00020 .00030 .000 .50 .00100 .00150 .00250 .0036 \quad 0.0053 \quad 0.01180 .02 n 00.0453$ $3.0+0010.00000 .00 n 10.00010 .00020 .00020 .00030,00070.00100 .00170 .00240 .00360 .0090 \quad 4.0136$

$5.0+0010.00000 .00000 .60010 .00010 .00010 .00020 .00040 .011060 .00100 .00150 .0 n 220.0049$

$7.0+0010.00000 .00000 .00000 .00010 .00010 .00010 .00030 .00040 .00070 .00110 .01616$

$1.0+0020.0000 \quad 0.00000 .00000 .00000 .00010 .00010 .00020 .00030 .00050 .00070 .0011$

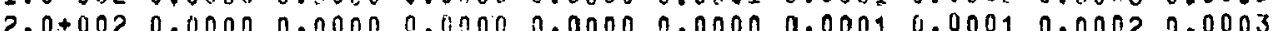

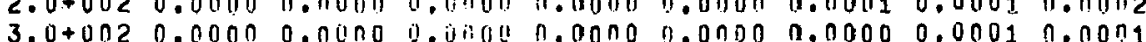

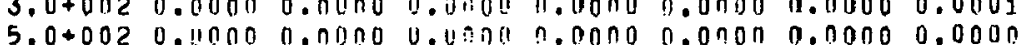

$7.0+0020.00000 .00000 .00000 .00000 .00000 .0000$

$1.0+0030.00000 .00000 .0000 \quad 0.00000 .0000 \quad 0.0000$ BET TA

$1.0-0030.88840 .99800 .90991 .00001 .00001 .00001 .00001 .00001 .00001 .00001 .00001 .00001 .00001 .00001 .00001 .0000$ $2.0=0030.88870 .0980$ i. 90991.110001 .00001 .00001 .00001 .00001 .00001 .00001 .00001 .00001 .00001 .00001 .00001 .0000 $3.0-0030.88860 .09800 .99991 .00001 .00001 .00001 .00001 .00001 .00001 .00001 .00001 .00001 .00001 .00001 .00001 .0000$

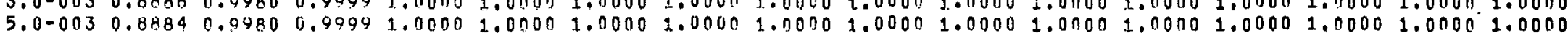

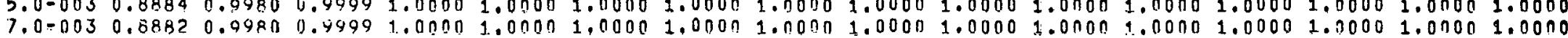

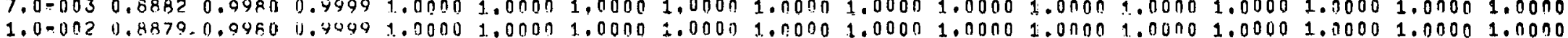
$2.0-0020.88690 .09800 .90991 .00001 .00001 .00001 .00001 .00001 .00001 .00001 .00001 .00001 .00001 .0000$ $3.0-0020.88540 .09800 .99491 .00001 .00001 .00001 .00001 .00001 .00001 .00001 .00001 .00001 .0000$

$5.0-0020.8840 \quad 0.99790 .99991 .00001 .00001 .00001 .00001 .00001 .00001 .00001 .00001 .0000$

$7.0=002 \quad 0.8820 \quad 0.99790 .99991 .00001 .00001 .00001 .00001 .00001 .00001 .00001 .0000$

$1.0=0010.57910 .09780 .9999 \quad 1.00001 .00001 .00001 .00001 .00001 .00001 .00001 .0000$

2.0-001 $0.86060 .99770 .99991 .000 ! 1.00001 .00001 .00001 .00001 .0000$

$3.0=0010.86020 .09750 .99991 .00001 .00009 .00001 .000 n 1.0000$

5.0-001 $0.84210 .99710 .9798 \quad 1.00001 .00001 .00001 .0000$

$7.0-0010.82470 .99670 .9998 \quad 1.00001 .00001 .0000$

$1.0+000 \quad 0.8000 \quad 0.9961 \quad 0.9996 \quad 1.00001 .00001 .0000$

$2.0+000 \quad 0.7273 \quad 0.994 \bar{\varepsilon} \quad 0.9997 \quad 1.0000$

$3,0+000 \quad 0.6667 \quad 0.9926 \quad 0.9496$

$5.0+000 \quad 0.5714 \quad 0.9884$

$7.0+000 \quad 0.5000$

$1,0+0,10,4211$ 
V.ALUES OF $F: O H N=B$ AINI NP= 2 BETA

1.0-0030.0010 0.0020 0.0030 0.0050 0.0070 0.01000.0200 0.0300 0.05000.06990.00990.19980.29980.49880.6872 2.0-0n3 $0.10100 .00200 .0 n 300.00500 .00700 .01000 .02000 .02990 .04990 .06990 .09980 .19970 .29960 .49850 .6870$

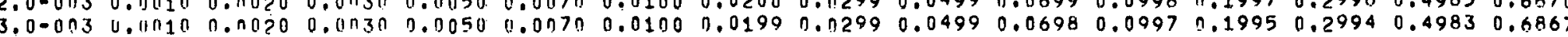

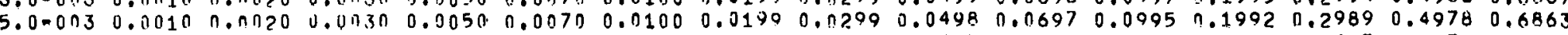

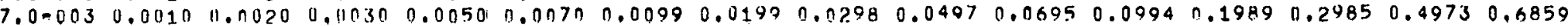

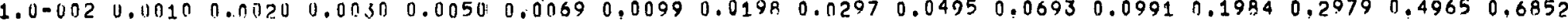

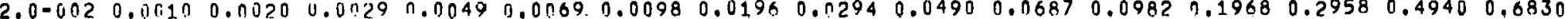
$3,0.0020 .0010 \quad 0.01190 .00290 .00490 .00680 .00970 .0194 \quad 0.02010 .04860 .06800 .09730 .1952 \quad 0.29360 .49140 .6808$ 50.0020 .00100 .00190 .00290 .00480 .00670 .00950 .01700 .02850 .04760 .06670 .09550 .19190 .28940 .48620 .6763 1.000 0.0910 .1838 0.2785 0.47300 .6645

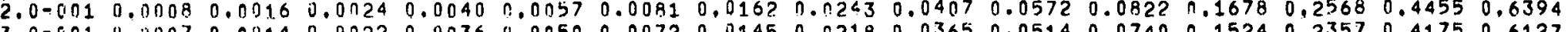

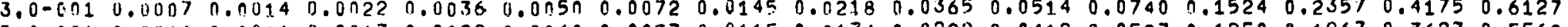

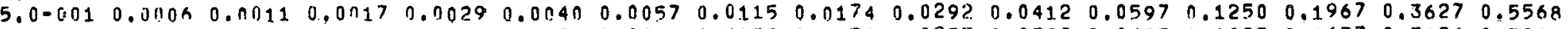

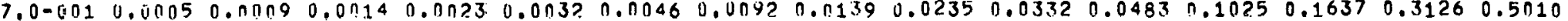

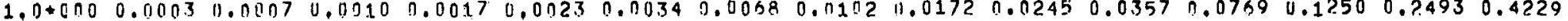

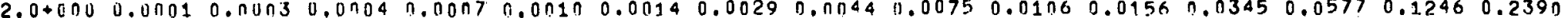
$3.0+000.00010 .00020 .40020 .00040 .00050 .0008 \quad 0.0016 \quad 0.00240 .0040 \quad 0.0058 \quad 0.00850 .01890 .03190 .07120 .1447$ $5.0+0000.00000 .00010 .00010 .00020 .00020 .00030 .00070 .00100 .00170 .00240 .00360 .00800 .01360 .03110 .0662$

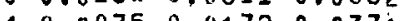

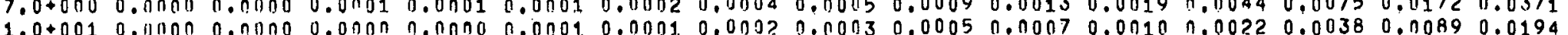

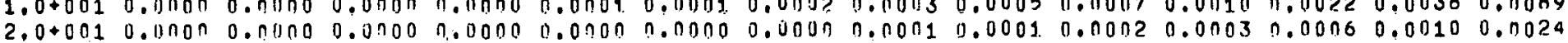
$3,0+0010.000 n 0.00000 .00000 .00000 .00000 .00000 .00000 .00000 .00010 .00010 .0001 .0 .00030 .0005$ $5.0+0010.0000 \quad 0.00000 .00000 .00000 .00000 .00000 .00000 .00000 .00000 .00000 .00000 .0001$

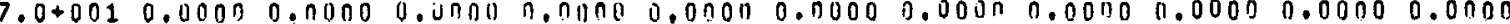

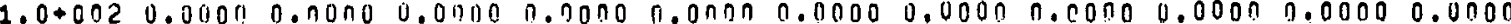
$2.0+0020.0000 \quad 0.00 n \pi \quad 0.0000 \quad 1.0000 \quad 0.0 n 0 n \quad 0.0000 \quad 0.0000 \quad 0.0000 \quad 0.0000$

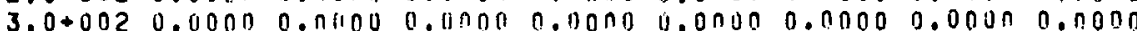

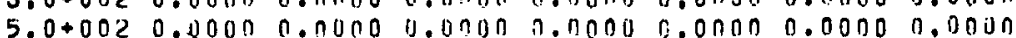

$3.0+0050.00010 .0000$ (1).

$1,0+0030.00000 .0000$ D.0000 n.,0000 0.000n 0.0000

$$
\text { ALTA }
$$

ALPHA $\quad 1.000 \quad 2.000 \quad 3.000 \quad 5.000 \quad 7.000 \quad 10.00 \quad 20.00 \quad 30.00 \quad 50.00 \quad 70.00 \quad 1.00 .0 \quad 200.0 \quad 300.0 \quad 500.0 \quad 700.0 \quad 1000$

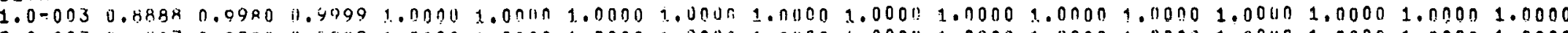

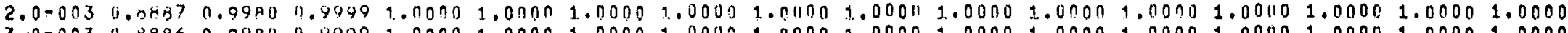
$3.0-0031.8886 \quad 0.9980 \quad 0.9099 \quad 1.00001 .00001 .00001 .00001 .00001 .00001 .00001 .00001 .00001 .00001 .00001 .00001 .0000$ $5.0-0030.88840 .9980 \quad 0.99991 .00001 .00001 .00001 .00001 .0 n 001.00001 .00001 .00001 .00001 .00001 .00001 .00001 .0000$

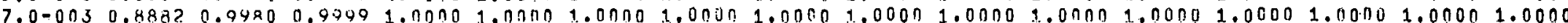

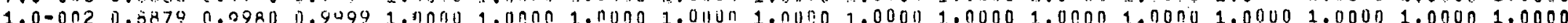

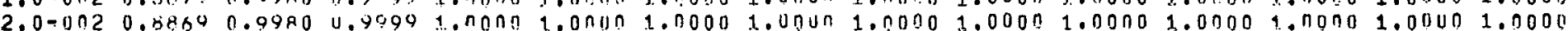

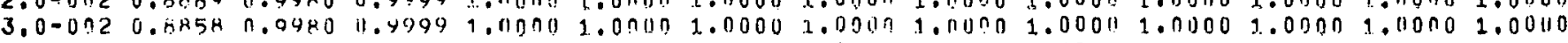

5.0-002 $0.08370 .9979 \pi .94992 .00001 .00 n \pi 1.0000 .00001 .001001 .00001 .00001 .00001 .0000$

$7.0-0020.84160 .99790 .90991 .00901 .00001 .00001 .00001 .001001 .000111 .00001 .0000$

1.0-001 0.8782 $0.0978 \quad 1.9099 \quad 1.110001 .00 n n 1.00001 .001191 .00001 .00001 .00001 .0000$

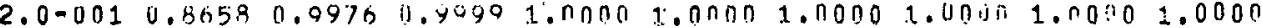

$3.0-0010.852110 .0973 \quad 1.9999 \quad 1.00001 .00001 .010001 .00101 .0000$

5.0-001 0.02050 .9460 11.4048 1.0000 1.00nก 1.0000 1.11000

$7.0-0010.78510 .0957$ 1). 90981.00001 .000071 .11000

$1.0+000 \quad 0.7273 \quad 0.0942 \quad 13.99971 .00001 .00001 .0000$

$2.0+000 \quad 0.53330 .0845 \quad 0.90931 .0000$

$3.0+000 \quad 0.36110 .9751 \quad 0.4947$

$5.0+0000.2051,0,04 ? 7$

$7.0+000 \quad 0.1231$

$1.0+001 \quad 0.0672$ 
VALUES OF F POH

ALPHA $0.0010 .0020 .0 \pi 30.005$ BETA

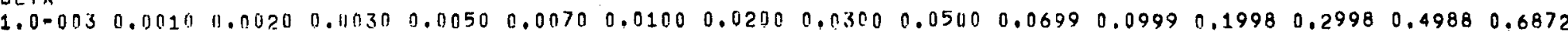

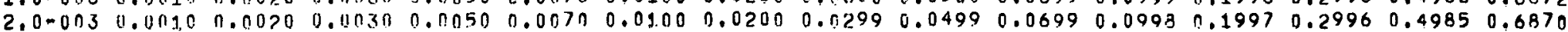

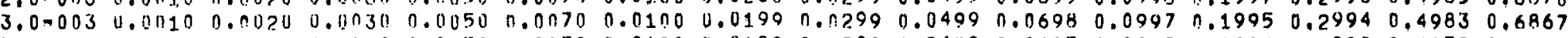
$5.0=003 \quad 0.0010 \quad 0.0020 \quad 0.0030 \quad 0.0050 \quad 0.0070 \quad 0.0100 \quad 0.01990 .02090 .04980 .06970 .0995 \quad 0.1992 \quad 0.2989 \quad 0.49780 .6863$

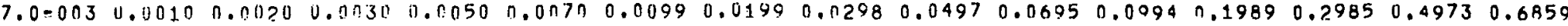

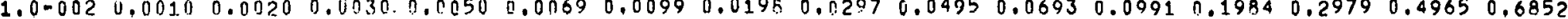

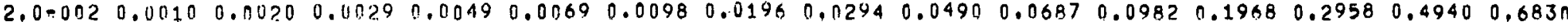
$3,0=0020.00110 .00190 .00290 .00490 .00680 .00970 .01940 .02910 .04860 .06800 .09730 .19520 .29360 .49140 .6808$ 06670.00550 .19190 .28930 .48620 .6763

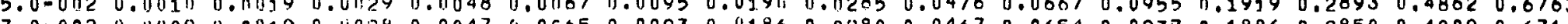

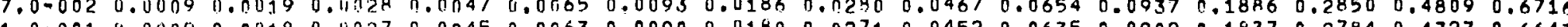

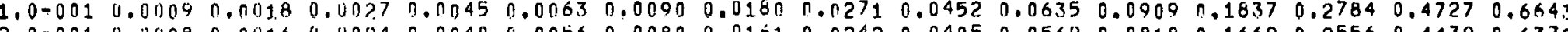

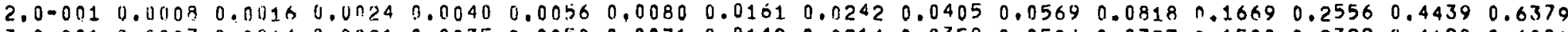
$\begin{array}{llllllllllllllll}3.0-001 & 0.0007 & 0.0014 & 0.0121 & 0.0035 & 0.0050 & 0.0071 & 0.0142 & 0.0214 & 0.0358 & 0.0504 & 0.0727 & 0.1500 & 0.2322 & 0.4128 & 0.6081\end{array}$

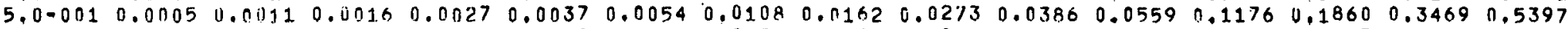
$\begin{array}{llllllllllllllllll}7.0001 & 0.0004 & 0.01) 08 & 0.0012 & 0.0020 & 0.0028 & 0.0040 & 0.0080 & 0.0121 & 0.0204 & 0.0289 & 0.0420 & 0.0898 & 0.1447 & 0.2823 & 0.4647\end{array}$ $\begin{array}{llllllllllllllllll}1.0+000 & 0.0003 & 0.0005 & 0.0008 & 0.0013 & 0.0018 & 0.0025 & 0.0051 & 0.0077 & 0.0130 & 0.0185 & 0.0270 & 0.0588 & 0.0968 & 0.1994 & 0.3547\end{array}$ $2.0+000 \quad 0.00010 .01010 .0002 \quad 0.00030 .00 n 50.00070 .00140 .00210 .00350 .00500 .00740 .01640 .02780 .06230 .1278$ $3.0+0000.00000 .00010 .40010 .04010 .00020 .00030 .00050 .00080 .00130 .00190 .00280 .04620 .01060 .02434 .0521$

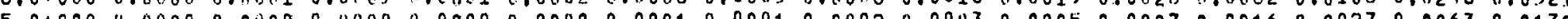
$5.0+000$.

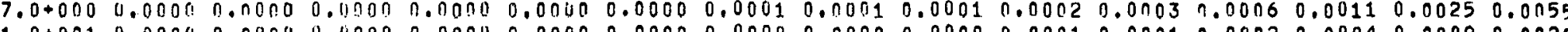
$1.0+0010.000\{0.00000 .00000 .00000 .00000 .00000 .00000 .00000 .00000 .00010 .0001000020 .00040 .00090 .0020$ $2.0+0010.09000 .00000 .00000 .00000 .0 n 000.00000 .00000 .00000 .00000 .00000 .00000 .00000 .00010 .0001$ $3.0+0010.00000 .01000 .00000 .00000 .00000 .00000 .00000 .00000 .00000 .00000 .00000 .00000 .0000$

$5.0+0010.0000 \cdot 0.011000 .00000 .00000 .00000 .00000 .00000 .00000 .00000 .00000 .00000 .0000$

$7.0+0010.00000 .0 \mathrm{U} 000.4000 \quad 0.0000 \quad 0.00000 .0000 \quad 0.0000 \quad 0.00000 .00000 .00000 .0000$

$1.0+0020.00000 .0000 \quad 0.00 n 0.00000 .00 n 00.00000 .00000 .00000 .00000 .00000 .0000$

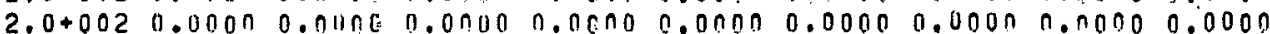

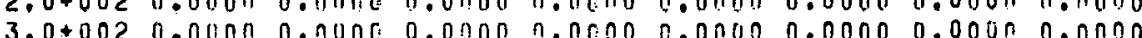

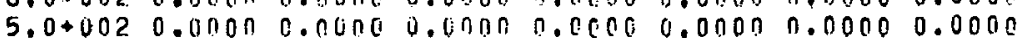

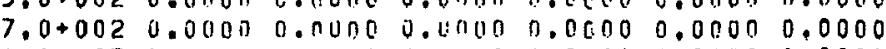

$1.0+0030.00000 .0000 \quad 0.00000 .00000 .00000,0000$ ALP BETA

$1.0-0030.88880 .9980 \quad 0.90991 .00001 .00001 .00001 .00001 .00001 .00009 .00001 .00001 .00001 .00001 .00001 .00001 .0000$ $2.0-0030.88870 .99800 .49991 .00001 .00001 .00001 .00001 .00001 .00001 .00001 .00001 .00001 .00001 .00001 .00001 .0000$ 3.0-003 0.8486 $0.9980 \quad 1.99991 .00001 .001101 .00001 .00001 .00001 .00001 .00001 .00001 .00001 .00001 .00001 .00001 .0000$

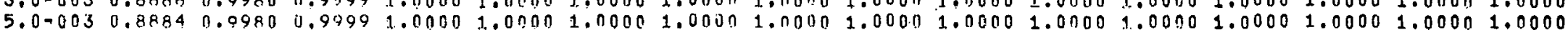
$3.0-0030.888 ? 0.99800 .99991 .00001 .00001 .00001 .00001 .00001 .00001 .00001 .00001 .00001 .00001 .00001 .00001 .0000$

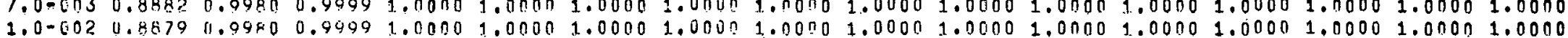

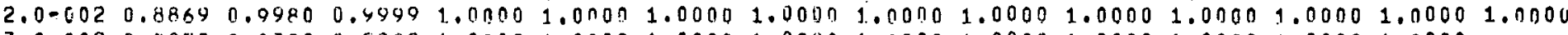
$3.0-0020.68580 .9980 \quad 0.99991 .00001 .00001 .00001 .00001 .00001 .00001 .00001 .00001 .00001 .0000$

$5.0-002 \quad 0.8437 \quad 0.99790 .99991 .00001 .00001 .00001 .00001 .00001 .00001 .00001 .00001 .0000$

$7.0=0020.8815 \quad 0.99790 .99991 .001001 .0000 \quad 1.00001 .00001 .011001 .00001 .00001 .0000$

$1.0=0010.87810 .99780 .99991 .00001 .00001 .00001 .00001 .00001 .00001 .00001 .0000$

2.0.001 $0.4651 \quad 0.99700 .9099 \quad 1.00 n 0 \quad 1.00001 .00001 .00001 .00901 .0000$

$3.0-001 \quad 0.8495 \quad 0.09720 .9999 \quad 1.00001 .00001 .0000 \quad 1.00001 .0000$

$5.0=0010.8101 \quad 0.9963 \quad 0.99981 .00001 .00001 .00001 .0000$

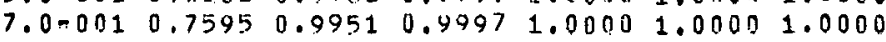

$1.0+000 \quad 0.6667 \quad 10.99220 .99961 .00001 .00001 .0000$

$2.0+000 \quad 0.3478 \quad 0.9714 \quad 0.99851 .0070$

$3.0+000 \quad 0.1667 \quad 0.9273 \quad 0.9960$

$5.0+000 \quad 0.04830 .7658$

$7.0+000 \quad 0.0196$

$1,0+0010,0071$ 


\begin{abstract}
VALUES OF F HOK IN= 8 ATI! $N H=4$ BETA

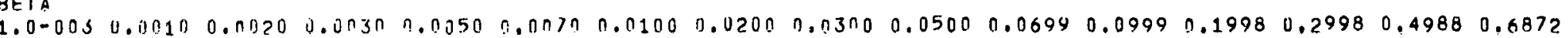

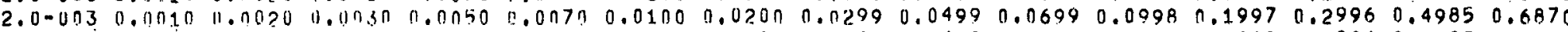

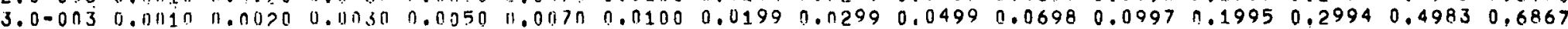

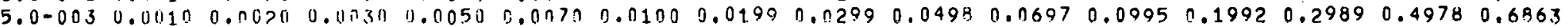

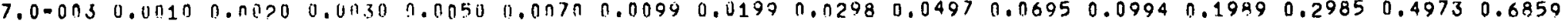

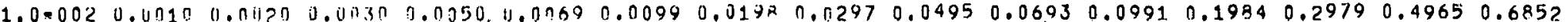
$2,0-0020.00100 .011200 .00200 .00490 .006900098001960 .02940 .04900 .06870 .09820 .19680 .29580 .49400 .6830$ 2.0-0 $\begin{array}{ll}0 & \\ 0\end{array}$

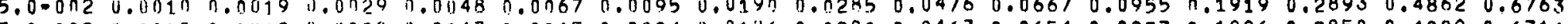

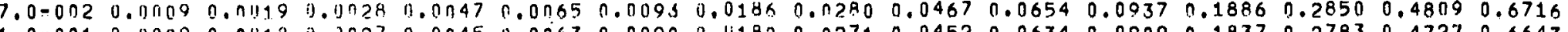

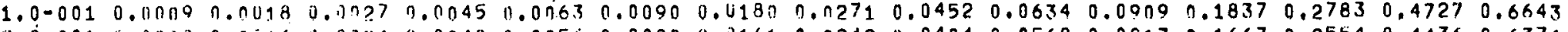

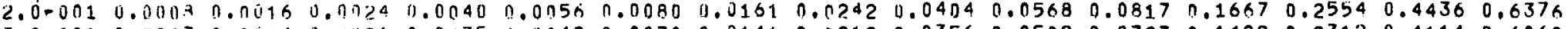

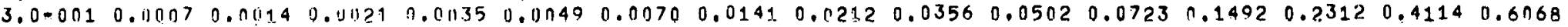

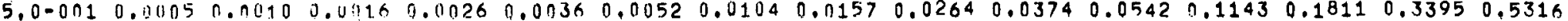

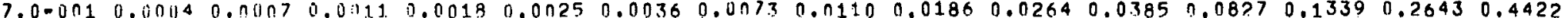

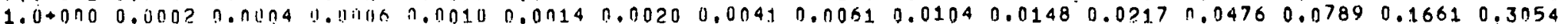
$2.0+0000.00010 .000111 .10010 .00020 .00020 .00030 .00070 .00100 .00170 .00240 .00360 .04800 .01360 .03110 .0662$

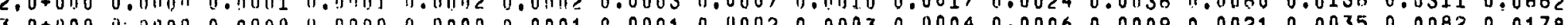

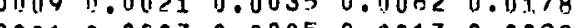
$5.0+000$ 0.

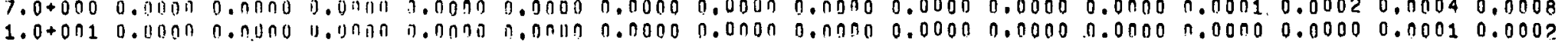

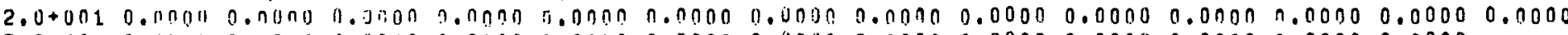
$3.0+0010.00000 .00000 .00000 .00000 .00000 .00000 .00000 .00000 .00000 .00000 .00000 .00000 .0000$
\end{abstract}

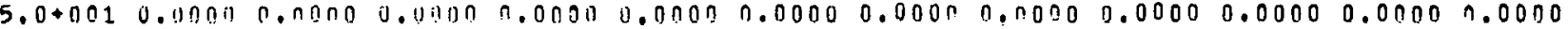

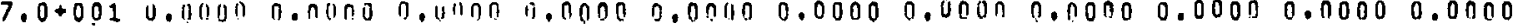

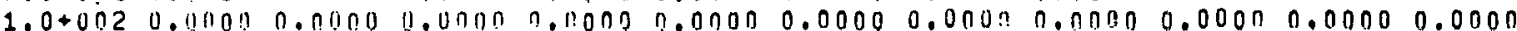

$2.0+0020.00000 .01300 .0000 .00000 .00000 .00000 .00000 .00000 .0000$

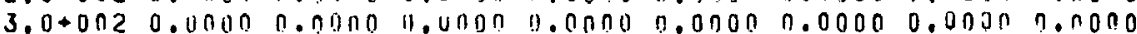

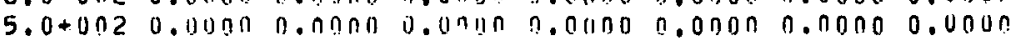

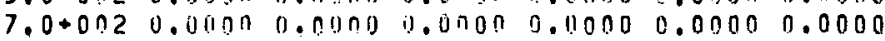

$1.0+0030.00000 .00000 .00000 .00000 .00000 .0000$ $B E T A$

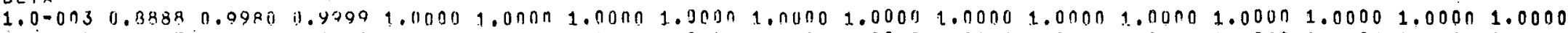
2.0 .0030 .89870 .99800 .90991 .00001 .00001 .00001 .00001 .00001 .00001 .00001 .00001 .00001 .00001 .00001 .00001 .0000 $3.0-0030.8 R 860.09800 .90991 .00001 .00301 .00001 .00001 .00001 .00001 .00001 .00001 .00001 .00001 .00001 .00001 .0000$ $5.0-0030.34840 .9980 .49091 .00001 .000 n 1.00001 .00001 .00001 .00001 .00001 .00001 .00001 .00001 .00001 .00001 .0000$ 7.0-003 0.88 .20 .09 .800 .49991 .0001 .00001 .00001 .00001 .00001 .00001 .00001 .00001 .00001 .00001 .00001 .00001 .0000

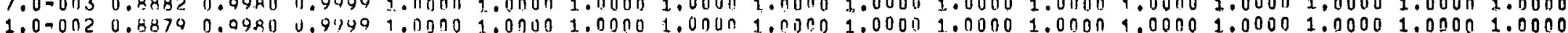

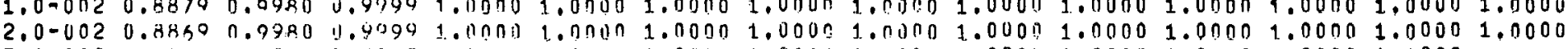
$3.0-0020.48540 .09800 .40991 .00001 .00001 .00001 .000 n 1.000 n 1.00001 .00001 .00001 .00001 .0000$

$5.0-0020.8+37 \quad 0.99790 .2099 \quad 1.00001 .00001 .00001 .00001 .00001 .00001 .00001 .00001 .0000$

$7.0-0020.8815 \quad 0.99790 .90991 .00001 .00001 .00001 .00001 .00001 .00001 .00001 .0000$

$1.0-0010.8780 \quad 0.9978 \quad 0.90991 .00001 .00001 .00001 .00001 .00001 .00001 .00001 .0000$

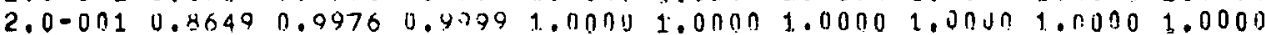

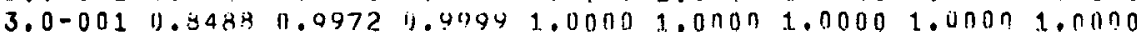

$5.0-001 \quad 0.005011 .0962$ 1).9998 1.0001) $1.00001 .0000 \quad 1.0000$

$7.0-001 \quad 0.74240 .9946 \quad 0.90971 .001101 .00001 .0000$

$1.0+000 \quad 0.01540 .9803$ ij. $9095 \quad 1.0000 \quad 1.00001 .0000$

$2.0+000 \quad 0.2051 \quad 0.0427 \quad 0.9909 \quad 0.9999$

$3.0+000 \quad 0.0620 \quad 11.8082 \quad$ i). .4979

$5.0+000 \quad 0.111010 .3950$

$7.0+000 \quad 0.0028$ 
VALUES OF F FOH IV $=8$ AMI NP= 5 BETA

$1.0 .0030 .00100 .0020 \quad 0.011300 .0450 \quad 0.0070 \quad 0.0100 \quad 0.0200 \quad 0.0300 \quad 0.05000 .06940 .09090 .19080 .2998 \quad 0.49880 .6872$ $2.0-0030.01100 .00200 .00300 .01500 .00700 .01000 .02000 .02990 .04990 .06990 .09980 .19970 .29960 .49850 .6870$ $3.0-0030.00100 .00>00.00300 .00510 .00700 .01000 .01990 .02990 .04990 .06980 .09970 .19950 .29940 .49830 .6867$ $5.0=003 \quad 0.110 .100 .00>0 \quad 0.0030 \quad 0.0050 \quad 0.0 n 70 \quad 0.0100 \quad 0.0199 \quad 0.02990 .04980 .0697 \quad 0.0995 \quad 0.1992 \quad 0.29890 .4978 \quad 0.6863$

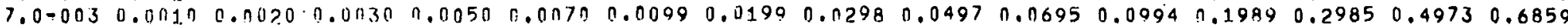

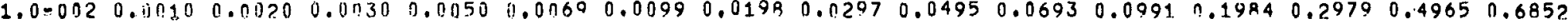

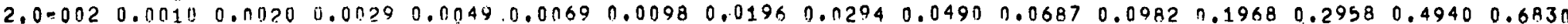
$3,0.0020 .00100 .00190 .00790 .00490 .00680 .00970 .01940 .02910 .04860 .06800 .09730 .19520 .29360 .49140 .6808$ 3.0 .0020 .01100 .00190 .00290 .00480 .00670 .00950 .01900 .02850 .04760 .06670 .09550 .19190 .28930 .48620 .6763

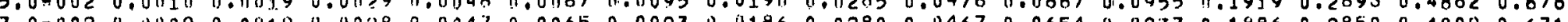

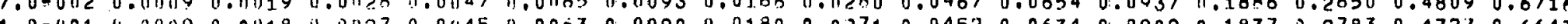

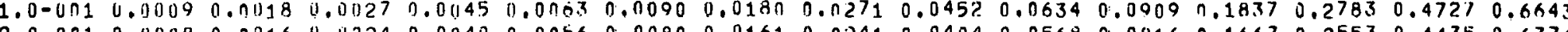

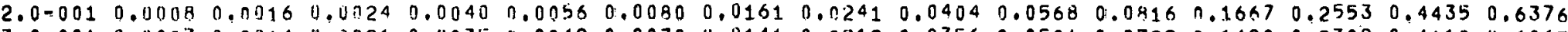
$3.0=0010.00070 .0014 \quad 0.0021 \quad 0.110350 .00490 .0070 \quad 11.01410 .0212 \quad 0.03560 .05010 .0722 \quad 0.1490 \quad 0.2309 \quad 0.4110 \quad 0.6063$

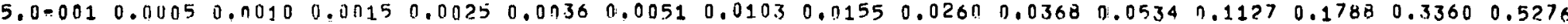
$7,0=001 \quad 0.0003 \quad 0.0007 \quad 0.0110 \quad 0.0017 \quad 0.0024 \quad 0.0034 \quad 0.00690 .01040 .0176 \quad 0.0250 \quad 0.0364 \quad n .0783 \quad 0.1272 \quad 0.2530 \quad 0.4278$

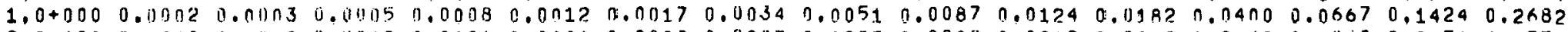

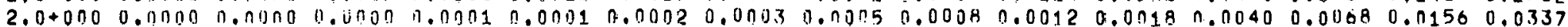

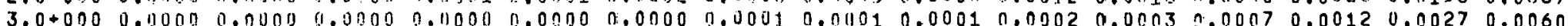

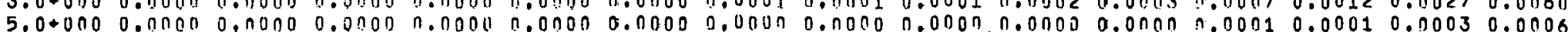

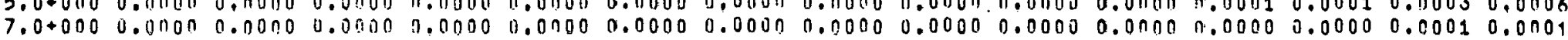

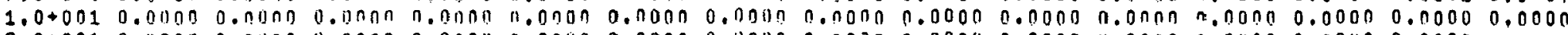

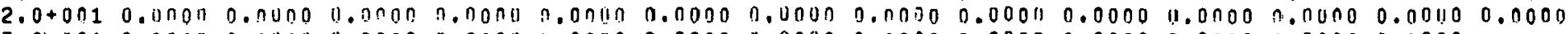

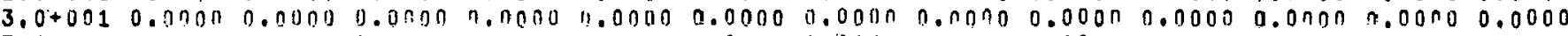

$5.0+0010.010000 .00000 .00000 .00000 .00000 .00000 .00000 .00000 .00000 .00000 .00000 .0000$

$7.0+0010.03000 .00000 .00000 .00000 .0000 \quad 0.00000 .00000 .00000 .00000 .00000 .0000$

$1.0+0020.0001) 0.0 n 000.0 n 00$ n.0n00 $0.00000 .00000 .000 n 0.00000 .00000 .00000 .0000$

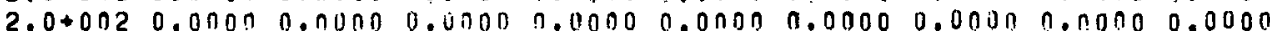

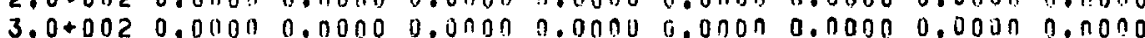

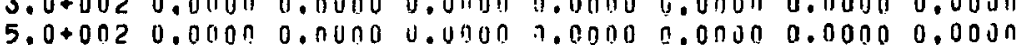

$7.0+0020.00000 .010000 .09000 .00000 .00000 .0000$

$1.0+0030.00000 .00000,00000.0000,0.00000,0000$

$$
\text { ALPH }
$$

1.000030 .88890 .09800 .90991 .00911 .00001 .00001 .00001 .00001 .00001 .00001 .00001 .00001 .00001 .00001 .00001 .0000 $2.0-0030.88370 .99800 .99991 .00011 .00001 .00001 .00001 .00001 .00001 .00001 .00001 .00001 .00001 .00001 .00001 .0000$ 3. $0.0030 .4886 \quad 0.99800 .90991 .00001 .00 n \pi 1.00001 .00001 .00001 .00001 .00001 .00001 .00001 .00001 .00001 .00001 .0000$ $5.0-0030.88840 .99800 .99991 .00001 .00001 .00001 .00001 .00001 .00001 .00001 .00001 .00001 .00001 .00001 .00001 .0000$

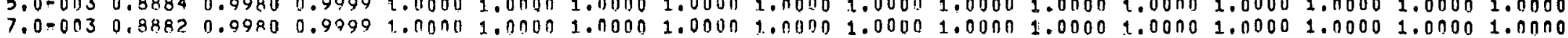

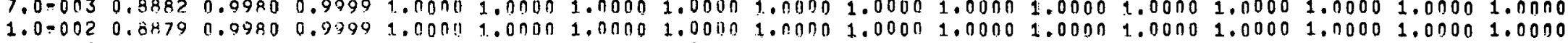
$2,0=0020.88690 .99800 .90992 .00001 .00001 .00001 .00001 .00001 .00001 .00001 .00001 .00001 .00001 .0000$ $3.0-0020.84580 .0980 \quad 0.99991 .00001 .00001 .00001 .00001 .00001 .00001 .00001 .00001 .00001 .0000$

$5.0-0020.88370 .99790 .99991 .00001 .00001 .00001 .00001 .00001 .000 n 1.00001 .00009 .0000$

$7.0=0020.38150 .99790 .49991 .00001 .00001 .00001 .00001 .00101 .00001 .00001 .0000$

$1.0-0010.8780 \quad 0.09780 .9999 \quad 1.00 n 01.00001 .00001 .00001 .011001 .00001 .00001 .00 n n$

2.0.001 $0.86490 .09760 .9999 \quad 1.00011 .000011 .00001 .00301 .011001 .0000$

3.090010 .84860 .99720 .99991 .00001 .000011 .04001 .00001 .0000

$5.0=001 \quad 0.8025 \quad 0.99620 .99941 .00001000001 .000001 .0000$

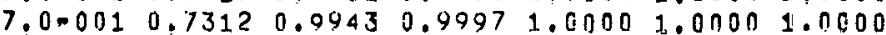

$1.0+090 \quad 0.5714 \quad 11.98840 .99941 .00001 .00001 .0000$

$2.0+000 \quad 0.1127 \quad 0.99 n 10.9936 \quad 0.5990$

$3.0+000 \quad 0.021 .5 \quad 0.58350 .9543$

$5.0+000 \quad 0.0020 \quad 0.1155$

$7.0+0 \cap 00.0004$

$1,0+0010.0001$ 


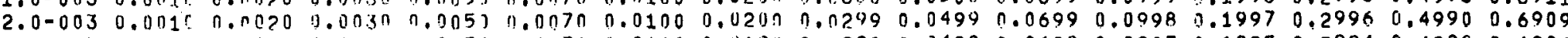

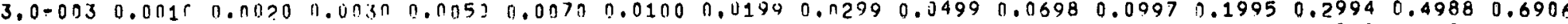

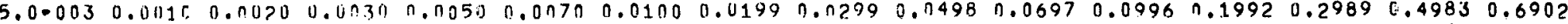

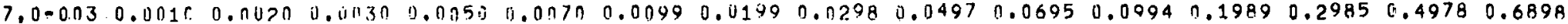

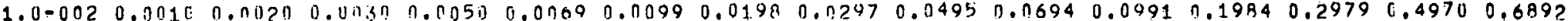

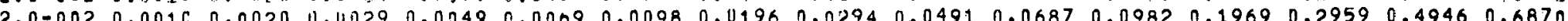
2.0.002 0.0010 0.0849

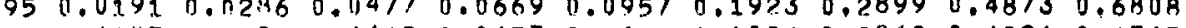

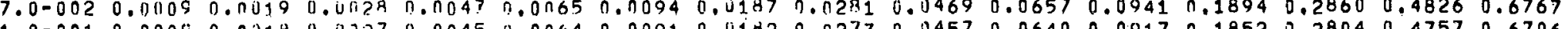

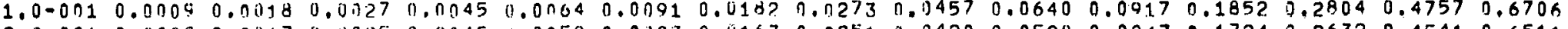

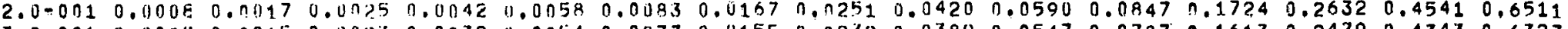
$3.0-0010.0014 \quad 0.00150 .0023 \quad 0.0 n 390.0054 \quad 0.0077 \quad 0.0155 \quad 0.02320 .03840 .05470 .0787 \quad 0.16130 .24790 .4343 \quad 0.6327$

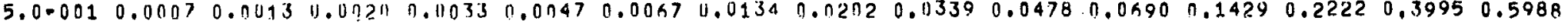
7.0-001 $0.0006 \quad 0.00120 .00180 .00290 .00410 .00590 .01190 .01790 .03000 .04240 .06130 .12820 .20130 .36990 .5684$ $\begin{array}{llllllllllllllllll}1.0+000 & 0.0005 & 0.0010 & 0.0015 & 0.0025 & 0.0035 & 0.0050 & 0.0101 & 0.0152 & 0.0256 & 0.0363 & 0.0526 & 0.1111 & 0.1765 & 0.3329 & 0.5282\end{array}$ $3.0+0000.10030 .00070 .111100 .00120 .00230 .00340 .00080 .01720 .01720 .02450 .03570 .07690 .12500 .24960 .4274$

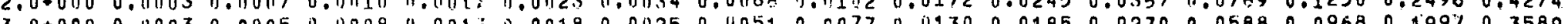
3.0+000 0.0003

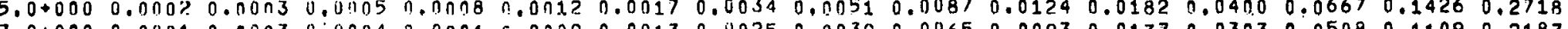
$7.0+000 \quad 0.00010 .00030 .00140 .00 n .60 .00090 .00130 .00250 .00390 .00650 .00930 .01370 .03030 .05080 .11090 .2187$

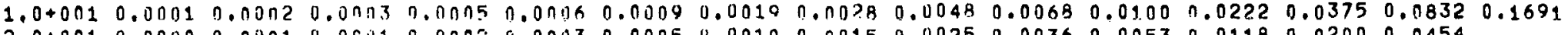

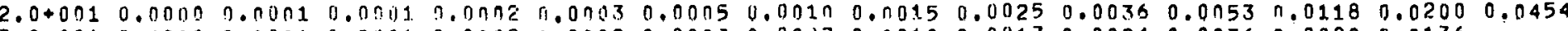
$3.0+0010.00000 .00010 .00 n 10.00 n 20.0 n 0 ? 0.00030 .0 n u 70.00100 .00170 .00240 .00360 .00800 .0136$

$5.0+0010.0000$ n.0u0u 0.101010 .00910 .00010 .00020 .0004 .0 .00060 .00100 .00150 .00220 .0049

$7.0+0010.00000 .00000 .00000 .00010 .00010 .00 n 10.00030 .00040 .00070 .00110 .0016$

$1.0+0020.00000 .00000 .00000 .00050 .00010 .00010 .000 ? 0.00130 .00050 .00070 .0011$

$2.0+0020.0000 \quad 0.00000 .01000 .0000,0.00000 .00010 .00010 .00020 .0003$

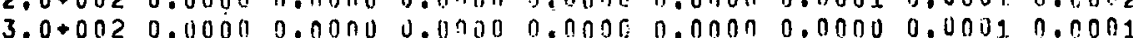

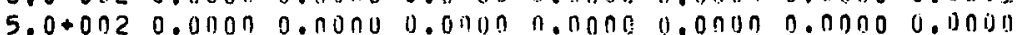

$7,0+0020.00000 .00000 .0000,0.00000 .00000 .0000$

$1.0+0030.00000 .00000 .00000 .00000 .00000 .0000$

BETA BETA

$1.0-0030.89990 .09001 .00031 .00001 .00001 .00001 .00001 .00001 .00001 .00001 .00001 .00001 .00001 .00001 .00001 .0000$ $2.0-0030.8998 \quad 0.99901 .00001 .001001 .00001 .00001 .000 n 1.00001 .00001 .00001 .00001 .00001 .00001 .00001 .00001 .0000$ $3.0 .0030 .8997 \quad 0.09901 .07001 .00001 .00001 .00001 .000 n \quad 1.00001 .00001 .00001 .00001 .00001 .00001 .00001 .000 n 1.0000$ $5.0-0030.899611 .09001 .00001 .00701 .00001 .00001 .00001 .011001 .0011001 .00001 .00001 .00001 .00001 .00001 .00001 .0000$ 7.0-003 0.89940 .99901 .0100 .1 .00001 .00001 .00001 .00001 .00001 .00001 .00001 .00001 .00001 .00001 .00001 .00001 .0000

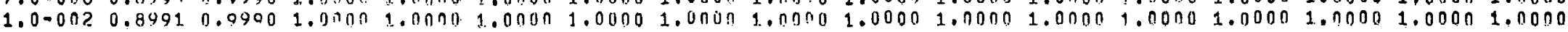
$2.0-0020.898 ? 0.99901 .00011 .011001 .00001 .00001 .00001 .00001 .00001 .00001 .00001 .00001 .00001 .0000$ 3.000020 .89730 .09901 .03001 .00001 .00001 .00001 .00001 .00001 .00001 .00001 .00001 .00001 .0000

$5.000020 .89550 .09901 .00001 .00001 .00001 .00001 .00001 .0 n=01.00001 .00001 .00001 .0000$

7.0 .0020 .89370 .99001 .00001 .00001 .00001 .00001 .00001 .00701 .00001 .00001 .0000

$1.0-0010.89110 .99891 .00001 .00001 .00001 .00001 .000 n 1.00101 .00001 .00001 .0000$

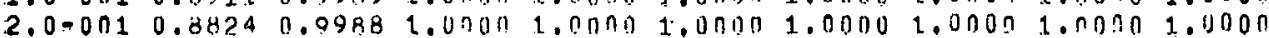

$3.0-0010.87380 .99871 .00001 .00001 .00001 .00001 .00001 .0000$

$5.0-0010.85710 .09850 .40091 .00701 .00001 .0000$ 1. unum

$\begin{array}{lllllll}7.0-001 & 0.8411 & 0.9983 & 0.8499 & 1.0070 & 1.0000 & 1.0000\end{array}$

$1.0+000 \quad 0.818 ? 0.9980 \quad 0.99991 .011001 .00101 .0000$

$2.0+000 \quad 0.7500 \quad 0.99710 .9999 \quad 1.190101$

$3.0+000 \quad 0.6923 \quad 0.0961 \quad 0.9090$

$5.0+000 \quad 0.51100 \quad 0.0942$

$7.0+010 \quad 0.5294$ 
VALUES OF F FOR $N=9$ ANM NF=?$$
N=9 \operatorname{Sin} N A P=
$$
BETA

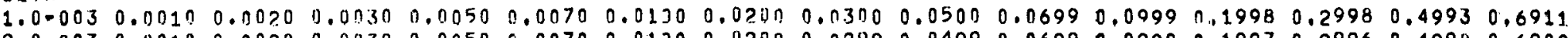
$2.0-0030.00100 .00200 .09300 .00500 .00700 .01300 .02000 .02090 .04990 .06990 .09980 .19970 .29960 .49900 .6909$ $3.0=0030.00100 .00200 .00300 .0050 \quad 0.00700 .01000 .01990 .02990 .04940 .06980 .09970 .19950 .29940 .49880 .6906$ 5.0-003 $0.00190 .0070 \quad 0.0130 \quad 0.0050 \quad 0.0070 \quad 0.0130 \quad 11.01990 .02990 .04980 .06970 .09950 .19920 .29890 .49830 .6902$

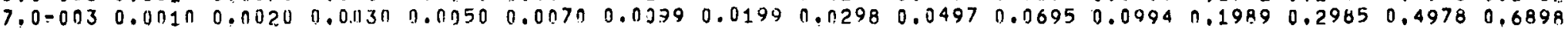
$\begin{array}{llllllllllllllll}1.0 .002 & 0.0010 & 0.0020 & 0.0030 & 0.0050 & 0.0069 & 0.0079 & 0.0143 & 0.0297 & 0.0495 & 0.0693 & 0.0991 & 0.1984 & 0.2979 & 0.4970 & 0.6891\end{array}$ $2,0-002 \quad 0.0010 \quad 0.0020 \quad 0.00290 .00490 .00690 .0098 \quad 0.01950 .02940 .0490 \quad 0.0687 \quad 0.0982 \quad 0.1968 \quad 0.2958 \quad 0.4945 \quad 0.687 n$

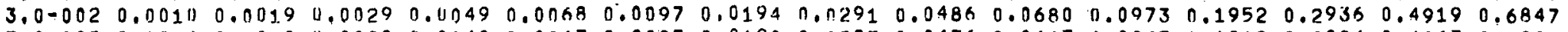
$5.0-0020.00100 .00190 .0020 \quad 0.00480 .00670 .00950 .0190 \quad 0.02550 .04760 .06670 .09550 .19190 .28940 .48670 .6803$ $7.0=0020.00090 .00190 .00280 .00470 .00650 .00930 .01850 .02800 .04670 .06540 .09370 .18970 .28510 .48150 .6757$ (1)

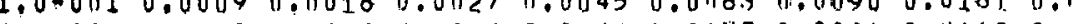
2.0 .0010 .00080 .00160 .01240 .01400 .01550 .00810 .01620 .02430 .04070 .05720 .03220 .16780 .25680 .44590 .6436

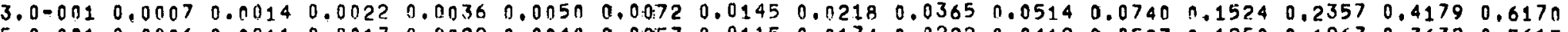
$\begin{array}{lllllllllllllllll}5.0001 & 0.0006 & 0.001 .1 & 0.0017 & 0.0029 & 0.0040 & 0.0057 & 0.0115 & 0.0174 & 0.0292 & 0.0412 & 0.0597 & 0.1250 & 0.1967 & 0.3632 & 0.5613\end{array}$ $7.0=0010.0005 \quad 0.00090 .0 n 14 \quad 0.0 n 23 \quad 0.0032 \quad 0.0046 \quad 0.00920 .01390 .02350 .03320 .04830 .10250 .16370 .31310 .5056$ $\begin{array}{llllllllllllllllll}1.0+000 & 0.0003 & 0.0007 & 0.0010 & 0.0017 & 0.0023 & 0.0034 & 0.0068 & 0.0102 & 0.0172 & 0.0245 & 0.0357 & 0.0769 & 0.1250 & 0.2496 & 0.4274\end{array}$ $2.0+000 \quad 0.0001 \quad 0.0003 \quad 0.0004 \quad 0.0007 \quad 0.00100 .00140 .00290 .00440 .00750 .0106 \quad 0.0156 \quad 0.03450 .0577 \quad 0.12480 .2424$ $3.0+0000.00010 .00020 .00020 .50040 .0005 \quad 0,00080.00160 .00240 .00400 .00580 .00850 .01890 .03190 .07130 .1469$ $5.0+0000.00000 .00010 .00010 .00020 .00020 .00030 .00070 .00100 .00170 .00240 .00360 .00800 .01360 .031 .0 .0674$

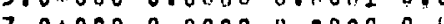

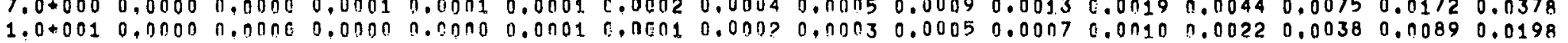

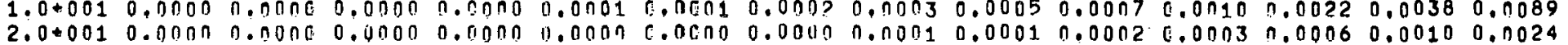

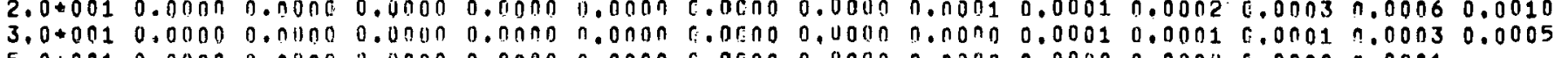

5.000010 .00000 .00000 .00000 .00000 .00000 .00000 .00000 .00000 .00000 .00000 .00000 .0001

$7.0+0010.00000 .00000 .00000 .00000 .00000 .00000 .00000 .00000 .00000 .00000 .0000$

$1.0+0020.00000 .00000 .00000 .00000 .0000 \quad c .00000 .00000 .00000 .00000 .00000 .0000$

$2.0+0020.00000 .00000 .0000,0.00000 .00000 .00000,00000.00000 .0000$

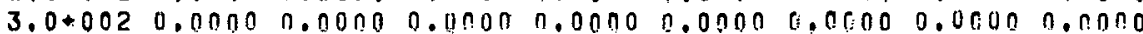

$5.0+0020.00000 .00000 .01000 \quad 0.0000 \quad 0.0000 \quad 0.00000 .0000$

$7.0+0020.00000 .00000 .00000 .00000 .00000 .0000$

$1.0+0030.00000 .00000 .00000 .00000 .00000 .0000$ BETA

LPHA $1.000 \quad 2.000 \quad 3.000 \quad 5.000 \quad 7.000 \quad 10.00 \quad 20.00 \quad 30.00 \quad 50.00 \quad 70.00 \quad 100.0 \% 200.0 \quad 300.0 \quad 500.0 \quad 700.0 \quad 1000$

$1.0=0030.89990 .9990 \quad 1.00001 .00001 .00001 .00001 .00001 .00001 .00001 .0000 \$ 0000 \$ 2.00001 .00001 .00001 .00001 .0000$ $2.0-0030.89980 .99001 .00001 .00001 .00001 .00001 .00001 .00001 .00001 .00001 .0000 \div 00001.00001 .00001 .00001 .0000$ $3.0-0030.8997 \quad 0.99901 .00001 .00001 .00001 .00001 .00009 .00001 .00001 .00001 .00001 .00001 .00001 .00009 .00001 .0000$ $5.0-0030.8995,0.9901 .00001 .00001 .00001 .08001 .00001 .00001 .00001 .00001 .00001 .00001 .00001 .00001 .00001 .0000$

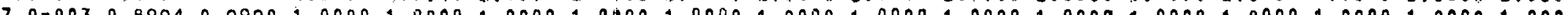
$1.0-002 \quad 0.8991 .0 .99901 .00001 .30 n 01.00001 .00001 .00401 .00001 .00001 .00001 .00001 .00001 .00001 .00001 .00001 .0000$ $2.0-0020.69920 .99901 .00011 .00901 .00001 .06001 .00001 .00001 .00001 .00001 .00001 .00001 .00001 .0000$

3.000020 .89720 .99901 .00001 .00001 .00001 .03001 .00001 .00001 .00001 .00001 .00001 .00001 .0000

5.000020 .89530 .99911 .00001 .00001 .00001 .01001 .00001 .00001 .00001 .00001 .00001 .0000

$7.0-0020.89330 .99891 .00001 .00001 .00001 .03001 .00001 .00001 .00002 .00001 .0000$

$1.0-0010.89020 .99891 .00001 .00001 .0000 \quad 1.03001 .00001 .00001 .00009 .00001 .0000$

$2.0-0010.87890 .99831 .00001 .0000 \quad 1.00001 .0 .7001 .00011 .00001 .0000$

$3.040010 .86620 .99851 .00001 .00001 .00001 .03001 .000 n 1.0000$

5.0-001 $0.83720 .99830 .99991 .00001 .001101 .03001,0000$

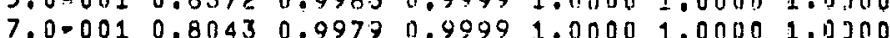

$1.0+000 \quad 0.7500 \quad 0.09710 .99991 .00001 .00001 .0300$

$2.0+000 \quad 0.56250 .99320 .9998 \quad 1.0000$

$3.0+000 \quad 0.4091 \quad 0.98740 .4996$

$5.0+000 \quad 0.22500 .9706$

$7.0+000 \quad 0.136$

$1.0+001 \quad 0.0750$ 

BET

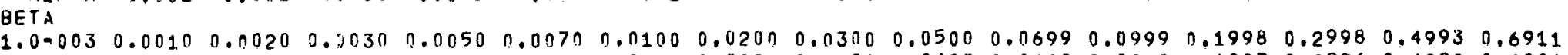

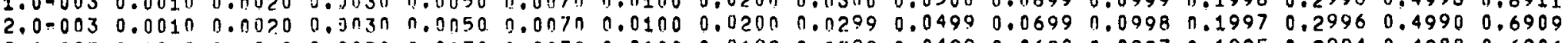

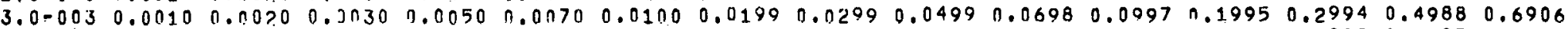

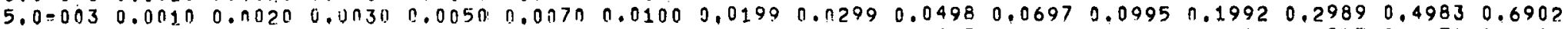

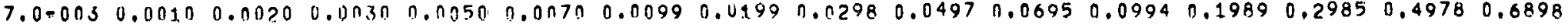

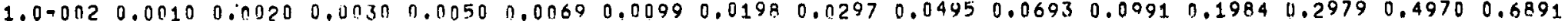
$2.0-0020.00100 .00204 .00290 .00490 .00690 .00980 .01960 .02940 .04900 .06870 .09820 .19680 .29580 .49450 .6870$ 2.0.002 0.010 0.00190 .00290 .00490 .00680 .00970 .01940 .02010 .04860 .06800 .09730 .19520 .29360 .49190 .6847 0.08930 .48670 .6807 年 7.0 .0020 .00090 .00190 .00280 .00470 .01650 .00930 .01450 .02800 .04670 .06540 .09370 .18860 .28500 .48140 .6756

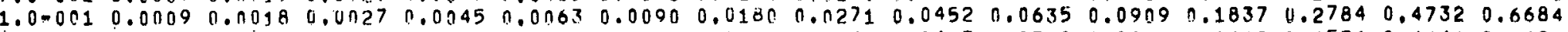

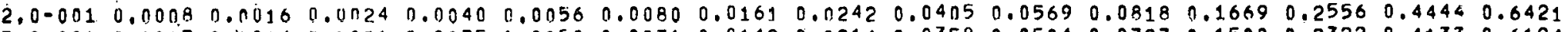
$3.0-0.010 .0007 \quad 0.01014 \quad 0.0021 \quad 0.00350 .0050 \quad 0.0071 \quad 0.01420 .02140 .0358 \quad 0.05040 .0727 \quad 0.1500 \quad 0.23220 .4133 \quad 0.6124$ $\begin{array}{lllllllllllllllll}5.0-001 & 0.0005 & 0.0011 & 0.0016 & 0.0027 & 0.0037 & 0.0054 & 0.0108 & 0.0152 & 0.0273 & 0.0386 & 0.0559 & 0.1176 & 0.1860 & 0.3474 & 0.5443\end{array}$ 7.0-0n1 $0.0004 \quad 0.0008 \quad 0.0 n 12 \quad 0.0020 \quad 0.0 n 280.0040 \quad 0.008 n \quad 0.0121 \quad 0.02040 .02890 .04200 .08980 .1447 \quad 0.2826 \quad 0.4692$

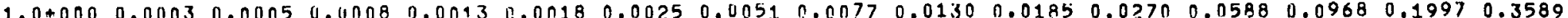
$2.0+0000.00010 .04010 .4002 \quad 0.00030 .00050 .00070 .00140 .00210 .00350 .00500 .00740 .01640 .0278 \quad 0.06240 .1299$

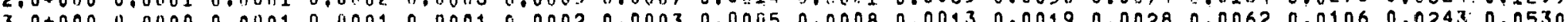
(1)

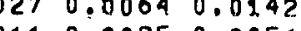

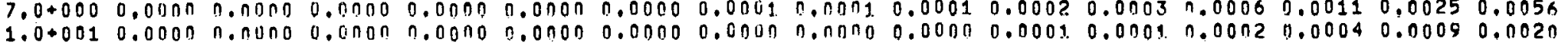

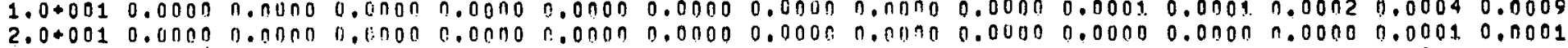
$3.0+0010.0000$ 0.0000 0.0000 0.0000 0.0000 0.0000 0.0000 0.00000.0000000000000000.00000.0000

$5.0+0010.0000 \quad 0.00000 .00000 .00000 .00000 .00000 .00000 .00000 .00000 .00000 .00000 .0000$

$7.0+001 \quad 0.0000 \quad 11.0000 \quad 0.110000 .00000 .00000 .00000 .00000 .00000 .00000 .00000 .0000$ $1.0+0020.00000 .01000 .00000 .00000 .00000 .0000 \quad 0.00000 .00000 .00000 .00000 .0000$ $2.0+0020.00000 .00000 .00000 .0000 \quad 0.00000 .00000 .00000 .00000 .0000$

$3,0+0020.00000 .00000 .00000 .0 \sin 0.00000 .0000 \quad 0.00000 .0000$

$5.0+0020.00000 .00000 .00000 .00100 .00000 .00000 .0000$

$7.0+0020.0000,0.00000,00000.00000 .00000,0000$

$1.0+0030.00000 .00000,00000.00900 .00000,0000$ BETA

1.0.003 0.8999 0.99901.00001.00001.00001.00001.00001.00001.00001.00001.00001.00001.00001.00001.00001.0000 $2.0-003 \quad 0.8998 \quad 0.09901 .00001 .00001 .00001 .00001 .00001 .00001 .00001 .00001 .00001 .00001 .00001 .00001 .00001 .0000$ $3.0-0030.89970 .99901 .00001 .00001 .00001 .00001 .00001 .00001 .00001 .00001 .00001 .00001 .00001 .00001 .00001 .0000$ 5.0003 0.8995 0.09001 .00001 .00001 .00001 .00001 .00001 .00001 .00001000001 .00001 .00001 .00001 .00001 .00001 .0000

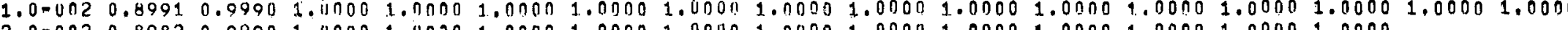
2.0-002 0.898? 0.99901 .00001 .00001 .00001 .00001 .00001 .00001 .00001 .00001 .00001 .00001 .00001 .0000 $3.0-0020.89720 .99001 .00001 .00001 .00001 .00001 .00001 .00001 .00001 .00001 .00001 .00001 .0000$ $5.0=0020.89530 .99901 .09001 .00001 .00001 .00001 .00011 .00001 .00001 .00001 .00001 .0000$

7.070020 .89330 .99891 .00001 .00001 .00001 .00001 .00001 .00001 .00001 .00001 .0000

$1.0=0010.89010 .99891 .00001 .00001 .00001 .00001 .00001 .00001 .00001 .00001 .0000$

$2.0-001 \quad 0.8782 \quad 0.9988 \quad 1.00001 .00001 .00001 .00001 .00001 .00001 .0000$

$3.0001 \quad 0.8640 \quad 0.99861 .00001 .00001 .00001 .00001 .00001 .0000$

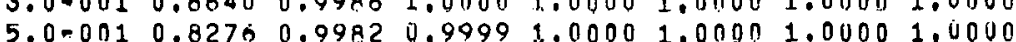

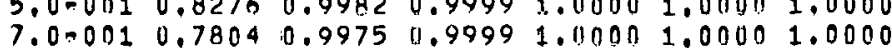

$1.0+000 \quad 0.69230 .99610 .99991 .00001 .00001 .0000$

$2.0+000 \quad 0.3750 \quad 0.98550 .99951 .0000$

$3.0+000 \quad 0.18370 .9623 \quad 0.9986$

$5.0+000 \quad 0.05450 .8676$

$7,0+000 \quad 0,0220$ 
VALUES OF F FOR $N=O A N D N P=4$

ALPF BETA

$1.04003 \quad 0.0010 \quad 0.0020 \quad 0.0030 .0 .0050 \quad 0.0070 \quad 0.0100 \quad 0.0200 \quad 0.0300 \quad 0.0500 \quad 0.06990 .09990 .1998 \quad 0.2998 \quad 0.4993 \quad 0.6911$

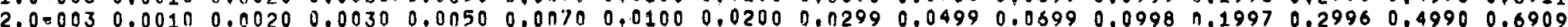

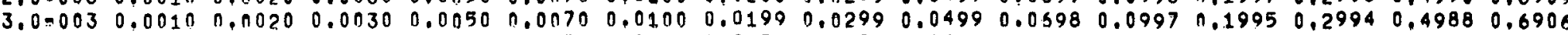
$5.0 .003 \quad 0.0010 \quad 0.0020 \quad 0.0030 \quad 0.0050 \quad 0.0070 \quad 0.0100 \quad 0.01990 .02990 .04980 .0697 \quad 0.0995 \quad 0.1992 \quad 0.29890 .4983 \quad 0.6902$ $7.0 .0030 .00110 .0020 \quad 0.0030 \quad 0.0050 \quad 0.0070 \quad 0.00990 .01990 .02980 .0497 \quad 0.0695 \quad 0.09940 .19890 .2985 \quad 0.4978 \quad 0.6898$

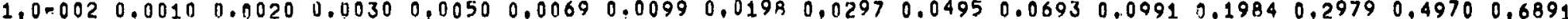
$2.05002 \quad 0.0010 \quad 0.0020 \quad 0.00290 .00490 .00690,00980.0196 \quad 0.0294 \quad 0.0490 \quad 0.0687 \quad 0.0982 \quad 0.1968 \quad 0.2958 \quad 0.4945 \quad 0.6870$ $3.01002 \quad 0.00100 .00190 .00290 .0049 \quad 0.0068 \quad 0.0097 \quad 0.0194 \quad 0.02910 .0486 \quad 0.0680 \quad 0.0973 \quad 0.1952 \quad 0.2936 \quad 0.49190 .6847$ 5.00020 .0010 .00190 .00290 .00480 .00670 .00950 .01900 .02850 .04760 .06670 .09550 .18190 .28930 .48670 .6802

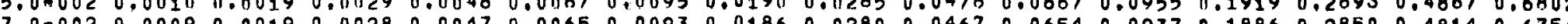
1.000010 .00090 .00190 .00280 .00470 .006530 .00930 .01860 .02800 .04670 .06540 .09370 .18860 .2850 .0 .48140 .6756 1.0 .0010 .00090 .10180 .00270 .10450 .00630 .00900 .01800 .02710 .04580 .06340 .09090 .18370 .27830 .47320 .6684

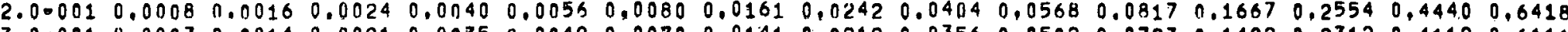

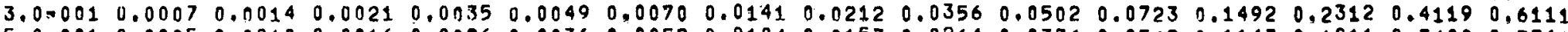
$5.000010 .0005 \quad 0.0010 \quad 0.0016 \quad 0.0026 \quad 0.0036 \quad 0.0052 \quad 0.0104 \quad 0.0157 \quad 0.02640 .03740 .0542 \quad 0.1143 \quad 0.18110 .3400 \quad 0.5361$ $\begin{array}{lllllllllllllllllll}7.0001 & 0.0004 & 0.0007 & 0.0011 & 0.0018 & 0.0025 & 0.0036 & 0.0073 & 0.0110 & 0.0186 & 0.0264 & 0.0385 & 0.0827 & 0.1339 & 0.2647 & 0.4467\end{array}$

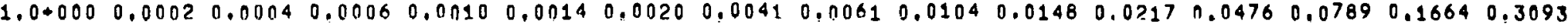

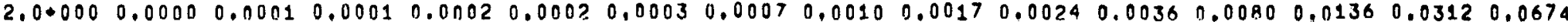
$3,0+0000,00000,00000.00000 .00000 .00010,00010.00020 .00030 .00040 .00060 .00090 .00210 .00350 .00820 .0182$

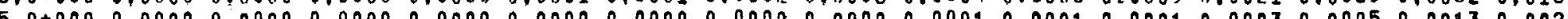
$7,0+000 \quad 0.0000 \quad 0.1000 \quad 0.0000 \quad 0.0000 \quad 0.00000 .00000 .00000 .00000 .00000 .00000 .010000 .00010 .00020 .00040 .0008$

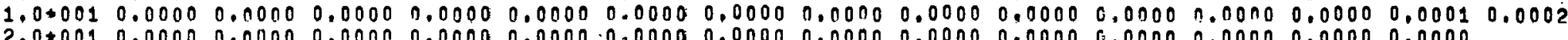
$2.000010 .0000 \quad 0.00000 .00000 .00000 .0000 .0 .00000 .00000 .00000 .00000 .00000 .00000 .00000 .00000 .0000$ $3,0+0010,00000,00000,00000,00000,00000,00000,00000,00000.00000 .00000,00000.00000 .0000$ $5.0+0010.0000 \quad 0.0000 \quad 0.0000 \quad 0,00000.00000 .0000 \quad 0.0000 \quad 0.00000 .00000 .00000,00000.0000$

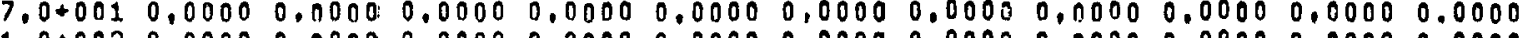
$1.000020 .00000 .00000 .00000 .00000 .0000 \quad 0.00000 .00000 .00000 .00000 .00000 .0000$ $2.00002 \quad 0.0000 \quad 0.0000 \quad 0.0000 \quad 0.0000 \quad 0.0000 \quad 0.0000 \quad 0.0000 \quad 0.00000 .0000$ $3.0+0020.00000 .00000,0000 \quad 0,0000 \quad 0,00000,0000.0 .00000 .0000$

$5.0+0020.00000 .00000 .00000 .00060 .00000 .00060 .0000$

$7.0+0020.00000 .00000 .00000 .00000 .00000 .0000$ $1,00030,00000,00000,00000,00000,00000,0000$ BET

ALPHA $1.000 \quad 2.000 \quad 3.000 \quad 5.000 \quad 7.000 \quad 10.00 \quad 20.00 \quad 30.00 \quad 50.00 \quad 70.00 \quad 100.0 \quad 200.0 \quad 300.0 \quad 500.0 \quad 700.0 \quad 1000$

$1.000030 .89990 .99901 .00001 .00001,00001.00001 .00001 .00001 .00001,00001.00001,00001.00001,00001.00001 .0000$ $2,0.0030,89980.99901 .00001 .00001 .00001 .00001 .00001 .00001 .00001 .00001 .00001 .00001 .00001 .00001 .00001 .0000$ $3.0-0030.89970 .99901 .00001 .00001 .00001 .00001 .00001 .00001 .00001 .00001 .00001 .00001 .00001 .00001 .00001 .0000$ $5.0=0030.89950 .99901 .00001 .00001 .00001 .00001 .00001 .00001 .00001 .00001 .00001 .00001 .00001 .00001 .00001 .0000$

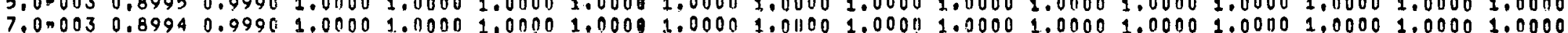

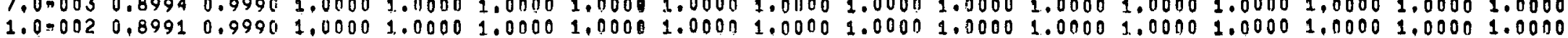

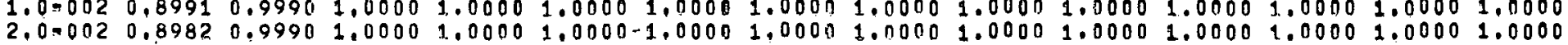
$3.0=0020.897 ? 0.99901 .00001 .00001 .001001 .00001 .00001 .011001 .000111 .00001 .00001 .00001 .0000$ $5,0.0020 .89530,99901,00001,00001,00001,00001,00001,00001.00001,00001.00001 .0000$ $7,0=0020.89330 .99891 .00001 .00011 .00001 .00001 .00001 .00001 .00001 .00001 .0000$ $1.0=0010.8901 \quad 0.99891 .00001 .00031 .00001 .00021 .00001 .00001 .00001 .00001 .0000$ $2,0=0010.8781 \quad 0.99881 .00001 .00001 .00001 .00031 .00001 .00001 .0000$ $3,0=0010.86330 .99861 .00001 .00001 .00001,00031.00001 .0000$ $5.0-0010.82290 .99810 .999 .91,00001,00001,00031,0000$

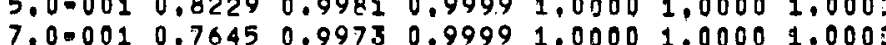

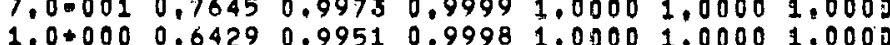
$\begin{array}{lllll}1.0+000 & 0.6429 & 0.9951 & 0.9998 & 1.0000 \\ 2.0+000 & 0.2250 & 0.9708 & 0.9990 & 1.0000\end{array}$ $3.0+000 \quad 0.0692 \quad 0.8941 \quad 0.9959$

$5,0+000 \quad 0.01140,5663$

$7.0+000 \quad 0.0032$

$1.0+0010.0008$ 


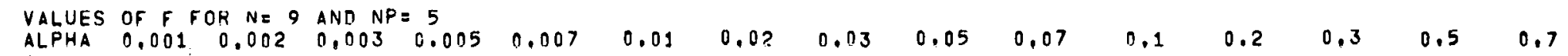
QETA

$1.000030 .0010 \quad 0.0020 \quad 0.0030 \quad 0.0050 \quad 0.0770 \quad 0.0100 \quad 0.0200 \quad 0.0300 \quad 0.0500 \quad 0.06990 .09990 .19980 .2998 \quad 0.49930 .6919$ $2.0=003 \quad 0.0010 \quad 0.00 ? 0 \quad 0.6030 \quad 0.0050 \quad 0.0070 \quad 0.0100 \quad 0.0200 \quad 0.02990 .04990 .06990 .0998 \quad 0.1997 \quad 0.2996 \quad 0.4990 \quad 0.6909$ $3,0=0030.0010 \quad 0,00200.003 n 0.0050 \quad 0.007 n \quad 0,0100 \quad 0.01990 .02990 .04990 .06980 .0997 \quad 0.19950 .29940,49880.6906$ $5,0.003 \quad 0.0010 \quad 0.0020 \quad 0.0030 \quad 0.0050 \quad 0.0070 \quad 0.0100 \quad 0.01990 .02990 .0498 \quad 0.0697 \quad 0.0995 \quad 0.1992 \quad 0.29890 .4983 \quad 0.6902$ $7.0 .003 \quad 0.00100 .0020 \quad 0.0030 \quad 0.0050 \quad 0.0070 \quad 0.00990 .0199 \quad 0.0298 \quad 0.0497 \quad 0.0695 \quad 0.0994 \quad 0.19890 .2985 \quad 0.4978 \quad 0.6898$

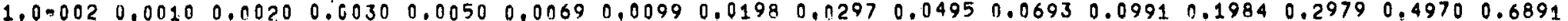

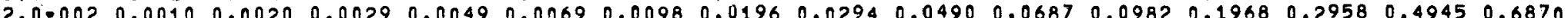
$3,0.0020 .00100 .00190 .00290 .00490 .00680 .00970 .09940 .02910 .04860 .06800 .09730 .19520 .29360 .49190 .0847$ $5.0-0020.00100 .00190 .00290 .00480 .00670 .00950 .01900 .02850 .04760 .06670 .09550 .19190 .28930 .48670 .6802$ 5.0 .0250 .0010 .019
$7,0.002$

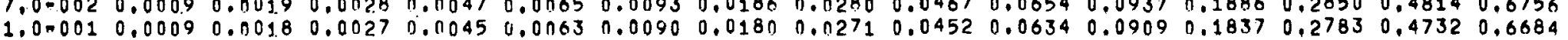

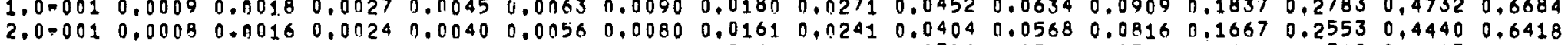
$3,0=0010,0007 \quad 0.00140 .0021 \quad 0,00350.00490,0070 \quad 0,01410,02120.0356 \quad 0.05010 .0722 \quad 0.1490 \quad 0.2309 \quad 0.41150 .6107$ $5.0=0010.0005 \quad 0.0010 \quad 0.0015 \quad 0.00250 .0036 \quad 0.0051 \quad 0.0103 \quad 0.01550 .02600 .03680 .0534 \quad 0.1127 \quad 0.1788 \quad 0.33640 .5321$ $\begin{array}{lllllllllllllllllll}7.0-001 & 0.0003 & 0.0017 & 0.0010 & 0.0017 & 0.0024 & 0.0034 & 0.0069 & 0.0104 & 0.0176 & 0.0250 & 0.0364 & 0.0783 & 0.1272 & 0.2534 & 0.4322\end{array}$

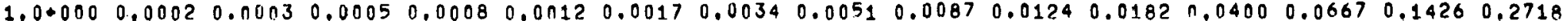
2.0 .0000 .00000 .00000 .00000 .00010 .00010 .00020 .00030 .00050 .00080 .00120 .00180 .00400 .00680 .01500 .0343 3.0 .0000 .00000 .00000 .00000 .00000 .00000 .00000 .00010 .00010 .00010 .00020 .00030 .00070 .00120 .00270 .0061

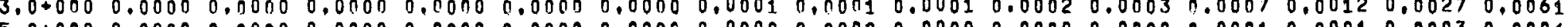
5.0 .000 .0 .00000 .00000 .0000 .

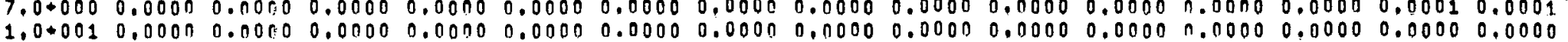

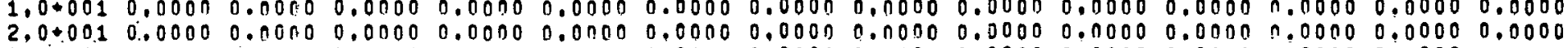
$3.000010 .0000 \quad 0.0000 \quad 0.00000 .00000 .0000 \quad 0.00000 .00000 .00000 .00000 .00000 .00000 .00000 .0000$ $5.0+0010.000 .00 .0000 \quad 0.0000 \quad 0.0000 \quad 0.0000 \quad 0.0000 \quad 0.00000 .00000 .00000 .00000 .0000 \quad 0.0000$ $7.0+0010.0000 \quad 0.00000 .0000 \quad 0.00000 .00000 .00000 .00000 .00000 .00000 .00000 .0000$ $1.0+0020.0000 \quad 0.0000 \quad 0.0000 \quad 0.0000 \quad 0.0000 \quad 0.0000 \quad 0.0000 \quad 0.00000 .00000 .00000 .0000$ $2.0+002 \quad 0.0000 \quad 0.0000 \quad 0.0000 \quad 0.0000 \quad 0,0000 \quad 0,0000 \quad 0.00000,0000 \quad 0.0000$

$3,0+0020,00000,00000,00000,00000,00000,00000,00000,0000$

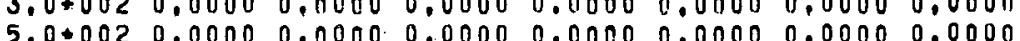

$7,000020,00000,00000,00000,00000,00000,0000$

$1,0+0030,00000.00000,00000.00000 .00000 .0000$

ALPHA. $1.000 \quad 2.000 \quad 3.000 \quad 5.000 \quad 7.000 \quad 10.00 \quad 20.00 \quad 30.00 \quad 50.00 \quad 70.00 \quad 100.0 \quad 200.0 \quad 300.0 \quad 500.0 \quad 700.0 \quad 1000$ BETA

1.0 .0030 .89990 .99901 .00001 .00001 .00001 .00001 .00001 .00001 .00001 .00001 .00001 .00001 .00001 .00001 .00001 .0000 $2.0=0030.89980 .99901 .00001 .00001 .00001,00001.00001 .00001 .00001,00001.00001 .00001 .00001,00001.00001 .0000$ 3.0 .0030 .89970 .09001 .00001 .00001 .00001 .00001 .00001 .00001 .00001 .00001 .00001 .00001 .00001 .00001 .00001 .0000 $5,0=0030.89950 .09901 .00001 .00001 .00001 .00001 .00001 .00001 .00001 .00001 .00001 .00001 .00001 .00001 .00001 .0000$

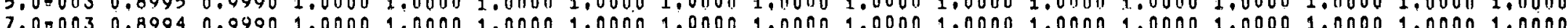

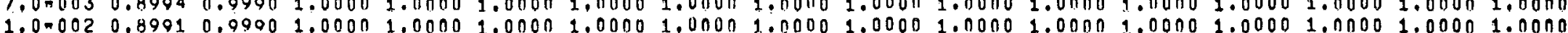

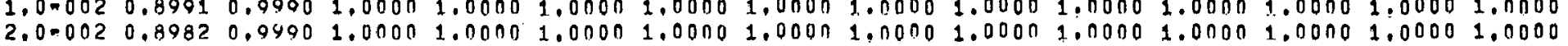
$3,0=0020.89720 .99901 .00001 .00001 .00001 .00001 .00001 .00001 .00001 .00001 .00001 .00001 .0000$ $5.0-0020.89530 .99901 .00001 .00001 .00001 .00001 .00001 .00001 .00001 .00001 .00001 .0000$

$7.0=002 \quad 0.89330 .99891 .00001 .00001 .00001 .00001 .00001 .00001 .00001 .00001 .0000$

$1.0=001 \quad 0.8901 \quad 0.09891 .00001 .00001 .00001 .00001 .00001 .00001 .00001 .00001 .0000$

2.0.001 $0.87810 .09881 .00001 .00001 .00001 .00001 .000 n 1.00001 .0000$

$3,0.0010 .86310 .09861,00001,00001,00001,00001.00001,0000$

$5,0=0010.82050 .99810 .99991 .00001 .00001 .00001 .0000$

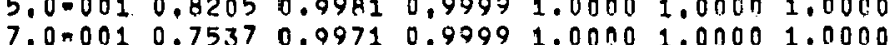

$1.0+000 \quad 0.00000 .09420 .99981 .00001,00001.0000$

$2.0+000 \quad 0.1250 \quad 0.94190 .9979 .1 .0010$

$3.0+000 \quad 0.02410 .73740 .9878$

$5.0+000 \quad 0.0023 \quad 0.2074$

$7.0+000 \quad 0.0005$

$1,0.0010,0001$ 
VALUES OF F FOR $N=13$ O AND $N P=1$

ALPHA 0,001 U.002 $0.003=1$ BETA

$1.0=003 \quad 0.0010 \quad 0.0020 \quad 0.0030 \quad 0.0050 \quad 0.0070 \quad 0.0100 \quad 0.0200 \quad 0.0300 \quad 0.0500 \quad 0.06990 .0999 \quad 0.1998 \quad 0.2998 \quad 0.4995 \quad 0.6937$ $2,0=0030.00100 .00200 .00300 .0050 \quad 0.00700 .0100 \quad 0.0200 \quad 0.02990 .04990 .06990 .09980 .19070 .29960 .49930 .6935$ 3,0-003 $0.0010 \quad 0.0020 \quad 0.0030 \quad 0.0050 \quad 0.0 n 70 \quad 0.0100 \quad 0.01990 .02990 .04990 .06980 .0997 \quad 0.19950 .29940 .4990 \quad 0.6933$ $5,0=003 \quad 0,00100.0030 \quad 0,0030 \quad 0.0050 \quad 0.0070 \quad 0.0100 \quad 0.01990,02090.0498 \quad 0.0697 \quad 0.0996 \quad 0.1992 \quad 0.2990 \quad 0.49850 .6920$ $7,0=0030.00100 .0020 \quad 0.00300 .0050 \quad 0.00700 .00990 .01990 .02980 .04970 .06950 .09940 .19890 .29850 .4980 \quad 0.6925$ $1,0=002 \quad 0.0010 \quad 0.0020 \quad 0.0030 \quad 0.0050 \quad 0.00690 .0094 \quad 0.01980 .02970 .04950 .06940 .0991 \quad 0.19840 .29790 .4973 \quad 0.6918$

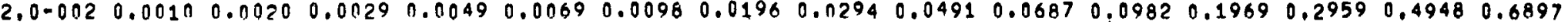
$3,0=002 \quad 0.00100 .00190 .0029 \quad 0.00490 .0068 \quad 0.0097 \quad 0,0194 \quad 0.02920 .04860 .06810 .00740 .1953 \quad 0.2938 \quad 0.4924 \quad 0.6876$ 5.00020 .00100 .00190 .00290 .00400 .00670 .00950 .01910 .02860 .04770 .06690 .00570 .19230 .28990 .48700 .6835 $5.0=0020.0010 .00190 .00290 .00480 .00670 .00950 .01910 .02860 .04770 .06690 .09570 .19230 .28990 .48760 .6835$

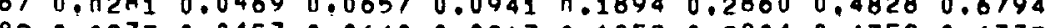

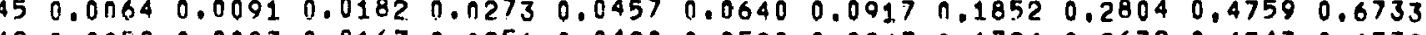

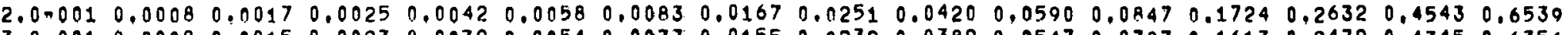
$\begin{array}{llllllllllllllll}3.0 .001 & 0.0008 & 0.0015 & 0.0023 & 0.0039 & 0.0054 & 0.0077 & 0.0155 & 0.0232 & 0.0389 & 0.0547 & 0.0787 & 0.1613 & 0.2479 & 0.4345 & 0.6356\end{array}$

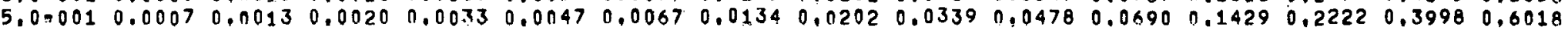

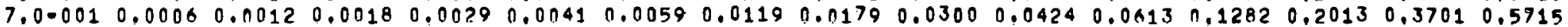

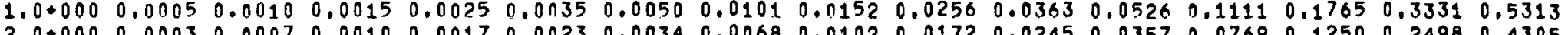

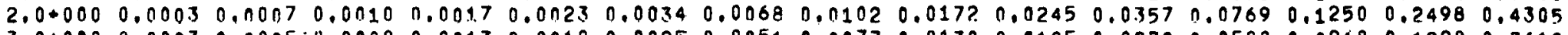
$3,0+000 \quad 0.00030 .0005 .0 .0008 \quad 0.00130 .0018 \quad 0.00250 .00510 .00770 .01300 .01850 .02700 .05880 .0968 \quad 0.1998 \quad 0.3618$ $5.0+0000.00020 .00030 .00050 .00080 .00120 .00170 .00340 .00510 .00870 .01240 .01820 .04000 .06670 .14270 .2743$ $7,0+000 \quad 0.00010 .00030 .00040 .00060 .00090 .00130 .00250 .00390 .00650 .00930 .01370 .03030 .05080 .11100 .2208$

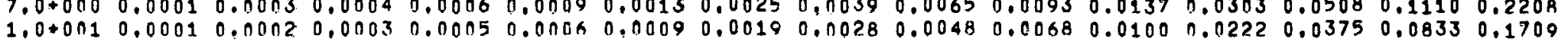

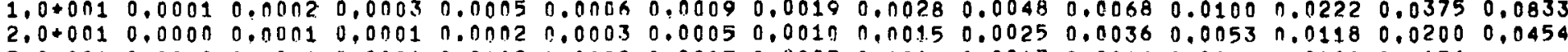
$3,0+0010.00000 .00010 .00010 .00020 .00020 .00030 .00070 .00100 .00170 .00240 .00360 .00800 .0136$

5.00010 .00000 .00000 .00010 .00010 .00010 .00020 .00040 .00060 .00100 .00150 .00220 .0049

$7.0+0010.00000 .00000 .00000 .00010 .00010 .00010 .00030 .00040 .00010 .00110 .0016$

$1.0+0020.00000 .00000 .00000 .00000 .00010 .00010 .00020 .00030 .00050 .00070 .0011$

$2.0+0020.00000 .00000 .00000 .00000 .0000 \quad 0.00010 .00010 .00020 .0003$

$3.0+0020.00000 .00000 .00000 .00000 .00000 .00000 .00010 .0001$

$5.0+0020.00000 .00000 .0000 \quad 0.00000 .00000 .00000 .0000$

$7,0+0020,00000.00000 .00000 .00000 .00000 .0000$

$1,000030,00000.00000,00000,00000,00000,0000$ BETA

ALPHA $1.000 \quad 2.000 \quad 3.000 \quad 5.000 \quad 7.000 \quad 10.00 \quad 20.00 \quad 30.00 \quad 50.00 \quad 70.00 \quad 100.0 \quad 200.0 \quad 300.0 \quad 500.0 \quad 700.0 \quad 1000$

$1.0,0030.9090 \quad 0,09951.00001 .00001 .00001 .00001 .00001 .00001 .00001 .00001 .00001 .00001 .00001 .00001 .00001 .0000$ $2,0-0030.90890 .09951,00001.00001 .00001 .00001 .00001 .00001 .00001 .00001 .00001 .00001 .00001 .00001,00001.0000$ $3,0=0030,908 \mathrm{H} \quad 0.99951 .00001 .00001 .00001 .00051 .000 n 1.00001 .00001 .00001 .00001 .00001 .00001 .00001 .00001 .0000$ $5,0=0030.90870 .09951 .00001 .00001 .00001 .00061 .00001 .00001 .00001 .00001 .00001 .00001 .00001 .00001 .00001 .0000$

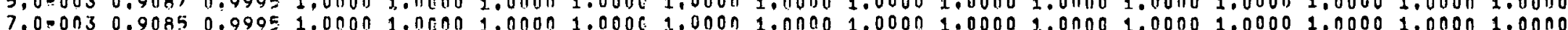

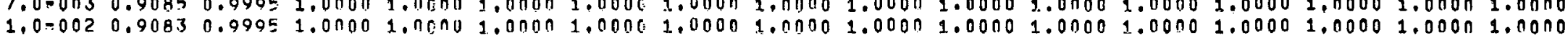

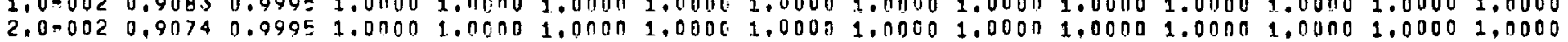

3.0 .0020 .90660 .99951 .00001 .00001 .00001 .00001 .00001 .00001 .00001 .00001 .00001 .00001 .0000

$5,0.0020 .90500 .09051 .00001,00001.00001,00001,00001.00001,00001,00001,00001.0000$

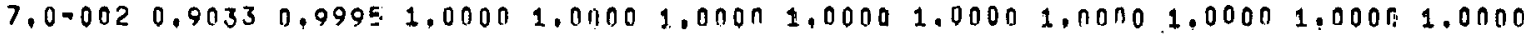

1.0 .0010 .90090 .99951 .00001 .00001 .00001 .00001 .00001 .00001 .00001 .00001 .0000

$2,0=0010.89290 .99941 .00001,0 \pi n \pi 1.00001 .00001 .00001 .00001 .0000$

$3,0-001 \quad 0.88500 .999 \mathrm{c} 1.00001 .00001 .00001 .00001 .00001 .0000$

$5,0=0010.86960 .09931,00001,00001,00001,00001,0000$

$7.0=001 \quad 0.8547 \quad 0.9992 \quad 1.00001 .00001 .00001 .0000$

$1.0+0000.83330 .09001 .00001 .00001 .00001 .0000$

$2,0.000 \quad 0,7692 \quad 0.99851,00001,0000$

$3.0+000 \quad 0.7143 \quad 0.99801 .0000$

$5.0+000 \quad 0.6250 \quad 0.9971$

$7,0+000 \quad 0,555$

$1,0+001 \quad 0,4762$ 


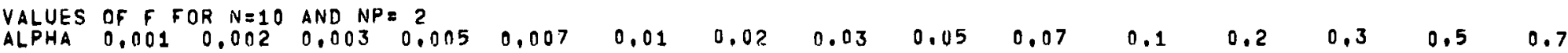
BETA

$1.0 .003 \quad 0.0010 \quad 0.0020 \quad 0.0030 \quad 0.0050 \quad 0.0070 \quad 0.0400 \quad 0.0200 \quad 0.0300 \quad 0.0500 \quad 0.06990 .0999 \quad 0.1998 \quad 0.2998 \quad 0.4995 \quad 0.6937$

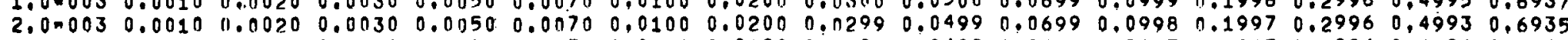
$3.0 .0030 .0010 \quad 0.0020 \quad 0.0030 \quad 0.0150: 0.0 n 7 n \quad 0.0100 \quad 0.01990 .02990 .04990 .06980 .0997 \quad 0.1995 \quad 0.29940 .4990 \quad 0.6933$ $5.000030 .0010 \quad 0.0020 \quad 0.0030 \quad 0.0050 \quad 0.0070 \quad 0.0100 \quad 0.0199 \quad 0.02990 .0498 \quad 0.0697 \quad 0.0995 \quad 0.1992 \quad 0.2989 \quad 0.49850 .6929$ $\begin{array}{lllllllllllllllllll}7.0 .003 & 0.0010 & 0.0020 & 0.0030 & 0.0050 & 0.0 n 7 n & 0.0099 & 0.0199 & 0.0298 & 0.0497 & 0.0695 & 0.0994 & 0.1989 & 0.2985 & 0.4980 & 0.0925\end{array}$

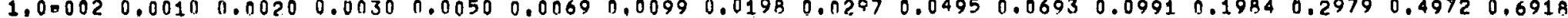

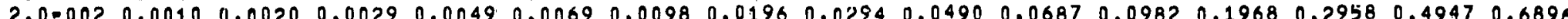
$3.0=0020.00100 .00190 .00290 .00490 .00680 .00970 .01940 .02910 .04860 .06800 .09730 .19520 .29360 .49210 .6874$

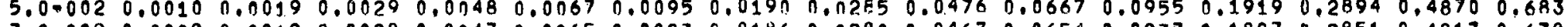
7.0 .0020 .00090 .00190 .00280 .00470 .00650 .00930 .01860 .02 .00 .04670 .06540 .09370 .18870 .28510 .48170 .6784

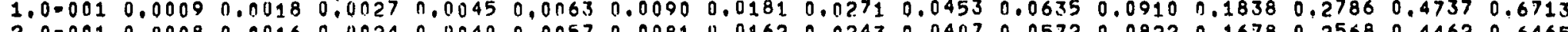

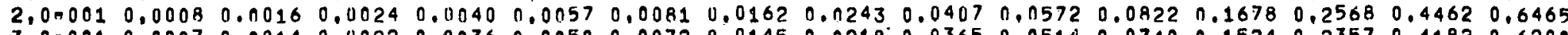
$3,0.001 \quad 0.0007 \quad 0.00140 .00220 .0036 \quad 0.0050 \quad 0.00720 .01450 .02180 .03650 .05140 .0740 \quad 0.15240,2357 \quad 0,4182 \quad 0.6200$

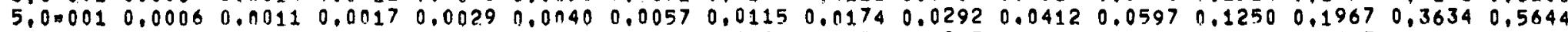
$\begin{array}{lllllllllllllllllll}7.0 .001 & 0.0005 & 0.0009 & 0.0014 & 0.0023 & 0.0 n 32 & 0.0046 & 0.0092 & 0.0139 & 0.0235 & 0.0332 & 0.0483 & 0.1025 & 0.1637 & 0.3133 & 0.5087\end{array}$ $1.0+000 \quad 0.0003 \quad 0.0007 \quad 0.00100 .0017 \quad 0.0 n 23 \quad 0.00340 .00680 .01020 .01720 .02450 .0357 \quad 0.07690 .1250 \quad 0.24980 .4305$ $2.0+000 \quad 0.0001 \quad 0.0003 \quad 0.0004 \quad 0.0007 \quad 0.0 n 10 \quad 0.0014 \quad 0.0 n 29 \quad 0.00440 .0075 \quad 0.0106 \quad 0.0156 \quad 0.0345 \quad 0.0577 \quad 0.1249 \quad 0.2447$ $3,0+0000.00010 .00020 .00020 .00040 .00050 .00080 .00160 .00240 .00400 .00580 .00850,01890.03190 .07140 .148$

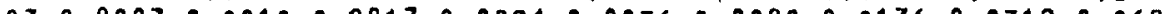
$5,0+0000.0000$.

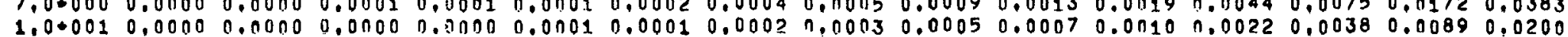

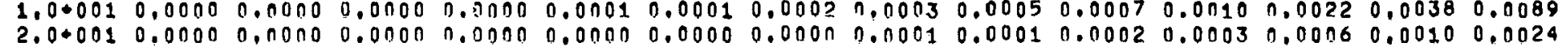
$3.0+0010.0000 \quad 0,00000.00000 .00000 .00000,00000.00000 .00000 .00010 .00010 .00010 .00030 .0005$

$5.0+0010,00000.00000 .00000 .00100 .00000 .00000,00000.00000 .00000 .00000 .00000 .0001$

$7.0+0010.00000 .00000 .00000 .00000 .00000 .00000 .00000 .00000 .00000 .00000 .0000$

$1.0+0020.00000 .00000 .00000 .00000 .00000 .00000 .00000 .00000 .00000 .00000 .0000$

$2.0+0020.000110 .0000 \quad 0.00000 .0 n n 0 \quad 0.0000 \quad 0.0000 \quad 0.00000 .00000 .0000$

$3.0+0020.00000 .0000 \quad 0.00000 .0000 \quad 0.001100 .00000 .00000 .0000$

$5,0+0020.00000 .00000 .0000 \quad 0.00000 .001100 .00000 .0000$

$7,0+0020.00000 .00000 .00000,00100,00000.0000$

$1,0+0030,00000.00000,00000,00000,00000,0000$

ALPHA $1.000^{\circ} 2.000 \quad 3.000 \quad 5.000 \quad 7.000 \quad 10.00 \quad 20.00 \quad 30.00 \quad 50.00 \quad 70.00 \quad 100.0 \quad 200.0 \quad 300.0 \quad 500.0 \quad 700.0 \quad 1000$ BETA

1.000030 .90900 .99951 .00001 .00001 .00001 .00001 .00001 .0000 .1 .00001 .00001 .00001 .00001 .00001 .00001 .00001 .0000 $2,0=0030,90890.09951 .00001,00001.00001,00001.00001 .00001 .000 ! 1.00001 .00001 .00001 .00001,00001,00001,0000$

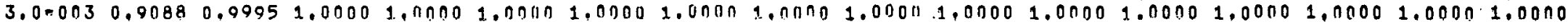
$5.0-0030.90870 .09051 .00001 .00001 .00001,00001.00001 .00001,00001,00001.00001 .00001,00001,00001,00001.0000$ $1.0-0030.90850 .09051 .00001 .00001 .00001 .00001 .00001 .00001 .00001 .00001 .00001 .00001 .00001 .00001 .00001 .0000$

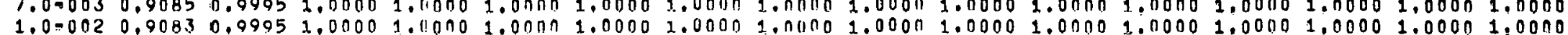

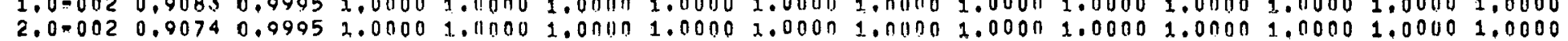

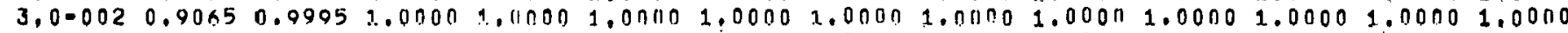
$5.0-0020.90480 .99951 .00001 .00001 .001101 .00001 .00001 .00001 .00001 .00001 .00001 .0000$ $7,0.0020 .90290 .09951 .00001 .00001 .00001 .00001 .00001 .00001 .00001 .00001 .0000$ $1.0 .0010 .9001 \quad 0.99959 .0 n 001.00001 .00001 .00001 .000 ? 1.00001 .00001 .00001 .0000$

$2.0=0010.8897 \quad 0.99942 .00001 .00001 .001101 .00002 .00001 .011001 .0000$

$3.0-001 \quad 0.8780 \quad 0.9993 \quad 1.00001 .00001 .00011 .00001 .00001 .0000$

$5.0=0010.85110 .0991 \quad 1.00001 .00001 .00011 \quad 1.00001 .0000$

7,0-001 $0,8203 \quad 3.99891,00001,011001,00001,0000$

$1.0+000 \quad 0.7692 \quad 0.9985$ 1.0000 $1.0 n 001,00001,0000$

$2,000000.5882,0.9966 \quad 0.9999,1,0100$

$3,0+000 \quad 0,4348 \quad 0.0937 \quad 0.9999$

$5.0+000 \quad 0.24390 .0851$

$7.0+000 \quad 0.1493$ 
VALUES OF F FOR NEIO AND NP:3 BETA

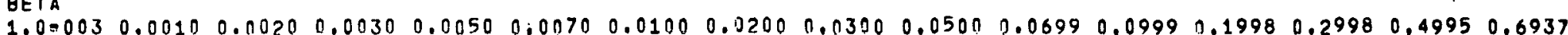

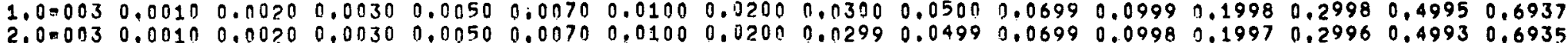

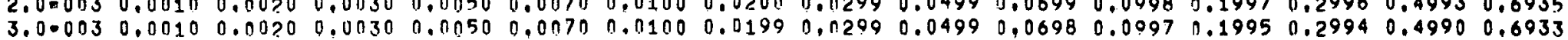

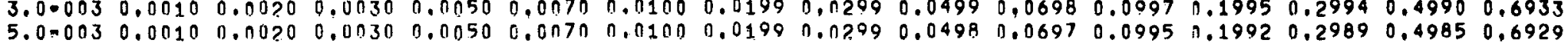

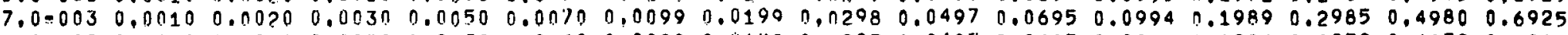

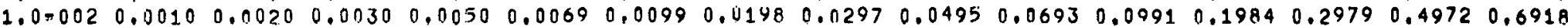

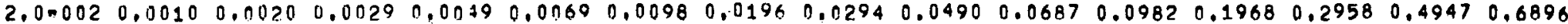

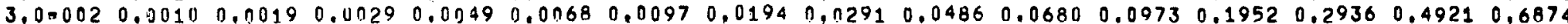
$5.0=0020.00100 .00190 .00290 .00480 .00670 .00950 .0190 .0 .02850 .04760 .06670 .09550 .19190 .28930 .48690 .6829$ $7.0-0020.00090 .00190 .00280 .00470 .00650 .00930 .01860 .02800 .04670 .06540 .09370 .18860 .2850 \quad 0.48160 .6783$

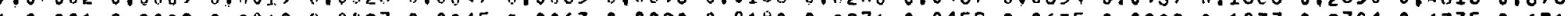
$1.0=0010.00090 .1030 .0127 .00450 .00630 .00900 .0180 .0 .0710 .04520 .06350 .09090 .18370 .27840 .47350 .6711$ $2.0 .0010 .00080 .00160 .00240 .0 n 40.00560 .00800 .01610 .02420 .04050 .05690 .08180 .16690 .25560 .44460 .6450$

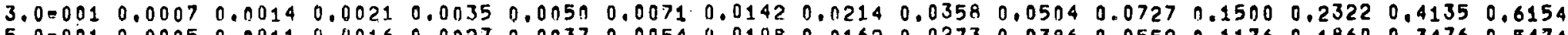
$5.0=0010.00050 .0011 \quad 0.0016 \quad 0.0027 \quad 0.0037 \quad 0.0054 \quad 0.01080 .01620 .0273 \quad 0.0386 \quad 0.05590 .1176 \quad 0.1860 \quad 0.3476 \quad 0.5474$

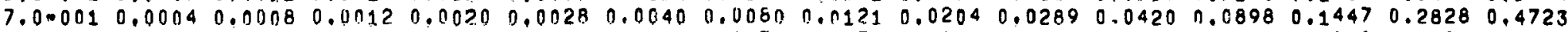

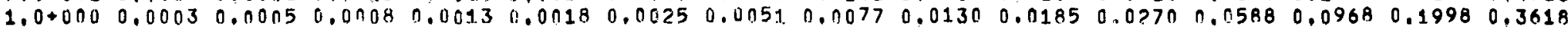
$2.0+000 \quad 0.0001 \quad 0.0001 \quad 0.0002 \quad 0.00 .03 \quad 0.0005 \quad 0.0007 \quad 0.0014 \quad 0.00210 .00350 .0050 \quad 0.00740 .0164 .0 .0278 \quad 0.06240 .1313$

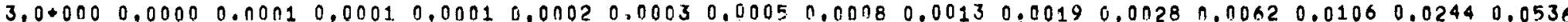
$5,0+0000,00000,0000.0 .00000 .00000 .00000 .00010 .00010 .00020 .00030 .00050 .00070 .00160 .00270 .00640 .0143$

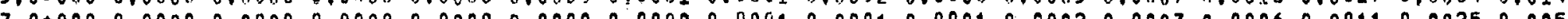

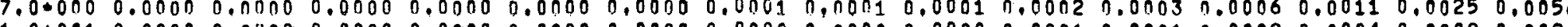

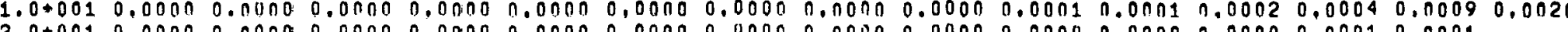

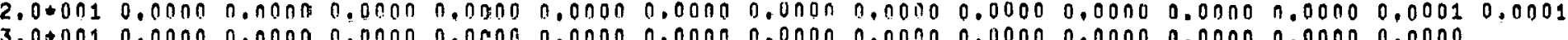

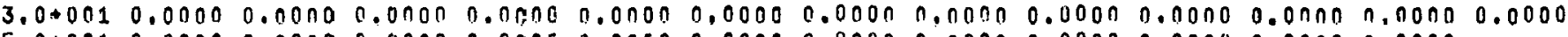
$5,0+0010.0000 \quad 0.00000 .00000 .00000 .00000 .00000 .00000,00000.00000,00000.00000 .0000$

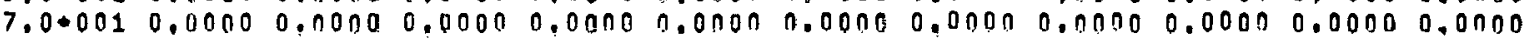
$1,0+0020,00000,00000.00000,00000.00000 .00000 .00000 .00000 .00000,00000.0000$ $2.0+0020.00000 .00000 .00000 .00000 .00000 .00000 .00000 .00000 .0000$

$3,0+002 \quad 0.0000 \quad 0.0000 \quad 0.0000 \quad 0.0000 \quad 0.0000 \quad 0.0000 \quad 0.0000 \quad 0.0000$

$5,0+0020,00000.00050 .00000 .0000,0,0000.00000,0000$

$7,0+0020,00000,0000,0,0000,00000,00000,0000$

$1.0+0030.0000 \quad 0.00000 .00000 .01000,00000.0000$ $B E T A$

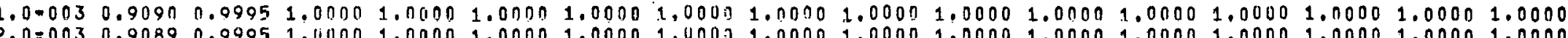

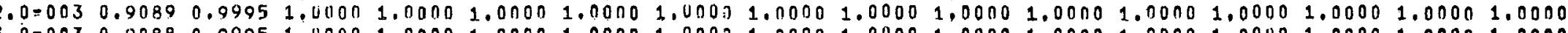

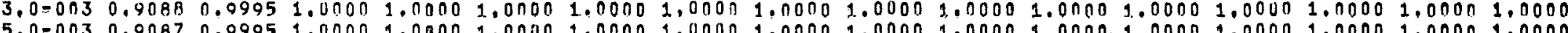
$7.0-0030.0085$. 09051.00001 .00081 .00001 .00001 .00001 .00001 .00001 .00001 .00001 .00001 .00001 .00001 .00001 .0000

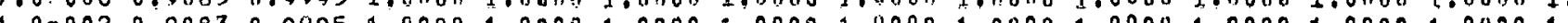

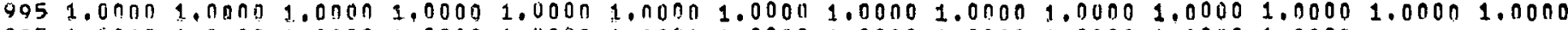

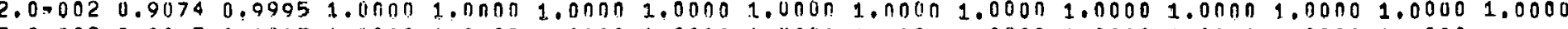

$3.0=0020.90650 .99951 .00001 .00001 .00001 .00001 .000 n 1.00001 .00001 .00001 .00001 .00001 .0000$

$5.0=0020,90480,99951.00001 .00001 .00001 .00001 .00001 .00001 .00001 .0000 \$ 1.00001 .0000$

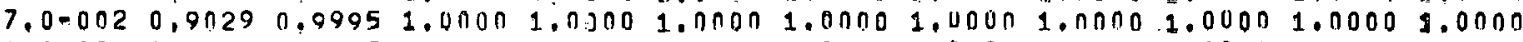

$1,0.0010 .90000 .99951 .00001 .03001 .000 n 1.00001 .00001 .00701 .00001 .00001 .0000$

$2.0=0010.88900 .99991 .00001 .01001 .00001 .00001 .00001 .00001 .0000$

$3.0=0010.87590 .999 .31 .00001 .03001 .00001 .00001 .00001 .0000$

$5.0=001 \quad 0.84210 .9991 \quad 1.0010 .01 .03001 .0000 \quad 1.00001 .0000$

$7.0=0010.79790 .99831 .00001 .03001 .00001 .0000$

$1.0+000 \quad 0.71430 .99871,00001.03001 .00001,0000$

$2,0+000 \quad 0.4000 \quad 0.09270 .9998,1.0000$

$3.0+000 \quad 0.2000 \quad 0.08030 .9995$

$5.0+000 \quad 0.0602 \quad 0.9292$

$7.0+000 \quad 0.0244$

1.0 .0010 .0089 


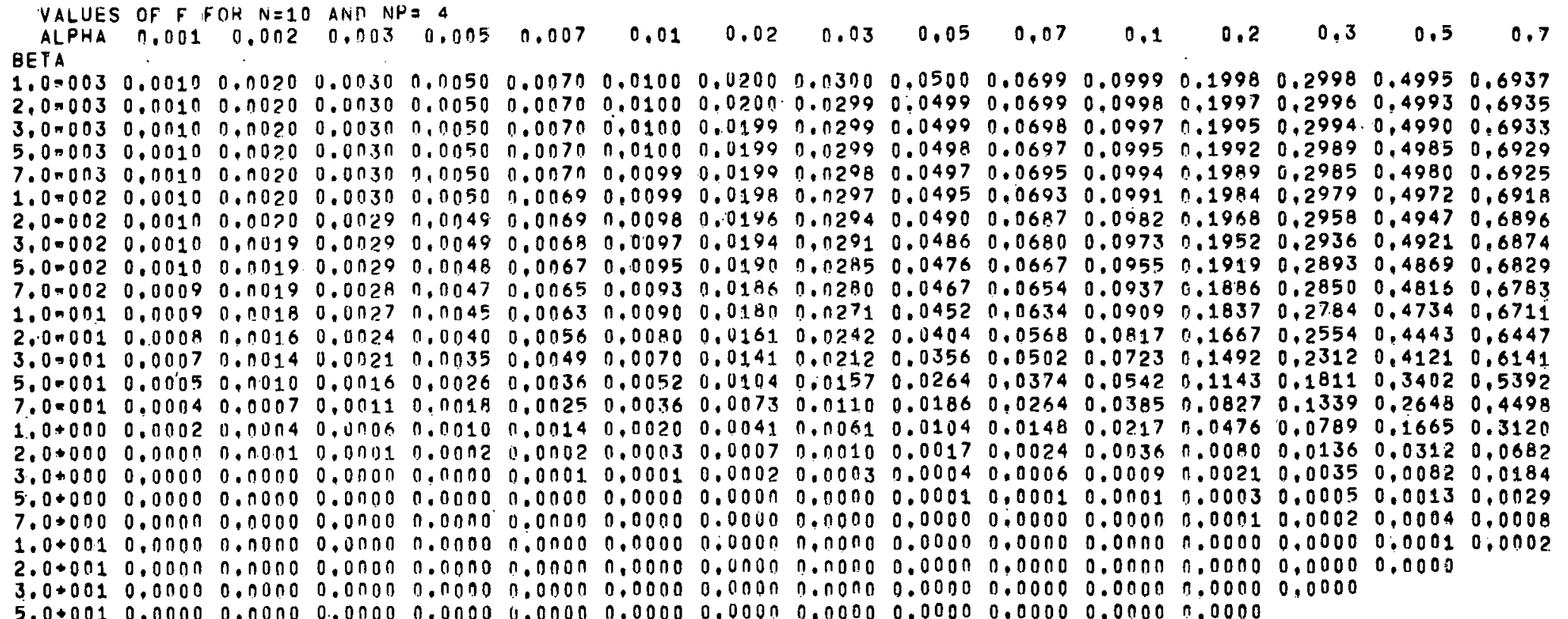

$3.0+0010.00000 .00000 .00000 .00000 .00000 .00000 .00000 .00 n 00.00000 .00000 .00000 .00000 .0000$

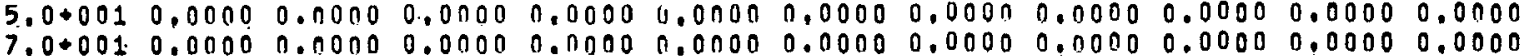

$1.0+0020.00000 .00000 .00000 .00000 .00000,00000.00000 .00000 .00000 .00000 .0000$

$2,0+0020.00000 .00000 .0000,0.0 n 00$ if.0n00 0.00000 .00000 .00000 .0000

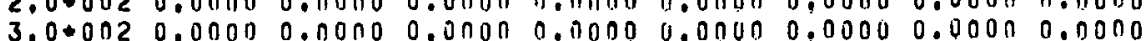

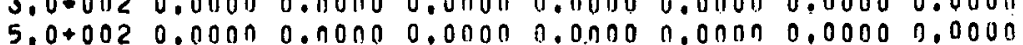

$7,0+0020,00000,00000,00000,00000,0000.0,0000$

$1.0+0030.00000 .00000 .00000 .00000 .0000 \quad 0.0000$

ALT PL BETA

$1.0-0030.9090 \quad 0.99951 .00001 .00001 .00001 .00001 .00091 .00001 .00001 .00001 .00001 .00001 .00001 .00001 .00001 .0000$ $2.0=0030.90890 .99951 .00001 .00001 .00001,00001.00001 .00001 .00001 .00001 .00001 .00001,00001,00001.00001 .0000$ $3,0=0030.90880 .99951 .00001 .00001 .00001 .00001 .00001 .00001 .00001 .00001 .00001 .00001 .00001 .00001 .00001 .0000$ $5,0-0030.90870 .99951 .00001 .00001 .00001 .00001 .00001 .00001 .00001 .00001 .00001 .00001 .00001 .00001 .00001 .0000$

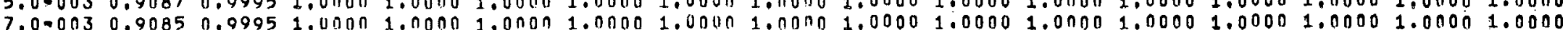

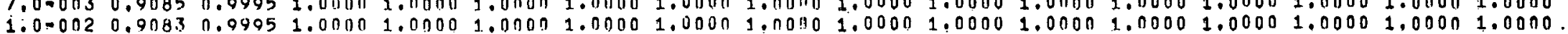

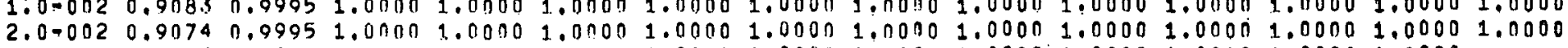
3.070020 .90650 .99951 .00001 .00001 .00001 .00001 .00001 .00001 .00001 .00001 .00001 .00001 .0000

$5.0=0020.90480 .99951 .00001 .00001 .00001,00001.00001 .00001 .00001 .00001 .00001 .0000$

$7.0=0020.90290 .99951 .00001 .001001 .00001 .00001 .00001 .00001 .00001 .00001 .0000$

$1.070010 .9000 \quad 0.99951 .00001 .00001 .00001 .00001 .00001 .00001 .00001 .00001 .0000$

2.070010 .88800 .99941 .00001 .00001 .00001 .00001 .00001 .00001 .0000

$3,0=0010.87530 .09031,00001.00001 .00001,00001.00001 .0000$

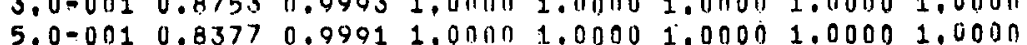

$\begin{array}{lllllll}5.0=001 & 0.8377 & 0.9991 & 1.0000 & 1.0000 & 1.0000 & 1.0000 \\ 7,0-001 & 0.7829 & 0.9486 & 1.0000 & 1.0000 & 1.0000 & 1.0000\end{array}$

$1,0+000 \quad 0.66670 .09760 .99991,00001.00001 .0000$

$2.0+000 \quad 0.24390 .9851$ ن.9097 1.0000

$3.0+000 \quad 0.0763 \quad 0.0442 \quad 0.9966$

$5.0+000 \quad 0.0126 \quad 0.7237$

$7.0+000 \quad 0.01136$ 
VALUES OF F FON N=10 ANO NP $=5$

(1). 002 O $0.043=0.005$ BETA

$1.0-0030.0010 \quad 0.0020 \quad 0.0030 \quad 0.0050 \quad 0.0070 \quad 0.01010 .0200 \quad 0.0300 \quad 0.0500 \quad 0.06990 .09990 .1998 \quad 0.2998 \quad 0.4995 \quad 0.6937$

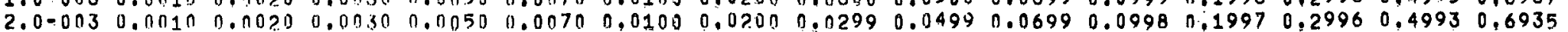

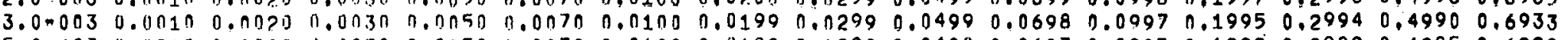

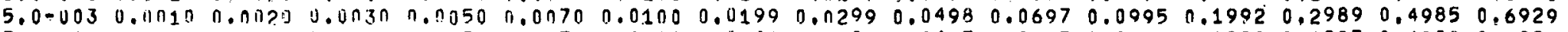
$7.0=003 \quad 0.00100 .00230 .00300 .0050 \quad 0.007 n \quad 0.00990 .0190 \quad 0.02980 .0497 \quad 0,0695 \quad 0.0994 \quad 0.19890 .2985 \quad 0.4980 \quad 0.6925$

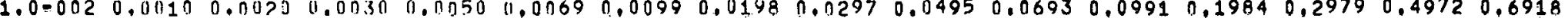
$\begin{array}{lllllllllllllllll}2.0 .002 & 0.0010 & 0.0020 & 0.0029 & 0.0049 & 0.0069 & 0.0098 & 0.0196 & 0.0294 & 0.0490 & 0.0687 & 0.0982 & 0.1968 & 0.2958 & 0.4947 & 0.6896\end{array}$ $3.0-0020.00100 .00170 .00290 .00490 .00680 .00970 .01940 .02910004860 .06800 .09730 .19520 .29360 .49210 .6874$

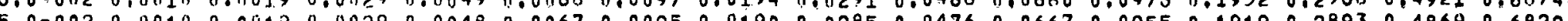
7.0.002 0.0n1.

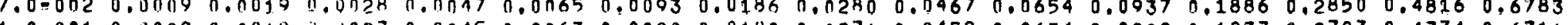

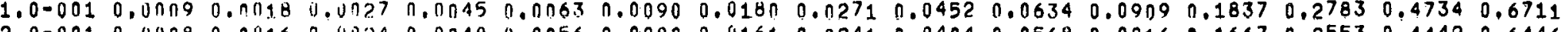

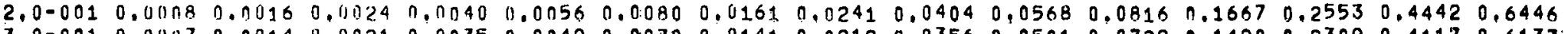

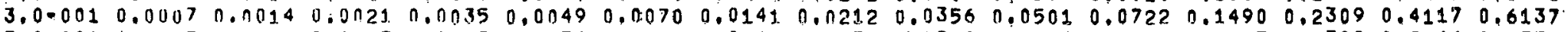
$\begin{array}{llllllllllllllllll}5,0=001 & 0.0005 & 0.001 .0 & 0.0 n 15 & 0.0025 & 0.0036 & 0,0.051 & 0.0103 & 0.0155 & 0.0260 & 0.0368 & 0.0534 & 0.1127 & 0.1788 & 0.3366 & 0.5353\end{array}$

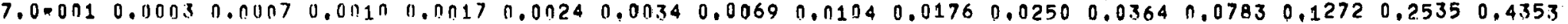
$\begin{array}{llllllllllllllll}1.0+000 & 0.000 ? & 0.0 n \cap 3 & 0.0 n 05 & 0.0008 & 1.0012 & 0.0017 & 0.0034 & 0.0051 & 0.0087 & 0.0124 & 0.0182 & 0.0400 & 0.0667 & 0.1427 & 0.2743\end{array}$

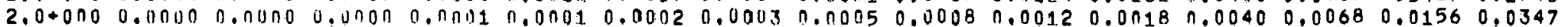
$3,0+0000,000 n 0.0100,0.0000 .0000 .00000 .00000 .00010 .00010 .00010 .00020 .00030 .00070 .00120 .00270 .0062$

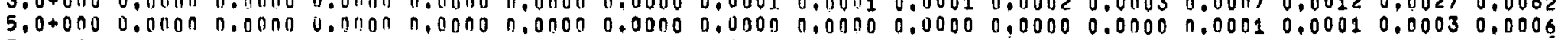

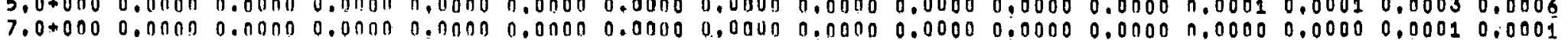

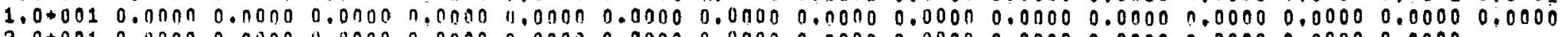
$2.0+0010.00000 .00000 .00000 .00000 .00000 .00000 .00000 .00000 .00000 .00000 .00000 .00000,00000.0000$

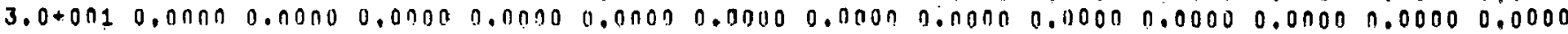

$5.0+0010.00000 .00000 .00000 .00200 .00000 .00000 .00000 .00000 .00000 .00000 .00000 .0000$

$7.0+0010.00000 .00000 .00000 .00000 .00000 .00000 .00000 .00000 .00000 .00000 .0000$

$1.0+0020.00000 .00000 .0000,0.00000,00000.00000 .00000,00000,00000,00000,0000$

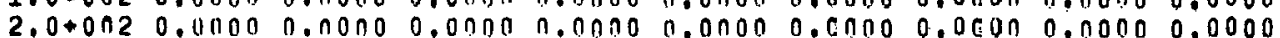

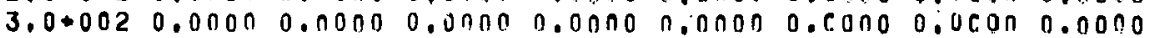

$5.000020 .00000 .00000 .00000 .00000,00000.00000 .0<00$

$7,0+0020.00000 .0000$ j, $00000,00000,0000,0000$

$1.000030 .00000 .0000 \quad 0.00000 .00000 .00000 .0000$ BETA

ALPHA $1.000 \quad 2.000 \quad 3.000 \quad 5.000 \quad 7.000 \quad 10.00 \quad 20.00 \quad 30.00 \quad 50.00 \quad 70.00 \quad 100.0 \quad 200.0 \quad 300.0 \quad 500.0 \quad 700.0 \quad 1000$

$1.0-0030.90900 .99951 .00001 .00001 .00001 .00001 .00001 .00001 .00001 .00001 .00001 .00001 .00001 .00001 .00001 .0000$

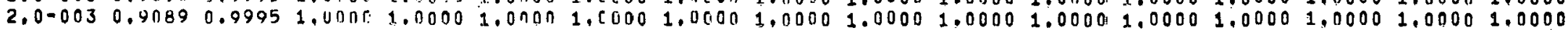
$3.0-0030.00890 .09951 .00001 .00001 .00001 .00001 .00001 .00001 .00001 .00001 .00001 .00001 .00001 .00001 .00001 .0000$ $5.0=0030.90870 .09951 .00001 .00001 .00001 .00001 .00001 .00001 .00001 .00001 .00001 .00001 .00001 .00001 .00001 .0000$

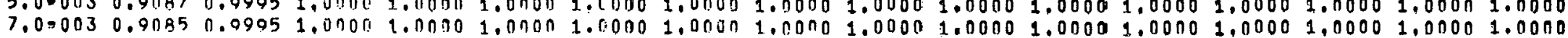

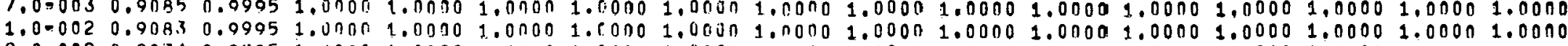
2.0-002 0.9074 0.9995 1.00001.00001.000n 1.00001.00001.00001.00001.00001.00001.00001.00001.0000 $3.0=0020,90650.99051,00001,00001.00001 .00001 .00001 .00001 .00001,00001.00001 .00001,0000$

$5.0=0020.90480 .99951 .00001 .00301 .000 n 1.00001 .00001 .00001 .00001 .00001 .00001 .0000$

7.0-002 $0.90290 .09951 .0 n n \pi 1.00901 .00001 .00001 .00001 .00001 .00001 .00001 .0000$

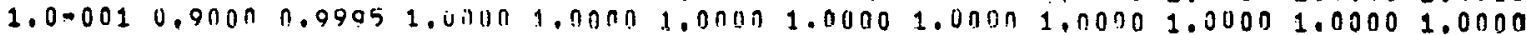

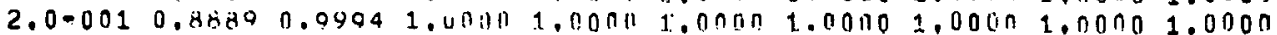

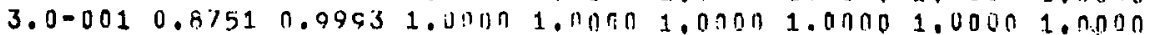

$5.0-0010.8355 \quad 0.99901 .0110109 .0060$ 1.0non 1.00001 .0000

7.0-001 0.7727 0.9986 1. iinun 1.011000 1.0non 1.0000

$1.0+000 \quad 0.6250 \quad 0.0971$ j.9090 $1.01000: .00001 .0000$

$2.0+0000.137 n \quad 0.07 r 1 \quad 0.94931 .0 n n \pi$

$3.0+0000.122 h 10.045010 .4050$

$5.0+000 \quad 0.11024 \quad 0.34 .8$

7. $0+0 \cap 0 \quad 0$. i) $\cap 05$

$1.0+0010.0)(; 011$ 
APPENDIX B

Extraction Factor $\alpha$ as a Function of $\mathrm{f}$, Scrub

Factor $\beta$, and Numbers of Stages.n. and $n^{\prime}$ 


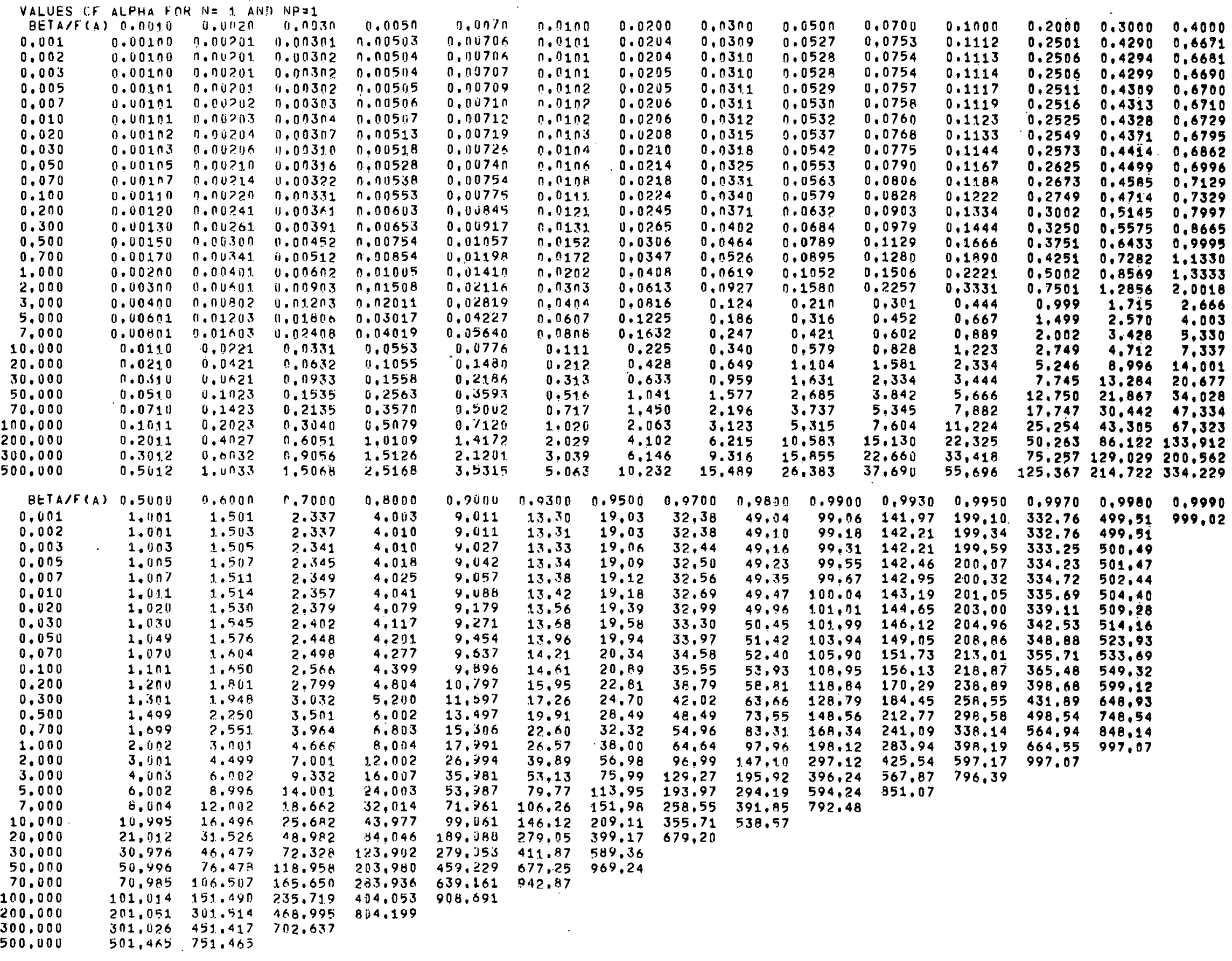


VALUES OF ALPHA FOR $N=1$ AND NP=2 PHA FOR N= 1 AND NP02 0.001 0.0050 $0.00100 \quad 0.002010 .003020 .00504$ $0.00100 \quad 0.002010 .00302 \quad 0.00504$ $0.001110 .002010 .00302 \quad 0.00505$ $0.001010 .00202 \quad 0.00303 \quad 0.00506$ $\begin{array}{lllll}0.001 C 1 & 0.00203 & 0.00304 & 0.00507\end{array}$ $\begin{array}{lllll}0.00182 & 0.00204 & 0.00307 & 0.00513\end{array}$ $\begin{array}{lllll}0.00103 & 0.00207 & 0.00310 & 0.00518\end{array}$ $\begin{array}{llll}0.00105 & 0.00211 & 0.00317 & 0.00529\end{array}$ $0.00108 \quad 0.00216 \quad 0.003240 .00540$ 0.001110 .002230 .003340 .00543 0.001390 .002790 .004180 .00628 0.001390 .003510 .0051870 .00680 0.001750 .003510 .005270 .00880 0.002190 .004390 .006590 .01100 0.003500 .006010 .009030 .01508 0.00700 n.01403 n.021n 0.03518 $0.013 n 10.02604 \quad 0.039110 .06531$ 0.031110 .062090 .093320 .15585 $\begin{array}{lllll}0.05702 & 0.11418 & 0.17158 & 0.28638\end{array}$ $\begin{array}{llll}0.1111 & 0.2224 & 0.3341 & 0.5575\end{array}$ $\begin{array}{llll}0.4213 & 0.8436 & 1.2665 & 2.1162\end{array}$ $\begin{array}{rrrr}0.9323 & 1.8654 & 2.81129 & 4.6816 \\ 2.5549 & 5.1089 & 7.6685 & 12.8260\end{array}$ $\begin{array}{rrrr}2.5549 & 5.1089 & 7.668 .5 & 12.8260 \\ 4.9792 & 9.9573 & 14.9622 & 24.9796\end{array}$ $10.1099 \quad 20.2494 \quad 30.3812 \quad 50.7517$

$40.2536 \quad 80.50631 .20 .9115 \quad 202.0272$ $90.3940181 .0311271 .7292453 .8580 \quad 636.2308 \quad 912.598$

BETA/F(A)
0.001
0.002
0.003
0.005
0.007
0.010
0.020
0.030
0.050
0.070
0.100
0.200
0.300
0.500
0.700
1.000
2.000
3.000
5.000
7.000
10.000$$
0.5000 \quad 0.6000 \quad 0.7000
$$

1,6000

7000

0.8000

0.8000
4.003

4.010
4.010

4.010
4.018

4.025

$1,007 \quad 1.511 \quad 2.34$

$\begin{array}{lll}1.011 & 1.514 & 2.357\end{array}$

$\begin{array}{lll}1.020 & 1.530 & 2.379\end{array}$

$1.030 \quad 1.547$

1.053

1.547
1.579

1.612

2.406

4,079

4,125

2.456
2.509

2.589

1.110

1.240
1.390

1.749

2.189

3.001

$1,8.60$

2.086

2.894

3.242
4.087

4.087
7.109

4.28

1.6 .343
30.320

$\begin{array}{lll}12.976 & 46.479 & 72.328\end{array}$

$\begin{array}{rrr}111.024 & 166.383 & 259.034\end{array}$

$421.143 \quad 631.348 \quad 961.445$

4,300

4.437

4.956
5.559

7.001

8,767
$12,0 \cup 2$

28,001

$\begin{array}{ll}51.972 & 117.00 \\ 23.902 & 279.05\end{array}$

9,011

9.011

9.042

9.057
9.088

9.089

9.27

9.469

9.988

11.163

12.506

15,163

19.100

63.050

117.005
279.053

$\begin{array}{rr}144.092 & 999.023\end{array}$

13.3

13.31

13.36

13.38

13.42
13.56

13.70
13.90

13.99
14.28

14.75

16.47
18.48

18.48
23.24

$23.24 \quad 33.23$

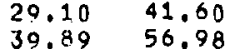

$9 ? .96 \quad 1.32 .94$

$172.73 \quad 246.83$

0.0204
0.0204

0.0310

0.0527

0.0754

0.0528

0.0205

0.0206
0.0206

0.0311

0.0529

$0.0311 \quad 0.053$ n

0.03150 .0537

0.0543

0.0210

0.0319

0.0554

0,0219

0.0326

0.0566

0.03430 .0584

0.0253

0.0383

0.0653

0.0732
0.0921.

$\begin{array}{ll}0.0541 & 0.0921 \\ 0.0677 & 0.1153\end{array}$

0.0677

$0.158 \mathrm{n}$

0.3684

0.1430

0.402

0.684

0.6328

0.959

$1.762 \quad 3.001$
3.432

5.841

22.1 .72
48.982

13.024

18.998

52.033

18.994
$.01 .502 \quad 153.987$

34.156

153.687

261.475
531.739

0,0754

0,0757

0,0760

0.0768

0,0776

0,0703

0.0835

0.0933

0.1318

0.1318

0.2257

0.5269

0.979

2.334

4.293
8.355

31.709

70.1310
192.017

192.017
374.268

0.1112

0.1113

0.1114

0.1119

0.1123

0.1133

0.1160

0.1194

0.1234

0.1377

0.1945

0.2435

0.7778

1.444

3,444

12,338

46.784

103,455
283,448

552.247

$0.2000 \quad 0.3000 \quad 0.4000$

$0.2501 \quad 0.4290 \quad 0.6671$

$0.2506 \quad 0.42940 .6681$

$\begin{array}{llll}0.2506 & 0.4299 & 0.6690\end{array}$

$0.25110 .4309 \quad 0.6700$

$0.25250 .4328 \quad 0.6738$

$0.25490 .4375 \quad 0.6805$

$\begin{array}{llll}0.2578 & 0.4418 & 0.6872\end{array}$

$0.2630 \quad 0.4509 \quad 0.7015$

$\begin{array}{lll}0.2687 & 0.4609 & 0.7167\end{array}$

0.31020 .47570 .7396

0.34740 .59500 .8264

$0.43750 .7501 \quad 1.9673$

0.54790 .03891 .1673

$0.7501 \quad 1.2856 \quad 2.0018$

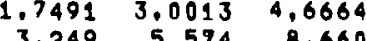

$3.249 \quad 5.594 \quad 8.660$

$\begin{array}{rrr}7.745 & 13.284 & 20.677 \\ 14.245 & 24.430 & 37.995\end{array}$ $27.757 \quad 47.578 \quad 74.037$ $105,287180.543280 .518$
232.769399 .171620 .606 638,184

0.000

931.152

170.000

100.000

200.000

500,000 

VALUES DF ALPHA FOR $N=1$ AND NP $=3$ 0 B 0.00 0.002

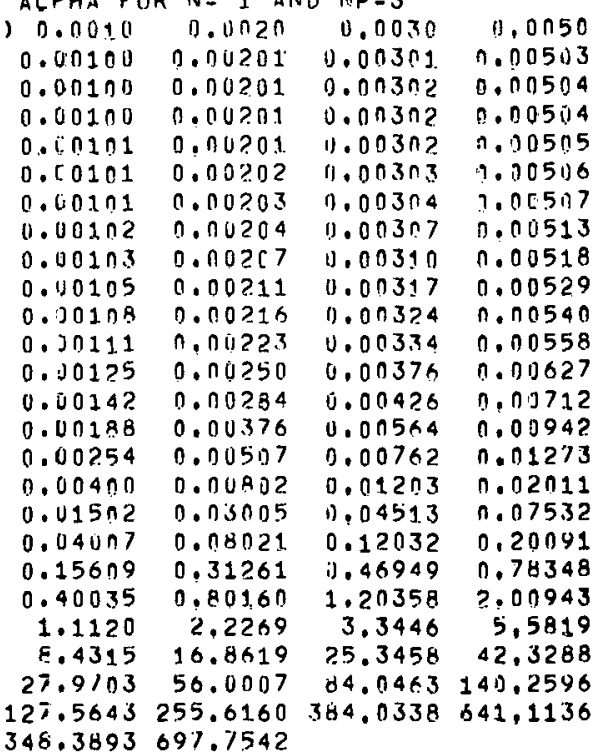
0.0079 0.0070 0.00705 0.00707 0.00710 0. no71n $0.11071 ?$ 0.071 . $0.1007<6$ $0.1007<2$ $0.007=7$ 0.0078 0,00860 0.00909 $0.0132 ?$ 0.01785 $0.023=0$ $0.1457=$ $0.24 ? 35$ 1.1005 2, B1B15 7.8352 59.3576 195.9002
898,9250$$
1.000
$$
10.000
20.000 30.000 50.000

\section{n. 1100} 0.0101 0.0101 $0.01 n$ r.oina n. n. $n .01 n$ n. 0106 90109 ก. 0112 n.n1? 0.0143 0.0100 0.0404 0.2516 0.4042 1.5755 11.224 0.0200 $0.02 \mathrm{j} 4$ 0.0204 0.0205 0.0205 0.0256 0.0206 0.0210 0.0215 0.0219 0.0255 0.0239 0.0383 0.0517 0.0816 0.3059 0.8159 70.000 100.000 200.000 500.000

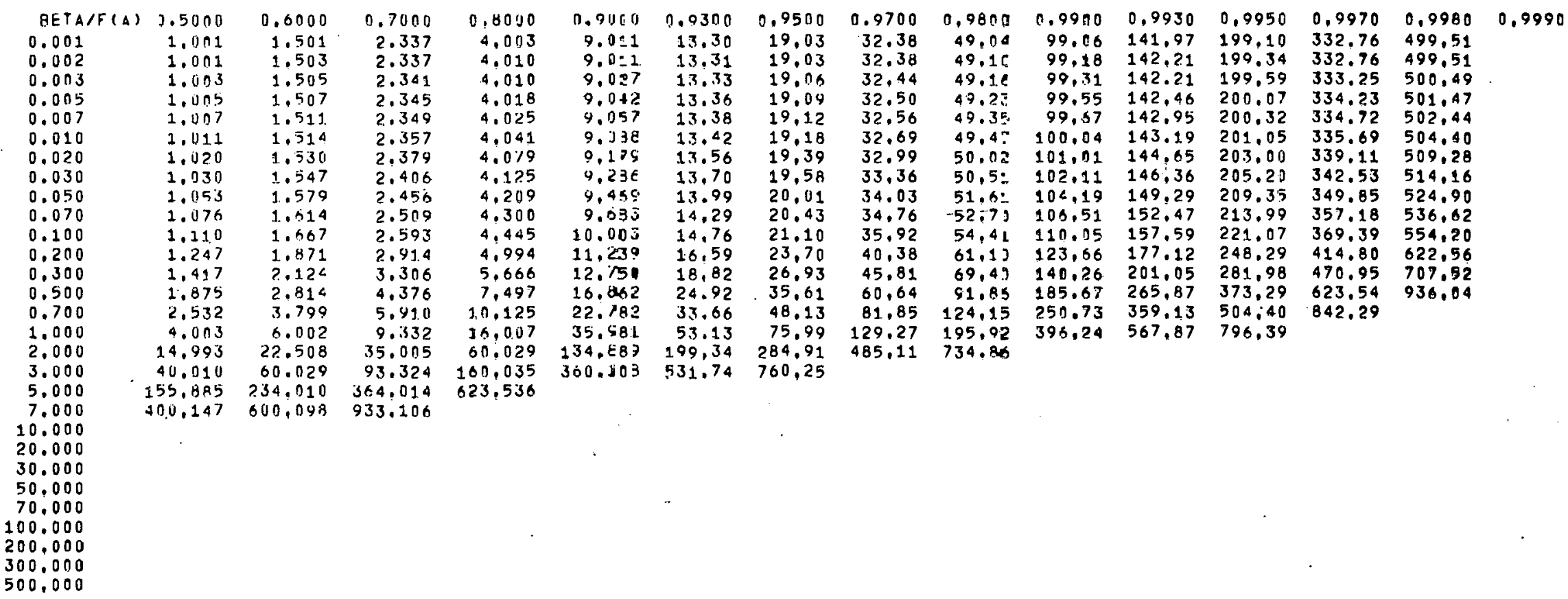
$0.0309 \quad 0.0527 \quad 0.0753 \quad 0.11120 .2501 \quad 0.4290 \quad 0.0671$ $0.0310 \quad 0.0528 \quad 0.0754 \quad 0.11140 .25060 .42900 .0690$ $\begin{array}{lllllll}0.0311 & 0.3529 & 0.0757 & 0.1117 & 0.2511 & 0.4309 & 0.6700\end{array}$ $\begin{array}{lllllll}0.0311 & 0.253 n & 0.0758 & 0.1119 & 0.2516 & 0.4318 & 0.6710\end{array}$

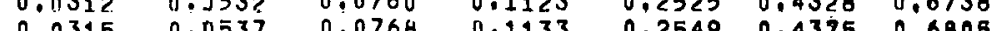
$\begin{array}{lllllll}0.0319 & 0.0543 & 0.0 .776 & 0.1145 & 0.2578 & 0.4418 & 0.6872\end{array}$ $\begin{array}{lllllll}0.0326 & 0.0554 & 0.0793 & 0.1169 & 0.2630 & 0.4509 & 0.7015\end{array}$ $0.03860 .0 .585 \quad 0.08370 .12340 .27780 .47620 .7406$ 0.114380 .07460 .10670 .13870 .31210 .53460 .8321 $\begin{array}{lllllll}0.0783 & 0.0987 & 0.1411 & 0.21783 & 0.4685 & 0.8035 & 1.2494\end{array}$ $0.0783 \quad 0.1332 \quad 0.1907 \quad 0.2816 \quad 0.6328 \quad 1.0853 .1 .6880$ $\begin{array}{lllllll}0.1237 & 0.6104 & 0.3012 & 0.4442 & 0.9995 & 1.7147 & 2.6656\end{array}$ $\begin{array}{rrrrrrr}0.4638 & 0.7892 & 1,1292 & 1.6671 & 3.7489 & 6.4288 & 10.0031 \\ 1.238 & 2.1 .05 & 3.009 & 4.445 & 10.003 & 17.137 & 26.058\end{array}$ $\begin{array}{lllllll}4.827 & .218 & 11.743 & 17.320 & 38.972 & 66.834 & 103.944\end{array}$ $12.36 \mathrm{~A} \quad 2: .043 \quad 30.107 \quad 44.465 \quad 100.038 \quad 171.510266 .846$ $\begin{array}{llllll}34.333 & 53.503 & 83.680 & 123.414 & 277.589 & 476.319 \\ 740.723\end{array}$ 260.499
863.770 $0.0310 \quad 0.0528 \quad 0.0754 \quad 0.1113 \quad 0.2506 \quad 0.42940 .6671$ 
VALUES OF $A L P H A$ FOR $N=1$ AND MP $=4$ BETA/F(A) 0.0010 0.0020 0.0030

$0.00100 .0020 \quad 0.003$

$0.001000 .00201 \quad 0.003010 .30503$

$0.003-0.00100 \quad 0.002010 .003020 .0504$

0.0050 .001010 .002010 .003020 .00505

0.0070 .00101 .0 .002020 .003030 .00506

0.0100 .002010 .002030 .003040 .00507

$\begin{array}{lllll}0.020 & 0.00102 & 0.00204 & 0.00307 & 0.00513\end{array}$

$0.030 \quad 0.00103 \quad 0.00207 \quad 0.00310 \quad 0.00518$

$\begin{array}{llllll}0.050 & 0.00105 & 0.00211 & 0.00317 & 0.00529\end{array}$

0.1000 .001110 .002230 .003340 .00558

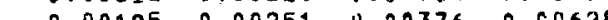

0.001250 .002510 .003760 .00628

$0.500 \quad 0.001940 .003880 .005830 .00973$

0,700

1,000

2,000

3.000

5,000

7.000

10.000

20,000

30.000

50,000
70.000

70,000
100,000

100,000

200.000

500,000

BETA/F
0,001
0,002
0,003
0,005
0,007
0,010
0,020
0,030
0,050
0,070
0,100
0,200
0,300
0,500
0,700
1,000
2,000
3,000
5,000
7,000
10,000
20,000
30,000
50,000
70,000
100,000
200,000
300,000
500,000
500
$0.00501 \quad 0.01002 \quad 0.015050 .02512$ $\begin{array}{llll}0.03111 & 0.06209 & 0.09332 & 0.15585\end{array}$ $\begin{array}{llll}0.12104 & 0.24251 & 0.36411 & 0.60801\end{array}$ $\begin{array}{rrrr}0.88158 & 1.56597 & 2.34894 & 3.92631 \\ 2.80289 & 5.61241 & 8.43147 & 14.07721\end{array}$ $\begin{array}{llll}11.1170 & 22.2635 & 33.4177 & 55.8176\end{array}$ $168.5799337 .6471506 .3481 \quad 846.1916$ 0.00706 0.00707 0.00709 0.0471 0.00712 0.00712 0.00726 0.00742 0.00783 0.00783 0.00881 0.01005 0.01365 0.01955 0.03524 0.21855 5.50560 5.50560
19.73059 19.73059
78.3090 $78.3090-112.3000$ $112.245 \quad 226.605$

0.0200
0.0204
0.0204
0.0205
0.0205
0.0206
0.0206
0.0208
0.0210
0.0215
0.0219
0.0227
0.0255
0.0291
0.0395
0.0566
0.1020
0.6328
2.4710
5.9464
7.1604
226.645

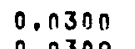
$0.03 n 9$ 0.0310 0.0311 0.0311 0.0311 0.031 .5 0.0319

\subsection{6} 0.0333
0.0343 0.0343 0.0386 0.0599 0.0858 0.1547 0,0594
3.741 $24.156 \quad 41.108$ $343.507 \quad 584.473$

0.0500
0.0527
0.0528
0.0528
0.0529
0.0530
0.0532
0.0537
0.0543
0.0554
0.0566
0.0585
0.0658
0.0750
0.1020
0.1458
0.2630
1.6308
6.368
41.108
1.47 .340
584.473

0,0700 0.0754 0.0754 0.0757 0.0758 0.0760 0.0768 0.0776 0,0793 0.0809 0.0940 0.0940 0.1075 0.1458 0.2088 0.3765 2.3337 58.808 210.816
836.426

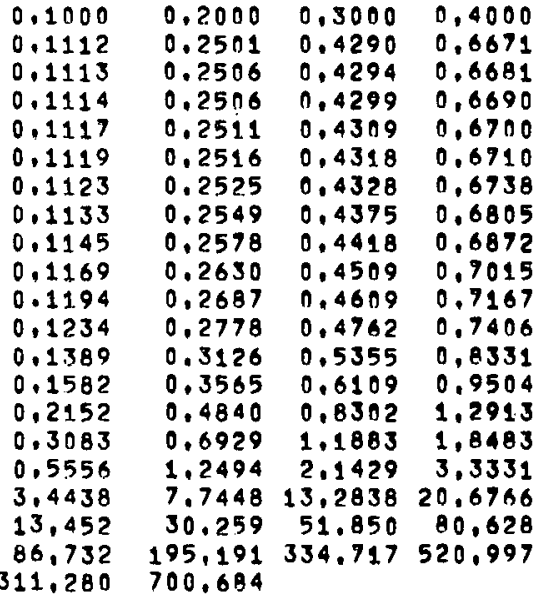

00.684 
VALUES CIF ALPHA FOR $N=1$ AND NP $=5$ BETALF (A) ODOURO 0.00200 .0030

0.002

0.003

0.005

0.007

0,01

0.020

0.030

0.050

0.070

0.100

0.200
0.300

0,500

0.700

1.000
2,000

2,000
3.000

3.000
5.000

7,000

10.000

20.000

30,000
50,000

50,000
70,000

00,00

200,000

300,000

500,000

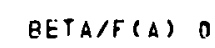

BETA

0.001

0.002

0,003

0,005

0.0010
0.020

0.030

0.030

0.050

0.100

0.100

0.500

1.000

3.000

5.000

10,000

0,000

30.000

50.000
70.000

70.000

100,000
200,000

200.000

300.000
500.000
0.5000

0.6000

1.001

1.005

1.007

1.011

1.030

1.053

1.112
1.249

1.427

1.96

6,002

364.014

$\begin{array}{ll}1.503 & 2.337 \\ 1.537\end{array}$

1,505

$1, \equiv 07$

1,514

1.547

1.579

1.614

1.667

2.143

2.952

4.415

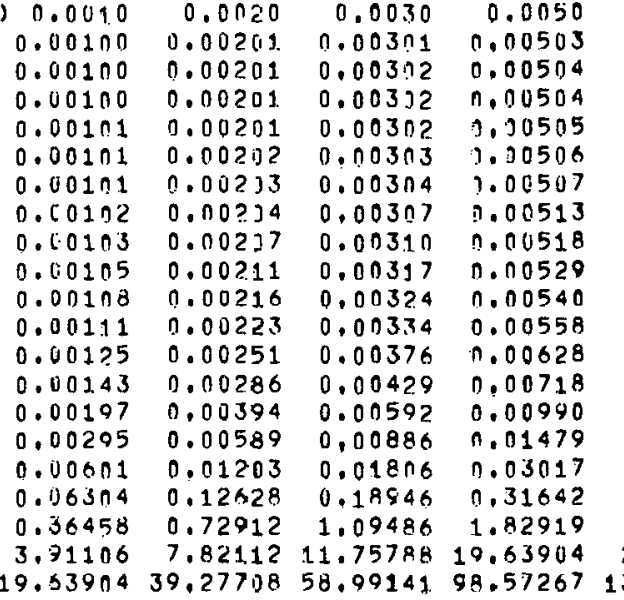

0,0072 0.0070 .9 0.00712 0.00719 0.00719 0,00726 0.0200 $\begin{array}{rrrrr}19.53904 & 39.27708 & 58.99141 .98 .57267 & 138,30652 \\ 111.2680 & 222.7791 & 334.2292 & 556,1059 & 783,6096\end{array}$

\section{0,8000 4,003 4,010 \\ 4.018 \\ 4,025 \\ 4,041 \\ 4,079 \\ 4,125
4.209 \\ 4,300 \\ 4,300
4,445 \\ 5,002 \\ 5,712 \\ 7.882
11.758}

24.003

$53.987 \quad 79,77$

$0,900000,9300$

$0.9500 \quad 0.9700 \quad 0.9$

$\begin{array}{lllllll}0.9800 & 0.9900 & 0.9930 & 0.9950 & 0.9990 & 0.9980\end{array}$

$\begin{array}{llllllll}32.38 & 49.04 & 99,06 & 141,97 & 199,10 & 332.76 & 499,51\end{array}$

$\begin{array}{llllllll}19.09 & 32,50 & 49.23 & 99.55 & 142,46 & 200.07 & 334.23 & 501.47\end{array}$

$\begin{array}{llllllll}19.12 & 32.56 & 49.35 & 99.67 & 142,95 & 200.32 & 334.72 & 502.44\end{array}$

$\begin{array}{llllllll}19.18 & 32.69 & 49.47 & 100.04 & 143,19 & 201.05 & 335.69 & 504.40\end{array}$

$19.39 \quad 32.99 \quad 50.02 \quad 101.01 \quad 144.65 \quad 203.00 \quad 339.11 \quad 509.28$

$\begin{array}{lllllllll}19,58 & 33,36 & 50,51 & 102,11 & 146,36 & 205,20 & 342,53 & 514.10\end{array}$

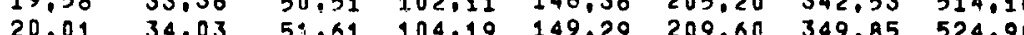

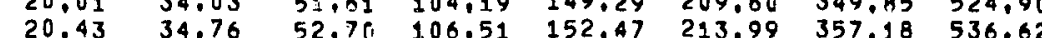

$21.10 \quad 35,92 \quad 54.47 \quad 110.05 \quad 157,59 \quad 221.07 \quad 369.30 \quad 554.20$

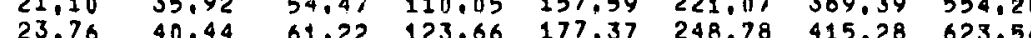

$23,76 \quad 40.44 \quad 61.22 \quad 123.66$ 177.37 248,78 415.28 623.54

$\begin{array}{llllllll}27.12 & 46.17 & 70.01 & 141.24 & 202.52 & 283.94 & 474.37 & 712.40 \\ 37.38 & 63,66 & 96,50 & 194,95 & 279.05 & 391.85 & 653.81 & 981.45\end{array}$

$\begin{array}{rrrrrrr}37.38 & 63.66 & 96.50 & 194,95 & 279.05 & 391.85 & 653.81 \\ 55.88 & 95.15 & 144.1 .7 & 291.26 & 417.24 & 585.45 & 977.54\end{array}$

0.683

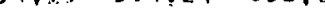
0,00705 0.00705 0.00705 0.00709 $0,007 E 7$ $0.007 E 7$ $0.00 E{ }_{1}$ 0.00881 $0.013 E 8$ 0.02074 0,04227 0.44421 2.56638 0.0100 0.0101 0.0204 $.01 n$ 0.0204 0.0205 0.01020 .0205 $0.0102 \quad 0.0206$ 0.04020 .0206 0.0208 0.0210 0.0215 0.0219 0.0255 0.0255 0.0299 0.0601 0.1225 1.2856 7.4282
0.0300 $0.03 n 9$ 0.0310 0.0310 0.0311 0.0312 0.0315 0.0319 0.0326 0.0333 0.0343 0.0442 0.0609 0.0909 0.1857 1.9484
11.254 11.254 $\begin{array}{rrr}39.4602 & 79.7739 & 120.789 \\ 98.1209 & 400.1471 & 605.957\end{array}$
0.0500 0.0528 0.0528 0.0529 0.0530 0.0532 0.0543 0.0554 0.056 $0.056 \mathrm{~h}$ 0.0658 0.0751 0.1036 0.1549 3,3141 19.151 205,689
0.0700 0.0754 0.0754 0.0757 0.0758 0,0760 0,0768 0,0776 0,0793 0,0809 0.0837 0.094 0.1074 0,1482 0.4518 27.390 7.0010
40.437 0.1000 0.1112 0.1114 0.1117 0.1119 0.1133 0.1145 0.1194 0.1234 0.2588 0.3269 $\begin{array}{llllllll}19,06 & 32.44 & 49.16 & 99,18 & 142.21 & 199.34 & 332.76 & 499.51\end{array}$

$0.3000 \quad 0.4000$

0.3000
$0.4204 \quad 0.6671$

0.53550 .7406

$1.0400 \quad 1.3123$

$2,5702 \quad 4,0026$

0.9990 


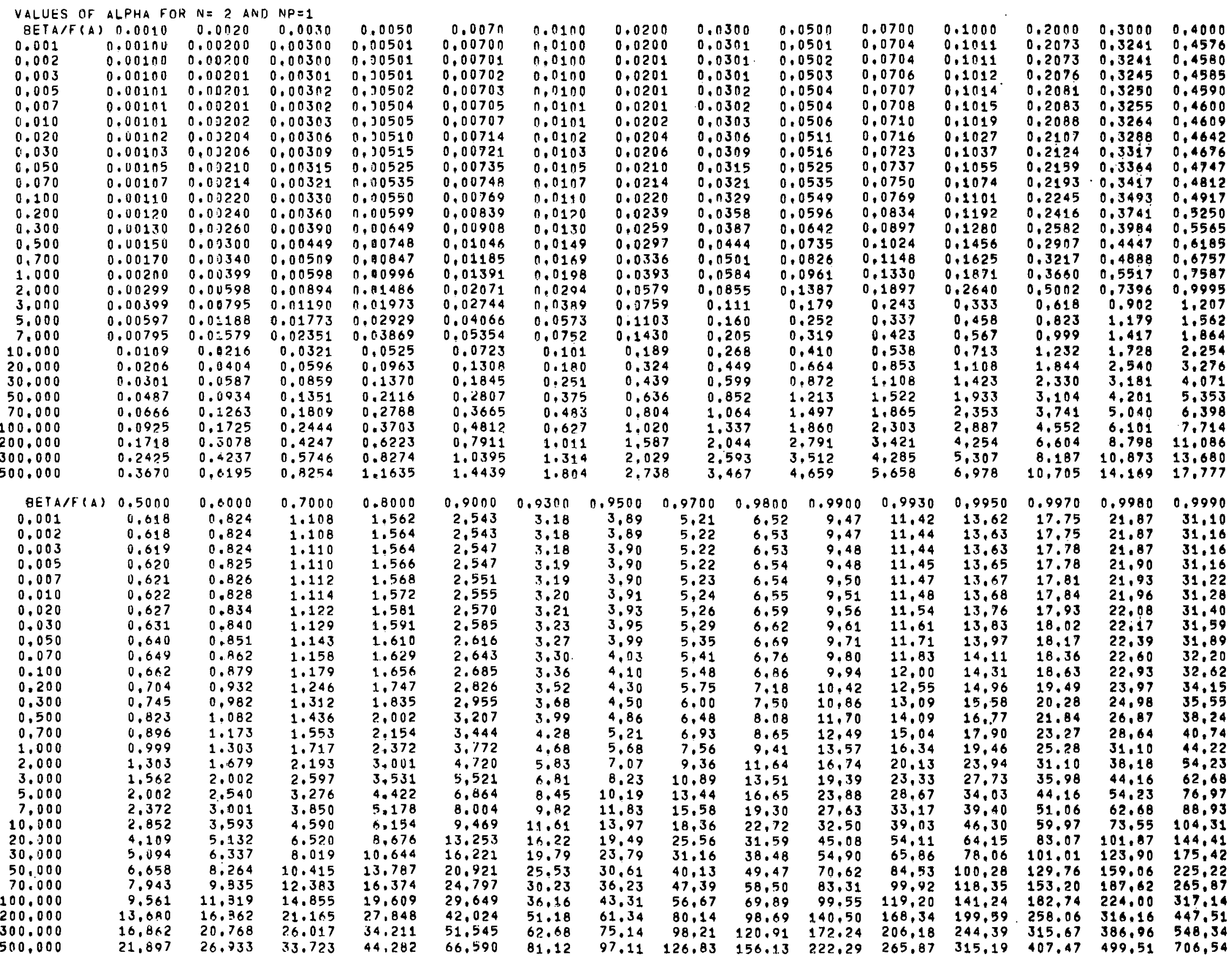


VALUES OF ALPHA FOR $N=2$ AND NP $=2$

DETA/F(A) 0.0010 0,0020 0.0030

$0.001 \quad 0.00100 \quad 0.00200 \quad 0.00300 \quad 0.00501$

$0.002 \quad 0.00100 \quad 0.00200 \quad 0.00300 \quad 0.00501$

$0.003 \quad 0.00100 \quad 0.00201 \quad 0.00301 \quad 0.00501$

$\begin{array}{llllll}0.005 & 0.00101 & 0.00201 & 0.00302 & 0.00502\end{array}$

$\begin{array}{lllll}0.007 & 0.00101 & 0.00201 & 0.00332 & 0.00504\end{array}$

$0.020 \quad 0.03102 \quad 0.00204 \quad 0.00306 \quad 0.00510$

$0.030 \quad 0.03103 \quad 0.00206 \quad 0.003090 .00516$

$0.050 \quad 0.00105 \quad 0.00210 \quad 0.003160 .00526$

$0.100 \quad 0.00111 \quad 0.00 ? 2$ ? 0.003330 .00554

$\begin{array}{lllll}0.200 & 0.00124 & 0.00248 & 0.00372 & 0.00619\end{array}$

$0.300 \quad 0.00139 \quad 0.00278 \quad 0.00417 \quad 0.00694$

$\begin{array}{llllll}0.500 & 0.00175 & 0.00350 & 0.00524 & 0.00372\end{array}$

0,700

1.000

2,000

3.000

7,000

20,000

30,000

50.000

70,000

100,000

200,000

300.000

500.000

BETA/FCA
0,001
0,002
0,003
0,005
0,007
0,010
0,020
0,030
0.050
0.070
0,100
0,200
0.300
0,500
0,700
1.000
2,000
3,000
5,000
7,000
10,000
20,000
30,000
50,000
70,000
100,000
200,000
300,000
500,000

$\begin{array}{llll}0.00219 & 0.00437 & 0.00655 & 0.001088\end{array}$

$\begin{array}{llll}0.00299 & 0.00598 & 0.00894 & 0.01486 \\ 0.00696 & 0.01383 & 0.02065 & 0.03405\end{array}$

$\begin{array}{llll}0.00696 & 0.01383 & 0.02065 & 0.03405 \\ 0.01285 & 0.02542 & 0.03768 & 0.06149\end{array}$

0.012850 .0254 ? 0.03708 1..06149

$0.0301130 .05866 \quad 0.08503 \quad 0.13701$

$\begin{array}{llll}0.1010 & 0.1873 & 0.2640 & 0.3989\end{array}$

$\begin{array}{llll}0.3193 & 0.5460 & 0.7320 & 1.0376\end{array}$

$\begin{array}{llll}0.5870 & 0.9537 & 1.2474 & 1.7205 \\ 1.1750 & 1.8158 & 2.3146 & 3.1157\end{array}$

$\begin{array}{llll}1.1750 & 1.8158 & 2.3146 & 3.115 \\ 1.7853 & 2.0961 & 3.3980 & 4.521\end{array}$

$2.7190 \quad 4.0255 \quad 5.0326 \quad 6.6424$

$\begin{array}{llll}5.8642 & 8.4925 & 10.5067 & 13.7263\end{array}$

$\begin{array}{rrrr}9.0266 & 12.9633 & 15.9769 & 20.798 \\ 15.3361 & 21.8973 & 26.9632 & 35.0046\end{array}$

$\begin{array}{llll}0.5000 & 0.6000 & 0.7090 & 0.8000\end{array}$

$\begin{array}{llll}0.618 & 0.824 & 1.108 & 1.562\end{array}$

$\begin{array}{llll}6.618 & 0.824 & 1.108 & 1.564\end{array}$

$\begin{array}{llll}0.619 & 0.824 & 1.110 & 1.564\end{array}$

$\begin{array}{llll}0.620 & 0.825 & 1.110 & 1.566 \\ 0.621 & 0.826 & 1.112 & 1.568\end{array}$

$\begin{array}{llll}0.622 & 0.828 & 1.114 & 1.572\end{array}$

$\begin{array}{llll}0.627 & 0.834 & 1.122 & 1.581\end{array}$

$0.632 \quad 0.841 \quad \$ .129 \quad 1.591$

$\begin{array}{llll}0.641 & 0.852 & 1.144 & 1.612 \\ 0.051 & 1.865 & 1.162 & 1.633\end{array}$

$\begin{array}{llll}0.051 & 0.865 & 1.162 & 1.633 \\ 0.066 & 0.884 & 1.185 & 1.665\end{array}$

0.781

$0.914 \quad 1.196 \quad 1.370 \quad 1.910$

$\begin{array}{llll}0.914 & 1.196 & 1.581 & 2.193 \\ 1.062 & 1.381 & 1.816 & 2.502\end{array}$

$1.062 \quad 1.381$

$1.313 \quad 1.679$

2.193

3.139

7.070

10,049
20.036

2.780

3.942
6.337

8.767

12.414

24.644
36.897

30.01 .5

50.019

(6.

$100.038 \quad 122.682 \quad 152 . \bar{c}$

$200.074 \quad 244.874 \quad 305.909$

$\begin{array}{lll}300.050 & 367.432 & 458.741 \\ 500.489 & 612.793 & 764.160\end{array}$
0,0070

$0.00700 \quad 0.0100$

$0,00701 \quad 0.010$

$0.00702 \quad 0.0100$

0.007050 .0100

$0.00707 \quad 0,0101$

0,00715

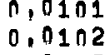

0.0103

0.0105
0.0107

0.00751

0,0077

0.0111

0.0124

0.00970

0,01520

0.0138

0.0217

0.0471

0.0294

$0.08450 \quad 0.1175$

$0.18446 \quad 0.2506$

0.30736

0.5165
1.2932

2.1086

0.4089

0.671
1.621

1.621
2.608
4.598

4.598
6.604

6.604
9.606

7,9584

19.670
29.710

$\begin{array}{ll}24,7355 & 29.71 .0 \\ 41,5354 & 49.775\end{array}$

$\begin{array}{ll}1.0 & 42.451 \\ 75 & 70.985\end{array}$
0.0200

0.0200

0.0201

0.0201

0.0202

0.0204

0.0206

0.0215
0.0222

0.0247

0,0276
0.0345

0,0429
0.0579

0.0579
0.1268

0.2178

0.4394

0.6891
1.085

2,475
3,888

6.734

9.591

28.153
0.0300

0.0301

0.0301

0.0301

0.0302

0.0304

0.0309

0.0315
0.0322

0.0332

0,0370

0.0413

0.0636
0.0855

0.1 .830

0.317
0.599

0.919

1.419
3.142

4.888
8.401

11.910

$\begin{array}{ll}11.167 & 22.56 \\ 34.760 & 45.50 \\ 52.339 & 68.42\end{array}$

$\begin{array}{rr}52.339 & 68.421 \\ 87.464 & 114.320\end{array}$

0.0 .537

0,0685

0.9044

$0 . \$ 387$
0.5864
0.5467

0.5864
0.467

0.467
0,872

1.303
1.967

4.231

6.520

15.672
0.0500

0,0704

$0.0503 \quad 0.0706$

$0.0504 \quad 0.0708$

$0.0506 \quad 0.0710$

$0.0511 \quad 0.0716$

$0.0526 \quad 0.0738$

$0.0615 \quad 0.0776$

0.06850 .0859

0.1442

0.1897

0.608

1.108

1.631
2.433

5.155

7,882
$+3,360$

$\div 3.360$

27.085

54.536

81.971
0.1000

0.1011

0.1012

0.1014

0.1019

0.1029

0.1038
0.1057

0.1079

0.1110
0.1227

$0.0955 \quad 0.1361$

0.11790 .1666

0.2023

0,2640
0,5136

0,802

1.423

2.067

3.047

6.360
0.683

16.343

22,996
32,990

66,346

99.671

5300
3.18

9500
3.89
3.89

7700
5.21
5.22
5.2

0.9800

3.52
6.53

0,9900

0,9930 6.383

$3.90 \quad 5.22$

5.53

$\begin{array}{ll}11.42 & 13.62\end{array}$

$\begin{array}{lll}9.47 & 11.44 & 13.63 \\ 9.48 & 11.44 & 13.63\end{array}$

$0.2000 \quad 0.3000 \quad 0.4000$

$0.2073 \quad 0.3241 \quad 0.4576$

$0.2073 \quad 0.3241 \quad 0.4580$

$0.2076 \quad 0.3245 \quad 0.4585$

$0.20810 .3250 \quad 0.4590$

0.2083 0.3255 0.4600

0.20880 .32640 .4609

0.21070 .3288 0.4647

0.21260 .33170 .4681

0.21640 .3374 0.4757

$\begin{array}{lll}0.2202 & 0.3431 & 0.4831\end{array}$

$0.2482 \quad 0.3841 \quad 0.5374$

$0.2730 \quad 0.4194 \quad 0.5851$

$0.32930 .5002 \quad 0.6900$

0.39320 .58990 .8073

$\begin{array}{lll}0.5002 & 0.7396 & 0.9995 \\ 0.9141 & 1.3028 & 1.7167\end{array}$

$\begin{array}{rrr}0.9141 & 1,3028 & 1,7167 \\ 1.371 & 1.914 & 2,486\end{array}$

$2.330 \quad 3.181 \quad 4.071$

$3.306 \quad 4.468 \quad 5.681$

$\begin{array}{rrr}4.788 & 6.113 & 8.111 \\ 9.774 & 12.948 & 16.252\end{array}$

$\begin{array}{lll}14.764 & 19.486 & 24.430\end{array}$

$\begin{array}{lll}14.766 & 32.563 & 40.742\end{array}$

$34.760 \quad 45.686 \quad 57.099$

$49.775 \quad 65.248 \quad 81.605$ $\begin{array}{lll}49.793 & 130.738 & 163.209\end{array}$ $2.543 \quad 3.18$

$2.547 \quad 3.19$

$2.551 \quad 3.19$

$2.555 \quad 3.20$

$2.585 \quad 3.21$

$\begin{array}{ll}2.585 & 3.23 \\ 2.620 & 3.27\end{array}$

$2.650 \quad 3.31$

$2.700 \quad 3.37$

$3.074 \quad 3.83$

$3.501 \quad 4.35$

$\begin{array}{ll}3.964 & 4.92 \\ 1.120 & 5.83\end{array}$

$\% .451 \quad 9.16$

$\begin{array}{ll}10.324 & 12.66 \\ 16.221 & 19.79\end{array}$

$\begin{array}{ll}16.221 & 19.79 \\ 27.141 & 27.02\end{array}$

$31.098 \quad 37.93$

$61.097 \quad 74.28$

$91.004 \quad 110.66$

3.90
$3.90 \quad 5.22$

3.54

6.54

$\begin{array}{lll}9.48 & 11.45 & 13.65 \\ 9.50 & 11.47 & 13.67\end{array}$

$3.91 \quad 5.24$

$3.96 \quad 5.29$

6.59

$9,51 \quad 11,48 \quad 13.68$

$0.62 \quad 11.54 \quad 13,76$

$\begin{array}{lll}9.62 & 11.61 & 13.83\end{array}$

4.00
4.05 5.35

4.05
$4.12 \quad 5.41$
4.38

6.70

6.77

$9.84 \quad 11.86$

13,99
14,14

$\begin{array}{llll}7.31 & 9.99 & 12.06 & 14.37\end{array}$

$\begin{array}{lll}7.78 & 11.24 & 13.56\end{array}$

$4.67 \quad 6.22$

$5.67 \quad 6.22$

$5.97 \quad 7.93$

$\begin{array}{rr}7.07 & 9.36 \\ 11.04 & 14.55\end{array}$

15.22

31.16

$32.44 \quad 42.45$

$\begin{array}{rr}45.44 \\ 88.93 & 59.4 \\ 116.15\end{array}$

$\begin{array}{rr}88.93 & 116.15 \\ 132.45 & 172.97\end{array}$ 
VALUES OF ALPHA FOR $N=$ ? AND $M P=3$

VALUES OF ALPHA FOR $N=$ ? AND $M P=3$
BETA/F (A) $0.0010 \quad 0.0020 \quad 0.0030$

0.002

0.003

0.005

0.007

0.010

0.020

0.03

0.050 .

0.070

0.100

0.200

0.300

0.500
0.700

0.700
1.000

1.000
2.000
3.000

1,000
3,000

5,000

7.000

10.000

20.000

50.000

70.000

100.000

200.000

300.000

500.000

$0.00300 \quad 0.00501$ $0.00100 \quad 0.00200 \quad 0.003000 .00501$ $\begin{array}{llll}0.00100 & 0.00201 & 0.00301 & 0.00501\end{array}$ $0.00101 \quad 0.00201 \quad 0.00302 \quad 0.00502$ $0.00101 \quad 0.002010 .00302 \quad 0.00504$ $0.001010 .002020 .00303 \quad 0.005105$ $0.001020 .042040 .00306 \quad 0.00510$ $0.00103 \quad 0.00206 \quad 0.003090 .00516$ $0.00105 \quad 0.00210 \quad 0.003160 .00526$ $0.00108 \quad 0.00215 \quad 11.003270 .00537$ 0.001110 .00222 u.00333 0.00555 $\begin{array}{llll}0.00125 & 0.00249 & 0.00374 & 0.00623\end{array}$ $0.00142 \quad 0.00283 \quad 0.00425 \quad 0.00707$ $0.00187 \quad 0.003740 .005610 .0093$ 0.003990 .00795 u.01190 0.01973 $\begin{array}{llll}0.01480 & 0.02920 & 0.04328 & 0.07043\end{array}$ $\begin{array}{llll}0.13858 & 0.07461 & 0.10858 & 0.17158\end{array}$ $\begin{array}{llll}0.13725 & 0.25014 & 0.34837 & 0.51646\end{array}$ $\begin{array}{llll}0.30641 & 0.52504 & 0.70528 & 1.00331\end{array}$ $0.6671 \quad 1.0739 \quad 1.3962 \quad 1.9141$ $2.4443 \quad 3.0383 \quad 4.5595 \quad 0.0244$ $4.8113 \quad 7.0010 \quad 8.6756 \quad 11.3612$ $10.8118 \quad 15.4687 \quad 19.0897 \quad ? 4.8270$ $\begin{array}{llll}18.1742 & 25.9257 & 31.8918 & 41.2912\end{array}$ $31.2895 \quad 44.4651$ 54.6579 71.,7007 $89.1733 \quad 126.3436 \quad 155.15 ? 2 \quad 200.5623$ $353.7604500 .4888613 .760979 \% .4807$

$0.007 n$ 0.00700 0.00701 0.00703 0.00705 0.00707 0,0071 0.00721 0.00737 0.0075 0.00777 0.00872 0.0099 0.01304 0175 0.112744 0.09642 0.22928 0.60141 1.25126

2.3413
7.2222 13.543 ? ?.9. 4902 49.0427 83.9243
237.0717 237.0717
436.2799 938.9649

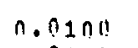
$0.010 \mathrm{c}$ 0.0100 0.0100 0.0101 0.010 0.0102 0.0103 0.0107 0.0111 0.0124 0.0186 . .0250 n.13.37 ก..3088 0.8512 2.897 8.737 16.313
35.371 58.808 $100.5 ? 6$ 284.425 $72.950 \quad 742.676$

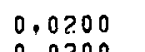
0.0201 0.0201 0.0201 0.0202 0.0202 0.0210 0.0215 0.0222 0.0249
0.0281 0.0369 0.0492 0.2459 0.5327 1.3524 2,4023 12.612 23.39 50.508 103.211 176.148 914.551

0.0300 0.0301 0.0301 0.0302 $0.03 n 4$ 0.0306 0.0315 0.0322 0.0372 0.0420 0.0731 0.1113 0.3450
0.720 1.753 3.051 .641

$0.9000 \quad 0.9300$ $\begin{array}{ll}2.543 & 3.18 \\ 2.543 & 3.18\end{array}$

2.54

2.54

2.551

2.555

2.570

2.620

2.650

2.700

2.887

3.108

3.104
3.638

3.638
4.300

4.500
5.521

11.132

11.132

$3.406 \quad 4.770 \quad 5.437 \quad 7.200$

$12.002 \quad 14.810 \quad 18.571 \quad 24.491$

$19.017 \quad 24.003 \quad 30.046$

$32.807 \quad 40.315 \quad 50.447 \quad 39.521$

$91.248 \quad 111.878 \quad 139.771 \quad 102.984$

$\begin{array}{llll}160.627 & 204.724 & 254.639 & 333.741\end{array}$

$59.480 \quad 75.01$

50,000

70,000

100.000

$\begin{array}{llll}356.090 & 436.768 & 545.411 & 713.379\end{array}$

$\begin{array}{lllllll}333.74 & 399.66 & 521.00 & 642.09 & 912.60\end{array}$

$\begin{array}{rrrr}0.9500 & 0.9700 & 0.9800 & 0.9900 \\ 3.89 & 5.21 & 6.52 & 9.47\end{array}$

5.21 6.52
6.53
6.53

6.53
6.54

3.90

3.19

3.20

3.90

3.91

3.23

3.31

3,60

3.87

4.51
5.32

4.81

3.62
.57 4,00
4.05
4,12 4,39 4.71
5.49 5.49 8.23 35,49

$53,93 \quad 70.50$

$86.73 \quad 113.22$ 5.23 5.24 5.35 5.51 5,87 30 30
5.10 13.51 26.63 6.54 6.59 6.63 6.77 7.34 9.47
9.47 9.47
9.48 9.48

9.51

9.56 9.62 9.84 10.00 10.53 13.13

15.34

19.39
38.106

3.106
62.44

$\begin{array}{ll}86.98 & 123.66 \\ 39.53 & 198.61\end{array}$ 0.0704

0.1000 $\begin{array}{llll}0.2076 & 0.3245 & 0.458\end{array}$ $\begin{array}{lllll}.0707 & 0.1014 & 0.2081 & 0.3250 & 0.4590\end{array}$ $\begin{array}{lllll}0.0710 & 0.1015 & 0.2083 & 0.3255 & 0.4600\end{array}$ $\begin{array}{lllll}0.0723 & 0.1038 & 0.2126 & 0.3317 & 0.4681\end{array}$ $\begin{array}{lllll}0.0738 & 0.1157 & 0.2164 & 0.3374 & 0.4757\end{array}$ $\begin{array}{lllll}0.0753 & 0.1079 & 0.2202 & 0.3431 & 0.4831\end{array}$ $0.0776 \quad 0.1111 \quad 0.22640 .3522 \quad 0.4955$ $0.08640 .12340 .24970 .3800 \quad 0.5403$ $0.0973 \quad 0.1382 \quad 0.27730 .42610 .5403$ $0.34790 .5260 \quad 0.7244$ $0.16370 .2290 \quad 0.43990 .6557 \quad 0.8922$ $0.2425 \quad 0.3331$ $0.6748 \quad 0.8946 \quad 1.4092 \quad 2.08572 .6999$ $2.963 \quad 3.692 \quad 5.765 \quad 7.699 \quad 9.713$ $5.010 \quad 6.185 \quad 9.515 \quad 12.597 \quad 15.713$ $8.660 \quad 10.629 \quad 16.160 \quad 21.318 \quad 26.719$ $24.674 \quad 30.107 \quad 45.38159 .602 \quad 74.403$ $45.381 \quad 55.107 \quad 85.38159 .602 \quad 74.403$ $97.474 \quad 118.592 \quad 178.101233 .277291 .260$ $161.256 \quad 196.5980 .10733 .279291 .260$ $\begin{array}{lllll}75.147 & 334.717 & 502.442 & 657.715 & 819.824\end{array}$ $.832944,82$

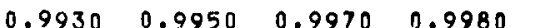
$\begin{array}{rrrrr}11.42 & 13.62 & 17.75 & 21.87 & 31.10\end{array}$ $\begin{array}{lllll}11.44 & 13.63 & 17.75 & 21.87 & 31.16\end{array}$ $\begin{array}{lllll}11.44 & 13.63 & 17.78 & 21.87 & 31.16 \\ 11.45 & 13.65 & 17.78 & 21.90 & 31.22\end{array}$ $\begin{array}{lllll}11.47 & 13.67 & 17.81 & 21.93 & 31.22\end{array}$ $\begin{array}{lllll}11.48 & 13.68 & 17.84 & 21.96 & 31.28\end{array}$ $\begin{array}{lllll}11.54 & 13.76 & 17.93 & 22.08 & 31.40\end{array}$ $\begin{array}{lllll}11.73 & 13.99 & 18.20 & 22.42 & 31.95\end{array}$ $\begin{array}{lllll}11.86 & 14.14 & 18.42 & 22.06 & 32.26 \\ 12.06 & 14.38 & 18.72 & 23.06 & 32.81\end{array}$ $\begin{array}{lllll}12.81 & 15.28 & 19.88 & 24.46 & 34.82\end{array}$ $\begin{array}{lllll}13.68 & 16.31 & 21.20 & 26.11 & 37.14\end{array}$ $\begin{array}{lllll}18.45 & 21.96 & 28.52 & 35.07 & 49.84\end{array}$ 
VALULS OF ALPHA FOR IV $=2$ AND APS $=4$ $0.050 \quad 0.001 n 5 \quad 0.00210 \quad 0.003160 .00526$ $\begin{array}{lllll}7.000 & 1.24745 & 1.92169 & 2.44431 & 3.28354\end{array}$ $\begin{array}{rrrrr}10.000 & 2.8710 & 4.2467 & 5.3072 & 6.9857 \\ 20.000 & 12.490 .3 & 17.0490 & 27.0194 & ? 8.6112\end{array}$ 30.000 50.000 70.000 100.000 200.000 300.000

500.000

\begin{tabular}{|c|c|c|c|c|c|c|c|c|c|c|c|c|c|c|c|}
\hline$B E T A / F: A)$ & $0.50 n 0$ & 0.0000 & 0.7000 & U. 0000 & $0.90 \cap u$ & 11.9300 & 0.9500 & 0.9700 & 0.9800 & $0.99 n 0$ & 0,9930 & 0.9950 & 0,9970 & 0,9980 & 0.9990 \\
\hline 0.001 & $\tilde{0.098}$ & 0.824 & 1.108 & 1.562 & 2.343 & 3.18 & 3.89 & 5.21 & 6.52 & 9.47 & 11.42 & 13.62 & 17.75 & 21.87 & 31.10 \\
\hline 0.002 & 4.018 & 0.824 & $1.1 \cap 0$ & 1.564 & 2.543 & 3.18 & 3.89 & 5.22 & 6.53 & 0.47 & 11.44 & 13.63 & 17.75 & 21.87 & $31: 16$ \\
\hline 0.003 & $1: .619$ & 0. ค2 4 & 1.110 & 1.564 & 2.547 & 3.18 & 3.90 & 5.22 & 6.53 & 9.48 & 11.44 & 13.63 & 17.78 & 21.87 & 31.16 \\
\hline 0.005 & 8.020 & 0.425 & 1.110 & 1.506 & 2.547 & 3.1 .9 & 3,90 & 5.22 & 6.54 & $9.4 \mathrm{E}$ & 11.45 & 13,65 & 17.78 & 21,90 & 31.22 \\
\hline 0.007 & $4,0>21$ & 0.826 & 1.112 & 1.568 & 2.251 & 3.19 & 3.90 & 5.23 & 6.54 & 9.50 & 11.47 & 13.67 & 17.81 & 21.93 & 31.22 \\
\hline 0.010 & $4.0 ? 2$ & 0.828 & 1.114 & 1.572 & 2.555 & 3.20 & 3.91 & 5.24 & 6.55 & 9.51 & 11.48 & 13.68 & 17.84 & 21.96 & 31.28 \\
\hline 0.020 & 0.677 & 0.434 & 1.122 & 1.581 & 2.570 & $3 . ? .1$ & 3.93 & 5.27 & 6.59 & 9.56 & 11.54 & 13.76 & 17.93 & 22.08 & 31.40 \\
\hline 0.030 & $4.63 ?$ & 0.841 & 1.129 & 1.591 & 2,285 & 3.23 & 3.96 & 5.29 & 6.53 & 9.62 & 11.61 & 13.83 & 18.02 & 22,17 & 31.59 \\
\hline 0.050 & 4.641 & ก. 25? & 1.144 & 1.612 & $2.02 \mathrm{U}$ & 3.27 & 4,00 & 5.35 & 6.70 & 9,71 & 11.73 & 13,99 & 18.20 & 22.42 & 31.95 \\
\hline 0.070 & 0.051 & 0.265 & $1.16 ?$ & 1.633 & 2.050 & 3.31 & 4.05 & 5.41 & 6.77 & 9.84 & 11.86 & 14,14 & 18.42 & 22.66 & 32.26 \\
\hline 0.100 & $(E, 6 \times)$ & ก. $* 5$ & 1.186 & 1.607 & 2.100 & 3.38 & 4.12 & 5.51 & 6.99 & 10.00 & 12.06 & 14.38 & 18.72 & 23.06 & 32.81 \\
\hline 0.200 & 4.724 & 0.058 & 1.280 & 1.791 & 2.891 & 3.60 & 4,40 & 5.88 & 7.34 & 10.63 & 12.83 & 15.28 & 19.88 & 24,46 & 34.82 \\
\hline $0.3 \cap 0$ & 0.704 & 1.045 & 1.390 & 1.941 & 3.116 & 3.8 .8 & 4.73 & 6.31 & 7.87 & 11.35 & 13.73 & 16,34 & 21.26 & 26.17 & 37.26 \\
\hline 0.500 & 0.979 & $1 . ? 76$ & 1.684 & 2.330 & 3.107 & 4.60 & 5.59 & 7.43 & 9.26 & 13.36 & 16.1 .0 & 19.15 & & 30.61 & 43,49 \\
\hline 0.700 & 1.230 & 1.500 & 2.093 & 2.868 & 1.521 & 5.59 & 6.78 & 8.98 & 11.16 & 16.07 & 19.33 & 23.00 & 29.86 & 36.71 & 52.16 \\
\hline 1.000 & 1.101 & 2.284 & 2.952 & 4.003 & 6.236 & 7.67 & 9.26 & 12.23 & 15.16 & 29.74 & 26.14 & 31.04 & 40.25 & 49.47 & 70,13 \\
\hline 2.000 & 5.1004 & 0.337 & 8.019 & 1.0 .644 & 10.221 & 19.79 & 23.79 & 31.16 & 38.48 & 54.90 & 65.86 & 78.06 & 101.01 & 123.90 & 175.42 \\
\hline 3.000 & $10,2 \cap 7$ & 12.979 & 16.313 & $21.51,1$ & 32,502 & 39.58 & 47.46 & 62.07 & 76.48 & 108.95 & 130.49 & 154.66 & 200.07 & 245.36 & 347.41 \\
\hline 5.000 & 27.452 & 33.723 & 42.2177 & 55.390 & 83.316 & 101.38 & 121.28 & 158.33 & 195.19 & 277.58 & 332.28 & 393.80 & 509.28 & 623.54 & 882.32 \\
\hline 7.000 & $52.4 \cap 0$ & $04 . ? 71$ & $B n .384$ & 105.409 & $15 \mathrm{H} .326$ & 192.51 & 230.10 & 300.54 & 369.97 & 525.38 & 629.39 & 745.61 & 964.36 & & \\
\hline 10.000 & $10<.920$ & $.12 n .541$ & $160.5 ? 3$ & 210.328 & 315.675 & 383.55 & 458,74 & 599.12 & 737.79 & & & & & & \\
\hline 20.000 & 409.993 & $5(12.44 ?$ & $A>6.445$ & 819.824 & & & & & & & & & & & \\
\hline $\begin{array}{l}30.000 \\
50.000 \\
70.000\end{array}$ & $91<.551$ & & & & & & & & & & & & & - & \\
\hline 100.000 & & & & & & & & & & & & & . & & \\
\hline 200.000 & & & & & & & & & & & & & & & \\
\hline $\begin{array}{l}300.000 \\
500.000\end{array}$ & & & & & & & & & & & & & & & \\
\hline
\end{tabular}

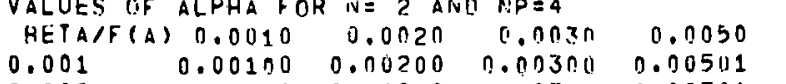
0.001 0.00100 0.00200 n.0.030 0.00501 $0.0020 .001 n 0.00200$ n. $0.033 n n$ n.00501

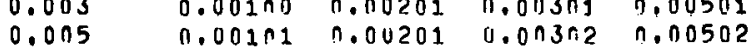
$\begin{array}{lllll}0.0 n 5 & 0.001 n 1 & 0.00201 & 0.0 n 3 n 2 & 0.00502 \\ 0.007 & 0.001 n 1 & 0.00201 & 0.003 n 2 & 0.00504\end{array}$ $0.010 \quad 0.00101$ n.0U202 0.0n3n3 0.00505 $0.020 \quad 0.001020 .042040 .003 n 60.00510$ $0.030 \quad 0.00103 \quad 0.00206 \quad 0.003 n 90.00516$ $0.070 \quad 0.001 n 8 \quad 0.002150 .003220 .00537$ $\begin{array}{lllll}0.100 & 0.00111 & 0.00222 & 0.003 .33 & 0.00555 \\ 0.200 & 0.00135 & 0.00250 & 0.00375 & 0.00624\end{array}$ $0.300 \quad 0.001420 .002450 .00427 .0 .00711$ $0.500 \quad 0.00194 \quad 0.003870 .00580 \quad 0.00965$ $\begin{array}{lllll}0.700 & 0.00277 & 0.00553 & 10.00427 & 0.01374\end{array}$ $\begin{array}{lllll}1.000 & 0.00408 & 0.00993 & 0.01483 & 0.02452\end{array}$ $2.000 \quad 0.03 ! 11$ 0.05R66 $11.08593 \quad 0.13701$ $3.000 \quad 0.1041 \mathrm{~N} \quad 0.201 \% 6 \quad$ U.?235? 0.42609 $5.000 \quad 0.21550 \quad 0.84738 \quad 1.11202 \quad 1.54308$

$0.007 n$ 0.00700 0.00709 .00702 0.0070 .3 0.00705 0.00715 0.00721 0.00753 0.00777 0,00994 0.01347 0,01919 0,03411 0.18446 0.54983 1.84976 3.97209 8.5704 33.9670 76.3559 $28.4586 \quad 40.4978 \quad 49.7141 \quad 94.3930$ $155.6405220 .3377370 .2644344,3659$ $317.6270 \quad 449.4634 \quad 551.2700 \quad 712.4026 \quad 843.2619$
0.0100 $n .0100$
$n .0100$ 0.0100 0.0100 0.0101 0.0102 0.0103 0.0105 0.0107 0.0125 0.0142 0.0192 0.0273
0.0482 $0.25 n 6$ 0.712 . 2.3528 4.8448 40.74 ? 91.493
253.175 253.175
405.606
0.0200 0.0200 $0.02 \mathrm{il}$ 0.0201 0.0201 0.0202 0.0204 0.0206
0.0210 0.0215 0.0222 0.0283 0.0381 0.0537 0.0933 0.4394 1.1483 3.5239
7.0773 7.0773 58.137 130.250 $\begin{array}{ll}360.108 & 443.604 \\ 704.590 & 867.676\end{array}$
0.0300 0.0301 $0.03 n 1$ 0.0301 $0.03 n$ 0.0302 $0.03 n 4$ 0.0306 0.130 0.0315
$0.03 ? 2$ 0.0333 0.0373 0.0423
0.0567 0.0795 0.1361 0.5994

1.497
4.437

8.8 ? 18.052 71.717
0.0500 0.0509 0.050 ? 0.0503 0.0504 0.0504 0.0506 0.0516 0.0526 0.0537 0.0620 0.0701 0.0933 0.1291 0.21 .64 0.8722 2,074
5.933 11.651 23.698 $112.122 \quad 136.353$ $\begin{array}{lll}98,614 & 250.733 & 304.444 \\ & 591.895 & 841.309\end{array}$ 
VALUES OF ALHHA FOH IN $=2$ ANO $M P=5$

\begin{tabular}{|c|c|c|c|c|c|c|c|c|}
\hline HETA/FCA & a) 0.0010 & 0.0020 & $0,00,20$ & 0.0050 & 0.0070 & 0.0100 & $0,0<00$ & 0.0300 \\
\hline 0.001 & 0.00100 & 0.00200 & 0.00300 & 0.00501 & 0.00700 & 0.0170 & 0.0200 & $0 . n 3 n 1$ \\
\hline & 0.00100 & $0.00 \geqslant 00$ & 0.00300 & 0.00501 & 0.00701. & 0.0100 & 0.0201 & 0.0301 \\
\hline 0.003 & 0.00100 & 0.00201 & 0.00301 & 0.00501 & 0.00702 & 0.0100 & 0.0201 & $0.03 n 1$ \\
\hline 0.005 & 0.00101 & 0.00201 & 13.0ก3ก? & 0.00502 & 0.00703 & 0.0100 & 0.0201 & $0.03 \cap 2$ \\
\hline 0.007. & $0.001 n 1$ & 0.00201 & 11.0030? & 0.01574 & $0.0 \cup 705$ & 0.9101 & 0.0201 & 0.0302 \\
\hline 0.010 & 0.00101 & 0.00202 & $0.003 \div 3$ & 0.00505 & 0.110707 & 0.0101 & 0.0202 & 0.0304 \\
\hline 0.020 & 0.00102 & 0,00204 & 0.00306 & 0,00510 & 0.00715 & 0.0102 & 0.0204 & 0.0306 \\
\hline 0.030 & 0.00103 & $0.00 \geqslant 06$ & $0.1503 \cap 9$ & 0.00516 & $0.0[1>21$ & 0.0103 & 0.0206 & $0.03 n 9$ \\
\hline 0.050 & $0.002 n 5$ & 0.00210 & 10.00316 & 0.00526 & 0.00737 & 0.0105 & 0.0210 & 0.0315 \\
\hline 0.070 & 0.00100 & $0.00 ? 15$ & $(1.00372$ & 0.00537 & 0.0075 .3 & $\left.n . n_{1} \cap 7\right)$ & 0.0215 & 0.0322 \\
\hline 0.100 & 0.00111 & $0.00 ? 22$ & 0.00333 & 0.00555 & 0.00777 & 0.0112 & 0.0222 & 0.0333 \\
\hline 0.200 & 0.00125 & 0.00250 & 0.00375 & 0.00624 & $0.0 \cup 874$ & 0.0125 & 9 & 373 \\
\hline 0,300 & 0.00143 & 0.00285 & 0.00428 & $0.0 \cup 712$ & $0.0 \cup 996$ & 0.0142 & 0.0283 & 0.0423 \\
\hline 0.500 & 0.00197 & 0.00393 & 0.00589 & 0.00979 & $0.0137 n$ & 0.0195 & 0. & \\
\hline 0,700 & 0.00293 & 0.00586 & 1). 000877 & 0.01456 & 0.02032 & 0.0299 & 0.0568 & 0.0839 \\
\hline 1.000 & 11.00597 & $0.011 .8 R$ & U.01.773 & 0.02929 & 0.114066 & 0.0573 & 0,11 & \\
\hline 2.000 & 0.05950 & 0.11335 & 0.16300 & 0.25253 & 0.35319 & 0.0414 & 0.7396 & 0.9823 \\
\hline 3.000 & $0.284 n 0$ & 0.48975 & 0.65951 & 0.94179 & 1.17878 & 1.4820 & 2.2726 & 2.891 \\
\hline 5.000 & 1.53927 & 2.34131 & 2.96310 & 3.95683 & $4.7 / 318$ & 5.8031 & 8.4407 & 10.507 \\
\hline 700 & 3.95083 & 5.78788 & 7.19932 & 9.43855 & 11.20960 & 13.5889 & 19.5170 & 24.125 \\
\hline 0.000 & 10.0042 & $14.428 ?$ & 17.7775 & 23.1486 & 27.4820 & 32.990 & 47.151 & 58.137 \\
\hline .000 & 57.5876 & $81.6 \cap 49$ & 1i) 0.1596 & 139.5175 & 153.6874 & 183.961 & 261.475 & 322.510 \\
\hline 30.070 & 158.0019 & 223.4998 & 274.6589 & 354.7370 & 420.0540 & 503.418 & 715.332 & 881.348 \\
\hline & 564.9418 & 794.31 .66 & 079.4922 & & & & & \\
\hline
\end{tabular}

$20.000 \quad 57.5876 \quad 81.6749$ 11) $9.1596 \quad 179.5175 \quad 153.6874 \quad 183.961 \quad 261.475$

30.000

50.000
70.000

100.000

200.000

300.000

500.000

\begin{tabular}{|c|c|c|c|c|c|c|c|c|c|c|c|c|c|c|c|}
\hline$B E T A / F(A)$ & 0.5000 & $0.0 \cap 00$ & 0.7000 & 0.8000 & 0.2000 & 0.9300 & 0.9500 & 0.9700 & 0.9800 & 0.9900 & 0,9930 & 0.9950 & 0.9970 & 0.9980 & 0.9990 \\
\hline 0.001 & 0.618 & 0.824 & $1.1 \operatorname{ng}$ & 1.562 & 2.543 & 3.18 & 3.89 & 5. & 6.52 & 9.47 & 11.42 & 13.62 & 17.75 & 21,87 & 31.10 \\
\hline & 0.618 & 0.924 & $1.1 \cap 8$ & 1.564 & 2.543 & 3.18 & 3.89 & & 6.53 & & 11.44 & 13. & 17.75 & & 16 \\
\hline 0.003 & 0.019 & 0.424 & 1.110 & 1.564 & 2.547 & 3.18 & 3.90 & 5. & 6.53 & 9.48 & 11.44 & 13.63 & 17.78 & 21,87 & 31 \\
\hline 0.005 & $0.6 \geqslant 0$ & 0.225 & 1.110 & 1.566 & 2.347 & 3.19 & 3,90 & & 6.54 & 9.48 & 11.45 & 13.65 & & 21.90 & \\
\hline .007 & 0.621 & 0.826 & 1.112 & 1.568 & 2.551 & 3.19 & 3.90 & 5. & 6.54 & 9. & 11,47 & 13.67 & & 21 & \\
\hline 10 & $0.0 ? 2$ & 0.824 & 1.11 .4 & 1.572 & 2. & 3.20 & 3.41 & 5. & 6.55 & & 11.48 & 13 & & 21 & 3 \\
\hline & 0.027 & 0.934 & $1.1 ? 2$ & 1. .581 & & 3.21 & 3.93 & 5.27 & 6.59 & 9. & 11.54 & 13 & & 22 & \\
\hline 0.030 & 0.032 & 0.941 & 1.129 & 1.591 & 85 & 3.23 & 3.95 & 5.29 & 6.63 & 9. & 11.61 & & & 22 & \\
\hline 0.0 & 0.041 & 0.252 & 1.144 & 12 & 2.620 & $3 . ? 7$ & 4.00 & 5.35 & 6.70 & 0. & 11.73 & & & 22 & \\
\hline 0.070 & 0.651 & $0 . P_{05}$ & 1.162 & 1.633 & 2.650 & 3.31 & 4.0 .5 & 5.41 & 6.77 & 9. & 11.86 & 14 & & & \\
\hline 0.100 & 11.067 & 0.885 & 1.186 & $1.66 \%$ & 2.100 & 3.38 & 4.1 .2 & 5.51 & 6.89 & 10.00 & 12.06 & 14 & 2 & 23 & \\
\hline 0.200 & 0.724 & 0.958 & 1.280 & 1,791 & 2.891 & 3.60 & 4 , & 5.88 & 7.34 & 10 & & & & & \\
\hline 03 & 0.795 & 1.047 & 1.392 & 1.941 & 3.1 & 3.88 & 4.74 & 6.31 & 7.88 & 11. & 13.74 & & & 26.20 & \\
\hline 0.500 & 0.990 & 1.289 & $1.7 n 1$ & 2.349 & 3.137 & 4.64 & 5.04 & 7.50 & 9.33 & 13 & & & & 30.85 & \\
\hline 0.7 & 1.286 & 1.659 & 2.166 & $? .96$ & 4.666 & 5.77 & 6.99 & 9.27 & 11.51 & & & & & & \\
\hline 1.000 & 2,002 & $2.54 \mathrm{n}$ & 3.276 & 4,422 & 6.864 & 8.45 & 10.19 & 13,44 & 16.65 & & 28.67 & & & & \\
\hline 2.000 & 7.451 & 9.240 & 11.636 & 15.397 & 23.032 & 28.43 & 34.09 & 44.65 & 55.09 & 78.43 & 94.06 & 111.51 & 144 & 176.88 & 250.24 \\
\hline 3.000 & 18,571 & 22.874 & 88.642 & 37.690 & 56.73 .3 & 69.03 & 82.70 & 107,97 & 132.94 & 189.33 & 226.69 & 268.80 & 347.41 & 425,54 & 602.05 \\
\hline 5.000 & 61.952 & 75.990 & 94.911 & 124,391 & 186.800 & 227.42 & 271.73 & 35 & 436.77 & 621.58 & 743.65 & 881.35 & & & \\
\hline .000 & 139.527 & $171 . n 21$ & $213.5 n 2$ & 279.542 & 419.078 & 510.7 .5 & 609.86 & 795.41 & 979.49 & & & & & & \\
\hline & 332 & 407 & 5 & 666.504 & 999,0 & & & & & & & & & & \\
\hline
\end{tabular}

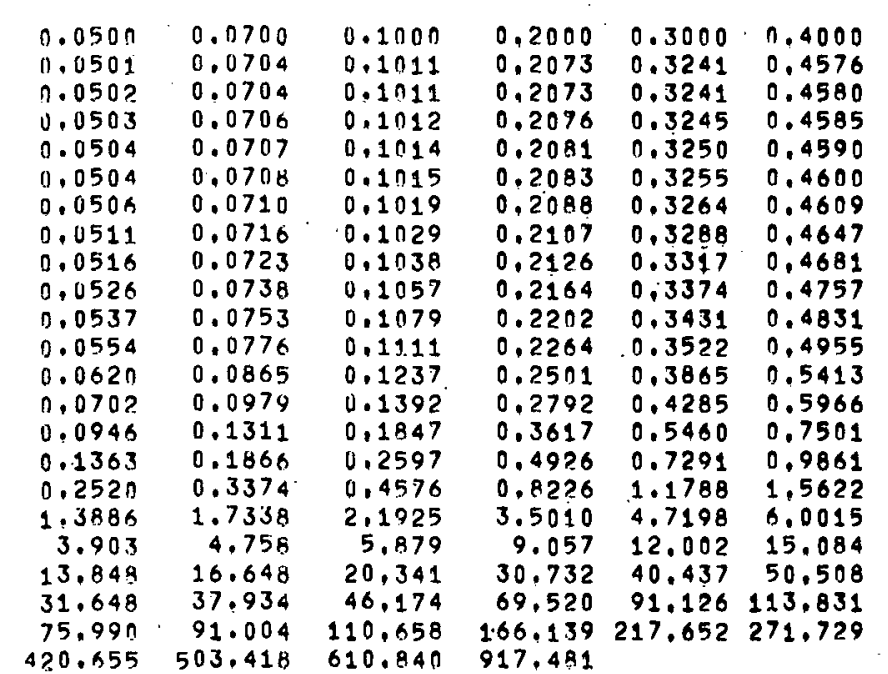

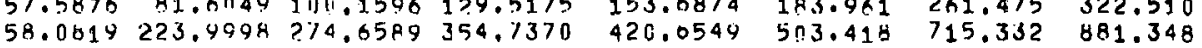
917,491

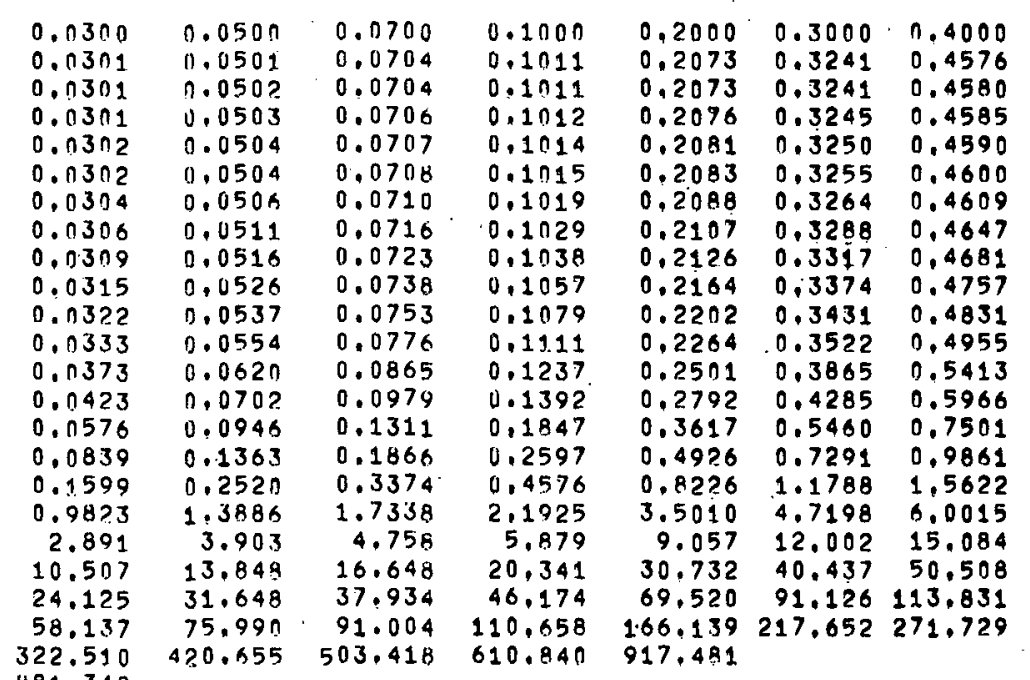

20.000

30.000

50.000
70.000

100,000

200.000

300.000
500.000 


\begin{tabular}{|c|c|c|c|c|c|c|c|c|c|c|c|c|c|c|c|}
\hline \multirow{2}{*}{$\begin{array}{l}\text { VALUES } \\
\text { BETA/Fi }\end{array}$} & \multirow{2}{*}{$\begin{array}{c}\text { ALPHA FC } \\
0.0 ! 190\end{array}$} & & $p=1+x$ & & \multirow[b]{2}{*}{$0,0 n 7 n$} & \multirow[b]{2}{*}{$0.01 n n$} & \multirow[b]{2}{*}{0.0200} & \multirow{2}{*}{\multicolumn{2}{|c|}{0.0370}} & \multirow[b]{2}{*}{$0.050 n$} & & & & & \\
\hline & & 0.0120 & $(1, n 03 n$ & 0.0050 & & & & & & & 0.0700 & 0.1000 & 0.2000 & 0.3000 & 0.4000 \\
\hline 0.001 & 0.00100 & $0.0620 n$ & $\dot{0.00300}$ & $r .00501$ & 0.00700 & n. B10 & 0.02. & $0 . n$ & & $0.050 !$ & 0.0701 & 0,1002 & 0.2014 & 0.3064 & 0.4185 \\
\hline 0.002 & 0.00100 & $n$. ก ט?an & 0. ถ०3กn & r.00501 & 0.00701 & 0.0100 & 0.02 & 0.0 & 301 & 0.0501 & 0.0702 & 0.1002 & 2016 & .3064 & 0.4189 \\
\hline 0.003 & $0.001 \cap 0$ & (). $\cap(1 \geq 01$ & 1). $1 \cap \cap 3 \cap 1$ & 00501 & 0.00702 & 0.0100 & 0.02 & 0.0 & 301 & 0.0509 & 0.0702 & .1004 & .2019 & 0,3069 & 0.4194 \\
\hline 0.005 & $0.001 \cap 1$ & 0.0020 .1 & 1.0n3?? & 0.00502 & 0.0070 .3 & 0.0100 & 0.02 & 0.0 & $\ln 2$ & 0.0503 & 0.0703 & 1,1006 & 2021 & 0.3074 & 1.4199 \\
\hline 0.007 & $0.401 n 1$ & $0.04>01$ & $0.0 n 3 n$ ? & 0.00504 & 1.00705 & 0.0101 & 0.02 . & 0.0 & 302 & 0.0503 & .0704 & .1007 & .2026 & 0.3078 & 0.4204 \\
\hline 0.010 & n. $\operatorname{yn} 101$ & ก.00>0? & $11,0 \times 3 \cap 3$ & $n .00505$ & 0.00707 & 0.0101 & 0.02 & 0.0 & 303 & 0.0505 & 0.0707 & 0.1010 & 0.2031 & 0.3083 & 0.4213 \\
\hline 0.020 & 0.00102 & i) $.00 \geq 04$ & i). 00306 & .00510 & 0.00714 & n.01n? & 0.02 & 0.0 & 306 & 0.0510 & 10.0713 & 0.1019 & 0.2045 & 0,3107 & 0,4242 \\
\hline 0.0 .30 & n. Unj.n 3 & G. $0.0 \cup \geqslant 10$ & $0.003 n 9$ & 9.ก 0515 & 0.00721 & 0.0103 & 0.02 & 0.0 & 309 & 11.0515 & 0.0720 & 0,1027 & 0,2062 & .3131 & .4266 \\
\hline 0,050 & 0.00105 & $0.00>10$ & 0.00315 & 0.00525 & 0.00735 & 0.0105 & 0.02 & $0 . n$ & 315 & 0.0524 & 9.0733 & 0.1045 & 0.2095 & 0.3174 & 0.4323 \\
\hline 0.070 & $0.101 \cap 07$ & n. ก४⿻14 & 1.กn3? & $\because .0$ of 535 & $0.0 \cup 748$ & 0.0107 & 0.02 & 0.0 & 320 & 0.0533 & 0.0746 & 0.1063 & 0.2126 & 0.3217 & 0.4380 \\
\hline 0.100 & 0.00110 & $n, 011 \geq 2 \pi$ & 0.00330 & .00550 & 0.00760 & $n .0110$ & 0.02 & 0,0 & 329 & 0.0547 & 0.0765 & 0,1091 & & 0.3283 & 456 \\
\hline 0.200 & 0.00130 & 1). . $n$ & 13.00360 & 1. nu599 & 0.00839 & $0.01>0$ & 0.02 & 0.0 & 358 & 0.0594 & 0.0828 & 0.1178 & 331 & .3493 & 0.4719 \\
\hline 0.300 & 0.00130 & $0.11 \cup 260$ & 0.00300 & 0.00649 & 0,00908 & 0.0130 & 0.02 & 0.0 & 386 & 0.0640 & $0.089 ?$ & 0.1265 & 482 & 1.3698 & 974 \\
\hline 0.500 & 0.00150 & $0.0 \cup 30 n$ & 0.00449 & 0.00748 & 0.01044 & 0.0149 & 0.02 & 0.0 & 44 & $0.073 ?$ & 0.1015 & 0.1 & & & 441 \\
\hline 0.700 & 0.00170 & $0.0 \cup 340$ & 0.00509 & 1.00847 & 0.01184 & $n .0169$ & 0.0 & 0.0 & & 0.0 & .1136 & 0. & & & \\
\hline 1.000 & $0.402 n 0$ & 0.04 .399 & 0.00598 & 4. 00094. & 0.01391 & 0.0198 & 0.03 & 0.0 & & 0.0953 & .1311 & 28 & 3426 & 0.4936 & 962 \\
\hline 2.000 & 0.010294 & 0.00548 & 0.00804 & 9.01435 & $0.020 \% 1$ & 0.02 .94 & 0.05 & 0.0 & 350 & 0.1368 & 0.1852 & 30 & 0,4523 & 0.0 & \\
\hline 3.000 & 0.40309 & 0.04795 & $u .0110 \mathrm{j}$ & $3 . n 10 \%$ & 0.02741 & $0.03 R E$ & 0.07 & & & & 0.234 & & & & \\
\hline 5.000 & 0.40597 & 0.ก118R & ij. 01773 & 3.02926 & $0.1,4060$ & 0.0572 & 0.10 & & & & 18 & & & 5 & \\
\hline 7.000 & 0.60705 & 0.111579 & U. 02351 & 9.03864 & 0.05342 & 0.0748 & 0.14 & & & 0 . & 0 & & & 8 & \\
\hline 10.000 & $0.01 \cap 9$ & $U . U>1 A$ & 0.0320 & 0.0524 & 0.0720 & $0: 100$ & 0.1 & & & 0.380 & 6.483 & & & & \\
\hline 20.000 & 0.0200 & 0.0404 & 0.0594 & 0.0956 & 0.1291 & 0.176 & 0.3 & & & 0.578 & 0.712 & & 1.7 & 1.661 & \\
\hline 30.000 & $0.03 \cap 1$ & 0.11505 & 0.0853 & 0.1 & $0.180 ?$ & 0.241 & 0,4 & & & 0.725 & .880 & & & & \\
\hline 50.000 & 0.11426 & U. 19 928 & 0.1334 & v. & 11.2643 & 0.350 & 0.5 & & & 0. & 9 & & & 2 & \\
\hline 70.000 & 0.0064 & $0.1 \geqslant 49$ & $1.7 .7 \times 8$ & l. $\ddot{c 608}$ & 0.3426 & 0.439 & 0.6 & & & 1.114 & 2,316 & & .2 & 7 & \\
\hline 00.000 & 0.0949 & U.1\%9n & 0.2355 & 0.3 & $0.43 / 1$ & 0.551 & 0.8 & & & & 1.543 & & & & \\
\hline 0.000 & 0.1682 & 0.2926 & 0.3913 & 0.5470 & $0.069 n$ & 0.817 & 1.1 & & & 880 & 2.063 & & & & \\
\hline 0.000 & $0.23 .3 \mathrm{H}$ & $0.39 n R$ & 0.5107 & 1. .0948 & $0.03 E 9$ & 1.007 & 1.4 & & & 2,105 & 2.429 & & 3.842 & 4,0 & 5,483 \\
\hline 10,000 & 0.3431 & 0.5451 & 0.0938 & 0.9151 & 1.0834 & 1.286 & 6 & & & 2,578 & 2.963 & & 28 & 20 & \\
\hline$A \in T A /$ & 0.5000 & non & 7000 & 68 & .9000 & & oton & & & .9900 & .993 & .9950 & 99 & 980 & 9990 \\
\hline 0.001 & 544 & 692 & 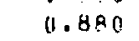 & & 1 & 1 & 27 & 2.19 & 3.27 & 4.25 & 4. & 8 & 7 & & 5 \\
\hline 0.002 & 54 & . $69 ?$ & & & & & & & & & & & & & \\
\hline 0.003 & & 0.493 & 0 & & & & 2, & 2 & & & 4.1 & & & & 65 \\
\hline 0.005 & 1.545 & 0.544 & 0.8 & & 1.0 & & 2. & 2.80 & 3. & 4. & 4.8 & 5.6 & 6 & & 0 \\
\hline 0.0 & 546 & 0.694 & & & 1 & & 2. & 2.8 & & & 4, & & & & \\
\hline 0.010 & 0.541 & 0.0 .56 & 0.88 & 1. & 1.667 & 1 . & 2. & 2.80 & 3.26 & 4, & 4.8 & 5. & 6. & 0 & \\
\hline 0.020 & 551 & $0.76 \mathrm{~B}$ & & & 1.675 & & 2. & 2.8 & & & 4. & & & & \\
\hline 0.030 & .54 & 0.703 & 10.893 & & 1.682 & & 2. & 2.8 & & & 4,5 & 5. & 6. & & \\
\hline 0.050 & $0.5 \circ 0$ & 0.711 & $0.90 ?$ & 1. & 1.696 & & 2, & 2.84 & & & 4, & 5. & & & \\
\hline 0.070 & & 19.719 & 0.911. & 1. & 1.7 & ?. & & 2.86 & & & $4:$ & 5. & & & \\
\hline 0.10 & דר & $19,7 \geq 0$ & 0.924 & & 1.130 & & 2. & 2.89 & & & 5. & & & & \\
\hline 0,2 & 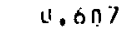 & $n$. & 0. & & & & & 2.99 & & 4. & 5.1 & $\theta_{0}+2$ & & & \\
\hline 0.3 & $53 \%$ & 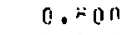 & 5 & & 1.050 & & & 3.09 & & & & & & & \\
\hline 0.5 & & $0 . \dot{x}<5$ & 1 & & & ?. & & 3.26 & & & 5. & & & & \\
\hline 0.7 & (4) & n. $9 \ddot{<1 .}$ & & & 2.476 & & & 3.42 & & & 5. & 6. & & & \\
\hline 1.0 & u & 0.9 & 1.2 & & $2 . c$ & & 2. & 3.6 & & & 6. & & & & \\
\hline 2.04 & & 3. . & $0^{\circ}$ & & 2.6 & & & 4.2 & & & & & & & \\
\hline 3.04 & i.150 & 1.359 & 1.68 & 2.1 & & & 3. & 4.68 & 5,44 & & 7. & & & & \\
\hline $5.0 n$ & & 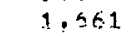 & & & 3.5 & & 4. & 5.42 & & & 9. & & & & \\
\hline 7.0 & $\therefore .577$ & 1.9 .77 & 2.2 & & 3,179 & & & 6,01 & & & & & & & \\
\hline 10.000 & $\therefore \sin B$ & 2.159 & 2.5 & 3.1 & 4.247 & 4. & 5. & 6.72 & 7.78 & & 11. & & - & & \\
\hline 24000 & 2.357 & $2 . \%$ & $3.27 ?$ & 4,0 & & 6. & & 8.43 & 9.74 & & & & & & \\
\hline 30.000 & 2.146 & $3 . ? 67$ & 3.79 .5 & 4,6 & 0.16 .9 & 7.08 & 8.02 & 9.65 & 11.1 .5 & 14 & 16.04 & & 21.41 & & 04 \\
\hline 50.000 & $5,3 ? 2$ & 3.405 & & & 7.052 & 8. & & & & & 19. & & & & \\
\hline 70.000 & 3.760 & $4.3^{3} 61$ & $5.1 ? 4$ & 0.2 & 8.248 & 9.45 & 10 . & 12.84 & 14.81 & 18.32 & 21.26 & 23 & 28.34 & & 41.05 \\
\hline 100.000 & 4.277 & 4.956 & 5.811 & 7.031 & 9.332 & 10.67 & & 14.49 & & 21.20 & 23.94 & & & & \\
\hline & 5.490 & 6.337 & 7.405 & 0.1 & 11. & & & 18.3 & 21 & 26 & 30 . & & 40 & & 28,20 \\
\hline 10 & 537 & 7.314 & 0.538 & 0 & 13. & & & & & & 34. & & & & \\
\hline 000 & $\triangle R 1$ & 8.737 & 10.186 & 2.201 & 1.6 .160 & 8.48 & 10 & 24.95 & 28.7 .3 & 36.41 & 41.11 & 46.05 & 54.36 & 62,68 & 79.8 \\
\hline
\end{tabular}




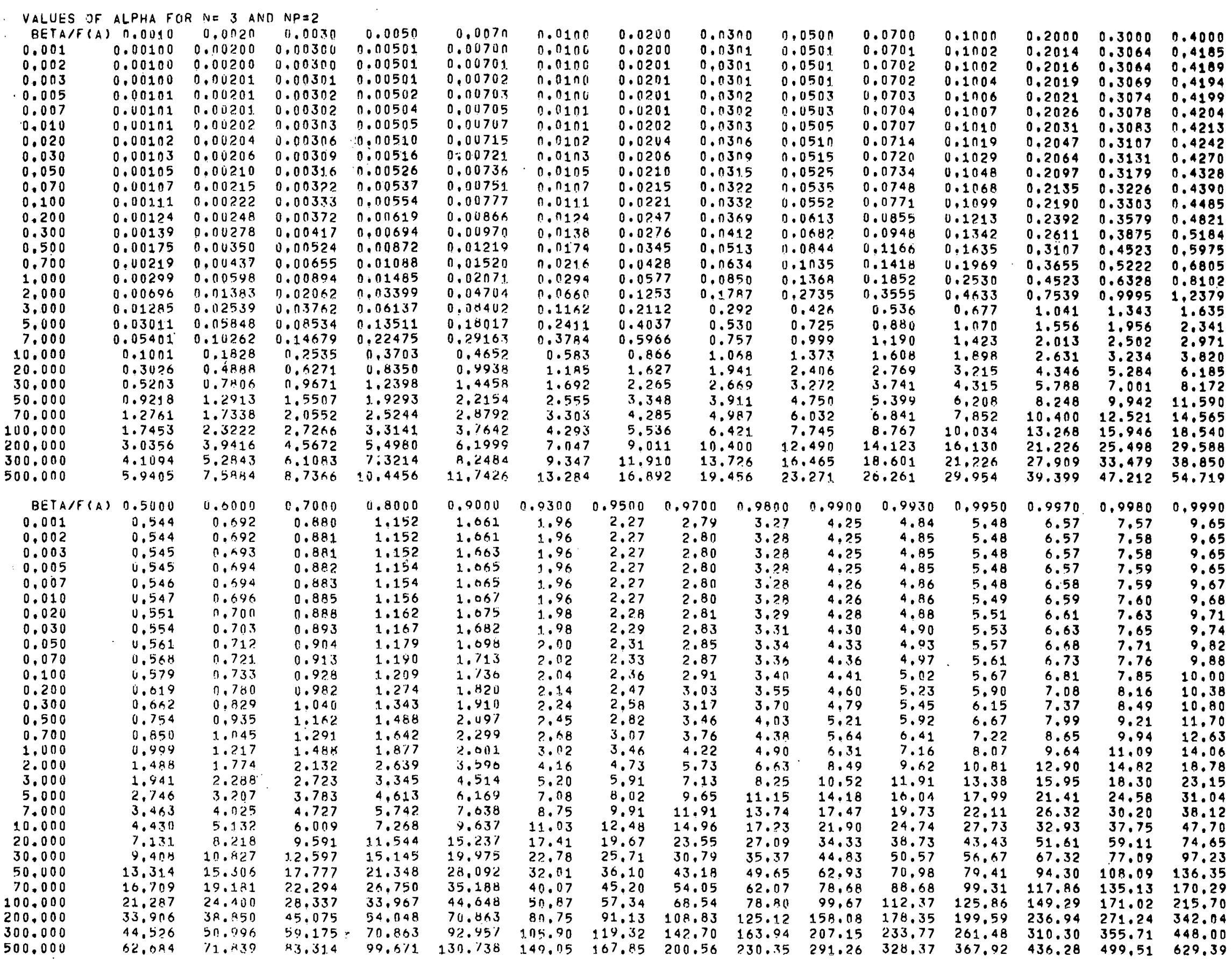


VALUES I: ALFHA + OK $N=3$ AND NP $=3$

0.005

0.007

0.010

0.030

0.050

0.070
0.100

0.100
0.200

0.300

0.500

0.700

1.000

2.000

3.000

5.000

1.000

10.000

30.000

50.000

$70.0 \cap 0$

1 10.000

300.000

51) 0000

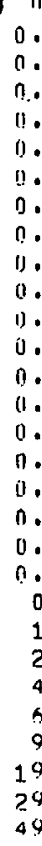

BET TA

0.001

0.001
0.002

0.003

0.005

$0.0 n$

0.010

0.020

0.030

0.050

0.100

0.100

0.300

0.300

0.700

1.000

2.000

3.000
5.000

$\%, 000$

10.000

0.40

30.000

50.090

70.000

100.000

290.000

500,000
แ. 007.00

0.00701

0.1100

ก. 100

n. ninn

0.00501

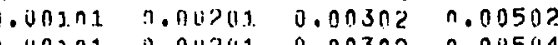

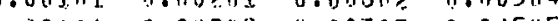

0.v01na nov?i14 u. un3no n.03510

0.00103 i.n0?15 0.003ng 0.00516

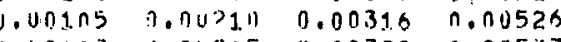

$\begin{array}{llll}0.0108 & 11.011215 & 0.00322 & 0.00537\end{array}$

$\begin{array}{llll}1) .40111 & 0.01222 & 0.00333 & n .00555 \\ 0.0 .0125 & 9.04249 & 0.00374 & 0.00623\end{array}$

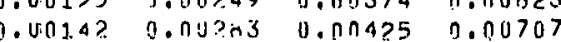

0.0187 n.01).374 0.0ก561 n.00933

0.00253 0.0145115 0.00755 0.01257

0.0199 n.0u705 0.01190 n.01970

0.01479 0.112017 0.04317 0.07nנ0

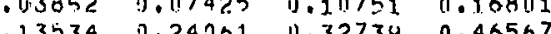

.291150 .272820 .073010 .81305

$\begin{array}{llll}0.5804 & 0.6579 & 1.0548 & 1.3447\end{array}$

$\begin{array}{llll}0.6137 & 2.1587 & 2.5397 & 3.0966 \\ 2.6384 & 3.4399 & 4.0026 & 4.8266\end{array}$

.6587 $5.9746 \quad 6.9718 \quad 8.2636$

$\begin{array}{rrrr}.6799 & 4.5978 & 9.8048 & 11.0968 \\ 9.6679 & 12.3172 & 14.1382 & 16.8314\end{array}$

$19.76101 \quad 24.41 .86 \quad 28.58 n 7 \quad 33.967 n$

$\begin{array}{llll}29.7106 & 37.5071 & 43.0002 & 51.1179 \\ 49.7141 & 62.0940 & 71.4393 & 95.3891\end{array}$

0.037113

$n \cdot 0100$

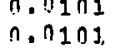

.007117

0.00721

0.00737

0.011553

0.01087 ?

0.00988

0.01304

0,02741

0.119571

0.51651

0.96897

1.5641
$3.516 ?$

$5.45 ?$ ?

9.3012

13.1312

38.0564

$\begin{array}{rr}38.0564 & 42.939 \\ 57.22 .14 & 64.637 \\ 95.6430 & 107.851\end{array}$

0.0200
0.0200

0.0300

$0,050 n$

0.0700

0.0501

0.0701

0.1000

0.1002

$0.0201 \quad 0.0301$

0.0501 .

0.0702

0.1004

$0.0201 \quad 0.03 n 2$

0.0503

0.0505

0.0704

0,1006

0.1107

0.1110
0.1019

0.1029

$0.0206 \quad 0.0309$

0.0210

0.0515

0.0525

0.0553

0.0720

0748

0.1048

0.1068
0.1100

0.0222

0.0332

0.0616

$0.0281 \quad 0.0420 \quad 0.0695$

0.0308

0.0859

0,0964

0.1220

0.12390 .1733

0.018

0.0492

0.0548

0.1178

0.1606

0.2214

0.31 .45

0.2367

$0.1103 \quad 0.1747$

0.4783

0.618

0.4681
0.833

1.596

1.257

1.900
2.856

2.360
3.497

0.5851

0.7 .339
1.209

2,181

2.71

$\begin{array}{ll}7.260 & 8.23\end{array}$

3,154
4,605

9,423

5.193
7.928

0.164

11.025
18.510

12.460

14.2 .45

$18.476 \quad 21.745$

26.017

$\begin{array}{lll}54.414 & 62.562 & 74.769\end{array}$

29.344

42.024

33,479

47.883

96.009

$\begin{array}{rrrrr}136.727 & 93.934 & 112.245 & 126.588 & 144.1 .66 \\ 136.017 & 187.35 & 210.816 & 240.113\end{array}$

$0.9300 \quad 0.9500$

$0.9700 \quad 0.9$ 187.

$0.51100 \quad 0.01100 \quad 0.7000 \quad 0.8000$

$\begin{array}{lll}0.492 & 0.880 & 1.152 \\ 0.692 & 0.881 & 1.152\end{array}$

$\begin{array}{lll}0.093 & 0.881 & 1.152\end{array}$

1.154

1.063

1.665

1.663

1.567

1.675

1.682

1.713

1.138

1.926

1.926
2.158

2.437

2.910

4.750

6.149
10.842

14.977

21.195
41.963

41.963
32.806

$32.800 \quad 77.82$
104.432

$47.82 \quad 27.30$

$\begin{array}{ll}71.47 & 53.93 \\ 11.0 .03\end{array}$

$20.0155 .22 .935 \quad 26.658 \quad 31.953$

$50.1914 \quad 5 \% .282 \quad 66.468 \quad 47.822$

70.008 वा.1.4n $92.957 \quad 111.258$

$\begin{array}{llll}104.038 & 114.564 & 132.691 & 159.058\end{array}$

104.432

$\begin{array}{rrrr}71.47 & 80.03 & 96.38 & 1.10 .66 \\ 118.84 & 133.91 & 160.04 & 183.06 \\ 1.66 .14 & 187.38 & 223.76 & 257.08\end{array}$

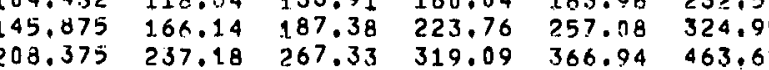

$\begin{array}{lllllll}416.260 & 474.37 & 534.67 & 639.18 & 732.01 & 926.27\end{array}$

$0,99,30.0$

0.9930
4.34

0.9950

5.48
5.48

$5.80 \quad 3, ? 8$

.80
2.80 3.28

$\begin{array}{rl}3.80 & 3.28 \\ 5.80 & 3.28\end{array}$

2.813 .29

$2.83 \quad 3.31$

$3.04 \quad 3.41$

3.41
3.55
3.73

3.73
4.13
4.61

4.61
5.44

$5.44 \quad 5.94$

6.98

11.06
15.49

$15.49 \quad 17.50$

33.72

47.58

$\begin{array}{rr}58 & 53.68 \\ 61 & 105.77\end{array}$

118.47

$\begin{array}{ll}157.94 & 176.88 \\ 261.36 & 293.7 .0\end{array}$

$366.46 \quad 410.40$

$300.050 \quad 343.507 \quad 308.194 \quad 476.319$

$\begin{array}{llll}494.51 ? & 5 \% 2.754 & 643.575 & 793.457\end{array}$

4.85
4.85

4.86

4.86

4.98

4.93
4.97

5.03

5.49

6.76

8.90
14.05

19.61

31.10

42.70

0.15
18.47
6.88

$0.2000 \quad 0.3000 \quad 0.4000$

$0.2014 \quad 0.3064 \quad 0.4185$

$\begin{array}{llll}0.2016 & 0.3064 & 0.4189\end{array}$

$0.2019 \quad 0.3069 \quad 0.4194$

$\begin{array}{lll}0.2021 & 0.3074 & 0.4199\end{array}$

$0.2026 \quad 0.3078 \quad 0.4204$

$0.20310 .3083 \quad 0.4213$

$0.20470 .3107 \quad 0.4242$

$0.2100 \quad 0.3179 \quad 0.4332$

$0.2135 \quad 0.3231 \quad 0.4390$

$0.2193 \quad 0.3307 \quad 0.4490$

$\begin{array}{lll}0.2401 & 0.3593 & 0.4840 \\ 0.2654 & 0.3927 & 0.5250\end{array}$

$\begin{array}{lll}0.3269 & 0.4733 & 0.6223\end{array}$

$0.4042 \quad 0.5718 \quad 0.7387$

$\begin{array}{lll}0.5441 & 0.7453 & 0.9418 \\ 1.1158 & 1.4325 & 1.7376\end{array}$

$\begin{array}{lll}1.738 & 2,173 & 2,589\end{array}$

$\begin{array}{lll}3.001 & 3.676 & 4.323 \\ 4.262 & 5.185 & 6.070\end{array}$

$6.162 \quad 7.451 \quad 8.691$

$12.460 \quad 14.993 \quad 17.442$

$18,754 \quad 22,538 \quad 26,170$

$\begin{array}{lll}31.343 & 37.629 & 43.611\end{array}$

$62.928 \quad 75.257 \quad 87.342$

$\begin{array}{lll}125.855 & 150.758 & 174.683\end{array}$

$\begin{array}{lll}188.844 & 226.197 & 261.964 \\ 314.698 & 376.710 & 136.768\end{array}$

$\begin{array}{rrr}0.9970 & 0.9980 & 0.9990\end{array}$

6.57
6.57
6.57

$\begin{array}{ll}6.57 & 7.58 \\ 6.57 & 7.58\end{array}$ 
VALUES TF ALPHA FOR $N=3 \quad A M O N P=4$

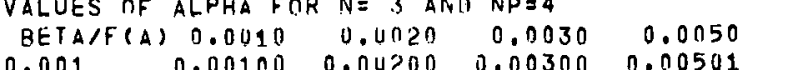

$0.001000 .00200 \quad 0.00300 \quad 0.00501$

0.001010 .002010 .003010 .00501

0.020

0.030

0.050

0.070

0.100

0.200

0.300

0,500

0.700

1.000

2,000

3.000

5.000

7.000

10.000

20.000

30.000

50.000

70.000

100.000

200.000

300.000

500.000

0.00

0.003

0.005

0.007

0.010

0.020

0.050

0.050

0.070

0.100

0,200

0,500

0.500

1.00

3.00

5.00

5,000

1.000

10,000

20.000

30.000

70,000

100,000

200.000

300,000
500,000

$\begin{array}{llll}0.001000 & 0.00201 & 0.003 n 2 & 0.00502\end{array}$ $0.001 n 8 \quad 0.00215 \quad 0.00322 \quad 0.00537$ 0.001110 .002220 .003330 .00555 $\begin{array}{llll}0.00194 & 0.00387 & 0.00580 & 0.00965\end{array}$ $\begin{array}{llll}0.00498 & 0.00991 & 0.01482 & 0.02452\end{array}$ $\begin{array}{llll}0.03011 & 0.05 R 48 & 0.08534 & 0.13511\end{array}$ $\begin{array}{llll}0.46520 & 0.70814 & 0.88267 & 1.14063\end{array}$

$\begin{array}{llll}0.967 n 7 & 1.34854 & 1.61366 & 2.00562\end{array}$

$\begin{array}{llll}1.8177 & 2.4138 & 2.8296 & 3.4361 \\ 5.1546 & 6.5966 & 7.6113 & 9.1029\end{array}$ $18.21147 \quad 23.0265 \quad 26.4445 \quad 31.4036$ $28.641 .7 \quad 36.2253 \quad 41.5354 \quad 49.2868$

$46.235158 .3 \mathrm{A1} \quad 66.9565 \quad 79.4077$

$16.8832 \quad 147.3397 \quad 168.8241200,3182$

$\begin{array}{llll}200.8045 & 253.1746 & 289.7956 & 343.9948 \\ 396.7201 & 500.4988 & 572.7543 & 680.1761\end{array}$

$\begin{array}{rrrr}0.5000 & 0.6000 & 0.7000 & 0.8000 \\ 0.544 & 0.692 & 0.880 & 1.152 \\ 0.544 & 0.692 & 0.881 & 1.152 \\ 0.545 & 0.693 & 0.881 & 1.152 \\ 0.545 & 0.494 & 0.882 & 1.154 \\ 0.546 & 0.694 & 0.883 & 1.154 \\ 0.547 & 0.696 & 0.885 & 1.156 \\ 0.551 & 0.700 & 0.888 & 1.162 \\ 0.554 & 0.703 & 0.893 & 1.167 \\ 0.561 & 0.712 & 0.904 & 1.179 \\ 0.569 & 0.721 & 0.913 & 1.192 \\ 0.579 & 0.734 & 0.928 & 1.209 \\ 0.022 & 0.783 & 0.986 & 1.278 \\ 0.672 & 0.840 & 1.053 & 1.358 \\ 0.797 & 0.984 & 1.219 & 1.556 \\ 0.961 & 1.1 .73 & 1.436 & 1.816 \\ 1.278 & 1.534 & 1.854 & 2.311 \\ 2.746 & 3.207 & 3.793 & 4.613 \\ 4.575 & 5.292 & 6.192 & 7.489 \\ 8.859 & 10.186 & 11.865 & 14.276 \\ 13.742 & 15.794 & 18.357 & 22.019 \\ 21.958 & 25.193 & 29.252 & 35.066 \\ 54.902 & 02.928 & 72.938 & 57.342 \\ 93.934 & 107.506 & 124.635 & 1.49 .293 \\ 185.181 & 212.037 & 245.850 & 294.190 \\ 289.307 & 331.299 & 384.034 & 459.717\end{array}$

0.020 .000000000001

$0.007 n$

0.0100

0.0100
0.0100

0.0200
0.0200

0.0200

$0,03 \cap 0$

$0.03 n 1$

$0.050 n$

0.0501

0.0700

0.0701

0.00702

0.0100

0.0201

0.0301

0.0501

0,0702

0.0703

0.0101

0.0201

0.0305

0.0503

0.0503

0.0704

0,00715

0.010

0.0303

0.0510

0.0707

0.05150 .0720

$0.0210 \quad 0.0315 \quad 0.0525$ $0.00105 \quad 0.00210 \quad 0.003160 .005260 .00737$

0.0115

0.00753

0.0107

0.0215

0.0315

0.0536

0.0734

0.0748

0.0772

0.02490 .037 $\begin{array}{llllll}0.00142 & 0.04285 & 0.00427 & 0.00711 & 0.00994 & 0.0142\end{array}$

0.01347

0.0192

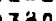

0.0422

0.0698

0.0861 $\begin{array}{llllll}0.0277 & 0.00553 & 0.00827 & 0.01374 & 0.01916 & 0.027\end{array}$

$\begin{array}{rrrr}9.0723 & 11.5290 & 13.2685 & 15,7938\end{array}$

0.034050 .0481

$0.49166 \quad 0.5137$

$1.33518 \quad 1.5679$

3.8958

10.2320

17.7164

35.1877
55.2683

88.9291

224.2439

$0.0790 \quad 0.1275$

0.1275

0.1275

0.1735

0.7253

0.2778

1.427

1.669

0.9056

1.11 .4

2.6503
4.445

11.590

20.036

3.4666

4.048

4.911

3.505

5.582

9.072
22.965

20.341

14.749
25.407

$50.3 \% 4$

16.984

57.8935 .005

39.460

09.154

77.943

100.282

$90.638 \quad 208.338$

122.010

438.721

549.

1.061

1.661
1.663

1.665

1.665

1.667

1.675

1.682

1.713

1.738

1.825

1.929

2.189
2.524

2.524

6.169

0.9300
1.96
1.96

1.96
1.96

1.96
1.96

1.96

1.98

2.100

2.02
2.04

2.14

2.26

2.55
$? .94$

3.67

0.9500

$2.27 \quad 0.9700$

2.27

2.79
2.80

0.9800
3.27

0.9900

0.9930

2.80
2.80

3.28
3.28

$\begin{array}{lll}2.27 & 2.80 & 3.28 \\ 2.27 & 2.80 & 3.28\end{array}$

$2.27 \quad 2.80 \quad 3.2$

$\begin{array}{lll}2.28 & 2.81 & 3.29 \\ 2.29 & 2.83 & 3.31\end{array}$

$\begin{array}{lll}2.31 & 2.85 & 3.34\end{array}$

$\begin{array}{lll}2.33 & 2.87 & 3.36 \\ 2.36 & 2.91 & 3.41\end{array}$

$2.47 \quad 3.04 \quad 3.56$

$2.60 \quad 3.20 \quad 3.74$

$\begin{array}{lll}3.93 & 3.59 & 4.1 .9\end{array}$

$4.19 \quad 5.08 \quad 5.90$

$\begin{array}{rrrr}11.36 & 12.84 & 15.40 & 11.15 \\ & 11.75\end{array}$

18.815
28.477

33.05 .37 .26

15.40
29.01

46.052

195.680

$\begin{array}{rrrr}57.52 & 59.24 & 70.74 & 81.24\end{array}$

$\begin{array}{lllll}185.181 & 212.037 & 245.850 & 294.190 & 385.499\end{array}$

$\begin{array}{lllll}289.307 & 331.299 & 384.034 & 159.717 & 603.028 \\ 465.577 & 532.715 & 617.676 & 738.770 & 968.262\end{array}$

$\begin{array}{llllll}438.72 & 494.63 & 590.33 & 678.0 ? & 857.91 & 967.29\end{array}$

4.25

4.25

$4, ?$

4.28

4.30

4.36

4.41

4.61

5.40

7.55

14.18

$22.54 \quad 25.47$

2.27
67.70

13.18
0.9950

5.48
5.48

5.48
5.48

5.48

5.48

5.51

5.53
5.57

5.61

5.68

5.68
5.93

6.21

6.21
6.92

6.92
7.84

9.62

17.99
28.55

53.44
81.97

81.97
130.01

322.02

$0.2000 \quad 0.3000 \quad 0.4000$

0.20140 .30640 .4185

$0.2016 \quad 0.3064 \quad 0.4189$

$0.2019 \quad 0.3069 \quad 0.4104$

$\begin{array}{lll}0.2021 & 0.3074 & 0.4199\end{array}$

$\begin{array}{lll}0.2026 & 0.3078 & 0.4204 \\ 0.2031 & 0.3083 & 0.4213\end{array}$

$\begin{array}{lll}0.2031 & 0.3083 & 0.4213 \\ 0.2047 & 0.3107 & 0.4242\end{array}$

$\begin{array}{lll}0.2047 & 0.3107 & 0.4242 \\ 0.2064 & 0.3131 & 0.4270\end{array}$

$0.2100 \quad 0.3179 \quad 0.4332$

$0.2100 \quad 0.31790 .4332$

0.21350 .32310 .4394

$0.21930 .3307 \quad 0.4490$

$0.2416 \quad 0.3598 \quad 0.4850$

$0.2684 \quad 0.39410 .5269$

$\begin{array}{lll}0.3345 & 0.4831 & 0.6347\end{array}$

$0.42940 .6042 \quad 0.7768$

$0.62230 .8407 \quad 1.0529$

$\begin{array}{rrr}1.5564 & 1.9560 & 2.3413 \\ 2.719 & 3.341 & 3.942\end{array}$

$\begin{array}{rrr}5.429 & 6.581 & 7.684 \\ 8.523 & 10.278 & 11.056\end{array}$ 
VALUES OF ALPHA FOR II = 3 ANI) NP=5

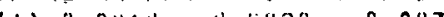

0.002

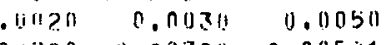

n. nOS U 1

0.0070

$n .01 n n$

0.0200 .00700 $n .0100$
$n .0100$ 0.v01no n.0lizan j.0n3no n.n05i . nonino novas i.0n3ne n.01501

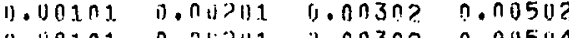

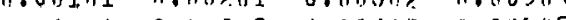

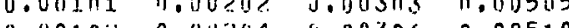

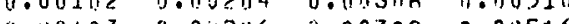
0.001nz n.cusur v.00309 n.nusik

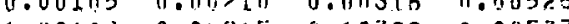

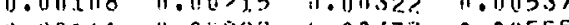

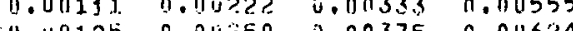

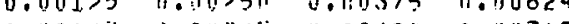
0.00143 0.013?25 il.03424 n. 111712 a. 00297 n.0usus u.nns89 0.00979

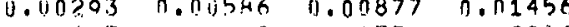
0.00597 0.0liter 1.01 .773 n. 0.059320 .11279 1..15990 0.24299 1..27112 $0.44421 \quad 0.57463 \quad 0.77214$ $1.13375 \quad 1.504157$ 1.85970 ?.29553 2.2955 .3
4.4204
5.0063 $4.4209 \quad 5.0033 \quad 6.5737 \quad 7.8822$ 14.6418 18.5404 21.2 .870 ग 25.3458

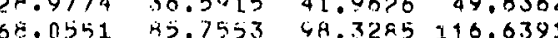
$15.20 ? 5$ 150.2644 117.2420204 .2244 $215.943 ? \quad 272.2175 \quad 311.7683 \quad 369.8737$

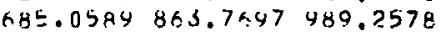
$0.0070 \pi \quad 0.01000 .0201$ 00705 .00715 0.00721 0.00753 0.0077 0.00874 0.00996 0.01368 0.04060 0.3145 ? 0.92367 2.02360 E. 8587 28.5976 $56.817 \mathrm{~h}$ 136.738 ?

$.01 n 1 \quad 0.0201$ $.0103 \quad 0.0206$ $0.01 n 5 \quad 0.0210$ 0.0215 . n.0142 0.0288 0.0 .572 0.4056

1.1044 3.0127 5.4598 1.0 .034 32.075 .2. 928 $147.340 \quad 186.774$ $258.057 \quad 325.928$ 0.0249 0.0386 0.0566 0.6328 1.5278 3.9253 7.0086
12.795 12.795 40.620
79.774 414.3072467 .042
0.0201
$0.0100 \quad 0.0201$ 50.000

70,000 $200.0 n$ उกฺ. 500.unu

\begin{tabular}{|c|c|c|c|c|}
\hline$B E T A / C(A)$ & U. & $0.611 \mathrm{i} n$ & 0.7000 & 0.3000 \\
\hline 0.001 & $U,>44$ & n..5? & 0.880 & 1.152 \\
\hline 0.002 & 0.544 & 0.592 & 0.881 & 1.152 \\
\hline 0.003 & 0.545 & $1 .+93$ & $0.8 \% 1$ & $1.15 ?$ \\
\hline 0.005 & 0.545 & $0 . \sin 4$ & 0.882 & 1.154 \\
\hline 0.007 & $10.5 \Delta 6$ & 0.294 & 0.883 & 1.154 \\
\hline 0.010 & 0.547 & 1.596 & 0.885 & 1.156 \\
\hline 0.020 & 0.551 & $0.70 n$ & $0.88 \mathrm{~B}$ & 1.162 \\
\hline 0.030 & 0.554 & 0.70 .3 & 0.893 & 1.167 \\
\hline 0.050 & $0.5 \times 1$ & $n .71 .2$ & 0.904 & 1.179 \\
\hline 0.070 & 0.264 & 0.7 .1 & 0.993 & 1.192 \\
\hline 0.100 & U. .574 & 0.734 & 0.928. & 1.209 \\
\hline 0.200 & U.0?2 & 11.763 & 0.986 & 1.278 \\
\hline $0.3 n 0$ & 0.072 & 0.441 & 1.053 & 1.358 \\
\hline 0.500 & 0.804 & 0.492 & 1.224 & 1.508 \\
\hline 0.700 & 0.900 & $1 . ? 05$ & 1.474 & 1.862 \\
\hline 1.000 & 1.304 & $1 . ค 61$ & 2.002 & 2.486 \\
\hline 2.000 & 3.500 & 4.1 .78 & $4.9 \cap 3$ & 5.948 \\
\hline 3.000 & 0.790 & 7.821 & 9.118 & $1 ? .980$ \\
\hline 5.000 & 15.307 & 17.686 & 20.555 & 24.644 \\
\hline $7.0 \cap 0$ & 26.628 & $3 n .549$ & 35.432 & 42.451 \\
\hline 10,000 & 47.141 & 54.719 & 63.496 & 75.990 \\
\hline 20.000 & $1 .<9,537$ & $1 \% 1.005$ & $198.6 n 9$ & $237.6 \% 2$ \\
\hline
\end{tabular}

10.000

30.000

50.000

$1 \% 1.005$

क83.10क 701.738 405.762

n.01n1 0.0202 $0.0125 \quad 0.0249$ $\begin{array}{lllll}462.600 & 608.887 & 603.85 & 789.66 & 477.30 \\ & & & & \end{array}$ $90.332 \quad 678.756$

$0.03 \cap 0$ $0.03 n 1$

0.0500 0.0501

0,0700 0.03010 .0501 0.03020 .0503 0.030

0.0503 $0.0303 \quad 0.0505$ 0.0309 0.0315 0,03 ? 2 0.0372 0.0423 0.0574 0.0834 0.1570
0.7987 1.827 4.567

8.111
14.749 14.749
46.723 0.0510
0.0515 0.0525 0.0536 0.0553 0.0617 0,0700 0.0939 1,1344 1.0525

2.273

5.536 17.686 09.437 $255.316 \quad 288.331$ $910.059 \quad 912.598$

0.0702 0,0702 0.0704 0.0707 0,0714 0.0734 0.0746 0.0861 0.0971 0.1821 0.3183

1.2474
2.610

6.284

1.1 .041 62.928 23.414
8.331

0.1000 0.1002 0.1102 0.1006 0.1007 0.1010 0.1119 0.1029 0.1068 0.11 .00
0.1222 0.1373 0.1804 0.4185 1.4878

7.207 12.612 22,782 40.504
327.882 573.731

$0.2000 \quad 0.3000 \quad 0.4000$ $0.201 .40 .3064 \quad 0.4185$ 0.20160 .30640 .4189 $0.20190 .3069 \quad 0.4194$ $0.2021 \quad 0.3074 \quad 0.4199$ $0.2026 \quad 0.3078 \quad 0.4204$ $0.2031 \quad 0.3083 \quad 0.4213$ $0.20470 .3107 \quad 0.4242$ 0.20640 .31310 .4270 0.21350 .32310 .4332 0.21350 .32310 .4394 $0.2406 \quad 0.3508 \quad 0.4850$ $0.2668 \quad 0.3946 \quad 0.5269$ $\begin{array}{llll}0.33388 & 0.4888 & 0.6404\end{array}$ $0.4466 \quad 0.0252 \quad 0.8016$ $0.6910 \quad 0.9246 \quad 1.1502$ $2,0972 \quad 2.6007 \quad 3.0852$ $4.117 \quad 5.010 \quad 5.872$ $9.561 \quad 11.529 \quad 13.406$ $16.648 \quad 19.975 \quad 23.210$ $94.95435 .920 \quad 41.657$ $184.205 \cdot 220.582 \quad 255.616$ $29.932 \quad 515.137 \quad 590.192$

\begin{tabular}{|c|c|c|c|c|c|c|c|c|c|c|}
\hline 9000 & . 9350 & 0.9500 & 0.9700 & $n, 98 \cap 0$ & 0.9900 & 0.9930 & 0.9950 & 0.9970 & 0.9980 & 0.9990 \\
\hline 601 & 1.96 & 2.27 & 2.79 & 3.27 & 4.25 & 4.84 & 5.48 & 6.57 & 7.57 & 9.65 \\
\hline a & & 2.27 & & & & & & & & \\
\hline .68 & 3.3 & 2.2 .7 & & 3.28 & $4, ? 5$ & 4 , & 5.48 & 6. & & \\
\hline .6 & 1.5 & 2.2 & 2. & 3.28 & 4,2 & & 5. & & & \\
\hline 0 & 1.96 & 2.27 & 2. & 3.28 & 4,3 & 4. & 5.48 & 6. & & \\
\hline 6 & 1.9 & 2.27 & 2. & 3.28 & 4,26 & 4. & 5.49 & & & \\
\hline .67 & 1.99 & 2.28 & 2. & 3.29 & $4, ? 8$ & 4. & 5.51 & & & \\
\hline 0 & & 2.2 & & 3.31 & 4 & & & & & \\
\hline os & 2.1 & 2.31 & 2. & 3.34 & 4 , & 4. & & & & \\
\hline 71 & ? & 2.33 & & a. & 4,36 & 4. & & & & \\
\hline 73 & 2.0 & 2.36 & 2. & 3.41 & 4,41 & 5. & 5. & 6. & & \\
\hline 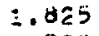 & 2. 1 & 2.47 & 3. & 3.56 & 4,61 & & & & & \\
\hline 92 & $? .2$ & 2.61 & 3.2 & 3.74 & 4,83 & 5. & 6. & 7. & & \\
\hline 90 & 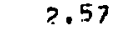 & 2 . & 3 . & 4.21 & 5.43 & & & & & \\
\hline $5 \varepsilon 2$ & 3.00 & 3.44 & 4.19 & 4.87 & 6.26 & 7.11 & 8.00 & & 11.01 & \\
\hline$x$ & 3.9 & 4.48 & 5.42 & 6.28 & 8,05 & & & & & \\
\hline 913 & 9.07 & 10.26 & 12,32 & 14 , ?. & 18.05 & 20. & & & & \\
\hline 20 & $\vdots 6.56$ & 18.69 & 22. & 25,77 & 32.69 & 36.90 & & 49 & 56 & \\
\hline & & 41.66 & 49. & 57.28 & 72.57 & 81.85 & $\$ 1$ & 108 & 124 & \\
\hline & 54 & 71.60 & 85.51 & 96,33 & 124,39 & 140 , & 157 & 186 & 213 & \\
\hline $0 / 1$ & 113.59 & .28 .05 & 152.71 & 975.166 & 222,05 & $250, ? 4$ & 280.52 & 332.76 & 381.10 & 480,23 \\
\hline
\end{tabular}




\begin{tabular}{|c|c|c|c|c|c|c|c|c|c|c|c|c|c|c|c|}
\hline BETA/F(A) & 0.01310 & $0.00 \geqslant 0$ & 0.0036 & 0.0050 & $0.0 n / 11$ & $\therefore, n 1 \cap 0$ & 0.020 & 0.0 & $\sin 0$ & $0.050 n$ & 0.0700 & $0.100 n$ & 0.2000 & 0.3000 & 4000 \\
\hline 0.001 & 0.00900 & $0 . n \cup 200$ & $0.003 n \pi$ & 0.00501 & 0.0070 ? & n. n1 no & 0.020 & 0.0 & $\ln 1$ & $11.050 n$ & 0.0701 & 0.1001 & $0.20 n 4$ & 0.3021 & 0.4070 \\
\hline 0,002 & 0.00100 & 0.00200 & $9.003 n 0$ & 0.00501. & n. ก0701. & $0.010 n$ & $0.02 i$ & 0.0 & $\operatorname{3ng} 1$. & 0.0501 & .0709 & $0.190 ?$ & 0.2007 & 0.3021 & 0.4070 \\
\hline 0.003 & 0.00100 & $0.00 ? 01$. & U.003 31 & $n .00501$ & $0.0070 ?$ & 0.0100 & 0.020 & $0 . n$ & $3 \cap 1$ & 0.0501 & .0702 & $0.100 ?$ & 0.2007 & 0.3026 & 0.4075 \\
\hline 0.005 & 0.00 .271 & $n, n \cup>01$ & $0.00 .30 \%$ & 0.005012 & 0.00703 & n.n1no & 0.021 & $0 . n$ & 312 & 0.0503 & .0703 & $0.1 \cap 05$ & 0.2011 & 0.3026 & 0.4080 \\
\hline 0.007 & 0.00101 & ח. ดบ201 & U. חOBsn? & 0.70504 & 0.00705 & $0.01 n 1$ & 0.020 & $n \cdot n$ & 312 & 1.0503 & 0.0704 & i். $1 \cap 06$ & 0.201 .4 & 0.3031 & 0.4085 \\
\hline 0.010 & (1) . $\mathrm{d}(01 \cap 1$ & n. กo? & 11.110303 & 0.705115 & $0.0 \cup 7 \cup 7$ & n. $\ln 1$ & $0.0 \geqslant 1$ & $0 . n$ & $3 \cap 3$ & 1.0505 & 0.0707 & 0.1010 & 0.2019 & 0.3040 & 0.4094 \\
\hline 0.020 & $0.001 \cap 2$ & $0.01 ; ? 04$ & 0.00306 & 0.70510 & 0.03714 & 0.0102 & 0.020 & 0.0 & 306 & 0.0510 & 0.0713 & $0.101 \mathrm{~A}$ & 0.2035 & 0.3059 & 0.4118 \\
\hline 0.030 & 0.00103 & 0.00206 & 0.00309 & 0,30515 & 0.00721 & $0 . n 1 \times 3$ & 0.020 & $0 . n$ & & 1.0515 & 0.0720 & $0.1 \cap 27$ & 0.2050 & 0.3083 & 0.4142 \\
\hline 0.050 & 0.00105 & $0.00 ? 10$ & 0.0031 .5 & 0.10525 & 0.00735 & 0.0105 & 0.021 & 0.0 & 314 & 0.0523 & 0.0733 & 0.1045 & 0.2083 & 0.3126 & 0,4194 \\
\hline 0.070 & a. U0107 & 0.002 .14 & 0.00321 & 0.00535 & .70748 & $n .01 n 7$ & 0.021 & $0 . n$ & & 0.0533 & 0.0745 & $0.1 \cap 63$ & $0 . ? 114$ & 0.3164 & 0.4242 \\
\hline 0.100 & (1) . 00019 & 0. & 4.0113311 & ח.\$0550 & 0.00769 & 0.09 .10 & 0.028 & $0 . n$ & 37.9 & 1.0547 & 0.0765 & $0.1 \cap 89$ & 0.2162 & 0,3226 & 0.4318 \\
\hline 0.200 & 11.001?0 & $0.011>411$ & 0.00360 & 0.00599 & 0.00330 & 0.0120 & 0.023 & $0, n$ & 358 & .0594 & 0.0828 & 0.1176 & 0.231 .2 & 0,3426 & 0.4552 \\
\hline 0.300 & 0.00130 & 0.04260 & 0.00390 & 0.10649 & 1.009118 & 0.0130 & 0.025 & 0.0 & 396 & $1.064 n$ & 0.0892 & 0.1 & 0.2459 & 0.3 & 0.4774 \\
\hline 0.500 & 0.00150 & $0.0 \cup 300$ & 0.00449 & 0.00748 & .01046 & 0.0149 & 0.02 & $0 . n$ & & $1.073 ?$ & 0.1014 & 0.1 & 0.2740 & 0.3 & 0,5184 \\
\hline 0.700 & 0.00170 & 0.00340 & 0.00509 & 0.00847 & . (1) 1184 & $0.01 \times 9$ & 0.03 & 0.0 & $\sin 0$ & .0821 & 0.1135 & 0.1589 & 0.2997 & 0.4299 & 65 \\
\hline 1.000 & $0.402 n \pi$ & 0.00390 & $0 . \cap 0598$ & $n .80994$ & .01391 & 0.0198 & 0.035 & $0 . n$ & & $1.095 ?$ & 0.1306 & 0.1821 & 360 & 0.4743 & 171 \\
\hline 2.000 & 0.00299 & $0.0 \cup 598$ & 1.00490 & ก. ก1485 & $0.1 .20 \% 1$ & 0.0204 & 0.05 & $0 . n$ & 349 & 1.1363 & 0.1845 & 0.2506 & 0.4375 & 0.5956 & 0.7415 \\
\hline 3.000 & 0.00509 & 0.011795 & 0.011913 & $n . n 19 \% n$ & 0.132741 & 0.0398 & 0.07 & 0. & 1.0 & 0.174 & 0.232 & 0.310 & 0.518 & 0.689 & 0.845 \\
\hline 5.000 & 0.00597 & 0.51188 & 0.01773 & 0.02926 & 0.04060 & 0.0572 & 0.10 & & 157 & 0.241 & 0 . & 0,5 & 0.644 & 0.831 & 0.999 \\
\hline 7.000 & 0.00795 & 0.01579 & 0.02351 & $n \cdot r$ & 0.0 & $n . n$ & & & & 0 . & & 0. & 0. & 0.939 & 1.118 \\
\hline 10.000 & $0.01 \cap 9$ & $0.0 ? 1 \mathrm{~A}$ & 0.0320 & 0.10524 & 0.0720 & 0.100 & 0.11 & & 254 & 0.371. & 0. & 0. & 0.856 & 1.066 & 1.257 \\
\hline 20.004 & 0.0206 & $0,0,404$ & 0.0594 & 0.0955 & 0.1289 & 0.175 & $0.3 i$ & & $4 n \pi$ & 0.540 & 0.661 & 0. & 1.112 & 2 & .570 \\
\hline 30.000 & 1.0301 & U. 0545 & 0.0853 & 0.1349 & 10.1795 & 0.239 & 0.3 & & $n 7$ & $0.67 ?$ & & 0. & & & \\
\hline 50,0 & & 11.11927 & 0.1 & 0.2042 & 0.2654 & 0.343 & 0 , & & 0 & 0.846 & 0.984 & 1.1 .44 & 1. & 1. & 2.078 \\
\hline 70.000 & 0.0664 & 0.2246 & 61 & 3.2640 & 0.3364 & 0.476 & 0.6 & & & 0.973 & 1.120 & 1. & 1. & 2. & 2.296 \\
\hline 100.000 & & 0.1485 & 36 & 0.3 & 0.4237 & 0.525 & 0 . & & 14 & 1. & 1.274 & 1. & 1. & 2. & 43 \\
\hline 00.000 & 0.1 .675 & 0.2898 & 0.381 .7 & 4.5222 & 0.0261 & 0.746 & 1.0 & & 85 & 1.431 & 1.612 & 1. & & & 3.093 \\
\hline 00,5 & 0.2331 & $0 . \overline{3} R_{13}$ & $0.480 \mathrm{H}$ & 0.6471 & 0.1615 & 0.893 & 1. & & 8 & 1.635 & 1.833 & 2,067 & 27 & 3.062 & 3.463 \\
\hline 00,000 & n. 3.309 & $0.521) .3$ & $0.64 \times 2$ & 0,8235 & $0.95 \cup 4$ & 1.007 & 1.6 & & 523 & 1.922 & 2.143 & 2.406 & 3.036 & & 3,980 \\
\hline BETA & .50 & 0.01 & -1 & 0. & 0 & 30 & 5 & & 0 & & 0.9930 & 0.99 & 0.9970 & 0.9980 & 0.9990 \\
\hline 0.001 & 0 & 5 & 4 & 0 & 1. & 1. & 1.73 & 2.05 & 2.32 & 2. & 3.15 & 3.46 & 3.98 & 4,44 & 5.34 \\
\hline 0.002 & & 0.645 & & & & & 1.7 & 05 & & & & & 3. & 4. & .35 \\
\hline 0.003 & 0 & $0 . \times 45$ & & & & & 1. & & 2.32 & 2 & 3. & 3.4 & 3.98 & 4.44 & 5.35 \\
\hline 0,005 & $0,5 ? 0$ & 0.546 & & & & & 1.7 & & 2.32 & & & 3 & 8 & 5 & \\
\hline 0.007 & $0.5 ? 1$ & 0.547 & 98 & 1 & & & 1.76 & 05 & 2.32 & 2.84 & 3. & 3. & 3.98 & 4.45 & 5.35 \\
\hline 0.010 & $0.5 ? 2$ & 0.648 & & & & & 1.74 & 2.05 & & & & & & & \\
\hline 0.020 & 0.525 & $n .651$ & 3 & 7 & 2 & 1.55 & 1.75 & 2.06 & 2.33 & 2.86 & 3 . & 3. & 3.99 & 4. & 5,37 \\
\hline 0.030 & 0.528 & 0.654 & 0.806 & 1 & 1.366 & & 1.75 & 06 & 2,34 & & 3. & 3. & 4.01 & 4. & 5.38 \\
\hline 0.050 & 0.534 & 0.560 & 0 & 1 & 1.375 & & 1,7 & 2.08 & 2.35 & 2.88 & 3. & & 4.03 & & 5.41 \\
\hline 0.070 & 0.538 & 1).667 & (1) & 1.0 & 1 . & & 1. & 2.09 & 2.36 & & 3. & & 4 & & 5.44 \\
\hline 0.100 & 0.347 & 0.676 & 0 & & 1 & & 1.79 & 2.10 & 2.38 & 2.92 & 3. & 3.55 & 4.08 & 4.55 & 5.48 \\
\hline 0,200 & 0.574 & 0.705 & 0 & 1.0 & $1.441 \mathrm{~J}$ & & 1.84 & 2. & 2.44 & 2.99 & 3. & 3. & 4.18 & 4. & 5.60 \\
\hline 0,300 & 0,598 & 0.733 & & 1. & 1.480 & & 1.88 & 2.22 & 2.50 & & 3.38 & 3.7 & 4.27 & 4. & 73 \\
\hline 0.5 & 0.644 & $0.7 \mathrm{~d} 3$ & & 1.1 & & & 1. & 2.31 & 2.61 & 3.18 & 3.5 & 3.5 & 4.44 & 4.95 & .94 \\
\hline 0.700 & 0.686 & 0.827 & 998 & $1 . ?$ & & & 2 . & 2.40 & 2.70 & & 3.6 & 3.5 & 4.59 & 5.12 & 6.14 \\
\hline 1.000 & $0.74 ?$ & 0.888 & & 1. & & & 2.1 & 2.51 & 2.83 & 3.45 & 3. & 4.1 & & & \\
\hline 2.000 & $0.8 \beta 8$ & 1.047 & & 1.4 & & & 2.42 & 2.83 & 3.19 & 3.86 & 4.25 & 4.6 & & 5.94 & 7.12 \\
\hline 3,000 & & 1.16 & & 1 & & & 2. & 3.0 & 3.44 & 4.17 & 4. & 5.0 & & 6.41 & .68 \\
\hline 5.000 & 1.169 & 1.352 & 1 & 1.8 & & 2.67 & 2.96 & 3.43 & 3.84 & 4.65 & 5. & 5. & 6. & & 8.52 \\
\hline 7.000 & 1. & 1.492 & & & & & 3. & 3.71 & 4.1 .6 & 5.02 & 5. & & 6.91 & & 9.19 \\
\hline 10.000 & 1.450 & 1. $.65 \mathrm{~B}$ & 1 & 2.246 & 2.841 & $3.1,7$ & 3.50 & 4.05 & 4.53 & 5.46 & 6. & 6. & & & 9.97 \\
\hline & 1.791 & 2.0 .29 & & 2.7 & 3.406 & & 4.18 & 4,82 & 5.38 & 6.47 & 7.1 .2 & 7.7 & 8.87 & 5 & 11.77 \\
\hline $0 \cap 0$ & 2.421 & 2.230 & 2.597 & 3.028 & 3.191 & 4.22 & 4.64 & 5.35 & 5.96 & 7.17 & 7. & & & & \\
\hline 50.000 & 2.345 & 2.639 & 2.994 & $3.4 \%$ & 4.536 & & 5.30 & 6.09 & 6.75 & 9.16 & 8.5 & 9. & & & .76 \\
\hline .000 & 2.5 & 2.9012 & & & & & 5. & 6. & 7.41 & & & 10. & & & \\
\hline & & 3.203 & & & 5.208 & & & 7.2 & 8,11 & 9.73 & 10. & 11. & 13.27 & & 17.56 \\
\hline 00.000 & 3.463 & 3.873 & 4.361 & 5.04 & 6.246 & & 7.59 & B.71 & & & & & & & \\
\hline & & 4.315 & $4.8 \times 5$ & & & & 8. & 8.01 & 10.75 & 12.87 & 14.1 & 15.40 & 17.53 & 19.43 & 23.15 \\
\hline 00.000 & 4.445 & 4.949 & .547 & 6.413 & 7.928 & 8.77 & 9.81 & 9.01 & 12.25 & 14.66 & 16.07 & 17.50 & 19.94 & 22.11 & 26.35 \\
\hline
\end{tabular}


VALUES OF ALPHA FOR IV $=4$ ANM NP $=2$

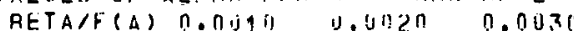
$0.0 n$ 0,003 0.005 0.007 0.010 0.02 0.030 0.050 0.07 0.1100 0.20 0.300 0.700 1.000
2.000 2. 0000
3.000 3.000 5.0110 7.090

10.000
20.400

20.000

30.000
50.000

70.00

100.000

200,000

300.000

5no.0.0

0.001

0.003

0.005

0.007

0.011

0.0 ?

0.030

0.051

0.100

0.100
0.200

0.300

0,500

0.700

1.000

3.000

3.000

5.000

7.0000
10.000

10.000
20.000

30.000

50.000

70.000

100.000

(200.00

350.000 0. 0.60100 0.10?01 1.00301 n.00501 n.00101 0.00201 i.003nz 0.00502

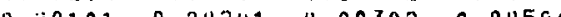

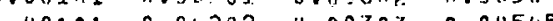

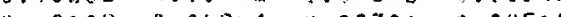

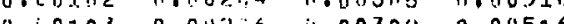
U. 0.001ns 0.0u?.10 0.023160 .00526

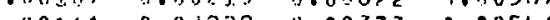
11.00134 $1.112 \% 4 R$ 11.0037? 1.011610

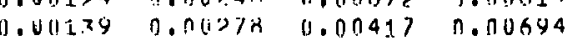
0.00175 n.00350 0.00524 0.00872 1.00\% n.mis c.0065 n.0108 n.un?ag 0.0u548 0.00304 0.01465 $\begin{array}{llll}0.130000 & 0.01333 & 0.0206 ? & 0.03349\end{array}$ a. ग1285 n.n2534 1j.03742 n.n6137

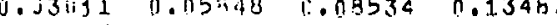
$0.35 .395 \quad 0.14750 \quad 0.14655 \quad 0.22308$ $\begin{array}{llll}0.1000 & 0.1421 & 0.251 .1 & 0.3622\end{array}$ $0.2983 \quad 11.4700 \quad 0.59040 .7606$ $0.4493 \quad 1.7177 \quad 0.8620 \quad 1.0643$ $\begin{array}{llll}0.8993 & 1.11106 & 1.2780 & 1.5259\end{array}$ $\begin{array}{llll}1.0091 & 1.411100 & 1.6060 & 1.89112\end{array}$ $\begin{array}{lllll}1.41177 & 1.7741 & 2.0094 & 2.3413\end{array}$ $\begin{array}{llll}2.1849 & 2.6770 & 3.0051 & 3.4745\end{array}$

$\begin{array}{llll}2.7 n A 6 & 3.3536 & 3.7642 & 4.3230 \\ 3 . \sin 3 & 4.4<51 & 4.95 \times 3 & 5.6734\end{array}$

0.7000

0.8000

1.01100 a. 145

0.045

$0.64 h$
0.647

ii, $i+46$

i. 031

$0 ., 555$

$n .561$

II. 579

0.71 .7

0.756

0.939

1.114

1.027

7.230

3.727

3.7 .77

5.4 .34

7.596

9.027

11.827
15.347
18.437

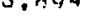

1.7.

18.437

24.191

0.999

1.001
1.001

$0.797 \quad 1.001$

$0.794 \quad 9.0113$

$0.800 \quad 1.003$

0.806

$0.814 \quad 1.020$

ח.

0.833

1.013
1.0313

1.041
1.087

1.137

0.998

1.009
1.101

1.236

1.652
2.006

2.59

3.099
3.715

5.315

6.551

10.110

12.12 .4

1.155

1.236

1.492

1.900
2.360

3.028

3.585
4.300

6.131
7.543

9.789
11.605

13.909

19.761
24.247
$0.00 \%$ 0.00700 00701 0.00702 $0.0070 x$ 0.00705 0.00707 0.10707 0.00715 0.00736 $0.1) 0751$ 0.110777 $0.049 / 0$ 0.01219

$0.0152 n$

0.04704

0.0839

0.17945

0.28829
0.4495

0.4495
0.6845

0.8845
1.2074
1.1033

2.097

2.5854

3.8062

$4.735 n$
6.2075

6.818

$\begin{array}{rr}.9300 & 0.9500 \\ 1.54 & 1.73 \\ 1.54 & 1.74\end{array}$

1.354

1.354

1.553

1.362

1.363

1.387

1.402

1.455

1.513

1.755

1.73

2.494

3,791

4.468

5.330

9.301

12.048

14.276

17.106
24.278

29.77

38.484

c.010n 0.0100

1100

$.01 n 1$

0.0101

.010

. 010

.01?

0.017

. 204

0.0660

0.1161
1.2302

0.3698

1.024

1.373

2.334

2.864

1.54
1.54

.55

1.55
1.55
1.55

1.56
1.57

1.58

1.60

1.762
1.85

1.98

1.98
2.78
3.32

3.3

4.96

5.92
8.37

10.28

13.30
15.76

26.78

4.64
$10.446 \quad 11.453$

$\begin{array}{ll}2.05 \\ 1.74 & 2.05\end{array}$

$\begin{array}{ll}.74 & 2.05\end{array}$

$1.74 \quad 2.05$

2.05

$\begin{array}{ll}1.75 & 2.06 \\ 1.75 & 2.07\end{array}$

$1.76 \quad 2.08$

$1.79 \quad 2.11$

$1.92 \quad 2.26$

2.062

42

3.09

6.50

1910.54

12.90

$0.9800 \quad 0.9900$

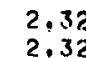

$\begin{array}{lll}2.84 & 3.15\end{array}$

$2.32 \quad 2.84 \quad 3.15$

$2.32 \quad 2.84 \quad 3.15$

2.33. $2.85 \quad 3.15$

$2.33 \quad 2.8 .5 \quad 3.16$

$2.34 \quad 2.85 \quad 3.17$

$2.37 \quad 2.90 \quad 3.21$

$2.39 \quad 2.72 \quad 3.24$

$\begin{array}{lll}2.55 & 3.1 .2 & 3.44 \\ 2.73 & 3.33 & 3.67\end{array}$

$2.91 \quad 3.54 \quad 3.90$

$4.01 \quad 3.86 \quad 4.25$

$\begin{array}{ll}7.17 & 6.28 \\ 8.87\end{array}$

8.32

$1: .73$

14.35
18.54

9.97

14.03

$18.17 \quad 18.82$

$26.17 \quad 24.28$

$\begin{array}{ll}26.23 & 28.73 \\ 31.34 & 34.33\end{array}$

$\begin{array}{lll}26.26 & 31.34 & 34.33 \\ 37.20 & 44.40 & 48.62\end{array}$

59.60
76.97

0.1000

0.1001

0.1002 
VALUES OIF ALPHA FOR IV $=4$ AND NP $=3$

\begin{tabular}{|c|c|c|c|c|}
\hline $\mathrm{FT}$ & & $0.11 n$ & 0.0030 & $1) .0050$ \\
\hline 001 & 0.00100 & 0.00200 & $0.003 n \pi$ & 0.30501. \\
\hline & נ) & ח. & $n .003 n 0$ & 1501 \\
\hline .003 & 0.110100 & $0 . \| 1 \geqslant 01$. & 0.07301 & 0.00501 \\
\hline & $0.001 \cap 1$ & $0.00 ? 01$ & $0.003 n$ ? & $0.0050 ?$ \\
\hline .007 & $0.0 \cap 101$ & ח.ก०201 & $(1,0 \cap 3 \cap ?$ & 0.00504 \\
\hline .010 & $0.0 n 1, n 1$ & 0.00202 & $0,0 \cap 3 \cap 3$ & 0.00505 \\
\hline 1.0 & $0,401,2$ & $0,0 \cup>04$ & 10.03306 & 0.00510 \\
\hline . & 0.00 & $0,0 \cup 206$ & $0.003 \cap 9$ & 0.00516 \\
\hline & 0.00105 & $0 . n \cup \geq 10$ & 0.00316 & 0.00526 \\
\hline 0 & $0 . i$ & 0.1 & 0.0 & 1537 \\
\hline 00 & 0.0 & $0, n \varphi ? 22$ & 3.0 & 0.010555 \\
\hline 0.2 & $0.001>5$ & 0.0 & 3.0 & 0.100623 \\
\hline & 0.0 & $0.0 \cup 283$ & 3.00425 & 0.00707 \\
\hline & 0.0 & 0.10 & 0.0 & 0.00933 \\
\hline .700 & 1). .0253 & 0.1010505 & 0.00756 & 0.01257 \\
\hline 1.000 & 0.0 & $0 . n$ & 0.01190 & \\
\hline 2.000 & 0.01479 & 0.112917 & 11004317 & 0.070118 \\
\hline 3.000 & 0.03352 & i). 0 & 0.10739 & $n .16753$ \\
\hline 5.000 & $(1.1$ & 0.23870 & 0.3 & 0.44994 \\
\hline .000 & 0.2 & 0.45556 & 0.5 & 0.74343 \\
\hline 000 & 0.5500 & 0.7787 & 0.9294 & 1.1387 \\
\hline 000 & & 1.6590 & 1.8979 & 2,2142 \\
\hline (viv & 1.9560 & 2.4100 & 2. & 3.1348 \\
\hline .000 & 3.0547 & 3.6943 & 4.1323 & 4.7427 \\
\hline 1000 & 4.1 & 4.0495 & 5.4064 & 3.1922 \\
\hline & & 6.4288 & & 8.1721 \\
\hline 0. & & 10 & & 094 \\
\hline & & $0 \cap 80$ & 16. & 7371 \\
\hline & & & $9,3<15$ & .9093 \\
\hline
\end{tabular}

$\begin{array}{llll}14.57 n 9 & 22.1110 & 24.5214 & 27.7093\end{array}$

$\begin{array}{rrr}0.007 n & 0.0100 & 0.0200 \\ 0.0070 n & 0.0100 & 0.0200 \\ 0.00701 & 0.0100 & 0.0201 \\ 0.00702 & 0.0100 & 0.0201 \\ 0.007133 & 0.0100 & 0.0201 \\ 0.00715 & 0.0101 & 0.0201 \\ 0.00707 & 0.0101 & 0.0202 \\ 0.00715 & 0.0102 & 0.0204 \\ 0.00721 & 0.0103 & 0.0206 \\ 0.00737 & 0.0105 & 0.0210 \\ 0.00753 & 0.0107 & 0.0215 \\ 0.00777 & 0.0111 & 0.0222 \\ 0.00872 & 0.0174 & 0.0248 \\ 0.00988 & 0.0101 & 0.0281 \\ 0.01304 & 0.0186 & 0.0308 \\ 0.01755 & 0.0249 & 0.0491 \\ 0.02741 & 0.0388 & 0.0754 \\ 0.019559 & 0.1315 & 0.2350 \\ 0.22046 & 0.2892 & 0.4609 \\ 0.54697 & 0.5605 & 0.9103 \\ 0.86455 & 1.0033 & 1.3066 \\ 1.2875 & 1.459 & 1.833 \\ 2.4519 & 2.719 & 3.318 \\ 3.4438 & 3.802 & 4.598 \\ 5.1924 & 5.712 & 6.871 \\ 6.7644 & 7.428 & 8.904 \\ 8.9197 & 9.789 & 11.712 \\ 15.1758 & 1.6 .618 & 19.883 \\ 20.6461 & 22.599 & 27.024 \\ 30.4117 & 33.296 & 39.704\end{array}$
$0.03 \cap 1$ 0.0301 0.0301 0.0302 0.0303 $0.03 \cap 6$ 0.0315 0.0322 0.0332 0.0371 0.0420 0.0548 0.0727 0.1111 0.3193 1.076 1.507 2.086
3.718 3.718
5.139 5.139
7.653 7.653 9.912
13.040 22.080 29.984
44.099

$0.050 n$
0.0500
0.0501
0.0501
0.0503
0.0503
0.0505
0.0510
0.0515
0.0525
0.0535
0.0553
0.0616
0.0694
0.0899
0.1176
0.1740
0.4518
0.760
1.310
1.791
2.440
4.300
5.910
8.782
11.361
14.916
25.254
34.272
50.447

0.0700 0.0701 0.0701 0.0703 0.0709 0.0707 0.0713 0,0734 0.0748 0.0772 0,0859 0.0964
0.1237 0.1601 0.1601
0.2319 0.5546 0.889 1.482
2.002 2.002
2.708 4.727 6.497 9.637
12.460 12,460
16,343 27.635
37.507 37.507
55.146
0.1000 0.1001 0.1002 0.11005 0.100 0.1010 0.101 .8 0.1048 0.1068 0.1068 0.1218 0.1361 0.1725
0.2200 0.3097 0.6795 1.043
1.684
2.254 3.024 5.246
7.192 10,644 13,757 18,052
30,503 30,503 60.822

\begin{tabular}{|c|c|c|c|c|c|c|c|c|c|c|}
\hline .9004 & .9300 & 0.9500 & 0.9700 & $0.98 n n$ & O 99. & 0.9930 & 0,9950 & 1.9970 & 0.9980 &, 9990 \\
\hline 1.35 & 1.54 & 1.73 & 2 & 2.32 & 2.84 & 3 . & 3.46 & 3.98 & 4.44 & 5 \\
\hline 1.3 & 1.5 & 1.7 & & & & 3 & & 3. & 4.44 & \\
\hline 1.3 & & 1,74 & & & & & 3. & & 4.44 & \\
\hline 1. & 1. & 1.74 & & & & & & 3. & 4.45 & \\
\hline & & 1. & & & & & & & & \\
\hline & 1. & 1,7 & & & & 3. & & & & \\
\hline & $j$ & 1.7 & & & & & & & & \\
\hline & 1.5 & 1. & & & & & 3. & 4. & & \\
\hline & 1.5 & 1. & & & & & & 4. & & \\
\hline & 1. & & & & & & & 4. & & \\
\hline & 9.1 & & & & & & & & & \\
\hline & 1. & & & & & & & & & \\
\hline & & & & & & & & & & \\
\hline 1. & 1. & 2. & 2 & & & & & & & \\
\hline & 2. & 2. & 2.6 & & & & & & & \\
\hline & 2. & & & & & & & & & \\
\hline & 3. & 3. & 4. & 4. & 5. & & & & & \\
\hline & 4. & 4. & 5. & 6 & 7, & 8 , & & 10 & & \\
\hline & & 7. & & & & & & & & \\
\hline & 8 & 9.07 & 10. & 11. & 13 & & & & & \\
\hline 9.728 & $\because$ & 11.79 & & & & & & & & \\
\hline 。 & i. & 19 & & 25 & 29 & & & & & \\
\hline & & & & & & & & & & \\
\hline$\therefore$ & 35. & 39.22 & 44 & 49.78 & & 65 & 70 & 80 & 05 & \\
\hline & & & & & & & & & & \\
\hline - & & & & & 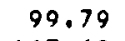 & 109 & 118.84 & 135.13 & & \\
\hline & & 110 & & 140.75 & & & & 227 & & \\
\hline & & & 171.7 & 190.5 & 227 & 248 & 3 & 307.86 & 340 & \\
\hline $984<>$ & & & 252.20 & 279.54 & 333.25 & 64 & 97 & 451.42 & 499. & \\
\hline
\end{tabular}

$0.2000 \quad 0.3000 \quad 0.4000$ $0.2004 \quad 0.3021 \quad 0.4070$ $0.20 n 7 \quad 0.3021 \quad 0.4070$ $0.2007 \quad 0.30260 .4075$ $0.2014 \quad 0.3031 \quad 0.4085$ $0.2019 \quad 0.3040 \quad 0.4094$ $0.2035 \quad 0.3059 \quad 0.4118$ 0.20850 .3131 .0 .4199 $0.2121 \quad 0.3179 \quad 0.4256$ $\begin{array}{lll}0.2178 & 0.3250 & 0.4342\end{array}$ $0.2383 \quad 0.3522 \quad 0.4661$ $0.2625 \quad 0.38320 .5022$ $0.3937 \quad 0.5432 \quad 0.6833$. 0.51840 .6891 . 0.8445 $0.9747 \quad 1.1998 \quad 1.4019$ $\begin{array}{lll}1.402 & 1.679 & 1.929\end{array}$ $\begin{array}{lll}2.166 & 2.540 & 2.883 \\ 2.849 & 3.314 & 3.945\end{array}$ $\begin{array}{lll}3.787 & 4.314 & 3.945 \\ 3.787 & 4.933\end{array}$ $6.497 \quad 7.482 \quad 8.386$ $\begin{array}{rrr}8.874 & 10.186 & 11.422 \\ 13.101 & 15.023 & 16.831\end{array}$ $\begin{array}{lll}16.923 & 19.395 & 21.684\end{array}$ $\begin{array}{lll}22.172 & 25.407 & 28.398\end{array}$ $\begin{array}{lll}37.385 & 42.817 & 47.883\end{array}$ $\begin{array}{lll}40.752 & 58.137 & 64.881\end{array}$ $0.7000 \quad 0.30110$ $0.796 \quad 0.999$ 0.797 1.0u1 $0.798 \quad 1.001$ $0.800 \quad 1.003$ $0.803 \quad 1.009$ $0.806 \quad 1.013$ $0.814 \quad 1.020$ $0.822 \quad 1.030$ $\begin{array}{ll}0.834 & 1.043 \\ 0.877 & 1.091\end{array}$ $\begin{array}{ll}0.877 & 1.091 \\ 0.976 & 1.144\end{array}$ $1.036 \quad 1.268$ 1.410 1.640
2.460 2.460
3.253 4.71 6.047

13.284

13.284
18.022 26.475 34,069 75.013 101.746 
VALUES OF ALPHA FOK IV $=4$ ANID NP $=4$

\begin{tabular}{|c|c|c|c|c|}
\hline$B \in T A$ & 0.0010 & 00 & 0.0030 & 0.0050 \\
\hline 0.001 & 0.00100 & $n .01300$ & 0.00300 & $0, n 0501$ \\
\hline 0.002 & 0,00100 & ח. DU? :0 & $0.003 n n$ & 0.00501 \\
\hline 0.003 & 0.00200 & 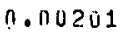 & 0.00301 & 0.00501 \\
\hline 0.005 & $0.001 n 1$ & $0 . \cap 0 ? \cap 1$ & 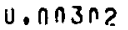 & 0.00502 \\
\hline $0.00 \%$ & 0.00101 & 0.00201 & $0.003 \cap 2$ & 0.00504 \\
\hline 0.010 & $0.0 \cap 1 \cap 1$ & 0. กบ2 is? & 11.00303 & 0.00505 \\
\hline 0.420 & 0.00102 & $0.00 ? 04$ & $1] .00306$ & $n .00510$ \\
\hline 0.030 & 0.00103 & $0.0 \cup 206$ & 0.00309 & 0.00516 \\
\hline 0,050 & 0.00105 & 0.00210 & 0.00396 & 3.00526 \\
\hline .070 & $0 . \ln 108$ & $0.0 \cup ? 15$ & $0.003 ? 2$ & 1.00537 \\
\hline 0.100 & 0.0 .0111 & $0.00 \geqslant 22$ & 0.00333 & 300555 \\
\hline 0,200 & 0.00125 & 0.0 is 250 & 0.00375 & 7.00624 \\
\hline 0.300 & 0.00142 & 0.0 (1) 35 & $0.004 ? 7$ & n.110711 \\
\hline 0.500 & 0.00104 & 0.00337. & 0.00580 & $n, 00965$ \\
\hline 0.700 & 0.00277 & $0.00553^{\circ}$ & 0.00827 & 0.01374 \\
\hline 1.000 & 0.00498 & 0.00991. & 0.01482 & 0.02452 \\
\hline 2.000 & 0.03011 & 0.05848 & 0.08534 & 0.13487 \\
\hline 3.000 & 0.10010 & 0.19543 & 0.26779 & n.38318 \\
\hline .000 & 0.74898 & 0.65855 & 0.79779 & 0.99186 \\
\hline .000 & 0,36264 & 1.14063 & 1.32183 & 1.57360 \\
\hline 0.000 & 1.4553 & 1.0235 & 2.0667 & 2,4100 \\
\hline 000 & 3.2488 & 3.9950 & 4.4527 & .1089 \\
\hline 000 & 5.1111 .2 & 0.1236 & $6.81 \cap 2$ & 7.7906 \\
\hline 50.000 & $8.6 \% 56$ & 10.5693 & 11.4985 & 13.1159 \\
\hline 0.000 & 12.2309 & 14.5960 & 16.1906 & 4489 \\
\hline 00.000 & 17.5038 & 20.9513 & 23.2096 & 26.4445 \\
\hline 00,000 & $35.37 n 8$ & 42.1457 & 46.6623 & 53.0710 \\
\hline 00.000 & 53.1320 & 63.2944 & 70.1 .303 & 79.6518 \\
\hline 0.00 & 88.685 & & 16.8832 & $32.93=$ \\
\hline
\end{tabular}

0.0100
0.0100

0.0200

0.0201

0.0201

0.0201

0.00705

0,00707

0.00715

0.00721

0.00753

0.00777

0.00874

0.00994

0.01916

0.03405

0.17945
0.47330

1.13110

1.75480

2.6579

5,5895

14,3061

20.0968

28.7943
57.7707

86.7319

$88.6850105 .5307 \quad 1.16 .8832 \quad 132.9354$

0.010

0.0101

0.0202

010

0.0204

0.0105

0.0210

$\begin{array}{ll}0.0105 & 0.0210 \\ 0.0111 & 0.0215\end{array}$

0.0175

0.0249

$0.0142 \quad 0.0282$

0.0380
0.0535

n.027

0.2302

0.3932

.291

0.8159

1.9637

1.6385
2.4252

3.581
7.382

9.146

11.178
18.724

15.672
22.019

26.292
37.629

31.526

37.629
75.379

0.0300

0.0301

0.1000

$0.2000 \quad 0.3000 \quad 0.4000$

0.0501

0.0302

0.0503

0.0503

0,0701

0.1002

0.1002

0.1005

0.1010

0.1018

0,0510

0.0306

0.0515

0.0713

0.0315

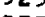

0.0734

0.1048

0.1068

$\begin{array}{lll}0.0553 & 0.0772 & 0.1099\end{array}$

$0.0517 \quad 0.0859$

0.1220

0.0697

0.0969

0.1368

0.2362

$0,1275 \quad 0.1728$

$\begin{array}{llll}0.1339 & 0.2085 & 0.2745 & 0.3612 \\ 0.5069 & 0.6719 & 0.7959 & 0.9408\end{array}$

0.973

1.194
2.200

1.358

1.551
2,738

3.177

3.509

4.628
9.439

5.094

8.218

14.230

10.339

2.738
3,903

5.651
11.437

17,198

20.829

23.820

26.078

29,191
41.780

$\begin{array}{ll}33.418 & 36.530\end{array}$

28,764

40,315

$95,643 \quad 104.676 \quad 115,418$

$0.2004 \quad 0.3021 \quad 0.4070$

$0,2007 \quad 0.3021 \quad 0.4070$

$0.2007 \quad 0.3026 \quad 0.4075$

$\begin{array}{lll}0.2014 & 0.3031 & 0.4080\end{array}$

$0.2019 \quad 0.3040 \quad 0.4094$

$0.2035 \quad 0.3059 \quad 0.4118$

0.20520 .30830 .4147

$0.2085 \quad 0.3131 \quad 0.4199$

$0.2121 \quad 0.3179 \quad 0.4256$

$\begin{array}{lll}0.2178 & 0.3250 & 0.4342 \\ 0.2387 & 0.3522 & 0.4666\end{array}$

$\begin{array}{lll}0.2635 & 0.3846 & 0.5041\end{array}$

$0.3288 \quad 0.4657 \quad 0.5966$

$\begin{array}{lll}0.4657 & 0.7263\end{array}$

$\begin{array}{lll}1.2837 & 1.5431 & 1.7815\end{array}$

$\begin{array}{lll}2.006 & 2.357 & 2.681\end{array}$

$\begin{array}{lll}3.436 & 3.980 & 4.491\end{array}$

$4,857 \quad 5,605 \quad 6,299$

$14.062 \quad 16.130 \quad 18.052$

$21.134 \quad 24.217 \quad 27.085$

$\begin{array}{lll}35.310 & 40.437 & 45.136\end{array}$

$49.409 \quad 56.611 \quad 63.172$

$\begin{array}{rrr}141.236 & 161.744 & 180.787\end{array}$

BETA/F (A) 0.5000 J.0000 $0.7000 \quad 0.8000$

$\begin{array}{lllll}0.9000 & 0.9300 & 0.9500 & 0.9700 & 0.96\end{array}$

0.9900

1.964

88,331

0,001

$\begin{array}{rrrr}0.519 & 0.545 & 0.796 & 0.999 \\ 0.519 & 0.445 & 0.797 & 1.001 \\ 0.599 & 0.645 & 0.797 & 1.001\end{array}$

0.002

1.001
1.001

1,352

1.354
1.354

1.354

0.00

$0.520 \quad 0.446 \quad 0.798 \quad 1.001$

$\begin{array}{llll}0.521 & 0.447 & 0.798 & 1.003 \\ 0.522 & 0.448 & 0.800 & 1.003\end{array}$

0.010

0.020

0,030

0.050

0.070

0.100

0.2011

0.300

0.500

0.700

1.000

2.000

3.000
5.000

5.000
7.000

7.000
10.000

10.000
20.000

20.000

30.000

50.000

70.000
100.000

200.000

300.000

$\begin{array}{lll}0.651 & 0.803 & 1.009\end{array}$

0.555

1,013

1.358

1.362

1.368
1.377

1.377
1.387

1.387
1.402

1.459

1.526

1.688
1.890

1.890
2.261

2.261
3.791

5.460
3.889

3.889
12.338

$\begin{array}{llll}3.0109 & 3.360 & 2.597 & 3.028 \\ 5.002 & 5.567 & 3.802 & 4.399\end{array}$

.0n1 7.775 8.721

$10.1103 \quad 13.102$

30.0172

$30.015 \quad 33.235 \quad 37.141$

$\begin{array}{ll}17.533 & 19.33 \\ 34.822 & 38.42\end{array}$

1.54
1.54

1.54
1.55
1.55

1.73
1.74
1.74

2,05
2,05
2,05

1.55

1,74

$\begin{array}{lll}2.05 & 2.32 & 2.84 \\ .05 & 2.32 & 2.84\end{array}$

0.9930
3.15

0.9950

$0.9970 \quad 0.9980 \quad 0.9990$

1.55

1,74

$\begin{array}{lll}2.05 & 2.32 & 2.84 \\ 2.05 & 2.84\end{array}$

3,15

3.46
3.46

3.15

3.46

3.46

3.46

$2.05 \quad 2.33 \quad 2.85 \quad 3.15$

1.57

1.75

$2.07 \quad 2.33 \quad 2.86$

3.16
3.17

1,77

1.60

1.66
1.73

1.91

2.13
2.53

1,86

$1.94 \quad 2.19$

2,13

2,37

2.81
4.64

6.05

6.65

$\begin{array}{lll}.08 & 2.35 & 2.8\end{array}$

$2.37 \quad 2.90$

$2.19 \quad 2.47 \quad 3.02$

$2.28 \quad \overline{2.57} \quad 3.14$

$\begin{array}{lll}2,49 & 2.81 & 3.42 \\ 2,76 & 3.11 & 3.77\end{array}$

$\begin{array}{lll}3.26 & 5.66 & 4.43 \\ 5.35 & 5.96 & 7.17\end{array}$

$7.64 \quad 8.51 \quad 10.20$

$\begin{array}{lllll}13.62 & 10,77 & 12,34 & 13.73 & 16.40\end{array}$

22.69

$\begin{array}{llllll}52.155 & 57.53 & 62.02 & 48.07 & 53.32 & 63.66 \\ & 95.64 .84 & 79.77 & 95.15\end{array}$

$\begin{array}{llll}70.018 & 75.370 & 61.93 n & 70.863\end{array}$

$100.038 \quad 110.658 \quad 123.658 \quad 141.480$

$200.074 \quad 229.314 \quad 247.315$

$300.1150 \quad 331.748 \quad 370.850 \quad 424.501$

$\begin{array}{rrrrrr}121.400 & 135.64 & 104.68 & 119,57 & 132.59 & 158.33 \\ 136,36 & 167,36 & 185,67 & 221.31\end{array}$

$\begin{array}{llllllll}173.463 & 191.04 & 209.11 & 238.89 & 264.89 & 316.16 & 345.95\end{array}$

3.17
3.19
3,21
3,24
3,34

3.24

3.34
3,47

3.78
4.16

4.87

7.87

11.18
17.99

17.99
24.86
35.19
69.64

$69.64 \quad 75.87$

3.98
3.98

3.98

3.98
3.98

3.98

3.99

3.99
4.01

3.48

3.50
3.52

3.55

3.67

3.81
4.14

.4 .56

5.34

8.60
12.20

19.61

27.05
38.30

75.87

4.03

4.06

4.22

4.38

4.75
5.22

6.11

9.80

13.89
22.32 


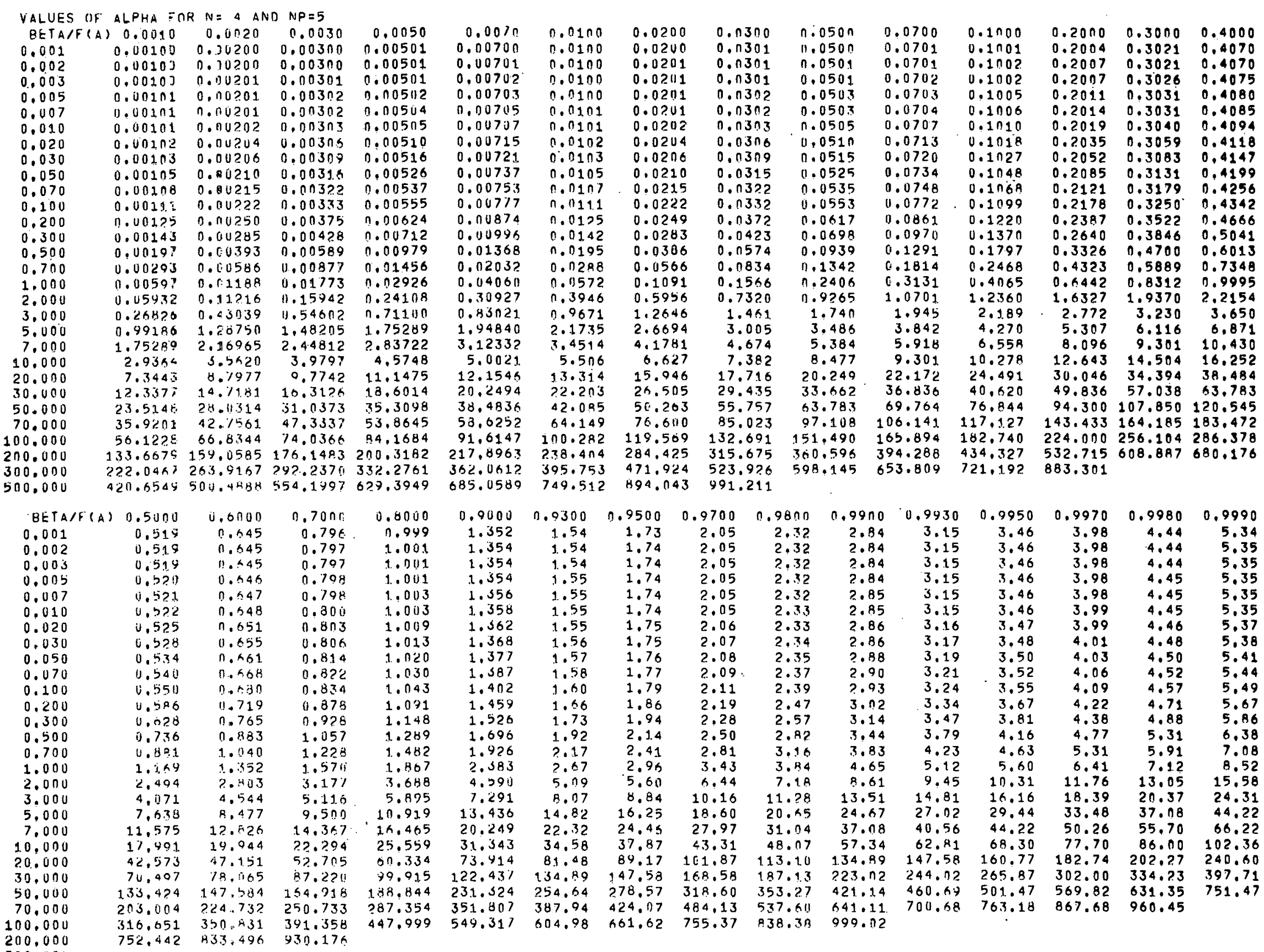




\begin{tabular}{|c|c|c|c|c|c|c|c|c|c|c|c|c|c|c|c|}
\hline$R \in T A / F(A)$ & 11.0110 & 19. $4 n 2^{n}$ & $0 . \cap \cup 30$ & 0.0050 & $0.00 ? 0$ & $9.01 \cap 0$ & \multirow{2}{*}{\multicolumn{2}{|c|}{$\begin{array}{l}0.0200 \\
0.0200\end{array}$}} & $0.03 \cap 0$ & 0.0500 & 0.0700 & 0.1000 & 0.2000 & 0.3000 & 0.4000 \\
\hline 0,001 & $0 ., 0100$ & $0.01,200$ & $0.003 n i$ & 0.01501 & ח0. & 0.0100 & & 0.0 & 301 & $n .0500$ & 0.0701 & $0.1 \cap 01$ & 0.2002 & 0. & 0,4027 \\
\hline 0.002 & $0 . \ln 1 \mathrm{n}$ & ח. ח ח ח & i) $\cap 030 ! n$ & 0.01501 & 0.30711 & 0.0100 & 0.02 & 0.0 & 301 & 1). .0501 & 0.0701 & 0,1002 & 0.2004 & 0.3012 & 0,4032 \\
\hline $0.013 \mathrm{~s}$ & 0. toinu & $n, \| \cup 2 n 1$. & 0.00301 & 0.104501. & 0. กบาบ? & 0.01 .00 & 0.0 ? & 0.0 & $3 n 1$ & 0.0501 & 0.07 .02 & 0.1002 & 0.2004 & 0.3012 & 0.4032 \\
\hline 0.005 & H. 00101 & $n .04 \geqslant 1) 1$ & $0.0030 ?$ & n. ก) 502 & 0.00753 & $0, n 1 \cap 0$ & 0.02 & 0.0 & 302 & (1. .0503 & 0.0703 & 0.1005 & .2009 & 0.3016 & 0.4037 \\
\hline $0.0 \cap 7$ & n.u03n1 & n.ก|ग? & $11.01030 \%$ & $n, n ! 5114$ & 0.00735 & 0.0101 & 0.02 & 0.0 & 302 & 0.0503 & 0.0704 & 0.1006 & 0.2011 & 0.3021 & 0.4042 \\
\hline 0.010 & 1). บ $0.1 \cap 1$ & ? & J. 010303 & $0.0 \cup 5 i 15$ & 0.00707 & ก. 8101 & 0.02 & $0 . n$ & 303 & 0.0505 & 0.0707 & 0.1010 & 0.2016 & 0.3026 & 0.4051 \\
\hline 0.020 & 1). $4 \cap 102$ & $0.1 ; \mid j>14$ & Q. 010356 & 0.011510 & 0.00714 & 0.0102 & $0.0 ?$ & 0.0 & 306 & 0.0510 & 0,0713 & 0.1018 & 0.2033 & 0.3045 & 0.4075 \\
\hline 0.030 & ". o010s & 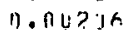 & $0.003 n a$ & 0.00515 & 0.00721 & 0.01 .03 & $0.0 ?$ & 0.0 & $3 \cap 9$ & 0.0515 & 0.0720 & 0.1027 & 0.2047 & 0.3069 & 0.4099 \\
\hline 0.050 & Sטחט ט ט & 0. & 0.010315 & $0.0 \cup 525$ & 0.00735 & 0.0105 & 0.02 & 0.0 & 314 & 0.0523 & 0,0733 & 0.1045 & 0.2081 & 0.3112 & 0.4147 \\
\hline 0.070 & 0.00107 & $n . n_{6}=1.4$ & 0.00379 & $n, 0 \cup 535$ & 0.00742 & $n .01 n \%$ & $0.0 ?$ & 0.1 & 320 & 0.053 .3 & 0.0745 & $0.1 \cap 63$ & 0.2112 & 0.3150 & 0.4194 \\
\hline 0.100 & ! & $n \cdot n_{1} ;=20$ & $0.01133 n$ & 0.00550 & $0 . \cap 0769$ & 0.01 .10 & 0.02 & 0.0 & 329 & 11.0547 & 0.0765 & 0.1089 & 0.2157 & 0,3212 & .4266 \\
\hline 0.201 & $(1 ., 0 \| 3.2 !$ & $0 .(10>40$ & U. $010.3 \times n$ & $n .00509$ & $0.0 \cup 830$ & $0.01>0$ & 0.0 ? & 0.0 & 358 & 0.0594 & 0.0828 & 0.1176 & 0.2309 & 0.3407 & 0.4490 \\
\hline $0.3 \div 0$ & 1]. $0(1), 3 !$ & ח. ח. & 0.jnsou & $n .010649$ & 0,00908 & 0.0131 & 0.02 & 0.0 & 386 & (1. 0640 & 0.0892 & 0.1263 & 0.2454 & 0,3593 & 0.4700 \\
\hline 0.500 & 0.40150 & 0.01300 & 1). $0 n+49$ & 0.00748 & 0,01045 & 0.0149 & 0.02 & 0.0 & 444 & (9) 0732 & 0.1014 & 0.1430 & 0.2730 & 0.3937 & 0,5088 \\
\hline $0.7 n 0$ & $0.001 .7 \mathrm{~J}$ & 0.00340 & ij. onsng. & $n .00947$ & 0.01184 & 0.0163 & 0.03 & 0.0 & 500 & 0.0821 & 0.1135 & 0.1589 & 0.2988 & 0.4247 & 432 \\
\hline $1.0 n 0$ & y. טo? & 0.01099 & (i. $11 \cap 50 \%$ & $n, n_{1} 994$ & 0.01391 & $n .0199$ & 0.03 & 0.0 & 582 & 0.0952 & 0.1308 & 0.1818 & 0.3341 & 0.4671 & 0.5889 \\
\hline 2.000 & (1).110204 & 1). & $(1.019894$ & $n .01465$ & $0.020 \% 1$ & 0.0294 & 0.05 & 0.0 & 849 & 0.1363 & 0.1842 & 0.2501 & 0.4323 & 0.5794 & .7091 \\
\hline 3.000 & 10.011 .509 & 0. nuigs & 11.011811 & $n . n 19 \%$ & 0.02741 & $n . r 3 \times 3$ & 0.07 & & 110 & 0.174 & 0.231 & 0.308 & 0.509 & 0.662 & 0.798 \\
\hline 5,000 & 0.011 .97 & $0.1118 \%$ & 14.01 .773 & $n, 02926$ & 0.114060 & 0.1572 & 0.10 & & & 3.240 & 0.312 & & 0.623 & & \\
\hline $7.11 \cap 0$ & $0.00 / 95$ & 0.115790 & 11.02351 & $n . n 3864$ & 0.05336 & 0.01747 & 0.14 & & 198 & 3.297 & 0.377 & 0.477 & 0.709 & 0.8 & \\
\hline 20.000 & {$[.01 \cap 9$} & i). $4 ? 1 \mathrm{~K}$ & 0.0 .3201 & 1).0524 & $i .0720$ & $0.1 \cap 0$ & 0.1 & & & 3,368 & 0.458 & 0.564 & 0.807 & 0,5 & 31 \\
\hline 20.000 & E. 1020n & $11 .(14) 4$ & 0.0504 & 1.0955 & 1.1280 & 0.175 & 0.3 & & 396 & 3.536 & 0.639 & 0.756 & 1.017 & 1.2 & \\
\hline 30.000 & $\therefore n \ln 1$ & i). & $0.035 x$ & 0.1349 & 0.1795 & 0.239 & 0.3 & & 497 & 3.648 & $0.75 A$ & 0,8 & 1.150 & 1.347 & .518 \\
\hline 50.000 & 1.0400 & 0.10027 & $0.133 \pi$ & 1.2040 & $0.2 \leqslant 49$ & 0.341 & 0.5 & & 639 & 3.800 & u. 913 & 1.041 & 1.329 & 1.539 & \\
\hline 70.000 & 1.0054 & 1.1246 & $\pi .170 .1$ & i). 2630 & 0.3245 & 0.42 .1 & 0.6 & & 738 & 3.905 & 1.022 & 1.156 & 1.457 & 1.679 & 73 \\
\hline 100.000 & 4.0918 & U.1+n? & 0.2331 & 1.3379 & 0.41 .00 & 0.515 & 0.7 & & 847 & $1.02 n$ & 1.143 & 1,284 & 1.598 & 1.833 & \\
\hline 10.000 & 0.1675 & $11,2+73$ & 0.3789 & 0.51 .17 & 9.6071 & 0.794 & 0.8 & & 074 & 1.261 & 1.394 & 1.549 & 1.902 & 2.162 & 2.399 \\
\hline $3 \cap 0.000^{\circ}$ & 0.2517 & 11.6779 & n.48?1 & 0.5201 & $0.127 ?$ & 0.839 & 1.0 & & 21.5 & 1.413 & 1.555 & 1,7 & 2 . & & 2.027 \\
\hline 00.000 & 0.3350 & 11.94 & $7.625 \%$ & .1 .7797 & v.8865 & $1.0 n 3$ & & & & 1.617 & 1.770 & 1.5 & & 9 & 2,944 \\
\hline$R \in T_{A} /=(A)$ & $3.5: 100$ & 1. क्षाओ? & Q. & 0.80110 & 0,9000 & 0 & 9500 & 700 & $0.98 n \pi$ & 0.9900 & 0.9930 & 0.0950 & 0.9970 & 0.9980 & 1.9990 \\
\hline 0.001 & b. bna & 0.2 .24 & 0.757 & 0.927 & 1.204 & 1.35 & 1.48 & 1.70 & 1.89 & 2.23 & 2.43 & 2.62 & 2.94 & 3.22 & 3,74 \\
\hline 0.002 & $4 . \sin \theta$ & 11.524 & i & & $t, 2<04$ & & 48 & 1.71 & 1.89 & 2.23 & 2.43 & 2.62 & 2.94 & 3.22 & 3,74 \\
\hline $0.0 \cap 3$ & ii. $>10$ & $n . \div 24$ & 0.757 & n.927 & 1.204 & 35 & 1.49 & 1.71 & $\because .89$ & 2.23 & 2.43 & 0.62 & 2.94 & 3.22 & 3,75 \\
\hline 0.005 & 1). .511 & $0.0 \% 5$ & 0.759 & 0.928 & 1.205 & 1.35 & .49 & 1.71 & 1.84 & 2.24 & 2.43 & 2.63 & 2.94 & 3.22 & 3,75 \\
\hline $0.00 \%$ & 4.511 & $n . s<5$ & $0.75 \mathrm{i}$ & 0.928 & 1.205 & 1.35 & 1.49 & 1.71 & $\therefore 89$ & 2.24 & 2.43 & 2.63 & 2.95 & 3.22 & 3,75 \\
\hline 0.010 & 0,512 & 11.626 & 0.754 & 0.929 & 1,205 & & .49 & 1.71 & $\therefore 89$ & 2.24 & 2.43 & 2.6 & 2.95 & 3,23 & 3,75 \\
\hline 0.020 & $0.5,5$ & $11 . n 29$ & $0.7 \times 3$ & 0.933 & 1,209 & 1.35 & .49 & 1.71 & $\therefore 90$ & 2.25 & 2. & 3.6 & 2.96 & 3,23 & 3,76 \\
\hline 0.030 & 6. & 0.432 & 0.765 & y & 1.213 & 1.36 & 1.50 & 1.72 & $=.94$ & & 2.44 & 2. & & 3.24 & 3.76 \\
\hline 0.050 & $1),>22$ & $0.33 \pi$ & 0.771 & 0.94 & 1.221 & 1.36 & 1.50 & 1.72 & .91 & & 2.46 & $?$. & 2.97 & 3.25 & 3,78 \\
\hline 0.070 & U.b? & $n, 243$ & 7 & 3 & 1.220 & & .5 & 9.73 & $1.9 \%$ & & 2.47 & & & 3. & 3,79 \\
\hline 11,100 & 0.535 & $(1,-5)$ & 0.78 .5 & 3.95 & 1.238 & 1.38 & 1.52 & 1.75 & 1.93 & & 2.48 & ?. & 3.01 & 3.29 & 3,82 \\
\hline 0.200 & 654 & $.6 \%$ & & & & & 1.5 & & $1.9 t$ & & 2. & & & & \\
\hline 0.300 & $0.3 \times 2$ & $n_{1} 7,1$ & n. 3.34 & 1.013 & 1.299 & 1.45 & 1.5 & 1.82 & 2.01 & & 2.58 & 2.78 & 3,12 & 3.41 & 3,96 \\
\hline 0.500 & $0.0 ? 3$ & 074 & 0.884 & 01 & & & 1.6 & 1.88 & $\Xi D E$ & & & & & & \\
\hline 0.7 & $1 . \Delta x(1)$ & $n .783$ & 0.924 & . & 1.410 & 1. .55 & 1.70 & 1.94 & $\geq .15$ & 2. & 2. & 2.95 & 3.31 & 3.61 & 9.19 \\
\hline $1.0 \cap 0$ & U. $/ \cap 4$ &.$\$ 34$ & & & 1.463 & & . & 2.02 & $2 . ? 5$ & & & & & & \\
\hline $2.00 \mathrm{U}$ & U.d.34 & 11.705 & 1.114 & 1.38 & 1.627 & 1.79 & 1.96 & 2.22 & $2.4 \mathrm{E}$ & $? .87$ & 3,11 & 3.34 & 3.74 & 4.08 & 4.73 \\
\hline 3.000 & अ?\% & 1.nng & & & 1.751 & 1.93 & 2.10 & 2.38 & $2.6=$ & 3.06 & 3.31 & 3.56 & & & 50 \\
\hline 5.000 & 1.1161 & 9.314 & 1.366 & $1.5 \% 7$ & 1.933 & 2.1 & 2.3 & & & 3.34 & 3.6 & & & & \\
\hline 7.00 & 1.162 & 1.308 & $1.47 n$ & 1.699 & $2.0 \% 4$ & 2.2 & 2. & & 3.05 & 3.5 & 3. & 4.1 & 4.61 & 5.02 & \\
\hline $10.0 \cap 0$ & 1.276 & 1.179 & 1.606 & 1.841 & 2.234 & $? .44$ & 2.65 & 2.99 & $3 . ? 7$ & & 4. & 4. & 4. & & \\
\hline 20.0 & . & 1. $x y$ ? & & & & & & & & & 4. & & 5. & & \\
\hline $30.0 \cap 0$ & 1.680 & 1.805 & 2.074 & 2.3 & 2.830 & 3.19 & 3. & 3.75 & 4.18 & 4.75 & 5.12 & 5.51 & & & 7.70 \\
\hline 50,00 & 1.907 & 2.101. & 2. & 2.6 & 3.156 & 3.4 & 3.72 & 4.16 & 4.55 & 5.2 & 5.69 & 6.11 & 6.79 & & 8.52 \\
\hline 70.000 & $2 .(16)$ & $2.2 \% 3$ & 2.5 & 2.8 & 3.398 & & & 4,47 & $4.8 B$ & & 6.1 & & & & \\
\hline 100 uno & 2.3 .40 & $? .40 .3$ & & & 3.6 & 3.99 & 4 . & 4.81 & 5.25 & 6.09 & 6.56 & 7.04 & 7.82 & 8.51 & 9.80 \\
\hline ou.unu & 2.621 & $2.4 \%$ & 3.173 & 3. & $4.24 \%$ & & & & 6.07 & & 7.57 & & & 9.79 & 11.28 \\
\hline 00.000 & 2.578 & 3.146 & $3.4 \times .7$ & 3.8 & 4.6 & 5. & 5. & 6.0 & 6.63 & 7.64 & 8.22 & R. & 0.79 & 10.63 & 12.25 \\
\hline no.000 & 3.219 & 3.516 & 3.473 & 4,338 & 5.155 & 5.59 & & & 7.33 & 8.48 & 9.12 & 9.77 & 10.86 & 11.80 & 13.59 \\
\hline
\end{tabular}




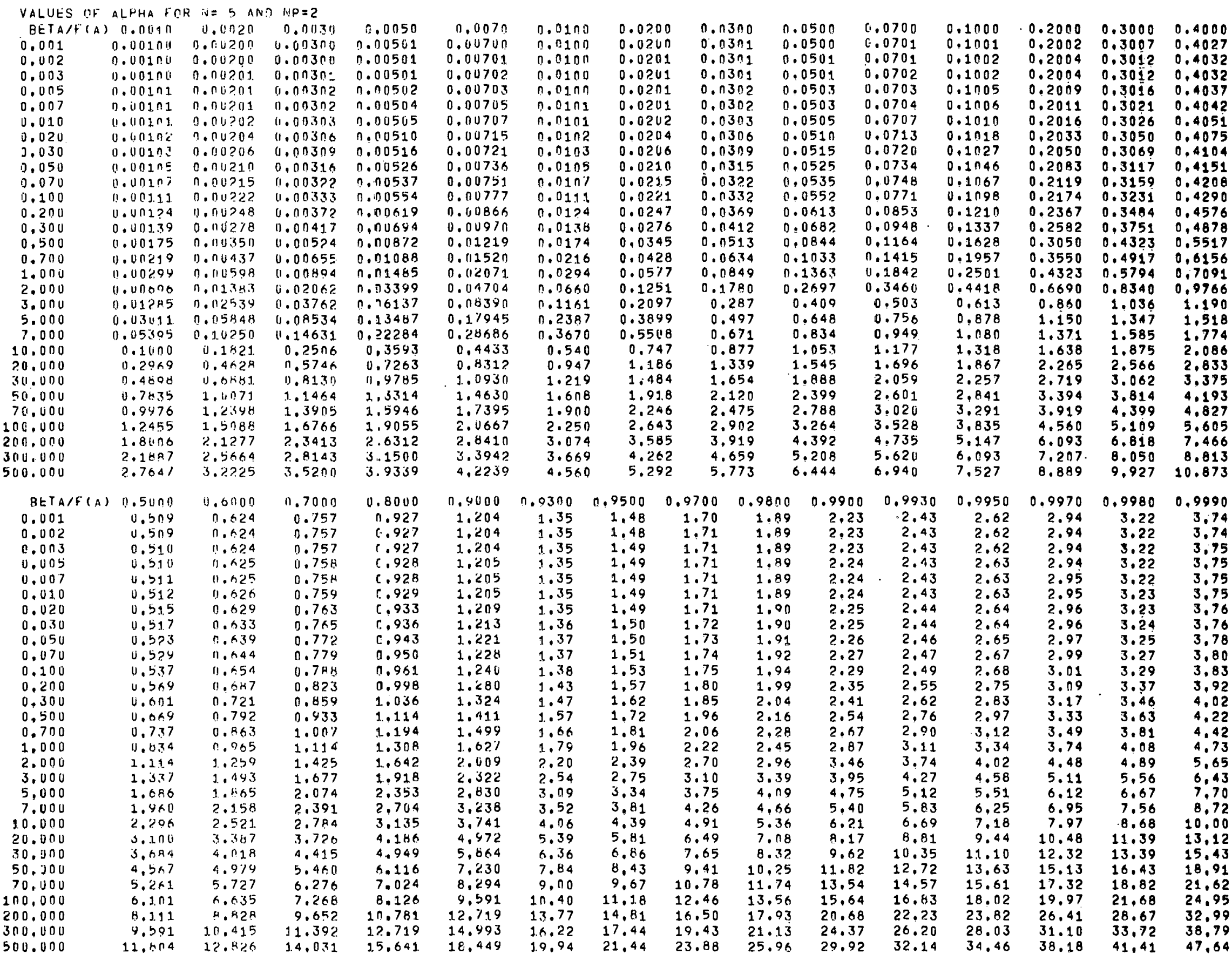


VALUES TIF ALPHA FOH IN $=5$ ANT NP $=3$

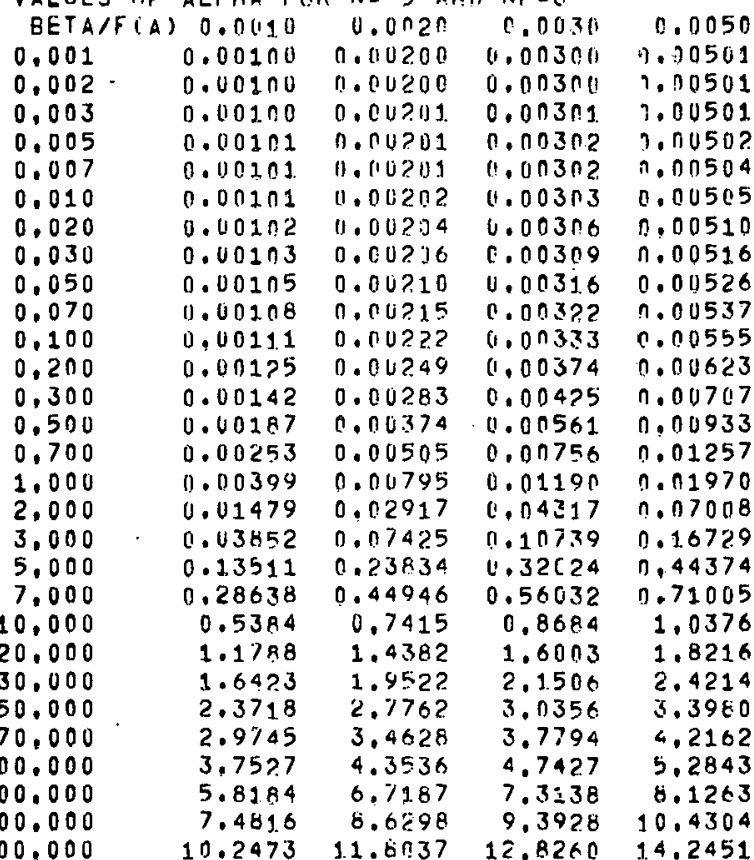

$\begin{array}{cc}\text { BETA/F(A) } & 0.5000 \\ 0.001 & 0.509 \\ 0.002 & 0.509 \\ 0.003 & 0.510 \\ 0.005 & 0.510 \\ 0.007 & 0.511 \\ 0.010 & 0.512 \\ 0.020 & 0.515 \\ 0.030 & 0.517 \\ 0.050 & 0.523 \\ 0.070 & 0.529 \\ 0.100 & 0.537 \\ 0.200 & 0.571 \\ 0.300 & 0.607 \\ 0.500 & 0.690 \\ 0.700 & 0.782 \\ 1.000 & 0.927 \\ 2.000 & 1.392 \\ 3.000 & 1.797 \\ 5.000 & 2.482 \\ 7.000 & 3.066 \\ 10.000 & 3.427 \\ 20.000 & 5.872 \\ 30.000 & 7.527 \\ 50.000 & 10.293 \\ 70,000 & 12.628 \\ 00.000 & 15.672 \\ 00.000 & 23.850 \\ 00.000 & 30.442 \\ 00.000 & 41.413\end{array}$

$0.0600 \quad 0.7000$ 0.324

0.625

0.625

0.026

0.029

$n, 633$

0.639

0.645

0.655

0.689

0.314

0.914

1.061

1.553

1.983

2.719

3.348

4.178

8.187

$11.178 \quad 12.231$

$13.711 \cdot 14.993$

17.015

33.851

44.953

0.927

0,927

$28.276 \quad 31.526$

36.103
49.104
0.0070

0.00700

0.00700

0.0100

0.0100

$0.0 \pm 00$

0.0200

$0.03 n$

0.0200

0.0301
0.0301

0.0301

0.00703

0,0100

0.0201

$.01 n 1$

0.0201

0.0070

.0101

0.0202

0.00721

0.00737

0.00753

0.00872

$0.01 n 3$

0.0105

0.0204

0.0210

$0.03 n 2$

0.0303

0.0306

0.0309

0.0315
0.0322

n.011.

0.01928

0.01304

0.0124

0.0141
0.0186

0.0186
0.0249

0.0274

0.0388

0.09559

0.53458

0.81496

1.1540

0.2883

$n .6376$

0.9294

1.284
2.154

2.6160

3.6574

4.5290

5.6734

2.837
3.949

4.898

6.116
9.378

$1.1628 \quad 12.017$

0.0222

$0.0248 \quad 0.0371$

$0.0281 \quad 0.0420$

$\begin{array}{ll}0.0491 & 0.0548 \\ 0.0727\end{array}$

$0.0754 \quad 0.1101$

0.4542

0.4542
0.8531

1.1673

2.536

3.314

5.666

7.07 .0

10.827
13.864

5.2521

15.084
$18.907 \quad 20.585$

$0.56 \varepsilon$

0,988

1.318

2.788

3.627

6.185

7.794

$0.900 \mathrm{v}$

0.9300

$\begin{array}{rr}0.9500 & 0.9700 \\ 1.48 & 1.70\end{array}$

$\begin{array}{lll}.788 & 13.131 & 14.123 \\ .084 & 15.801 & 18.083 \\ .585 & 22.904 & 24.613\end{array}$

$0.0500 \quad 0.0700$

$0,07.01$

0.0501

0.0709

0.0503

.0703

0.0704

0.0505

0.0707

0.0515

.0720

0.0734

0.0535

0.0616

0.0772

0.0858

$0.0899 \quad 0.1237$

$0.1176 \quad 0.1601$

$\begin{array}{ll}0.1740 & 0.2314 \\ 0.4456 & 0.5422\end{array}$

0.726

1.169

0.835

$3.135 \quad 3.39$

3.390

6.905

6.032

7.428

1.204
1.204

1.204

1.205
1.205

1.205

1.209

1.213
1.221

1.228

1,24

1.284

1.438

1,438

1.751

2.402

4.025

4.025
4.918

6.055

9.240
11.819

16.099

19,700

24.430
37.141

47.395

64.393

1,48
1,48

1.48
1.49

1.49

1.49

1.49

1.50

1.50
1.51

1.53

1.63

1.75

1.88

2.10
2.84

2.84
3.53

4,72

5.75
7.11

7.11
10.77

10.02

17.41

26.44

$\begin{array}{ll}51.24 & 55.02 \\ 69.64 & 74.89\end{array}$

1.70
1.71

1.71
1.71

0.9810
1.89
1.89
1.89

0.9900
2.23

$1.71 \quad 1.89$

$1.71 \quad 1.89$

1,71

1.73

1.74
1.75

1.80

1.99

2.14

2.38
3.20
3.96

3.96

6.42
7.93

7.93

12.00
15.34

20.83

25.50

48.01

2.29 $\begin{array}{rrrr}48.01 & 52.16 & 60.09 & 64.52 \\ 61.22 & 65.59 & 76.60 & 82.34 \\ 83.31 & 90.52 & 104.19 & 112.00\end{array}$

$\begin{array}{llll}1.90 & 2.25 & 2.44 & 2.64 \\ 1.94 & 2.44 & 2.64\end{array}$

$\begin{array}{llll}1.91 & 2.26 & 2.46 & 2.65\end{array}$

$\begin{array}{llll}1.99 & 2.35 & 2.55 & 2.76\end{array}$

$2.42 \quad 2.63 \quad 2.84$

$\begin{array}{llll}36 & 2.76 & 2.99 & 3.23\end{array}$

$\begin{array}{llll}3.06 & 3.31 & 3.56\end{array}$

$5.02 \quad 5.41 \quad 5.80$

$\begin{array}{rrrr}8.10 & 8.71 & 9.35\end{array}$

$\begin{array}{lll}9.07 & 15.07 & 16.74\end{array}$

$\begin{array}{ll}13.21 & 20.68\end{array}$

11.51
17.38

17.38
22.14

30.05

36.77
45.56

119.81

$0.2000 \quad 0.3000 \quad 0.4000$

$0.2002 \quad 0.3007 \quad 0.4027$

$0.2004 \quad 0.3012 \quad 0.4032$

$0.2004 \quad 0.3042 \quad 0.4032$

$0.201990 .3016 \quad 0.4037$

$0.2016 \quad 0.3026 \quad 0.4051$

$0.2033 \quad 0.3050 \quad 0.4075$

$0.2050 \quad 0.3000 \quad 0.4104$

$0.2050 \quad 0.3069 \quad 0.4104$

$0.2033 \quad 0.3117 \quad 0.4156$

$0.21190 .3159 \quad 0.4208$

$\begin{array}{lll}0.2174 & 0.3231 & 0.4290 \\ 0.2379 & 0.3498 & 0.4595\end{array}$

$\begin{array}{lll}0.2616 & 0.3798 & 0.4936\end{array}$

$\begin{array}{lll}0.3198 & 0.4499 & 0.5708\end{array}$

$\begin{array}{lll}0.3899 & 0.5317 & 0.6586\end{array}$

$\begin{array}{lll}0.5088 & 0.6624 & 0.7978 \\ 0.9056 & 1.0853 & 1.2398\end{array}$

$1.240 \quad 1.444 \quad 1.621$

$\begin{array}{lll}1.2405 & 1.444 & 1.621 \\ 1.785 & 2.261\end{array}$

$2.238 \quad 2.536 \quad 2.803$

$\begin{array}{lll}2.826 & 3.184 & 3.509\end{array}$

$\begin{array}{lll}5.651 & 6.318 & 5.39 \\ 5 & 6.925\end{array}$

$\begin{array}{lll}7.745 & 8.645 & 9.469\end{array}$

$9.515 \quad 10.613 \quad 11.621$

$\begin{array}{lll}11.819 & 13.192 & 14.428\end{array}$

$23.026 \quad 25.682 \quad 28.062$

$31.343 \quad 34.944 \quad 38.178$

$\begin{array}{llll}0.9970 & 0.9980 & 0.9990\end{array}$

$\begin{array}{lll}2.94 & 3.22 & 3.74\end{array}$

$2.94 \quad 3.22 \quad 3.75$

$2.94 \quad 3.22 \quad 3.75$

$2.95 \quad 3.22 \quad 3.75$

$2.95 \quad 3.23 \quad 3.75$

$2.96 \quad 3.23 \quad 3.76$

$2.97 \quad 3.25 \quad 3.78$

$2.99 \quad 3.27 \quad 3.80$

$3.09 \quad 3.29 \quad 3.83$

$\begin{array}{lll}3.18 & 3.47 & 4.03\end{array}$

$3.38 \quad 3.69 \quad 4.28$

$\begin{array}{lll}3.98 & 4.34 & 5.02\end{array}$

$\begin{array}{lll}5.26 & 5.73 & 6.62\end{array}$

$\begin{array}{rrr}8.46 & 7.02 & 8.11 \\ 8.55 & 9.30 & 10.72\end{array}$

$\begin{array}{lll}10.37 & 11.27 & 12.98\end{array}$

$19.27 \quad 20.92 \quad 15.98$

$24.55 \quad 26.63 \quad 30.61$

$33.36 \quad 36.16 \quad 41.60$

$\begin{array}{lll}40.80 & 44.28 & 50.87\end{array}$

$76.51 \quad 54.84 \quad 63.05$

$97.72 \quad 83.07 \quad 95.52$

$\begin{array}{lll}132.69 & 144.17 & 165.65\end{array}$ 


\begin{tabular}{|c|c|c|c|c|c|c|c|c|c|c|c|c|c|c|c|}
\hline & Pt & & P: & & & & & & & & & & & & \\
\hline BETAI & 0.019910 & 0.0020 & 0.0030 & 0,0050 & $0.007 ?$ & $0.01 \cap 0$ & 0.020 & $0 . n$ & $3 \cap 0$ & 0.0500 & 0.0700 & 0.1000 & 0.20 & 0.30 & 0.400 \\
\hline 0.001 & 0.00100 & $0.00 \geqslant 010$ & 0.00300 & 0.00501 & 0.00700 & 0.0100 & 0.020 & 0.0 & $3 n 1$ & 0.0500 & 0.0701 & 0.1001 & $0, ?$ & 0.3 & \\
\hline 0.002 & $0.00 .1 \cap 0$ & $0 . n \cup \geq 00$ & $0.0 n 3 \cap 0$ & .00501 & .00701 & 0.0100 & 0.020 & 0.0 & 301 & 0.0501 & 0.0701 & .1002 & .2004 & .3012 & 0.4032 \\
\hline 0.003 & 0.00100 & $0.10 \cup ? 01$ & $0.0 \cap 3 \cap 1$ & .00501 & .00702 & 0.0100 & 0.020 & 0.0 & 301 & 0,0509 & 0.0702 & 0.1002 & & & 0,4032 \\
\hline 0.005 & 1).001n1 & 0.00201 & 0.00302 & .00502 & .0070 .3 & $n .01 \cap 0$ & 0.020 & $0 . n$ & $3 \cap 2$ & 0.0503 & 0.0703 & 1.1005 & 2009 & .3016 & .4037 \\
\hline $0.00 \%$ & $0.001 \cap 1$ & $0.0 \cup ? 01$ & $0.003 \cap 2$ & 0.00504 & .00705 & $n \cdot 0_{1} n \mathcal{1}$ & 0.020 & 0.0 & 302 & $\pi .0503$ & 0.0704 & 0.1006 & 0.2011 & 0.3021 & 0.4042 \\
\hline 0.010 & 1). $001 n 1$ & $0.00 \geq 02$ & $0.0 \cap 3 \cap 3$ & 0.00505 & .00707 & $n, n 1 n 1$ & 0.020 & 0.0 & $3 \cap 3$ & 0,0505 & 0,0707 & 0.1010 & 016 & 0,3026 & 0.0051 \\
\hline 0.0 & 0.00102 & 0.00204 & 0.00306 & .00510 & .00715 & 0.0102 & 0.320 & 0.0 & 306 & 0. & 0.0713 & .1018 & 0. & & 0.0 \\
\hline 0. & 0.00103 & 0.00206 & 0.00309 & .00516 & .00721 & 0.0103 & 0.020 & 0.0 & 309 & 0.0515 & 0.0720 & 0.1027 & 0 & 0.3069 & 0.4104 \\
\hline 0,0 & 0.00105 & 0.00210 & 0.00316 & 1.00526 & .00737 & 0.0105 & 0.021 & 0.0 & 315 & 0.0525 & 734 & 0.1048 & & .3117 & 0.4156 \\
\hline 0.070 & $0.001 n 8$ & $0,0 \| \geq 15$ & $0.0133 ? 2$ & 0.00537 & .00 & 0.0107 & 0.021 & 0.0 & 322 & 0. & 748 & 0.1067 & 0.2119 & & \\
\hline 0.100 & 0.00111 & $0.0 \cup \geq 22$ & 0.90333 & 0.00555 & .00777 & 0.0111 & 0.022 & 0.0 & & 0.1 & 772 & 0.1099 & 0.2174 & & 0.4290 \\
\hline 0.200 & 0.00 & $0.0 \cup \geqslant 50$ & 0.00375 & $n .00624$ & 0.00874 & 0.0125 & 0.024 & 0.0 & 372 & 0 . & 859 & 0.1 & & & \\
\hline 0. & 0.0 & $0.0 u \geqslant 85$ & 0.00427 & .1 & 94 & 0.0142 & 0.028 & & & & & & & & \\
\hline 0. & 0.00 & 0.00387 & 0.00580 & .00 & .01347 & 0.0192 & 0.038 & 0.0 & 565 & & 72 & 0,17 & & & 04 \\
\hline 0.700 & 0.0 & 0.00553 & 0.0082 .7 & 0.01 & 01 & 0.0272 & 0.053 & 0.0 & 790 & & 728 & & & & \\
\hline 1.0 & 0.0 & 0.00991 & 0.01482 & $n, n$ & .0 & 0.0 & 0.0 & 0.1 & & & & & & & \\
\hline 2.000 & 0.0 & 0.05244 & 0.08534 & $n \cdot 1$ & $0.1 / 945$ & 0.2387 & 0.3 & 0,4 & 974 & & 58 & $0:$ & & 166 & 83 \\
\hline 3.000 & 0.1 & 0.19519 & 0.26731 & 0.37984 & 0.46567 & 0.5641 & 0.773 & 39 & 905 & 82 & 007 & 1.350 & 15 & 1.914 & 2.128 \\
\hline 5.000 & 0.4 & 0.63566 & 0.75773 & 0.91986 & 1.03192 & 1.1559 & 1. & & & & 1.975 & & & & \\
\hline 7.000 & 0.81305 & 1.03755 & 1.17878 & 1.36 & 1,4 & 1. & 1.8 & 2. & & & & & & & \\
\hline 10.000 & 1.2799 & 1.5469 & 1.7196 & & 2.1162 & 2. & 2 & & 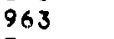 & & 3.600 & 3. & 4.651 & 5.216 & 5. \\
\hline 20. & 2. & 2.9517 & & & & & & 5. & & & & 6. & & & \\
\hline 30. & & $4.17 \times 1$ & 4. & & & 5. & 6. & & & & 104 & 9 & 76 & 04 & \\
\hline 50,000 & 5.5437 & 0.41159 & 6.9704 & 7.7 & B. & 8. & 10 & 24 & & & 13.482 & & & & \\
\hline 70.000 & 14 & 5.4467 & 9.1791 & 10.2 & 10. & 1.1. & & 11 & & & 86 & & & & \\
\hline 0. & 9.8048 & $11.3 \cap 01$ & 12.2767 & & 14. & & & & & & & & & & \\
\hline & 17. & 19.8221 & 21 & & & & & & & & & & & & 83 \\
\hline 0 & 2.3 .9 & 27.4820 & 29. & & & & & & & & & & 50 & & 319 \\
\hline 10. & $36.04 ? 2$ & 41.4743 & 45.0144 & 49.8972 & 53.3762 & 57 & 66 & & & & 177 & 92.835 & 109.193 & 121,644 & 132.935 \\
\hline BETA/ & 0.5000 & 0.0000 & D. & 0 & 0.9 & .93 & $n .950$ & 00 & 1) & .9 & .9 & 0. & 0.99 & 1.9 & 0.9 \\
\hline 0.001 & & 0.624 & & & & & & & & & & & & & \\
\hline & & .0 .524 & & & & & 1. & .71 & 19 & & 2 & & 2. & & 3 \\
\hline 0. & & 0.924 & & & & & 1. & 1 & 1. & & & & & & \\
\hline 0.1 & 0 & 0.525 & & 0 & 1. & & 1. & 1.71 & & & 2 & & 2 & & 75 \\
\hline 0.0 & & 0.525 & & & & & & & & & & & & & \\
\hline 0.010 & & $n, 626$ & & & & & 1. & 1 & & & 2 , & & & & \\
\hline & & 9 & & & & & & & & & 2 , & & & & \\
\hline & & 0,6 & & & & & 1 , & & 1 & & 2 . & & & & \\
\hline & & 9 & & & & & 1. & & 1 & & & & & & \\
\hline 0. & & $0 . \times 45$ & & & & & 1. & 1.7 & 1.92 & & & & & & \\
\hline 0. & & 0.655 & & & & & 1. & 1.7 & 1 & & & & & & \\
\hline 0. & & $0 . \$ 89$ & & & & & 1. & 1.8 & 1.99 & & & & & & \\
\hline & & 0.729 & & & & & & 1,8 & 2 & & & & & & \\
\hline 0. & & 0.824 & & & & & 1. & & 2.21 & & & & & & \\
\hline 0.700 & & 0.939 & & & & & 1. & 2 & 0 & & & & 3.68 & & \\
\hline 1. & & 159 & & & & & & & & & & & & & \\
\hline 2.0 & & a & & & & & & & 400 & & 5 & & & & \\
\hline 3. & & 2.570 & & & & & & & & & & & & & \\
\hline 5.0 & & 3,87 & & & & & & & & & & & & & \\
\hline 7.0 & 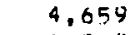 & $5 . n 78$ & 5.5 & 6.2 & & & & & 45 & 12 & & & & & \\
\hline$\simeq 0,0$ & & 7 & & & & & & & & & & & & & \\
\hline 20. & 10 & 11.919 & & 14 & 17 & & & & 2 & & & & & & \\
\hline & & 18 & & & & & & & & & & & & & \\
\hline 50. & & 24 & & 3 & 35 & & & & & & & & & & \\
\hline 0 & & 19 & & & & & & & & & & & & & \\
\hline 0.0 & & $43 . \pi$ & & & & & & & & & & & & & \\
\hline 10 & & 75.013 & & & & & & & 1.5 & & & & 31 & & .12 \\
\hline & & 103.822 & & & & & & & & & & & & & \\
\hline 0.000 & 144,166 & 156.373 & .777 & 1.90 .308 & 223.756 & 241.82 & 01 & & 31 & 361.57 & 388.43 & 415.77 & 460.69 & 499.51 & 574,71 \\
\hline
\end{tabular}




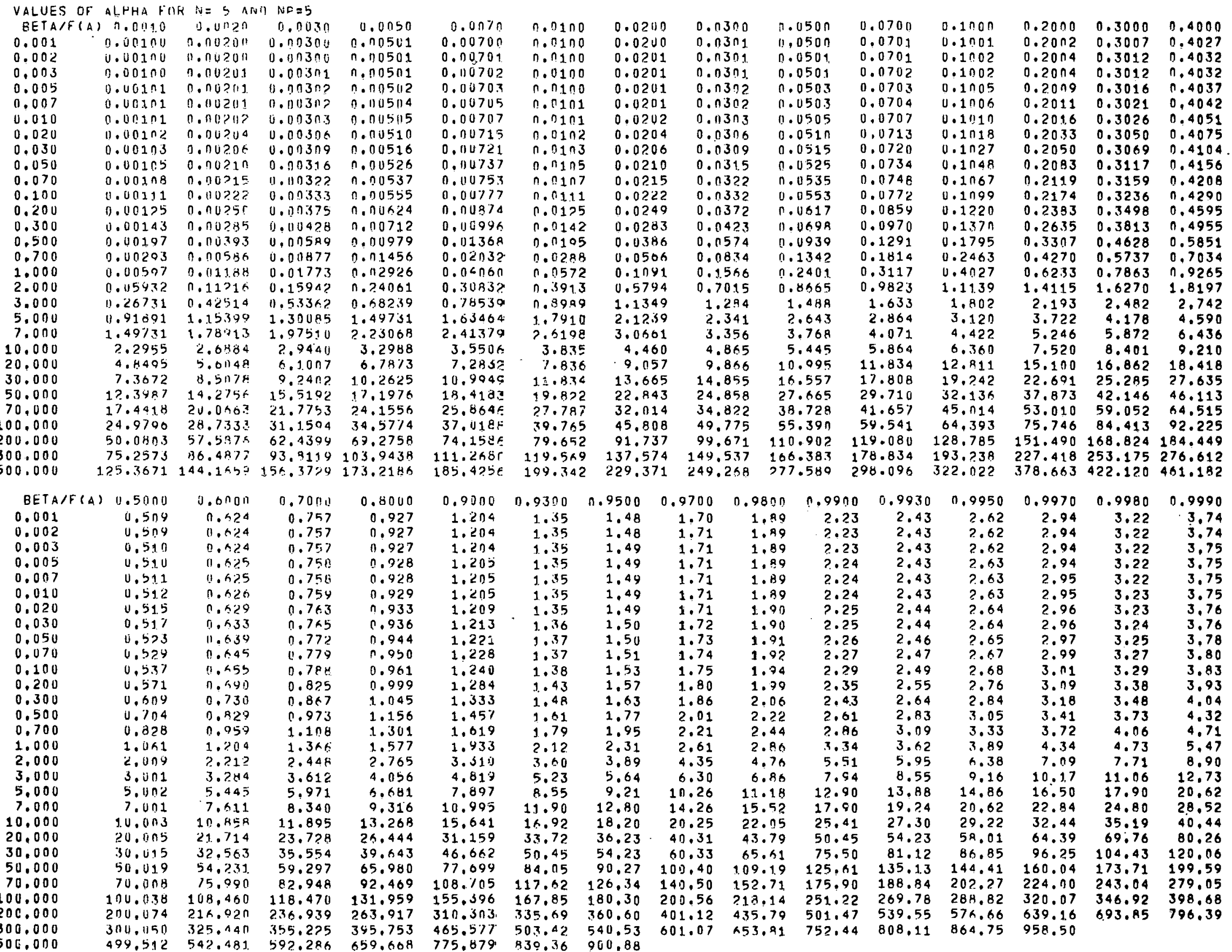




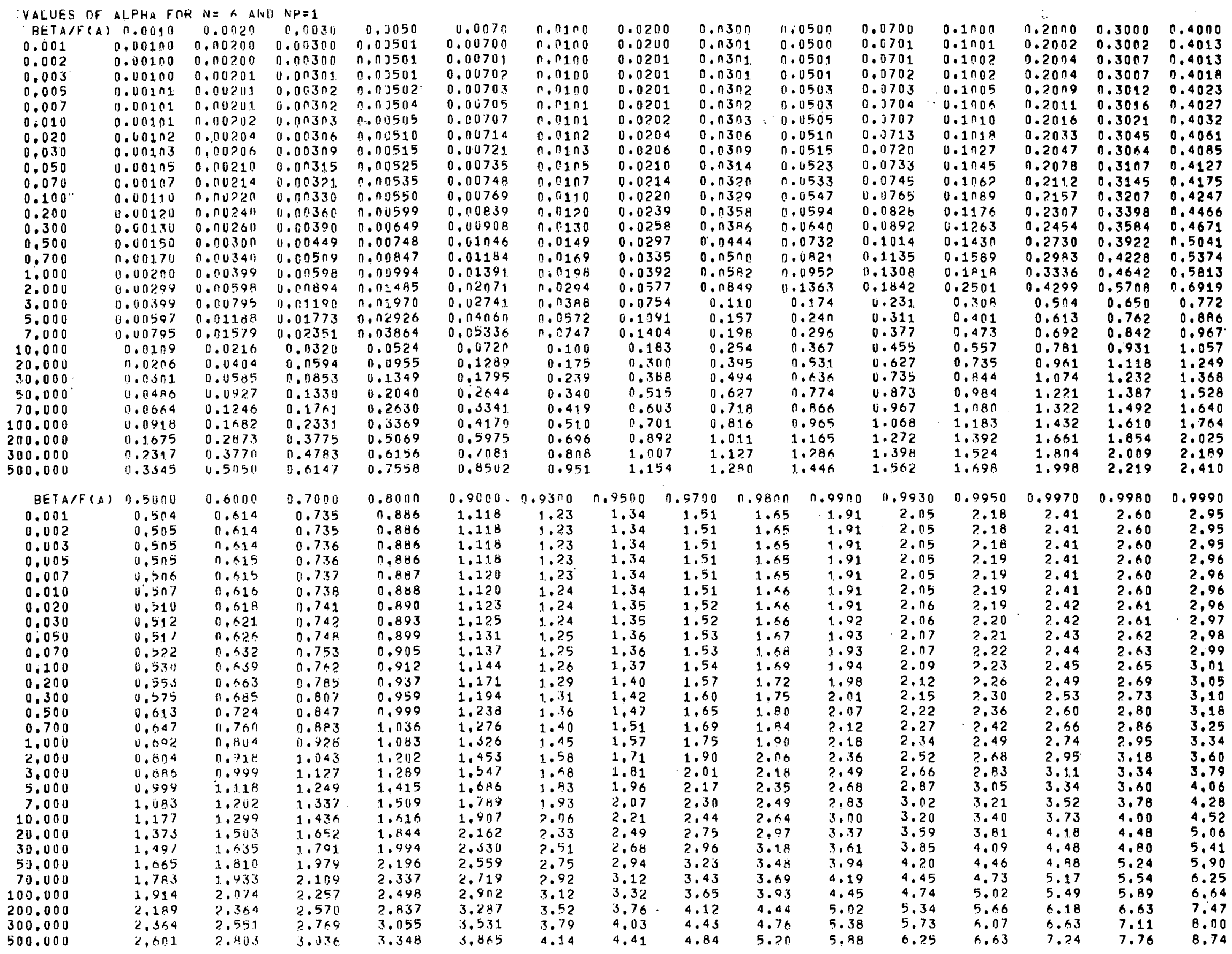



VALUES OF ALPHA FOR IN $=6$ AND NP $=2$ 0.00200 u.0n300 0.00501 0.001000 .00010 .001 $0.005 \quad 0.031010 .002010 .003 n ? 0.00502$ $\begin{array}{lllll}0.007 & 0.031 n 1 & 0.04201 & 0.003 n 2 & 0.04504\end{array}$ $0.010 \quad 0.001 n 10.002020 .003 n 30.00505$ $0.030 \quad 0.001030 .002060 .00309$ 0.00516 $0.050 \quad 0.001050 .002100 .03160 .000526$ $0.00105 \quad 0.00210 \quad 0.0031 .6 \quad 0.00526$ 0.00107 0.0u2.15 0.013220 .00537 0.001110 .0030 . 0.0333 .00554 $0.001240 .00248,0.003720 .00619$ $0.001390 .002 i 8 \quad 0.00417 \quad 0.00694$

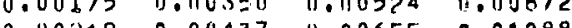
$0.002190 .00437 \quad 11.00655 \quad 0.01088$ $0.00299 \quad 0.00508 \quad 10.0 .0894 \quad 0.01485$ 0.000960 .013630 .030 .20 .03399 0.01285 0.12539 J.0.37A2 .006137

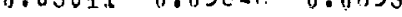
$0.05395 \quad 0.10250 \quad 0.14631 \quad 0.22200$ $\begin{array}{llll}0.1000 & 0.1821 & 0.2506 & 0.3584\end{array}$ $\begin{array}{llll}0.2964 & 0.4600 & 0.5670 & 0.7072\end{array}$ $\begin{array}{llll}0.4459 & 0.0729 & 0.7454 & 0.9294\end{array}$ $\begin{array}{llll}11.7596 & 11.9537 & 1.07 n 1 & 1.2227\end{array}$ i). $9466 \quad 1.1464 \quad 1.2684 \quad 1,4286$ $\begin{array}{llll}1.15 ? 1 & 1.3659 & 1.4916 & 1.6652\end{array}$ $\begin{array}{llll}1.5650 & 1.8292 & 1.9827 & 2.1925\end{array}$

$\begin{array}{llll}1.8731 & 2.1468 & 2.3184 & 2.5549 \\ 2.2841 & 2.01107 & 2.8029 & 3.0775\end{array}$ 200.000 300.000

\begin{tabular}{|c|c|c|c|c|}
\hline EETA/F(A) & 0.5 & 0.6000 & 0.7000 & $0 ; 8000$ \\
\hline 0.001 & $c .504$ & 0.614 & 0.735 & 0.886 \\
\hline & $6.5 \cap 5$ & 0.614 & 0.7 .35 & 0.886 \\
\hline 0.003 & 6.505 & 0.514 & 0.736 & 0.886 \\
\hline 0.005 & C.spis & 0.615 & 0.736 & 0.886 \\
\hline 0.007 & $1 . \sin 6$ & 0.015 & 0.737 & 0.887 \\
\hline 0.010 & $i, b n 7$ & 0.616 & 0.738 & 0.888 \\
\hline 0.020 & 6.510 & 0.616 & 0.741 & 0.891 \\
\hline 0.030 & 0.512 & 0,621 & 0.743 & 0.894 \\
\hline 0.050 & 4. 517 & 0.627 & 0.749 & 0.900 \\
\hline 0.070 & 0.023 & 0.633 & 0.755 & 0.906 \\
\hline 0.100 & 0.532 & 0.641 & $0.7<3$ & 0.914 \\
\hline 0.200 & 0.561 & 0.672 & 0.795 & 0.946 \\
\hline 0.300 & 0.593 & 0,703 & 0.826 & 0.979 \\
\hline 0,500 & 4.656 & 0.767 & 0.891 & 1.043 \\
\hline 0,700 & 0.717 & $n .830$ & 0.954 & 1.110 \\
\hline 1.000 & 0.804 & 0.918 & 9.043 & $1: 202$ \\
\hline 2.000 & $\therefore 0143$ & 1.1 .62 & 1.295 & 1.465 \\
\hline 3.000 & $\therefore .220$ & 1.350 & 1.492 & 1,673 \\
\hline 5.000 & i.497 & 1.035 & 1.791 & 1.994 \\
\hline 7.000 & 1.105 & 1.852 & 2.021 & 2.242 \\
\hline 10,000 & 1.952 & 2.112 & 2.299 & $? .543$ \\
\hline 20.000 & 2.517 & 2.715 & 2.944 & 3.245 \\
\hline 30.000 & 2.914 & 3.135 & 3.394 & 3.737 \\
\hline 50.000 & 3.493 & 3.757 & 4.064 & 4,468 \\
\hline 70.000 & 3.934 & 4.224 & 4.567 & 5,017 \\
\hline 00.000 & 4.460 & $4.7 \mathrm{a1}$ & 5.162 & 5,673 \\
\hline & $5.6 \times 6$ & h. 078 & 6.558 & 7,192 \\
\hline 100.000 & 5.5 .13 & 6.986 & 7.535 & 8,264 \\
\hline 00.000 & 7.160 & 8.309 & 3.966 & 9.820 \\
\hline
\end{tabular}

$0.01070 \quad 0.010$ 0,00701 0.00702 0.00703 0.00705 0.00715 0,00721 0.00751 0.0078 0.00970 0.01219 0.01520
0.02071 0.04704 0.08390 0.17945 0.4400 0,4409 1.0262 1.3256 1.5393 2.3375 2.7190 0.0100 0.0100 0.010 0.0101 0.0101 0.0103 $0.01 n 5$ 0.0107 0.0111 0.0138 0.0174 0.0216
0.0294 0.0660 0.1161 0.2387 0.3660 0.903 1.129 1.438 1.601 1.918 $2.9 n 2$ 3.490
0.0200 0.0200 0.0201 0.0201 0.0201 0.0202 0.0204 0.0206 0.0210 0.0221 0.0247 0.0276 0.0428 0.0577 0.1251
0.2097 0.2097 0.5441 0.726 1.343 1.673 2.200 2,849 3.957
0.0300 0.0301 0.030 0.0301
0.0302 0.0302 0.0306 0.0309 0.0315 0.0332 0.0412 0.0513 0.0634 0.0849
0.1780 0.287

0.494

0.658
0.843

1.226

1.474

1.822

2.379

3.070

3.551
4.254
0.0500

0.0500

0.0501

0.0501

0.0503

0.0503

0.0510

0.0525

0.0535

0.0552

0.0613
0.0682

0.0844

0.1033

0.1363
0.2692

0.407

0.636
0.804

0,992

1.390

2.025

2.303

3,379

3.379
3.903
4.666
0.0700 0.0701 0.0701 0.0702
0.0703 0.0704 0.0707 0,0713
0,0720 0.0734 [. 0748 0.0771
$\mathrm{C} .0853$ C. 0948 $r, 1164$
$[.1415$ [. 1842 c. 3455 0.498 0.735 0.905 1.095 1.505 1.780
2.170 2.463 2:807 3,604 4.155
4.972

$0.9000 \quad 0.9300 \quad 0.9500 \quad 0.9700 \quad 0.9800 \quad 0.9900 \quad 0.9930$

$\begin{array}{llllll}1.118 & 1.23 & 1.34 & 1.51 & 1.65 & 1.9\end{array}$

1,118

1,118

1,118

1,120

1.123

1.125

1.133

1.13

1.148

1.215

1.284

1.354 1,453
1.741 1,971 2.330 2.612 2.952 3.749 4.315 5.139 5.77 6.520
8.264 8.264
9.484 9.484
11.270
1.23
1.23

1.23

1.23

1.24
1.24

1.24

1.25
1.26

1.26

1.30
1.33

9.41
1.48

1.48
1.58

1.88

2.13
2.51

2.81

3.17
4.02

4.62

5.50
3.18

6.97

8.83

10.13
12.05

1.34

1.34

$\begin{array}{ll}1.51 & 1.65 \\ 1.51 & 1.65\end{array}$

$1.51 \quad 1.65$

$\begin{array}{lll}.34 & 1.51 & 1.65\end{array}$

$1.35 \quad 1.52 \quad 1.66$

1.36

1.36

1.41

1.52

1.60
1.71

2.02

$\begin{array}{lll}2.28 & 2.52 & 2.7 \\ 2.68 & 2.96 & 3.1\end{array}$

$3.99 \quad 3.30 \quad 3.55$

$4.28 \quad 4.70 \quad 5.05$

$\begin{array}{lll}4.92 & 5.39 & 5.80 \\ 5.85 & 6.41 & 6.89\end{array}$

$\begin{array}{lll}6.57 & 7.19 & 6.89\end{array}$

$\begin{array}{lll}9.38 & 10.26 & 11.03\end{array}$ $\begin{array}{lll}10.77 & 11.77 & 12.64 \\ 12.80 & 14.00 & 15.01\end{array}$ $\begin{array}{rrr}7.41 & 8.13 & 8.72\end{array}$

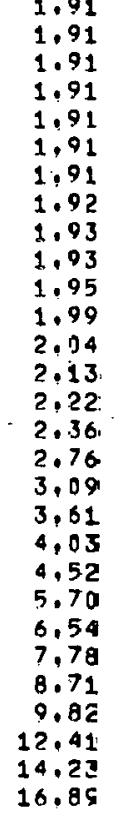

0.1000

0.1001

0.1002

0.1005

0.1006

0.1018

0,1027

0.1046

0.1067

0.1210

0.1337

0.1628

0.1957

0.2501
0.4394

0.602

0.844
1.015

1.637

1.926
2.337

2,646
3,017

3.858

4.453
.5 .315

0,9950
2.18.

2.18

2.18
2.18
2.18

2.19

2,1

2.19

2.20

2.21 
VALUES UF ALFHA FOR $N=6$ ANII $N F=3$ BETA/F(A) 0.0010 O. 0020 0.01030 $0.001 n 00.00200 \quad 0.003 n n \quad 0.00501$ 0.001000 .002010 .003010 .00501 0.00101 n.00201 $0.003 n 2$ n.00502 0.001ni n.00201 0.0n3n? 0.00504 0.00101 .0 .002020 .003030 .00505 0.001020 .00204 l. $00306 \quad 0.011510$ 0.001030 .00206 0.0030 0.00516 $0.001050 .04210 \quad 4.003160 .00526$ 0.001080 .00215 0.0.32 0.00537 0.01110 .00222 u.00333 0.00555 0.00125 0.00249 v0.01374 0.00523

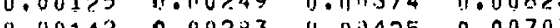
$0.00187^{\circ} 0.003740 .005610 .00933$ 0.002530 .005050 .017500 .00357 0.00253 n.00505 0.015 0.01257 0.01479 0.02017 0.04317 0.07008

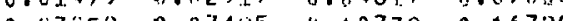
0.03518 0.0.385 0.19739 n.0.6729 0.135110 .23810 0.3197n 0.44135 0.285900 .44708 0.55365 0.59384 0.53270 .72150 .0350 $1.0987 \quad 1.3147 \quad 1.4325$ $1.4649 \quad 1.6095$ $2.0456 \quad 2.2917$ $2.4290 \quad 2.7647$ $2.9593 \quad 3.3522$ $4.2849 \quad 4.8342$ $\begin{array}{ll}5.2990 & 5.9710 \\ 6.8941 & 7.7701\end{array}$

1.8464

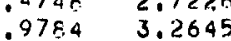
$3.6040 \quad 3.9492$ $5.1923 \quad 5.6734$ $8.3247 \quad 9.0723$

300.000

BETA/F (A)

0.001

0.002

0.003

0.007

0.010

0.020

0.050

0.070

0.100

0.200

0,500

0.700

2.000

3.000

5.000

10,000

20.000

30.000

50.000

100.000

100.000

200.000

500.000

0.8000 0.886
0.886 0.886
0.886 0.886
0.886 0.887 0.888 0.891 0.894 0.900 0.906 0.915 0.948 0.984 1.064 1. 152 1.269 1.722 2.097 2.71 .8

3.2 .15

3.85

5.498
6.757

8.767

8.767
10.385 12.445 17.655
21.653 21.653
28.001
$0.007 n$ 0.00701 0.00702 0.00705 0.04707 0.00715 0.0072 0.00753 0.00777 0,00872 0.0098 0.01304 0,01755 0.0271 0,04559 0.2199 0.52885

0.78730
1.0758 1. 71,86 2.1811 . 2.8944 3.4705

4.1933

6.0091

9.67 .17 n.0100 .0100 $0 . n 1 n 1$ n.01n

n.01n

0.0105

0.0107

n. 0111

0.012

0.0186

0.0219

ก.131.5 0.2878 0.5261
0.8884

1.183

1.950
2.337

3.093

3.699

6.398

7.882
10.217

0.0200
0.0200
0.0201
0.0201
0.0201
0.0201
0.0202
0.0204
0.0206
0.0210
0.0215
0.0222
0.0248
0.0281
0.0368
11.0491
10.0754
11.2343
0.4514
0.8207
1.0891
1.398
2.124
2.662
3.509
4.186
5.048
7.215
8.874
11.514

$0.03 n 0$ $0.03 n 1$ $0.03 n$ $0.03 n$ ? $0.03 n 2$ $.0 .03 n$ $0.03 n$ o

0.0315

0.03 ?
0.0332

0.0371

$0.042 \pi$

0.054

0.0727

0.1101

0.3169
0.560

0.938

1.211
1.532

1.532
2.299

2.875

3.776
$4.5 n 6$

5.422

7.745 9.530
12.353
0.0500 0.0500 0.0501 0. 0503 0.0503 0. $051 \mathrm{n}$ 0.0515 0.0535 0.0553 0.0616 0.0694 0.117 0.1176
0.1740 0.4433 0.706 1.091
1.373 1.715 3.543 4.148 4.941 5.948
8.493 10.430
13.513
0.0700 0.0701

$0.070 \mathrm{~s}$ 0.0703 0.0704 0.0707 0.072 0.0734 0.0748 0.0772 0.0964 0.1237

0.1601

0.2314

0.305

1.196

1.488
1.844

2.715

4.415

4.415
5.261

6.322

9.011
0.9000
1.118
1.118
1.118
1.118
1.120
1.120
1.123
1.125
1.133
1.139
1.148
1.183
1.221
1.305
1.400
1.547
2.029
2.444
3.139
3.715
4.445
6.322
7.160
10.049
11.926
14.276
20.249
24.627
32.075$$
1134
$$

1.34
1.34
1.34
1.34
1.34
1.34
1.35
1.35
1.36
1.36
1.38
1.41
1.45
1.54
1.65
1.81
2.34
2.81
3.59
4.24
5.07
7.18
8.81
1.41
3.53
16.19
22.93
28.15
36.35

.9700
1.51
1.51
1.51
1.51
1.51
1.51
1.52
1.52
1.53
1.54
1.55
1.59
1.63
1.73
1.84
2.01
2.59
3.09
3.94
4.65
5.56
7.87
9.65
12.49
14.79
17.72
25.10
30.73
39.77

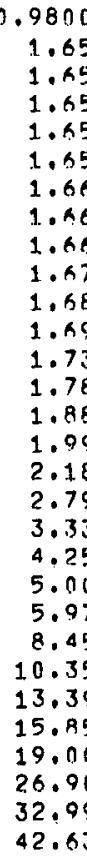

0.9900
1.91
1.91
1.91
1.91
1.91
1.91
1.91
1.92
1.93
1.93
1.95
1.9
2.04
2.1
2.2
2.49
3.17
3.7
4.8
5.6
4.7
9.53
11.6
15.0
17.8
21.3
30.28
37.08
47.94

0,1000 0.1001 $0.1 \cap 02$ 0.1005 .1010 0,1027 $0.1 \cap 48$ 0.11667 0.1218 0.1361

0.1723

0.2197

0.6414

0.0414
0.915 1. 314 1.619 1,990 2.91 .7 4.727 0.628 5.764
9.637 11.834
15.336 $\begin{array}{rr}0.9930 & 0.9950 \\ 2.05 & 2.18\end{array}$

2.05

2.05

2.05
2.05

2.05

2,06

2.06

2.07

2.09

2.14

2.31

2.44

3.66
3.38

4.03

5.11

6.02
7.17

10.13
12.40

16.04

18.97
22.69

22.69
32.14

39.40

50.93

2.18
2.18
2.19

2.19
2.19

?.19

$? .19$
2.20

2.21

2.22

2.28

2.46

2.60
2.83

4.28
5.42

0.9950
2.18
2.18

3.59

5.42
6.38
7.60 
VALUES OF ALPHA FOR NO $A$ AIVN $N P=4$

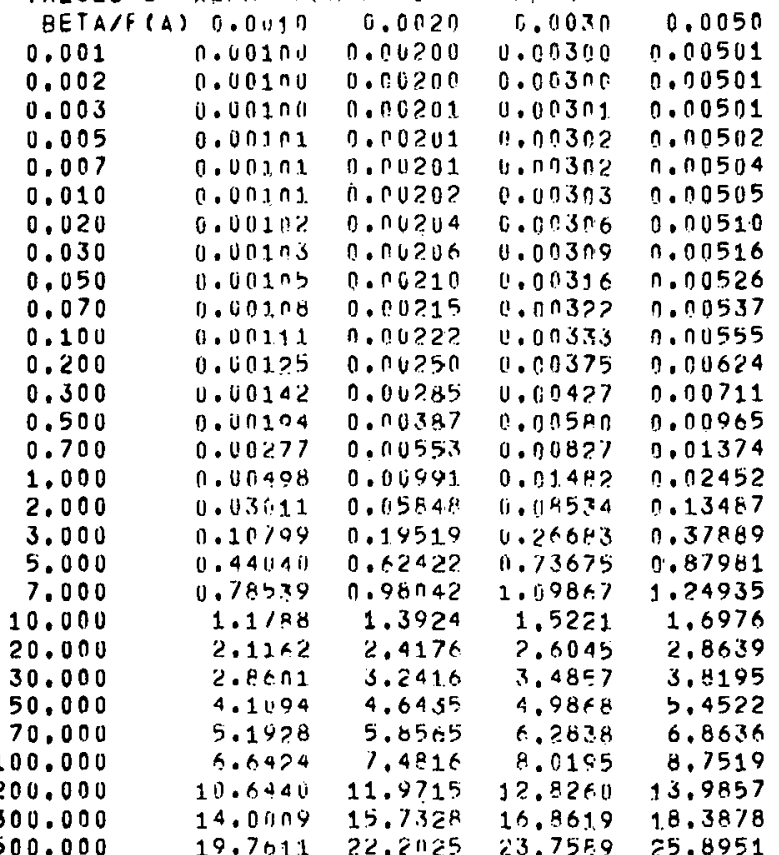

\begin{tabular}{|c|c|c|c|c|}
\hline$B E T A / F$ & n.5 & $0.610 \mathrm{an}$ & 0.70 r. & 0.8000 \\
\hline 0.001 & $0 . \operatorname{sn} 4$ & 0.624 & 0.735 & 0.886 \\
\hline & 0,505 & 0.014 & 0.735 & 0.886 \\
\hline $0.0 \cap 3$ & U.ts & 0.614 & 0.736 & ก. คВ 6 \\
\hline 0.005 & $0,5 \cap 5$ & 0.615 & 0.736 & $\pi .886$ \\
\hline 0. & U. $2 \pi x$ & $0 . \wedge 15$ & (i. .737 & $! 1.867$ \\
\hline 0.010 & $0 . \sin 7$ & 0.616 & 0.738 & 0.868 \\
\hline 0.020 & 0,510 & 0.618 & $0.7<1$ & 0,891 \\
\hline 0.030 & 0.5 .13 & 0.601 & $0.7<3$ & 0.894 \\
\hline 0.050 & U.517 & 0.627 & $0.7<9$ & 0.900 \\
\hline 0.070 & 0,523 & $11.6 \pm 3$ & 11.755 & 0.906 \\
\hline 0.100 & 0.5 .32 & fi. 641 & 0.763 & 10.915 \\
\hline 0.200 & $v .563$ & 0.674 & 0.797 & 0.948 \\
\hline 0.300 & 0,509 & 0.710 & 0.833 & 0.986 \\
\hline 0,500 & 0.683 & 0.750 & $0.9 ? !$ & 1.074 \\
\hline 0.700 & $0.7 \times 3$ & 0.856 & $1.0 ? 2$ & 1.179 \\
\hline 1.000 & 0,948 & $1, n \in 4$ & 1.104 & 1.358 \\
\hline 2.000 & 1.407 & $1.6 \div 5$ & 1.791 & 1.994 \\
\hline 3.000 & 1,483 & 2.147 & 2.337 & 2.585 \\
\hline 5.000 & $2.8 ? 2$ & 3.009 & 3.291 & 3.623 \\
\hline 7.000 & 3.554 & $3.9=0$ & 4.132 & 4.537 \\
\hline 10.000 & 4,539 & 4.865 & 5.254 & 5.765 \\
\hline 20.000 & 7.253 & 7.775 & 9.396 & 9.179 \\
\hline 30.000 & $9.5>0$ & 111.217 & 13.010 & 12.063 \\
\hline 50,000 & $13,4.36$ & 14.358 & 1.5 .519 & 16.984 \\
\hline 000 & 16 . Wh2 & 18.052 & 19.475 & 21.287 \\
\hline 000 & 21.409 & 22.904 & 24.674 & 27.024 \\
\hline & $34,11 ? 4$ & $.4 \in 9$ & .77 & 939 \\
\hline 00.000 & 44.648 & $47.4 \bar{E}$ ? & 51.484 & 56.306 \\
\hline 00 & 62. $\operatorname{tin} 6$ & 67.201 & $7 \% .450$ & 70.286 \\
\hline
\end{tabular}

$0.007 n$ 0.00701 . 0.00702 0.00705 0.00707 0.00715 0.00721 0.00753 00777 0.00874 0.00994 0.01347 0.01916 0.03405 0.17945 0.46281 0.97470 1.35426 3.0470 $4.056 n$ 5.7803 7.2756 14.8096 19.4550 $32.746 \quad 35.127$

$\begin{array}{llllll}0.9000 & 0.9300 & 0.9500 & 0.9700 & 0.9810 & 0.9900\end{array}$ $\begin{array}{lllll}1.1 .18 & 1.23 & 1.34 & 1.51 & 1.35\end{array}$ 1.118 1.118 1.1 .18 1.120 1.120 1.125 1.133 1,139 1,148 1.183 1.223 1.316 1,429 1,623 2.330 2,997 4,186 5.223 6.627 5.59 13.833 19,456
24,400 30,976 49,226
64.515 64,515
90,160

$0.0200 \quad 0.0300$

$0.0301 \quad 0.0500$

$0.02010 .0301 \quad 0.0501$

$0.0201 \quad 0.03 n 2$

0.0501
0.0503

$0.0201 \quad 0.0302$

0.0503

0.0204

0.0306

0.0510

0.0525

0.0535

0.0215

0.0335

0.0617

0.0925

0.1275

$0.0380 \quad 0.0565$

0.05350 .079

0.2083

0.3884

0.7501
1.2875

1.7052

0.866

1. 0:17

1.593
2.063

2.677

5.757
8.157

3.084

10.247

$\begin{array}{llll}1.23 & 1.34 & 1.51 & 1.65\end{array}$

$\begin{array}{llll}1.23 & 1.34 & 1.51 & 1.55 \\ 1.23 & 1.34 & 1.51 & 1.55\end{array}$

$\begin{array}{llll}1.23 & 1.34 & 1.51 & 1.55 \\ 1.24 & 1.34 & 1.51 & 1.56\end{array}$

$\begin{array}{llll}1.24 & 1.35 & 1.52 & 1.36\end{array}$

$\begin{array}{llll}1.24 & 1.35 & 1.52 & 1.56 \\ 1.25 & 1.36 & 1.53 & 1.57\end{array}$

$\begin{array}{llll}1.26 & 1.36 & 1.54 & 1.58 \\ 1.26 & 1.38 & 1.55 & 1.59\end{array}$

$\begin{array}{llll}1.30 & 1.41 & 1.59 & 1.73\end{array}$

$\begin{array}{llll}1.34 & 1.45 & 1.63 & 1.76 \\ 1.44 & 1.55 & 1.74 & 1.99\end{array}$

$\begin{array}{llll}1.55 & 1.68 & 1.87 & 2.03\end{array}$

$\begin{array}{llll}1.76 & 1.29 & 2.10 & 2.27 \\ 2.51 & 2.68 & 2.96 & 3.1 .8\end{array}$

$\begin{array}{llll}3.22 & 3.43 & 3.77 & 4.06\end{array}$

$\begin{array}{llll}5.59 & 4.77 & 5.23 & 5.52\end{array}$

$\begin{array}{rrrr}11.25 & 7.54 & 8.25 & 8.96 \\ 11.97 & 13.09 & 14.05\end{array}$

$\begin{array}{llll}4.76 & 15.10 & 17.17 & 18.39\end{array}$

$\begin{array}{llll}46.02 & 22.08 & 24.13 & 25.86 \\ 30.03 & 30.23 & 32.38\end{array}$

$\begin{array}{llll}33.05 & 35.07 & 38.36 & 41.11\end{array}$

$52.52 \quad 55.76 \quad 60.94 \quad 65.25$
0.0701

0.0701

0.0703

0.0704
0.00707

0.0707

0.0713
0.0720

0,0734

0.0748

0.0772
0,0859

0.0969

0.1272

0.1728
0.2735

0.2735
0.7348

1.120
1.717

2.85
4.636

8.676
110.888

13.848

$20.768 \quad 22.080$

$\begin{array}{ll}27.269 & 28.977 \\ 38.423 & 40.803\end{array}$

0.1000

$0.2000 \quad 0.3000 \quad 0.4000$

$\begin{array}{llll}0.1001 & 0.2002 & 0.3002 & 0.4013\end{array}$

$\begin{array}{llll}0.1002 & 0.2004 & 0.3007 & 0.4013\end{array}$

$\begin{array}{llll}0.1005 & 0.2009 & 0.3012 & 0.4023\end{array}$

$\begin{array}{llll}0.1006 & 0.2011 & 0.3016 & 0.4027\end{array}$

$\begin{array}{llll}0.1010 & 0.2016 & 0.3021 & 0.4037\end{array}$

$0.1027 \quad 0.2050 \quad 0.30640 .4085$

$\begin{array}{llll}0.1048 & 0.2083 & 0.3112 & 0.4137\end{array}$

$\begin{array}{llll}0.1067 & 0.2119 & 0.3155 & 0.4189\end{array}$

$\begin{array}{llll}0.1099 & 0.2174 & 0.3226 & 0.4270\end{array}$

$\begin{array}{llll}0.1368 & 0.2625 & 0.3798 & 0.4907\end{array}$

$\begin{array}{llll}0.1774 & 0.3264 & 0.4557 & 0.5727\end{array}$

$\begin{array}{llll}0.2355 & 0.4104 & 0.5498 & 0.6700\end{array}$

$\begin{array}{llll}0.3574 & 0.5632 & 0.7110 & 0.8340\end{array}$

$\begin{array}{llll}1.236 & 1.0739 & 1.2322 & 1.3676\end{array}$

$\begin{array}{llll}1.236 & 1.490 & 1.673 & 1.831 \\ 1.858 & 2.177 & 2.414 & 2.620\end{array}$

$2.379 \quad 2.765 \quad 3.051 \quad 3,306$

$\begin{array}{llll}3.066 & 3.547 & 3.903 & 4.224\end{array}$

$\begin{array}{llll}4.964 & 5.712 & 6.269 & 6.764 \\ 6.551 & 7.527 & 8.248 & 8.889\end{array}$

$\begin{array}{llll}9.271 & 10.629 & 11.651 & 12.551\end{array}$

$\begin{array}{lllll}11.636 & 13.345 & 14.611 & 15.733\end{array}$

$\begin{array}{llll}14.794 & 16.953 & 18.571 & 20.005\end{array}$

$\begin{array}{llll}16.96 & 29.575 & 29.51 .831\end{array}$

$\begin{array}{llll}43.550 & 49.836 & 54.536 & 58,747\end{array}$

$0.9970 \quad 0.9980 \quad 0.9990$

$\begin{array}{lr}1.91 & 0.9930 \\ 2,05\end{array}$

0.9950

$9.91 \quad 2.05 \quad 2.18$

2.41
2.41

2.41
2.41

$2.60 \quad 2.95$

$1.01 \quad 2.05$

$1.91 \quad 2.05$

$1.91 \quad 2.05$

$1.91 \quad 2.06$

$\begin{array}{ll}1.92 & 2.06 \\ 1.93 & 2.07\end{array}$

$1.93 \quad 2.07$

1.95

1.99
2.04

2.17

2.32
2.59

3.61
4.59

4.59
6.34

7.90
9.99

0.99
15.82

$20.71 \quad 16.80$

$29.13 \quad 30.92$

$36.47 \quad 38.73$

$\begin{array}{lll}36.47 & 38.73 & 40.99 \\ 46.26 & 49.10 . & 51.97\end{array}$

2.18
2.19

2.19

2.19

2.20

2.22

2.09
2.14
2.19

2.19

2,49

3,85

4.89
6.75

.75
8.40

8.89

2.23
2.28
2.34

2.48

2.95

4.19

15

11.24
17.78

23.30
32.75

2.41

2.41

2.42

2.42

2.44

2.46
2.51

2.72

2.72
2.91

3.24

4.48
5.67

7.81

9.70
12.26

12.26
19.39

$\begin{array}{ll}14.76 \\ 0.77 & 23.33\end{array}$

$\begin{array}{lll}25.38 & 27.18 & 30.55\end{array}$

$\begin{array}{lll}44.65 & 47.76 & 42.88 \\ & 53.68\end{array}$

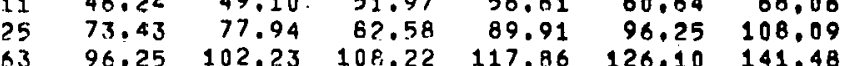
$\begin{array}{rrrrrrrrrr}96.86 & 102.72 & 112.37 & 120.42 & 135.38 & 143.68 & 151.98 & 165.65 & 177.37 & 199.10\end{array}$ 


\begin{tabular}{|c|c|c|c|c|c|c|c|c|c|c|c|c|c|c|c|c|}
\hline$B E T A / F(A)$ & $0.0(10)$ & $0.002 \%$ & 0.0030 & 0.0050 & $0.007 \%$ & $n \cdot 0.1 \cap 0$ & 0.0200 & \multicolumn{2}{|c|}{$0.03 n 0$} & \multicolumn{2}{|c|}{$0.050 n$} & 0.0700 & $0.1 \cap 00$ & 0.2 & .3000 & 0.4000 \\
\hline 0.001 & 0.00100 & 1. 10200 & 4. - ก30is & 0.00501 & 0.00700 & $n \cdot 0100$ & \multirow{2}{*}{\multicolumn{2}{|c|}{$\begin{array}{l}0.0200 \\
0.0201\end{array}$}} & \multirow{2}{*}{$\begin{array}{l}0.03 \cap 1 \\
0.03 n 9 .\end{array}$} & \multirow{2}{*}{\multicolumn{2}{|c|}{$\begin{array}{l}0.050 n \\
0.0501\end{array}$}} & \multirow{2}{*}{$\begin{array}{l}0.0701 \\
0.0701\end{array}$} & 0.1001 & 0.2002 & 0.3002 & 0.4013 \\
\hline 0.002. & 0.00100 & & $0.0030 n$ & 000501 & & $n .01 n 0$ & & 0.0 & & & & & 0.1002 & 0.2004 & 0.3007 & 0.4013 \\
\hline 0.003 & 0.00100 & 0.00201. & . & 00501 & $0,0 \cup 70 ?$ & $n .31 \cap 0$ & \multirow{2}{*}{$\begin{array}{l}0.0201 \\
0.0201\end{array}$} & & 0.0301 & \multicolumn{2}{|c|}{$0.050 \mathrm{~s}$} & 0.0702 & 0.1002 & 0.2004 & $0.300 ?$ & 0.4018 \\
\hline 0.005 & $0.001 n 1$ & 0.00201 & 0.0म3ก? & .00502 & $0,0070.8$ & $n . ? 100$ & & $0 . n$ & 302 & 0.0 & 503 & 0.0703 & 0.1005 & 0.2009 & 0.3012 & 0.4023 \\
\hline 0.007 & 0.00101 & 0.00201 & (i!, (ii) sח? & .00504 & 0.00735 & ח. & 0.02 & $0 . n$ & $3 \cap 2$ & & 1503 & 0.0704 & $0.1006^{\circ}$ & 0.2011 & 0.3016 & 0.4027 \\
\hline 0.010 & 0.00101. & 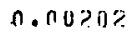 & r, onisns & $.0 \cup 5115$ & $0,0070 \%$ & $n, n 101$ & 0.02 & 0.0 & 303 & 0.0 & 505 & 0.0707 & $0.1 \cap 10$ & 0.2016 & 0.3021 & 0.4037 \\
\hline 0.020 & 0.00102 & $n .0 \cup 204$ & $0,0 \pi 3 \cap 0$ & .00510 & 0.00715 & 0.0102 & 0.02 & $0 . n$ & 306 & & 1510 & 0.0713 & 0.1018 & 0.2033 & 0.3045 & 0.4061 \\
\hline 0.030 & $0.001 \cap 3$ & $0 . n 02.06$ & $0.0 \cap 3 \cap 9$ & .00516 & $0,00721$. & $n \cdot 0,1 \cap 3$ & 0.02 & 0.0 & $3 n 9$ & 0.1 & 1515 & 0.0720 & $0.1 \cap 27$ & 0.2050 & 0.3064 & .4085 \\
\hline 0.050. & 0.00105 & $0.0 \cup 21 n$ & 0.00316 & .00526 & 0,00737 & 0.0105 & 0.02 & 0.0 & 31.5 & & 1525 & 0.0734 & 0.1048 & 0.2083 & 0.3112 & 0.4137 \\
\hline $0.070^{\circ}$ & 0.00108 & 0.011215 & (1.)ก3?? & .00537 & 0.00753 & 0.0107 & 0.02 & 0.0 & $3 ? 2$ & 0.0 & 5535 & 0.0748 & 0.1067 & 0.2119 & 0.3155 & 0.4189 \\
\hline 0.100 & 0.00111 & 0.11222 & $(1,04333$ & .00555 & 0.00777 & 0.0111 & 0.022 & 0.0 & 332 & & & 0.0772 & 0.1099 & 0.2174 & 0.3 & \\
\hline j).200 & $0.001>5$ & 0.00250 & 6,010375 & .00624 & $0,0 \cup 874$ & $n .01 \geqslant 5$ & 0.02 & 0.0 & 372 & 0.1 & J17 & 0.0859 & 0,1220 & 0.2381 & 0.3493 & 0.4566 \\
\hline 0.300 & 0.00143 & 0.00285 & ט. 014 ? & .00712 & 0.00996 & 0.0142 & 0.02 & 0.0 & 423 & & & 0.0970 & 0.1 & & & 0.4907 \\
\hline 0.500 & 0.00107 & 0.00393 & $\therefore .0 \cap 560$ & 1.00979 & 0.01368 & n.0105 & 0.03 & 0.0 & 574 & 0.0 & 1939 & 0.1291 & 0.1795 & $0.33 \cap 3$ & 4600 & 0.5765 \\
\hline 0.700 & 0.00293 & 0.010586 & 4.07877 & $n .01456$ & 0.0273 ? & 0.0298 & 0.05 & 0.0 & 334 & 0.1 & & 0.1814 & 0.2463 & 0.4251 & & 0.6862 \\
\hline 1.000 & 0.00507 & 0.11788 & $(1.119 .773$ & 1). 02926 & 0.04060 & 0.0572 & 0.10 & 0.1 & G & 0. & 01 & 112 & 0.4008 & 0.6128 & & \\
\hline 2.000 & 0.05932 & $0.1121 \mathrm{~K}$ & $11.1594 \%$ & 0.24001 & 0.30784 & 10.3809 & 0.5 & 0.6 & 953 & 0.8 & & 332 & 1.0434 & 1.2837 & & 1. \\
\hline 3.000 & $0.26 \% 31$ & 0.42323 & $11.5 ? 700$ & $n .66714$ & 0.70060 & $n \cdot 8617$ & 1.06 & 05 & 183 & & 45 & 1.457 & 1,587 & 1.875 & 36 & 2.269 \\
\hline 5.000 & 0.67005 & 1.07578 & 1.23593 & 1.35235 & 1.45916 & 1.5774 & 1.82 & & 983 & & 200 & 2.353 & 2.532 & 2.940 & & 3.509 \\
\hline 7.000 & 1.352 .35 & $1.5774 ?$ & 1.71665 & 1.011548 & 2.0561 .3 & 2.1849 & 2.49 & & 692 & & 971 & 3.169 & 3.398 & 3.926 & & 4.659 \\
\hline 10,000 & $1.952 ?$ & 2.2345 & 2.1100 & 2.6541 & 2.8221 & 3.017 & 3.4 & & 84 & & $04 \hat{B}$ & 4.315 & 4.513 & 5.315 & & 6.299 \\
\hline 20.000 & 3.6226 & 4.1552 & 4.4604 & 4.88 & $5.169 ?$ & $5.5 \mathrm{no}$ & 6.2 & & & & & 7.775 & 8.309 & 9.545 & 10.461 & 11.270 \\
\hline 30.000 & 5.22 .33 & 5.6947 & 5.3219 & 6.9018 & 7.3139 & 7.775 & 3.7 & & 8 & 1,0 & & 10.949 & 11.697 & $13.4 n 6$ & & 24 \\
\hline 50.000 & ล.00ก5 & 4.1129 & 0.750 .1 & 10.5440 & 11.2696 & 11.972 & 13.4 & 14. & 74 & & & 801 & 17.961 & 20.585 & & 247 \\
\hline 70.000 & 10.7508 & 12.0936 & $12.963 \mathrm{~s}$ & 14. & 14. & 35. & & & & & & 94 & 23 & 27.268 & & 36 \\
\hline 00.000 & 14.5350 & 16.3431 & 17.50 & 19, & 20. & 21. & 24 & 25 & & & & 46 & 32.075 & 36.71 .4 & & \\
\hline 0.000 & $26.017 ?$ & 29.2215 & 31.2815 & 34.0891 & $36.1 \cap 33$ & $38.3 n 1$ & 43. & 46 & & & & 20 & 57.221 & 65.492 & 71,717 & 77.210 \\
\hline 00.000 & 36.5305 & 41.0471 & 43.9157 & 47.8220 & 50.6296 & 53.712 & 60.4 & 64. & & & & & 80.262 & 91.859 & 100.526 & 108.216 \\
\hline $0,0,000$ & 56.01007 & 62.9282 & 0.7 .3227 & 73.3041 & 77.5766 & P.2.3.37 & 92.5 & 99 & & 108 & 460 & & 122.926 & 140.748 & 32 & 50 \\
\hline BETA! & $50 \mathrm{ln} 0$ & $\operatorname{con} 00$ & 700 i) & 0 & 0 & 9300 & 0 & & & & & 0.9930 & .9950 & .9970 & 9980 & 390 \\
\hline 0.001 & $\sin 4$ & 0.614 & 0.735 & .886 & 1.8 & 1.23 & 1.34 & 1.51 & & 65 & 1.91 & 2.05 & 2.18 & 2.41 & 2.60 & .95 \\
\hline 0.002 & & .514 & & & & & 1.3 & 51 & & 5 & & & & & & 15 \\
\hline 0.003 & 5 & 0.014 & "t & n & & & 1. & 1. & & & & & & & & \\
\hline 0.005 & $0.5 n j$ & $\because .015$ & $0.73 \mathrm{~h}$ & 0.8 & 1 . & 1. & 1.34 & 1.5 & & 65 & 1.9 & & $?$ & 2.41 & & 2.96 \\
\hline 0.007 & $0 . \operatorname{sns}$ & 0.615 & 0.737 & n. & 1.120 & j. & 1.34 & 1.5 & & 65 & i. & & & 2. & & 26 \\
\hline 0.010 & 0.507 & 0.616 & 0.734 & 0.848 & 1.220 & 1. & 1,3 & 1.5 & & ho & 1,9 & 2. & 2 & 2.41 & & 2 \\
\hline 0.020 & 0.510 & $n .618$ & 0. & 0 & 1. & i. & 1.3 & 1.5 & & 66 & 1. & & & 2 & & 6 \\
\hline 0.030 & 0.513 & 0.62 .1 & 0.743 & 0.8 & 1.125 & 1.24 & 1.3 & 1.52 & & 60 & 1.9 & 2. & 2. & 2.42 & 2 & 2.97 \\
\hline 0.050 & 11.517 & 1). A?? & :3. $74 n$ & 0 & & & 1. & 1. .53 & & 67 & 1. & & & 2.43 & & 2.98 \\
\hline 0.070 & $0.5>3$ & 0.633 & 0.756 & 0.906 & 1. & 1.226 & 1. & 1.54 & & 68 & 1. & & 2. & 2.44 & & 2.99 \\
\hline 0.100 & 0.532 & $0 . \$ 4.1$ & 0.763 & 0015 & & & 1. & 1.5 & & & & 2. & ?. & 2.46 & & 3.01 \\
\hline 0.20 & 0.56 .3 & 0.074 & $0.70 \%$ & & & & 1.41 & 1.59 & & 73 & 1 , & & & & & 3.07 \\
\hline 0.300 & 0. & 0.711 & 1.834 & & & & 1. & 1.6 & & & & & & & & 3.15 \\
\hline 0.5 & & $n$. Ru|n & 3.9 & & & & 1. & 1.7 & & & & & & & & 14 \\
\hline 0,70 & 0.709 & 0.913 & $i, n$ & & & & 1. & 1.8 & & 0 & 2. & 2. & 2. & 2. & 3. & 3.58 \\
\hline 1.0 & 4.099 & 1. & 1.2 & & & & 1. & & & & & & & & & \\
\hline 2.000 & 1.701 & $1.301 !$ & 2.1163 & 2,2 & 2. & 2.86 & 3.1 & 3.3 & & $\times 2$ & 4.09 & 4. & 4.63 & 5.16 & 5i & 6.12 \\
\hline 3.000 & $2.45 ?$ & 2.643 & 7. & & & & 4. & & & & & & & & & \\
\hline 5.000 & 3.172 & 4.1745 & $4.37 t$ & $4,8.1$ & 5.5 & 5. & 6.5 & 6.9 & & 41 & 8. & 8 . & 9.41 & 10 & 9 & 12.37 \\
\hline 7.000 & & 5364 & 5.796 & 6.3 & & 7.8 & 8. & $\therefore 0$ & & & 10. & & & & & \\
\hline 10.000 & 6.749 & 7.237 & $7.8 \mathrm{~F}$ & . & & $1 n .0$ & 11.9 & $1<\cdot<0$ & & & 14 & & & & & 74 \\
\hline 20.000 & $1.2 .0 \times .5$ & 17.9 .18 & $13.9 \div 2$ & $15 . ? 5$ & 7.472 & 18.66 & 19.8 & & & & $2 k$. & 27. & 20.40 & & & 38,54 \\
\hline 30.000 & 16.55 .3 & 15.144 & & & & & 27. & $3 n .38$ & & & 36 & & & & & 93 \\
\hline 50.000 & & $2 \% .707$ & 29.933 & 3 & 37 & 40.07 & 42.5 & 46.4 & & & & & & & & \\
\hline 70.000 & 34.3014 & 30.030 & 39.6 & 43.366 & 49. & 53.01 & 56. & & & & 74 & & & & & .07 \\
\hline 00.000 & 40.206 & . $\$ 9 ?$ & 376 & 58.341 & 66.834 & 71. & 75.75 & 82.83 & & & 99.79 & & & 2.19 & & \\
\hline 0.000 & $.5 n 1$ & 48.411 & 55.155 & 104.066 & 119.203 & 127.32 & 35.13 & & & & 1.77 .86 & & & & & \\
\hline 0.000 & $115.0 n 1$ & $123.4 i) ?$ & 133.420 & 146.119 & $16 \% .1$ & 179.35 & 89.53 & 206.91 & & $=0$ & 249.27 & 264.99 & 280.03 & 304.93 & .42 & 46 \\
\hline 10.000 & $171, .569$ & $16 ?$. & खn4.श口4 & $2<3.51 ?$ & $256.1 \cap 4$ & 27.29 & 89.50 & 316.65 & & $\circ 6$ & 381.59 & 405.52 & $42 n, 96$ & 467.04 & 499.51 & 561.04 \\
\hline
\end{tabular}


VALUES OF ALPHA FOR IV $=7$ ANI NP=1 BETA/F (A) O.DO1O U.0020 0.0030

\begin{tabular}{|c|c|c|c|c|}
\hline & & & & \\
\hline 1 & $0.002 \cap 0$ & $0.0 \cup 2011$ & $(0,0 i) 3 \cap(i$ & 0.00501 \\
\hline & 0.00100 & 0.00200 & 0.03301 & 0.00501 \\
\hline .005 & 0.00inu & 0.00201 & 0.0)3ก1 & 0.00501 \\
\hline 0.005 & $0.001 \cap 1$ & n.0u?01 & 0.0 ก 0 ? & 0.00502 \\
\hline 0.007 & 0.00101 & $0.00 \geqslant 0 j$ & $0.093 \cap 2$ & 0.005114 \\
\hline 0.010 & 0.00101 & $0.00 ? 02$ & 4.013303 & n. OOSU5 \\
\hline 0.020 & 0.00102 & $0.0 \cup ?)^{4}$ & $0.0103 \cap 0$ & 0.00510 \\
\hline 0.030 & 0.00103 & 0.00206 & $0.0 \cap 309$ & 0.00515 \\
\hline 0.050 & 0.00105 & 0.00210 & 0.03315 & 0.00525 \\
\hline 0.070 & 0.009 .07 & $n .00214$ & $0.0 \cap 3>1$ & n. ก11535 \\
\hline 0.100 & 0.001110 & 0.00220 & $0.0 n 330$ & 0.011550 \\
\hline 200 & 0.00120 & 0.00240 & $0.0113 \times 1 ;$ & $0.0 \cup 599$ \\
\hline 0.300 & 0.00130 & 0.002611 & 0.00390 & 0.00649 \\
\hline 0.500 & 0.00150 & 0.00300 & 0.00449 & 0.00748 \\
\hline 0.700 & 0.00170 & $0.0 \cup 340$ & 0.00509 & 0.00847 \\
\hline 1,000 & D. Uก?חD & 0.00399 & 0.017598 & $n .00994$ \\
\hline 2.000 & 0.00209 & 0.00598 & $11.3 \cap 894$ & 0.01485 \\
\hline 3.000 & 0.00509 & 0.00795 & 0.01190 & 0.01970 \\
\hline 5.000 & 0.00597 & 0.01138 & 0.01773 & 0.02926 \\
\hline 7.000 & 0.00705 & 0.01579 & 0.02351 & 0.03864 \\
\hline 0.000 & $0.02 n 9$ & 0.0216 & 0,0320 & 0.0524 \\
\hline 20.000 & 0.0206 & 0.04174 & 0,0504 & 0.0955 \\
\hline 30.000 & $0.0 \sin 1$ & 0.0585 & 0.0853 & 0.1349 \\
\hline 30.000 & 0.0486 & 0.0927 & 0.9331 & 0.2040 \\
\hline 10.000 & 0.0664 & 0.1246 & 0.17 .61 & 0.2830 \\
\hline 10.000 & 0.0918 & 0.1682 & 0.2331 & 0.3369 \\
\hline 10.000 & 0.1675 & 0.2873 & 0.3770 & 0.5050 \\
\hline 0.000 & 0.2394 & 0.3765 & $0.47 \mathrm{KA}$ & 0.6009 \\
\hline 10.000 & 0.3341 & 0.5031 & 0.6090 & 0.7415 \\
\hline BETA & 0.5000 & 0.0000 & 0.7000 & 0.8000 \\
\hline 0.001 & $0,50 ?$ & 0.608 & 0.722 & 0.860 \\
\hline & 0,502 & 0.608 & 0.722 & 0.860 \\
\hline 0.003 & $0.5 n 3$ & 0.608 & $0.7 \geqslant 3$ & 0.861 \\
\hline 0.005 & $0.5 \cap 3$ & 0.600 & $0.7 ? 3$ & 0.861 \\
\hline 0.007 & $U .5 \cap 4$ & $0.6[9$ & $(1.724$ & 0.802 \\
\hline 0.010 & $0 .>\cap 5$ & 0.610 & 0.724 & 0.862 \\
\hline 0.020 & U. $5 \cap 7$ & 0.013 & 0.727 & 0.865 \\
\hline 0.030 & $0,5.10$ & 0,615 & $0.73 n$ & 0.867 \\
\hline .050 & 0,515 & 0.620 & 0.735 & ก.872 \\
\hline .070 & 0,519 & 0.025 & 0.740 & 0.877 \\
\hline 100 & U.5?7 & 1). 632 & 0.746 & 0.864 \\
\hline 200 & 0.549 & 0.655 & 0.76 .9 & c.906 \\
\hline 300 & 0,570 & 0.676 & 0.789 & 0.926 \\
\hline 500 & 0.607 & 0.713 & 0.825 & 0.961 \\
\hline & & & 0.858 & 0.992. \\
\hline 1.000 & $0,6 \& 2$ & 0.786 & 0.499 & 1.034 \\
\hline & 0.786 & 0. คBด & 0.999 & 1.135 \\
\hline 0 & 0.860 & 0.961 & 1.072 & 1.207 \\
\hline 000 & 0.9 & 1,062 & 1.175 & 1.312 \\
\hline 000 & 1,034 & 1.135 & 1.247 & $\begin{array}{l}1.389 \\
\end{array}$ \\
\hline & 1,1 & 1,2 & 1.329 & 1.474 \\
\hline & 1,2 & .385 & 1.505 & 1.658 \\
\hline & $1,3 \times 1$ & 1.490 & 1.614 & $1.7 \% 4$ \\
\hline & 1.514 & (1) & 1.761 & 1.929 \\
\hline & 1,608 & 1.725 & 1.862 & 2.036 \\
\hline 100 & 1,711 & 1.835 & 1,975 & 2.158 \\
\hline & 1.926 & 2.059 & 2.212 & 2.410 \\
\hline & 2,055 & 2.196 & 2.357 & 2.566 \\
\hline & 2.234 & 2.383 & 2.555 & 2.780 \\
\hline
\end{tabular}
0.00700 0.00701 0,00702 0.00703 0.00705 0.00707 0.00714 0.00721 0.00755 0.0748 0.00030 0.00903 0.101046 0.01391 0.02071

0.02741

$0.0406 \pi$

0,05336 0.0720 0.1289
0.1795

0.2644

0.3336 0.5927 0.6077
0.8283

0.0100

$0.0100 \quad 0.0200$

$0.0100 \quad 0.0201$

$0.01 n 0 \quad 0.020$

$0.0101 \quad 0.0201$

0.0101

0.0103

0.0105

n.0107

0.0120

1). 0130

0.0169

0.0198

0.0234

0.0572

ก.074

0.100
0.175

0.2 .3

0.340

0.418

0.686

0.790
0.919

0.0000

\section{1,062}

$1: 062$

1. 064

1.064
1.064
1.066

1.064

1.066

1.068
1.070

1.076

1.076
1.080

1.087

1.108
1.129

1.129
1.165

1.196

1.238

1.343
1.421

1.421
1.532

1.012

1.705

1.904

2,1032
2,204

2,322

2,456
2,738

2.738
2,714

2.714
3.150

$\begin{array}{rrrr}.9300 & 0.9500 & 0.9700 & 0,7800 \\ 1.16 & 1.25 & 1.39 & 1.50 \\ 1.16 & 1.25 & 1.39 & 1.50 \\ 1.16 & 1.25 & 1.39 & 1.50 \\ 1.16 & 1.25 & 1.39 & 1.50 \\ 1.16 & 1.25 & 1.39 & 1.51 \\ 1.16 & 1.26 & 1.39 & 1.51 \\ 1.17 & 1.26 & 1.40 & 1.51 \\ 1.17 & 1.26 & 1.40 & 1.51 \\ 1.17 & 1.26 & 1.40 & 1.52 \\ 1.18 & 1.27 & 1.41 & 1.52 \\ 1.18 & 1.28 & 1.42 & 1.53 \\ 1.21 & 1.30 & 1.44 & 1.55 \\ 1.23 & 1.32 & 1.46 & 1.58 \\ 1.26 & 1.36 & 1.50 & 1.82 \\ 1.30 & 1.39 & 1.54 & 1.55 \\ 1.34 & 1.43 & 1.58 & 1.70 \\ 1.45 & 1.55 & 1.70 & 1.83 \\ 1.53 & 1.63 & 1.79 & 1.91 \\ 1.64 & 1.75 & 1.91 & 2.05 \\ 1.73 & 1.83 & 2.01 & 2.15 \\ 1.82 & 1.94 & 2.11 & 2.26 \\ 1.03 & 2.15 & 2.35 & 2.51 \\ 2.17 & 2.29 & 2.49 & 2.66 \\ 2.35 & 2.48 & 2.70 & 2.88 \\ 2.47 & 2.61 & 2.84 & 3.03 \\ 2.61 & 2.76 & 3.00 & 3.20 \\ 1.91 & 3.07 & 3.33 & 3.55 \\ 3.09 & 3.27 & 3.54 & 3.77 \\ 3.34 & 3.53 & 3.83 & 4.07\end{array}$

0.0300

0.0301

0.0301

0.0302

0.0202

0.0206

0.0210
0.0214

0.0220

0.0258
0.0297

0.0335

0.0392

0.0754

0.1404

0.300

0.388
0.513

$0.598 \quad 0.620$

$\begin{array}{ll}0.690 & 0.7106\end{array}$

$0.866 \quad 0.971$

$\begin{array}{ll}0.967 & 1.072 \\ 1.095 & 1.200\end{array}$
0.0500

0.0503

0.0503

0.0505

0.0510

0.0523

0.0533
0.0547

0.0594

0.0640

0.0732
0.0821

0.10952

0.1363

0.1 .74
0.240

0.296

0.367
0.528

0.629

0.759

0.931

1.102
1.205

1.205
1.337
0.0701

0,0702

0.0703

0.0704

0.0707

0.0720

0.0745

0.0765

0,0828
0.0892

0.1014

0.1135
0.1308

0.1842

0.231
0.311

0.376

0.454

0.621

0.722
0.849

0.932

1.020

1.297
1.432

0.1000

0.1001

0.1002

0.1005

0.1006

0.1018

0.1027

0.1045

0.1089

0.1176

0.1263
0.1 .430

0.1589

0.1818

0.2501
0.308

0.400

0.472
0.554

0.722

0.822
0.948

$.1,030$

1.118
1.293

1.400
1.539

1,71

J. 99.30

0.9950

$1.71 \quad 1.81 \quad 1.92$

$1.71 \quad 1.82 \quad 1.92$

$1.71 \quad 1.82$

$1.71 \quad 1,82$

$1.71 \quad 1.8$

1,7

1,72
1,73
1.73
1,76

1,76

1,7

1.87

1.92

2.05
2,15
2.30

2.40

2,50
2,53
2,80

2,97

3.20

3,37

3.94

4.19
4.52

$0.2000 \quad 0.3000 \quad 0.4000$

$0.2002 \quad 0.3002 \quad 0.4008$

$0.2004 \quad 0.3007 \quad 0.4008$

$0.2004 \quad 0.3007 \quad 0.4013$

$0.2009 \quad 0.3012 \quad 0.4018$

$0.20110 .3016 \quad 0.4023$

$\begin{array}{llll}0.2016 & 0.3021 & 0.4027\end{array}$

$0.20330 .3040 \quad 0.4051$

$0.2078 \quad 0.3102 \quad 0.4123$

$0.2112 \quad 0.3145 \quad 0.4170$

$0.2157 \quad 0.3202 \quad 0.4237$

$0.2307 \quad 0.3398 \quad 0.4452$

$\begin{array}{lll}0.2454 & 0.3579 & 0.4657\end{array}$

$0.2726 \quad 0.3918 \quad 0.5022$

$\begin{array}{lll}0.2983 & 0.4223 & 0.5346\end{array}$

$0.33360 .4628 \quad 0.5765$

$\begin{array}{lll}0.4294 & 0.5670 & 0.6824\end{array}$

$\begin{array}{lll}0.502 & 0.642 & 0.757 \\ 0.607 & 0.747 & 0.860\end{array}$

$\begin{array}{lll}0.682 & 0.742 & 0.860 \\ 0.821 & 0.932\end{array}$

$\begin{array}{lll}0.764 & 0.901 & 1.011\end{array}$

$0.928 \quad 1.062 \quad 1.175$

$\begin{array}{lll}1.026 & 1.162 & 1.274\end{array}$

$1.234 \quad 1.375 \quad 1.495$

$\begin{array}{lll}1.326 & 1.471 & 1.593 \\ 1.511 & 1.665 & 1.797\end{array}$

$\begin{array}{lll}1.625 & 1.785 & 1.922 \\ 1.776 & 1.945 & 2.093\end{array}$

$\begin{array}{llll}0.9970 & 0.9980 & 0.9990\end{array}$

$\begin{array}{lll}2.109 & 2.23 & 2.49 \\ 2.09 & 2.23 & 2.49\end{array}$

$\begin{array}{lll}2.09 & 2.23 & 2.49 \\ 2.09 & 2.23 & 2.49\end{array}$

$\begin{array}{lll}2.09 & 2.23 & 2.49 \\ 2.09 & 2.23 & 2.50\end{array}$

$2.09 \quad 2.23 \quad 2.50$

$2.10 \quad 2.24 \quad 2.50$

$\begin{array}{lll}2.10 & 2.24 & 2.51 \\ 2.11 & 2.25 & 2.51\end{array}$

2.26

$2.12 \quad 2.27 \quad 2.53$

$2.18 \quad 2.33 \quad 2.60$

$2.23 \quad 2.38 \quad 2.66$

$2.28 \quad 2.43 \quad 2.71$

$2.49 \quad 2.66 \quad 2.96$

$\begin{array}{lll}2.61 & 2.78 & 3.09\end{array}$

$2.91 \quad 3.09 \quad 3.44$

$\begin{array}{lll}3.05 & 3.25 & 3.61\end{array}$

$\begin{array}{lll}3.37 & 3.58 & 3.97 \\ 3.57 & 3.80 & 4.22\end{array}$

$3.85 \quad 4.09 \quad 4.54$

$4.05 \quad 4.30 \quad 4.77$

$\begin{array}{lll}4.26 & 4.53 & 5.12 \\ 4.73 & 5.02 & 5.56\end{array}$

$\begin{array}{lll}5.02 & 5.33 & 5.90 \\ 5.41 & 5.74 & 6.36\end{array}$ 


\begin{tabular}{|c|c|c|c|c|c|c|c|c|c|c|c|c|c|c|c|}
\hline $\begin{array}{l}\text { VALUES OF } \\
\text { RETA/F (A) }\end{array}$ & $\begin{array}{c}\text { AltrHA FO } \\
n=0101 \mathrm{~d}\end{array}$ & 1.011211 & $n, 00,30$ & 0.0050 & $0.00 / 0$ & $n .010 n$ & $(0.020 n$ & 0.03 & $3 \cap n$ & .10500 & 0.0700 & 1). 1000 & 0.2000 & 0.3000 & 0.4000 \\
\hline 0.001 & 0.00100 & n. กu>u? & 0.01300 & 0.00501 & 0.06700 & 0.0100 & $0.020 n$ & 0.03 & 301 & $.050 n$ & 0.0701 & $0.1 \div 01$ & 0.2002 & 3002 & 0.4008 \\
\hline 0.002 & 0.00100 & n.nu?0: & U. & 0.00501 & 0.007111 & P. Dino & 0.0201 & 0.03 & 301 & .0501 & 0.0701 & 0.1 nos & .2004 & 1.3007 & 0.4008 \\
\hline 0.003 & $0.0(0101)$ & $0 . n$ Q 0.01 & H. nosisn & ה.00501 & $0.0070 ?$ & $0.01 n 0$ & 0.0201 & $0 . n 3$ & 301 & .0501 & 0.0702 & $0.1 \cap 0 ?$ & 1.2004 & .3007 & 0.4013 \\
\hline 0.005 & 0.00101 & $0.00 ? 01$ & Q. $1103 n \%$ & 0.00502 & 0.00703 & $r . n 100$ & 0.0201 & 0.03 & 3ก? & .0503 & 0.0703 & 0.1005 & $0.20 \cap 9$ & .3012 & 0.4018 \\
\hline 0.007 & 0.40 .101 & ח. $)(1 \geqslant 0.1$ & $11003 n ?$ & ถ. 0115114 & 0,00705 & n.ก101. & 0.0201 & 0.03 & 3 ก? & .0503 & 0.0704 & $0.1 \cap 06$ & 0.2011 & 1.3016 & 0.4023 \\
\hline 0.01 .0 & $\pi \cdot 0010$ & ח. 0, & 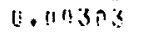 & 0.065115 & 0.04707 & n.l101 & 0.0202 & 0.03 & $3 \cap 3$ & .0505 & 0.0707 & 0.1010 & 0,2016 & 1.3021 & 0.4027 \\
\hline 0.020 & ט.un1n? & 1). $n \cup 104$ & 11.010305 & 0.01510 & 0.00715 & n.310 & $0.02 \downarrow 4$ & $0 . n 3$ & $3 n 6$ & $.051 n$ & 0.0713 & $u \cdot 1 \cap 18$ & 0.2033 & 1.3045 & 0.4051 \\
\hline 0.030 & i). 00203 & 0.00006 & 0.011399 & 1.00516 & 0.00721 & 0.0103 & $0.026 \mathrm{~K}$ & 0.03 & 3ng & .0515 & 0.0720 & $0.1 \cap 27$ & 0.2050 & 0.3064 & 0.4080 \\
\hline 0.050 & 0.00105 & $0 . n \cup>10$ & 0.110316 & 0.00526 & 0.00736 & 0.0105 & $0.02 .1 n$ & 0.03 & 315 & .0525 & 0.0734 & $0.1 \cap 46$ & 0.2083 & 0.3107 & 0.4127 \\
\hline 0.070 & 0.00107 & $0.00 \% 15$ & แ.กค3?? & 0.00537 & 0.00751 & $0.010 \%$ & 0.0215 & .0 .03 & $3 ? 2$ & .0535 & 0.0746 & 0.1067 & 0.2119 & 55 & 0.4180 \\
\hline 0.100 & 0.60191 & $0, n \varphi \geqslant ? ?$ & (1.0.1933: & 9. 00554 & 0.00777 & 0.0111 & $0.02 \% 1$ & $0 . n 3$ & 3.32 & .0552 & 0.0771 & $0.1 \ln 98$ & 0.2171 & 0.3 & 0.4261 \\
\hline 0.200 & 0.00124 & $0.01024 R$ & $\because . \cap n 37 \%$ & 9.111619 & 0.00866 & 0.9174 & 0.026 .7 & 0.03 & 369 & .0613 & 0.0853 & 0.1210 & 0.2347 & 0,3469 & 0.4538 \\
\hline 0.300 & 0.40239 & $0.0427 \%$ & 1.07417 & 0.00694 & 0.00970 & 0.0138 & 0.1276 & 0.04 & 412 & .0582 & 0.0948 & 0.1337 & 0.2578 & 0.3736 & 0.4821 \\
\hline 0.500 & 0.001 .75 & $0.0035 n$ & $1: .010520$ & $1.0087 ?$ & 0.01219 & n. 0174 & 0.0345 & 0.05 & 51.3 & .0844 & 0.1164 & 0.1628 & 0.3045 & 94 & 0.5422 \\
\hline 0.700 & 0.00219 & 0.00437 & 0.011655 & 1.01088 & 0.01520 & 0.0216 & 0.0428 & 0.06 & 6.34 & $.1 \cap 33$ & 0.1415 & 0.1957 & 0.3541 & .4859 & 0.6004 \\
\hline 1.000 & 0.00209 & 0.00598 & $0.0 \operatorname{lin} 804$ & .01485 & $0.02 n \%$ & $n .0204$ & 0.0577 & 0.08 & 849 & .1363 & 0. & 0.2501 & 0.4294 & & 0.6824 \\
\hline 2.000 & 0.00006 & 0.0238 .4 & $11.6 \geq 04 ?$ & 0.03349 & 0.04704 & $n .0600$ & 0.1251 & 0.17 & 780 & $.269 ?$ & 0.3450 & 0.4385 & 0.6491 & 863 & 0.8989 \\
\hline 3.000 & (1.01245 & 0.02538 & 0.0376 .2 & 0.06237 & $0.0839 \mathrm{n}$ & f. .1161 & 10.2097 & 0.2 & 287 & 0.407 & 0.496 & 0.598 & 0.807 & 0.943 & 1.053 \\
\hline 5.000 & 0.03011 & 0.05848 & U. 1) 453.3 & ค. 15487 & 0.11945 & 0.2387 & 0.3879 & 0.9 & 492 & 0.629 & 0.722 & 0.822 & 1.026 & 1.162 & 1.274 \\
\hline 7.000 & 0.05305 & $0.1025 n$ & 0.14631 & 9.22260 & 0.28686 & 0.3655 & 0.5413 & 0.0 & 650 & $8 n$ & 0. & & 1.179 & & 1.434 \\
\hline 10.000 & 0.1000 & 3.1821 & $\pi .2506$ & 0.3584 & 0.4340 & 0.5 .32 & 0.715 & 0.8 & 821 & 56 & 1.043 & 1. & 1.350 & 1.495 & 1.621 \\
\hline$\therefore 0.000$ & $0.29+4$ & 1).458 & 11.5638 & 0.6967 & 0.7835 & 0.875 & 1.051 & 1.1 & 6 & & 1.387 & 1. & 1.724 & 1.890 & 2.032 \\
\hline & $0.464 \pi$ & i] .664 .4 & $10.764 \%$ & u. & 10.9847 & 1.074 & 1. & 1.3 & 1 & & 1.6114 & 1.719 & 1.971 & .154 & 2.311 \\
\hline 50.000 & $0.745 \mathrm{~s}$ & $9.9 \geqslant 18$ & 1.0274 & 1.1521 & 1.2379 & $1,3.31$ & $1.5 \% 0$ & 1.6 & 638 & 97 & 1.910 & 2.036 & 2.322 & 28 & 2.711 \\
\hline 70.000 & 141 & $\because .6491$ & 1.1902 & 1. & 1.4134 & 1.51 .1 & 1.713 & 1.6 & & 19 & & 2. & 2. & 07 & 3.005 \\
\hline 100. & 1.0930 & $\div .2684$ & $1.375 \%$ & 1.5 & 1.6098 & 1.715 & 1.933 & 2.0 & 70 & 2.254 & 2. & 2, & & 3. & 3.348 \\
\hline & 1.4406 & $\therefore .6442$ & $1.766 \%$ & 1.92 & 2.0361 & 2.158 & 2.418 & 2.5 & 2 & 2.803 & 2.963 & 3. & 3.558 & 3.858 & 4.125 \\
\hline 10 & $1.67 \times 5$ & 1. 8902 & ?. n?no & 2.2002 & 2.3222 & 2.460 & $2.7 \div 6$ & 2.5 & 925 & 3.177 & 3.352 & & 4. & 4.354 & 4.651 \\
\hline 10.000 & 1.9942 & 2.2345 & 2.363 .3 & 2.5454 & 2.1260 & 2.893 & 3.211 & 3.4 & 421 & 3.707 & 3.911 & $4,1,48$ & 4.692 & 5.071 & 5.414 \\
\hline BETA/ & 0.5 L & 0.6000 & 0.70011 & 0.8000 & 0.9000 & $93 \cap 0$ & 9500 & 00 & 980 & 0.9900 & 0.9930 & 0.9950 & .9970 & .9980 & 9.9990 \\
\hline 0.001 & $0 . \sin 2$ & $0.60 \%$ & 22 & & 1. & 1 & 1.25 & 1.39 & 1.50 & 1.71 & 1.81 & 1.5 & $n 9$ & 2.23 & 2.49 \\
\hline 0.002 & $\sin 2$ & $0.60 \mathrm{~B}$ & 22 & $n$ & & & 1.25 & 1.39 & 1.50 & & 1.81 & 1 . & 2.19 & & 2.49 \\
\hline 0.003 & $0.5 \mathrm{~ns}$ & $0.00 \mathrm{~B}$ & $n .733$ & 0. & 1.064 & & & & $5 n$ & & 1.82 & 1 & 2.0 & & 2.49 \\
\hline 0.005 & $0.5 n 3$ & 0.609 & 1.723 & $n .8$ & 1.064 & 1. & 1.25 & 1.39 & 1.50 & & 1.82 & 1. & 2.09 & & 2,49 \\
\hline 0.007 & $0.2 n 4$ & 0.409 & 6.720 & 0.8 & 1.0 & 1. & 1.2 & 1.39 & 1.51 & 1. & 1.82 & 1. & 2.09 & 2. & 2.50 \\
\hline 0.010 & $0 . \operatorname{sns}$ & $0 . A 11$ & 6.174 & & 1. & & 1.26 & 1.39 & 1.51 & & 1.82 & 1. & 2.09 & & 2.50 \\
\hline 0.0 & 0.507 & $n .61 .3$ & D. & & & & 1.26 & 1.40 & 1.51 & 1. & 1.82 & 1. & 2.10 & 24 & 2.50 \\
\hline 0.030 & 0.510 & 0.616 & 0.730 & 0. & 1.070 & 1.17 & 1.26 & 1.40 & 1.59 & 1.71 & 1.82 & 1.5 & 2.10 & 2.24 & 2.51 \\
\hline 0.050 & & $n, 62 n$ & & & & & 1.26 & 1.41 & 1.52 & 1. & 1.83 & .. & 2 & & 2,51 \\
\hline 0.070 & $0.5>0$ & I). .25 & $0.7 \Delta 1$ & 0.878 & 1.682 & 1 & 1.27 & 1.41 & $1.5 ?$ & 9.73 & 1.84 & 1. & 2.1 & 2.26 & 2.52 \\
\hline 0.100 & $0.5 \geqslant 9$ & 0.435 & 0.749 & 0.88 & $1,1 B 9$ & & 1.28 & 1.42 & 1.53 & 1.74 & 1.85 & 1.9 & 2.13 & 2 & 2.54 \\
\hline 0.200 & 0.557 & 0.063 & 1.776 & $n$ & 18 & J. & 1.31 & 1.45 & 1.56 & $1 . .77$ & 1.88 & 1. & & 2 & 2.58 \\
\hline 0,300 & 0.508 & 0.693 & $0.8 n \theta$ & 0 & 1.146 & & 1.34 & 1.48 & 1.60 & 1.80 & & & & & 2.63 \\
\hline 0.500 & 0.648 & 0.753 & 0.855 & 0.999 & 1.204 & 1. & 1.40 & 1.54 & 1.66 & 1.88 & 1.99 & 2. & & & 2.72 \\
\hline 0.700 & $0.7 n 6$ & 0.810 & $n .9 ? 1$ & 1.05 & 1,261 & & 1.46 & 1.61 & 1.73 & 1.95 & 2.07 & & 2. & 2.53 & 2.82 \\
\hline 1.000 & 0.786 & 0.889 & $1 ! .994$ & & 1. & & 1.55 & 1.70 & 1.83 & & 2.18 & & & & 2.96 \\
\hline 2.000 & 4.909 & $1.10 ?$ & 1.21 .3 & 1.352 & 1374 & 2. & 1.79 & 1.96 & 2.1 .4 & 2.3 & 2.49 & ?. & 2.84 & 3. & 3.37 \\
\hline 3.000 & 2.56 & 1.259 & 1.375 & 1.5 & 1.1 & 1. & & 2 & & & 2 . & & & & 3.70 \\
\hline 5.000 & 1.381 & 1.490 & 1.61. & 1.7 & 2.0 & & 2.29 & 2.49 & 2.60 & $? .9$ & 3.14 & 3. & & 3.80 & 4.22 \\
\hline 7.000 & 1.547 & 1.661 & 1.795 & 1.9 & 2.2 & 2. & 2,52 & 2.75 & $2.9 ?$ & $3 . ?$ & 3. & 3. & 3. & 4.1 & 4.61 \\
\hline 10.000 & 1,740 & 1.864 & 2.016 & 2.1 .5 & 2.494 & 2.65 & 2.80 & 3.04 & 3.24 & 3.61 & 3.81 & 4.01 & 4.32 & 4.60 & 5.09 \\
\hline 20.000 & $2,9,73$ & 2.318 & $2.4 \mathrm{AB}$ & 2.7 & 3.066 & 3.26 & 3.44 & 3.73 & 3.96 & 4.41 & 4.65 & 4.8 & 5. & & 6.20 \\
\hline 30.000 & 2.467 & 2.627 & 2.814 & 3.058 & 3,463 & 3.67 & 3.87 & 4.19 & 4.47 & 4.96 & 5.23 & 5.50 & 5. & 6.29 & 6.97 \\
\hline 50.000 & 2.887 & 3.074 & 3.297 & 3.566 & & & 4.51 & 4.88 & 5.19 & 5,75 & 6.06 & s. & 6. & 7. & 8.07 \\
\hline 70.000 & 3.296 & 3.402 & 3.638 & 3.94 & 4.453 & 4.72 & 4.97 & 5.38 & 5.72 & 6.34 & 6.69 & 7.03 & 7.58 & & 8.89 \\
\hline 100.000 & $3.5 \times 2$ & 3.787 & $4.04 \varepsilon$ & & & & & 5.98 & 6.35 & 7.04 & 7.42 & 7.79 & 8.40 & 8.92 & \\
\hline & .384 & $4 . \wedge 51$ & 4.972 & 5.3 & 6.0 & 0.42 & 6.77 & $7 \div 31$ & 7.78 & 8.61 & $9, n 7$ & 9. & & 89 & \\
\hline & 4.941 & 5.246 & $5.5 n s$ & & & & 7.62 & & 8.75 & 0.68 & 10.20 & 10.72 & 11.54 & 25 & 10.34 \\
\hline 00.000 & 5.750 & $6.1 \cup 1$. & 6.5015 & 7.047 & 7.928 & 8.39 & 8,54 & 9.55 & 10.14 & 11.24 & 11.83 & 12.43 & 13.39 & 14.20 & 15.70 \\
\hline
\end{tabular}




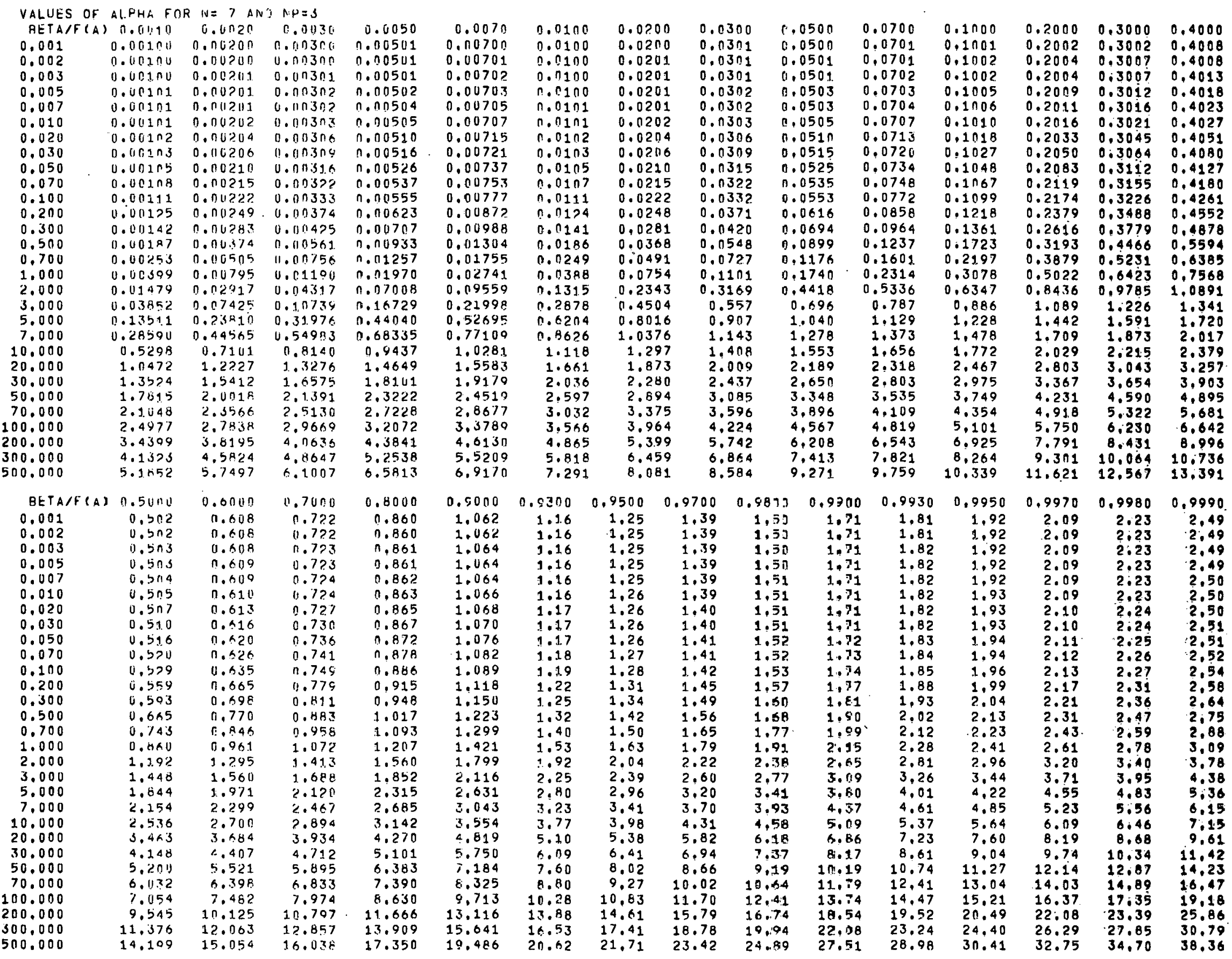




\begin{tabular}{|c|c|c|c|c|}
\hline $\begin{array}{l}\text { VALUES OF } \\
B E T A / F(A)\end{array}$ & $\begin{array}{c}\text { ALPHE. FOR } \\
0.01 .10\end{array}$ & $\begin{array}{l}N=7 \text { AN } \\
0.0020\end{array}$ & $\begin{array}{l}N P=4 \\
\cap .0030\end{array}$ & 0.0050 \\
\hline 0.001 & 0.00100 & 0.00200 & 0.00300 & 0.00541 \\
\hline $.0,002$ & 0.00100 & $0.0020 ?$ & 0.00300 & 0.00501 \\
\hline 0.003 & 0.00100 & 0.00201 & 0.00301 & 0.00501 \\
\hline 0.005 & 0.00101 & 0.00201 & $0.0 \times 3 \times 2$ & 0.00502 \\
\hline 0.007 & 0.00101 & 0.0020 .1 & $0.003 n 2$ & 0.00504 \\
\hline 0.010 & 0.00101 & $0 . n 0202$ & $0.0 \cap 3 \cap 3$ & 0.00505 \\
\hline 0.020 & $0.001 \cap 2$ & 0.00204 & 11.00306 & 0.00510 \\
\hline 0.030 & $0.001 \cap 3$ & 0.00206 & 0.00309 & 10.00516 \\
\hline 0.050 & 0.00105 & 0.00210 & 0.00316 & 0.00526 \\
\hline 0.070 & $0.001 \cap 8$ & 0.00215 & 11.0032? & 0.00537 \\
\hline 0.100 & 0.00111 & 0.00222 & 11.00333 & 0.00555 \\
\hline 0.200 & 0.00125 & 0.011250 & 0.00375 & $\cap .0 \cap 624$ \\
\hline 0.300 & 0.00242 & $0.0 \cup 285$ & $11,00<27$ & 0.04711 \\
\hline 0.500 & 0.011194 & 0.00387 & $(1.00580)$ & 0.00965 \\
\hline 0.700 & 0.00277 & 0.00553 & 0.00827 & 0.01374 \\
\hline 1.000 & 0.00408 & 0.00091 & $0.01<82$ & 0.02452 \\
\hline 2.000 & 0.03011 & 0.05448 & 0.08534 & $n .13487$ \\
\hline 3.000 & 0.10799 & 0.19519 & 0.25683 & $n .37841$ \\
\hline 5,000 & 0.43545 & 0.61950 & $0.72<36$ & 0.85501 \\
\hline 7.000 & 0.76918 & 0.94465 & 1.11471 .7 & 1.17497 \\
\hline 10.000 & 4.1158 & 1.2932 & 1.4000 & 1.5412 \\
\hline 20.000 & 1.8073 & 2.0972 & 2.238 .3 & 2.4290 \\
\hline 30.000 & 2.4252 & $2,7 n 37$ & $2.879 ?$ & 3.1157 \\
\hline 50.000 & 3.3217 & 3.0917 & 3.9243 & 4.2391 \\
\hline 70.000 & 4.0636 & 4.51 .38 & 4.7684 & 5.1699 \\
\hline 100.000 & 5.0249 & 5.5666 & 5.9101 & 6.3753 \\
\hline 200,000 & 7.5503 & 8,5552 & 8.8587 & 9.5454 \\
\hline 300.000 & $9.5 \sin 6$ & $10.5 \times 77$ & 11.2006 & 12,0783 \\
\hline 500.000 & 12.8413 & 14.199 .3 & $15 . n 600$ & 16.2211 \\
\hline$B E T A / F(A)$ & $0.50 \cap 0$ & 0.6000 & 0.7000 & 0.8000 \\
\hline 0.001 & $0.5 n 2$ & $0.6 \cup 8$ & 0.72 .2 & 0.860 \\
\hline 0.002 & $0.5 n 2$ & $0.60 \mathrm{~B}$ & 0.722 & 0.860 \\
\hline 0.003 & $0.5 n 3$ & 0.608 & 0.723 & 0.861 \\
\hline 0.005 & $0 . \sin 3$ & $\pi .509$ & 0.723 & 0.861 \\
\hline 0.007 & $u, b \cap 4$ & 11.609 & 11.724 & D.80? \\
\hline 0.010 & 0.505 & 0.610 & 0.724 & 0.863 \\
\hline 0.020 & 0.507 & 0.513 & 0.7377 & 0.865 \\
\hline 0.030 & 0.510 & 0.616 & 0.730 & 0.867 \\
\hline 0.050 & 0.516 & $0 .+20$ & 0.736 & $0.8 \% 2$ \\
\hline 0.070 & $0.5 ? 0$ & 0.626 & 0.741 & 0.878 \\
\hline 0,100 & $0.5 ? 9$ & 0.535 & 0.749 & 0.856 \\
\hline 0.200 & 0.559 & 0.665 & 0.781 & 0.916 \\
\hline 0.300 & 0,504 & $n .700$ & 0.8 .13 & 0.948 \\
\hline 0.500 & 0.0 .74 & 11.779 & 0.800 & 1.026 \\
\hline 0.700 & $0.1 / 66$ & 0.869 & 0.980 & 1.1 .16 \\
\hline 1.000 & $0,9: 16$ & 1.0 .17 & 1.127 & 1.265 \\
\hline 2.000 & 1.3 .81 & 1.490 & 1.614 & $1.7 \% 4$ \\
\hline 3.000 & 1.766 & 3.092 & 2.036 & 2.223 \\
\hline .000 & 2,399 & 2.555 & 2.738 & 2.918 \\
\hline 7.000 & 2.929 & 3.116 & 3.333 & 3.61 .9 \\
\hline 10.000 & 3,052 & 3.842 & $4,1 \cap ?$ & 4.445 \\
\hline 20.000 & $5.42 ?$ & 5.757 & 6.134 & 6.642 \\
\hline 30.000 & $6.8 \times 4$ & 7.283 & 7.760 & 8.401. \\
\hline 50.000 & 9.225 & 9.789 & 1.1 .430 & 11.285 \\
\hline 70.000 & 11,209 & 1.1 .880 & 12.673 & 13.696 \\
\hline 100.000 & 13.172 & 14.596 & 1.5 .550 & 16.811 \\
\hline 200.000 & 20.524 & 21.745 & 23.179 & 25.041 \\
\hline 300.000 & 25.005 & 27.452 & 29.252 & 31.587 \\
\hline 500.000 & 34.089 & 56.775 & 30.216 & 42.329 \\
\hline
\end{tabular}

\begin{tabular}{|c|c|c|c|c|c|c|}
\hline $\begin{array}{l}0.0070 \\
0.00700 \\
0.00701 \\
0.00702 \\
0.00703\end{array}$ & $\begin{array}{l}0.0100 \\
0.0100 \\
0.0100 \\
0.0100 \\
0.0100\end{array}$ & $\begin{array}{l}0.0200 \\
0.0200 \\
0.0201 \\
0.0201 \\
0.0201\end{array}$ & $\begin{array}{l}0.0300 \\
0.0301 \\
0.0301 \\
0.0301 \\
0.0302\end{array}$ & $\begin{array}{l}0.0500 \\
0.0500 \\
0.0501 \\
0.0501 \\
0.0503\end{array}$ & $\begin{array}{l}0.0700 \\
0.0701 \\
0.0701 \\
0.0702 \\
0.0703\end{array}$ & $\begin{array}{l}0.1000 \\
0.1001 \\
0.1002 \\
0.1002 \\
0.11005\end{array}$ \\
\hline 0.010705 & 0.0101 & 0.0201 & $0.03 \cap 2$ & 0.0503 & 0.0704 & 0.1 NOB \\
\hline 0.00707 & 0.0101 & 0.0202 & 0.0303 & 0.0505 & 0.0707 & $0.1 n_{1} n$ \\
\hline 0.00715 & $0.101 \cap 2$ & 0.02114 & $0.03 \cap 6$ & $n .051 n$ & 0.0713 & $0.1 n 18$ \\
\hline 0.00721 & 0.0103 & 0.0206 & 0.0309 & 0.0515 & 0.0720 & $0.1 \cap 27$ \\
\hline 0.00737 & 0.0105 & 0.0210 & 0.0315 & 0.0525 & 0.0734 & 0.1048 \\
\hline 0.00753 & 0.0107 & 0.0215 & 0.0322 & 0.0535 & 0.0748 & 0.1067 \\
\hline 0.00777 & 0.0111 & 0.0222 & 0.0332 & 0.0553 & 0.0772 & 0.1090 \\
\hline 0.00874 & 0.0125 & 0.0249 & 0.0372 & 0.0617 & 0.0859 & 0.1220 \\
\hline 0.110994 & 0.11142 & 0.0282 & 0.0422 & 0.0697 & 0.0969 & 0.1368 \\
\hline 0.01347 & 0.0192 & 0.0360 & 0.0565 & 0.0925 & $0.127 \%$ & 0.1771 \\
\hline 0.01916 & 0.0272 & 0.0535 & 0.0790 & 0.1275 & 0.1728 & 0.2355 \\
\hline 0.03405 & $n . n 4 B 1$ & 0.0926 & 0.1339 & $0.2 n 8.3$ & 0.2735 & 0.3574 \\
\hline 0.17945 & $0.23 R 7$ & 0.3879 & 0.491 .7 & $0.629 n$ & 0.7224 & $0.821 k$ \\
\hline 0.10138 & 0.5536 & 0.7368 & 0.843 & 0.977 & 1.066 & 1.164 \\
\hline 0.03909 & 1.00300 & 1.2074 & 1.314 & 1.455 & $1.55 \mathrm{~s}$ & 1.667 \\
\hline 1.26270 & $1.356 ?$ & 1.5469 & 1.665 & 1.825 & 1.941 & 2.067 \\
\hline 1.6365 & 1.743 & 1.964 & 2.101 & $2.29 ?$ & 2.425 & 2,578 \\
\hline 2.5626 & 2.711 & 3.020 & 3.219 & 3.49 .3 & 3.684 & 3.011 \\
\hline 3.2835 & 3.467 & 3.838 & 4.102 & 4.445 & 4.689 & 4.064 \\
\hline 4.4527 & 4.705 & 5.216 & 5.544 & $6.00 ?$ & 6.322 & 6.696 \\
\hline 5.4369 & 5.734 & 6.352 & 6.757 & 7.299 & 7.694 & B. 142 \\
\hline 6.6958 & 7.062 & $7.8 \geq 1$ & $8.3 n 9$ & 8.981 & 9.469 & 10.018 \\
\hline 10.0336 & 10.568 & 11.697 & 12.429 & 13.421 & 14.123 & 14.947 \\
\hline 12.6887 & & 14.794 & 15.702 & 16.953 & 17.839 & 18.876 \\
\hline 17.0450 & 17.930 & 19.853 & 21.073 & 22.752 & 23.942 & 25.315 \\
\hline
\end{tabular}

$0.2000 \quad 0.3000 \quad 0.4000$ $0.2 .0020 .3002 \quad 0.4008$ $0.20040 .3007 \quad 0.4008$ $0.2004 \quad 0.3007 \quad 0.4013$ 0.20190 .30120 .4018 $0.2016 \quad 0.30210 .4023$ $0.2033 \quad 0.3045 \quad 0.4051$ $0.2050 \quad 0.3064 \quad 0.4080$ $\begin{array}{lll}0.2083 & 0.3112 & 0.4127\end{array}$ $0.21190 .3155 \quad 0.4180$ $\begin{array}{lll}0.2174 & 0.3155 & 0.4180 \\ 0.2138 & 0.4261\end{array}$ $0.2381 \quad 0.3488 \quad 0.4557$ $0.2625 \quad 0.3794 \quad 0.4888$ $0.3264 \quad 0.4547 \quad 0.5680$ $0.4099 \quad 0.5470 \quad 0.6624$ $0.5594 \quad 0.7005 \quad 0.8130$ $1.0262 \quad 1.1616 \quad 1.2741$ $\begin{array}{lll}1.373 & 1.520 & 1.646 \\ 1.914 & 2.093 & 2.0250\end{array}$ $\begin{array}{lll}2.357 & 2.566 & 2.749\end{array}$ $\begin{array}{lll}2.925 & 3.177 & 3.398 \\ 4.415 & 4.781 & 5.101\end{array}$ $\begin{array}{lll}4.415 & 4.781 & 5.101 \\ 5.597 & 6.063 & 6.467\end{array}$ $\begin{array}{lll}7.535 & 8.157 & 8.691\end{array}$ $9.164 \quad 9.912 \quad 10.568$ $\begin{array}{lll}11.270 & 12.185 & 12.979 \\ 16.801 & 18.174 & 19.364\end{array}$ $\begin{array}{lll}16.801 & 18.174 & 19.364 \\ 21.226 & 22.935 & 2.4 .430\end{array}$ $\begin{array}{lll}21.226 & 22.935 & 24.430 \\ 28.459 & 30.732 & 32.746\end{array}$

$\begin{array}{lllllllllll}0.9000 & 0.9300 & 0.9500 & 0.9700 & 0.9600 & 0.9900 & 0.9930 & 0.9950 & 0.9970 & 0.9980 & 0.9990\end{array}$

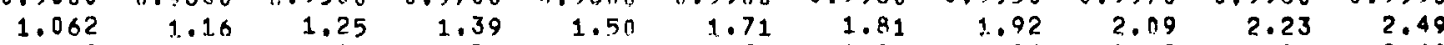

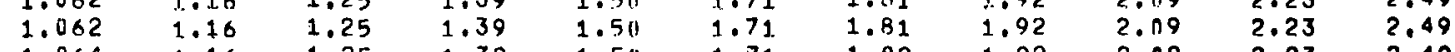

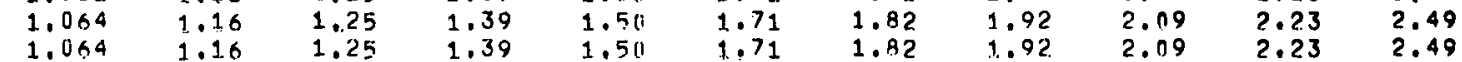

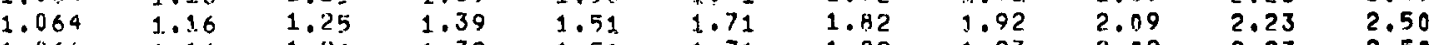

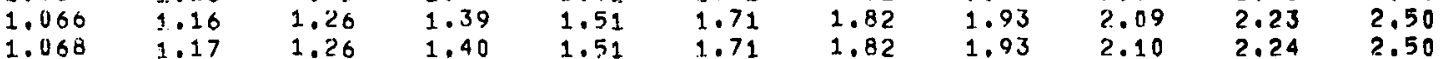

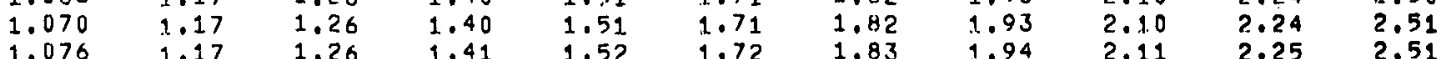

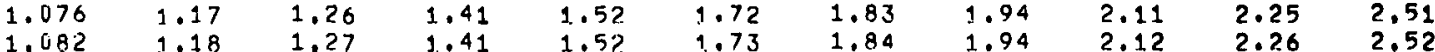

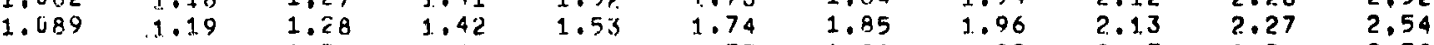
$\begin{array}{lllllllllll}1.120 & 1.22 & 1.31 & 1.45 & 1.57 & 1.77 & 1.88 & 1.99 & 2.17 & 2.31 & 2.58\end{array}$ $\begin{array}{lllllllllll}1.152 & 1.25 & 1.24 & 1.49 & 1.00 & 1.81 & 1.93 & 2.04 & 2.22 & 2.36 & 2.64 \\ 1.230 & 1.33 & 1.43 & 1.57 & 1.69 & 1.91 & 2.02 & 2.14 & 2.33 & 2.48 & 2.76\end{array}$

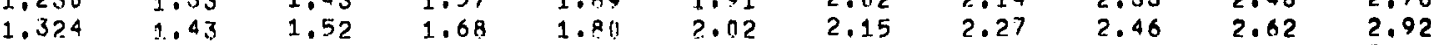

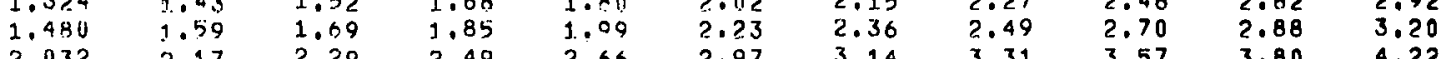

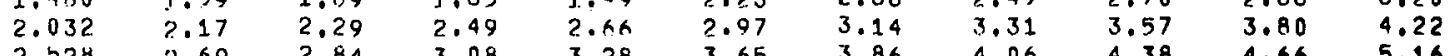

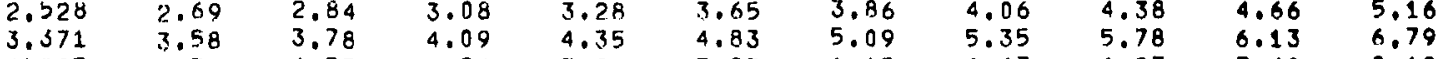

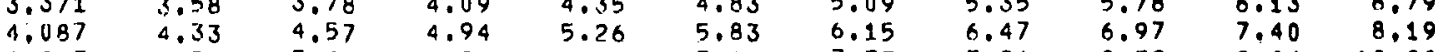

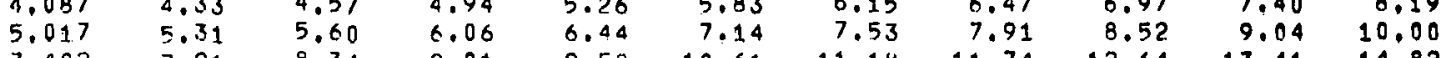

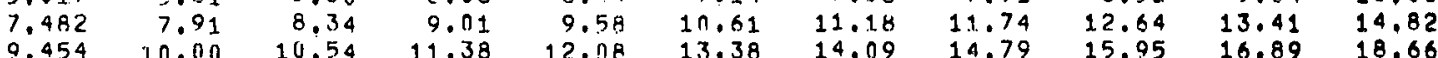

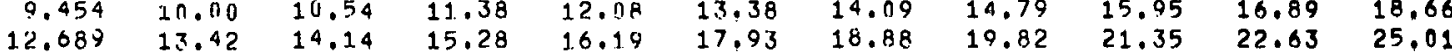

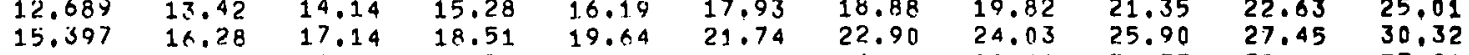

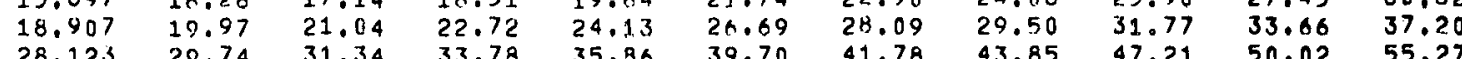

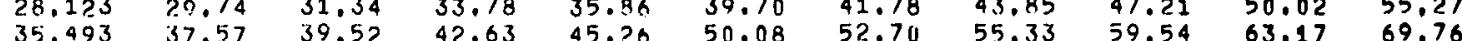

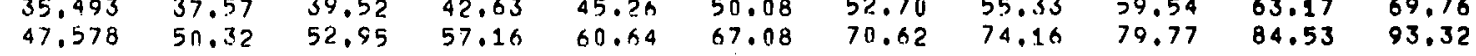


VALUES OF ALPHA FOR W= 7 AND R:F $=5$

\begin{tabular}{|c|c|c|c|c|}
\hline BET & & . 1 & n. & $\mathrm{ii.00}$ \\
\hline 0.001 & o. & $0.0 ! ? 00$ & 1). ก:936 & 0.0115131 \\
\hline & ניח ט. ט. & ונוס? ניח, & 11.01300 & 0.00501 \\
\hline 0.003 & 0.01010 .0 & 0.00201 & 0.00301 & n.00501 \\
\hline 0. & o. Unan & $0.0 \cup ? 01$ & $0.0 \cap 3 \cap 2$ & \\
\hline & 0.001 .01 & $0 . \cap \cup>01$ & 11.09302 & n.005i \\
\hline 0.010 & $0 . \ln 1 n 1$ & 0. กu>n? & 1.00303 & $0.0051 ! 5$ \\
\hline & 0.00102 & $0 . n \cup 204$ & "). & $n .705 .0$ \\
\hline 0.0 & $0.601 \cap 3$ & $0.00 \geqslant 0 \mathrm{~K}$ & 0.010350 & 0.00516 \\
\hline & 0.00105 & $0 . n(i \geqslant 10$ & 0.00 & \\
\hline & 0.00108 & $0 . \cap \cup>15$ & $i$ i. 1 & 537 \\
\hline & 0.00111 & ก. 11 U? 2? & $11 . .1$ & $n, 0$ \\
\hline 0. & 0.0 & 0.00250 & 11. & 0.0 \\
\hline & 0.019 .43 & $0.0 \cup 245$ & 11, & 0.0 \\
\hline & 0.0 & 0.110393 & 11.1 & n. $009 \% 9$ \\
\hline 0. & 0.00203 & 0.00586 & (1. & 0.01456 \\
\hline 1.000 & 0. Uก597 & 0.011 .48 & 0.01973 & 0.02926 \\
\hline 2. & 0.0 & $0.11 \geqslant 16$ & 11.15942 & $n .24061$ \\
\hline 00 & 0.26731 & 0.42228 & $n .525 \mathrm{na}$ & 0.65951 \\
\hline 500 & 0.85406 & $1.0 \angle 810$ & 1.12919 & 1.26080 \\
\hline & $1.261) A 0$ & 1.44 .3911 & 1.55 & 1.7 \\
\hline 00 & 1.7305 & 1.9560 & 34 & 2.272 \\
\hline & 3.0 & $5.34+4$ & & 3.85 \\
\hline 10 & 4.0 & 4.5290 & 4. & \\
\hline & 5.9 & 6.5066 & 7.0 & 26 \\
\hline & 7.61 & 8.4315 & 8.9350 & 9.6309 \\
\hline & & $10.9 n 34$ & 11.5 & \\
\hline & 16.2821 & 17.9911 & 19.7562 & 20.5240 \\
\hline & & & 25.5240 & $=7.4920$ \\
\hline & 1 & $.7 k 116$ & 1.8357 & 33 \\
\hline
\end{tabular}
0.00700 0.00701 0.007112 0.100743 0.010705 0.100707 0.10715 0.00737 0.00757 0.10777 0.110879 0.11369 0.02032 $0.04 n 6 n$ 0.30736 0.74629

1.34851

1.80630
2.3985 4.0484 5.4598 7.9279 10.1090 $21.561 \%$ 28.8249
41.5964

$n \cdot n 100$ 0.0100 0.010

0.0200 0.0200 $0.03 n n$ $n . n 100$
$n . n 1 n 1$ n. 0101 n. n1n2 0.0103 ก.ก1n? n. 01111 N. 0125 1.. 01.42 0.0195
0.0288 ก. 0572 0. 3894 0.8388 1.4458
1.9179 1.9179
2.540 2.540
4.277 5.757
8.355 8.355 10.659
13.787 $=2.691$ $0.03 n 1$ 0.02010 .0302 0.02020 .0303 0.02114 $0.03 n s$ 0.0206 0.0215 0.0222 0.0249 0.0386 0.0566
0.1091 $0.56 \% 0$ 1.0147 1.0423
2.1544 2.1544
2.833 4.743 6.383
9.255 11.804
15.252 25.102 33.601
48.432

$0.03 n g$
$0.03 ? 2$ 0.0332 0.0372 0.0423 0.0574 0.0834 0.1566
0.6757 1.120$$
2.303
$$

\subsection{8}

6.787

9.835
12.536

16.191

35.676

70.391
13.794 $\begin{array}{lll}n .01 n n & 0.0201 & 0.03 n 1\end{array}$ 0.0500 0.0501 0.0503 0.0503 0.0510 0.0515 0.0535 0.0553 0.0617 0.0930 0.1342 0.8121

1.255
1.920

2,505 3.276 7.337 10.613
13.528 17.503 28.764 55.51 ?
0.0700
0.0701
0.0701
0.0702
0.0703
0.0704
0.0707
0.0713
0.0720
0.0734
0.0748
0.0772
0.0859
0.0970
0.1291
0.1814
0.3117
0.9027
1.347
2.048
2.650
3.463
5.757
7.730
11.178
14.245
18.418
30.290
40.498
58.381 $0.9400 \quad 0.0300 \quad 0.9500 \quad 0.9700 \quad 0.9870 \quad 0.9700 \quad 0.9930$ $\begin{array}{lllll}1.062 & 1.16 & 1.25 & 1.39 & 1,50 \\ 1.062 & 1.16 & 1.25 & 1.39 & 1.50\end{array}$ 1.062
1.064 1.064
1.064 1.064 1.066 1.066
1.068 1.068

1.076 1.082$$
1.120
$$

1.152
1.234

$$
1.339
$$

1.532

2.2 200

3.001
4.293 4,293
5.460 5.460
7.039 7.039
11.560 15.458 22.294
28.367 $\begin{array}{lllll}36.653 & 38.73 & 40.74 & 43.98 & 46.72\end{array}$ $\begin{array}{rrrrr}60.151 & 63.54 & 66.96 & 72.21 & 76.60 \\ 80.584 & 85.112 & 89.42 & 96.50 & 102.48\end{array}$ 1.71
1.71
1.71
1.71
1.71
1.71
1.71
1.71
1.72
1.73
1.74
1.77
1.81
1.51
2.04
2.00
3.21
4.11
6.12
7.76
9.99
16.34
21.84
31.26
40.61
51.67
84.78
113.22
163.21 $\begin{array}{ll}1.71 & 1.81 \\ 1.71 & 1.81\end{array}$ $\begin{array}{llll}1.16 & 1.25 & 1.39 & 1.50 \\ 1.16 & 1.25 & 1.39 & 1.50\end{array}$ $\begin{array}{llll}1.16 & 1.25 & 1.39 & 1.50\end{array}$ $\begin{array}{llll}1.16 & 1.25 & 1.39 & 1.51\end{array}$ $1.17 \quad 1.26 \quad 1.40 \quad 1.51$ $\begin{array}{llll}1.17 & 1.26 & 1.40 & 1.51 \\ 1.17 & 1.26 & 1.41 & 1.52\end{array}$ $\begin{array}{llll}1.18 & 1.27 & 1.41 & 1.5 \text { ? }\end{array}$

$\begin{array}{llll}.22 & 1.31 & 1.45 & 1.57\end{array}$

$\begin{array}{llll}.25 & 1.34 & 1.49 & 1.5\end{array}$

$\because .14 \quad 1.43 \quad 1.58 \quad 1.70$

$\begin{array}{llll}0.64 & 1.75 & 1.69 & 1.91\end{array}$

$\begin{array}{llll}2.43 & 2.57 & 2.79 & 2.77\end{array}$

$\begin{array}{llll}3.28 & 3.36 & 3.65 & 3.98\end{array}$

$\begin{array}{llll}4.55 & 4.80 & 5.19 & 5.52\end{array}$

$\begin{array}{llll}5.78 & 6.09 & 6.59 & 7.00\end{array}$
$7.45 \quad 7.85 \quad 8.49 \quad 9.11$ $12.2 .3 \quad 12.89 \quad 13.91 \quad 14.78$ $\begin{array}{lll}17.23 & 18.60 & 19.73\end{array}$

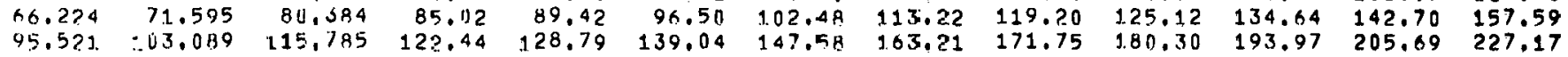

0.1000

0.1001

0.1002

0.1005

0.1006

0.1110

0.1018

0.1027

0.1067

0.1099

0.1220

0.1370

0.1795

0.2463

0.9995

1.452

2.181

3,673

6.093

8,187

11.834
15.069

15.069

32.014

42,817
61,707

$0.2000 \quad 0.3000 \quad 0.4000$

$0.2002 \quad 0.3002 \quad 0.4008$

$\begin{array}{lll}0.2004 & 0.3007 & 0.4008\end{array}$

$\begin{array}{lll}0.2014 & 0.3007 & 0.4013\end{array}$

$0.2009 \quad 0.3012 \quad 0.4018$

$0.20110 .3016 \quad 0.4023$

$\begin{array}{lll}0.2016 & 0.3021 & 0.4027\end{array}$

$0.2033 \quad 0.3045 \quad 0.4051$

$0.2050 \quad 0.30040 .4080$

$0.2083 \quad 0.3112 \quad 0.4127$

0.21190 .31550 .4180

$\begin{array}{lll}0.2381 & 0.3488 & 0.4557\end{array}$

$\begin{array}{llll}0.2630 & 0.3798 & 0.4898\end{array}$

$\begin{array}{llll}0.3298 & 0.4585 & 0.5727\end{array}$

$0.4242 \quad 0.5622 \quad 0.6776$

$\begin{array}{lll}0.6071 & 0.7472 & 0.8598 \\ 1.2036 & 1.3428 & 1.4691\end{array}$

$\begin{array}{lll}1.680 & 1.844 & 1.987\end{array}$

$2.486 \quad 2.704 \quad 2.894$

$\begin{array}{lll}3.192 & 3.463 & 3.703 \\ 4.148 & 4.491 & 4.796\end{array}$

$\begin{array}{lll}6.864 & 7.428 & 7.928\end{array}$

$9.210 \quad 9.957 \quad 10.613$

$\begin{array}{lll}13.299 & 14.382 & 15.336\end{array}$

$\begin{array}{lll}16.953 & 18.327 & 19.517\end{array}$

$\begin{array}{lll}21.897 & 23.667 & 25.193 \\ 35.981 & 38.850 & 41.413\end{array}$

$48.127 \quad 51.972 \quad 55,390$

$69.398 \quad 74.891 \quad 79,774$

$\begin{array}{llll}0.950 & 0.9970 & 0.9980 & 0.9990\end{array}$

$\begin{array}{lllll}1.81 & 1.92 & 2.09 & 2.23 & 2.49 \\ 1.82 & 1.92 & 2.09 & 2.23 & 2.49\end{array}$

$\begin{array}{llllll}171 & 1.82 & 1.92 & 2.09 & 2.23 & 2.49 \\ 1.71 & 1.82 & 1.92 & 2.09 & 2.23 & 2.49\end{array}$

$\begin{array}{lllll}1.82 & 1.92 & 2.09 & 2.23 & 2.50\end{array}$

$\begin{array}{llll}1 & 1.82 & 1.93 & 2.10\end{array}$

$\begin{array}{llllll}71 & 1.82 & 1.93 & 2.10 & 2.24 & 2.51\end{array}$

$\begin{array}{llllll}73 & 1.84 & 1.94 & 2.11 & 2.25 & 2.51 \\ 1.94 & 2.12 & 2.26 & 2.52\end{array}$

$\begin{array}{llllll}1.74 & 1.85 & 1.96 & 2.13 & 2.27 & 2.54\end{array}$

$\begin{array}{llllll}81 & 1.93 & 2.04 & 2.22 & 2.36 & 2.04\end{array}$

$\begin{array}{lllll}2.15 & 2.33 & 2.49 & 2.77\end{array}$

$\begin{array}{llllll}10 & 2.43 & 2.56 & 2.78 & 2.96 & 3.95\end{array}$

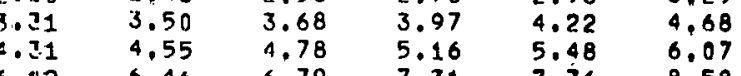

$\begin{array}{llllll}6.12 & 6.46 & 6.79 & 7.31 & 7.76 & 8.58\end{array}$

$\begin{array}{rrrrrr}7.36 & 8.17 & 8.58 & 9.26 & 9.82 & 10.86\end{array}$

$\begin{array}{llllll}9.39 & 10.52 & 11.06 & 11.90 & 12.63 & 13.96\end{array}$

$\begin{array}{llllll}1.84 & 23.00 & 24.16 & 25.99 & 27.57 & 30.44\end{array}$

$\begin{array}{llllll}1.26 & 33.11 & 34.76 & 37.45 & 39.70 & 43.85\end{array}$

$\begin{array}{llllll}1 & 42.15 & 44.22 & 47.64 & 50.45 & 55.76\end{array}$

$\begin{array}{rrrrrr}1.67 & 54.35 & 57.10 & 61.46 & 65.13 & 71.96 \\ 4.78 & 89.30 & 93.69 & 100.77 & 106.87 & 117.98\end{array}$

200.000

500,000

44.535 89.66? 


\begin{tabular}{|c|c|c|c|c|}
\hline $\begin{array}{l}\text { VALUES OF } \\
\text { BETA/F (A) }\end{array}$ & $\begin{array}{l}\text { ALPHA FO } \\
0.01190\end{array}$ & $\begin{array}{l}N=\& A N I \\
0.002 \pi\end{array}$ & $\begin{array}{l}N P=1 \\
0.0030\end{array}$ & 0.0050 \\
\hline 0.001 & 0.00101 & 0.00200 & U.nก300 & 0.00501 \\
\hline 0.002 & 0.00100 & $0.00>00$ & חח300. ט. & 0.00501 \\
\hline 0,003 & $0.001 \cap 0$ & 0.00201. & $0.003 n 3$ & 0.00501 \\
\hline 0.005 & 0.00101 & 0.00201 & $0.003 n ?$ & 0.01502 \\
\hline 0.007 & 0.00101 & ก. กบ?อ1 & $0.0 n 3 n z$ & $n .00504$ \\
\hline 0.010 & O.OnIn1 & 0.00202 & $0.003 \cap 3$ & 0.00505 \\
\hline 0.020 & 0.001 .02 & 0,00204 & 0.00306 & 0.00510 \\
\hline 0.030 & $0.001 \cap 3$ & 0.00206 & $0 . n \cap 3 n 9$ & 0.00515 \\
\hline 0.050 & 0.00105 & 0.00210 & 0.00395 & 0.00525 \\
\hline 0.070 & 0.00107 & $0.00 ? 14$ & $0.0 \cap 321$ & 0.00535 \\
\hline 0.100 & $0.0011 \mathrm{ij}$ & $0.04 ? 20$ & $0.0 \cap 330$ & 0.00550 \\
\hline 0.200 & 0.00120 & 0.00240 & 0.00360 & 0.00599 \\
\hline 0.300 & 0.00 .330 & 0.00200 & 0.110390 & 0.00649 \\
\hline 0.500 & 0.00150 & 0.00300 & 0.00449 & 0.00748 \\
\hline 0.700 & $0.0 \cap 170$ & $0.0034 \pi$ & 0.00509 & 0.00847 \\
\hline 1.000 & $0.0 \cap 2 n 0$ & 0.00399 & 0.00598 & $n .00994$ \\
\hline 2,000 & 0.011299 & ก. ก U59 & 0.010804 & 0.01485 \\
\hline 3.000 & 0.00399 & 0.00785 & 11.0 .1190 & 0.01970 \\
\hline 5.000 & 0.00597 & 0.011 .88 & 0.01773 & 0.02926 \\
\hline 7.000 & 0.60705 & 0.01579 & 0.02351 & $n .03864$ \\
\hline 10.000 & 0.0109 & 0.0216 & 0.0320 & 0.0524 \\
\hline 20.000 & 0.0206 & 0.0404 & 0.0594 & 0.0955 \\
\hline 30.000 & $0.0 \sin 1$ & 0.0585 & 0.085 .3 & 0.1349 \\
\hline 50.000 & 0.0486 & 0.0927 & 0.1336 & 0.2040 \\
\hline 70.000 & 0.0064 & 0.1246 & 0.1761 & 0.2630 \\
\hline 00,000 & 0.091 .8 & 0.1682 & 0.2331 & 0.3369 \\
\hline 00.000 & 11.1675 & 0.2873 & 0.3770 & 0.5031 \\
\hline 00,000 & $n .2314$ & 0.3765 & 0.4757 & 0.6061 \\
\hline 00.000 & 1.3541 & U.502? & 11.6051 & 0.7329 \\
\hline$B E T A / F(A)$ & 0.51100 & 0.0000 & 0.7000 & 0.8000 \\
\hline 0.001 & $0.5 \cap 1$ & 0.604 & 0.715 & 0.844 \\
\hline 0.002 & $0.5 \cap 1$ & 0.605 & $0.71,5$ & 0.844 \\
\hline 0.003 & 0.502 & 0.505 & 11.716 & 0.844 \\
\hline 0.005 & $0.5 n 2$ & 0.605 & 0.796 & 0.845 \\
\hline 0.007 & U.jng & 0.006 & 0.71 .7 & 0.845 \\
\hline 0.010 & 0,503 & 0.607 & 0.717 & 0.845 \\
\hline 0,020 & $0.5 n 6$ & 0.609 & 0.720 & .0 .847 \\
\hline 0.030 & 0.509 & 0.612 & 0.721 & 0.850 \\
\hline 0.050 & 0.514 & 0.017 & 0.726 & 0.854 \\
\hline 0.070 & 0.518 & 0.621 & {$[.131$} & 0.859 \\
\hline 0.100 & 0.525 & 0.528 & 0.738 & 7. 865 \\
\hline 0,200 & 0,548 & 0.650 & 0.759 & 0.805 \\
\hline 0.300 & 0.568 & 0,670 & 0.778 & 0.904 \\
\hline 0,500 & 0.604 & 0.705 & 0.812 & 0.936 \\
\hline 0.700 & 0.636 & 0.736 & C. .841 & 0.963 \\
\hline 1.000 & 0.071 & 0.775 & $5, .879$ & 0.999 \\
\hline 2.000 & 0.775 & $0 . \Omega>0$ & 0.971 & 1.089 \\
\hline 3.000 & ĩ. 343 & 0.936 & 1.030 & 1.152 \\
\hline 5,000 & 0.936 & 1,1126 & $1,1 \geq 3$ & 1.244 \\
\hline 7.000 & 1). 999 & 1.099 & 1.186 & 1.307 \\
\hline 10,000 & $7.971)$ & 1.100 & 1.257 & 1.379 \\
\hline 20.000 & $1,21,3$ & 1.303 & $1.4 n_{4}$ & 1.530 \\
\hline 30.000 & 1.209 & 1.342 & 3.495 & 1.625 \\
\hline 50.000 & 1.413 & 1.509 & 1.610 & 1.751 \\
\hline 10.000 & 1.400 & 1.589 & 1.698 & 1.839 \\
\hline 00.000 & 1.576 & $1.6 \% 5$ & 1.789 & 1.933 \\
\hline 00.000 & 1.747 & 1.454 & 1.975 & $? .132$ \\
\hline 000 & 1.854 & 1.067 & 2.003 & 2.257 \\
\hline 00.000 & 1.904 & 2.1.1? & $\because .04$. & 2.421 \\
\hline
\end{tabular}

\begin{tabular}{|c|c|c|c|c|}
\hline $\begin{array}{l}0.0070 \\
0.00700 \\
0.00701 \\
0.00702 \\
0.00703\end{array}$ & $\begin{array}{l}0.0100 \\
n .0100 \\
0.0100 \\
n .01100 \\
n .0100\end{array}$ & $\begin{array}{l}0.0200 \\
0.0200 \\
0.0201 \\
0.0201 \\
0.0201\end{array}$ & $\begin{array}{l}0 . n 3 \cap 0 \\
0 . n 301 \\
0.03 n 1 \\
0.0301 \\
0 . n 302\end{array}$ & $\begin{array}{l}0.0500 \\
0.0500 \\
0.0509 \\
0.0501 \\
0.050 .3\end{array}$ \\
\hline 0.00705 & $n .0101$ & 0.0201 & $0 . n 3 n 2$ & 0.0503 \\
\hline $0.0 \cup 707$ & $n .01 n 1$ & 0.0202 & $0.03 n 3$ & 0.0505 \\
\hline $\begin{array}{l}0.00714 \\
0.00721 .\end{array}$ & $\begin{array}{l}n .0102 \\
0.0103\end{array}$ & $\begin{array}{l}0.02 \cup 4 \\
0.0206\end{array}$ & $\begin{array}{l}0.03 \cap 6 \\
0.0309\end{array}$ & $\begin{array}{l}0.0510 \\
0.0515\end{array}$ \\
\hline 0.00735 & 0.0105 & 0.0210 & 0.0314 & 0.0523 \\
\hline $\begin{array}{l}0.00748 \\
0.00769\end{array}$ & $\begin{array}{l}0.0107 \\
0.0110\end{array}$ & $\begin{array}{l}0.0214 \\
0.0220\end{array}$ & $\begin{array}{l}0 . n 3 ? 0 \\
0.03 ? 9\end{array}$ & $\begin{array}{l}0.0533 \\
0.0547\end{array}$ \\
\hline 0.00830 & $0 . n 1>0$ & 0.0239 & 0.0358 & 0.0594 \\
\hline 0.00908 & 0.0130 & 0.0258 & 0.0386 & $0.064 n$ \\
\hline 0.01046 & 0.0149 & 0.0297 & 0.0444 & $0.073 ?$ \\
\hline 0.01184 & 0.0169 & 0.0335 & $0.05 n n$ & 0.0821 \\
\hline 0.01391 & n. ก1०B & 0.0392 & 0.0582 & $0.095 ?$ \\
\hline $0.02 \pi 71$. & $n .12294$ & 0.0577 & 0.0849 & 0.1363 \\
\hline 0.02741 & 0.0388 & 0.0754 & 0.110 & 0.174 \\
\hline 0.04060 & 0.0572 & 0.1031 & 0.157 & $0,24 n$ \\
\hline 0.05336 & 0.0747 & 0.1404 & 0.198 & $0.2 .9 n$ \\
\hline 0.0720 & 0.100 & 0.133 & 0.254 & 0.367 \\
\hline 0.1289 & 0.175 & 0.300 & 0.304 & 0.527 \\
\hline 0.1795 & 0.239 & 0.387 & 0.401 & 0.625 \\
\hline 0.2644 & 0.340 & 0.511 & 0.617 & $0.74 \mathrm{R}$ \\
\hline 0.5336 & 0.41 .8 & 0.506 & 0.700 & 0.827 \\
\hline 0.4161 & 0.506 & 0.684 & 0.785 & 0.908 \\
\hline 0.5899 & 0.680 & 0.849 & 0.944 & 1.062 \\
\hline 0.6910 & 0.779 & 0.941 & 1.034 & $1.15 n$ \\
\hline 6 & $0.80 \%$ & 1. .055 & 1.146 & 1.263 \\
\hline
\end{tabular}

0.0700
0.0701
0.0701
0.0702
0.0703
0.0704
0.0707
0.0713
0.0720
0.0733
0.0745
0.0765
0.0828
0.0892
0.1014
0.1135
0.1308
0.1842
0.231
0.311
0.376
0.453
0.61
0.715
0.83
0.90
0.98
1.141
1.23
1.34

0.1000 0.1001 0.1102 0.1005 $0.1 \operatorname{nn} 6$ 0.1010 0.1018 0.1027 0.1045 0.1062 0.1062 U. 1176 0.1263 0.1430 0.1431 0.1589 0.1918 0.2501 0.400 0.400
0.471

0.471
0.552

0.715

0.80

0.923

0,998

1.076

1.22 .5

1.318
1.134

1.026
1.026

1.026
1.026

1.028

1.028
1.028

1.028
1.430

1.032

1.038

1.041

1.066

1.116

1.143

1.268

1.288
1.433

1.427

1.493

1.570
1.132
1.433

2.067

2.170

2. 281

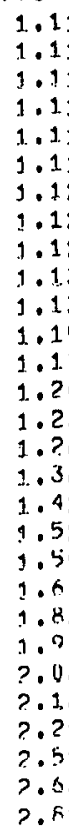

$0.9500 \quad 0.97$

1.1 .9

$.9700 \quad n .98$

$.980 n$
1.40

n.99no

0.9930

0.9950

.9950
1.75
1.75

1.19
1.1 .9

1.19

1.31

1.31
1.31
1.31

1.40

1.57
1.57
1.57
1.57

1.66
1.66

1.75
1.75
1.75

1.75

1.75

1.20

1.31
1. 31
j. 31

1.41

1.57
1.57
1.57

$1.49 \quad 1.58$

$1.49 \quad 1.58$

$1.42 \quad 1.58$

$1.42 \quad 1.59$

$1.45 \quad 1.62$

$1.46 \quad 1.63$

1.53
1.57

1.67

1. 174

1.85

1.93

$2.0 ?$
2.29
2.33
2.44

2.33
2.44

2.41.

$3 . n \mathrm{n}$

3.1 .7
3.39
1.67

.68

1.68

1.71
1.73

1.73
1.76
1.79
1.84

1.84

1.95

2.15

2.24

2.34

2.55
2.69

2.88

3.01

3.46

3.64
3.90
1.75
9.76

1.76

1.79

1.81

1.85

1.93

2.04

2.25

2.35

2.45
2.67

2.82

3.15

3.30

3. 51

4.07
$0.2000 \quad 0.3000 \quad 0.4000$ $0.2002 \quad 0.3002 \quad 0.4003$ 0.20140 .30020 .4008 $\begin{array}{lll}0.2004 & 0.3007 & 0.4008\end{array}$ $0.2011 \quad 0.3096 \quad 0.4018$ $0.2016 \quad 0.3021 \quad 0.4027$ $0.20330 .3040 \quad 0.4051$ 0.20470 .30640 .4075 $\begin{array}{lll}0.2078 & 0.3102 & 0.4118\end{array}$ $\begin{array}{lll}0.2112 & 0.3145 & 0.4166\end{array}$ $\begin{array}{lll}0.2157 & 0.3202 & 0.4232\end{array}$ $0.23 n 7 \quad 0.3398 \quad 0.4447$ $\begin{array}{lll}0.2454 & 0.3579 & 0.4647\end{array}$ $0.2983 \quad 0.42180 .5327$ $0.3336 \quad 0.4619 \quad 0.5746$ $\begin{array}{llll}0.4290 & 0.5651 & 0.0767\end{array}$ $\begin{array}{lll}0.501 & 0.638 & 0.747\end{array}$ $\begin{array}{lll}0.604 & 0.739 & 0.843\end{array}$ $\begin{array}{lll}0.677 & 0.807 & 0.909 \\ 0.755 & 0.881 & 0.980\end{array}$ $\begin{array}{lll}0.916 & 1.026 & 1.123\end{array}$ $0.994 \quad 1.112 \quad 1.209$ $\begin{array}{lll}1.112 & 1.223 & 1.320\end{array}$ $\begin{array}{lll}1.175 & 1.295 & 1.396 \\ 1.253 & 1.375 & 1.478\end{array}$ $\begin{array}{lll}1.410 & 1.537 & 1.644\end{array}$ $\begin{array}{lll}1.505 & 1.635 & 1.747 \\ 1.627 & 1.764 & 1.883\end{array}$

$\begin{array}{lll}0.9970 & 0.9980 & 0.9990\end{array}$ $\begin{array}{lll}1.88 & 1.99 & 2.20 \\ 1.88 & 1.99 & 2.20\end{array}$ $\begin{array}{lll}1.88 & 1.99 & 2.20 \\ 1.88 & 1.99 & 2.20 \\ 1.88 & 1.99 & 2.20\end{array}$ $\begin{array}{lll}1.98 & 1.99 & 2.20\end{array}$ $\begin{array}{lll}1.88 & 2.00 & 2.20 \\ 1.89 & 2.00 & 2.20\end{array}$ $\begin{array}{lll}1.89 & 2.00 \quad 2.21\end{array}$ $1.89 \quad 2.01 \quad 2.21$ $1.91 \quad 2.02 \quad 2.23$ $\begin{array}{lll}1.93 & 2.05 & 2.25\end{array}$ $\begin{array}{lll}1.99 & 2.11 \quad 2.33\end{array}$ $2.03 \quad 2.15 \quad 2.36$ 2.20
$2.20 \quad 2.42$ $2.29 \quad 2.42 \quad 2.66$ $2.55 \quad 2.81$ $2.63 \quad 2.78 \quad 3.04$ $\begin{array}{lll}2.86 & 3.02 & 3.32\end{array}$ $3.23 \quad 3.40 \quad 3.73$ $\begin{array}{lll}3.53 & 3.55 & 3.90\end{array}$

$3.87 \quad 4.07 \quad 4.46$
$\begin{array}{lll}4.07 & 4.29 & 4.70 \\ 4.35 & 4.58 & 5.02\end{array}$ 
VALUES OF ALPHA FOR $N=R$ ANIJ. MH $=2$

\begin{tabular}{|c|c|c|c|c|}
\hline BETA/F C & 0.0010 & $0.00 ? n$ & 0.0030 & 0.0050 \\
\hline 0.001 & 0.00100 & 0.00200 & $0,003 \cap 0$ & 0.0050 \\
\hline & $0.001 \cap 0$ & 0.00 .200 & 0.00300 & 0.00501 \\
\hline & $0.001 \cap 0$ & 0.00201 & 0.00301 & 501 \\
\hline & $0.001 \cap 1$ & 0.00201 & $0.003 n 2$ & 0.00502 \\
\hline & 0.00101 & 0.00201 & $0.003 \cap ?$ & 0.00504 \\
\hline & 0.00101 & 0.00202 & 0.00303 & $0.005 n 5$ \\
\hline & 0.00102 & 0.00204 & 0.003ne & 0.00510 \\
\hline & 0.00103 & 0.00206 & 0.013119 & 0.00516 \\
\hline & $0.001 \cap 5$ & 0.0 & 0.00316 & 0.00526 \\
\hline 0. & 0.00107 & 0.00 .215 & 0.00322 & 0.00537 \\
\hline & 0.0 & 0.0 & 1100 & 0. \\
\hline & 0.0 & 0.00248 & i) .00372 & 0.00619 \\
\hline & 0.0 & 0.00278 & $0.0 \cap 41 \%$ & 1694 \\
\hline & 0.00175 & 0.00 .350 & 0.0 & $n .0$ \\
\hline 0 & 0.6 & 0.0 & 0.00655 & 088 \\
\hline 1.000 & 0.00299 & 0.00598 & $0.0 \cap 894$ & 0.0 \\
\hline 2.000 & 0.00606 & 0.0 & 1). 112062 & $n .1$ \\
\hline 3.000 & 0.01295 & 0.02539 & 1). 0.3762 & 0.06137 \\
\hline 000 & $0.03 \cup 11$ & 0.05848 & 0.08534 & 0.1 \\
\hline 000 & 0.05395 & 0.10 .250 & 0.14631 & 0.22260 \\
\hline 1.000 & 0.11000 & 0.1821 & 0.2501 & 579 \\
\hline & 0.2964 & 0.4580 & 0.5613 & 0.6900 \\
\hline & $0.4 B .31$ & 0.6 & 0.7 & 0.8789 \\
\hline 0 & $0.73 \times 8$ & 0.8948 & 0.9900 & 144 \\
\hline 10 & 0.89 .32 & 1.0491 & 9.1387 & \\
\hline 000 & 1.051 .0 & 1.2 & 1. & 15 \\
\hline . & 1.3581 & 1.5202 & 1. & 491 \\
\hline 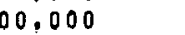 & 1.5498 & 1.7205 & 1. & 1. \\
\hline & $0 \times 3$ & $1.494 ?$ & 2. & 2.2688 \\
\hline BET & 0.5 & 0.0 & 0 & 00 \\
\hline 0,001 & & & 5 & \\
\hline 0,002 & & & & 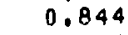 \\
\hline & & & & \\
\hline 0.005 & $0.5 n 2$ & 0.6 & 16 & 0 \\
\hline 0.007 & & & & \\
\hline 0,010 & 0 & 7 & $n$. & 0.845 \\
\hline 0.020 & & & 0. & \\
\hline .030 & & & 0.722 & 0.850 \\
\hline & & & & \\
\hline & & 0.1 & & \\
\hline & 8 & & D. & \\
\hline & & & & \\
\hline & $\pi$ & & & \\
\hline & & & 0.847 & \\
\hline & 0 & & & \\
\hline & 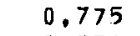 & 0.870 & 0.5 & \\
\hline & 17 & & & \\
\hline & 1,1 & & 1.2 & \\
\hline & 7 & & 1. & \\
\hline & 1.4 & & 1.6 & \\
\hline & 1.598 & 1.699 & $1 . t$ & 1.960 \\
\hline & & & & \\
\hline & 1.177 & 2.3 & 2.4 & 2.631 \\
\hline & & & 2.8 & \\
\hline & 2.7 & 2.0 & 3.0 & 3,295 \\
\hline & 3,009 & 3.1 & 3.3 & \\
\hline & 3.615 & 3.810 & 4.041 & 4.331 \\
\hline & 4.01 .8 & 4.2 & 4.48 .3 & \\
\hline & ๖৭0 & 4.8 .34 & 5.116 & 5.4 \\
\hline
\end{tabular}
$0.0070 \quad 0.01 n 0$ $0.007020 .0100 \quad 0.0201$ 0.00721 $0.0294 \quad 0.0577$ 0.0100
0.0100 $\quad 0.0200$ $0.00701 \quad 0.0100 \quad 0.0201$ $0.00703 \quad 0.0100 \quad 0.0201$ 0.00705 0,00715 0.00751 0.00777 0.00970 0,01219 0.01520 0.02071 0.04704 0.08390 0,17945 0.2 .8585 0.4394 0.955 1.1788 1.3276 1.4916
1.8387 1.8387
2.0628
2.3795 $\begin{array}{ll}0.0101 & 0.0201 \\ 0.0101 & 0.0202\end{array}$ $0.0102 \quad 0.0204$ $0.0103 \quad 0.0206$ $0.0107 \quad 0.0215$ 0.01110 .0221 $0.0124 \quad 0.0247$ $\begin{array}{ll}0.0138 & 0.0276 \\ 0.0174 & 0.0345\end{array}$ $0.0216 \quad 0.0428$ $0.1161 \quad 0.2097$ $0.2387 \quad 0.3875$ $0.3655 \quad 0.5393$ $0.530 \quad 0.707$ 1.036 1.259 1.410 1.577 1.937
2.170 1.190 $\begin{array}{ll}1.190 & 1.284 \\ 1.417 & 1.516\end{array}$ $1.576 \quad 1.679$ $\begin{array}{ll}1.755 & 1.864 \\ 2.139 & 2.265\end{array}$ $\begin{array}{ll}2.139 & 2.265 \\ 2.391 & 2.532 \\ 2.749 & 2.906\end{array}$

0.0300
0.0301
0.0301
0.0301
0.0302
0.0302
0.0303
0.0306
0.0309
0.0315
0.0322
0.0332
0.0369
0.0412
0.0513
0.0634
0.0849
0.1780
0.287
0.491
0.045
0.807
1.108
1.284
1.516
1.679
1.864
2.265
2.532
2.906

$0.050 n$ 0.0500 0.0501 0.0503 0.0503 0.0510 0.0515 0.0535 0.055 ? 0.0682 0.0844 0.1033 0.1363
$0.269 ?$

0.407 0.625
0.775 0.92 1.225 1.404 1.816 2.013 2.723
3.120
0.070
0.070
0.070
0.070
0.070
0.070
0.070
0.071
0.072
0.073
0.074
0.077
0.085
0.094
0.116
0.141
0.184
0.345
0.49
0.71
0.85
1.00
1.30
1.48
1.73
1.91
2.11
2.55
2.85
3.27

$0.9000 \quad 0.0300 \quad 0.9500 \quad 0.9700 \quad 0.9800 \quad 0.9900 \quad 0.9930$
$1.026 \quad 1.11 \quad 1.19 \quad 1.31 \quad \div 40$

1.026

1.028

1.028

1.028
1.030

1.030
1.032

1.038

1.038
1.041

1.049

1.074

1.099
1.150

1.200

1.268

1.463

1.612

1.833
2,002

2,002
2,200

2,200
2,639

2.639
2.936

2.936
3.356

3,065

4.018

4.811

5,338
6.085

$\begin{array}{llll}1.11 & 1.19 & 1.31 & \because .31\end{array}$

$1.11 \quad 1.19 \quad 1.31$

$\begin{array}{lll}1.11 & 1.19 & 1.31 \\ 1.12 & 1.20 & 1.31\end{array}$

1.20

$\begin{array}{llll}1.12 & 1.20 & 1.32 & \because .4\end{array}$

$1.13 \quad 1.21 \quad 1.33 \quad 0.43$

$\begin{array}{llll}1.16 & 1.24 & 1.36 & \vdots .45 \\ 1.18 & 1.26 & 1.38 & \vdots .48\end{array}$

$\begin{array}{llll}1.18 & 1.26 & 1.38 & \vdots .48 \\ 1.24 & 1.32 & 1.44 & \vdots .54 \\ 1.29 & 1.37 & 1.49 & \vdots .59\end{array}$

$\begin{array}{llll}1.29 & 1.37 & 1.49 & \div .59 \\ 1.36 & 1.44 & 1.57 & \vdots .67\end{array}$

$\begin{array}{llll}1.55 & 1.64 & 1.78 & \div .89 \\ 1.71 & 1.80 & 1.94 & 2.00\end{array}$

$\begin{array}{llll}1.71 & 1.80 & 1.94 & 2.06 \\ 1.94 & 2.04 & 2.20 & 2.33\end{array}$

$\begin{array}{llll}2.12 & 2.22 & 2.39 & 2.53 \\ 2.32 & 2.44 & 2.62 & 2.77\end{array}$

$\begin{array}{llll}2.32 & 2.44 & 2.62 & 2.77 \\ 2.78 & 2.92 & 3.13 & 3.3\end{array}$

3.09

3.54
3.86

3.86
4.23

5.76

5.61
6.90

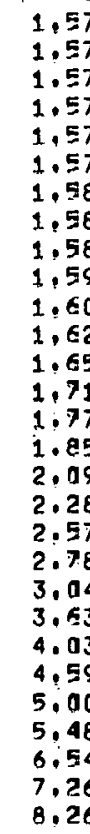

0.1000

0.1001

0.1002

0.1005

0.1006

0.1010

0.1018
0.1027

0.1046

0.1067

0.1210

0.1337

0.1628
0.1957

0.2501

0.4380

0.595
0.808

0.948

1.095
1.392

1.581

1.581
1,839

2,021

2,234
2,700

3,009
3,444
$0.2000 \quad 0.3000 \quad 0.4000$

$0.2002 \quad 0.3002 \quad 0.4003$

$0.2014 \quad 0.3002 \quad 0.4008$

$0.2004 \quad 0.3007 \quad 0.4008$

$0.20190 .3012 \quad 0.4013$

0.20110 .30160 .4018

0.20160 .30210 .4027

$0.2033 \quad 0.3045 \quad 0.4051$

$0.2050 \quad 0.3064 \quad 0.4075$

$0.2083 \quad 0.3107 \quad 0.4127$

$0.21190 .3155 \quad 0.4175$

0.21710 .32260 .4256

$0.2367 \quad 0.3469 \quad 0.4528$

$0.2578 \quad 0.3736 \quad 0.4821$

$0.3045 \quad 0.4290 \quad 0.5403$

$0.35360 .4850 \quad 0.5975$

$\begin{array}{llll}0.4290 & 0.5651 & 0.6767\end{array}$

$\begin{array}{lll}0.6433 & 0.7749 & 0.8789\end{array}$

$0.794 \quad 0.919 \quad 1.019$

$\begin{array}{lll}0.994 & 1.112 & 1.209 \\ 1.127 & 1.247 & 1.347\end{array}$

$1.274 \quad 1.398 \quad 1.501$

$1.585 \quad 1.720 \quad 1.835$

$\begin{array}{lll}1.785 & 1.929 & 2.055\end{array}$

$2.063 \quad 2.227 \quad 2.368$

$\begin{array}{lll}2.265 & 2.440 & 2.593 \\ 2.498 & 2.688 & 2.852\end{array}$

$3.009 \quad .3 .230 \quad 3.425$

$\begin{array}{lll}3.348 & 3.596 & 3.810 \\ 3.827 & 4.109 & 4.354\end{array}$

$0.9970 \quad 0.9980 \quad 0.9990$

$0.9980 \quad 0.9990$
$1.99 \quad 2.20$

$1,66 \quad 1.75$

$\begin{array}{ll}1.66 & 1.75 \\ 1.66 & 1.75\end{array}$

$\begin{array}{ll}1.66 & 1.75\end{array}$

$1.66 \quad 1.75$

$1.66 \quad 1.75$

$\begin{array}{ll}1.66 & 1.7 .5 \\ 1.67 & 1.75\end{array}$

$\begin{array}{ll}1.67 & 1.75\end{array}$

$\begin{array}{ll}1.67 & 1.76 \\ 1.68 & 1.76\end{array}$

$1.68 \quad 1.77$

$1.71 \quad 1.80$

1.83

1.89
1.96

2.04

2.30

2.51

2,82
3,06

3.06
3.34

3.98

4,41

5.02

5.48
6.00

6.00

7.93

9.03

$\begin{array}{rrr}1.88 & 1.99 & 2.20 \\ 1.88 & 1.99 & 2.20 \\ 1.88 & 1.99 & 2.20 \\ 1.88 & 1.99 & 2.20 \\ 1.88 & 1.99 & 2.20 \\ 1.88 & 2.00 & 2.20 \\ 1.89 & 2.00 & 2.20 \\ 1.89 & 2.00 & 2.21 \\ 1.89 & 2.01 & 2.22 \\ 1.90 & 2.01 & 2.22 \\ 1.91 & 2.02 & 2.23 \\ 1.94 & 2.06 & 2.27 \\ 1.97 & 2.09 & 2.30 \\ 2.04 & 2.16 & 2.38 \\ 2.10 & 2.23 & 2.45 \\ 2.20 & 2.33 & 2.55 \\ 2.47 & 2.61 & 2.87 \\ 2.68 & 2.84 & 3.11 \\ 3.02 & 3.18 & 3.49 \\ 3.27 & 3.45 & 3.78 \\ 3.57 & 3.77 & 4.12 \\ 4.25 & 4.48 & 4.90 \\ 4.71 & 4.97 & 5.43 \\ 5.37 & 5.66 & 6.18 \\ 5.85 & 6.16 & 6.73 \\ 6.41 & 6.75 & 7.37 \\ 7.64 & 8.05 & 8.78 \\ 8.46 & 8.92 & 9.74 \\ 9.64 & 10.16 & 11.09\end{array}$




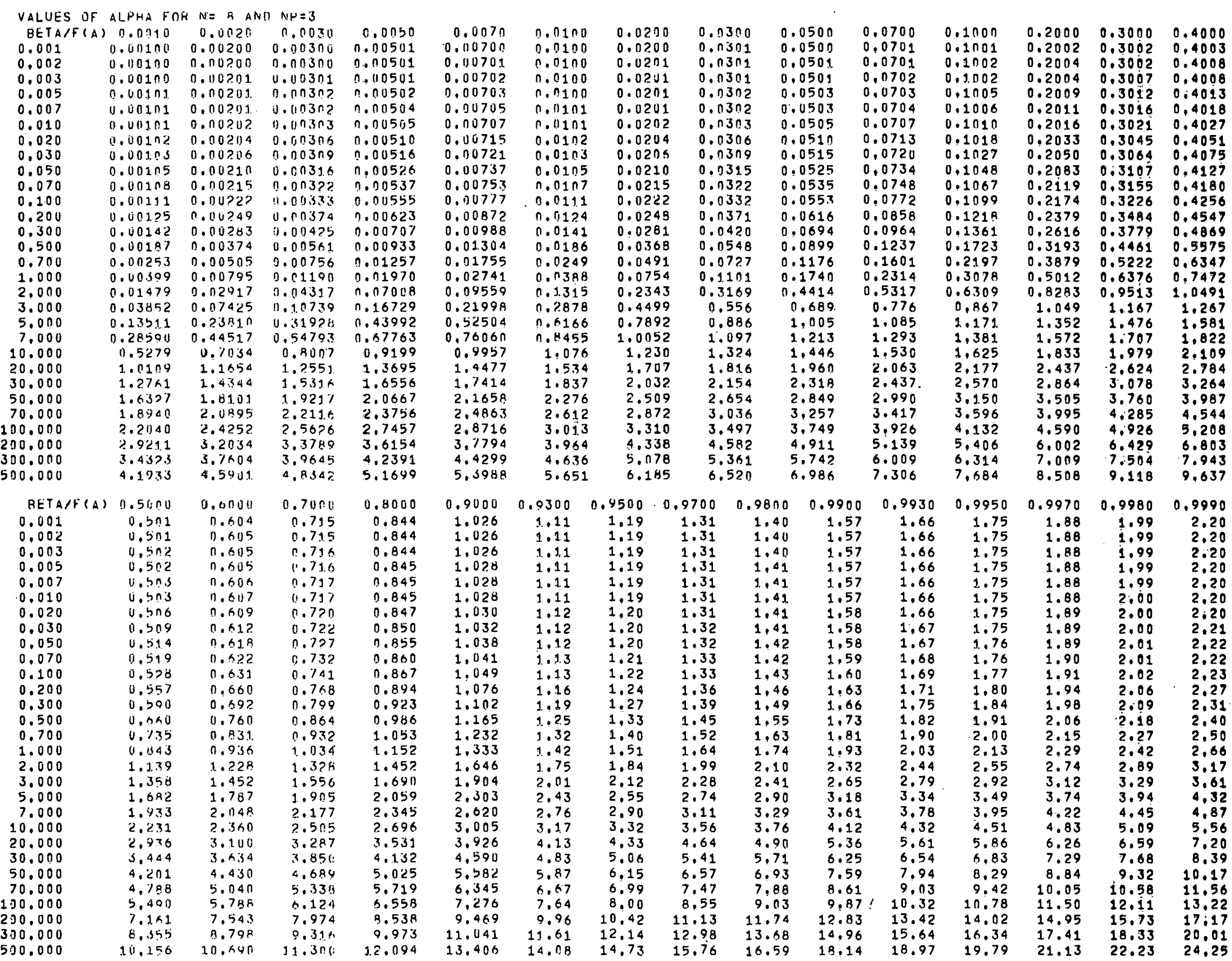


VALUES OF ALPHA FOR $N=\triangle A$ ANII INP $=4$

\begin{tabular}{|c|c|c|c|c|}
\hline BETA $/=(A)$ & 0.0414 & ט. & $f_{1}, \pi(1) 3 n$ & 0.0050 \\
\hline 0.001 & 0.00100 & 0.00200 & i) ansn: & ก.01) 5 il 1 \\
\hline 0.002 & 0.00100 & $0.00>0 n$ & $0.003 \cap 0$. & n.00501 \\
\hline 0.003 & $0.0 \cap 1, n i 0$ & 0.04201. & d.nn3ni & 0.70501 \\
\hline 0.005 & o.unint. & 0.00201. & Q. 10 ก $3 \cap 2$ & $n .011502$ \\
\hline 0.007 & n. $001 \cap 1$ & 0. กט?ग1 & $0.1103 n 2$ & $0.005 \pi 4$ \\
\hline 0.010 & $0.4 \cap 1,1$ & בווקיטחם ח & u. innsos & 0.005115 \\
\hline 0.020 & 0.00102 & $10.012 \mathrm{UA}$ & D.01130s & ก. กำ $51 !$ \\
\hline 0.030 & $0.001 n 3$ & 0.00200 & $0.0 n 3 n 0$ & 0.010516 \\
\hline 0.050 & 0.00105 & 0.00210 & ด. 00316 & D. nu5zh \\
\hline 0.070 & $0.0111 \mathrm{n}$ & $0.0 \cup 215$ & i]. ถคว?: & ก. 011537 \\
\hline 0.100 & 0.001 .11 & 0.110227 & U. 10nss: & 0.00555 \\
\hline 0.200 & 0.00125 & 0.00250 & $4.9037 \mathrm{~s}$ & $n . n \cup 624$ \\
\hline 0.300 & 0.00142 & 0.011285 & $0.0104 \%$ & 0.00711 \\
\hline 0.500 & 0.00194 & ก.กบ347 & $0.0052 !$ & n. 00965 \\
\hline 0.700 & 0.110277 & 0.00553 & (1.098? & 0.01374 \\
\hline 1.000 & 0.110007 & 0.00991 & 1). 91402 & 0.02452 \\
\hline 2.000 & $0.03 \div 11$ & $0.0 \supset R \triangle A$ & 0.03534 & 0.13487 \\
\hline 3.000 & 0.10109 & 0.19519 & i1. 24643 & $0.3 / 811$ \\
\hline 5.000 & 0.4360 .7 & 0.61109 & 1.71 .073 & 0.93800 \\
\hline 7.000 & $0.75 n \times 9$ & 0.92081 & 1.01094 & 1.12538 \\
\hline 10.000 & $1.07 ? 0$ & 1.2265 & 1.3161 & 1,4344 \\
\hline 20,000 & 1.70 .33 & 1.8445 & 1. $09 R \quad$ & 2.1468 \\
\hline 30.000 & $2.1+68$ & $2.3 \times$ iा 4 & 2.4977 & $\therefore .6710$ \\
\hline 50.000 & 2.8206 & $3.10+1$ & 3.2707 & 3,50136 \\
\hline 70.000 & $3.38 ? 7$ & $3.70 \% 0$ & 3.9111 & 4.1741 \\
\hline 00.000 & 4.0789 & 4.4504 & 4.7005 & 5.0249 \\
\hline 00.000 & $5.8 \% 60$ & 6.3753 & $6.711 . n$ & 7.1688 \\
\hline 100.000 & 7.1 SRO & 7.8364 & $n .74 \mathrm{KA}$ & 0.8129 \\
\hline 100.000 & $9.301 ?$ & 10,1537 & 10.6896 & 11.4069 \\
\hline$B E T A / F(A)$ & 0.5000 & 1.6000 & $r_{i} .7 \cup 0 i_{i}$ & 0.5000 \\
\hline 0,001 & U. 5n 1 & 0.604 & 0.7 .15 & 0.844 \\
\hline 0.002 & $0 . \sin 1$ & 0.605 & 0.795 & 0.844 \\
\hline 0.003 & $4.5 n 2$ & $0 . \cap 05$ & 0.716 & $n, 844$ \\
\hline 0.005 & $0 . \sin 2$ & 0.6015 & $11.71 \mathrm{~h}$ & 0.845 \\
\hline 0.007 & U. bns & $n .606$ & $0.71 \%$ & 0.845 \\
\hline 0.010 & U. & 0.607 & $\int_{i} .797$ & 0.845 \\
\hline 0.020 & $0.5 n 6$ & 7.509 & 0.720 & 0.847 \\
\hline 0.030 & $0 . \operatorname{lng}$ & $0.51 ?$ & 0.732 & 0.850 \\
\hline 0.050 & U. $\$ 14$ & $0 . A 1 \%$ & {$[1,7 \geqslant ?$} & 0.855 \\
\hline 0.070 & 0.519 & $0.52 ?$ & 0.737 & n.8O0 \\
\hline 0,100 & $0.3>8$ & 0.631 & 0.741 & 0.867 \\
\hline 0.200 & 0.557 & 0. BSO & $0.7+6$ & 0.894 \\
\hline 0.300 & 0.591 & 0.593 & 0.800 & 0.924 \\
\hline 0.500 & 0.569 & 0.767 & 0.879 & 0.994 \\
\hline 0.700 & 0.757 & n.R5? & 0.958 & 1.072 \\
\hline 1.000 & 0.804 & 0.936 & 1. . 1) & 1.202 \\
\hline .000 & 1.209 & $1.39 ?$ & 1.405 & 1.625 \\
\hline 3.000 & 1.019 & 1.720 & $1.83 \%$ & 1.967 \\
\hline .000 & 2.124 & 2.246 & $2.38 \%$ & 2.570 \\
\hline 7.000 & 2.532 & 2.577 & 2.849 & 3.051 \\
\hline 10,000 & 3.0151 & 3.7 .19 & $3.4 n 9$ & 3.651 \\
\hline 20.000 & 4.354 & 4.590 & A.das & 5.2118 \\
\hline 30.000 & $3.3 \times 1$ & 5.451 & $5.97 \%$ & 5.4116 \\
\hline 50.000 & 0.955 & 7.321 & 7.745 & 8.294 \\
\hline 70.000 & 8.248 & B.691 & 9.179 & 9.835 \\
\hline 00.000 & 9.381 & $10.4 i j(1$ & 10.995 & 11.773 \\
\hline 00.000 & 14.016 & 14.764 & 1.5 .619 & 13.709 \\
\hline 00 & $1 / .108$ & 18.1 .13 & 14.120 & 20.463 \\
\hline 00.000 & $? 2.235$ & 23.393 & 24.735 & 26.475 \\
\hline
\end{tabular}

$0.0010 \quad 0.0100 \quad 0.02000$

$0.0100 \quad 0.0200 \quad 0.03 n 0 \quad 0.050 n$

0.110701 0.01010 0.0201

$0.0301 \quad 0.0500$

0.0700

0.0701

0.0709

$0.03 n 1$

0.0501

n.n1no 0.0241

0.0302

11.0501

0.11070 .3

$0, n 1 . n 1$

0.0271

0.0302

$\pi .050$

0.0703

0.0704

0.00707

0.0102

$0 . \operatorname{lin} n$

$0.051 n$

0.0707

0.0713
0.0720

(1)

$0.01155 \quad 0.0206$

$0.03 \div 9$

0.0325

0.0525

$\pi .0535$

0.00777

n.01n 0.0215

0.0335

11. 0553

0.0734

0.0748

0.0859

0.00994

$0.0125 \quad 0.0249$

$0.04 ? 2$

[..0697

0.0969

0.0192

0.0360

$0.05 \times 5$

0.0925

$\begin{array}{llll}0.01916 & 0.0272 & 0.0535 & 0.0791\end{array}$

0.0491
0.04045090

0.0926

0.1339

$0.1 ? 75$

0.1272

0.2735

0.5517

0.7282

0.4977
0.827

1.19976

$0.9976 \quad 1.1521$

$1.2709 \quad 1.4401$

1.537

1.890
2.753

.625 ?

0.2735

1.028

1.761

2.143

3.104

3.835
4.987

$2.0420 \quad 3.230$

4.445

5.244

6.345

5.658
6.795
9.667

4.3608

$546 ? \quad 5.498 \quad 6.017$
$.4816 \quad 7.836$

$\begin{array}{lll}7.836 & 8.569\end{array}$

$11.9145 \quad 12.460 \quad 13.619$

11.084
14.352

5.925

10.110

12.414
16.068

0.1 non

0.1001

0.1 n?

0.1 nos

i. 1005

$0.1 n 1 . n$
$0.1 . n 18$

$0.1 \cap 27$

0.1348

0.1067
$0.1 \ln 99$

$0.122 \pi$

0.1368 .

0.1771

0.357

$0.8 \cap 83$

1.114

1.539

1.864

2,265

4.033

5.246

6.230
7.474

10.613

$.2000 \quad 0.3000 \quad 0.4000$

$0.2002 \quad 0.3002 \quad 0.4003$

0.20040 .30020 .4008

$0.2074 \quad 0.3007 \quad 0.4008$

$0.20190 .3012 \quad 0.4013$

$0.2011 \quad 0.3016 \quad 0.4018$

$0.2016 \quad 0.3021 \quad 0.4027$

$0.2033 \quad 0.3045 \quad 0.4051$

$0.2050 \quad 0.3064 \quad 0.4075$

$0.2083 \quad 0.3107 \quad 0.4127$

$\begin{array}{llll}0.2119 & 0.3155 & 0.4180\end{array}$

$\begin{array}{lll}0.2384 & 0.3226 & 0.4256 \\ 0.2381 & 0.3488 & 0.4547\end{array}$

$0.26250 .3794 \cdot 0.4878$

0.32640 .45420 .5660

$0.4094 \quad 0.54510 .6566$

$0.55750 .6938 \quad 0.8007$

$\begin{array}{rrr}1.398 & 1.1120 & 1.2093\end{array}$

$1.740 \quad 1.883 \quad 2.006$

$\begin{array}{lll}2.093 & 2.254 & 2.399\end{array}$

$\begin{array}{lll}2.532 & 2.723 & 2.887\end{array}$

$\begin{array}{lll}3.634 & 3.9173 & 4.132\end{array}$

$\begin{array}{lll}5.826 & 6.238 & 6.604\end{array}$

$6.909 \quad 7.405 \quad 7.836$

$\begin{array}{rrr}8.279 & 8.874 & 9.378\end{array}$

$\begin{array}{lll}11.773 & 12.597 & 13.314 \\ 14.443 & 15.458 & 16.343\end{array}$

$\begin{array}{lll}14.443 & 15.458 & 16.343 \\ 18.693 & 19.975 & 21.134\end{array}$

$\begin{array}{lllllllllll}0.9300 & 0.95110 & 0.9700 & 0.98 n 11 & 0.9900 & 0.9930 & 0.9950 & 0.9970 & 0.9980 & 0.9990\end{array}$

$\begin{array}{llllll}1.026 & 1.11 & 1.19 & 1.31 & 1.40 & 1.57 \\ 1.020 & 1.11 & 1.19 & 1.31 & 1.40 & 1.57\end{array}$

$1.020 \quad 1.11 \quad 1.19 \quad 1.31 \quad 1.40 \quad 1.57$

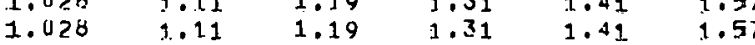

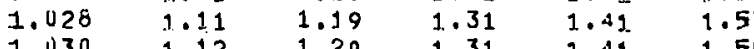

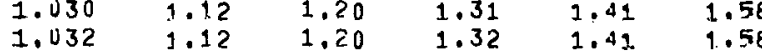

$\begin{array}{llllll}1.038 & 1.12 & 1.20 & 1.32 & 1.4 \% & 1.58\end{array}$

$1.041 \quad 1.13 \quad 1.21 \quad 1.33$

$1.476 \quad 1.16 \quad 1.22 \quad 1.33$

$\begin{array}{llll}1.104 & 1.16 & 1.24 & 1.36\end{array}$

$1.171 \quad 1.26$

1.26

1.385

1.035

2.223

$1.34 \quad 1.42$

$\begin{array}{ll}1.47 & 1.56 \\ 1.94 & 2.04\end{array}$

$2.35 \quad 2.47$

$\begin{array}{ll}3.02 & 3.17 \\ 3.58 & 3.75\end{array}$

3.598

$4 . ?$

5.18

7.100

4.38
6.88
7.47

$9.194 \quad 3.57$

$\begin{array}{ll}13.040 & 11.45 \\ 13.041\end{array}$

$\begin{array}{ll}18.510 & 19.43 \\ 22.691 & 23.52\end{array}$

4.48
6.37

7.81

10.11
11.99

11.99
1.4 .34

20.31

29.513

$\begin{array}{rr}1.66 & 1.75 \\ 1.66 & 1.75 \\ 1.66 & 1.75 \\ 1.66 & 1.75 \\ 1.66 & 1.75 \\ 1.66 & 1.75 \\ 1.66 & 1.75 \\ 1.67 & 1.75 \\ 1.67 & 1.76 \\ 1.68 & 1.76 \\ 1.69 & 1.77 \\ 1.71 & 1.80 \\ 1.75 & 1.84 \\ 1.83 & 1.92 \\ 1.93 & 2.02 \\ 2.10 & 2.20 \\ 2.69 & 2.82 \\ 3.23 & 3.38 \\ 4.12 & 4.31 \\ 4.87 & 5.09 \\ 5.81 & 9.07 \\ 8.23 & 8.58 \\ 10.08 & 10.52 \\ 13.04 & 13.60 \\ 15.43 & 13.10 \\ 18.48 & 19.27 \\ 26.17 & 27.30 \\ 32.07 & 3.48 \\ 41.41 & 43.24\end{array}$

1.8
1.8
1.8
1.8
1.8
1.8
1.8
1.8
1.8
1.9
1.9
1.9
1.9
2.0
2.1
2.3
3.1
3.62
4.6
5.4
6.4
9.16
11.2
14.5
17.2
20.5
29.1
35.6
46.11

$\begin{array}{rr}1.99 & 2.20 \\ 1.99 & 2.20 \\ 1.99 & 2.20 \\ 1.99 & 2.20 \\ 1.99 & 2.20 \\ 2.00 & 2.20 \\ 2.00 & 2.20 \\ 2.00 & 2.21 \\ 2.01 & 2.22 \\ 2.01 & 2.22 \\ 2.02 & 2.23 \\ 2.06 & 2.27 \\ 2.10 & 2.31 \\ 2.19 & 2.41 \\ 2.30 & 2.53 \\ 2.49 & 2.74 \\ 3.18 & 3.49 \\ 3.81 & 4.17 \\ 4.86 & 5.31 \\ 5.73 & 6.26 \\ 6.83 & 7.46 \\ 9.65 & 10.54 \\ 11.83 & 12.92 \\ 15.28 & 16.68 \\ 18.08 & 19.73 \\ 21.65 & 23.61 \\ 30.61 & 33.42 \\ 37.57 & 40.99 \\ 48.49 & 52.89\end{array}$

2.20
2.20
2.20 
VALUES OF ALPHA FRIK IV $=$ \& ATS R.P $=5$

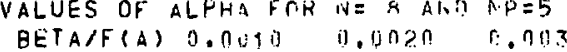

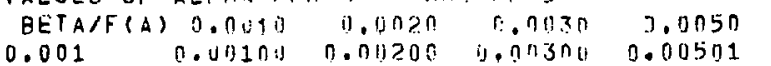

0. 1010no 0.00200 i.

0.00100 0.00201 0.00501

$0.005 \quad 0.401010 .110201$ u. 0n3n. n. nu501

0.007

0.010

0.020

0,050

0,050
0.070

$\therefore .100$

0.200

0.300

0.500
0.700

1.000

2.000
3.000

5.000

7.000
10.000

10.000
20.000

30.000

50.000

70.000

100.000

\begin{tabular}{|c|c|c|c|c|}
\hline$B E T A / F(A)$ & 0.5 un & 0.6000 & r.7ung & $\$ .3000$ \\
\hline 0.001 & $0.5 n 1$ & 0.5 i 4 & 0.715 & 0.844 \\
\hline $\begin{array}{l}0.002 \\
0.003\end{array}$ & $0.5 n 1$ & 0.605 & 0.71 .5 & $\begin{array}{r}0.844 \\
0.844\end{array}$ \\
\hline $\begin{array}{l}0.003 \\
0.005\end{array}$ & $0.5 \cap 2$ & $n .405$ & $\begin{array}{l}0.716 \\
0.716\end{array}$ & $\begin{array}{l}0.844 \\
0.845\end{array}$ \\
\hline $\begin{array}{l}0.005 \\
0.007\end{array}$ & $\begin{array}{l}0.5 n 22 \\
0.5 n 3\end{array}$ & $\begin{array}{l}0.005 \\
0.606\end{array}$ & $\begin{array}{l}0.716 \\
11.717\end{array}$ & $\begin{array}{l}1.845 \\
0.845\end{array}$ \\
\hline $\begin{array}{l}0.007 \\
0.010\end{array}$ & $\begin{array}{l}0.5 n 3 \\
0,5 n s\end{array}$ & $\begin{array}{l}n .606 \\
n .507\end{array}$ & 0.717 & $\begin{array}{l}0.845 \\
0.845\end{array}$ \\
\hline 0.020 & U. & 0.609 & $0.7 \bar{E}$ & 0.847 \\
\hline 0.030 & $0.5 \cap 9$ & n.A12 & 0.722 & 0.850 \\
\hline 0,050 & 0.514 & 0.618 & 0.727 & 0.855 \\
\hline 0.070 & 0,519 & 0.622 & 0.732 & 0.800 \\
\hline 0.100 & $0,5 ? 8$ & 0.6 .31 & 0.741 & 0,867 \\
\hline 0.200 & 0.557 & 0.600 & 0.766 & 0.894 \\
\hline 0.300 & 0.592 & 0.694 & 0.800 & 0.925 \\
\hline 0.500 & 0.673 & 0.771 & 0.875 & 0.996 \\
\hline 0.700 & 0.770 & 0.865 & 0.965 & 1.085 \\
\hline 1.000 & 0.936 & 1.025 & 1.123 & 1.244 \\
\hline 2.000 & 1.463 & 1.558 & 1.667 & $1.8 \mathrm{i} 6$ \\
\hline 3.000 & 1.905 & $? .021$ & 2.151 & 2.315 \\
\hline 5.000 & 2.050 & 2.799 & 2.971 & 3.192 \\
\hline 7.000 & 3.287 & 3.467 & 3.676 & 3.942 \\
\hline 10.000 & 4.125 & 4.354 & $4.6 \cap 5$ & 4.941 \\
\hline 20,000 & $6,4 \cap 6$ & 6.749 & 7.138 & 7.653 \\
\hline 30.000 & 8.279 & 8.721 & 9.275 & 9.881 \\
\hline 50.000 & $11.4 ? 2$ & 12.033 & 12.71 .9 & 13.619 \\
\hline 70.000 & 14.12 .3 & 14.871 & 15.733 & 16.831 \\
\hline 00.000 & 17.626 & $18,+0.1$ & 19.670 & 21.043 \\
\hline & 27.529 & 28.73 .3 & 30.381 & 32.502 \\
\hline 000 & 35.249 & 37.080 & 39.155 & 41.902 \\
\hline & 48,403 & 51.057 & 5.3 .926 & $57.71 n$ \\
\hline
\end{tabular}

0.001010 .00211 n.0n3n\% n.00504

0.00202 0.00201 0.0030s 0.00510

0.00103 0.00206 0.00300 9.00516

$0.001050 .002110 .00310 \quad 0.00526$

0.010108 0.001215 0.0n3?2 n.n015.57

$0.110111 \quad 0.00222 \quad 0.00333 \quad 9.70555$

$0.00143 \quad 0.00235$ D.00428 0.00712

$0.001970 .00393 \quad 0.00589$ n.n097

0.40597 0.n11HE 0.01773 n.n292

$0.05432 \quad 0.11216 \quad 11.1594 \%$ n.24061

0.265830 .42160 u.424n

$0.83794 \quad 0.99508$ 1.0933? 1.19786

1.197 a $^{2} 1.35426$ 1..44772 1.56979

$\begin{array}{llll}1.5904 & 1.7759 & 1.0826 & 2.0247 \\ 2.5969 & 2.8525 & 3.0127 & \$ .2225\end{array}$

0.7139
$2.05 \% 5$

4.7950 3.7?2?

5.7350 5.1775

3.9253

4.1933

0.4211

5. $45 ?$ ?

.8260
.2222

8.4772

.0571

$11.4374 \quad 12.4903 \quad 13.1 .464 \quad 14.0314$

14.7701 10.1?95 RK.984त 1.8.1132 2ट.2331 23.423? ?4.9796

$0.007 n$ 0.00700 0.00701 0.00703 0.00705 0,00707

0.00715

0.00721

0.00737

0.00753

0.74879

1) 0996

0.01368

0,02032

0.30736

7577

1.27415
1.55180

2.1239

3.3713

4.3841

7.5350

9.4538

14.0419

18.9066
26.0477 $n, 01 n \pi$

n. nino

ก. 0100

nonino

0.1101

0.0103

. 105

0.0107

0.1125

C. C. 142

0.0298

0.0572

0.3889
0.8235

1.3543

1. $.74,34$

3.5 .35

4.598

6.368
7.807

9.896

15.3 .36

19.761
77.268
$0.030 n$

$0.03 n 1$
$0.03 n 1$

0.0301

$0.030 \%$

0.0303

0.0306

0.0309

0.03 ? 2

0.037

0.0372
0.042 .3

0.0574

0.0834

0.1566
0.6770

1.076

1.619
2.051

2.005

4.094

5.307
7.352

9.103

11.392

17.655

22.752
31.343 $\begin{array}{rrr}0.0500 & 0.0700 & 0.1000 \\ 0.0500 & 0.0701 & 0.1001 \\ 0.0501 & 0.0701 & 0.1007 \\ 0.0501 & 0.0702 & 0.1002 \\ 0.0503 & 0.0703 & 0.1005 \\ 0.0503 & 0.0704 & 0.1006 \\ 0.0505 & 0.0707 & 0.1010 \\ 0.0510 & 0.0713 & 0.1018 \\ 0.0515 & 0.0720 & 0.1027 \\ 0.0525 & 0.0734 & 0.1048 \\ 0.0535 & 0.0748 & 0.1067 \\ 0.0553 & 0.0772 & 0.1099 \\ 0.0617 & 0.0859 & 0.1220 \\ 0.0698 & 0.0979 & 0.1370 \\ 0.0939 & 0.1291 & 0.1795 \\ 0.1342 & 0.1814 & 0.2 .463 \\ 0.2401 & 0.3112 & 0.4003 \\ 0.7987 & 0.8827 & 0.9709 \\ 1.192 & 1.272 & 1.360 \\ 1.753 & 1.848 & 1.956 \\ 2.208 & 2.322 & 2.448 \\ 2.799 & 2.936 & 3.093 \\ 4.384 & 4.590 & 4.834 \\ 5.681 & 5.948 & 6.253 \\ 7.867 & 8.233 & 8.645 \\ 9.728 & 10.186 & 10.705 \\ 12.185 & 12.750 & 13.406 \\ 18.876 & 19.731 & 20.738 \\ 124.339 & 25.468 & 26.719 \\ 33.540 & 35.066 & 36.836\end{array}$
0.9930
1.66

0.9950

.9950
1.75
1.75
1.75

$1.026 \quad 9.11$

1.028

1,028

1.031

1.032

1.038
1.041

1. 049

1.076

1.104
1.175

1.265

1.427

2.589

3.551
4.384

4.384

5.483
8.477

10.949
15.084

15.084
18.632

23.301
35.981

35.981
46.418

63.905
1. 11

1.11
1.11
9.12

1.12

1.12
1.12

1.13

1.16

1.1 .9

1.35

1.52
2.14

$? .73$

4.61

5.76
4.90

$11.50 \quad 9.33$

$15.85 \quad 16.59$

19.58
24.46

37.8

48.74
67.08

1.19
1.19
1.19
1.19
1.19
1.19
1.20
1.20
1.20
1.21
1.22
1.24
1.27
1.34
1.43
1.60
2.25
2.86
3.92
4.83
6.03
9.33
12.03
16.59
20.46
25.59
39.52
50.93
70.1 .3

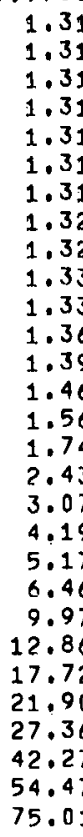

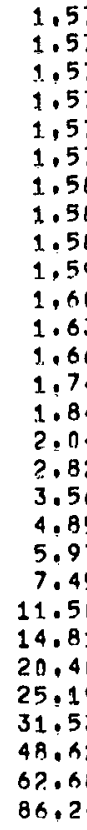

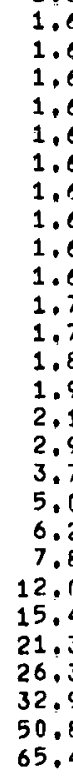

1.66
1.66

1.66

.66

1.67

1.67

1.69

1.71

1.83
1.94
2.15

1.94
2.15
2.96

2.96

5.09

6.25

. 03

15.49

21.35

32.93

65.49
90.27
1.75

1.75

1.75
1.75

1.75
1.76

1.76

1.80

1.84
1.93
2.04

2.04
2.25

3.10
3.90

5.31

8. 14

12.55

22.26

27.51
34.39

53.07

$\begin{array}{rrrr}69.42 & 72.94 & 76.72 & 83.68 \\ 94.18 & 100.40 & 105.65 & 115.17\end{array}$ 


\begin{tabular}{|c|c|c|c|c|c|c|c|c|c|c|c|c|c|c|c|}
\hline $\begin{array}{l}\text { VALUES OF } \\
\text { BETA/F(A) }\end{array}$ & $\begin{array}{l}\text { ALHHA FC } \\
0.01190\end{array}$ & $\begin{array}{l}N=9 \quad A M D \\
0.0020\end{array}$ & 0.073. & & & & & & & & & & & & \\
\hline $\begin{array}{l}B E T A A(A) \\
0.001\end{array}$ & $\begin{array}{r}0.011 .10 \\
0.00100\end{array}$ & $\begin{array}{r}0.0020 \\
0.00206\end{array}$ & $\begin{array}{l}n .0730 \\
0.00 .30 \%\end{array}$ & $\begin{array}{r}1.0050 \\
0.00501\end{array}$ & $\begin{array}{r}0.0070 \\
0.04700\end{array}$ & $\begin{array}{l}n .0100 \\
0,0100\end{array}$ & $\begin{array}{l}0.020 \\
0.020\end{array}$ & $\begin{array}{l}0.0 \\
0.0\end{array}$ & $\begin{array}{l}3 n n \\
301\end{array}$ & $\begin{array}{l}.3500 \\
1.3500\end{array}$ & $\begin{array}{l}0.0700 \\
0.0709\end{array}$ & $\begin{array}{l}0.1000 \\
0.1001\end{array}$ & $\begin{array}{l}0.2000 \\
0.2002\end{array}$ & $\begin{array}{l}0.3000 \\
0.3002\end{array}$ & $\begin{array}{l}0.4000 \\
0.4003\end{array}$ \\
\hline 0,002 & 0.00100 & $0.00 ? 110$ & $0.003 n$ & 0.00501 & 0.00701 & 0.0100 & 0.020 & 0.0 & $\ln 1$ & .3501 & 0.0701 & 0.1002 & 0.2004 & 0.3002 & 0.4003 \\
\hline 0.003 & 0.00100 & $0.00 \% 01$ & 0.00301 & 1.00501 & $0.0070 ?$ & $n .01 \cap 0$ & 0.020 & 0.0 & 301 & $.350 \mathrm{~g}$ & 0.0702 & 0.1002 & 0.2004 & 0.3007 & 0,4008 \\
\hline 0.005 & $0.001 n 1$ & $0.0 \cup 2 \cup 1$ & J.0ก3ก? & 0.00502 & 0.0070 .3 & 0.0100 & 0.020 & $0 . n$ & 302 & 1.0503 & 0.0703 & $0.1 \cap 05$ & 0.2009 & 0.3012 & 0,4013 \\
\hline 0,007 & 0.00101 & 0.00201 & 0.01302 & 0.00504 & 0.00705 & n.cins. & 0.020 & $0 . n$ & $30 ?$ & .0503 & 0.0704 & 0.1006 & 0.201 .1 & .3016 & .4018 \\
\hline 0.010 & 0.00101 & 0.00202 & 0.011303 & 0.00505 & .00707 & 0.0101 & 0.020 & $0 . n$ & $3 n 3$ & .0505 & 0.0707 & $0.1 n 1 n$ & 0.2016 & 1.3021 & .4023 \\
\hline 0.020 & 0.00102 & $0 . \cap \cup 204$ & 0.00308 & 0.00510 & 0.00 .714 & 0.0102 & 0.020 & $0 . n$ & 306 & $.051 n$ & 0.0713 & 0.1018 & $0,20.33$ & .30 .40 &, 4046 \\
\hline 0,030 & $0.001 n 3$ & 0.00206 & $0.003 \cap 9$ & 0.00515 & 0.00721 & $n .0103$ & 0.020 & 0.0 & $\ln 9$ & 1.051 .5 & 0.0720 & 0.1 .27 & 0.2047 & 0,3064 & 1.4070 \\
\hline 0.050 & 0.00105 & $0.00 \geq 10$ & 0.00315 & 0.00525 & 0.00735 & 0.0105 & 0.021 & $0 . n$ & 314 & 1.0523 & 0.0733 & $0.1 \cap 45$ & 0.2078 & 0.3102 & 0.4118 \\
\hline 0.070 & 0.00107 & $0.0 \cup 21.4$ & $0.0 \cap 3 \geq 1$ & 0.00535 & 0.00749 & 0.0107 & 0.021 & 0.0 & 300 & 1,0533 & 0.0745 & $0.1 n 62$ & 0.2112 & 0.3145 & 0.4166 \\
\hline 0.100 & 0.00110 & 0.00220 & a.onszn & 0.00550 & 0.010767 & 0.0110 & 0.022 & 0.0 & 32.9 & .0547 & 0.0765 & $0.1 n 89$ & 0.2157 & 0.3202 & 0.4232 \\
\hline 0.200 & $0.001 ? 0$ & 0.00240 & 0.00360 & 0.00599 & 0.04839 & 0.0100 & 0.023 & $0 . n$ & 358 & 1.0594 & 0.0828 & 0.1176 & 97 & 0.3398 & 1.4447 \\
\hline 0.300 & 0.00430 & $0.00 \% 60$ & 0.00390 & 0.00649 & $0.0 \cup 9 \cup 8$ & 0.0130 & 0.025 & 0.0 & 346 & $1.064 n$ & 0.0892 & 0.126 .3 & 0.2454 & 0,3579 & 0.4647 \\
\hline 0.500 & 0.00150 & 0.00300 & $0.0 ? 449$ & 0.00748 & 0.01046 & 0.0149 & 0.029 & 0.0 & 444 & .0732 & 0.1014 & 0.1430 & 0.2726 & 0.3913 & 0.5002 \\
\hline 0.700 & 0.00170 & 0.00340 & $0.705 \% 9$ & 0.00847 & 0,01184 & 0.0169 & 0.033 & 0.0 & $5 \cap 0$ & 1.0821 & 0.1135 & 0.1589 & 0.2983 & 0.4218 & 0.5317 \\
\hline 1.000 & $0.002 \cap 0$ & 0.00399 & 0,00508 & 0.00994 & 0,01391 & 0.0198 & 0.039 & 0.0 & $58 ?$ & $.095 ?$ & 0.1308 & 0.1818 & 0.3331 & 0.4619 & 0.5727 \\
\hline 2,000 & 0.00299 & 0.00598 & $\mathrm{u} .01094$ & 0.01485 & 0.0 .21071 & 0.0294 & 0.057 & 0.0 & 449 & 1.1363 & {$[., 1842$} & 0.2501 & 0.4285 & $0.564 i$ & 0,6729 \\
\hline 3.000 & 0.00309 & 0.04795 & リ. 01.19? & n.ก1970 & 0.02741 & n.0398 & 0.075 & & 1.0 & 0.174 & 0.231 & 0.308 & 0.500 & 0.636 & 0.741 \\
\hline 5.000 & 0.00507 & 0.01188 & 0.01 .773 & 0.02926 & $0.0406 \pi$ & 0.8572 & 0.109 & & & $4 n$ & 0.311 & 00 & 0. & .732 & 0.832 \\
\hline 7,000 & 0.00795 & 0.01579 & 0.02351 & $n .03864$ & 0.05336 & 0.0747 & $0.14 n$ & & 98 & 0.296 & 0.376 & 0.471 & 0.673 & 0.798 & 0.1 \\
\hline 10.000 & 0.0109 & 0.0216 & 0.0320 & 0.052 .4 & $0.072 n$ & 0.100 & ii. 18 & & 254 & 0.366 & 0.453 & 0,551 & 0.748 & 0.867 & 0,959 \\
\hline 0.000 & 0.0206 & 0.04114 & ก. 1599 & 0.0955 & 0.1280 & i). 175 & 0.30 & & 34 & 0.526 & 0.616 & 0.710 & 0.889 & 0.999 & 1.087 \\
\hline 30.000 & $0.0 .5 \cap 1$ & 0.0585 & 0.0853 & 11.1349 & 0.1795 & 0.239 & $0.3 \mathrm{H}$ & & 490 & 0.623 & 0.710 & 0.799 & 0.971 & 1.078 & 1.164 \\
\hline 50.000 & 1.0486 & 0.0327 & 0.1330 & 0.2040 & 0.2644 & 0.340 & 0.51 & & 615 & 0.742 & 0.823 & 0.906 & 1.068 & 1.175 & 1.261 \\
\hline 70,000 & 3.0064 & 0.1246 & 0.176 .1 & 0.2630 & 0.5336 & 0.4 .18 & r..54 & & & 6.817 & 0.893 & 73 & 1. & .238 & 326 \\
\hline 00.000 & 3.0918 & $0.168 ?$ & 0.2331 & 0.3364 & 0.4161 & $0.50 \mathrm{~A}$ & 0.6 & & & ๕.892 & & & & & \\
\hline 0.000 & 0.1075 & 0.2873 & 0.3770 & 0.5031 & $0.588 n$ & $0.67 \%$ & 0.8 & & 5 & 1.032 & 1.102 & 1.179 & 1. & 6 & 37 \\
\hline & 0.2394 & $0 . \$ 765$ & $0 .<757$ & 0.0042 & 0.6872 & 0.771 & 0,9 & & & & & & & & 623 \\
\hline 00 & 0.3341 & 0.5012 & 0.6042 & 0.7272 & 0.8045 & 0.892 & 1.0 & & & 1.211 & 1.280 & 1.358 & 1.522 & .637 & .736 \\
\hline BETA $/ \mathrm{H}$ & $50 \cap 0$ & onon & 7000 & .80 & 9000 & $e$ & 9500 & & & co & 930 & ,9950 & 9970 & .9980 & .9990 \\
\hline 0.001 & 0.501 & 603 & 710 & 8.32 & 995 & $\pi$ & .1 .5 & $1 .<5$ & $1 \ldots$. & 1.67 & .55 & 1.62 & 1.73 & .83 & .99 \\
\hline 0.002 & $\operatorname{sn} 1$ & 603 & $c$ & $?$ & & & 1 & & & & 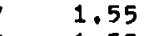 & I. & & & 9 \\
\hline 0.003 & 501 & 603 & 0 & ? & 1 & $n$ & 15 & 1.25 & 35 & 47 & .5 & 1.6 & 1. & 1. & 9 \\
\hline 0.005 & $0.5 n 2$ & 0.604 & 711 & 833 & 1.001 & .08 & .15 & 1.25 & $1,3=$ & 1.47 & 1,5 & 1.6 & 1.74 & 1.83 & .99 \\
\hline 0.007 & 0.502 & 0.004 & 711 & 0.8 .33 & & 1.08 & 1.1 & 1.25 & & 1.47 & 1. & 1.. & 1. & 1. & 9 \\
\hline 0.010 & 0.503 & 0.605 & 0.712 & 0,834 & 1.001 & 1.08 & 1.15 & 1,25 & 1.35 & 1.28 & 1.5 & 1.62 & 1.74 & 1.83 & .00 \\
\hline 0.020 & $0.5 \cap 6$ & 0,607 & 715 & 83 & 1.003 & 1.0 & 1.1 & 1.26 & 1,3 & 1,4 & 1. & 1.6 & 1.74 & 3 & 0 \\
\hline 0.030 & $0 . \sin \theta$ & 0.610 & 0.71 .7 & 0.838 & 1,005 & 1.0 & 1.15 & 1.26 & 1,34 & 1.48 & 1.5 & 1.63 & 1.74 & 1.83 & .00 \\
\hline 0.050 & 0.543 & 0.615 & $7 \supset 1$. & 0.843 & 008 & $\therefore$ & 1.16 & 1.26 & 1.34 & 1,48 & 1 & 1. & 1.75 & 1. & 1 \\
\hline 0 & 0.517 & 0.619 & 0.725 & 0.8 & 1 & 1.09 & 1.16 & & 1.34 & & & 1.63 & 1.75 & 1.84 & 01 \\
\hline 0.100 & $0.5 ? 4$ & 0.626 & $0.73 ?$ & 0.852 & 1.019 & 1.09 & 1.17 & 1.27 & 1.35 & $1,+9$ & & 1.64 & 1.75 & 1.85 & $0 ?$ \\
\hline 0,200 & P & 0.647 & 752 & 0.8 & 1. & 1. & 1.1 & 1.29 & $1.3^{7}$ & 1. & . & 1.0 & & 1.87 & 4 \\
\hline 0.300 & 567 & 0.667 & 0 & 0.8 & 1 & & 1. & & 1.38 & & 1.60 & $10 t-2$ & & 9 & \\
\hline .500 & $0.0 \cap 2$ & 0.701 & 0.803 & 0.9 & 1.0 & 1.1 & 1.2 & 1. & 1.4: & 1.56 & 1.63 & 1. & 1.83 & 1.92 & .10 \\
\hline & .634 & 0.7 & 0.8 & & & & 1.20 & & 1.4 & & 1.6 & 1 & $1,8-2$ & 48 & 3 \\
\hline .000 & 0.673 & $0.76 E$ & 0.865 & 0.97 & 10 & 1.2 & 1.2 & 1.3 & & & & & & & \\
\hline 00 & 0.768 & 0.857 & $04 \mathrm{c}$ & 105 & 1.2 & 1.2 & 1.3 & 1.47 & 1.55 & & 1. & 1. & 1. & 0 & \\
\hline .000 & 0.832 & $0,91.8$ & 007 & 1. & & & 1.4 & 1.53 & & & & & & & \\
\hline 000 & & 0.990 & 087 & 1.18 & 1.352 & & 1.5 & 1.6 & & & 1 , & 2. & 2.17 & 2.28 & 2,48 \\
\hline 000 & 0 & 1.057 & & & & & 1. & & & & & & & & \\
\hline 00 & 1.040 & 1.120 & & 131 & 1 & & 1.6 & 1.7 & 1.84 & & & & & 2. & \\
\hline & & & & & & & & & & & & & & & \\
\hline 100 & 1.242 & 1.322 & 1.410 & 1.520 & 1.696 & 1.78 & 1.87 & 1.99 & 2.13 & 2.29 & 2.39 & 2.49 & 2.65 & 2.78 & 3.02 \\
\hline 50.000 & 1.341 & & & & 1.808 & & & & & & & & & & \\
\hline 0.000 & 1.406 & 1.490 & 1.581 & 1.699 & 1.884 & 1.9 & 2.07 & 2. & 2.33 & $2 !+3+2$ & 2.64 & & & 3.07 & 3.33 \\
\hline & 1.478 & 1.562 & 1.658 & 1.778 & 1,971 & 2.0 & 2.16 & 2.3 & & & 2.7 & 2.8 & 3.0 & 3,20 & 3,46 \\
\hline & 1.623 & 1.713 & 1.812 & 1.941 & 2,147 & & 2. & & & & 2,5 & 3.11 & 3.30 & 3.46 & 3.75 \\
\hline & .711 & 1.804 & 1.907 & 2.04 & 2.254 & & & & & & 3.13 & 3.26 & 3.6 & 3.63 & 3.93 \\
\hline 800 & .827 & 1.926 & 2.032 & 2.173 & 2,399 & & 2 & & & 7 & 3.33 & 3.46 & 3.67 & 3.85 & 4.17 \\
\hline
\end{tabular}




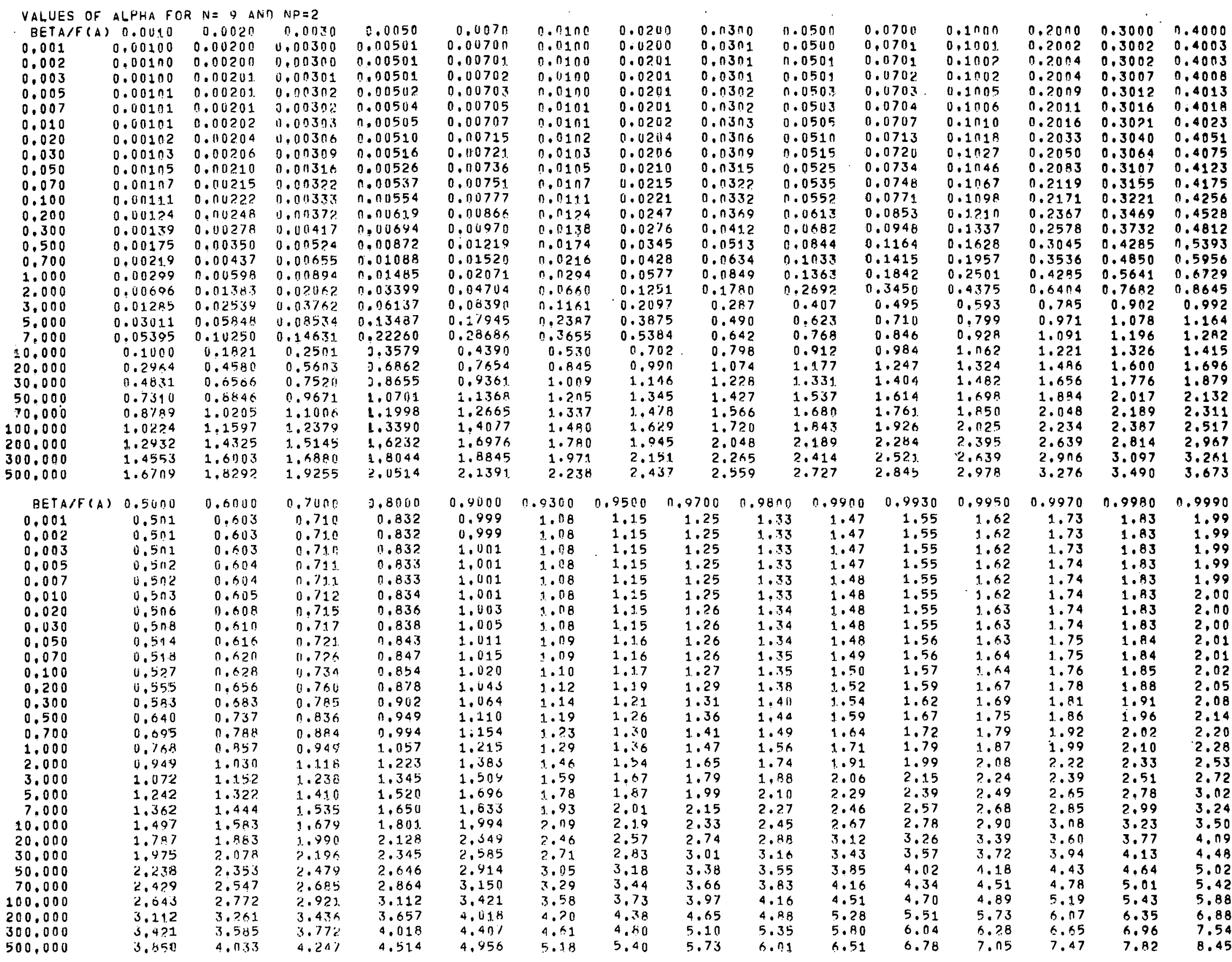




\begin{tabular}{|c|c|c|c|c|c|c|c|c|c|c|c|c|c|c|c|}
\hline $\begin{array}{l}\text { VALUES OF } \\
\text { RETA/F (A) }\end{array}$ & $\begin{array}{c}A L H H A F O \\
0.0 ! 10\end{array}$ & U. $0 ? \geqslant 0$ & 0,$01131 ;$ & 3.0050 & $0.0 \cap 70$ & $0 . n 1 \cap 0$ & 0.0200 & & $0.03 n 0$ & 0.0500 & 0.0700 & 0.1000 & 0.2000 & 0.3000 & 0.4000 \\
\hline 0.001 & 0.60100 & $0.00 ? 010$ & $0.00 .3 n \pi i$ & 0.00501 & 0.007000 & 0.7100 & 0.02. & 0.1 & $3 \cap 1$ & 0.0500 & 0.0701 & 0.1001 & $0.20 n 2$ & 0.3002 & 0.4003 \\
\hline 0.002 & 0.00100 & 1) . no? ? ? & u.no3nti & 0.011501 & 0.00701. & 0.0100 & 0.02 & 0.8 & 301 & 0.0501 & 0.0701 & 0.1002 & 0.2004 & 0.3002 & 0.4003 \\
\hline 0.003 & 0.110100 & ח. ח ט ? 1 & 0.00309 & 0.00501 & 0.00702 & 0.0100 & 0.02 & 0.1 & 301 & $0.050 j$ & 0.0702 & 0.1002 & 0.2004 & 0.3007 & 0.4008 \\
\hline 0.005 & 0. เ 0101 & $\cap . \cap \cup \geq 01$ & a.nก3n? & $0.0050 ?$ & 0.0070 .3 & $n . n 1 n \theta$ & 0.02 & 0.1 & $3 \cap 2$ & ח.0503 & 0.0703 & 0.1005 & 0.2009 & 0.3012 & 0.4013 \\
\hline 0.007 & 0.0030 .1 & $0.0(121)]$ & 2. & 0.00504 & 5 0,0070 & $n .01 n q$ & 0.02 & 0.1 & 3n? & 0.0503 & 0.0704 & 0.1006 & 0.201 .1 & 0.3016 & 0.4018 \\
\hline 0.010 & $0.001 . n 1$ & $0.0 \cup \geq 11 ?$ & $0.7 n 3113$ & 0.011505 & 0.110707 & $n .0101$ & 0.02 & 0.1 & $3 \cap 3$ & 0.0505 & 0.0707 & 0.1010 & 0.20 .16 & 0.3021 & 0.4023 \\
\hline 0.020 & 0. unin? & $0.00>044$ & 0.010306 & n.00510 & 0.110715 & 0.0102 & 0.02 & 0.0 & 306 & 1).051n. & 0.0713 & 0.1018 & 0.2033 & 0,3045 & 0.4051 \\
\hline 0.030 & 0.0n1ns & $0.0 \| \geq 06$ & $0.0 \cap 3 \cap 0$ & 0.011516 & 0.00721 & 0.0103 & 0.02 & 0.1 & $3 n 9$ & 0.0515 & 0.0720 & 0.1027 & 0.2050 & 0.3064 & 0.4075 \\
\hline 0.050 & ก.Uก1n5 & $0 . n i 1 \% 10$ & 0.00316 & $0.3152 \mathrm{~A}$ & 0.00737 & 0.0105 & 0.02 & 0.1 & 315 & 0.0525 & 0,0734 & $0.1 \cap 48$ & 0.2083 & 0.3107 & 0.4123 \\
\hline 0.070 & $0.0 \cap 108$ & $0.00 ? 15$ & $0.0 n 32 \%$ & 0.00537 & 0.00753 & $n . n 1 n 7$ & 0.02 & 0.8 & 322 & $n .0535$ & 0,0748 & 0.1067 & 0.2119 & 0.3 & 0.4175 \\
\hline 0.100 & 0.011111 & $0.0 \cup>2 ?$ & 0.71133 .9 & $n .70555$ & 0.00777. & 0.0111 & 0.02 & 0.0 & 3.32 & 0.0553 & 0.0772 & 0.1099 & 0.21 .74 & 0.3226 & 0.4256 \\
\hline 0.200 & 0.011125 & 0.010249 & $0,011.574$ & 0.00623 & $0.0087 ?$ & n.01?4 & 0.02 & 0.1 & 371 & $0.061 \mathrm{~A}$ & 0.0858 & 0.121 .8 & 0.2379 & 0.3484 & 0.4542 \\
\hline 0.300 & 0.011142 & 0.00253 & 0.011425 & 0.00707 & 0.00988 & 0.0141 & 0.02 & 0.0 & 420 & 0.0694 & 0,0964 & 0.1361 & 0.26 .16 & 0.3779 & 0.4859 \\
\hline 0.500 & 0.00187 & 0.00 .374 & 0.010563 & 0.019933 & 0.01304 & 0.0196 & 0.0 & 0.0 & 548 & 0.0899 & 0,1237 & 0.1723 & 0.3193 & 0.4456 & 0.5565 \\
\hline 0.700 & 0.00253 & 0.00505 & 0.011756 & 0.01257 & 0.01755 & 0.0249 & 0.04 & 0.0 & $7 ? 7$ & 0.1176 & 0.1601 & 0.2197 & 0.3879 & 0.5212 & 0.6318 \\
\hline 1.000 & 0.40309 & 0.06795 & 0.01160 & $n .91070$ & 0.02741 & 0.0398 & 0.07 & 0.1 & 1n 1 & $0.174 n$ & 0.2314 & 0.3078 & 0.5002 & & 406 \\
\hline 2.000 & 11.01479 & 0,02017 & $0.0431 \%$ & 0.01008 & 0.09559 & 0.1315 & 0.23 & 0.3 & 69 & 0.4414 & 0.5308 & 0.6290 & 8 & 0.9323 & 1.0205 \\
\hline 3.000 & $0.0335 ?$ & $0.0 / 425$ & 0.1 .97 .34 & 0.16729 & 0.21998 & $n .2878$ & 0.44 & & 55 & 0.685 & 0.768 & 0.854 & 1.020 & 1.127 & 1.213 \\
\hline 5.000 & 0.13511 & 0.23510 & $0.319 ? 6$ & 0.43945 & 0.52408 & 0.5147 & 0.78 & & & $0.98 ?$ & 1.053 & 1.129 & & & .484 \\
\hline 7.000 & 0.285011 & 0.44517 & i).54607 & 0.67381 & 0.75392 & $n .834 n$ & 0.98 & & 62 & 1.167 & 1.236 & 1. & 1. & 1.589 & .684 \\
\hline 10.000 & 0.5269 & 1.6986 & $0.79 ? .1$ & 0,9027 & 4.9728 & 1.043 & 1. 1 & & & $1.36 \%$ & 1. & 1,5 & 1.694 & & 1,922 \\
\hline 20.000 & $0.96 \times 1$ & 1.1734 & $1.20 .3 n$ & 1. & 1.3695 & 1.442 & 1. & & & & 83 & & & & \\
\hline 30.000 & 1.2227 & 1.35x1 & 1.4401 & $1.546,9$ & 1.6194 & 1.698 & 1.8 & & 60 & 2.093 & 2.185 & 2.292 & 8 & 2.696 & 2.841 \\
\hline 50.000 & $1.5 ? 59$ & $1.6 \% 47$ & 1. $.766 ?$ & 1.8864 & 1.9675 & 2.059 & 2.2 & & & 2. & 2.627 & 2 , & & & 994 \\
\hline 70.000 & 1.7453 & $\left.1 . y_{i}\right) 55$ & 2.01150 & 2.1391 & $2 .<<69$ & 2.320 & $\ddot{2} .5$ & & 62 & 2.837 & 2.959 & 3. & & 3.623 & 8.814 \\
\hline 00.000 & 1.9991 & 2.1773 & 2.2879 & 2.4329 & 2.5359 & 2. & 2. & & & 3.215 & 3.352 & 3. & 3. & & 15 \\
\hline 00.000 & $2.5 / 72$ & $2.70_{14}$ & $2.92 F \%$ & 3.1119 & 3.2378 & 3.379 & 3.6 & & & 4.087 & 4,262 & 4. & 4. & & 5,467 \\
\hline 0.0000 & $2.97 \div 7$ & 3.2225 & 3.3789 & 3.5849 & $3.7 \geqslant 90$ & 3.898 & 4.2 & & & 4.705 & 4.895 & 5. & 5. & & 6.276 \\
\hline 00.000 & 3.55116 & 3.4500 & 4,1331 & 4.2773 & 4.4527 & 4.6 .36 & & & & 97 & 5.834 & & & 8 & 7,466 \\
\hline BETA & 0.5000 & 0.60011 & $0.700 \mathrm{ij}$ & 4.8000 & $0.96 r .0$ & 0 & .9500 & & 3.90 & .9900 & 0.9930 & 0.9950 & .9970 & 0.9980 & 990 \\
\hline 0.001 & $5 n 1$ & 0.003 & 710 & n & 0. & 1 & 1.1 & 1.25 &.,- 33 & 1.47 & 1.55 & 1.62 & .73 & 1 & 1.99 \\
\hline & & 60.3 & 10 & & & & 1.1 & & & & & 1. & .73 & 3 & 1.99 \\
\hline 0.003 & $b ! 1$ & ח.6u3 & .71 .11 & $n, 8$ & 1.0071 & 1 & 1.15 & 25 & 73 & 1.47 & 1.55 & 1.62 & 1.73 & 1.83 & 1.99 \\
\hline 0,005 & $5 n ?$ & 0.504 & 711 & 0 & 1,0 & 1.08 & 1.15 & 1.25 & & & 1. & 1.62 & 1.74 & 1.83 & 1.99 \\
\hline 0.007 & $\sin 2$ & ח. Gij 4 & 711 & 3 & 1.001 & & 1.15 & 1.25 & -. 33 & & 1.5 & 1.62 & 1.74 & 1.83 & 100 \\
\hline 0.010 & u. $\supset$ n & 0.6015 & 71.2 & 0. & $1, \cos 1$ & 1.08 & $1.15^{\circ}$ & 1.25 & \pm .33 & & 1.5 & 1. & 1.74 & 1.83 & 2.00 \\
\hline 0.020 & $1.5 \cap 6$ & $n$, hidg & 715 & 0.8 & 1,003 & 1.08 & 1.15 & 1.26 & 2.34 & 1. & 1.5 & 1.6 & 1.74 & 1.8 & 2.00 \\
\hline 0.030 & $0.5 \cap 8$ & 0.510 & 717 & $n$ & 1. & & 1.15 & 1.26 & 2.34 & 1. & 1. & & 1. & & \\
\hline 0.050 & 514 & 0.516 & 721 & $n, 8$ & 1.6 & 1. & 1.1 & 1.26 & 1.34 & 1. & 1. & 1. & 1. & 1 & \\
\hline 0.070 & 918 & $\pi . A 20$ & 726 & 0.847 & & & 1. & 1.2 & \pm .35 & & & & & & \\
\hline 0.100 & ๖>7 & 11.529 & .734 & 0.854 & 1.020 & 1.10 & 1.17 & 1.27 & 1.35 & 1.50 & 1.5 & 1.64 & 1.76 & 1.B5 & 2. \\
\hline 0.200 & $55 \%$ & 0.557 & 701 & & & & 1.19 & 1.029 & 1.38 & & 1. & & & & \\
\hline 0. & $5 \% B$ & 0.587 & 0.789 & & 1. & 1. & 1.22 & 1.32 & 1.40 & 1.55 & 1.6 & 1.70 & 1.81 & 1,91 & 2.08 \\
\hline 0,500 & 650 & 0.753 & 0.859 & 0 & 1.8 & 1. & 1.27 & 1.38 & 1.46 & 1.61 & 1.6 & 1.76 & 1.88 & & \\
\hline 0.7 & 129 & 0.421 & 1.914 & & & & 1, & 1.4 & 1.52 & & & & & & \\
\hline 1,000 & 0.0332 & n.9.16 & 1120 & & 1. & & 1. & 1.5 & 1.62 & & 1.5 & 1. & $\cdots$ & & 336 \\
\hline 2.00 & & 1.181. & $1 .<t$ & & 1. & & 1. & 1.8 & 1.92 & & & & & & \\
\hline 3,000 & 1.201 & 1.373 & 1.46 & $1.5 \%$ & 1.7 & & 1.5 & 2.0 & 2.17 & & 2 , & 2. & 2. & & 3.11 \\
\hline 5.00 & 8 & 1.656 & & & 2. & $?$. & 2. & 2. & 2.55 & & & & & & \\
\hline 7. & 1.776 & 1.871 & $97>-2$ & & 2.3 & 2. & 2. & 2.7 & 2.86 & & 3. & 3.37 & 3. & 3 & 4.06 \\
\hline 10.000 & $2.0>1$ & $2.12^{4}$ & 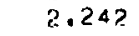 & & & & 2. & 3.0 & $3 . ? 2$ & & & & & & \\
\hline & 2.52 .5 & 2.715 & 2.56 & 3.04 & 3.3 & 3.50 & $3.0,5$ & 3.89 & 4.08 & 4, & 4.6 & 4.79 & 5.08 & 5.32 & 5,76 \\
\hline 30.000 & 2.482 & 3.127 & 3.291 & & & 4.03 & 4,20 & 4.46 & 7.68 & & 5. & 5.49 & & & \\
\hline 50.000 & 3.558 & 3.750 & 3. & 4. & 4.582 & 4. & 4.99 & 5.3 & 5.57 & 6.03 & 6.28 & 6.53 & & 7.25 & 7.84 \\
\hline 70.000 & & 4.136 & 1.407 & & 5.147 & & 5.60 & & 5.24 & & 7.04 & & & & \\
\hline & & 4.735 & & & 5. & a. & 6.32 & 6.72 & 7.04 & 7.62 & 7.94 & 8.25 & 8.74 & 9 & 90 \\
\hline & $5.7 ? 7$ & h. no? & & $\because 7$ & & & & & $3.9 n$ & & & & & & \\
\hline & 6.574 & 6.937 & 7.2 & 7.649 & 8.4 .31 & $A . B_{1}$ & 9.16 & 9.73 & 13.20 & & 11 & 11.94 & & & \\
\hline & 7.4 .21 & 0.147 & 2.599 & 9.149 & 10.098 & $1 n .46$ & & & $1 \geq .1 ?$ & & 13.6 & $14,1,8$ & 15,02 & 15.70 & 16.98 \\
\hline
\end{tabular}


VALUES OF ALPHA FZK IV $=9$ ANT NHE

\begin{tabular}{|c|c|c|c|c|}
\hline$E E T A / F C A$ & $\eta .0 ! 17$ & 0.0 .020 & $n, n 0,3 n$ & 0.0050 \\
\hline 0.001 & 0.00100 & 0.014200 & 0.0113001 & 0.00501 \\
\hline 0.002 & 0.001 Ba & ח. & v.0n3nu & 0.00501 \\
\hline 0.003 & (1.01100 & 0.00201 & $0.0 \pi 3 n y$ & 0.00501 \\
\hline 0,005 & 0.00101 & 0.00201 & $0.0030 \%$ & $0.01050 ?$ \\
\hline 0.007 & $0.010 \ln 1$ & $0.0 \cup ? n 1$ & ? & $0.005(14$ \\
\hline 0.010 & $(1.00101$ & ? & 0.00303 & 0.00505 \\
\hline 0,020 & $0.001 \cap 2$ & 0.00204 & $0.003 r .0$ & 0.00510 \\
\hline 0.030 & 0.00103 & 0.00206 & 0.00309 & 0.00516 \\
\hline 0.050 & 0.010105 & 0.00210 & 0.07316 & 0.00526 \\
\hline 0.070 & 0.001 . & $0, \cap 0>15$ & $10.003 ? 2$ & 0.00537 \\
\hline 0.100 & $0.0 n 111$ & 0.110222 & 0.00333 & 0.00555 \\
\hline 0.200. & $0.001>5$ & 0.00250 & 0,00375 & 0.00624 \\
\hline 0.300 & 0.00142 & 0.00285 & 0.01427 & 0.00711 \\
\hline 0.500 & 0.00104 & 0.00 .387 & 0.00580 & 0.00965 \\
\hline 0.700 & 0.40277 & 1). 010553 & 0.00827 & ก. $0113 / 4$ \\
\hline 1.000 & 0.00493 & 0.00991 & $n .0148 ?$ & 0.02452 \\
\hline 2.000 & 0.03011 & $0.115 \pi 48$ & 0.0534 & 0.13447 \\
\hline 3.000 & 0.10709 & 0.14519 & 0.26683 & 0.3779 .3 \\
\hline 5.000 & 0.43691 & $0.61>78$ & 0.71100 & n.92735 \\
\hline 7.000 & $0.752 n 1$ & 0.00365 & 0.99614 & 1.08914 \\
\hline 10.000 & 1.0415 & 1.1769 & 1.2570 & 1.3581 \\
\hline 20.000 & $1.5+550$ & $1.737 \mathrm{~A}$ & $1.931 \mathrm{~J}$ & 1.9522 \\
\hline 30.000 & 1.9502 & $2.1 \geqslant 17$ & 3.2345 & 2.3795 \\
\hline 50.000 & 2.5015 & 2.71 .90 & $2.85 ? 5$ & 3.0280 \\
\hline 70.000 & 2.9326 & 3.1805 & 3.3360 & 3.5429 \\
\hline 100.000 & $3.40 ? 8$ & 3.7566 & 3.9339 & 4.1781 \\
\hline 200.000 & 4.7655 & 5.1623 & 5.1044 & 5,7345 \\
\hline 300.000 & 5.7345 & $6.20 \% 5$ & 6.4974 & 6.8865 \\
\hline 500.000 & 7.2298 & 7.6211. & 8.1873 & 8.6756 \\
\hline EETA/F (A) & $0.50 r_{1} 0$ & U. 0000 & 0.70013 & 0.8000 \\
\hline 0.001 & 0.501 & 0.603 & $0.71 n$ & 0.832 \\
\hline 0,002 & $0.5 n 1$ & n.to3 & 0.710 & 0.832 \\
\hline 0.003 & $0.5 n !$ & 0.603 & 0.711 & 0.832 \\
\hline 0.005 & 0.572 & 0.604 & 0.711 & 0.833 \\
\hline 0.007. & $0.5 n 2$ & 11.604 & 0.71 .1 & 0.833 \\
\hline $0.010^{\circ}$ & $0.5 n 3$ & 0.005 & $0.71 \%$ & 0.834 \\
\hline 0.020 & $0.50 \mathrm{~B}$ & n.tog & 0.715 & 0.836 \\
\hline 0.030 & $0.5 \mathrm{Bn}$ & 0.610 & 0.717 & 0.838 \\
\hline 0.050 & 0,514 & 0.516 & 0.7 .21 & 0.843 \\
\hline 0.070 & 0.51 .0 & $n .+.2 n$ & $n .776$ & 0.847 \\
\hline 0.100 & $6,5 ? 7$ & 0.629 & 0.734 & 0.854 \\
\hline 0.200 & 4.551 & 0.658 & $0.76 ?$ & 0.880 \\
\hline 0.300 & 0.500 & 0.689 & 0.791 & 0.907 \\
\hline 0.500 & 0.665 & 0.761 & 0.258 & 0.971 \\
\hline 0.700 & 0.750 & 0.840 & 0.933 & $1 . n 41$ \\
\hline 1.000 & 0.580 & 0.963 & 1.051 & 1.158 \\
\hline 2,000 & 1,242 & 1. $32 ?$ & $1.41 \pi$ & 1.520 \\
\hline 3.000 & 1.510 & 1.400 & 1.698 & 1.820 \\
\hline 5.000 & 1,933 & 2.036 & 2.147 & 2.296 \\
\hline 7.000 & 2.265 & 2.379 & $? .509$ & 2.677 \\
\hline 10.000 & 2.673 & 2.803 & 2.955 & 3.146 \\
\hline 20.000 & 3.676 & 3.850 & $4.05 \mathrm{~A}$ & 4.315 \\
\hline 30.000 & 4,472 & 4.536 & 4.872 & 5.185 \\
\hline 50.000 & 5.582 & 5.841 & 0.146 & 0.528 \\
\hline 70.000 & 6.497 & 6.803 & 7.154 & 7.604 \\
\hline 100.000 & 7.038 & 7.989 & 2.401 & 8.920 \\
\hline 200.000 & 10.430 & 10.903 & 1.1. $4 \mathrm{KA}$ & 1.2.185 \\
\hline 300.000 & $12.5 n 6$ & 13.085 & $13.75 \%$ & 14.611 \\
\hline 500.000 & $15,7,33$ & 16.465 & $17.28 \mathrm{~s}$ & 18.357 \\
\hline
\end{tabular}

0.01171 0.00701 0.04702 0.00703 0.00705 0.00707

0.00715 0.00721 0.00737 0.00753 0.0077 0.10874 0.00994 0.01347 0.03405 0.27945 0.4014 .3 0.89983

1.15399

1.4267
2.0361

2.4786

3.1500

3.6841

4.5383

5,9557

7.1535

9.0113
0.0100

0.0100

.0100

. 1 1n

$0.01 n 1$

ก.1)1n?

0.0103

0.0105
$n .0107$

ก. ก111

0.1125
0.0142

0.0192

0.127

0.0481
0.2387

0.5508

0.9728

1.2246
1.499

2.132

2.595

3.297
3.842

3.842
4.521

6.278

7.451
9.303

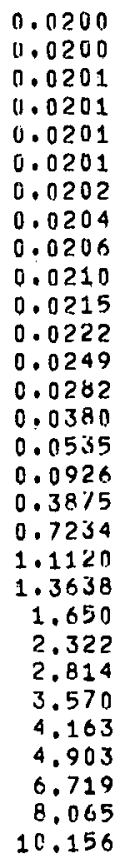

0,9000

0.999
0.999

1. 001

1. 1001

1.001

1.003

1.005

1.011

1.015
1.020

1.043

1.070
1.131

1.200

1.316

1.096
2.017

1.01
2.532

2.532
2.944

3.444

4.735
5.099

5.099
7.161

8.325

13.345

16.007
20.097 $\begin{array}{ll}1.08 & 1.1 .5 \\ 1.08 & 1.15\end{array}$

1.08

1.08
1.08
1.08

1.08

1.09
1.09

1.10

1.12
1.15

1.15

1.39

1.78
2.12

2.065

3.08
3.62

4.95

7.48

7.71
10.22

10.22
13.94
$0.03 n 0$ 0.0301

0.0301

0.0302

$0.03 \cap 2$

0.0316

0.0309

0.0322

0.0332
0.0372

0.0372

0.0565
0.0790

0.0790
0.1339

0.4898

0.817
1.194

1.446

1.741
2.440

2.440
2.955

3.745

4.369
5.139

5.139
7.047

$10.652 \quad 8.981$

0.0700

0.0701

0.0702

0.0703

0.0704

0.0707

0.0713

0.0720

0.0748

0.0748

0.0859

0.0965
0.1272

0.1272
0.1728

0.2735

0,7101

1.003
1.366

1.368
1.633

1.952

2.715
3.280

4.146

4.842

5.689
7.791

7,791
9,347

9.347
11.758

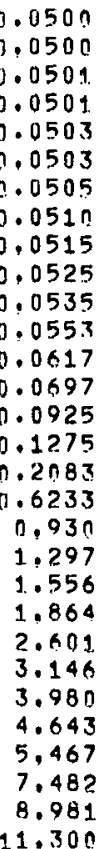

0.1000 0.100 ? 0.1002 0.1006 0.1010 0.1018 0.1027

0.1048

0.1048
0.1067

0.1099

0.1368

0.1771

0.2355
0.3570

0.7987

1.080

1.448
1.719

2.048

2,845

4,338

5,055

5,948

8,142
9,774 9,774
12,292

0.9950

9950
1.62
1.62 1.33
1.3

0.9910

0.9930 1,55
1,55 1.47
1.47 1.55
1.55

1.55

16.71
20.98
1.25

(.25

1.25

1.26

1.26

1.15

1.16

1.19

1.22

1.35

1.46
1.87

2.21

3.21

5.15

6.1

7.79
9.06

10.63

14.50
17.38

17.38
21.84
$1.33 \quad 1.48$

1.34

1.34

1.35

1.35

1.38

1.40

1.46

1.54

1.67
2.10

2.48

3,0

3.59
4.21

5.74

6.89
8.68

10.08

11.83

16.13
19.33

19.33
24.78

1.48
1.48

1.48

1.48
1.49

1.50

1,52

1,61

1.69

2.29

2.70

3.36

3.90
4.57

6.22

7.47

10.90

12.80
17.44

20.92
26.26

1.55
1.55

1.55

1.56
1.56
1.57

1.60

1.63
1.69

1.69
1.77

1.91

2.81

3.50

4.06
4.76

6.48

9.78

$\begin{array}{rr}9.77 & 10.16\end{array}$

$11.36 \quad 11.80$

$18.17 \quad 18.88$

$\begin{array}{lll}21.26 & 27.36 & 28.40\end{array}$

1.62
1.62
1.62

$\begin{array}{llll}9.63 & 1.74 & 1.83 & 2.00 \\ 1.63 & 1.74 & 1.83 & 2.00\end{array}$

$\begin{array}{llll}1.63 & 1.75 & 1.84 & 2.01\end{array}$

$\begin{array}{llll}1.64 & 1.75 & 1.84 & 2.01 \\ 1.64 & 1.76 & 1.85 & 2.02\end{array}$

$\begin{array}{llll}1.67 & 1.79 & 1.88 & 2.05\end{array}$

$\begin{array}{llll}1.70 & 1.82 & 1.91 & 2.09\end{array}$

$\begin{array}{llll}1.85 & 1.98 & 2.08 & 2.26\end{array}$

$\begin{array}{llll}1.99 & 2.12 & 2.23 & 2.43\end{array}$

$\begin{array}{llll}2.493 & 3.65 & 2.78 & 3.02 \\ 3.93 & 3.26 & 3.54\end{array}$

$\begin{array}{llll}3.64 & 3.87 & 4.05 & 4.38\end{array}$

$\begin{array}{llll}4.22 & 4.48 & 4.69 & 5.08\end{array}$

$\begin{array}{llll}4.94 & 5.24 & 5.49 & 5.94 \\ 6.73 & 7.14 & 7.47 & 8.08\end{array}$

$\begin{array}{llll}8 & 8.55 & 8.97 & 9.68\end{array}$

$\begin{array}{lll}10.75 & 11.25 & 12.17\end{array}$

$\begin{array}{lll}12.51 & 13.09 & 14.14 \\ 14.66 & 15.34 & 10.59\end{array}$

$\begin{array}{lll}14.66 & 15.34 & 16.59 \\ 19.97 & 20.92 & 22.60\end{array}$

$\begin{array}{lll}19.97 & 20.92 & 22.60 \\ 23.94 & 25.07 & 27.09\end{array}$

$\begin{array}{ll}25.07 & 27.09 \\ 31.46 & 34.03\end{array}$ 
VALUES OF ALPHA FOK IV $=9$ ANII NHE $=5$

\begin{tabular}{|c|c|c|c|c|}
\hline BETA, & & (1) & (1, ก)30 & 0 \\
\hline 0,001 & 0.00100 & 0.00200 & $0.0030 i$ & $n, n 0501$ \\
\hline & 0.00100 & 0.00200 & $0.0 \cap 3 \cap 5$ & 0.00501 \\
\hline & $0.001 n$ i & .00201 & 0.00301 & 150 \\
\hline & $0.4 n 101$ & ก. ००श01 & $0.0 \times 3 n 2$ & 0.0050 \\
\hline & $0 . \ln 1 n 1$ & 1201 & 10.07302 & .00504 \\
\hline 0.010 & $0,001 \cap 1$ & . ก४202 & $0.0 \cap 303$ & 0.00505 \\
\hline & 0.01102 & & 0,00306 & 0.00510 \\
\hline 0. & 0.00103 & $.00 ? 06$ & $0.0 \cap 3 \cap 9$ & 0.00516 \\
\hline 0 & 105 & 10 & 1). 0 & $n . n$ \\
\hline & 8 & 0 . & .0 & $n . n$ \\
\hline 0 & $0.1)(1111$ & 0.1 & $0.0 \times 3.33$ & $n .0$ \\
\hline 0.200 & 0.01125 & $0 . n(125 n$ & 0.00375 & 0.00624 \\
\hline 0.300 & 0.110143 & 0.1 & 0.00428 & 0.00712 \\
\hline 0.500 & 0.011107 & 0.1 & 0.01589 & 0.011979 \\
\hline 0.700 & $0.00 \% 93$ & $n . n$ & $11.0087 \%$ & 0.02456 \\
\hline 1.000 & 0.00507 & 0.01 .188 & $0 . n 1773$ & 0.02926 \\
\hline 2.000 & 0.1154 .32 & 0.11216 & (i.1594? & $0.740 \mathrm{~A}$ \\
\hline 3.000 & 0.26083 & 0.4 & 0.52313 & 0.65092 \\
\hline 5.000 & 0.82640 & 0.07279 & 1.05290 & 1.15399 \\
\hline & 1.153599 & 1.7694. & 1.35952 & 1.47251 \\
\hline 10. & 1.4973 & 1.0461 & 1.7357 & 1.8540 \\
\hline 0 & 2.3146 & 8 & & \\
\hline & 2.9440 & 3. & $3.34 P_{4}$ & \\
\hline una & .5 & 4.2 & 4.4 & 4.7655 \\
\hline & & 28 & & \\
\hline & $5.8 \% 94$ & 01 & & 620 \\
\hline & 19 & 30 & & \\
\hline - & 19.918 .5 & 11.80 .37 & 12.3682 & 13,1007 \\
\hline & $14.55 n 2$ & 15.7328 & it. 4652 & 17.4418 \\
\hline
\end{tabular}

$B E T A / F(A)$ D

0.001
0.002

0.002
0.003

0.005

0.007

0.010

0.020

0.030
0.050

0.050
0.070

0.070
0.100

0.100
0.200

0.200
0.300

0.500

0.700

1.000
2.000

2.000
3.000

5.000

7.000

10.000

20.000

30.000

70.000

100.000

200.000

300.000

500.000

\begin{tabular}{|c|c|c|c|}
\hline & & & \\
\hline 0.501 & 0.603 & 0.710 & $\begin{array}{r}0.832 \\
0.833\end{array}$ \\
\hline $0.5 n 1$ & 0.603 & 0.710 & 0.832 \\
\hline $0.5 n 2$ & $0.6 \% 4$ & 0.711 & $n .833$ \\
\hline$U, 5 \cap 2$ & 0.604 & 0.711 & 0.833 \\
\hline $0 . \sin 3$ & 0.605 & 0.712 & 0.934 \\
\hline 0.506 & 0.608 & 0.715 & 0.836 \\
\hline $0.5 \cap 8$ & 0.610 & 0.71 .7 & 0.838 \\
\hline U.514 & 0.516 & 0.721 & 0.843 \\
\hline 0.57 .4 & 0.620 & 0.726 & 0.847 \\
\hline $0.5 ? 7$ & 0.429 & 0.734 & 0.854 \\
\hline 0.557 & 0.658 & $0.7+2$ & 0.880 \\
\hline 0.590 & 0.689 & 1.791 & 0.907 \\
\hline 0.069 & 0.764 & 0.862 & 0.973 \\
\hline 0.763 & 0.853 & 0.946 & 2.053 \\
\hline 0.498 & 0.990 & 1.087 & 1.192 \\
\hline 1.383 & 1.465 & 1. .556 & 1.673 \\
\hline 1.755 & 1.848 & 1.950 & 2.090 \\
\hline 2.357 & 2.475 & 2.608 & 2.784 \\
\hline $2.8 \circ 0$ & 2.997 & 3.158 & 3.364 \\
\hline $3 . \operatorname{s!n} 1$ & 3.673 & 3.865 & 4.109 \\
\hline 5.185 & 5.429 & 5.712 & 6.078 \\
\hline $0.5 \geqslant 0$ & 6.8 .25 & 7.170 & 7.627 \\
\hline 0.601 & 9.0 का & 9.561 & 10,156 \\
\hline 0.401 & 10.980 & $11.5 \Delta 4$ & 12.261 \\
\hline 2.811 & 13,406 & 14.092 & $14: 962$ \\
\hline 8.676 & 10.761 & $20.7 \mathrm{Eg}$ & 22.050 \\
\hline S.OA7 & 24.706 & 26.017 & 27.635 \\
\hline $1,4 \times 5$ & 32.929 & 34.577 & 35.714 \\
\hline
\end{tabular}

0.00700

0.00701

ก. 0100

0.0100
$n .01100$

$0.00703 \quad 0.0110$

0.00707

0.110707

0.00721

0.00737

0.00753

0.00874

0.00996

0.01364
0.022032

0.114060

0.030736

0.73199

1.54308

1.5331

2.5250

4.5993

5.5939

7.2367

10.6424
13,6042

18.113 ?

$n \cdot \ln 101$

$n .0101$
0.010 ?

0.0103

$0.01 n 5$

n. 011.1

0.0125

0.0142
0.0195

ก. 0288

0.0572
0.3889

0.8130

1. 2894

1.6194
2.025
3.051

3.051

3.858

5.162

6.246
7.638

11.285

14.154
18.846

0.0200

0.03013
$0.03 n 1$

0.0507

0.0201

0. 0301

0.0500
0.0501

0.0201

0.0301
0.0302

0.0501

0.0503
0.3503

0.0202

$0.03 n 3$

0.0204
0.0246

0.0306

0.0319
0.0315

0.0210

0.0222

0.0249

0.0386

0.0332

0.0372

0.0423
0.0574
0.0834

$0.0506 \quad 0.0834$

$\begin{array}{ll}0.1091 & 0.1566 \\ 0.5641 & 0.6671\end{array}$

0.9613

0.6671
1.045

1.7758

1.514

$2.208 \quad 2.322$

$\begin{array}{ll}3.314 & 3.478 \\ 4.178 & 4.384\end{array}$

5.590

4.384
5.854

$6.764 \quad 7.093$

$12.216 \quad 12.795$

15.336
20.371

16.038
21.348

c. 3505

0.0511
0.0515

0.0525

0.0535
0.0553

0.0617

0.0698

0.0939

0.2401

0.7902

1.1 .48

2,002

2.475

3.699
4.659

6.230

9.210

13.574
17.045

0.9500

$0.9300 \quad 0.9500$

$\begin{array}{lll}16.038 & 22.660 & 23.576\end{array}$

.0700

0.1000

$0.2000 \quad 0.3000 \quad 0.4000$

$\begin{array}{lll}0.2002 & 0.3002 & 0.4003\end{array}$

0.10020 .20040 .30020 .4003

$\begin{array}{lllll}0.0703 & 0.1005 & 0.2009 & 0.3012 & 0.4013\end{array}$

$0.0704 \quad 0.1006 \quad 0.20110 .30160 .4018$

$0.0720 \quad 0.1027 \quad 0.2050 \quad 0.30640 .4051$

0.07340 .1048

$0.0748 \quad 0.1067$

0.07720 .1099

0.085

0.129

0.1814

0.1220

0.1370

0.2463

0.8684

1.219
1.707

2.093

0.3999
0.9494

1.795
1.795

1.795
2.196

2,708

4,1133

6.780

8,187

10.018

18.510

$\begin{array}{ll}0.499 & 1.08 \\ 0.999 & 1.08\end{array}$

$\begin{array}{ll}0.999 & 1.08 \\ 1.001 & 1.08\end{array}$

1.001

1.001
1.003

1.005

1.005

1.015

1.020

1,045

1.070

1.133
1.211

1.352

1.858

2.307

3.062

3.696

4.514
6.058

8.355

11.132

13.436
16.404

24.125

30.259
40.193

$1.08 \quad 1.15$

1.08

1.08

1.09
1.199

1.10
1.12

1.1 .2
1.15

1.21

1.29
1.43

1.95
$? .42$
3.20

2.42
3.20

3.87

4.72
. .96

4.96
1.74
11.62

11.62
14.03

$25.19 \quad 26.81$

$31.59 \quad 32.87$

$0.9700 \quad 0.9800$

0.9900

$\begin{array}{ll}1.25 & 1.33 \\ 1.25 & 1.33\end{array}$

1.25

$1.25 \quad 1.33$

$\begin{array}{lll}1.15 & 1.25 & \$ .33 \\ 1.5 & 1.26 & 1.34\end{array}$

$1.1 .5 \quad 1.26 \quad 1.36$

$1.16 \quad 1.26 \quad 1.36$

17

1.271 .35

1.22

1.28

1.50

2.04
2.52

3.34
4.03

4.92

7.25

.099 .65

$1.30 \quad 1.38$

$1.32 \quad 1.40$

$1.46 \quad 1.55$

$1.62 \quad 1.71$

$\begin{array}{ll}2.18 & 2.29 \\ 2.69 & 2.83\end{array}$

$3.55 \quad 3.73$

$4,28 \quad 4.49$

5.22
7.70

$\begin{array}{ll}9.65 & 11.11 \\ 2.84 & 13.45\end{array}$

$15.49 \quad 16.22$

$27.85 \quad 29.16$

$\begin{array}{ll}34.88 & 36.53 \\ 46.36 & 43.55\end{array}$
0.9930

0,9950

1.55
1.55
1.55
1.55
1.55
1.55
1.55
1.55
1.56
1.56
1.57
1.60
1.63
1.70
1.79
1.96
2.60
3.20
4.22
5.08
6.18
9.09
11.41
15.16
18.30
22.29
32.81
41.11
54.66

1.62
1.62
1.62
1.62
1.62
1.62
1.63
1.63
1.63
1.64
1.64
1.67
1.79
1.77
1.87
2.06
2.71
3.33
4.38
5.28
6.43
9.45
11.85
15.73
19.00
23.18
34.09
42.70
56.79

1.62
1.62
1.62
1.62
1.62
1.62
1.63
1.63
1.63
1.64
2.64
1.67
1.79
1.77
1.87
2.06
2.71
3.33
4.38
5.28
6.43
9.45
11.85
15.73
19.00
23.18
34.09
42.70
56.79

.

$\begin{array}{lll}0.9970 & 0.9980 & 0.9990\end{array}$

$\begin{array}{lll}1.73 & 1.83 & 1.99\end{array}$

$\begin{array}{lll}1.74 & 1.83 & 1.99\end{array}$

$\begin{array}{lll}1.74 & 1.83 & 1.99 \\ 1.74 & 1.83 & 2.00\end{array}$

$1.74 \quad 1.83 \quad 2.00$

1.74

1.75

1.76

1.82

1.89

1.99
2.17

2.88

3.54

4.65

5.59
6.81

10.00

12.54

16.68
20.13

24.52

36.10
45.26

60.09

$1.84 \quad 2.01$

$\begin{array}{ll}1.88 & 2.02 \\ 1.01 & 2.05\end{array}$

$\begin{array}{ll}1.99 & 2.17 \\ 2.09 & 2.28\end{array}$

$2.28 \quad 2.48$

$\begin{array}{ll}3.02 & 3.28 \\ 3.71 & 4.02\end{array}$

5.86
7.13

$13.1 .3 \quad 14.18$

$17.44 \quad 18.85$

$21.04 \quad 22.75$

$\begin{array}{ll}25.68 & 27.73 \\ 37.75 & 40.80\end{array}$

$\begin{array}{ll}47.33 & 51.12 \\ 62.93 & 67.93\end{array}$
$1.85 \quad 2.01$

$1.91 \quad 2.09$

$\begin{array}{ll}3.71 & 4.02 \\ 4.87 & 5.28\end{array}$

$10.48 \quad 11.33$ 


\begin{tabular}{|c|c|c|c|c|}
\hline VALUES OF & AL_PHA & & & \\
\hline$B E T A / F(A)$ & noouso & U. 0 nृ? & $n . n 10,3 !$ & 0.0050 \\
\hline $\begin{array}{l}0.001 \\
0.002\end{array}$ & $\begin{array}{l}0.001001 \\
0.00100\end{array}$ & $\begin{array}{l}0.010200 \\
0.00200\end{array}$ & $\begin{array}{l}x .00304 \\
0.01300\end{array}$ & $\begin{array}{l}0.011501 \\
0.011501\end{array}$ \\
\hline 0.003 & 0.00100 & 0.00201 & 0.00301 & 0.011501 \\
\hline 0.005 & $0.001 \cap 1$ & 0.00201. & $0.0130 ?$ & 0.00502 \\
\hline 0.007 & $0.001 n t$ & 0.00201 & [3. Bas? & 7.0115114 \\
\hline 0.010 & 0.00101 & $0.0020 ?$ & fi.consns & 0.101505 \\
\hline 0.020 & $0.001 n 2$ & 0.011204 & 0.011306 & $n .010510$ \\
\hline 0.030 & 0.00103 & 0.01206 & - $0.0 \cap 309$ & 0.010515 \\
\hline 0.050 & 0.00105 & 0.00210 & 0.00315 & 0.00525 \\
\hline 0.070 & 0.031 .07 & 0.00214 & $\pi, \cap \cap 3 ? 1$ & 0.00535 \\
\hline 0.100 & 0.00110 & 0.00220 & $11.0133 \pi$ & $n .011550$ \\
\hline 0.200 & 0.0 ה 1.20 & $0.0(1240$ & $1,0 n .3 \times 1$ & 0.011599 \\
\hline 0,300 & 0.001 .30 & 0.010260 & 11.00396 & 0.00649 \\
\hline 0.500 & 0.00150 & $0,00.300$ & 0.00449 & 0.00748 \\
\hline 0.700 & 0.0 .1770 & 0.00340 & 10.00509 & 0.00847 \\
\hline 1.000. & $0.0 n 200$ & 0.00399 & 1. On50K & $n .0 n 994$ \\
\hline $2.000^{\circ}$ & 0.00209 & 0.00598 & 4.008890 & 0.01485 \\
\hline 3.000 & 0.00399 & 0.00795 & if. $n 1,100$ & $n . n 3.970$ \\
\hline 5.000 & 0.00597 & 0.01188 & {$[.01 .77 .3$} & 0.02926 \\
\hline 7.000 & 0.00795 & 0.01579 & 0.02351 & 0.03864 \\
\hline 10.000 & 0.0109 & 0.0216 & 0.0320 & 0.0524 \\
\hline 20.000 & $0.02 n 6$ & 0.0404 & 0.0594 & 0.0955 \\
\hline 30.000 & $0.03 n 1$ & 0.0585 & 0.0853 & 0.1349 \\
\hline 50.000 & 0.0486 & $0.19 \geqslant 27$ & 0.93311 & $1,2.2040$ \\
\hline 70,000 & 0.0664 & 0.1246 & $0.17 \times 1$ & 0.2630 \\
\hline 00,000 & 0.0918 & 0.1582 & 0.2332 & 0.3364 \\
\hline 000,000 & 0.1675 & 0.2373 & 0.3770 & 0,5031 \\
\hline & 0.2314 & 0.3760 & 0.4752 & $n .6032$ \\
\hline 00,000 & 0.3341 & $0.501 ?$ & 0.6023 & 0.7234 \\
\hline$B E T A / F(A)$ & 0.5000 & 0.6000 & 0.7000 & 0.8000 \\
\hline 0.001 & 0.500 & 0.501 & $0.7 n 7$ & 0.824 \\
\hline 0,002 & $0.5 n 1$ & 0.502 & 0.707 & 0.824 \\
\hline 0,003 & $0.5 \cap 1$ & 0.502 & 0.707 & $n .824$ \\
\hline 0.005 & 4.501 & 0.502 & $0.7 \cap B$ & 0.824 \\
\hline 0.007 & U.Sก? & 0.603 & $11.7 n 8$ & $0.8 \% 5$ \\
\hline 0.010 & $0.5 \cap 3$ & 0.604 & $0.7 n 9$ & 0.825 \\
\hline 0,020 & 0.505 & 0.606 & 0.711 & 0.827 \\
\hline 0.030 & $0.5 n 8$ & 0.609 & 0.713 & 0.830 \\
\hline 0.050 & 0.513 & 0.614 & 0.718 & 0.834 \\
\hline 0.070 & 0.517 & $0.61 \%$ & 0.723 & 0.838 \\
\hline 0.100 & 0.524 & $0.6 \geq 5$ & 0.726 & 0.844 \\
\hline 0.200 & 0,546 & $f .446$ & 11.747 & 0.861 \\
\hline 0.300 & $0.5 \times 6$ & 0.6655 & 0.765 & 0.877 \\
\hline 0.500 & 0.601 & 0.699 & 0.790 & 0.905 \\
\hline 0.700 & 0.632 & 0.726 & ค. ค2? & $0.9 ? 8$ \\
\hline 1.000 & 0.071 & 0.763 & 0.855 & 0.959 \\
\hline 2.000 & $0.7 \times 3$ & 0.347 & 0.934 & 1.034 \\
\hline 3,000 & $0.8 ? 4$ & 0.905 & $0.98 \mathrm{t}$ & 1.083 \\
\hline 5,000 & $0.9 n 5$ & 0.980 & 1.06 .1 & 1.154 \\
\hline 000 & 0.959 & 1.034 & 1.11.0 & 1.205 \\
\hline 10,000 & 1.09 .7 & 1.089 & 1.167 & 1. 261 \\
\hline 20.000 & 1.131 & 1.202 & $1.27 \%$ & $1.3 \% 3$ \\
\hline 30.000 & $1,2 n 0$ & 1.270 & 1.347 & $1.44 ?$ \\
\hline 50.000 & 1.286 & 1.356 & 1.434 & 1.534 \\
\hline & 1.343 & 2.215 & 1.495 & 1.546 \\
\hline 00,000 & $1.4 n 6$ & 1.478 & 1.560 & 1.663 \\
\hline$: 00,000$ & 1.5 .32 & 1.008 & $1.69 ?$ & 1.801. \\
\hline 300.000 & $1.6 n 6$ & 1.664 & 1.774 & 1.864 \\
\hline 000.000 & 1.705 & $1.7 \times 7$ & .1 .879 & 1.994 \\
\hline
\end{tabular}

\begin{tabular}{|c|c|c|}
\hline$n 7 n$ & $.11 n 0$ & 0.0200 \\
\hline OC70n & 0.0100 & .0260 \\
\hline 00701 & $n .0100$ & 0.0201 \\
\hline 0.00702 & n.0100 & 0.0201 \\
\hline 0.00703 & . n1no & 0.0201 \\
\hline 0.00705 & $0 . n 1 \cap 1$ & 0.0201 \\
\hline 0.04707 &.$n_{1} n_{1}$ & 0.0202 \\
\hline 0.110714 & ก. ח102 & 0.0204 \\
\hline 0.110721 & 0.01 & 0.0206 \\
\hline 0.110735 & 0.01 & 0.0210 \\
\hline 0.110748 & $n .0107$ & 0.021 .4 \\
\hline 0.010769 & $n .0110$ & 0.0220 \\
\hline 0.110839 & $0.01 \geq 0$ & 0.0239 \\
\hline 0.40908 & 0.0130 & 0.0258 \\
\hline 0.01046 & 0.0149 & 0.0297 \\
\hline 0.111184 & $0.01 \times 9$ & 0.0335 \\
\hline 0.01391 & $\cap . \cap 1 O B$ & 0.0392 \\
\hline $0.112 n>1$ & $n . n 2.94$ & $0.05 \% 7$ \\
\hline 0.02749 & 1.0388 & 0.0754 \\
\hline $0.64 \pi 60$ & 0.0572 & 0.1091 \\
\hline & 47 & 404 \\
\hline 0.0720 & 0.100 & 0.143 \\
\hline & 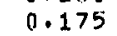 & 0.300 \\
\hline 0.1795 & 0.239 & 0.387 \\
\hline 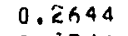 & 0.340 & 0.511 \\
\hline 0.3336 & 0.418 & 0.593 \\
\hline 0.4161 & $0.5 n 5$ & 0.678 \\
\hline $0.58 \% n$ & 0.675 & 0.829 \\
\hline 0.6843 & 0.745 & 1). .909 \\
\hline & 0.871 & 1.00 \\
\hline
\end{tabular}

3nn $\quad 0.050 n$ 0.0301

$0.0301 \quad 0.0509 \quad 0.0709$

$0.0503 \quad 0.0703$

$0.03 n 2 \quad 0.0503 \quad 0.0704$

0.0303

0.0505

$0.03 n 9$

0.0515

0.0707

0.031 .0 .05230 .0726

$0.0320 \quad 0.05330 .0745$

0.0329

0.0358

0.0547

0.0765

$11.064 h \quad 0.0892$
0.0732

0.0444 O.073?

0.0892
0.1014

0.0582

0.0849

0.0821

0.1135

0.1308
0.1842

0.157

0.1363

0.174

0.231

0.198

0.296

0.319

0.394

0.365

$\begin{array}{ll}0.490 & 0.525 \\ 0.622 & 0.622\end{array}$

0.6140 .73

0.693

0.809

$0.771 \quad 0.881$

$\begin{array}{ll}0.911 & 1.01 \mathrm{j} \\ 0.986 & 1.082\end{array}$

0.453

0.615
0.706

0.815

0.882
0.949

1.074

1.144
1.232

0,1000

4.1 no

0.100 ?

0.1005

0.1 nos

0.1018

0.1127

$0.1 n 62$

0.11889

0.1176

0.1430

0.1589

0.2501

0.308

0.400
0.471

0.471
0.551

0.706

0,792

0,893

$0.95 \mathrm{~K}$

1.020

1,943
1,213

1.21 .3
1.301

0.20020 .30020 .4003

$0.2004 \quad 0.3002 \quad 0.4003$

$0.2004 \quad 0.3007 \quad 0.4008$

0.20110 .30160 .4013

$0.2016 \quad 0.30210 .4023$

$0.20330 .3040 \quad 0.4046$

0.20470 .30640 .4070

$0.2078 \quad 0.3102 \quad 0.4118$

0.21120 .31450 .4166

0.2370 .32020 .4232

$0.2357 \quad 0.33980 .0447$

$0.2726 \quad 0.35790 .4642$

0.27260 .39130 .5002

0.2930 .42130 .5317

0.33310 .46190 .5727

$\begin{array}{rrr}0.4285 & 0.5632 & 0.6710 \\ 0.500 & 0.634 & 0.737\end{array}$

$\begin{array}{lll}0.601 & 0.728 & 0.824\end{array}$

$\begin{array}{lll}0.671 & 0.791 & 0.882\end{array}$

$\begin{array}{lll}0.743 & 0.857 & 0.943\end{array}$

$\begin{array}{lll}0.879 & 0.980 & 1.061 \\ 0.953 & 1.051 & 1.129\end{array}$

$\begin{array}{lll}1.043 & 1.139 & 1.215\end{array}$

$\begin{array}{lll}1.1112 & 1.196 \quad 1.272\end{array}$

$\begin{array}{lll}1.164 & 1.257 & 1.333\end{array}$

$\begin{array}{lll}1.284 & 1.377 & 1.457 \\ 1.354 & 1.450 & 1.530\end{array}$

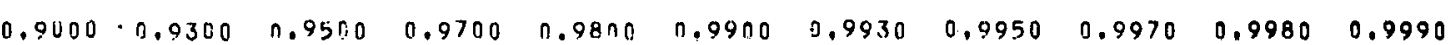

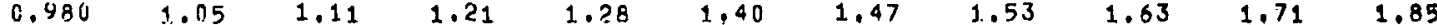

$0.980 \quad 1.05 \quad 1.11 \quad 1.21 \quad 1.28$

$0.980 \quad 1.05 \quad 1.1 .1 \quad 1.21 \quad 1.28$

$0.982 \quad 1.05 \quad 1.12 \quad 1.21 \quad 1.28$

$0.982 \quad 1.05 \quad 1.12 \quad 1.21 \quad 1,28$

$0.984 \quad 1.05$. 1.1.2 $1.21 \quad 1.28$

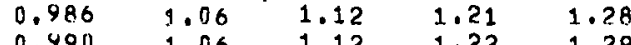

$0,994 \quad 1.06 \quad 1.12 \quad 1.22 \quad 1 . ? 9$

$\begin{array}{lllll}0.498 & 1.07 & 1.3 .3 & 1.22 & 1.30\end{array}$

$\begin{array}{lllll}1.015 & 1.08 & 1.35 & 1.24 & 1.31\end{array}$

$\begin{array}{lllll}1.028 & 1.10 & 1.16 & 1.25 & 1.32\end{array}$

$1.055 \quad 1.12 \quad 1.18 \quad 1.28$

$\begin{array}{lllll}1.076 & 1.14 & 1.21 & 1.30 & 1.35\end{array}$

$\begin{array}{lllll}1.076 & 1.14 & 1.21 & 1.30 & 1.37 \\ 1.104 & 1.17 & 1.23 & 1.33 & 1.40\end{array}$

$\begin{array}{lllll}1.175 & 9.24 & 1.30 & 1.40 & 1.47\end{array}$

$\begin{array}{lllll}1.225 & 1.29 & 1.36 & 1.45 & 1.53\end{array}$

$\begin{array}{lllll}1.295 & 1.36 & 1.43 & 1.52 & 1.60 \\ 1.347 & 1.42 & 1.48 & 1.58 & 1.66\end{array}$

$\begin{array}{lllll}1.402 & 1.42 & 1.48 & 1.58 & 1.64\end{array}$

$\begin{array}{lllll}1.520 & 1.59 & 1.66 & 1.77 & 1.85\end{array}$

$\begin{array}{lllll}1.593 & 1.77 & 1.74 & 1.85 & 1.94 \\ 1.688 & 1.77 & 1.84 & 1.95 & 2.04\end{array}$

$\begin{array}{lllll}1.755 & 1.93 & 1.91 & 2.02 & 2.1 .2 \\ 1.625 & 1.91 & 1.99 & 2.10 & 2 . ? 0\end{array}$

$\begin{array}{llll}1.925 & 9.91 & 1.99 & 2.10 \\ 1.971 & 9.06 & 2.14 & ? .27\end{array}$

$2.181 \quad 2.28$

$\begin{array}{lll}2.24 & 2.37 & 2.46 \\ 2.36 & 2.51 & 2.62\end{array}$

1.40
1.40
1.40
1.40
1.40
1.41
1.41
1.41
1.42
1.42
1.43
1.45
1.47
1.50
1.53
1.60
1.66
1.74
1.80
1.86
2.190
2.09
2.21
2.29
2.38
2.56
2.67
2.82

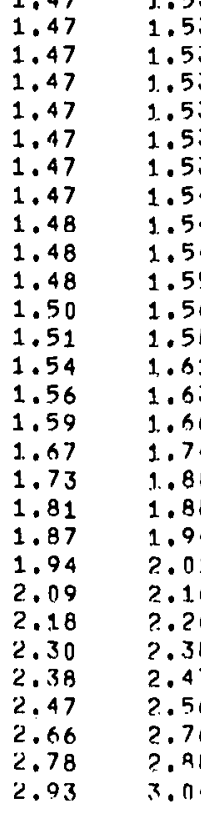

1.63
1.63

1.63

1.63

1.63

1.63

1.63

1.63

1.64

1.64

1.66
1.58

1.71

1.73

1.76

1.84
1.91

1.71

1.71

1.71

1.71

1.71

1.71
1.72

1.72

1.74

1.76

1.81

1.85

1.93
1.99

2.06

2.09

2.13

2.15
2.23

2.3

2.3

2.49
2.63

2.63
2.72

2.83

3.04
3.17

1.85
1.85

1.85
1.85

1.85

1.85
1.85

1.85
1.85

1.86
1.86

1.86
1.87

1.88

1.90
1.93

1.96

1.99

2.15

2.25
2,32

2.40

2.57

2.83

2,93
3,04

3.27
3.41

3.60 
VALUES IF ALPHA FOH $N=20$ ANI) $N P=2$

\begin{tabular}{|c|c|c|c|c|}
\hline BETA & $n, 0$ & $0.0 \cap 2 \pi$ & 0.0030 & 0,0050 \\
\hline 0.001 & $0.001 n 1)$ & $0.0020 !$ & D. & n.00501. \\
\hline 0.002 & 0.00100 & 0.00200 & 0.00300 & 0.00501 \\
\hline & & & & \\
\hline 0.005 & $0.001 \cap 1$ & 0.00201 & $0.003 \cap 2$ & 0.00502 \\
\hline 0.007 & $0.001 \cap 1$ & $0 . n \cup ? .1 .1$ & $0 . n \cap 3 \cap 2$ & 0.00504 \\
\hline 0.010 & 0.00101 & $0.0020 ?$ & $0 . \cap \cap 3 \cap 3$ & 0.00505 \\
\hline 0.020 & $0.001 . \cap 2$ & 0.00204 & $0.003 n t$ & 0.00510 \\
\hline 0.030 & $0.001 n 3$ & 0.00205 & $0.0 \sin \theta$ & $n .00516$ \\
\hline 0.050 & 0.00105 & $0.0 \cup 210$ & 0.00316 & 0.00526 \\
\hline 0.070 & $0.001 n 7$ & $0 . \cap \cup \geqslant 1.5$ & $0.0 \cap 322$ & 0.00537 \\
\hline 0.1 .00 & 0.00111 & D. मט?2? & 0.00333 & $n .00554$ \\
\hline 0.200 & 0.00124 & $0.016 ? 48$ & 0.011372 & 0.00619 \\
\hline 0.300 & 0.001 .39 & 0.00278 & 0.01141 .7 & 0.00694 \\
\hline 0.500 & 0.00175 & $0.01035 n$ & 0.00574 & 0.00872 \\
\hline 0.700 & 0.00219 & 0.00437 & 0.00655 & 0.01088 \\
\hline 1.000 & 0.00209 & 0.00598 & $0.0 \cap B 94$ & $n .01485$ \\
\hline 2.000 & 0.00606 & 0.0236 .3 & $1,0 ? 0 \times \%$ & $n .13399$ \\
\hline 3.000 & 0.01295 & 0.02539 & 0.113762 & $n .06137$ \\
\hline 5.000 & $0.03 \cup 11$ & 0.05848 & 0,09534 & 0.13487 \\
\hline 7.000 & 0.05305 & 0.10250 & 0.14631 & 0.22260 \\
\hline 0.000 & 0.1000 & 0.1821 & 0.2501 & 0.3579 \\
\hline 0.000 & $0.29 \times 4$ & 0.4576 & 0.5594 & 0.6843 \\
\hline 30.000 & $0.46 ? 1$ & 0.6547 & $0.747 ?$ & 0.8560 \\
\hline 50.000 & 9.7272 & 0.8731. & $(1.951 .3$ & 1.0453 \\
\hline 70.000 & 0.8674 & 0.9095 & 1.0720 & 1.1616 \\
\hline 00.000 & 1.0014 & 1.1254 & 1.1950 & 1.2856 \\
\hline 00.000 & 1.2436 & 1.3657 & 1.4382 & 1.5316 \\
\hline 00.000 & $1.38<7$ & 1.5107 & $1.567 n$ & 1.6861 \\
\hline 00.000 & $1.5 \% 97$ & $1.7115 ?$ & 1.7872 & 1.8940 \\
\hline$B E T A / F(A)$ & $0.5 \mathrm{un!}$ & 0.0000 & 0.7000 & 0.8000 \\
\hline 0.001 & $0.5 \cap 0$ & 0.50 .1 & 0.707 & 0.824 \\
\hline 0.002 & $0,5 \cap 1$ & 0.602 & $0.7 \cap 7$ & 0,824 \\
\hline 0.003 & $0.5 \cap 1$ & 0.602 & $0.7 n 7$ & 0.824 \\
\hline 0.005 & $0.5 \cap 1$ & $0.50 ?$ & $0.7 \mathrm{nB}$ & 0.824 \\
\hline 0.007 & 0,502 & $0.6 \cup 3$ & 0.706 & 0.825 \\
\hline 0.010 & $11.5 \times 3$ & 0.604 & $0.7 \cap 9$ & 0.825 \\
\hline 0.020 & 0.505 & 0.606 & 11.711 & 0.828 \\
\hline 0,030 & $0.5 n 8$ & 0.600 & 1).714 & 0.830 \\
\hline 0,050 & 0.593 & 0.614 & 0.719 & 0.834 \\
\hline 0.070 & 0.318 & 0.019 & 0.723 & 0.839 \\
\hline 0.100 & 0,527 & 0.127 & $0.73 n$ & 0.845 \\
\hline 0.200 & 0.555 & 0.654 & 0.755 & 0.867 \\
\hline 0.300 & $0.5 \times 2$ & 0,080 & 0.780 & 0.889 \\
\hline 0,500 & 0.639 & 0.733 & $0.82 \mathrm{~B}$ & 0.934 \\
\hline 0.700 & $0.69 ?$ & 0.783 & 0.873 & 0.977 \\
\hline 1.000 & $0.7 \times 3$ & 0.847 & 0.934 & 1.034 \\
\hline 2,000 & 0.934 & 1.009 & $1,0 F \%$ & 1.181 \\
\hline 3.000 & 1.647 & 1.1 .18 & $1.19 \mathrm{~A}$ & 1. .289 \\
\hline 5.000 & $1.2 n 0$ & 1.270 & 1.347 & 1.442 \\
\hline 7,000 & $1,3 \cap 5$ & 1.377 & 1.455 & 1. .555 \\
\hline 10,000 & 1.423 & 1.495 & 1.577 & 1.682 \\
\hline 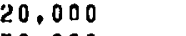 & 1.671 & 1.751 & 1.843 & 1.956 \\
\hline 30,000 & $1,6,31$ & 1.918 & 2.013 & 2.135 \\
\hline 50.000 & 2,1148 & 2.143 & 2.2511 & 2,383 \\
\hline 70.000 & $2.2 \cap 4$ & 2.303 & 2.418 & 2.559 \\
\hline 00.000 & 2.379 & 2,486 & 2.608 & 2.701 \\
\hline 00.000 & $2.7 \times 1$ & 2.879 & 3.017 & 3.192 \\
\hline 00.000 & 3.005 & 3.139 & 3.287 & 3.474 \\
\hline 0.000 & 3.315 & 3.490 & 3.654 & 3.865 \\
\hline
\end{tabular}

$0.007 n$ 0.00701
0.00702 0.00703 0.00705 0.00707 0.00721 0.00736 0.00751 0.00866 0.00970 0.01219 $0.02 n 71$ 0.04704 0.08390 0.17945 0.28686
0.4391 0.4390
0.7606 0.9218 1.1044 1.2207 1.5946 1.7529
1.9675
$0.03 n 0$ 0.0100 $n, 0100$ $n .0100$
0.0101 $n . n 101$ $n .0102$
$n .0103$ 0.0105 ก. 0107 0.0111
0.0124 0.0138 0.0174 0.0216
0.0294 0.0294
$n .0660$ 0.1161 0.2397
0.3655 0.3655
0.529 0.836 0.988 1.167 1.284 $1.4 \cap 8$ 1.665
1.827
2.048 1.975
2.212 0.0200 0.0201 0.0201 0.0201 0.0202
0.0204 0.0206 0.0210 0.0215 0.0247 0.0276 0.0345
0.0428 0.0577 0.1251
0.2097 0.2097 0.3875 0.700 0.973 1.114
1.289 1.406
1.535 1.804
0.0500 0.0500 0.0501 0.0501 0.0503 0.0505 0.0510 0.0515 0.0525 0.0535 0.0552 0.0613 0.0682 0.0844

0.1033

0.1363

0.2692 $\begin{array}{ll}0.287 & 0.406 \\ 0.490 & 0.622\end{array}$

0.640

0.792 1.047 1.362 1. 482 1.614 1.892
2.070
2.315

0.622
0.763
0.899

1.141 1.278
1.457 1.579
1.717 1.717 2.1 .93 2.452

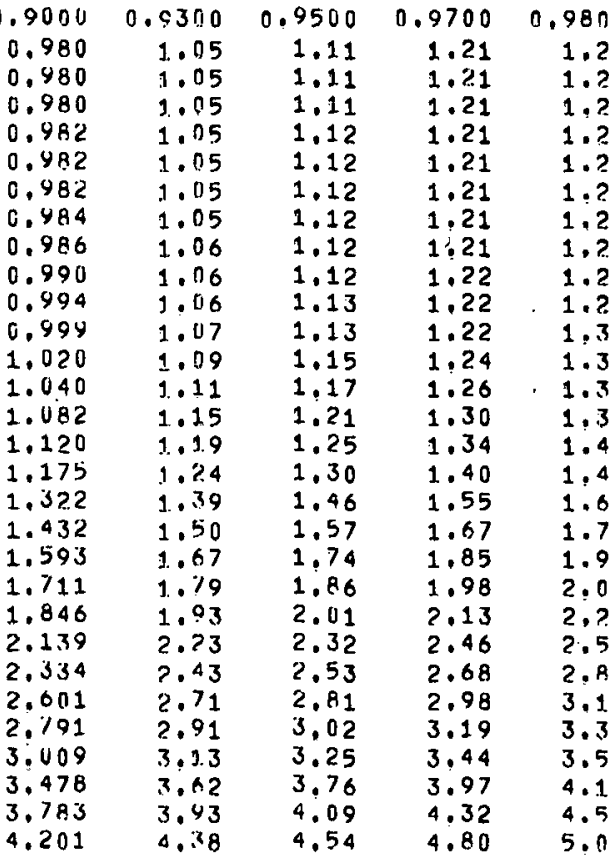

$800 \quad 0.9900$

0.0700 0.070 0,0709 0,0703 0,0703 0.0707 0.0713 $0.072 \pi$ 0.0734 0.0779 0.0853 0.0946 0.1164 0.1415 0.1842
0.3450 0.495

0.706

0.838 0.967 1.204 1.522 1,648 1.789 2.090 2.280
2.547
0.1000 0.1001 .1002 1005 0.1006 $0.1 \cap 18$ 0.1027 0.1046 0.1098 0.1210 0.1337 0.1628 0.1957 0.2501
0.4375

0.592
0.792 0.915 1.038
1.270 1.410 1.595
1.724 1.869 2,181 2.379
2.654

0.9950 $\begin{array}{ll}1.40 & 1.47 \\ 1.40 & 1.47\end{array}$ 1.40 1.40 1.40 1.40 1.41
1.41
1.41 1,42 1.42 1,44 1,46
1,50 1,54 1.60 1.67 1,90 2.0 2.23
2.40
2.77
3.01 2.77
3.01 3.01 3.59 3.87 4.45
4.84 4.84
5.38
1.47

1.47

1.47

1.47

1.48
1.48
1.49 1.49 1.51
1.53 1.61
1.67 1.84 1.98
2.18 2.3 2.50
2.88
3.13 3,48
3,73 3,73
4,01 4,01
4,62 5.02
5.58 1.68 1.74 1.92
2.05 2.05
2.26 2,41 .59 2.98 3.24 3.24
3.60
3.86
4.16
$0.2000 \quad 0.3000 \quad 0.4000$ $\begin{array}{lll}0.2002 & 0.3002 & 0.4003\end{array}$ $0.2004 \quad 0.3002 \quad 0.4003$ $0.2004 \quad 0.3007 \quad 0.4008$ $0.2009 \quad 0.3012 \quad 0.4013$ $0.20110 .3016 \quad 0.4018$ $0.2016 \quad 0.3021 \quad 0.4023$ $0.2033 \quad 0.3040 \quad 0.4051$ $\begin{array}{llll}0.2050 & 0.3064 & 0.4075\end{array}$ $0.21190 .3155 \quad 0.4123$ 0.21710 .32210 .4251 $0.2367 \quad 0.3469 \quad 0.4528$ $\begin{array}{lll}0.2578 & 0.3732 & 0.4812\end{array}$ 0.30450 .42850 .5393 $\begin{array}{lll}0.3536 & 0.4840 & 0.5947\end{array}$ $\begin{array}{lll}0.4285 & 0.5632 & 0.6710 \\ 0.6385 & 0.7625 & 0.8550\end{array}$ $\begin{array}{rrr}0.780 & 0.890 & 0.975\end{array}$ $0.953 \quad 1.051 \quad 1.129$ $\begin{array}{lll}1.064 & 1.158 & 1.234\end{array}$ $1.181 \quad 1.274 \quad 1.350$ $\begin{array}{lll}1.413 & 1.511 & 1.593 \\ 1.558 & 1.661 & 1.749\end{array}$ $1.753 \quad 1.865 \quad 1.960$ $\begin{array}{lll}1.892 & 2.009 & 2.109\end{array}$ $2.048 \quad 2.173 \quad 2.280$ $\begin{array}{lll}2.379 & 2.521 & 2.643 \\ 2.597 & 2.749 & 2.879\end{array}$ $0.9970 \quad 0.9980 \quad 0.9990$ $\begin{array}{llll}1.53 & 1.63 & 1.71 & 1.85\end{array}$ $\begin{array}{llll}1.53 & 1.63 & 1.71 & 1.85\end{array}$ $\begin{array}{llll}1.53 & 1.63 & 1.71 & 1.85\end{array}$ $1.53 \quad 1.63 \quad 1.71 \quad 1.85$ $1.53 .1 .63 \quad 1.71 \quad 1.85$ $\begin{array}{llll}1.53 & 1.63 & 1.71 & 1.85 \\ 1.54 & 1.63 & 1.71 & 1.85\end{array}$ $\begin{array}{llll}1.54 & 1.63 & 1.71 & 1.86\end{array}$ $\begin{array}{llll}1.55 & 1.65 & 1.73 & 1.86\end{array}$ $\begin{array}{llll}1.57 & 1.67 & 1.75 & 1.89\end{array}$ $\begin{array}{llll}1.59 & 1.69 & 1.77 & 1.91 \\ 1.63 & 1.74 & 1.82 & 1.96\end{array}$ 4.16
4.79 5.20
5.77 
VALUES OF ALFHA FOR IN $=1 \|$ ANI NOF $=3$

\begin{tabular}{|c|c|c|c|c|}
\hline$B E T A / F(A$ & 11.0010 & $0.00>n$ & 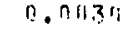 & 0.0050 \\
\hline 0.001 & 0.00 in 0 & 0.56200 & 0.00306 & 0.04501 \\
\hline 0.002 & d. bos nu & $0.0 \cup 200$ & $0.003 \cap 0$ & $\pi .00501$ \\
\hline 0.003 & $0,0(13,00=$ & $0.00 ? 0.1$ & $0.0 \cap 301$ & 0.00501 \\
\hline 0.005 & 0.001 .01 & 0.00201 & $0.003 n ?$ & $0.0050 ?$ \\
\hline 0.007 & $0.0(1) n 1$ & 0.601120 .1$. & ? & 0.001514 \\
\hline 0.010 & $0.0 \cap 2 \cap 1$ & ח. ח. & $1.003 n 3$ & 0.00505 \\
\hline 0.020 & 0.00102 & $0.0(1) 20.4$ & 0.0300 & 0.00510 \\
\hline .030 & 0. ves.ns & $0.0020 \%$ & 0.00300 & $0 . \cap 2516$ \\
\hline 0.050 & d) U U 1, 5 & 0.00210 & $0.0031 \%$ & 0.011526 \\
\hline 0.070 & 0.0njes & 0.00215 & $0.0732 \%$ & 0.00537 \\
\hline 0.100 & 0.00111 & $0.00 ? 2 ?$ & 0.110333 & $n .00555$ \\
\hline 0.200 & 0.010125 & 0.00749 & 0.111374 & n. 00623 \\
\hline 0.300 & 0.00142 & 0.00283 & $0.004 ? 5$ & 0.012707 \\
\hline 0.500 & 0.00187 & 0.00374 & 0.00561 & 0.00933 \\
\hline 0,700 & 0.00253 & 0.00505 & 0.00756 & $\pi .01257$ \\
\hline 1.000 & 0.00300 & 0.00795 & $0.0119 !$ & $\cap . \cap 1970$ \\
\hline 2.000 & 0.01479 & 0.0 .2917 & 0.114317 & $n .07008$ \\
\hline 3.000 & 0.03652 & 0.07425 & $(0.10734$ & 9.1 .6729 \\
\hline $5.000:$ & 0.13511 & $0.2381 \pi$ & 0.31 .928 & $\pi .43945$ \\
\hline $7,000^{\circ}$ & 0.28 & 0.44517 & $(8.54607$ & 0.57190 \\
\hline 0,000 & $0.52 k 9$ & 0.6957 & 0.7863 & 0.8903 \\
\hline 000 & 0.96 & $1.093 \mathrm{~B}$ & 1.1635 & 1.2532 \\
\hline 30.000 & $1.16 n \%$ & 1.3509 & 9.3 & 1.4649 \\
\hline 50.000 & 1.4477 & 1.3755 & 1.5537 & 1.7548 \\
\hline 70.000 & 1.6346 & 1.7720 & 1.45 & 1.9637 \\
\hline 1.000 & 1.8483 & 1.9980 & 2.0895 & 2.2116 \\
\hline 1.000 & 2.3222 & 2.5053 & 2.41 & 2.7609 \\
\hline .000 & $2.64<5$ & 2.8487 & 2.9745 & 3.1386 \\
\hline 0.000 & 3.1119 & 3.3446 & 3.4495 & 3.6841 \\
\hline BETA & 0.50 & 0.6000 & $n .7000$ & 00 \\
\hline 0.001. & $0.5 n 0$ & 01 & $y$ & 824 \\
\hline & & & & 824 \\
\hline 0.003 & 0.5 & r.6is? & 0.7 & .824 \\
\hline 0.005 & 0.3 & 0.602 & & 324 \\
\hline 0.007 & $\dot{0.5 n 2}$ & $n \cdot A \| 3$ & $n$. & 9.82 .5 \\
\hline 0.010 & 0.503 & 1.604 & 0. & 25 \\
\hline 0.020 & 0.505 & $n .013 \mathrm{~h}$ & 0.7 & 0.828 \\
\hline 0.030 & $0,5 \cap 0$ & 009 & & 830 \\
\hline 0.050 & 0.5 & .614 & $n .7$ & .834 \\
\hline 0.070 & 0.5 .18 &.$\therefore 19$ & H & . 839 \\
\hline 0.100 & & & & .845 \\
\hline 0.200 & 4.5 & 55 & & 868 \\
\hline 07 & & & & \\
\hline 0.500 & 0.6 & 0.748 & 0 & .947 \\
\hline 0.700 & & & & 1.003 \\
\hline 1.000 & 0.524 & .905 & & \\
\hline 2.000 & $1.1: 72$ & 1.1 & & 14 \\
\hline 3.000 & 1.2 & 1.314 & 1. & 1.490 \\
\hline 5.000 & 1.484 & $1.55 \mathrm{~K}$ & & 1.749 \\
\hline & 1.6 & 1.741 & 1.8 & 1.945 \\
\hline 10. & & & & 2.1 .77 \\
\hline .000 & 2.3 & 2.440 & 2.559 & 2.708 \\
\hline 000 & 2.6 & $? .772$ & & $.0 \% 4$ \\
\hline .000 & 3.1 & 3.253 & 3.4 & 3.604 \\
\hline 1.000 & $3.4 \times 3$ & 3.612 & 3.7 & 3.995 \\
\hline .000 & 3.405 & 4.033 & 4.224 & 4.460 \\
\hline & 4,798 & 4.996 & $5.22\}$ & 5.521 \\
\hline & $5.4 ? 2$ & 5.658 & 5.918 & 5.253 \\
\hline .000 & 0.315 & 6.61? & 0.9 .17 & 7.306 \\
\hline
\end{tabular}

0.0079 0,00701 0,00702 0.00703 0.00705 0.110707 0.110715 $0,0.0721$ 0.00737 $0.0 \cup 753$ 0.0087 0.008 . 0.01304 0.01755 0955 21998 0.5240 0.74915 0.9556 1.3123 1.8235 2.0399 2.2917 2.8639 3.2530 3,8138 n. n.1no ก. 0100 0.0100 0.0100 $.01 n 1$ .0101 0.0102 0.0105 n.71n 0.0 .124 0.0141 - D.tag 0.0388 ก.1315 ก.? 0.6137

1.0?0 1,375 1.595
1.900 2.124 2.383
2.975 2.975
3.375 3.957 0.0260 0.0201 0.0201

0.0201

0.02014

0.0206

0.0210

0.0215
0.0222

0.0248

0.0368

0.0491

0.0754
0.2343

0.4445

0.7759

0.9633

1.144
1.501

1.732

2.055

2.292

2.570

3.200
3.634

3.634
4.254
0.0300 0.0301 0.0301 0.0302 $0.03 n 2$ $0.03 n 3$ 0. 0.0315 0.0322 0.0371 0.0420 0.0548 $0.07 ? 7$
0.1111 $0.31 \times 9$ 0.554
0.862 1.038 1.217
1.579 1. 816 2.151
2.399 2.688 3.345
3.795 4.445

$0.050 n$
0.0500
0.0501
0.0501
0.0503
0.0503
0.0505
0.0510
0.0515
0.0525
0.0535
0.0553
0.0616
0.0694
0.0899
0.1176
0.1740
0.4414
0.682
0.963
1.131
1.308
1.682
1.929
2.274
2.540
2.841
3.535
4.010
4.697
109

0.9000

0.980
0.980

0.980

0.982
0.482

0,982

0.984

0.986

0.990

0.999

1.020

1.003

1.146

1.225

1.457

1.918

2.128

2.379

2.952

3.919

4.346

6.002
6.787

7.928

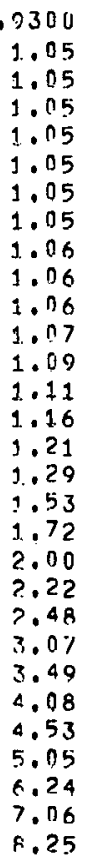

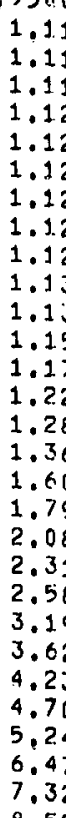

1.11
1.11
1.11
1.12
1.12
1.12
1.12
1.1 .2
1.12
1.13
1.13
1.15
1.17
1.22
1.28
1.36
1.60
1.79
2.08
2.31
2.58
3.19
3.62
4.23
4.70
5.24
6.47
7.32
8.55

9700
1.21
1.21
1.21
1.21
1.21
1.21
1.21
1.21
1.22
1.22
1.22
1.25
1.27
1.32
1.37
1.45
1.70
1.90
2.21
2.44
2.73
3.38
3.82
4.48
4.96
5.53
6.83
7.73
9.03

$9800 \quad 0.9900$

1.28
1.28

1.28

1.28
1.28

1.28
1.28
1.28

1.28
1.29

1.29

1.30

1.32

1.34
1.39

1.34
1.44
1.53
1.78

1.78

1,99
2,31
2,55

. 35

.52

4.99

4.67
5.18

5.77

7.13
8.07

9.42

1.40
1.40
1.40
1.40
1.40
1.40
1.41
1.41
1.41
1.42
1.42
1.44
1.46
1.52
1.57
1.66
1.93
2.15
2.49
2.76
3.07
3.79
4.29
5.02
5.56
6.20
7.65
8.66
11.11
0.0701 0.0702 0.0703 0.0704 $0.070 \%$

0.0713

0.0720

0.0748

0.0772

0.0858

0.1237

0.1601

0.2314
0.5308

0.763

$1.02 \varepsilon$

1.194
1.371

1.753

2.000

2.368
2.634

2.952

3.669

4.163
4.872 0.1002 0.100 ? 0.1005 0.1005 0.1018 0.1027 0.1048 0.1067

0.109 0.1361 0.1723 0.21978 0.6271

0,845

1.261

1.442

1.931

2.471

2,749

3,074
3,820

4.331

5.071

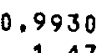

$0.20000 .3000 \quad 0.4000$ $0.2002 \quad 0.3002 \quad 0.4003$ $0.00040 .3002 \quad 0.4003$ $0.2009 \quad 0.30920 .4013$ 0.20110 .30160 .4013 $0.2016 \quad 0.3021 \quad 0.4023$ $\begin{array}{llll}0.2033 & 0.3040 & 0.4051\end{array}$ $\begin{array}{llll}0.2050 & 0.3064 & 0.4075\end{array}$ $\begin{array}{llll}0.2083 & 0.3107 & 0.4123\end{array}$ $\begin{array}{llll}0.2119 & 0.3155 & 0.4175\end{array}$ $0.21740 .3226 \quad 0.4256$ $\begin{array}{llll}0.2379 & 0.3484 & 0.4542\end{array}$ 0.26160 .37790 .4859
$0.3193 \quad 0.4456$ $\begin{array}{llll}0.3193 & 0.4456 & 0.5565\end{array}$ $\begin{array}{lll}0.3879 & 0.5212 & 0.6299\end{array}$ $0.50120 .6338 \quad 0.7368$ $0.999 \quad 1.097 \quad 1.173$ $1.240 \quad 1.333 \quad 1.411$ $1.404 \quad 1.501 \quad 1.583$ $\begin{array}{lll}2.0106 & 2.128 & 2.234\end{array}$ $\begin{array}{lll}2.288 & 2.425 & 2.543\end{array}$ $2.692 \quad 2.852 \quad 2.986$ $2.994 \quad 3.169 \quad 3.318$ $\begin{array}{lll}3.348 & 3.543 & 3.707 \\ 4.155 & 4.392 & 4.598\end{array}$ $4.705 \quad 4.972 \quad 5.208$ $5.506 \quad 5.818 \quad 6.085$ $0.9970 \quad 0.9980 \quad 0,9990$ $\begin{array}{lll}1.63 & 1.71 & 1.85 \\ 1.63 & 1.71 & 1.85\end{array}$ $\begin{array}{lll}1.63 & 1.71 & 1.85 \\ 1.63 & 1.71 & 1.85\end{array}$ $\begin{array}{lll}1.63 & 1.71 & 1.85 \\ 1.63 & 1.71 & 1.85\end{array}$ $\begin{array}{lll}1.63 & 1.71 & 1.85\end{array}$ $\begin{array}{lll}1.63 & 1.71 & 1.85\end{array}$ $\begin{array}{lll}1.63 & 1.71 & 1.85 \\ 1.63 & 1.71 & 1.86\end{array}$ $\begin{array}{lll}1.64 & 1.72 & 1.86 \\ 1.65 & 1.73 & 1.87\end{array}$ $\begin{array}{lll}1.65 & 1.73 & 1.87 \\ 1.67 & 1.75 & 1.89\end{array}$ $\begin{array}{lll}1.67 & 1.75 & 1.89 \\ 1.69 & 1.77 & 1.92\end{array}$ $\begin{array}{lll}1.75 & 1.83 & 1.98 \\ 1.81 & 1.90 & 2.05\end{array}$ $\begin{array}{lll}1.81 & 1.90 & 2.05 \\ 1.91 & 1.99 & 2.15\end{array}$ $\begin{array}{lll}1.91 & 1.99 & 2.15 \\ 2.20 & 2.31 & 2.48\end{array}$ $\begin{array}{lll}2.45 & 2.56 & 2.76 \\ 2.83 & 2.96 & 3.18\end{array}$ 
VALUES OF ALPHA FOR $N=30$ ANII $N P=4$

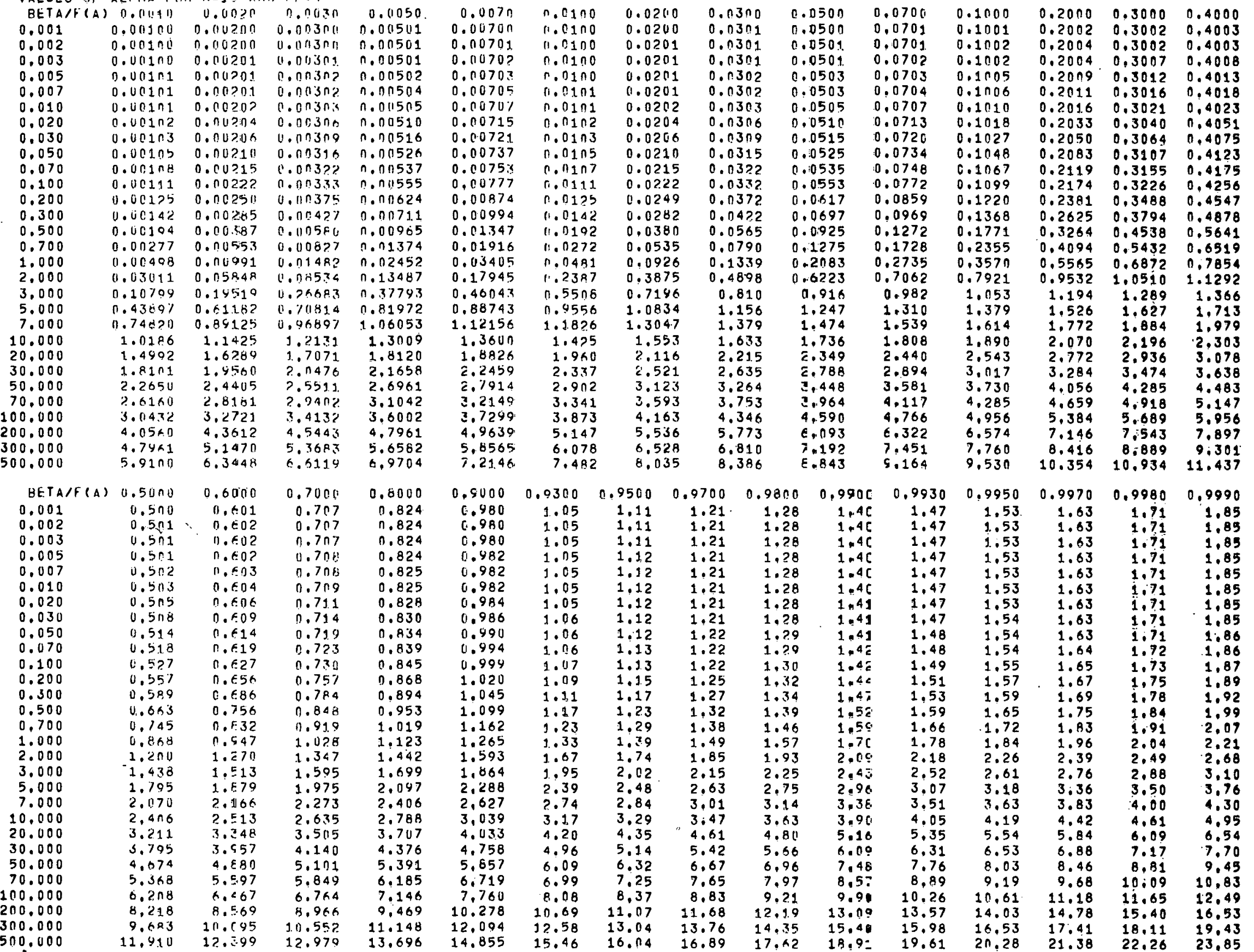


VALUES OF ALPHA FOH $N=1$ O AND $N P=5$ BETA/F(A) 11.0 ind 0.002000 .0031

0.002

0.003

0.005

0.007

0.030

0.050

0,050

0.070

0.20

0.300

0.500

0.700

1.000
2,000

3,000

5.000

7.000
10.000

20.000

30.000

50.000

70.000

100.000

200.00

300.000
500.000

$0.601000 .00200,0.00300$

0.000101 0.00201 il.003n? 0.00502

0.001010 .00201 J.0030. 0.00504

0.00101 0.00202 D.00303 n.00505

0.001090 .002150 .003230 .00537

$0.001110 .00222 \quad 0.00333 \quad 0.00555$

$0.001250 .00250 \quad 0,003750.00624$

$0.001430 .002850 .00426 \quad 0.00712$

$\begin{array}{llll}0.00107 & 0.00393 & 0.00589 & 0.00979\end{array}$

$0.002930 .00586 \quad 0.0 n 8770.01456$

0.00597 0.0118a D.0.11773 0.02926

$0.26683 \quad 0.42180 \quad 0.523930 .64997$

$0.2127 \% \quad 0.95562 \quad 1.03001 \quad 1.11965$

$1.11965 \quad 1.23982 \quad 1.31030 \quad 1.40194$

$\begin{array}{llll}1.4229 & 1.5507 & 1.6270 & 1.7262\end{array}$

$\begin{array}{llll}2.1124 & 2.2803 & 2.3833 & 2.5168 \\ 2.6274 & 2.8258 & 2.951 \% & 3.1157\end{array}$

$3.4323 \quad 3.6879$ 3.450! 4.0560

$4.0665 \quad 4.3841 \quad 4.5748 \quad 4.8190$

$4.9029 \quad 5.2691 \quad 5.4903 \quad 5.7879$

$\begin{array}{llll}6.9657 & 7.4969 & 7.8059 & 8.2331\end{array}$

$\begin{array}{rrrr}8.5841 & 9.2097 & 9.5911 & 10.0947 \\ 11.1116 & 11.9105 & 12.4140 & 13.0701\end{array}$

BETA/F(A) $0.50000 .6000 \quad 0.70000 .0000$

0.001

0.002

0.003

0.005

0.007

0.010

0.020

0.030

0.050

0.070

0.100
0.200

0.300

0.500

0.700

1.000

2.000

3.000

5.000

7.000

0.000

30.000

30.000

50.000

0.00

100.000

20.000

500.000

$17.296 \quad 17.430 \quad 18.75 .4$

19.792
25.540 0,00700 0,00701 .0070 ? 0.00705 0.00707 0,00715 0,00721 0.00737 0.00753

0.00874

0.00996 0.01368 0.04060 0.30736 0.72817 1.46298 1.7949 2.6083
3.2263

4.2010

4.9944

5.9862

8.5078
10.4456

10.4456
13.5279
0.0100 0.010 0.010 0.0100

0.0101

0.0101

0.0103

0.0105

0.0107

0.0175

0.0142

0.0195

0.0572

11.388

1.2417

1,5278
1.869

2.711

3.348

4.361

5,178

8.8 ?

4.016
4.016

0.9000

0.9300
1.05

0.98

0.980
0.982

0.98

0.982

0.984

0.986

0.49

0.999

1.020

1.102

1.102
1.171

1.295
1.730

2.105

2.719

3.226

5.490

6.734

8.721

12.368

17.533

21.470

1.05
1.05
1.05

1.05

1.05

1.05

1.06

1.06
1.06
1.07

1.09

1.11

1.17
1.24

.36

1.81
2.20

2.84
3.36

4.03

5.71

9.07
10.75
12.06

12.86

18.24
22.32

27.86

$0.0200 \quad 0.0300$ $0.0201 \quad 0.0301$

0.0201

0.0201

0.0202

0.0303

$0.0210 \quad 0.0315$

$0.0215 \quad 0.0322$

$0.0240 \quad 0.0332$

$0.0283 \quad 0.0423$

$0.0386 \quad 0.0574$

0.0566

0.1091

0.5632

0.0664

0.6643

1.022
1.438

$1.6613 \quad 1.743$

2,025

3.604

4.689

5.567
6.673

9.484

11.636
15.054

2.116

3.051
3.764

4.895

5.811
6.963

9.881

12,139
15,702

$0.050 n$

0.0501

0.0501
0.0503
0.0503

0.0503

0.0510

0.0515

0.0525

0.0553

0.0617

0.0698

0.1342

0.2401

0.7844

1.535

1,85 ?

3.226

3.980

6,131

7.352

10.430
12.795

13.812

$16.557 \quad 17.167 \quad 17.839$

$\begin{array}{rrrrr}1.11 & 0.9700 & 0.9800 & 0.9900 & 0.9930 \\ 1.21 & 1.28 & 1.40 & 1.47\end{array}$

1,11

1.12

1.12

1.12

1,12

1.13

$1,1.5$

1.18

1.30

1.43

2.28

2.94

4.17

5.93
7.27

9.41

13,33

18.48
23.15

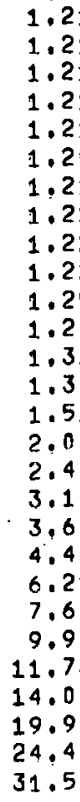

.21
.21

1,28

1.28
1.28

1.28
1,28
$1 ., 28$

1.28

1.28 1.4

$1.29 \quad 1.42 \quad 1.48$

1.32
1.34

1.34

1.40
1.47

1.60

2.09

3.25

3.85

6.53

8.100

$9.93 \quad 1.0 .35$

$14.06 \quad 14.67$

$\begin{array}{lll}29.02 & 31.59 & 32.03\end{array}$

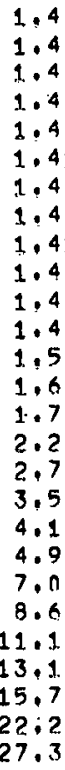

$1.44 \quad 1.5$

$1.47 \quad 1.53$

$.74 \quad 1.81$

1.81
2.35
2.83
3.63

4.29

5.13

8.92

11.53

13.65
16.31

23.12

36.59

(n) 
APPENDIX C

Parameter $g$ as a Function of Extraction Factor $\alpha$, Scrub Factor $\beta$, and Numbers of Staǵes $n$ and $n^{\prime}$ 

BETA

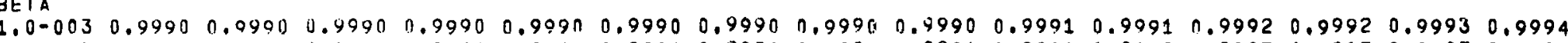
$\begin{array}{llllllllllllllll}2.0-003 & 0.998 n & 0.0980 & 0.9980 & 11.9980 & 0.9980 & 0.9980 & 0.998 n & 0.9981 & 0.9981 & 0.9981 & 0.9982 & 0.9983 & 0.9985 & 0.9987 & 0.9988\end{array}$ $\begin{array}{lllllllllllllllll}3.0-003 & 0.9971 & 0.9970 & 0.9770 & 0.9970 & 0.997 n & 0.9970 & 0.9971 & 0.9971 & 0.8972 & 0.9972 & 0.9973 & 0.9975 & 0.9977 & 0.9980 & 0.9982\end{array}$ $\begin{array}{lllllllllllllllll}5.0-003 & 0.995 n & 0.9950 & \text { i).995i } & 0.9950 & 0.9951 & 0.9951 & 0.9951 & 0.9952 & 0.9953 & 0.9953 & 0.9955 & 0.9959 & 0.9962 & 0.9967 & 0.9971\end{array}$ $\begin{array}{lllllllllllllllll}7.0-003 & 0.9931 & 0.0931 & 0.9931 & 0.9931 & 0.9931 & 0.9931 & 0.9932 & 0.9032 & 0.5934 & 0.9935 & 0.9937 & 0.9942 & 0.9946 & 0.9954 & 0.9959\end{array}$ $1.0-0020.99010 .04010 .90010 .99010 .90020 .99020 .99030 .09040 .49060 .99070 .99100 .99170 .99240 .99340 .9942$

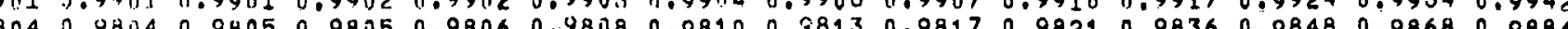

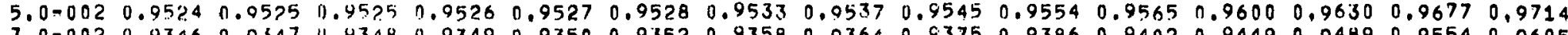

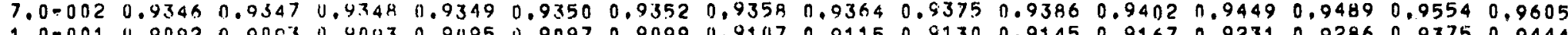
$\begin{array}{lllllllllllllllll}1.000 & 0.909 ? & 0.9093 & 0.9093 & 0.9095 & 0.9 n 97 & 0.9099 & 0.9107 & 0.0115 & 0.9130 & 0.9145 & 0.91 .67 & 0.9231 & 0.9286 & 0.9375 & 0.9444\end{array}$ $\begin{array}{llllllllllllllllllll}2.0-001 & 0.8335 & 0.83 .36 & 0.8337 & 0.8340 & 0.8343 & 0.8347 & 0.8361 & 0.8374 & 0.8400 & 0.8425 & 0.8462 & 0.8571 & 0.8667 & 0.8824 & 0.8947\end{array}$ $\begin{array}{lllllllllllllllllll}3.0-001 & 0.7694 & 0.7696 & 4.7698 & 0.7701 & 0.7705 & 0.7710 & 0.7727 & 0.7744 & 0.7778 & 0.7810 & 0.7857 & 0.8000 & 0.8125 & 0.8333 & 0.8500\end{array}$ $\begin{array}{lllllllllllllllll}5.0-001 & 0.6669 & 0.6671 & 0.6673 & 0.6678 & 0.6682 & 0.6689 & 0.6711 & 0.6732 & 0.6774 & 0.6815 & 0.6875 & 0.7059 & 0.7222 & 0.7500 & 0.7727\end{array}$ $\begin{array}{llllllllllllllll}7.0=001 & 0.5885 & 0.5887 & 0.5890 & 0.5894 & 0.5899 & 0.5906 & 0.5930 & 0.5954 & 0.6000 & 0.6045 & 0.6111 & 0.6316 & 0.6510 & 0.6818 & 0.7083\end{array}$

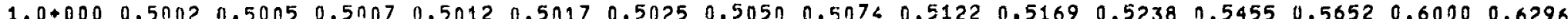
1.00000 .33360 .33380 .33400 .33440 .33490 .33550 .33770 .3390 .34430 .34850 .35480 .37500 .39390 .42860 .4595

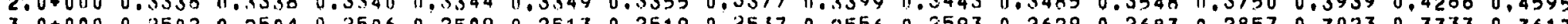
3.0 .000 .

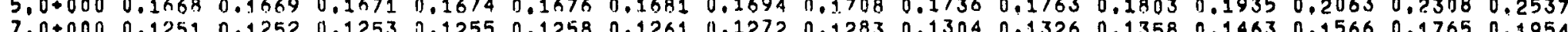

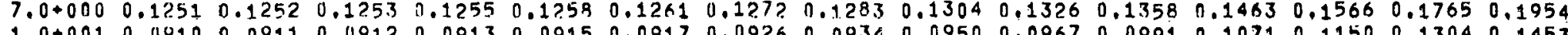
$\begin{array}{llllllllllllllllllll}1.0+001 & 0.0910 & 0.0911 & 0.0912 & 0.0913 & 0.0915 & 0.0917 & 0.0926 & 0.0934 & 0.0950 & 0.0967 & 0.0991 & 0.1071 & 0.1150 & 0.1304 & 0.1453\end{array}$

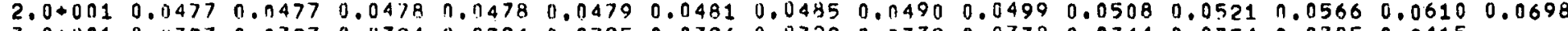
$\begin{array}{llllllllllllllll}3.0+001 & 0.0323 & 0.0323 & 0.0324 & 0.0324 & 0.0325 & 0.0326 & 0.0329 & 0.0332 & 0.0338 & 0.0344 & 0.0354 & 0.0385 & 0.0415\end{array}$

$\begin{array}{llllllllllllllllll}5.0+001 & 0.0196 & 0.0196 & 0.0197 & 0.0197 & 0.0197 & 0.0198 & 0.0200 & 0.0202 & 0.0206 & 0.0210 & 0.02150 .0234\end{array}$

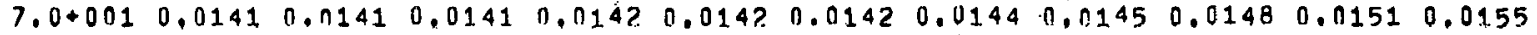

$1.0+0020.00990 .00090 .00990 .0100 \quad 0.0100 \quad 0.01000 .01010 .01020 .01040 .01060 .0109$

$2.0+0020.011500 .00500 .00500 .00500 .00500 .00500 .00510 .00510 .0052$

$3.0+0.020 .00330 .00330 .00330 .00330 .00330 .00340 .00340 .0034$

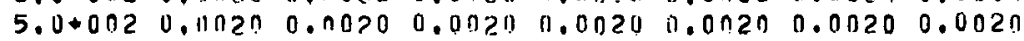

$7.0+0020.00140 .00140 .0014 \quad 0.00140 .0 n 140.0014$

$1.0+0030.00100 .00100 .00100 .00100 .00100 .0010$

ALPHA $1.000 \quad 2.000 \quad 3.000 \quad 5.000 \quad 7.090 \quad 10.00 \quad 20.00 \quad 30.00 \quad 50.00 \quad 70.00 \quad 100.0 \quad 200,0 \quad 300.0 \quad 500.0 \quad 700.0 \quad 1000$ BETA

$1.0-003 \quad 0.99950 .9997 \quad 1.99980 .99980 .99990 .99991 .00001 .00001 .00001 .00001 .00001 .00001 .00001 .00001 .00001 .0000$ $2.0=0 n 30.999 n \quad 0.9993 \quad 0.99950 .99970 .99980 .9998 \quad 0.99990 .99991 .00001 .00001 .00001 .00001 .00001 .00001,00001.0000$ $3.0=003 \quad 0.0945 \quad 0.0990 \quad 0.9993 \quad 0.9995 \quad 0.9996 \quad 0.9997 \quad 0.9990 \quad 0.99990 .99991 .00001 .00001 .00001 .00001 .00001 .00001 .0000$

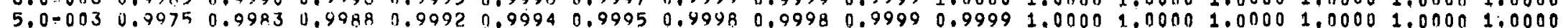

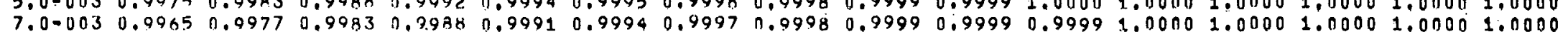

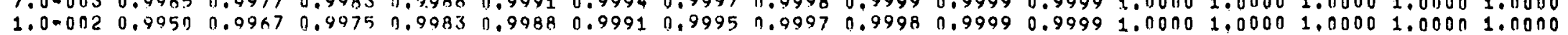

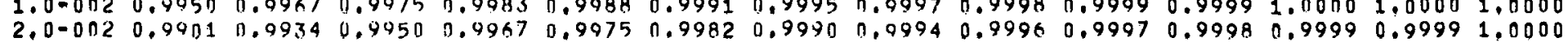
$\begin{array}{llllllllllllllll}3.0-002 & 0.985 ? & 0.0901 & 0.9926 & 0.9950 & 0.9963 & 0.9973 & 0.9986 & 0.9990 & 0.9994 & 0.9996 & 0.9997 & 0.9999 & 0.9999\end{array}$ $\begin{array}{llllllllllllllll}5.0-002 & 0.9754 & 0.9836 & 0.9877 & 0.0917 & 0.9938 & 0.9955 & 0.9916 & 0.9984 & 0.9990 & 0.9993 & 0.9995 & 0.9998\end{array}$

$\begin{array}{lllllllllllllll}7.0 .002 & 0.9662 & 0.9772 & 0.9828 & 0.9885 & 0.9913 & 0.9937 & 0.9967 & 0.9977 & 0.9986 & 0.9990 & 0.9993\end{array}$

$\begin{array}{llllllllllllll}1.0-001 & 0.9524 & 0.9677 & 0.9756 & 0.9836 & 0.9877 & 0.9910 & 0.9953 & 0.996 B & 0.9980 & 0.9986 & 0.9990\end{array}$

$2.0-001 \quad 0.9091 \quad 0.9375 \quad 0.9524 \quad 0.9677 \quad \pi .9756 \quad 0.9821 \quad 0.9906 \quad 0.9936 \quad 0.9961$

$\begin{array}{lllllllll}3.0-001 & 0.8696 & 0.0091 & 0.9302 & 0.0524 & 0.9639 & 0.9735 & 0.9850 & 0.9904\end{array}$

$\begin{array}{lllllllll}5.0-001 & 0.8000 & 0.8571 & 0.8889 & 0.0231 & 0.9412 & 0.9565 & 0.9767\end{array}$

$\begin{array}{llllllll}7.0-001 & 0.7407 & 0.81 \cap 8 & 0.8511 & 11.8955 & 0.9195 & 0.9402\end{array}$

$1.0+000 \quad 0.6667 \quad 0.7500 \quad 0.8000 \quad 0.8571 \quad 0.8840 \quad 0.9167$

$2.0+000 \quad 0.5000 \quad 0.0000 \quad 0.0667 \quad 11.7500$

$3.0+000 \quad 0.41000 .5000 \quad 11.5714$

$5.0+000 \quad 0.2857 \quad 0.3750$

$7.0+000 \quad 0.2222$

$1,0+001 \quad 0.1667$ 
$1.0=003 \quad 0.9990 \quad 0.0990 \quad 0.9990 \quad 0.9990 \quad 0.0990 \quad 0.9990 \quad 0.9990 \quad 0.0990 \quad 0.9990 \quad 0.99910 .99990 .9992 \quad 0.0992 \quad 0.9993 \quad 0.9994$ $1,0=003$
$2,0.9980$

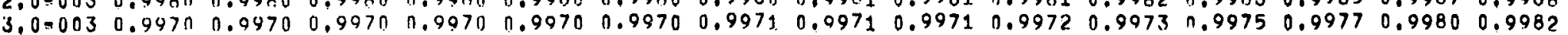
$\begin{array}{llllllllllllllllll}5.0-003 & 0.9950 & 0.9950 & 0.9950 & 0.99511 & 0.9950 & 0.9950 & 0.9951 & 0.9951 & 0.9952 & 0.9953 & 0.9955 & 0.9958 & 0.9961 & 0.9967 & 0.9971\end{array}$

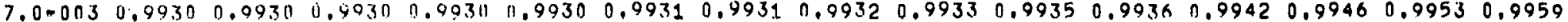

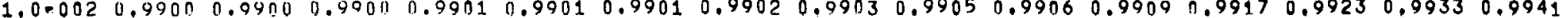

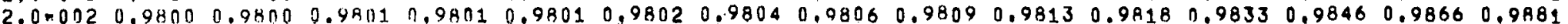

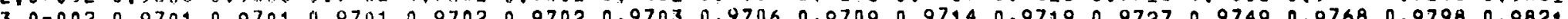

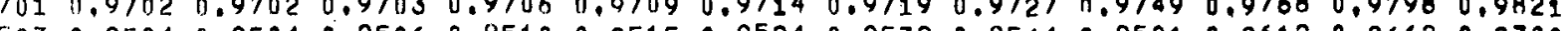
$5.0 .0020 .05020 .95 n 20.95030 .9540 .95040 .95060 .951 n 0.05150 .95240 .95320 .95441 .95810 .96120 .96620 .9700$ (1)

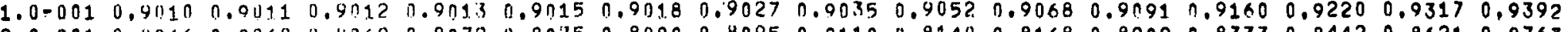

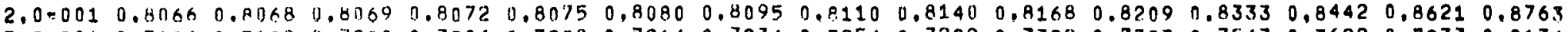
$\begin{array}{lllllllllllllllll}3,0=001 & 0,7196 & 0.7198 & 0,7200 & 0.7204 & 0.7208 & 0.7214 & 0.7234 & 0.7254 & 0.7292 & 0,7329 & 0.7383 & 0.7547 & 0.7692 & 0.7937 & 0.8134\end{array}$ $\begin{array}{lllllllllllllllll}5.0=001 & 0.5717 & 0.5719 & 0.5722 & 0.5726 & 0.5731 & 0.5739 & 0.5763 & 0.5787 & 0.5833 & 0.5879 & 0.5946 & 0.6154 & 0.6341 & 0.6667 & 0.6939\end{array}$ $\begin{array}{lllllllllllllllll}7.0=001 & 0.4569 & 0.4571 & 0.4574 & 0.4579 & 0.4584 & 0.4591 & 0.4615 & 0.4440 & 0.4684 & 0.4735 & 0.4803 & 0.5021 & 0.5221 & 0.5576 & 0.5882\end{array}$ $\begin{array}{llllllllllllllll}1.0+000 & 0.3336 & 0.3338 & 0.3340 & 0.3344 & 0.3349 & 0.3355 & 0.3377 & 0.3399 & 0.3443 & 0.3485 & 0.3548 & 0.3750 & 0.3939 & 0.4286 & 0.4595\end{array}$ $2.0+000 \quad 0.1430 \quad 0.1431 \quad 0.14320 .14350 .1437 \quad 0.14410 .1453 \quad 0.14650 .14890 .15130 .15490 .16670 .17810 .2000 \quad 0.2208$

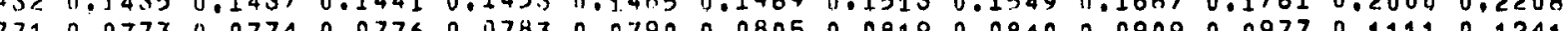
$3.0+0000.077 n 0.07710 .07710 .07730 .07740 .07760 .07830 .07050 .08050 .08190 .08400 .09090 .09770 .11110 .1241$ $5.0+0000.03230 .03230 .03240 .03240 .03250 .03260 .03290 .03320 .03380 .03440 .0354 n .03850 .04150 .04760 .0536$ $\begin{array}{llllllllllllllll}7.0+000 & 0.0176 & 0.0176 & 0.0176 & 0.0176 & 0.0177 & 0.0177 & 0.0179 & 0.0181 & 0.0184 & 0.0187 & 0.0193 & 0.0210 & 0.0227 & 0.0261 & 0.0295\end{array}$

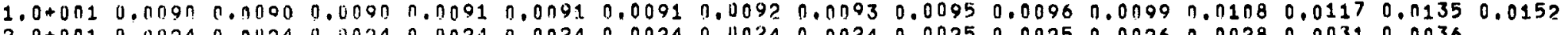
$2.0+0010.0 n 240.01124 \quad 0.00240 .00240 .00240 .00240 .00240 .00240 .00250 .00250 .0026 \quad 0.00280 .00310 .0036$ $3.0+0010.01110 .00110 .0 n 11 \quad 11.001 .10 .00110 .00110 .00110 .00110 .00110 .00110 .00120 .00130 .0014$

$5.0+0010.00040 .00040 .00040 .00040 .00040 .00040 .00040 .00040 .00040 .00040 .00040 .0005$

$7.0+0010.00020 .00020 .00020 .00020 .00020 .00020 .00020 .00020 .00020 .00020 .0002$

$1.0+0020.00010 .00010 .0001 .0 .00010 .00010 .00010 .00010 .00010 .00010 .00010 .0001$

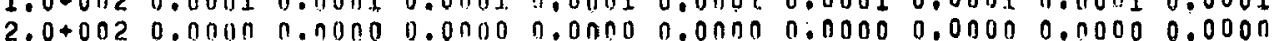

$3.0+0020.000 n \pi .000011 .0000,0,0000,00000.00000 .00000 .0000$

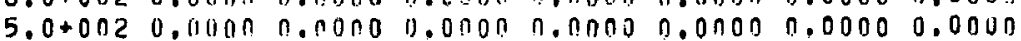

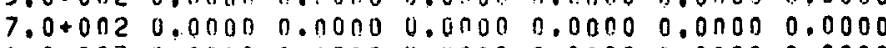

$1,0+003 \quad 0.00000 .0000 \quad 0.01000 .00000 .00000 .0000$ BETA

$1.0=0030.9905 \quad 0.0907 \quad 4.99970 .9998 \quad 1099990.99991 .00001 .00001 .00001 .00001 .00001 .00001 .00001 .00001 .00001 .0000$

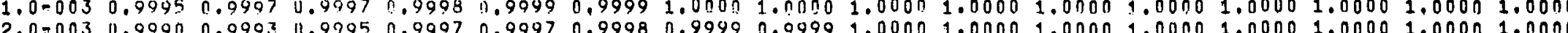
$2,0=003$ (1.090,

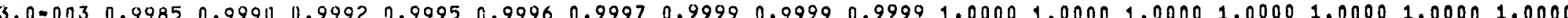
$5.0-0030.99750 .99830 .99870 .90920 .99940 .99950 .99980 .99980 .99990 .99991 .00001 .00001 .00001 .00001 .00001 .0000$ 7.0-003 0.99650 .09770 .99820 .998310 .99990 .99940 .99970 .99980 .99990 .99990 .99991 .00001 .00001 .00001 .00001 .0000 1.0-002 $0.9950 \quad 0.996$ E 19.9975 .0 .99830 .99870 .99910 .99950 .99970 .99980 .99990 .99990 .99991 .00001 .00001 .00001 .0000

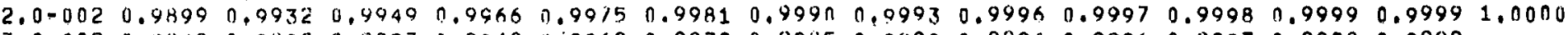
$\begin{array}{lllllllllllllll}3.0-002 & 0.9848 & 0.9892 & 0.9923 & 0.9949 & 0.996 ? & 0.9972 & 0.9985 & 0.9990 & 0.9994 & 0.9996 & 0.9997 & 0.9998 & 0.9999\end{array}$

$\begin{array}{lllllllllllllll}5.0-002 & 0.9744 & 0.9828 & 0.9470 & 0.9913 & 0.9935 & 0.9952 & 0.9975 & 0.9983 & 0.9990 & 0.9993 & 0.9995 & 0.9997\end{array}$

$\begin{array}{lllllllllllllllll}7.0=002 & 0.9639 & 0.975 E & 0.9 R 16 & 0.9977 & 0.9907 & 0.9932 & 0.9964 & 0.9976 & 0.9985 & 0.9989 & 0.9993\end{array}$

$\begin{array}{lllllllllllllll}1.0 & 0.01 & 0.9479 & 0.0646 & 0.9732 & 0.9920 & 0.9964 & 0.9901 & 0.9948 & 0.9965 & 0.9978 & 0.9985 & 0.9989\end{array}$

$\begin{array}{lllllllllll}2.0-001 & 0.8929 & 0.9255 & 0.9434 & 0.0615 & 17.9709 & 0.9786 & 0.9887 & 0.9073 & 0.9953\end{array}$

$3,0=0010.83690 .8850$ U. $41120.9390 \quad 0.95350 .96580 .98180 .9876$

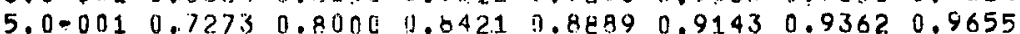

$7,0-0 \cap 10.6270 \quad 0.7160 \quad 0.77070 .83450 .8705 \quad 0.9024$

$1.0+000 \quad 0.50000 .60000 .6667 \quad 0.7500 \cdot 0.8000^{\circ} 0.8462$

$2.0+0000.250 n 0.33320 .40000 .5000$

$3.0+000 \quad 0.14290 .200017 .2500$

$5.0+000 \quad 0.0625 \quad 0.09 n 5$

$7.0+000 \quad 0.0345$

$1.0+001 \quad 0.017$ 


\begin{abstract}
VALUES OF G $F$ GIR $N=1$ AND $N P=3$
BETA

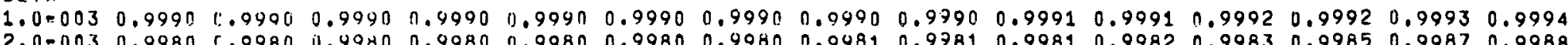

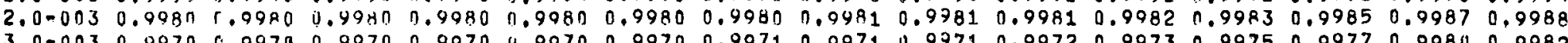

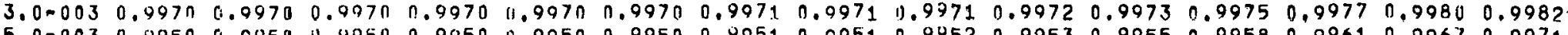

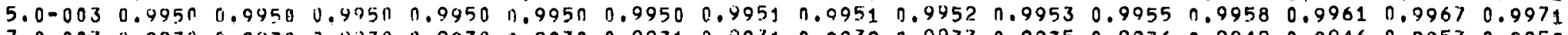
$\begin{array}{llllllllllllllll}7,0-003 & 0.9931 & 0.9930 & 0.9430 & 0.9930 & 1,993 n & 0.9931 & 0.9931 & 0.0932 & 0.9933 & 0.9935 & 0.9936 & 0.9942 & 0.9946 & 0.9953 & 0.9959\end{array}$ 1.0-002 $0.4900 \quad 0.09090 .9900 \quad 0.9900 \quad 0.99010 .99010 .99020 .99030 .99050 .99060 .99090 .99170 .99230 .99330 .9941$

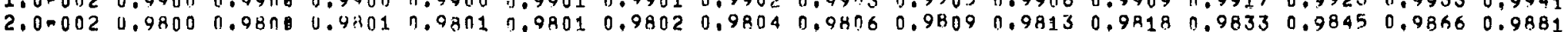

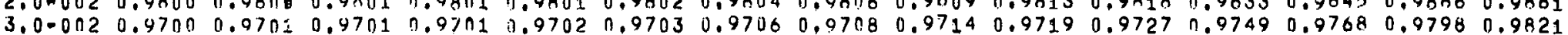

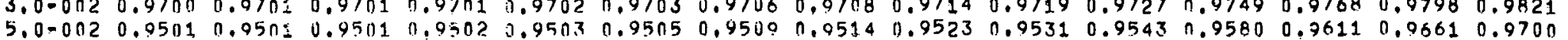

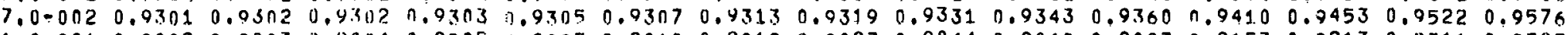
$1.0-001 \quad 0.9 n 02 \quad 0.90 n 3 \quad 0.9 n 04 \quad 0.9 n 05 \quad 3.9 n 07 \quad 0.9010 \quad 0.9010 \quad 0.9027 \quad 0.9044 \quad 0.9060 \quad 0.9083 \quad 0.9153 \quad 0.9213 \quad 0.93110 .9387$

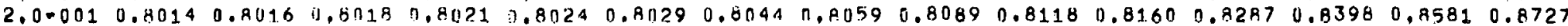
$\begin{array}{lllllllllllllllllll}3.0-001 & 0.7059 & 0.7061 & 0.7 n 63 & 0.7 n 68 & 0.7072 & 0.7078 & 0.7098 & 0.7118 & 0.7157 & 0.7196 & 0.7251 & 0.7421 & 0.7571 & 0.7325 & 0.8030\end{array}$ $\begin{array}{lllllllllllllllllll}5.0=001 & 0.5336 & 0.5333 & 0.5341 & 0.5346 & 7.5351 & 0.5358 & 0.5383 & 0.54 \pi 7 & 0.5455 & 0.5501 & 0.5570 & 0.5783 & 0.5977 & 0.6316 & 0.6602\end{array}$ $\begin{array}{llllllllllllllll}7.0 .001 & 0.395 \pi & 0.3953 & 0.3955 & 0.3360 & 0.3965 & 0.3972 & 0.3995 & 0.4019 & 0.4065 & 0.4111 & 0.4178 & 0.4391 & 0.4589 & 0.4946 & 0.5258\end{array}$ $1.00000 .25020 .25040 .05060 .2509-25130.25190 .25370 .25560 .25930 .26290 .26830 .28570 .30230 .33330 .3617$

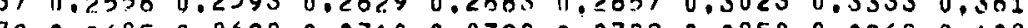

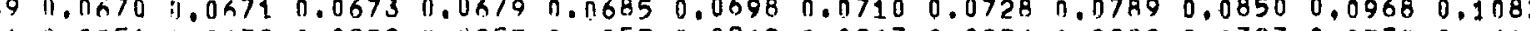

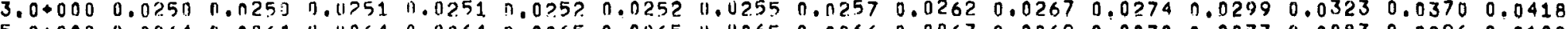

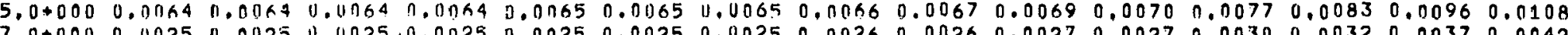
$7,0+000 \quad 0.0025 \quad 1.0025 \quad 0.0 n 25.0 .0025 \quad 0.0 n 25 \quad 0.0025 \quad 0.0 n 25 \quad 0.0026 \quad 0.0026 \quad 0.0027 \quad 0.0 n 27 \quad 0.0030 \quad 0.0032 \quad 0.0037 \quad 0.0042$

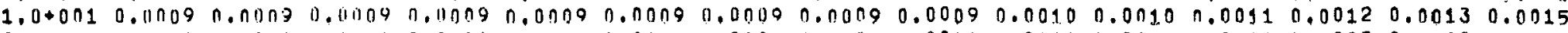

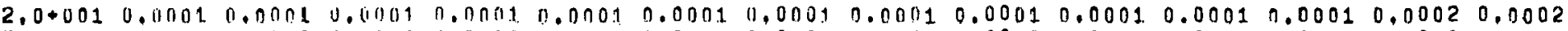

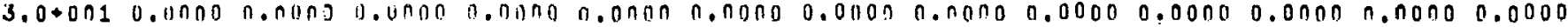

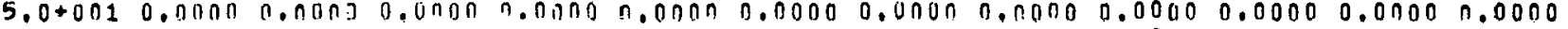

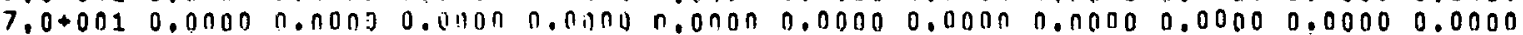

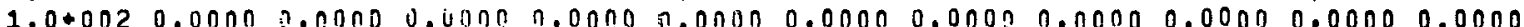

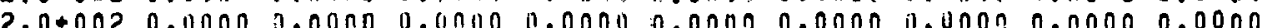

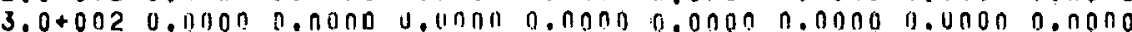

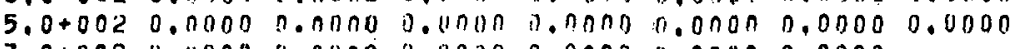

$7,0+002 \quad 0.00000 .001000 .0000 \quad 0.0000 \quad 0.0000 .0 .0000$

$1.0+0030.0000$ 0.000n 0.00000 .00000 .0000 n.0000 ETA

$1.0=0030.99950 .99970 .9997 \quad 0.99980 .99990 .9999 .1,00401.00001 .00001 .00001 .00001 .00001 .00001 .00001 .00001 .0000$ 1.0-003 0.9990 0.99930 .99950 .99970 .99970 .99980 .99990 .99991 .00001 .00001 .00001 .00001 .00001 .00001 .00001 .0000

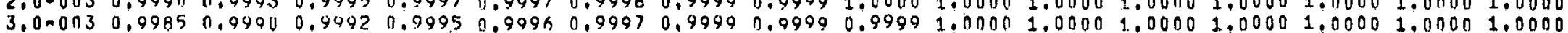

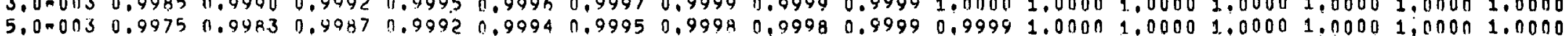

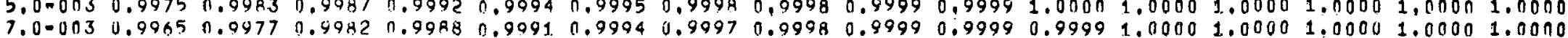

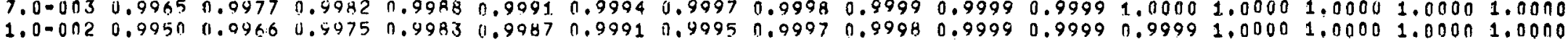

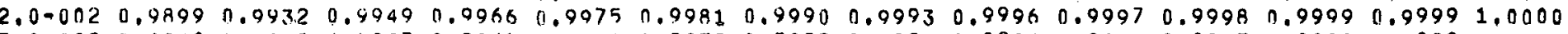

$\begin{array}{llllllllllllll}3.0=002 & 0.9848 & 1.0898 & 0.4923 & 0.9949 & 0.9961 & 0.9972 & 0.9985 & 0.9990 & 0.9994 & 0.9996 & 0.9997 & 0.9998 & 0.9999\end{array}$

$\begin{array}{lllllllllllll}5.0-002 & 0.9744 & 0.9878 & 0.9870 & 0.9913 & 0.9935 & 0.9952 & 0.9975 & 0.9983 & 0.9990 & 0.9993 & 0.9995 & 0.9997\end{array}$

$\begin{array}{lllllllllllll}7.0 .002 & 0.9637 & 0.0755 & 0.9815 & 0.9876 & 0.9907 & 0.9932 & 0.9964 & 0.9976 & 0.9985 & 0.9989 & 0.9993\end{array}$

$\begin{array}{lllllllllllll}1.0=001 & 0.9474 & 0.9643 & 0.973 n & 0.5818 & 0.9863 & 0.9900 & 0.9947 & 0.9964 & 0.9978 & 0.9984 & 0.9989\end{array}$

$\begin{array}{lllllllllll}1.0 & 0=001 & 0.9897 & 0.0236 & 0.9416 & 0.9603 & 0.9699 & 0.9780 & 0.9883 & 0.9921 & 0.9952\end{array}$

$3.0-001 \quad 0.4275 \quad 0.8790 \quad 0.9156 \quad 0.9350 \quad 0.95050 .96350 .98050 .9867$

$5.0 .0010 .69570 .7742 \quad 0.0205 \quad 0.8727 \quad 0.90140 .92630 .9600$

$\begin{array}{lllllll}7,0-001 & 0.5661 & 0.6618 & 0.7229 & 0.7965 & 0.8392 & 0.8777\end{array}$

$1.0+000 \quad 0.4000 \quad 0.5000 \quad 0.5714 \quad 0.6667 \quad 0.7273 \quad 0.7857$

$2.0+000 \quad 0.1250 \quad 0.1765 \quad 0.22220 .3000$

$3.0+000 \quad 0.04880 .07140 .1930$

$5,0+000 \quad 0,0127 \quad 0.0190$

$7.0+000 \quad 0.0050$ 

BETA

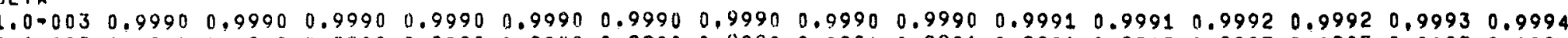

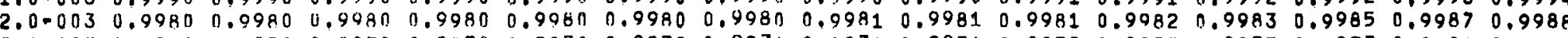
$\begin{array}{llllllllllllllll}3.0 .003 & 0.9970 & 0.0970 & 0.997 n & 0.9970 & 0.997 n & 0.9970 & 0.9971 & 0.0971 & 0.9971 & 0.9972 & 0.9973 & 0.9975 & 0.9977 & 0.9980 & 0.9982\end{array}$ $\begin{array}{lllllllllllllllll}5,0.003 & 0.9950 & 0.9950 & 1.9950 & 0.9950 & 1.995 n & 0.9950 & 0.9951 & 0.9951 & 0.9952 & 0.9953 & 0.9955 & 0.9958 & 0.9961 & 0.9967 & 0.997\end{array}$ $\begin{array}{lllllllllllllllll}7.0 .003 & 0.9931 & 0.9930 & 0.9930 & 0.0930 & 0.9930 & 0.9931 & 0.9931 & 0.0932 & 0.9933 & 0.9935 & 0.9936 & 0.9942 & 0.9946 & 0.9953 & 0.9959\end{array}$

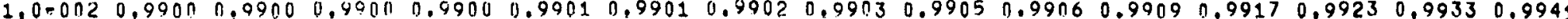
$2,0=002 \quad 0.9800 \quad 0.9800 \quad 0.9801 \quad 0.9801 \quad 0.9801 \quad 0.9802 \quad 0.9804 \quad 0.9806 \quad 0.98090 .9813 \quad 0.98180 .9833 \quad 0.98450 .9866 \quad 0.9881$ 3.000020 .97000 .07014047010 .97010 .97020 .97030 .97060 .97080 .97140 .97190 .97270 .97490 .97680 .97900 .9821

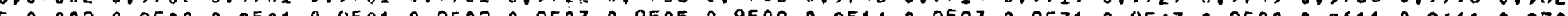

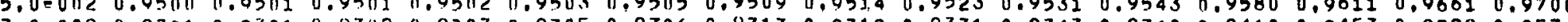

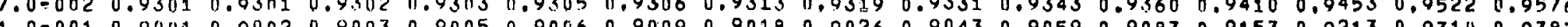

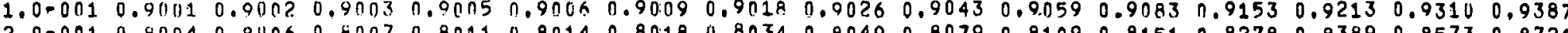
$2.0=0010.8004 \quad 0.8006 \quad 0.8007 \quad 0.8011 \quad 0.8 n 14 \quad 0.8018 \quad 0.8034 \quad 0.80490 .8079 \quad 0.8109 \quad 0.8151 \quad 0.8278 \quad 0.8389 \quad 0.8573 \quad 0.8720$ $\begin{array}{lllllllllllllllllll}3.0=001 & 0.7119 & 0.7021 & 0.7023 & 0.7027 & 0.7 n 32 & 0.70038 & 0.7058 & 0.7079 & 0.7118 & 0.7157 & 0.7213 & 0.7384 & 0.7536 & 0.7792 & 0.8000\end{array}$ $\begin{array}{llllllllllllllll}5.0-0 & 0.5164 & 0.516 .6 & 0.5169 & 0.5174 & 0.5179 & 0.5186 & 0.5211 & 0.5235 & 0.5283 & 0.5330 & 0.5399 & 0.5614 & 0.5810 & 0.6154 & 0.6445\end{array}$ $\begin{array}{lllllllllllllllll}7.00001 & 0.3608 & 0.3611 & 0.3413 & 0.3618 & 0.3622 & 0.3629 & 0.365 ? & 0.3675 & 0.3719 & 0.3763 & 0.3829 & 0.4036 & 0.4230 & 0.4583 & 0.4895\end{array}$ $\begin{array}{llllllllllllllll}1.0+000 & 0.2002 & 0.2003 & 0.2005 & 0.2008 & 0.2011 & 0.2016 & 0.2032 & 0.2048 & 0.2079 & 0.2110 & 0.2157 & 0.23 n 8 & 0.2453 & 0.2727 & 0.2982\end{array}$ $1.0+0000.43230 .03230 .63240 .03240 .03250 .03260 .03200 .03320 .03380 .03440 .03540 .03850 .04150 .04760 .0536$ $3.0+0000.00830,0030.00830,00830.00830 .00830 .00840 .00850 .00870 .0080 .00910 .00990 .01070 .01230 .0140$

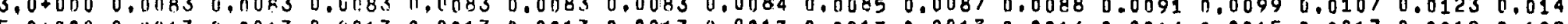
$5.0+0000.0013$ (1.0013 0.01130 .00130 .0013 n.0013 0.00130 .00130 .00130 .00140 .00140 .00150 .00170 .00190 .0022 $7.0+0000.0004 \quad 0.0004 \quad 0.00040 .00040 .00040 .00040 .00040 .00040 .00040 .00040 .0004 \quad 0.00040 .00050 .00050 .0006$ $1.0+0 n 10.00 n 10.00010 .0 n 010.00010 .0 n 010.00010 .00010 .00010 .00010 .00 n 10.00010 .00 n 10.00010 .00010 .0002$ $2.0+0010.00000 .00000 .60000 .00000 .00000 .00000 .00000 .00000 .00000 .00000 .00000 .00000 .00000 .0000$

$3.0+0010.00000 .0000 \quad 0.4000,0.00000 .00000 .00000 .00000 .00000 .00000 .00000 .00000 .00000 .0000$

$5.0+0010.00000 .0000 \quad 0.6000 \quad 0.0000 .0 .0000 \quad 0.00000 .00000 .00000 .00000 .00000 .00000 .0000$

$7,0+001.0 .00000 .00000 .00000 .00000 .00000 .00000 .00000 .00000 .00000 .00000 .0000$

$1,0+0020.00000 .00000 .00000 .00000 .00000 .00000 .00000 .00000 .00000 .00000 .0000$

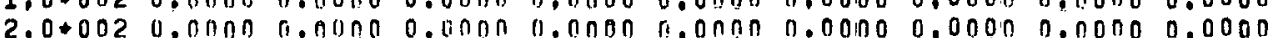

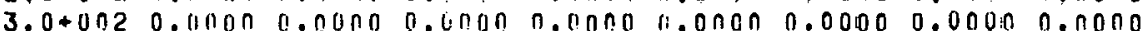

$5,0+0020.00000 .00000 .611000,00000.00000,00000.0000$

$7.0+0020.00000 .00000 .00000 .00000 .00000 .0000$

$1,0+0030,0.0000 .00000 .00000 .00000 .00000 .0000$

ALPHA $1.000 \quad 2.000 \quad 3.000 \quad 5.000 \quad 7.000 \quad 10.00 \quad 20.0 n \quad 30.00 \quad 50.00 \quad 70.00 \quad 100.0 \quad 200.0 \quad 300.0 \quad 500.0 \quad 700.0 \quad 1000$ BETA

$1.000030 .9995 \quad 1.9997 \quad 0.9997 \quad 0.99980 .9999 \quad 0.99991 .00001 .00001 .00001 .00001 .00001 .00001 .00001 .00001 .00001 .0000$ $2.0=003 \quad 0.9990 \quad 0.09930 .59950 .9997 \quad 0.9997 \quad 0.99980 .99990 .09991 .00001 .00001 .00001 .00001 .00001 .00001 .00001 .0000$

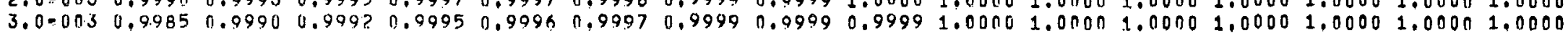

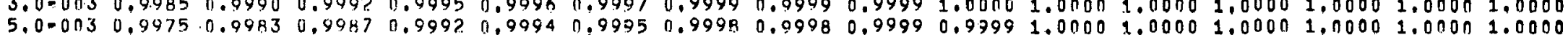

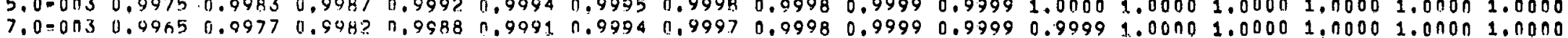
$1.0-002 \quad 0.9950 \quad 0.9966 \quad 0.9975 \quad 0.9983 \quad 0.99870 .97910 .99950 .99970 .99980 .99990 .99990 .99991 .00001 .00001 .00001 .0000$ $2.0-002 \quad 0.9899 \quad 0.0932 \quad 0.99490 .9966 \quad 0.9975 \quad 0.9951 \quad 0,9990 \quad 0.9993 \quad 0.9996 \quad 0.9997 \quad 0.9998 \quad 0.99990 .99991 .0000$

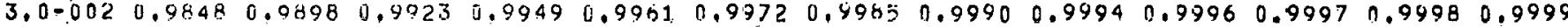

$\begin{array}{llllllllllllllll}5.0-002 & 0.9744 & 0.0828 & 0.9870 & 0.9913 & 0.9935 & 0.9952 & 0.9975 & 0.9983 & 0.9990 & 0.9993 & 0.9995 & 0.9997\end{array}$

$\begin{array}{llllllllllllll}7.0=002 & 0.9637 & 0.9755 & 0.9815 & 7.9876 & 0.9907 & 0.9932 & 0.9964 & 0.9976 & 0.9985 & 0.9989 & 0.9993\end{array}$

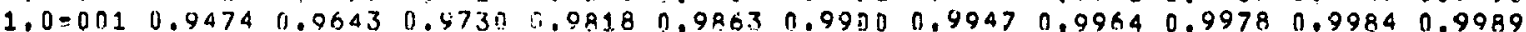

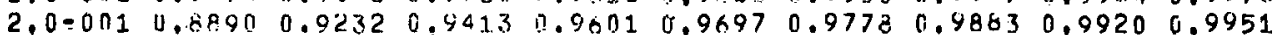

$\begin{array}{lllllllll}3,0.001 & 0.8247 & 0.4759 & 0.9039 & 0.9338 & 0.9495 & 0.9623 & 0.980 ? & 0.9865\end{array}$

5.0-001 $0.68090 .7619 \quad 0.81 .010 .66490 .8951 \quad 0.92150 .9573$

$7,0-001$
0.5301 $0.6285 \quad 0.60290 .77190 .8186 \quad 0.86 .12$

$1.0+000 \quad 0.3333 \quad 0.4286 \quad 0.5000 \quad 0.6000 \quad 0.6667 \quad 0.7333$

$2.0+000 \quad 0.0625 \quad 0.0909 \quad 0.11 .76 \quad 0.1667$

$3,0+000 \quad 0.013640 .02440,0323$

$5.0+000 \quad 0.0026 \quad 0.0038$

$7.0+000 \quad 0.0007$

$1.0+0010.0002$ 


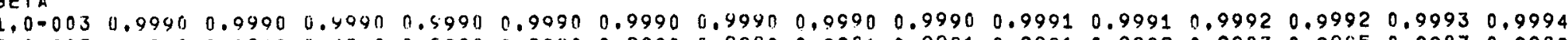

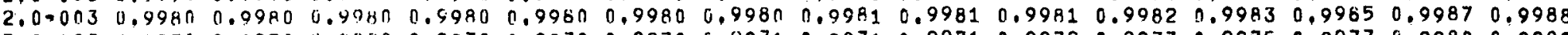
$\begin{array}{lllllllllllllllll}3.0=0 n 3 & 0.9970 & 0.9970 & 1.9070 & 0.9970 & 0.9970 & 0.9970 & 0.9971 & 0.9971 & 0.9971 & 0.9972 & 0.9973 & 0.9975 & 0.9977 & 0.9980 & 0.998 ?\end{array}$ $\begin{array}{lllllllllllllllll}5.0-003 & 0.9950 & 0.0950 & 0.9450 & 0.9950 & 0.9950 & 0.9950 & 0.9951 .0 .9951 & 0.9952 & 0.9953 & 0.9955 & 0.9958 & 0.9961 & 0.9967 & 0.9971\end{array}$

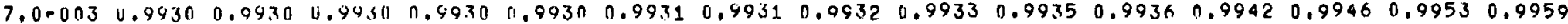

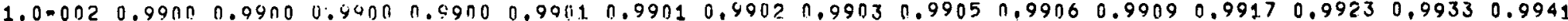

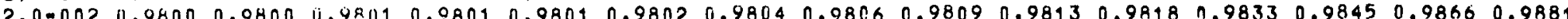
2.0-0n2 $0.9700 .07 n 10.97010 .9701097420 .97030 .97060 .97080 .97140 .97190 .97270 .97490 .97680 .97980 .9821$

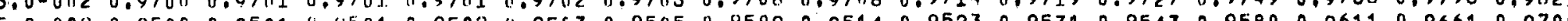

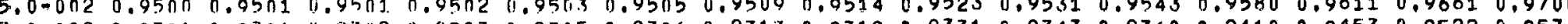
7.0 .0020 .9301 .0 .03010 .930 ? $0.93 n 3 \quad 1.93050 .93060 .93130 .93190 .9331$ 0.9343 0.93601 .94100 .94530 .95220 .9576

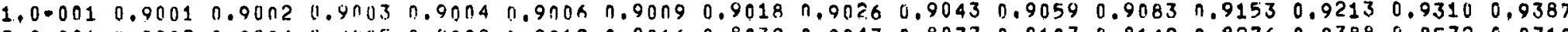

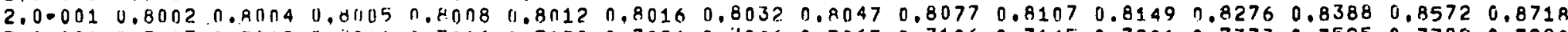

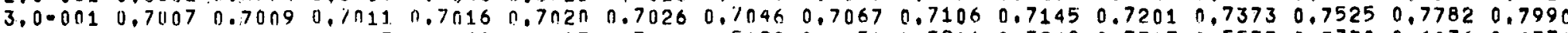
$\begin{array}{llllllllllllllllllll}5.0-001 & 0.51182 & 0.5084 & 0.5 n 47 & 0.5092 & 0.5 n 97 & 0.5104 & 0.5129 & 0.5153 & 0.5201 & 0.5248 & 0.5317 & 0.5533 & 0.5730 & 0.6076 & 0.6370\end{array}$ $\begin{array}{lllllllllllllllll}7.0-001 & 0.340 ? & 0.34 n 4 & 0.3407 & 0.3411 & 0.3416 & 0.3422 & 0.3445 & 0.3467 & 0.3510 & 0.3553 & 0.3617 & 0.3820 & 0.4011 & 0.4359 & 0.4669\end{array}$ $\begin{array}{lllllllllllllllll}1.0+000 & 0.1668 & 0.1669 & 0.1671 & 0.1674 & 0.1676 & 0.1681 & 0.1694 & 0.1708 & 0.1736 & 0.1763 & 0.1803 & 0.1935 & 0.2063 & 0.2308 & 0.2537\end{array}$

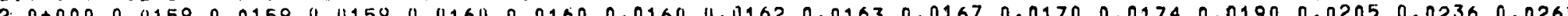
2.0+000 0.0027.0.0

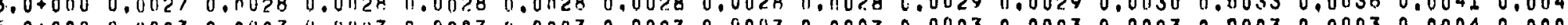

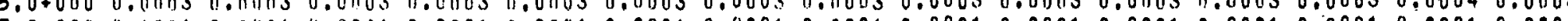

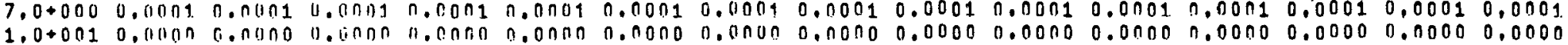

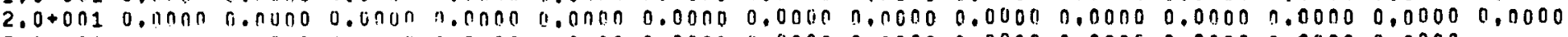
$3.0+0010.01000 .00000,601100.00000 .0000,0.00000 .00000 .00000 .00000 .00000 .00000 .00000,0000$

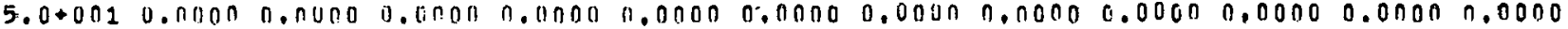

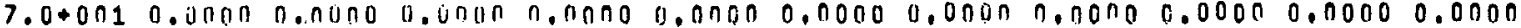

$1.0+0020.00000 .00000 .00000 .00000 .00000 .00000 .00000 .00000 .00000 .00000 .0000$

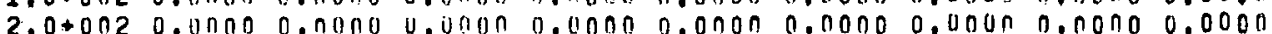

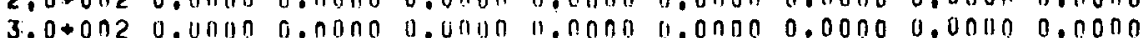

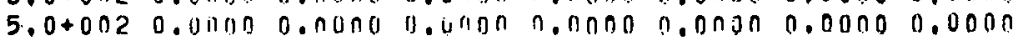

$7,0+0020.00000 .00000,4000,0,00000,0090 \quad 0,0000$

$1,0+0030.110000 .00000 .0000,0,00000.00000 .0000$

$$
\text { BETA }
$$

$1.0-003 \quad 0.9995 \quad 0.9997 \quad 0.9097 \quad 0.0998 \quad 0.99990 .9999 \quad 1.00001 .00001 .00001 .00001 .00001 .00001 .00001 .00001 .00001 .0000$ $2.00003 \quad 0.9990 \quad 0.9993 \quad 0.9995 \quad 0.0997 \quad 0.9997 \quad 0.99980 .9999 \quad 0.09991 .00011 \quad 1.00001 .00001 .00001 .00001 .00001 .00001 .0000$

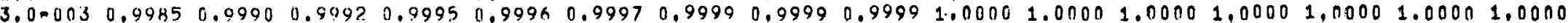
$5,0-0030.09750 .0983 \quad 0.99870 .99920 .9994 \quad 0.99950 .9994 \quad 0.09980 .99990 .99991 .00001 .00001 .00001 .00001 .00001 .0000$ 7.0-003 $0.09650 .09770 .908 ? 0.49880 .90910 .99940 .99970 .09980 .99990 .99990 .99991 .00001 .00001 .00001 .00001 .0000$ 1.0-002 $0.9950 .09660 .40750 .4983 \quad 0.99870 .99910 .99950 .99970 .99980 .99990 .99990 .99991 .00001 .00001 .00001 .0000$ $2.0-0020.98990 .09320 .99490 .49660 .99750 .99810 .9990 \quad 0.99930 .9996 \quad 0.99970 .9998 \quad 0.99990 .99991 .0000$ $\begin{array}{llllllllllllll}3.0-002 & 0.9448 & 0.0896 & 0.9923 & 0.9949 & 0.9961 & 0.9972 & 0.9985 & 0.9990 & 0.9994 & 0.9996 & 0.9997 & 0.9998 & 0.9999\end{array}$ $\begin{array}{llllllllllllll}5,0=0 & 0.9744 & 0.987 .8 & 0.9870 & 0.9913 & 0.9935 & 0.9952 & 0.9975 & 0.9983 & 0.9990 & 0.9993 & 0.9995 & 0.9997\end{array}$

$\begin{array}{llllllllllllll}7.0 .002 & 0.9637 & 0.9755 & 0.9815 & 0.9876 & 0.99117 & 0.9932 & 0.9964 & 0.9976 & 0.9985 & 0.9989 & 0.9993\end{array}$

$\begin{array}{lllllllllllll}1.0 .001 & 0.9474 & 0.0643 & 0.9730 & 0.9818 & 0.9863 & 0.9900 & 0.9947 & 0.9964 & 0.9978 & 0.9984 & 0.9989\end{array}$

$\begin{array}{lllllllllll}2.0 .001 & 0.8489 & 0.0231 & 0.9412 & 0.9600 & 0.9697 & 0.9778 & 0.980 ? & 0.9920 & 0.9951\end{array}$

$3,0.0010 .82390 .87530 .903411 .93350 .94430 .96260 .9800 \quad 0.9864$

$5.0=0010.0737 \quad 0.7559 \quad 0.8050 \quad 0.8610 \quad 0.8920 \quad 0.9191 \quad 0.9559$

$\begin{array}{lllllll}7.0-001 & 0.5075 & 0.4071 & 0.6733 & 0.7556 & 0.8047 & 0.8500\end{array}$

$1.0+000 \quad 0.2857 \quad 0.3750 \quad 0,44440.54550 .6154 \quad 0.6875$

$2,0+000 \quad 0.01313 \quad 0,0462 \quad 0,0606 \quad 0.0882$

$3,0+000 \quad 0,00550,00820,0109$

$5,0+000 \quad 0.0005 \quad 0.0008$

$7.0+000 \quad 0.0001$ 
VALUES OF G FOK $N=2$ ANII $N P=1$ BETA

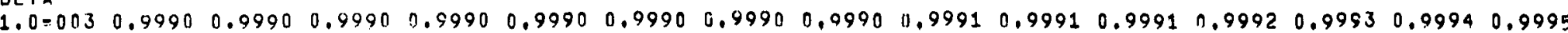

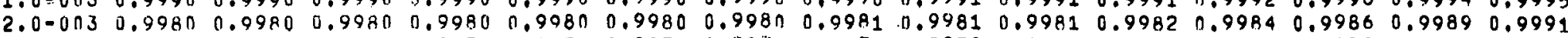
$\begin{array}{llllllllllllllll}3.0 .003 & 0.9970 & 0.9970 & 0.9970 & 0.9970 & 0.9970 & 0.9970 & 0.9971 & 0.9971 & 0.9972 & 0.9972 & 0.9973 & 0.9976 & 0.9978 & 0.9983 & 0.9986\end{array}$ $\begin{array}{lllllllllllllllll}5.0 .003 & 0.9950 & 0.9950 & 0.4950 & 0.9950 & 0.9951 & 0.9951 & 0.9951 & 0.9952 & 0.9953 & 0.9954 & 0.9955 & 0.9960 & 0.9964 & 0.9972 & 0.9977\end{array}$ $\begin{array}{llllllllllllllllll}7,0-003 & 0.9931 \cdot 0.0931 & 0.9931 & 0.9931 & 0.9931 & 0.9931 & 0.9932 & 0.9933 & 0.9934 & 0.9935 .0 .9937 & 0.9944 & 0.9950 & 0.9960 & 0.9968\end{array}$ $\begin{array}{lllllllllllllllll}1,0-002 & 0.9901 & 0.0901 & 0.4901 & 0.9901 & 0.9902 & 0.9902 & 0.9903 & 0.9904 & 0.9906 & 0.9908 & 0.9911 & 0.9920 & 0.9929 & 0.9943 & 0.9955\end{array}$ $2,0-002 \quad 0.9804 \quad 0.98040 .98040 .98050 .98050 .98060 .98080 .9810 \quad 0.98140 .98170 .98230 .98410 .98580 .98870 .9010$ $3,0-0020.97090 .97090 .97100 .97100 .971100 .97120 .97140 .97170 .97230 .97280097370 .97640 .97890 .90310 .9865$

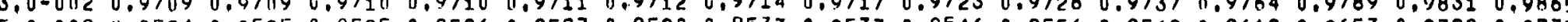

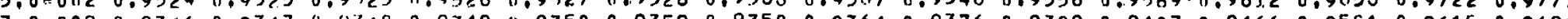

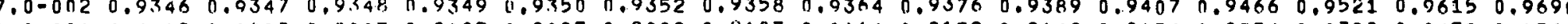
$\begin{array}{llllllllllllllllll}1.0001 & 0.9092 & 0.9093 & 0.9 n 93 & 0.3095 & 0.9 n 97 & 0.9099 & 0.9107 & 0.9116 & 0.9132 & 0.9149 & 0.91 .74 & 0.9254 & 0.9329 & 0.9459 & 0.9563\end{array}$

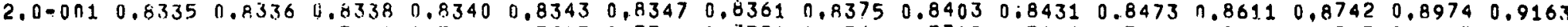
$\begin{array}{llllllllllllllllll}3,0.001 & 0.7694 & 0.7696 & 0.7498 & 0.77 n 1 & 0.7705 & 0.7710 & 0.7728 & 0.7746 & 0.7782 & 0.7818 & 0.7872 & 0.8052 & 0.8225 & 0.8537 & 0.8795\end{array}$ $\begin{array}{llllllllllllllllllll}5.0-001 & 0.6669 & 0.6671 & 0.6673 & 0.6678 & 0.6632 & 0.6689 & 0.6711 & 0.6734 & 0.6779 & 0.6825 & 0.6894 & 0.7126 & 0.7354 & 0.7778 & 0.8141\end{array}$ $\begin{array}{llllllllllllllllll}7.0=001 & 0.5885 & 0.5887 & 0.5490 & 0.5894 & 0.5999 & 0.5907 & 0.5931 & 0.5956 & 0.6006 & 0.6056 & 0.6133 & 0.6392 & 0.6651 & 0.7143 & 0.7578\end{array}$ $\begin{array}{lllllllllllllllll}1.0+000 & 0.5003 & 0.5005 & 0.5008 & 0.5013 & 0.5 n 1 R & 0.5025 & 0.5050 & 0.5076 & 0.5128 & 0.5180 & 0.5261 & 0.5536 & 0.5816 & 0.6364 & 0.6865\end{array}$ 2.0+000 0.3336 0.33380 .33400 .33440 .33490 .33560 .33780 .34010034480 .34960 .35690 .38270 .41000 .46670 .5227 $3.0+0000.25020 .25040 .25060 .25090 .25130 .25190 .25380 .25570 .25970 .06300 .27010 .02950 .31060 .36840 .4220$

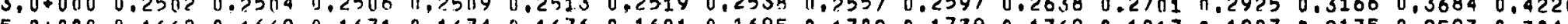
$5.0+0000.16680 .16690 .16710 .16740 .16760 .16810 .1695 n .17 n-9.17390 .17690 .1217 n .19870 .21750 .25930 .3046$ $\begin{array}{llllllllllllllll}7.0+000 & 0.1251 & 0.1252 & 0.1253 & 0.1255 & 0.1258 & 0.1261 & 0.127 ? & 0.1284 & 0.1307 & 0.1331 & 0.1369 & 0.1505 & 0.1657 & 0.2000 & 0.2383\end{array}$

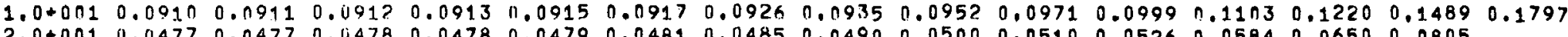

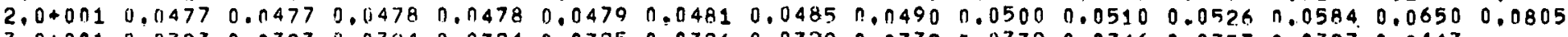
$\begin{array}{lllllllllllllll}3,0+001 & 0.032 .3 & 0.0323 & 0.0324 & 0.0324 & 0.0325 & 0.0326 & 0.0329 & 0.0332 & 0.0339 & 0.0346 & 0.0357 & 0.0397 & 0.0443\end{array}$

$5.0+0010,0196 \quad 0.0196 \quad 0.0197 \quad 0.0197 \quad 0.0197 \quad 0.0198 \quad 0.02000 .0202 \quad 0.0206 \quad 0.021 .0 \quad 0.0717 \quad 0.0242$

$\begin{array}{llllllllllllllllllll}7.0+001 & 0.0141 & 0.0141 & 0.0141 & 0.0142 & 0.014 ? & 0.0142 & 0.0144 & 0.0145 & 0.0148 & 0.0151 & 0.01 .56\end{array}$

$1.0+002.0 .00990 .00990 .00990 .0100 \quad 0.0100 \quad 0.0100 \quad 0.01010 .01020 .01040 .01060 .0110$

$2,0+0020.0050 \quad 0.00500 .00500 .0050 \quad 0.0050 \quad 0.0050 \quad 0.0051 \quad 0.00510 .0052$

$3,0+002 \quad 0.00330 .00330 .00330 .00330 .00330 .00340 .00340 .0034$

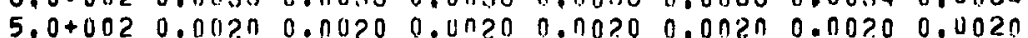

$7.0+0020.00140 .00140 .00140 .00140 .00140 .0014$

$1,0+0030.00100 .00100 .00100 .00200 .00100 .0010$ BET

ALPHA $1.000 \quad 2.000 \quad 3.000 \quad 5.000 \quad 7.000 \quad 10.00 \quad 20,00 \quad 30.00 \quad 50.00 \quad 70.00 \quad 100.0 \quad 200.0 \quad 300,0 \quad 500.0 \quad 700.0 \quad 1000$

1.000030 .99970 .99990 .99991 .00001 .00001 .00001 .00001 .00001 .00001 .00001 .00001 .00001 .00001 .00001 .00001 .0000 2, $0=0030.99930 .99970,90980,99991.00001 .00001 .00001 .00001 .00001,00001.00001 .00001 .00001 .00001 .00001 .0000$ $3,0=0030.9990 \quad 0.99960 .99980 .99990 .99991 .00001 .00001 .00001 .00001 .00001 .00001 .00001 .00001 .00001 .00001 .0000$ $5.0=0030.99830 .99930 .99960 .99980 .99991 .00001 .00001 .00001 .00001 .00001 .00001 .00001 .00001 .00001 .00001 .0000$

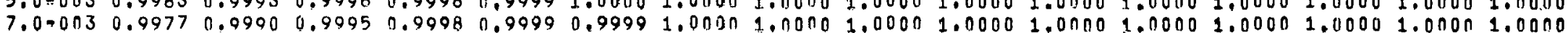

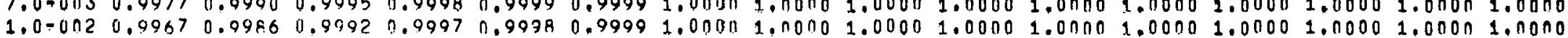

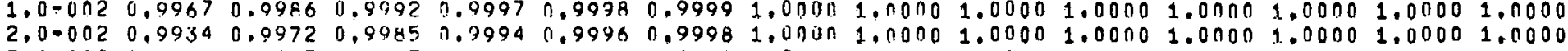
$3.0-0020.99010 .9957 \quad 0.99770 .99900 .99750 .99970 .99991 .00001 .00001 .00001 .00001 .00001 .0000$

$5,0=0020.98360 .99290 .99620 .99840 .99910 .99950 .99900 .99991 .00001 .00001 .00001 .0000$

$7,0.002 \quad 0.9772 \quad 0.0901 \quad 0.9946 \quad 0.9977 \quad 0.9988 \quad 0.9994 \quad 0.9998 \quad 0.99991 .00001 .00001 .0000$

$1,0=001 \quad 0.9677 \quad 0.98590 .9924 \quad 0.9968 \quad 0.9982 \quad 0.9991 \quad 0.9998 \quad 0.99991 .00001 .00001 .0000$

$\begin{array}{llllllllll}2.0-001 & 0.9375 & 0.0722 & 0.9848 & 0.9936 & 0.9905 & 0.9982 & 0.9995 & 0.9998 & 0.9999\end{array}$

$3.0-0010.90910 .95890 .9774 \quad 0.990 / 4 \quad 0.9948 \quad 0.9973 \quad 0.9993 \quad 0.9997$

$5,0.0010 .85710 .93330 .96300 .98410 .99130 .99550 .9938$

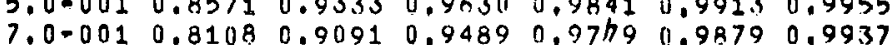

$1,0+000 \quad 0.7500 \quad 0.8750 \quad 0.9286 \quad 0.94880 .98280 .9911$

$2,0+000 \quad 0.6000 \quad 0.7778 \quad 0.8467 \quad 0.9894$

$3,0+000 \quad 0.5000 \quad 0.70 n 0 \quad 0.8125$

$5.0+000 \quad 0.3750 \quad 0.5833$

$7.0+000 \quad 0.3000$

$1,0+001 \quad 0,230$ 
GET SETA

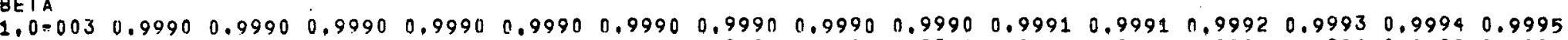

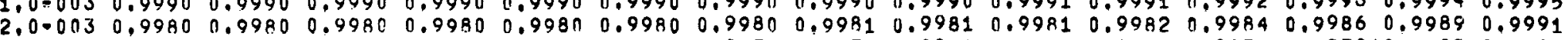

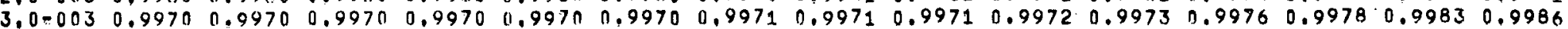
$\begin{array}{llllllllllllllll}5.0-003 & 0.9950 & 0.9950 & 0.9950 & 0.9950 & 0.9950 & 0.9950 & 0.9951 & 0.9951 & 0.9952 & 0.9953 & 0.9955 & 0.9960 & 0.9964 & 0.9971 & 0.9977\end{array}$

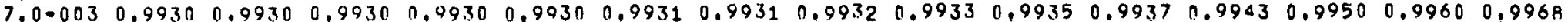

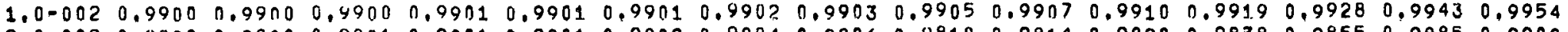
$\begin{array}{llllllllllllllll}1.0 .002 & 0.9800 & 0.9800 & 0.9801 & 0.9801 & 0.9801 & 0.9802 & 0.9804 & 0.9806 & 0.9810 & 0.9814 & 0.9820 & 0.9838 & 0.9855 & 0.9885 & 0.9908\end{array}$ $3,0-0020.97010 .97 n 10.97010 .97020 .97020 .97030 .97060 .97090 .97150 .97210 .97290 .97570 .97830 .98260 .9861$

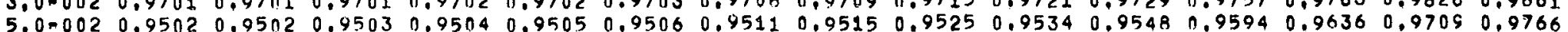

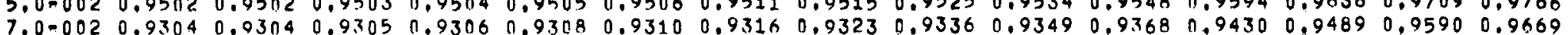

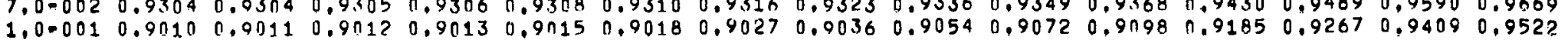

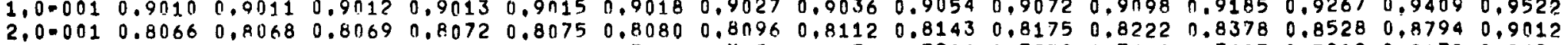
$\begin{array}{llllllllllllllllll}3.0001 & 0.7196 & 0.7198 & 0.7200 & 0.7204 & 0.7208 & 0.7214 & 0.7235 & 0.7255 & 0.7296 & 0.7338 & 0.7400 & 0.7607 & 0.7809 & 0,8178 & 0.8488\end{array}$ $\begin{array}{llllllllllllllllll}5.0=001 & 0.5717 & 0.5719 & 0.5722 & 0.5727 & 0.5731 & 0.5739 & 0.5764 & 0.5789 & 0.5839 & 0.5890 & 0.5968 & 0.6231 & 0.6495 & 0.7000 & 0.7449\end{array}$ $\begin{array}{lllllllllllllllll}7.0=001 & 0.4569 & 0.4571 & 0.4574 & 0.4579 & 0.4584 & 0.4591 & 0.4616 & 0.4642 & 0.4693 & 0.4746 & 0.4826 & 0.5103 & 0.5388 & 0.5952 & 0.6479\end{array}$ $\begin{array}{lllllllllllllllll}1.0+000 & 0.3336 & 0.3338 & 0.3340 & 0.3344 & 0.3349 & 0.3356 & 0.3378 & 0.3401 & 0.3448 & 0.3496 & 0.3569 & 0.3827 & 0.4100 & 0.4667 & 0.5227\end{array}$ $2,000000.14300 .14310 .14320 .14350 .14370 .14410 .14530 .14660 .14920 .15190 .15610 .17430 .18810 .22580 .2674$ 3.000000 .07700 .07710 .07710 .07730 .07740 .07760 .07840 .07910 .08060 .08220 .08470 .09370 .10380 .12730 .1543

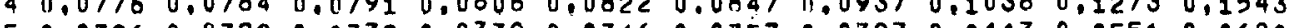
$5,040000.03230 .03230 .03240 .03240 .03250 .03260 .03290 .03320 .03390 .03460 .03570 .03970 .04430 .05510 .0680$ $7,0+0000.01760 .01760 .01760 .01760 .01770 .01770 .01790 .01810 .01840 .01880 .01940 .02170,02420,03030.0376$

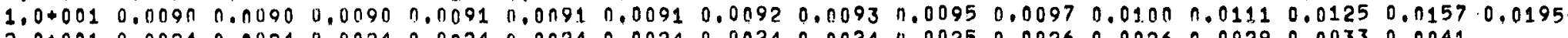
$2.0+0010.00240 .00240 .0 n 24 \quad 0.0024 \quad 0.0024 \quad 0.00240 .00240 .0024 \quad 11.00250 .00260 .0026 \quad 0.00290 .0033 \quad 0.0041$

$3,0+0010.00110 .00110 .00110 .00110 .00110 .00110 .00110 .00110 .00110 .00120 .00120 .00130 .0015$

$5,0+0010.00040 .00040 .00040 .00040 .00040 .00040 .00040 .00040 .00040 .00040 .00040 .0005$

$7,0+0010.00020 .00020 .00020 .00020 .00020 .00020 .00020 .00020 .00020 .00020 .0002$

$1.0+0020.00010 .00010 .00010 .00010 .00010 .00010 .00010 .00010 .00010 .00010 .0001$

$2,0+0020,0000$ 0.0000 $0.00000,00000,00000,00000,00000,00000,0000$

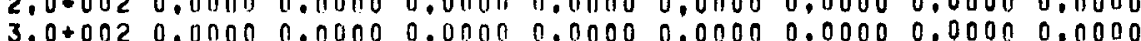

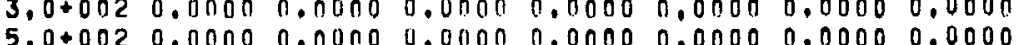

$7,0+0020.00000,00000,0000,0,0000,00000,0000$

$1,0.0030,00000,0000,00000,00000,00000,0000$

ALPHA $1.000 \quad 2.000 \quad 3.000 \quad 5.000 \quad 7.000 \quad 10.00 \quad 20.00 \quad 30.00 \quad 50.00 \quad 70.00 \quad 100.0 \quad 200.0 \quad 300.0 \quad 500.0 \quad 700.0 \quad 1000$ BETA

$1.0-0030.99970 .99990 .99991 .00001 .00001,00001.00001,00001.00001,00001,00001.00001,00001,00001,00001.0000$ 2.0 .0030 .99930 .99970 .99980 .99991 .00001 .00001 .00001 .00001 .00001 .00001 .00001 .00001 .00001 .00001 .00001 .0 .000

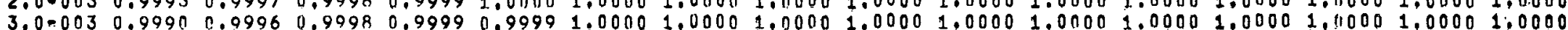

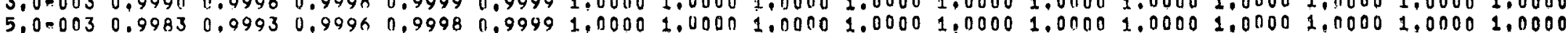

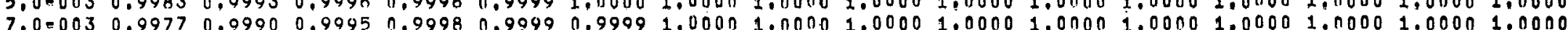

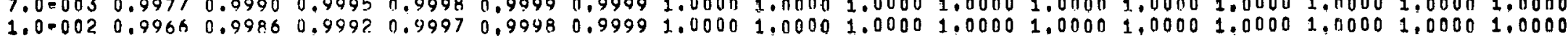

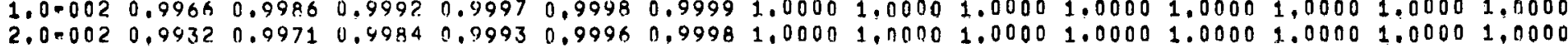
$3.0=002 \quad 0.9898 \quad 0.9956 \quad 0.9976 \quad 0.9990 \quad 0.9995 \quad 0.9997 \quad 0.99991 .00001 .00001 .00001 .00001 .00001,0000$

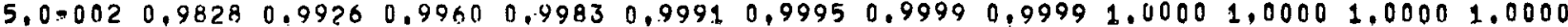

$7.0=0020.9756 \quad 0.9894 \quad 0.9943 \quad 0.9976 \quad 0.9987 \quad 0.9993 \quad 0.9998 \quad 0.99991 .00001 .00001 .0000$

$1,0=0010.9646 \quad 0.0845 \quad 0.9916 \quad 0.9965 \quad 0.9981 \quad 0,9990 \quad 0,9997 \quad 0.99991 .00001 .00001 .0000$

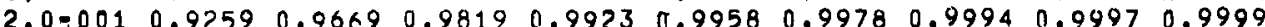

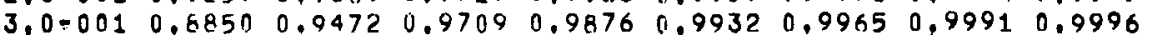

$\begin{array}{lllllllll}5.0=001 & 0.6000 & 0.9032 & 0.9455 & 0.9764 & 0.9870 & 0.9933 & 0.9982\end{array}$

$7,0=001 \quad 0,7160 \quad 0.9547 \quad 0,9161 \quad 0.96300,97950,9894$

$1.0 .000 \quad 0.6000 \quad 0.7778 \quad 0.8667 \quad 0.93940 .96610 .9823$

$2.0 .000 \quad 0.33330 .53850 .68420 .8376$

$3,0+000 \quad 0,2000 \quad 0.36840 .5200$

$5,0+000 \quad 0.0909 \quad 0.1892$

$7.0+000 \quad 0.0508$

$1.0+001 \quad 0.0265$ 
VALUES OF G FOR $N=2$ AND $N P=3$ BETA

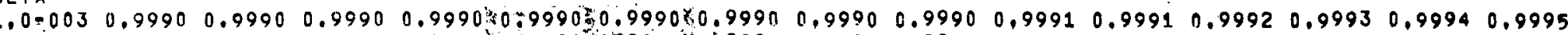

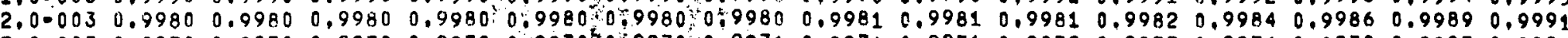
$3,0=003 \quad 0.9970 \quad 0.9970 \quad 0.997 n \quad 0.9970 \cdot 0.9970 \times 0.997000 .9971 \quad 0.9971 \quad 0.9971 \quad 0.9972 \quad 0.9973 \quad 0.9976 \quad 0.9978 \quad 0.9983 \quad 0,9986$ $\begin{array}{llllllllllllllll}5.07003 & 0.9950 & 0.9950 & 0.9950 & 0.9950 & 0.9950 & 0.9950 & 0.9951 & 0.9951 & 0.9952 & 0.9953 & 0.9955 & 0.9960 & 0.9964 & 0.9971 & 0.9977\end{array}$

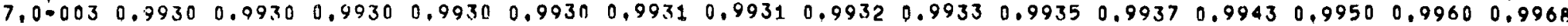
$1.07002 \quad 0.7900 \quad 0.9900 \quad 0.9900 \quad 0.9900,0.9901-0.9901,0.9902 \quad 0.9903 \quad 0.99050 .9907 \quad 0.9910 \quad 0.9919 \quad 0.9928 \quad 0.9943 \quad 0.9954$

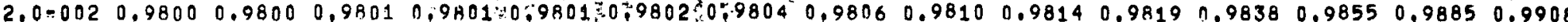

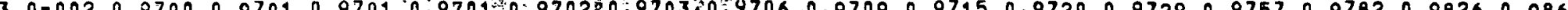

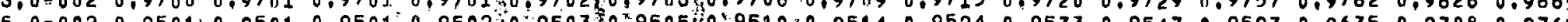
$5.0=0020.9510 .05120 .95010 .95020 .95030 .950540 .95130 .95140 .95240 .9533 .0 .9547 n .95930 .96350 .97080 .9765$ . 0.0020 .93010 .93020 .93020 .93030 .930570 .93070 .93130 .93200 .93330 .934640 .93650 .94280 .94860 .95880 .9668 $1.0=0010.9002 .0 .9003 \quad 0.9004 \quad 0.90050 .90070 .90100 .90190 .90280 .90460 .90640 .90910 .91780 .9260 \quad 0.9404 \quad 0.9518$

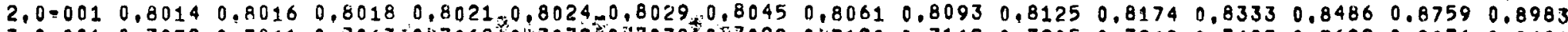

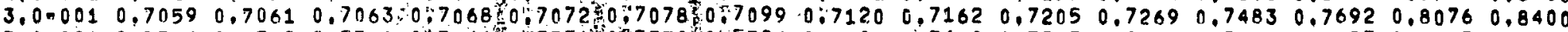

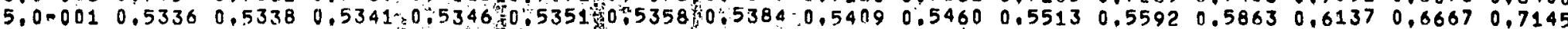

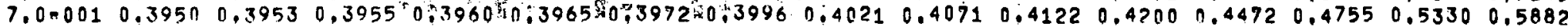

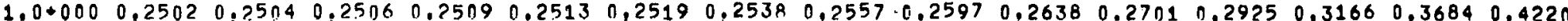
$2.0+000 \quad 0.06670 .06680 .06690 .06700 .06710 .06730 .06790 .06860 .06990 .07130 .07350 .08140 .09030 .11110 .1353$ $3,0.0000 .02500 .0250$ 0.0251 0.02510 .02520 .02520 .02550 .02500 .02630 .02680 .02770 .03080 .03440 .04290 .0532

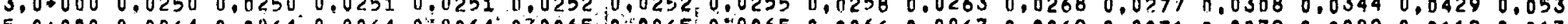
5.0.000 0.0064

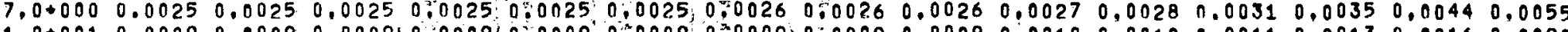
$1.0+0010.00090,00090.0009 \times 0.000910 .00090,00090700090,00090.00090,00100.00100 .00110 .00130 .00160 .0020$ $2,0+0010.00010 .00010 .00010 .00010 .00010 .00010 .00010 .00010 .00010 .00010 .00010 .00010 .00020 .0002$ $3,0+0010.0000 \quad 0.00000 .00000 .00000 .00000 .00000 .00000 .00000 .00000 .00000 .00000,00000,0000$

$5,0+0010.0000 \quad 0,0000,0,0000.0,0000,0.0000,0,0000,0,0000,0,00000,00000,00000.00000,0000$

$7,0+0010,00000,0000.0,00000,0000,0,0000,0,0000,0,0000,0,00000.00000,00000,0000$

$1.0+0020.00000 .00000 .0000: 0,00000 \% 00000 \% 00000700000000000000000.0000 .0 .0000$

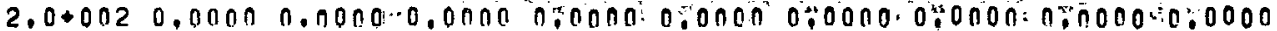

$3,0+0020.00000 .00000 .0000 \quad 0,00000,0000 \quad 0,00000,00000.0000$

$5,000020,00000,0000.0,00000,00000,00000,00000,0000$

$7.0+0020.00000 .00000,00000.0000 .0 .00000,0000$

$1,0+0030,00000,0000,0,00000,00000,00000 \% 0000$

$$
\text { BETA }
$$
BETA

ALPHA $1.000 \quad 2.000 \quad 3.0 .00 \% 5 \% 000 \% 7000510,00 \quad 20.00 \quad 30000.50 .00 \quad 70.00 \quad 100.0 \quad 200.0 \quad 300.0 \quad 500.0 \quad 700.0$

$1,0=0030.99970 .99990 .99991 .00001 .00001 .00001 .00001 .00001 .00001 .00001 .00001 .00001 .00001 .00001 .00001 .0000$ $2,0=0030.99930 .99970 .9998,0.9999,1.00001,00001.00001 .00001 .00001 .00001 .00001 .00001 .00001 .00001 .00001 .0000$ $3.000030 .9990 \quad 0.09960 .99980 .99990799991 \% 00001 \% 00001.0000: 1.00001 .00001 .00001 .00001 .00001 .00001 .00001 .0000$

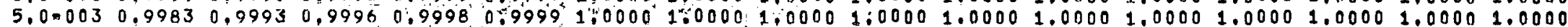

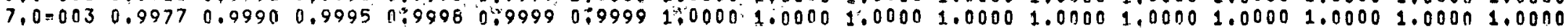
$1.0=002 \quad 0.996 \pi \quad 0.9986 \quad 0.9992 .0 .99970 .99980 .99991 .00001 .00001 .00001 .00001 .00001 .00001 .00001 .00001 .00001 .0000$ $2,0=0020.9932 \quad 0.9971 .0 .99840 .9993 \quad 0.9996 \quad 0.99981 .00001 .00001 .00001 .00001 .00001 .00001 .00001 .0000$ $3,0=002 \quad 0.9898 \quad 0.9956 \quad 0.9976 .0,9990.0,9995,0,9997.0,9999.1 .00001 .00001 .00001 .00001 .00001 .0000$

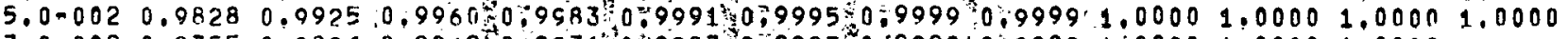

$7.0-002 \quad 0.97550 .9894-0.994250,997610099870099930,999900.9999,1,00001.00001 .0000$

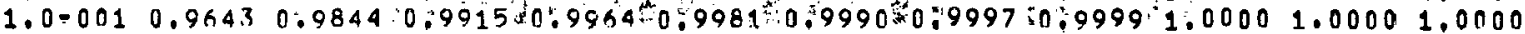

$\begin{array}{llllllllllll}2.00001 & 0.9236 & 0.965 E & 0.9813 & 0.9921 & 0.9957 & 0.9978 & 0.9994 & 0.9997 & 0.9999\end{array}$

$3.0=001,0.8780 \quad 0.0438 \quad 0.9689 \quad 0.9667 \quad 0.9927 \quad 0.9963 \quad 0.9990 \quad 0.9996$

$5,0=001 \quad 0.7742 \quad 0.8885 \quad 0.936 .9 .0 .97250 .9849 .0 .99220 .9979$

$7.0=001 \quad 0.6618 \quad 0.8203 \quad 0.894500,95290 \% 9738 \times 0.9864$

$1.0+000 \quad 0.5000 .0,7000 \quad 0,8125,0.9118,0,950010 \% 9737$

$2,0+000 \quad 0.1765 \cdot 0.3333 \quad 0.4815,0 \% 889$

$3.0+000 \quad 0.0714 \quad 0.15 ? \bar{c} \quad 0.2500$

$5.0+000 \quad 0.0190 \quad 0.043 c$

$7.0+000 \quad 0.0075$

$1.0+001 \quad 0.0027$ 


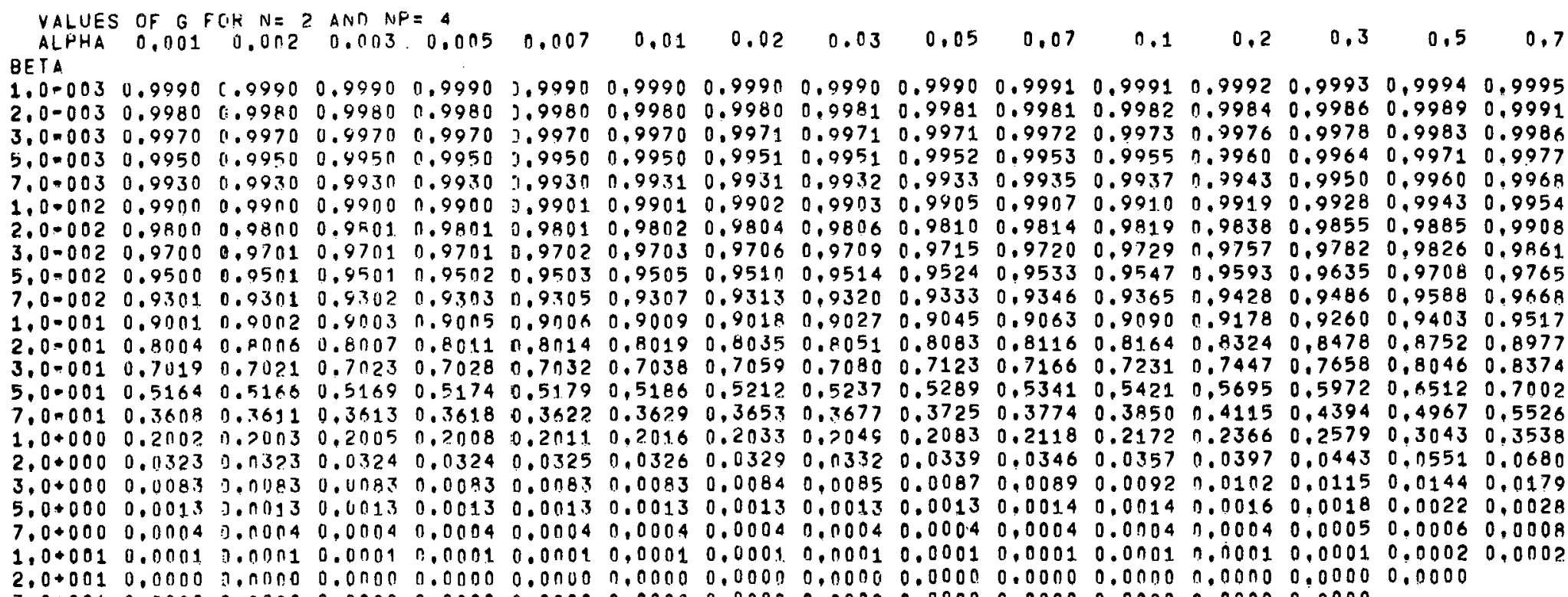


VALUES OF G FOR $N=2$ AND NP= 5 BETA

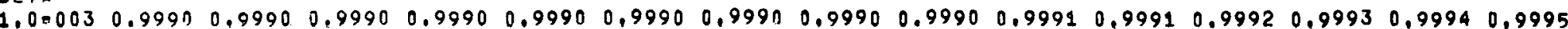

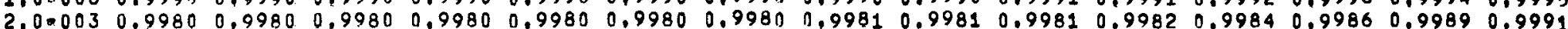

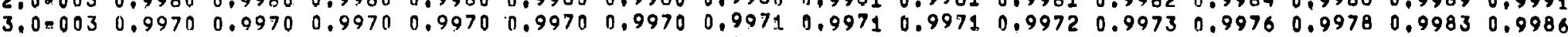

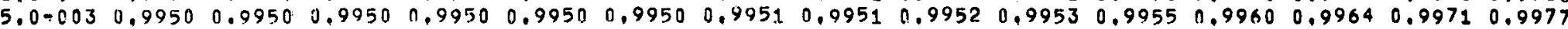
$\begin{array}{llllllllllllllll}7.07003 & 0.9930 & 0.9930 & 0.9930 & 0.9930 & 0.9930 & 0.9931 & 0.9931 & 0.9932 & 0.9933 & 0.9935 & 0.9937 & 0.9943 & 0.9950 & 0.9960 & 0.9968\end{array}$

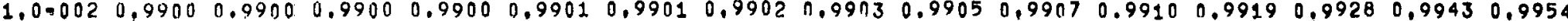
$\begin{array}{llllllllllllllll}2,0-002 & 0.9800 & 0,9800 & 0.9801 & 0,9801 & 0,9801 & 0,9802 & 0.9804 & 0,9806 & 0.9810 & 0,9814 & 0.9819 & 0,9838 & 0,9855 & 0,9885 & 0.9908\end{array}$ $\begin{array}{llllllllllllllll}3.0=002 & 0.9700 & 0.9701 & 0.9701 & 0.9701 & 0.9702 & 0.9703 & 0.9706 & 0.9709 & 0.9715 & 0.9720 & 0.9729 & 0.9757 & 0.9782 & 0.9826 & 0.9861\end{array}$

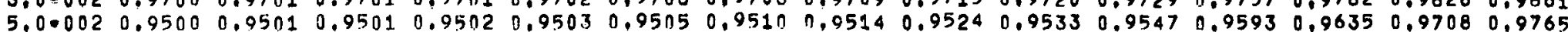
$5.0 .0020 .9500 \quad 0.95010 .95010 .95120 .95030 .95050 .9510 \quad 1.95140 .9540 .95330 .95470 .95930 .96350 .97080 .9765$

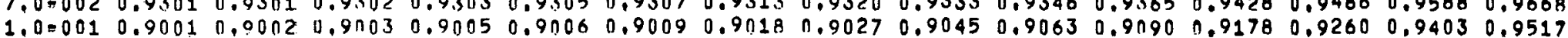

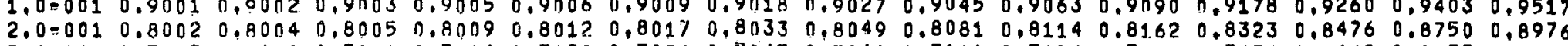

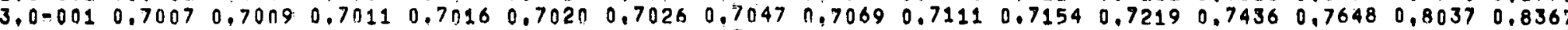
$\begin{array}{llllllllllllllll}5.0-001 & 0.508 ? & 0.5084 & 0.5087 & 0.5092 & 0.5 n 97 & 0.5104 & 0.5130 & 0.5155 & 0.5207 & 0.5260 & 0.5340 & 0.5614 & 0.5893 & 0.6437 & 0.6933\end{array}$ $\begin{array}{lllllllllllllllllllll}7.0 & 001 & 0.340 \% & 0.3405 & 0.3407 & 0.3411 & 0.3416 & 0.3423 & 0.3445 & 0.3469 & 0.3516 & 0.3564 & 0.3638 & 0.3898 & 0.4173 & 0.4741 & 0.5301\end{array}$

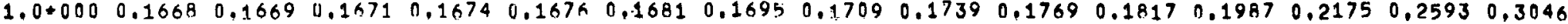

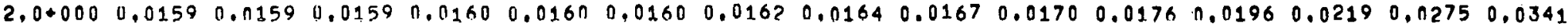
$3,0+000 \quad 0.00270 .0028 \quad 0.00280 .00280 .00280 .00280 .00280 .00280 .00290 .00300 .00300 .00340 .00380 .00480 .0060$ $5,0+0000.00030 .00030 .00030 .00030 .00030 .00030 .00030 .00030 .00030 .00030 .00030 .00030 .00040 .00040 .0006$

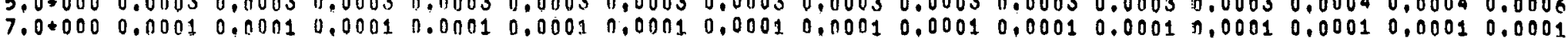

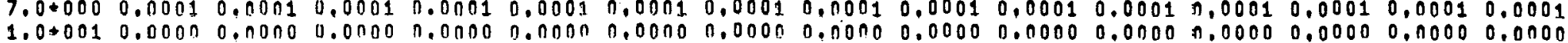
$2.0+0010.00000 .00000 .00000 .00000 .00000 .00000 .00000 .00000 .00000 .00000 .00000 .00000 .00000 .0000$

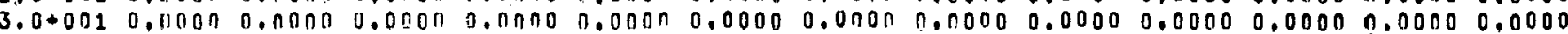

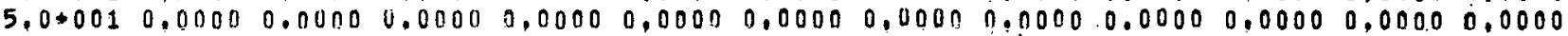

$7.0+0010.0000 \quad 0.00000 .00000 .00000 .00000 .00000 .00000 .00000 .00000 .00000 .0000$

$1.000020 .00000,0000.0,00000.00000,0000 \quad 0,00000.00000,00000.00000,00000,0000$

2.0.002 0.0000 $0.0000 \quad 0.0000 \quad 0.0000 .0 .0000 \quad 0,00000.0000 \quad 0.00000 .0000$

$3,0+002 \quad 0.00000 .0000 \quad 0.00000 .0000,0.00000 .0000 \quad 0.0000 \quad 0.0000$

$5.0+002 \quad 0.00000 .00000 .00010 .0000 \quad 0.00000 .00000 .0000$

$7,0+0020,00000.00000 .0000,0,00000,00000,0000$

$1,0 \neq 0030,00000,00000,00000,00000,0000.0,0000$

ALPHA $1.000 \quad 2.000 \quad 3.000 \quad 5.000 \quad 7.000 \quad 10.00 \quad 20.00 \quad 30.00 \quad 50.00 \quad 70.00 \quad 100.0 \quad 200.0 \quad 300.0 \quad 500.0 \quad 700.0 \quad 1000$ BETA

1.000030 .99970 .99990 .99991 .00001 .00001 .00001 .00001 .00001 .00001 .00001 .00001 .00001 .00001 .00001 .00001 .0000 $2,0=0030.99930 .9997 \quad 0.99980 .99991,00001.00001 .00001 .00001,00001,00001.00001 .00001,00001,00001.00001,0000$ $3.0-0030.99900 .09960 .99980 .99790 .99901 .00001 .40001 .00001 .00001,00001.00001 .00001,00001.00001 .00001 .0000$

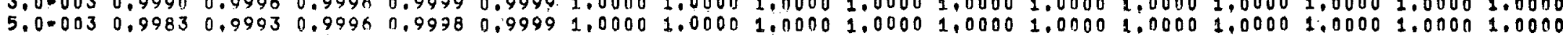

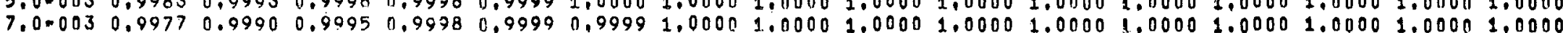

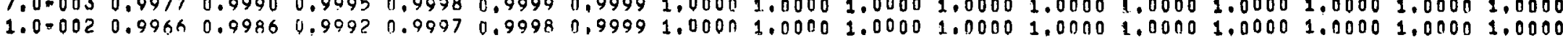

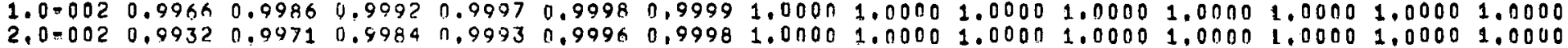
$3.0=0020.9898 \quad 0.9956 \quad 0.9976 \quad 0.9990 \quad 0.9995 \quad 0.9997 \quad 0.99991 .00001 .00001 .00001 .00001 .00001 .0000$

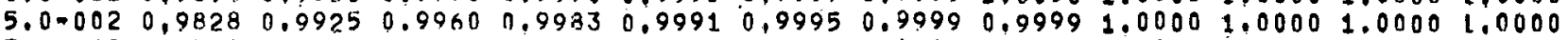
$\begin{array}{llllllllllll}7.0002 & 0.9755 & 0.9894 & 0.9942 & 0.9976 & 0.9987 & 0.9993 & 0.9998 & 0.9999 & 1.0000 & 1.0000 & 1.0000\end{array}$ $\begin{array}{lllllllllll}1.0-001 & 0.9643 & 0.9844 & 0.9915 & 0.99 \times 4 & 0.9981 & 0.9990 & 0.9997 & 0.9999 & 1.0000 & 1,00001,0000\end{array}$ $\begin{array}{lllllllllll}2.0 .001 & 0.9231 & 0.9655 & 0.9811 & 0.9920 & 0.9956 & 0.9978 & 0.9994 & 0.9997 & 0.9999\end{array}$

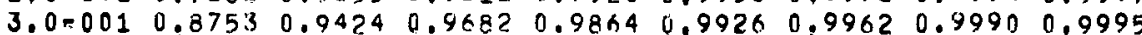

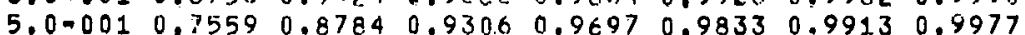

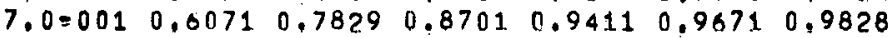

$\begin{array}{lllllll}1,0+000 & 0,3750 & 0.5833 & 0,7222 & 0.8611 & 0.9194 & 0.9569\end{array}$

$2.0+000 \quad 0.04620 .1014 \quad 0.17330 .3333$

$3,0+000 \quad 0,0082 \quad 0.0189 \quad 0.0346$

$5,0+000 \quad 0.0008 \quad 0.0018$

$7,0+000 \quad 0.5002$

$1.0+0010.600 .0$ 


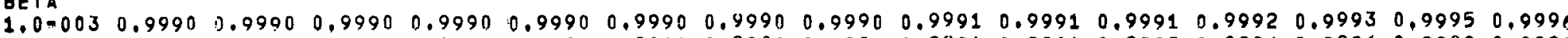
$\begin{array}{llllllllllllllll}2.0=003 & 0.9980 & 0.9980 & 0.9980 & 0.9980 & 0.9980 & 0.9980 & 0.9980 & 0.9981 & 0.9981 & 0.9981 & 0.9982 & 0.9984 & 0.9986 & 0.9989 & 0.9992\end{array}$ $\begin{array}{llllllllllllllll}3,0.003 & 0.9970 & 1.9970 & 0.9970 & 0.9970 & 0.9970 & 0.9970 & 0.9971 & 0.9971 & 0.9972 & 0.9972 & 0.9973 & 0.9976 & 0.9979 & 0.9984 & 0.9988\end{array}$

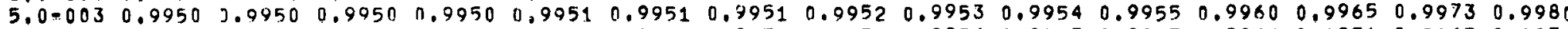
$\begin{array}{llllllllllllllllll}7.0-003 & 0.9931 & 3.9931 & 0.9931 & 0.9931 & 0.9931 & 0.9931 & 0.9932 & 0.9933 & 0.9934 & 0.9935 & 0.9937 & 0.9944 & 0.9951 & 0.9963 & 0.9972\end{array}$

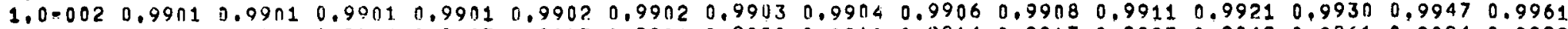
$\begin{array}{llllllllllllllll}2,0.002 & 0.9804 & 0.9804 & 0.9804 & 0.9805 & 0.9805 & 0.9806 & 0.9808 & 0.9810 & 0.9814 & 0.9817 & 0.9823 & 0.9842 & 0.9861 & 0.9894 & 0.9922\end{array}$ $\begin{array}{llllllllllllllll}3,0-002 & 0.9709 & 0.0709 & 0.9710 & 0.9710 & 0.9711 & 0.9712 & 0.9714 & 0.9717 & 0.9723 & 0.9729 & 0.9737 & 0.9765 & 0.9793 & 0.9843 & 0.9883\end{array}$

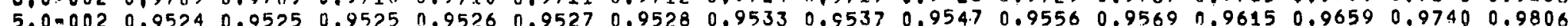

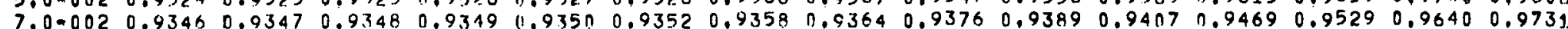

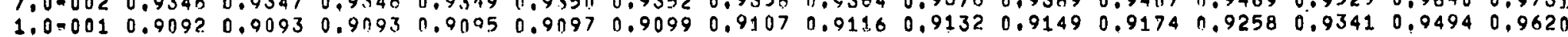
$\begin{array}{lllllllllllllllll}2.0-001 & 0.8335 & 0.8336 & 0.8338 & 0.8340 & 0.8343 & 0.8347 & 0.8361 & 0.8375 & 0.8403 & 0.8432 & 0.8474 & 0.8619 & 0.8763 & 0.9036 & 0.9268\end{array}$ $\begin{array}{lllllllllllllllll}3.0001 & 0.7694 & 0.7696 & 0.7698 & 0.7701 & 0.7705 & 0.7710 & 0.7728 & 0.7746 & 0.7782 & 0,7819 & 0.7874 & 0.8062 & 0.8253 & 0.8621 & 0.8941\end{array}$ $\begin{array}{llllllllllllllllll}5,0.001 & 0.6669 & 0.0671 & 0.6673 & 0.6678 & 0.6682 & 0.6689 & 0.6711 & 0.6734 & 0.6780 & 0.6826 & 0.6896 & 0.7140 & 0.7392 & 0.7895 & 0.8351\end{array}$ $\begin{array}{lllllllllllllllllll}7.0-001 & 0.5885 & 0.5887 & 0.589 n & 0.5894 & 0.5899 & 0.5907 & 0.5931 & 0.5956 & 0.6006 & 0.6057 & 0.6135 & 0.6407 & 0.6693 & 0.7282 & 0.7835\end{array}$ $\begin{array}{llllllllllllllllll}1.0+000 & 0.5003 & 0.5005 & 0.5008 & 0.501 .3 & 0.5018 & 0.5025 & 0.5051 & 0.5076 & 0.5128 & 0.5181 & 0.5263 & 0.5552 & 0.5863 & 0.05522 & 0.717 n\end{array}$ $2,0+0000.33360 .33380 .33400 .33440 .33490 .33560 .33780 .34040 .34480 .34960 .35710 .38420 .41470 .48390 .558$ 3.00000 .25020 .25040 .25060 .25090 .25130 .25190 .25380 .25580 .25970 .26380 .27030 .29380 .32080 .38460 .4578

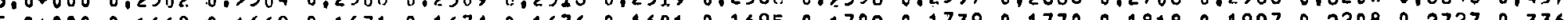

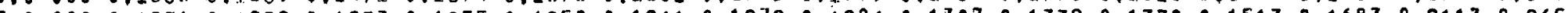

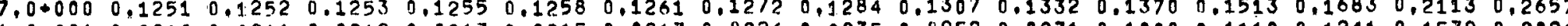

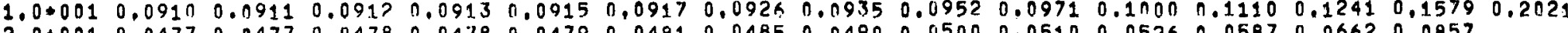

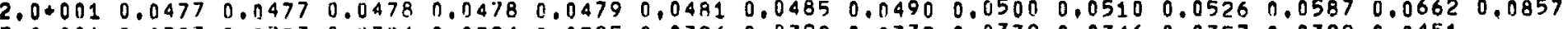

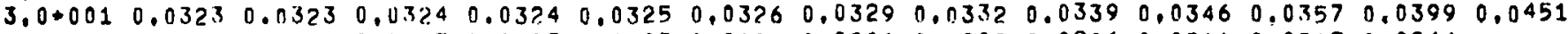

$\begin{array}{llllllllllllllll}5.0+001 & 0.01 .96 & 0.0196 & 0.0197 & 0.0197 & 0.0197 & 0.0198 & 0.0200 & 0.0202 & 0.0206 & 0.0211 & 0.0217 & 0.0244\end{array}$

$\begin{array}{llllllllllllllllll}7.0+001 & 0.0141 & 0.0141 & 0.0141 & 0.0142 & 0.0142 & 0.0142 & 0.0144 & 0.0145 & 0.0148 & 0.0151 & 0.0156\end{array}$

$1.00002 \quad 0.00990 .00990 .00990 .0100 \quad 0.0100 \quad 0.01000 .01010 .01020 .01040 .01060 .0110$

$2.0+0020.0050 \quad 0.0050 \quad 0.0050 \quad 0.0050 \quad 0.0050 \quad 0.0050 \quad 0.0051 \quad 0.00510 .0052$

$3.0+0020.00330 .00330 .00330 .00330 .00330 .00340,00340.0034$

$5,0+0020.002 \pi 0.00200 .00200 .00200 .00200,00200.0020$

$7,0.0020 .00140 .00140 .00140 .00140 .00140 .0014$

$1.0+003 \quad 0,00100.00100 .00100,00100.00100,0010$

$A L P H$ BETA

$1.0-0030.99980 .99991 .00001 .00001 .00001 .00001 .00001 .00001 .00001 .00001 .00001 .00001 .00001 .00001 .00001 .0000$ $2.0=0030.9995 \quad 0.99991 .00001 .00001 .00001 .00001 .00001 .00001 .00001 .00001 .00001 .00001 .00001 .00001 .00001 .0000$ $3.0-0030.99930 .9998 .0 .99991 .00001 .00001,00001.00001 .00001 .00001,00001.00001 .00001 .00001 .00001 .00001 .0000$ $5,0=0030.99880 .09970 .99991 .00001 .00001,00001.00001,00001.00001,00001.00001 .00001 .00001,00001,00001.0000$ $7.0-0030.9983 .0 .09950 .99981 .00001 .00001 .00001 .00001 .00001 .00001 .00001 .00001 .00001 .00001,00001.00001 .0000$ $1.0-0020.99750 .99930 .99980 .99991 .00001,00001,00001.00001 .00001 .00001 .00001 .00001 .00001 .00001 .00001 .0000$ $2.000020 .9951) 0.99870 .99950 .99991 .00001 .00001 .00001 .00001 .00001 .00001 .00001 .00001 .00001 .0000$ $3.0 .002 \quad 0.99260 .9980 \quad 0.9993 \quad 0.9998 \quad 0.99991 .00001 .00001 .00001 .00001 .00001 .00001 .00001 .0000$

$5,0=002 \quad 0.98770 .99670 .99880 .99970 .99991 .00001 .00001 .00001 .00001 .00001 .00001 .0000$

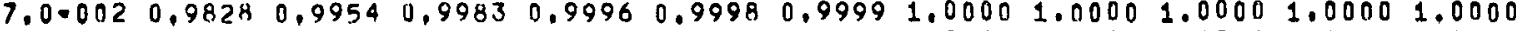

$1.0 .0010 .9756 \quad 0.99340 .99750 .9994 \quad 0.9998 \quad 0.99991 .00001 .00001 .00001 .00001 .0000$

$\begin{array}{lllllllllll}2.0 .001 & 0.9524 & 0.9868 & 0.9950 & 0.9987 & 0.9995 & 0.9998 & 1.0000 & 1.0000 & 1.0000\end{array}$

$3,0=0010.93 n 20.08040 .9926 \quad 0.9981 \quad 0.99930,9997 \quad 1,00001.0000$

$5,0,0010,88890,9677 \quad 0,9877 \quad 0,9968 \quad 0,9988 \quad 0,9996 \quad 0,9999$

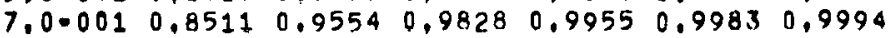

$1,0+000 \quad 0,8000 \quad 0.93750 .97560 .99360 .9975 \quad 0,9991$

$2,0+000 \quad 0.6667 \quad 0.8824 \quad 0.9524 \quad 0.9873$

$3,0+000 \quad 0.57140 .8333 \quad 0.9302$

$5,04000 \quad 0.4444 \quad 0.7500$

$7.0+000 \quad 0.3636$ 
VALUES OF G FOR $N=3$ AND NPS 2

ALPHA 0.0010 .0020 .00320 .005 BETA

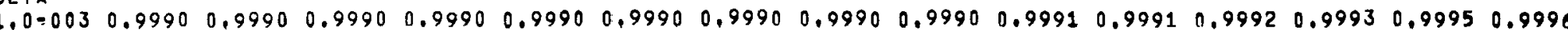

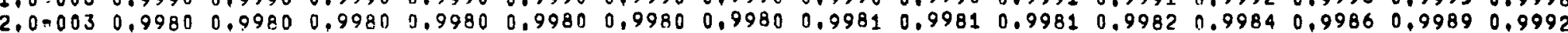

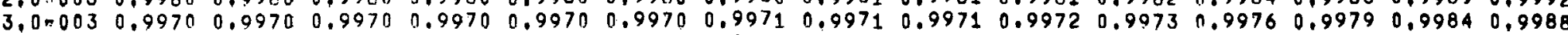

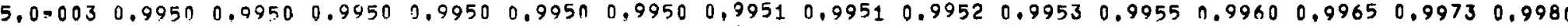

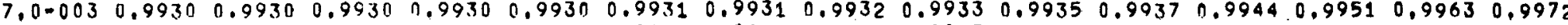
$\begin{array}{llllllllllllllll}1.0 .002 & 0.9900 & 0.9900 & 0.9900 & 0.9901 & 0.9901 & 0.9901 & 0.9902 & 0.9903 & 0.9905 & 0.9907 & 0.9910 & 0.9920 & 0.9929 .0 .9946 & 0.9960\end{array}$

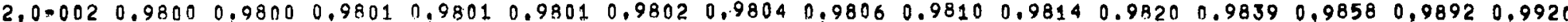

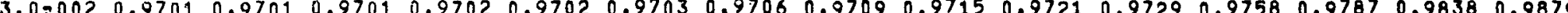
5.0 .0020 .95020 .95020 .95030 .95040 .95050 .95060 .95110 .95150 .95250 .95340 .95490 .95960 .96430 .97280 .9797 $70=0020.93040 .93020 .93050 .93060 .93080 .93100 .93160 .93230 .93360 .93490 .93600 .94340 .94980 .96160 .9713$

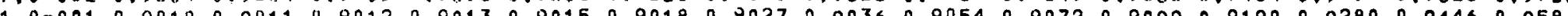

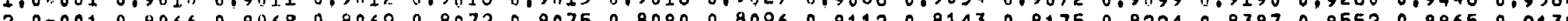

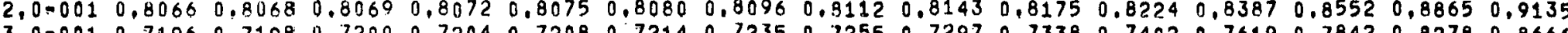
$\begin{array}{lllllllllllllllll}3,0=001 & 0.7196 & 0.7198 & 0.7200 & 0,7204 & 0.7208 & 0.7214 & 0,7235 & 0,7255 & 0.7297 & 0,7338 & 0.7402 & 0.7619 & 0.7842 & 0,8278 & 0,8666\end{array}$ $5.0=001 \quad 0.5717 \quad 0.571 .90 .5722 \quad 0.5727 \quad 0.5731 \quad 0.57390 .5764 \quad 0.57890 .58390 .5891 \quad 0.5970 \quad 0.6246 \quad 0.6539 \quad 0.7143 \quad 0.7716$ $\begin{array}{lllllllllllllllll}7,0-001 & 0.4569 & 0.4571 & 0.4574 & 0.4579 & 0.4584 & 0.4591 & 0.4616 & 0.4642 & 0.4694 & 0.4747 & 0.4828 & 0.5119 & 0.5435 & 0.6117 & 0.6804\end{array}$

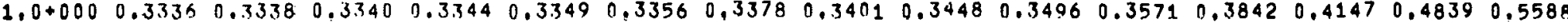

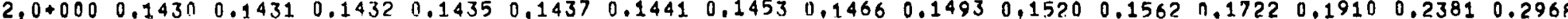
$3,0+0000.07700 .07710 .07710 .07730 .07740 .07760 .07840 .07910 .08060 .09220 .09470 .09420 .10560 .13510 .1743$ .

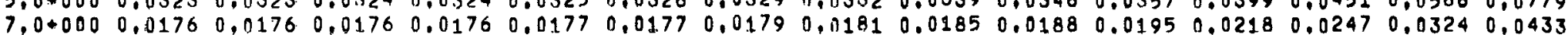

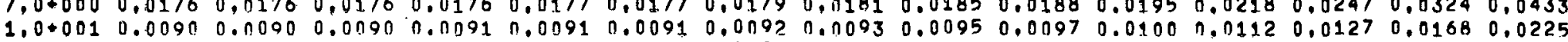
$2.0+0010.00240 .00240 .00240 .00240 .00240 .00240 .00240 .00240 .00250 .00260 .00260 .00300 .00340 .0044$ $3,0+0010.00110 .00110,00110,00110.00110,00110,00110.00110 .00110,00120.00120,00130.0015$

$5,0+0010,00040,00040.00040,00040.00040 .00040,00040.00040 .00040,00040,00040.0005$

$7,0+0010,00020.00020 .00020,00020.00020,00020.00020 .00020 .00020,00020.0002$

$1,0+0020,00010,00010,00010,00010,00010,00010,00010.00010 .00010,00010.0001$

$2,0+0020,00000,00000,0000,0,0000,0,0000,0,00000,00000.00000,0000$

$3,0+0020,00000.00000 .0000,0,0000,0,0000,00000,00000.0000$

$5,0+0020,00000.0000 \quad 0.00000,0001) 0.0000 \quad 0,00000,0000$

$7,0+0020,00000.00000,0000 \quad 1,00000.00000,0000$

$1,0+0030,00000,00000,0000,0,00000,00000,0000$

$$
\text { ALTA }
$$
BETA

1.000030 .99970 .09991 .00001 .00001 .00001 .00001 .00001 .00001 .0000 .1 .00001 .00001 .00001 .00001 .00001 .00001 .0000 $2,0=0030,79950.99990 .99991 .00 .001 .00001,00001.00001 .00001 .00001,00001.00001 .00001,00001.00001 .00001 .0000$ $3,0=0030.999 ? \quad 0,99980.99991 .00001 .00001,00001.00001 .00001 .00001 .00001 .00001 .00001 .00001 .00001 .00001 .0000$ $5,0.0030 .99870 .09970 .99991 .0000 \quad 1.00001 .00001 .00001 .00001 .00001 .00001 .00001 .00001 .00001 .00001 .00001 .0000$ $7,0-0030.99820 .09950 .99981 .00001 .00001 .00001 .00001 .00001 .00001 .00001 .00001 .00001 .00001 .00001 .00001 .0000$ $1,0=0020.99750 .09930 .99970 .99791 .00001,00001.00001 .00001 .00001 .00001 .00001 .00001 .00001 .00001 .00001 .0000$ $2,0=0020.99490 .99860 .9995 \quad 0.99996 .99991 .00001 .00001 .00001 .00001 .00001 .00001 .00001 .00001 .0000$ $3.0 .0020 .99230 .99790 .99920 .9978 \quad 0.99991 .00001 .00001 .00001 .00001 .00001 .00001 .00001 .0000$ $5,0.0020 .9870 \quad 0.99650 .99870 .99770 .99991 .00001 .00001 .00001 .00001 .00001 .00001 .0000$

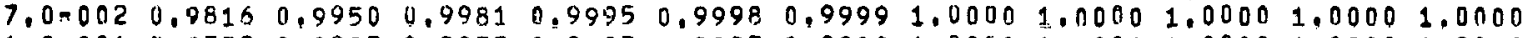

$1.000010 .9732 \quad 0.99 ? 7 \quad 0.9973 \quad 0.9973 \quad 0.9997 \quad 0.99991 .00001 .00001 .00001 .00001 .0000$

$2,0.0010 .9434 \quad 0.9843 \quad 0.9940 \quad 0,99.350 .9994 \quad 0.99981 .00001 .00001 .0000$

$3,0=001 \quad 0.9112 \quad 0.9747 \quad 0.9903 \quad 0.9975 \quad 0.9990 \quad 0.9996 \quad 1.00001 .0000$

$5.0 .0010 .8421 \quad 0.95240 .48160 .99520 .99810 .99930 .9999$

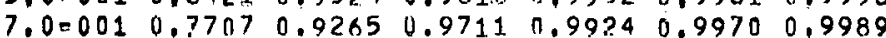

$1,0+000 \quad 0.66670 .88240 .9524 \quad 0.98730 .9950 \quad 1,9982$

$2,0+000 \quad 0.400 \pi \quad 0.71430,8696 \quad 0,9630$

$3,0+000 \quad 0.250110 .5556 \quad 0.7692$

$5,0+000 \quad 0,: 176 \quad 0.3333$

$7.0+000 \quad 0.0667$

$1,0+001 \quad 0,0351$ 


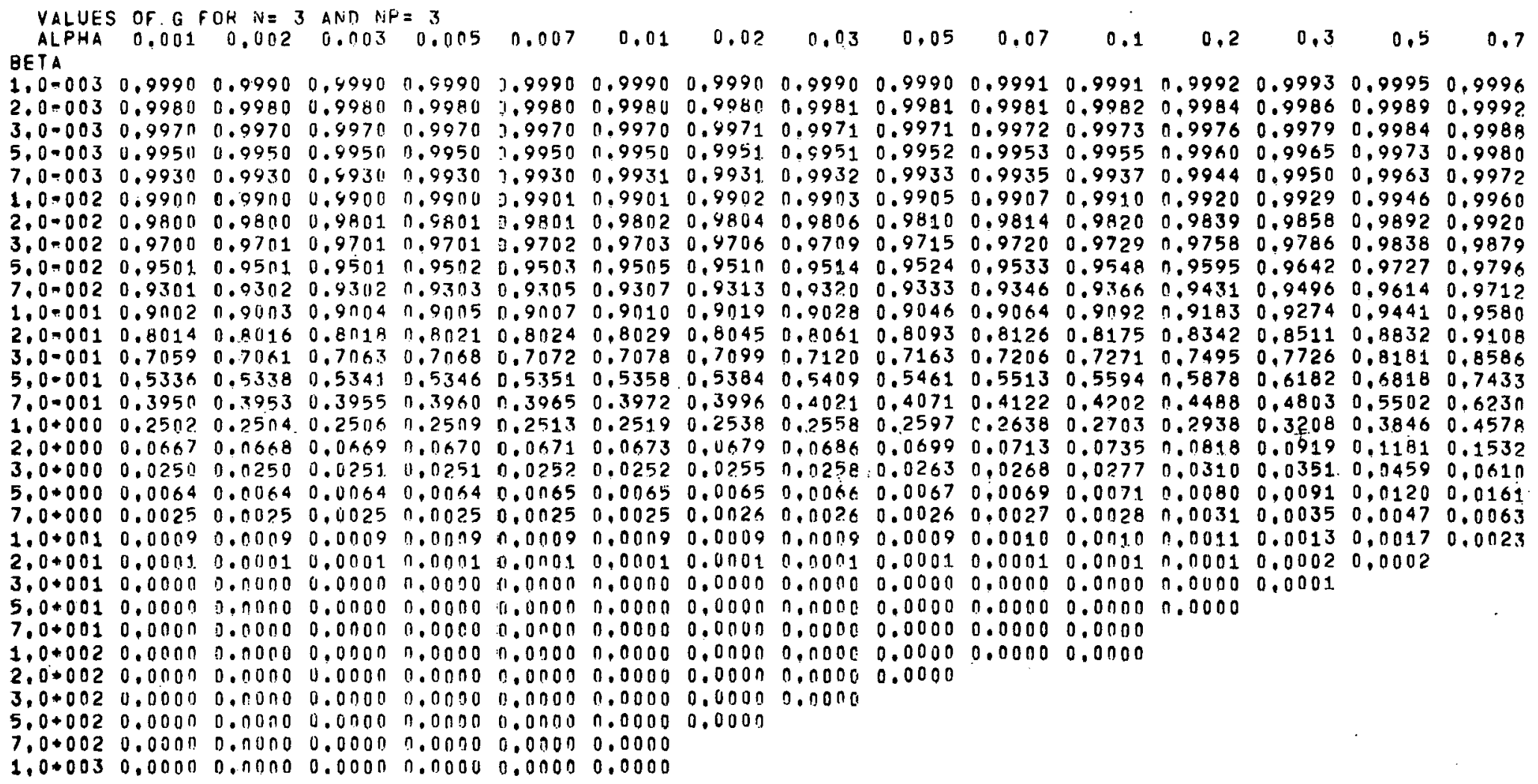



VALUES OF G FOF $N=3$ ANII NP= 4 BETA

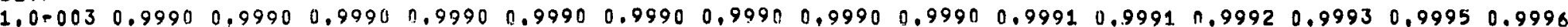

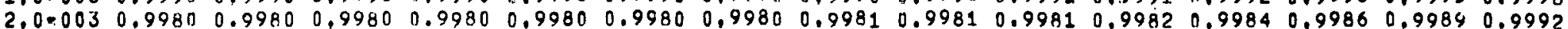
$3,0=0030.9970 \quad 0.99700 .9970 \quad 0.9970 \quad 0.9970 \quad 0.99700 .99710 .99710 .99710 .99720 .99730 .99960 .99790 .99840 .9988$

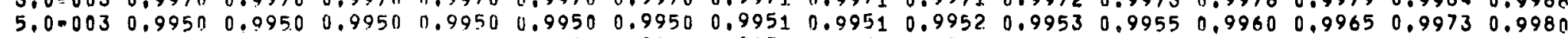
$\begin{array}{llllllllllllllll}7,0=003 & 0.9930 & 0.9930 & 0.9930 & 0.9930 & 0.9930 & 0.9931 & 0.9931 & 0.9932 & 0.9933 & 0.9935 & 0.9937 & 0.9944 & 0.9950 & 0.9963 & 0.9972\end{array}$ $1,0=002 \quad 0.9900 \quad 0.9900 \quad 0.9900 \quad 0.9900 \quad 0.99010 .9901 \quad 0.9902 \quad 0.99030 .99050 .9907 \quad 0.9910 \quad 0.9920 \quad 0.99290 .9946 \quad 0.9960$ $2.0=002 \quad 0.9800 \quad 0.9800 \quad 0.9801 \quad 0.9801 .0 .9801 \quad 0.9802 \quad 0.9804 \quad 0.9806 \quad 0.9810 \quad 0.9814 \quad 0.9820 \quad 0.9839 \quad 0.9858 \quad 0.9892 \quad 0.9920$ $\begin{array}{lllllllllllllllll}3,0=002 & 0.9700 & 0.9701 & 0.9701 & 0.9701 & 0.9702 & 0.9703 & 0.9706 & 0.9709 & 0.9715 & 0.9720 & 0.9729 & 0.9758 & 0.9786 & 0.9838 & 0.9879\end{array}$ $\begin{array}{lllllllllllllllll}5,00002 & 0.9500 & 0.95 n 1 & 0.9501 & 0.9502 & 0.9503 & 0.950 .5 & 0.951 n & 0.9514 & 0.9524 & 0.9533 & 0.9548 & 0.9595 & 0.9642 & 0.9727 & 0.9796\end{array}$

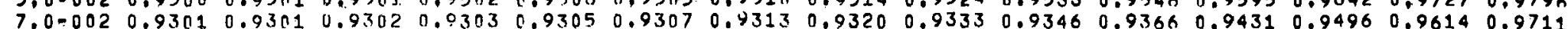
1,0-001 $0.90010 .90020 .9003 \quad 0.9005 \quad 0.9006 \quad 0.90090 .90180 .90270 .90450 .90640 .90910 .91830 .92730 .94410 .9580$

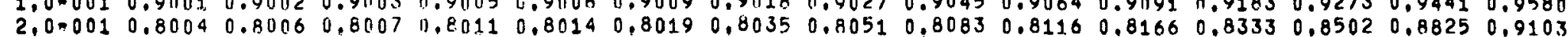

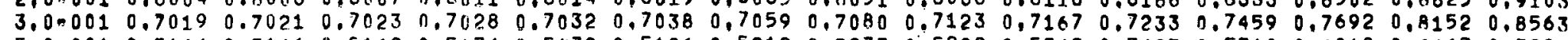
$\begin{array}{llllllllllllllll}5.0-001 & 0.5164 & 0.5166 & 0.5169 & 0.51 .74 & 0.5179 & 0.5186 & 0.5212 & 0.5237 & 0.5289 & 0.5342 & 0.5423 & 0.5710 & 0.6018 & 0.6667 & 0.7299\end{array}$

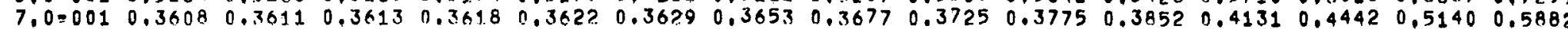
$\begin{array}{llllllllllllllllll}1.0+000 & 0.2002 & 0.2003 & 0.2005 & 0.2008 & 0.2011 & 0.2016 & 0.2033 & 0.2049 & 0.2083 & 0.2119 & 0.2174 & 0.2378 & 0.2616 & 0.3191 & 0.3877\end{array}$

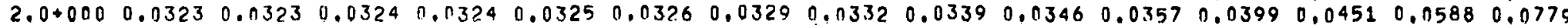
$\begin{array}{llllllllllllll}3,0+000 & 0.0083 & 0.0083 & 0.0083 & 0.0083 & 0.0083 & 0.0083 & 0.0084 & 0.0085 & 0.0087 & 0.0089 & 0.00920 .01030 .0117 & 0.0154 & 0.0207\end{array}$ $5,0+0000.00130 .00130 .00130 .00130 .00130 .00130 .00130 .00130 .00130 .00140 .00140 .00160 .00180 .00240 .0032$ $7,0+0000.00040 .00040 .00040 .00040 .00040 .00040,00040.00040 .00040,00040.00040 .00040 .00050,00070,0009$ $1,0+0010.0009 .0 .00010,00010,00010.00010,00010,00010,00010,00010,00010,00010.00010 .00010,00020,0002$ $2,0+0010.00000 .00000 .00000,00000.00000 .00000,00000,00000.00000,00000.00000 .00000 .00000,0000$ $3,0+0010,00000.00000,00000,00000.00000,00000,00000,00000.00000 .00000 .00000 .00000 .0000$ $5,0+0010,00000,00000,00000,00000,00000,00000,00000,00000,00000,0000000000,0000$ $7.0+0010.00000 .0000 \quad 0.0000 \quad 0.00000 .00000 .00000 .00000 .00000 .00000 .00000 .0000$ $1,0+0020.00000 .00000 .00000 .00000 .00000,00000,00000.00000 .00000 .00000 .0000$

$2,0+0020.00000 .00000 .0000 \quad 0.0000 .0 .0000 \quad 0.00000 .00000 .00000 .0000$

$3,0+002 \quad 0.0000 \quad 0.0000 \quad 0.0000 \quad 0.0000 \quad 0.0000 \quad 0.0000 \quad 0.0000 \quad 0.0000$

$5,0+0020.00000 .00000 .00000 .00000 .00000 .00000,0000$

$7,0+0020,0000 \quad 0.0000 \quad 0,00000,00000,0000 \quad 0,0000$

$1,0+0030,00000,0000,00000,00000,00000,0000$ BETA

ALPHA $1.000 \quad 2.000 \quad 3.000 \quad 5.000 \quad 7.000 \quad 10.00 \quad 20.00 \quad 30.00 \quad 50.00 \quad 70.00 \quad 100.0 \quad 200.0 \quad 300.0 \quad 500.0 \quad 700.0 \quad 1000$ $1.0-0030.99970 .09991 .00001 .00001 .00001 .00001 .00001 .00001 .00001 .00001 .00001 .00001 .00001 .00001 .00001 .0000$ $2,0=003 \quad 0.9995 \quad 0,09990.99991 .00001 .00001,00001.00001 .00001 .00001 .00001 .00001 .00001 .00001 .00001 .00001 .0000$ $3.0=003 \quad 0.999 ? \quad 0.9998 \quad 0.9997 \quad 1.00001 .00001,00001.00001 .00001 .00001 .00001 .00001 .00001,00001.00001 .00001 .0000$ $5,0-0030.99870 .09970 .99991,00001.00001 .00001 .00001 .00001 .00001,00001.00001 .00001 .00001 .00001 .00001 .0000$

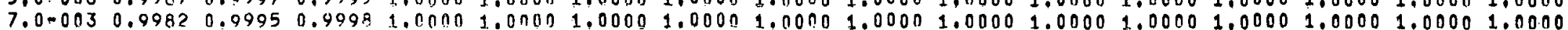

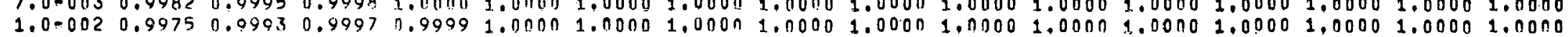

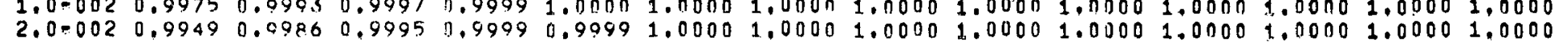

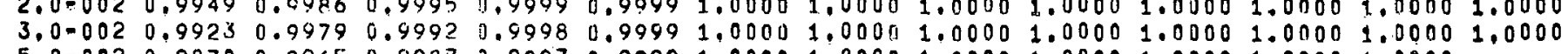

$5.0=002 \quad 0.9870 \quad 0.9965 \quad 0.9987 \quad 3.99970 .99991 .00001 .00001 .00001 .00001 .00001 .00001 .0000$

$7,0=0020,98150.99500,9981.9,99950,99980.99971 .00001 .00001 .00001 .00001 .0000$

$1,0=0010.97300 .99260 .9972,9.99930 .9997 \quad 0.99991 .00001 .00001 .00001 .00001 .0000$

$2.0 .001 \quad 0.94130 .9836 \quad 0.9938 \quad 0.9984 \quad 0.9994 \quad 0.999 .91 .00001 .00001 .0000$

$\begin{array}{lllllllll}3,0 \div 001 & 0.9039 & 0.0724 & 4.9895 & 0.9973 & 0.9989 & 0.999 .6 & 0.9999 & 1.0000\end{array}$

$\begin{array}{lllllllll}5,0-001 & 0,8101 & 0.941 .2 & 0,9771 & 0,9940 & 0.9977 & 0,9992 & 0,9999\end{array}$

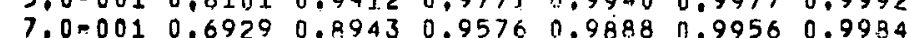

$1,0+000 \quad 0.5000 \quad 0.78950 .90910 .9750 \quad 0.99010 .9954$

$2,0+000 \quad 0.1176 \quad 0.3333 \quad 0.5714 \quad 0.8397$

$3,0+000 \quad 0.03230 .1 .1110 .2500$

$5,0+000 \quad 0,00510.0189$

$7,0+000 \quad 0.0014$

$1,0+001 \quad 0,0004$ 

BETA

1.0-003 $0.9990 \quad 0.9990 \quad 0.9990 \quad 0.9990 \quad 0.9990 \quad 0.999100 .9990 \quad 0.9990 \quad 0.9990 \quad 0.9991 \quad 0.9991 \quad \pi .9992 \quad 0.9993 \quad 0.9995 \quad 0.9996$

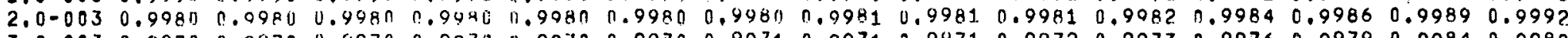
$\begin{array}{llllllllllllllll}3.0-003 & 0.9970 & 0.8970 & 0.9970 & 0.9970 & 0.997 n & 0.9970 & 0.9971 & 0.0971 & 0.9971 & 0.9972 & 0.9973 & 0.9976 & 0.9979 & 0.9984 & 0.9988\end{array}$

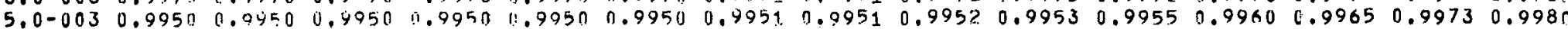
$\begin{array}{llllllllllllllll}7.0 .003 & 0.9930 & 0.0930 & 4.7930 & 11.9930 & 0.9930 & 0.9931 & 0.9931 & 0.0932 & 0.9933 & 0.9935 & 0.9937 & 0.9944 & 0.9950 & 0.9963 & 0.9972\end{array}$

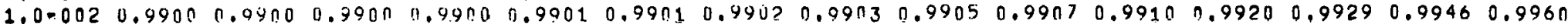
$2,0-0020.98000 .090040 .98010 .98010 .98010 .98020 .98040 .98060 .98100 .98140 .98200 .98390 .98580 .98920 .9920$ 3.00020 .97001107010 .97010 .97010 .97020 .97030 .97060 .97090 .97150 .97200 .97290 .97580 .97860 .98380 .9879

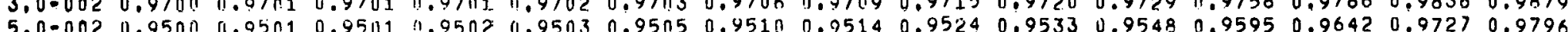

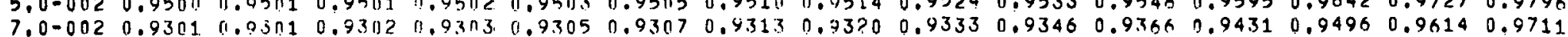

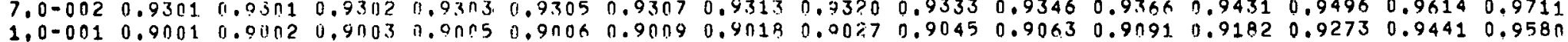

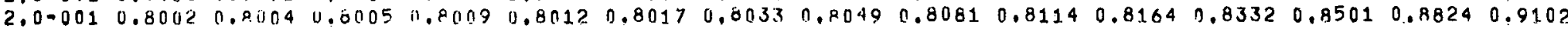
$\begin{array}{llllllllllllllllll}3.0-001 & 0.7007 & 0.7309 & 0.7011 & 0.7016 & 0.7 n 20 & 0.7026 & 0.7047 & 0.7069 & 0.7112 & 0.7155 & 0.7221 & 0.7448 & 0.7682 & 0.8143 & 0.8556\end{array}$

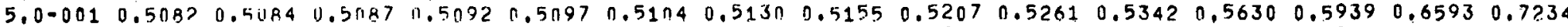

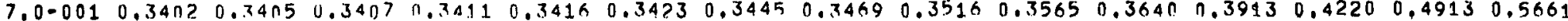

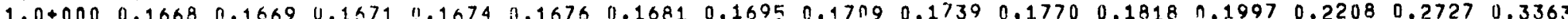
$3.0+0000.01540 .01590 .01590 .0140 .01600 .01600 .09620 .01640 .01670 .01700 .01760 .01970 .02230 .02940 .0393$ (1)

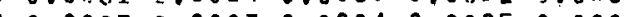

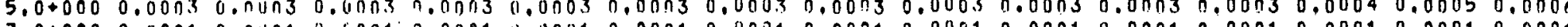

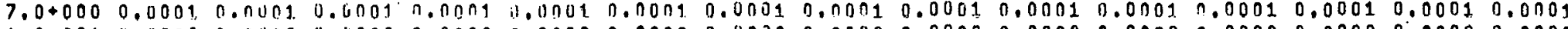

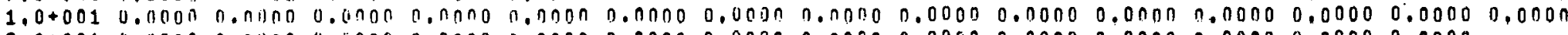

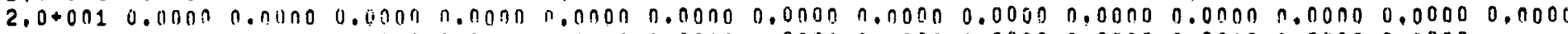
$3,0+0010.0000,0.00000 .0000,0.0000,0.000 n 0.00000 .00000 .00000 .00000 .00000 .000 n 0.00000 .0000$

$5,0+0010.00000 .04000 .00007 .00000 .00000 .00000 .00000 .00000 .00000 .00000 .00000 .0000$

$7.0+0010.00000 .00000 .00000 .00000 .00000 .00000 .00000 .00000 .00000 .00000 .0000$

$1.0+0020.00000 .00000 .00000 .00000 .00000 .00000 .00000 .00000 .00000 .00000 .0000$

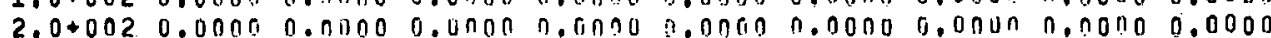

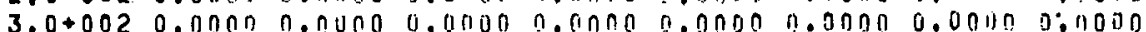

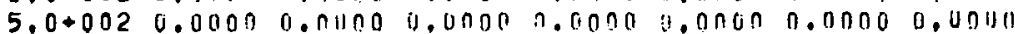

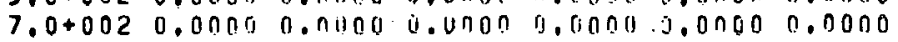

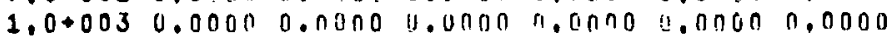

ALPHA 1.000 2.100 3.000 5.000 7.000 $10.0020 .0030 .0050 .00 \quad 70.00 \quad 100.0 \quad 200.0 \quad 300.0 \quad 500.0 \quad 700.0 \quad 1000$ BETA

$1.0-0030.99970 .09091 .00001 .00001 .00001 .00001 .00001 .00001 .00001 .00001 .00001 .00001 .00001 .00001 .00001 .0000$ 2.0-003 $0.99950 .99990 .99991 .01001 .00001 .00001 .000 n 1.00031 .00001 .00001 .00001 .00001 .00001 .00001 .00001 .0000$ $3.0-0030.99920 .99980 .99991 .00901 .00001 .00001 .00101 .00001 .00001 .00001 .00001 .00001 .00001 .00001 .00001 .0000$

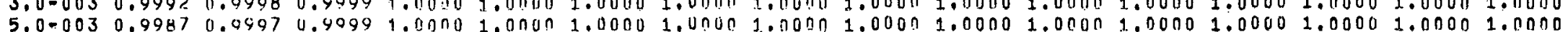

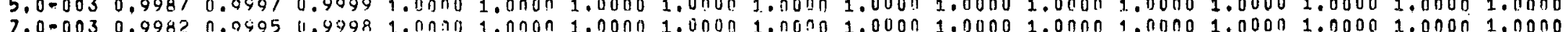

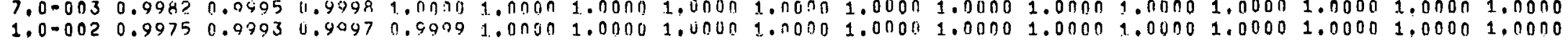
$2.0-002 \quad 0.99490 .9586 \quad 0.99950 .9099 \quad 0.9999 \quad 1.00001 .60001 .00001 .00091 .00001 .00001 .00001 .00001 .0000$

3.0-002 0.99230 .99790 .999 ? 0.99980 .99991 .000111 .00007 .00901 .00001 .00001 .00001 .00001 .0000

$5.0=0020.987 i 1 \quad 0.9965 \quad 0.9987 \quad 0.99770 .99991 .00001 .00001 .00001 .00001 .00001 .00001 .0000$

$7.0=0020.98150 .9950 \quad 0.9981 \quad 0.99950 .99980 .99991 .0001 \quad 1.00301 .00001 .00001 .0000$

$1.0=0010.97310 .9476 \quad 1.99720 .9993 \quad 0.99970 .99991 .00001 .00001 .00001 .00001 .0000$

$2.0-0010.94120 .3636 \quad j .9934 \quad 0.9394$ i. 99940.9998 1.000n 1.010001 .0000

$3,0=0010.90340 .0723$ U. 90940.0973 i. 90990.99960 .99991 .0000

$5.000010 .8050 \quad 0.9303 \quad 11.9764 \quad 11.9938 \quad 0.9076 \quad 0.99910 .4949$

$7.0=0010.67330 .88540 .9537 \quad 0.98770 .9952 \quad 0.9983$

$1.0+000 \quad 0.4444 \quad 0.7500 \quad 0.8389 \quad 0.9689 \quad 0.9877 \quad 0.9955$

$2.0+000 \quad 0.0606 \quad 0.1948 \quad 11.3927 \quad 11.71 .56$

$3.0+000 \quad 0.01090 .0397 \quad 0.0993$

$5.0+000 \quad 0.00107 .0038$

$7,0+0000.000$ ?

$1.0+0010.0000$ 
VALUES OF G FOH iN $=4$ aNi $N F=1$

$$
\text { ALPHA }
$$

1.0-003 $0.0991 \quad 0.0490 \quad 0.9990 \quad 0.9990 \quad 0.9990 \quad 0.9990 \quad 0.9990 \quad 0.0990 \quad 0.9991 \quad 0.9991 \quad 0.9991 \quad 1.9992 \quad 0.9993 \quad 0.9995 \quad 0.9996$ 2.000030 .09800 .0980499800 .99500 .99800 .99800 .59800 .09810 .99810 .99810 .99829 .99840 .99860 .9990 .0 .9993

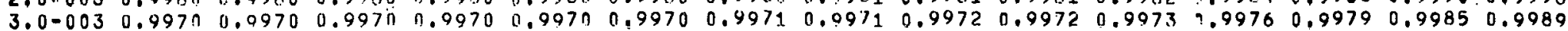
$5.0=003 \quad 0.9950 \quad 0.0950 \quad 0.9950 \quad 0.9950 \quad 0.9051 \quad 0.9951 \quad 0.9959 .0 .99520 .9953 \quad 0.99540 .99559 .9960 \quad 0.99650 .99740 .9982$ $\begin{array}{llllllllllllllll}7,0-003 & 0.9931 & 0.0931 & 0.9931 & 0.9931 & 7 j .9031 & 0.9931 & 0.9932 & 0.0933 & 0.9934 & 0.9935 & 0.9937 & 1.9944 & 0.9951 \quad 0.9964 & 0.9975\end{array}$

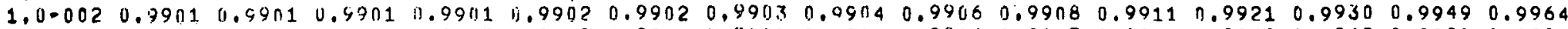

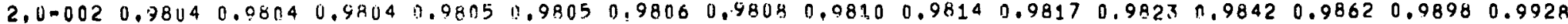

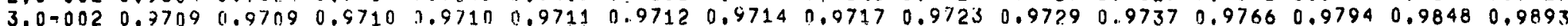
$5,0=0020.95240 .05250 .95250 .95260 .95270 .95280 .95330 .95370 .95470 .95560 .95690 .96150 .96610 .97480 .9823$

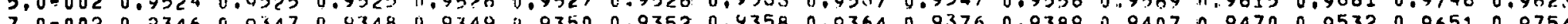
(1)

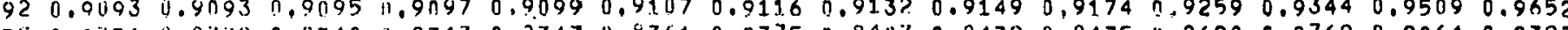

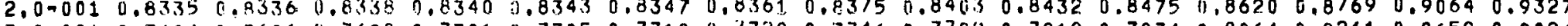

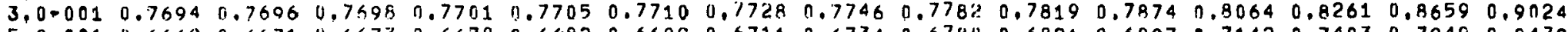
$\begin{array}{llllllllllllllllll}5,0.001 & 0.6669 & 0.6671 & 0.6673 & 0.6678 & 0.6682 & 0.6689 & 0.6711 & 0.5734 & 0.67811 & 0.6820 & 0.6897 & 0.7142 & 0.7403 & 0.7949 & 0.847 ?\end{array}$ $\begin{array}{lllllllllllllllll}7.0 .001 & 0.5885 & 0.5887 & 0.589 n & 0.5894 & 0.5899 & 0.59 n 7 & 0.5931 & 0.5956 & 0.6006 & 0.6057 & 0.6135 & 0.6490 & 0.6706 & 0.7346 & 0.7985\end{array}$ $\begin{array}{llllllllllllllll}1.0+000 & 0.50 n .3 & 0.50 n 5 & 0.5008 & 0.5013 & 0.5018 & 0.5025 & 0.5051 & 0.5076 & 0.5128 & 0.5181 & 0.5263 & 0.5555 & 0.5876 & 0.6596 & 0.735 n\end{array}$

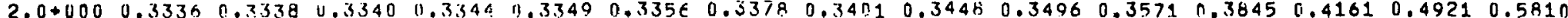
$3.0+000 \quad 0.25020 .25040 .2506: 1.2504 \quad 1.25130 .25190 .25380 .25580 .25970 .26390 .2703 \quad 0.29410 .3220 \quad 0.39240 .4803$ $5.0+0000.16680 .16650 .16710 .16740 .16760 .16810 .16950 .17090 .17390 .17700 .18180 .19990 .22180 .27930 .3568$

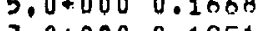

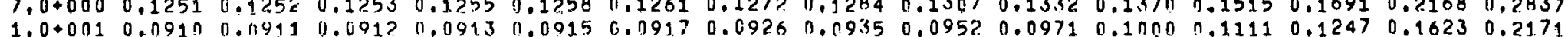

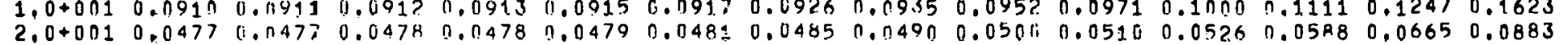

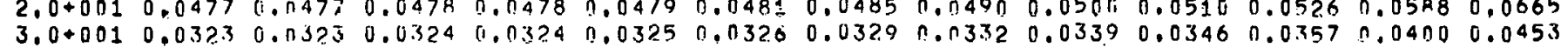

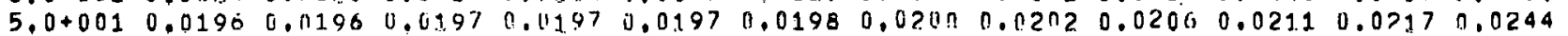

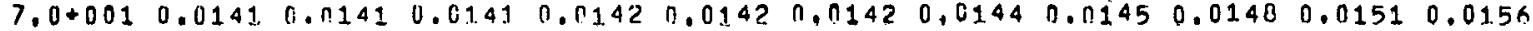

$1.0+0020.00990 .00990 .00990 .010 \pi 0.01000 .01000 .01010 .01020 .01040 .01060 .0110$

$2,0+002 \quad 0.005 n \quad 0.0050 .0050 \quad 0.0053 \quad 0.0050 \quad 0.0050 \quad 0.0 n 51 \quad 0.0051 .0 .005 \%$

$3.0+002 \quad 0.00330 .0033 \quad 0.00330 .00330 .00330 .00340 .00340 .0 .034$

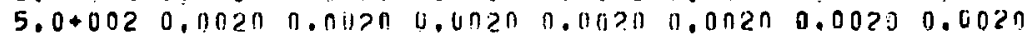

$7.0+0020.00140 .01140 .00140 .00140 .00140 .0014$

$1,0+0030.00100 .00100 .00100 .00100,00100,0010$

$$
\text { BETA }
$$

ALPHA $=00002.003 \quad 3.0005 .0110 \quad 7.000 \quad 10.03 \quad 20.00 \quad 30.00 \quad 50.00 \quad 70.00 \quad 1.00 .0 \quad 200.0 \quad 300.0 \quad 500.0 \quad 700.0 \quad 1000$

$1.0-0030.99981 .00001 .00001 .00001 .00001 .00001 .00001 .000001 .00001 .00001 .00001 .00001 .00001 .00001 .00001 .0000$ 2.0-003 $0.9996 \quad 0.99991 .00001 .60 n 01.00001 .00001 .00001 .00001 .00001 .00001 .00001 .00001 .00001 .00001 .00001 .0000$ $3,0=0030.99940 .99991 .00001 .00001 .00001 .00001 .00001 .00001 .00001 .00001 .00001 .00001 .00001 .00001 .00001 .0000$ $5,0-0030.99900 .99931 .00001 .00001 .0 n 001.00001 .00001 .00001 .00001 .00001 .00001 .00001 .00001 .00001 .00001 .0000$ $5,0-003$
$1,0=0020980$

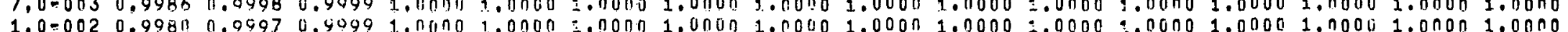

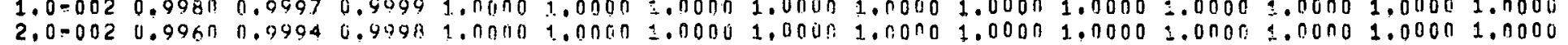
$3,0-0020.99400 .05900 .99951 .00001 .0000=00001.00001 .00001 .00001 .0000 \div .0000=000001.0000$ $5.0-0020.9901,0.99840 .99960 .99991 .0000 \div 00001.00001 .00001 .00001 .00009 .0000200000$

$7.0-0020.98620 .09770 .90940 .99992 .0000$ i.00001 $1.00001 .00001 .00001 .00002 .00 n 0$ $1.0-0010.98040 .09080 .99920 .99991 .00001 .00001 .00001 .00001 .00001 .00001 .0000$ $2.0=0010.96150 .09300 .9983 \quad 0.9997 \quad 19.99991 .00001 .00001 .00001 .0000$

$3,0-0010.94340 .09040 .9975$ n.s456 0.99491 .00001 .00001 .0000

$5.0-0010.9091 \quad 0.9841 \quad 0.4959 \quad 0.9934 \quad 0.9998 \quad 1.00001 .0000$

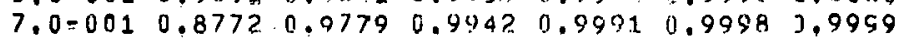

$1.0+000 \quad 0.8333 \quad 0.96980 .99180 .99470 .9994 \quad 9.9959$

$2,0+000 \quad 0.7143 \quad 0.9354 \quad 0.9437 \quad 0.9974$

$3.0+000 \quad 0.625 \pi \quad 0.919 .3 \quad 0.9758$

$5.0+000 \quad 0.5000 \quad 11.81611$

$7.0+000 \quad 0.4167$

$1,0+001 \quad 0.3333$ 


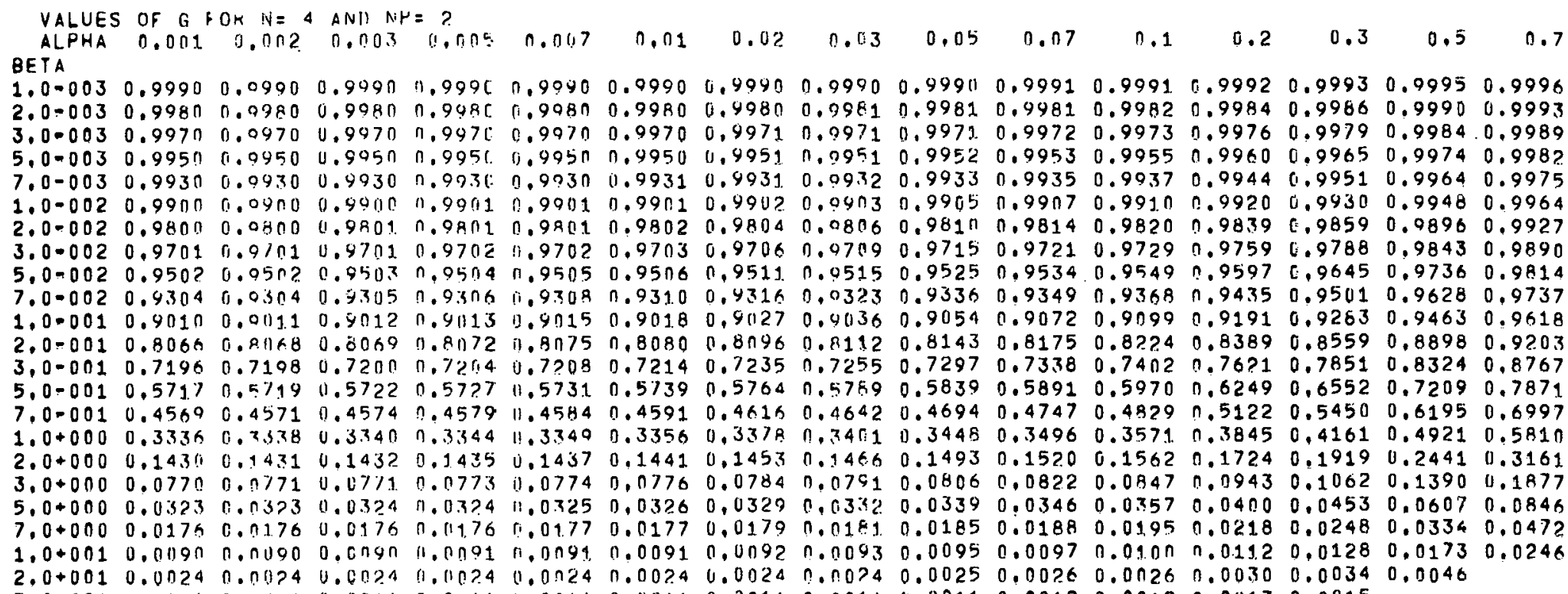

1.0.003 $0.9990 \quad 0.0990 \quad 0.999 n \quad 0.999 C \quad 0.9990 \quad 0.0990 \quad 0.9990 \quad 0.9990 \quad 0.9990 \quad 0.9991 \quad 0.9991 \quad 0.9992 \quad 0.9993 \quad 0.9995 \quad 0.9996$

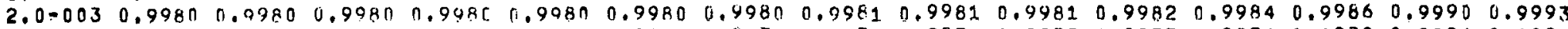
$\begin{array}{lllllllllllllll}3.0 .003 & 0.997 n & 0.0970 & 0.9970 & 0.9976 & 0.9970 & 0.9970 & 0.9971 & 0.9971 & 0.9973 & 0.9972 & 0.9973 & 0.9976 & 0.9979 & 0.9984 .0 .9989\end{array}$

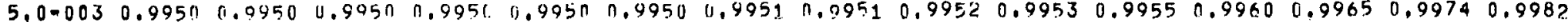

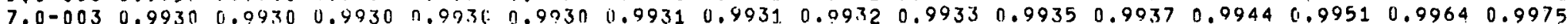
$1.0-0020.99000 .09000 .99000 .99010 .90010 .99010 .99020 .09030 .99050 .99070 .99100 .99200 .99300 .99480 .9964$ 1.00020 .900 .906 $3.0 .002 \quad 0.97010 .97 n 1 \quad 0.9701 \quad 0.9702 \quad 1.9702 \quad 0.9703 \quad 0.9706 \quad 0.97490 .971$ 5.0-002 0.950? $0.95 n 20.950 ? n .9504 \pi .95050 .95 n 60.9511 .0 .05150 .95250 .9534 .0 .95490 .9590 .96450 .97360 .9814$

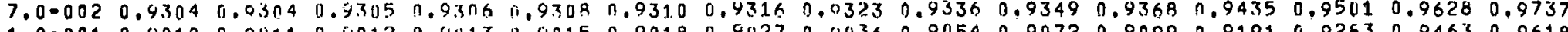

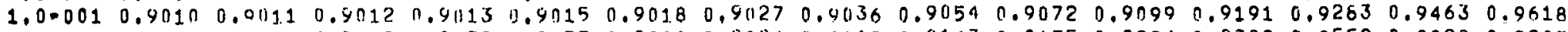

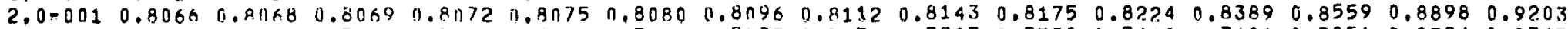
$\begin{array}{llllllllllllllllll}3,0-001 & 0.7196 & 0.7198 & 0.7200 & 0.7204 & 0.7208 & 0.7214 & 0.7235 & 0.7255 & 0.7297 & 0.7338 & 0.7402 & 0.76 ? 1 & 0.7851 & 0.8324 & 0.8767\end{array}$ $\begin{array}{lllllllllllllllll}5.0-001 & 0.57177 & 0.5719 & 1.5722 & 0.57 ? 7 & 11.5731 & 0.5739 & 0.5764 & 0.5759 & 0.5839 & 0.5891 & 0.5970 & 0.6249 & 0.6552 & 0.7209 & 0.7871\end{array}$ $\begin{array}{llllllllllllllllllll}7.0-001 & 0.4569 & 0.4571 & 0.4574 & 0.4579 & 11.4544 & 0.4591 & 0.4616 & 0.4642 & 0.4694 & 0.4747 & 0.4829 & 0.5122 & 0.5450 & 0.6195 & 0.6997\end{array}$ $1.0+000 \quad 0.3336 \quad 0.33380 .3340 \quad 0.3344 \quad 13340 \quad 0.33560 .33780 .34510 .34480 .34960 .35710 .38450 .41610 .49210 .5810$

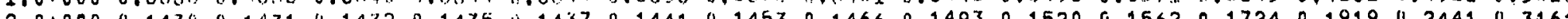

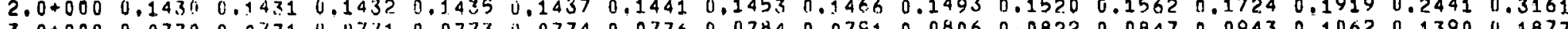

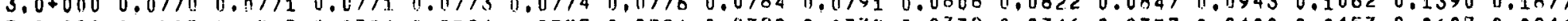
$5.0+0000.03230 .03230 .03240 .0 .32411 .0 .3250 .03260 .03290,03320.03390,03460.03570 .04100 .04530 .06070 .0846$ $7,0+000 \quad 0.0176 \quad 0.0176 \quad 0.0176 \quad 0.01 .76 \quad 1.01770 .01770 .01790 .01810 .01850 .01880 .01950 .02180 .02480 .03340 .0472$

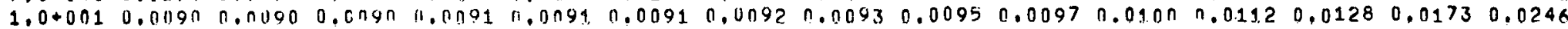
$2.0+0010.00240 .0024 \quad 0.00240 .0024 \quad 0.0024 \quad 0.00240 .00240 .00240 .00250 .00260 .00260 .00300 .00340 .0046$

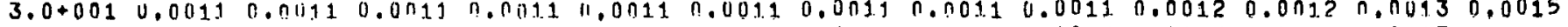

$5.0+0010.00040 .011040 .0004$ i).00n4 li.0n04 0.0004 0.0n04 0.0004 0.0004 0.00040.0004 0.0005

$7.0+0010.000 \% 0.01020 .00020 .1101020 .00020 .00020 .00420 .00020 .00020 .00020 .0002$ $1.0+0020.00010 .00010 .00010 .0001$ 1.0001 $0.00010 .00010 .00010,00010,00010,0001$

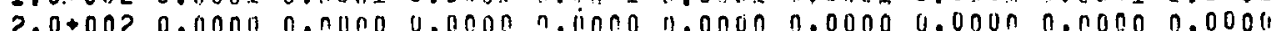

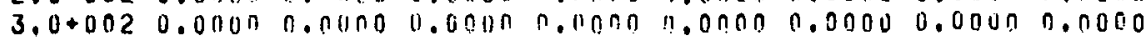

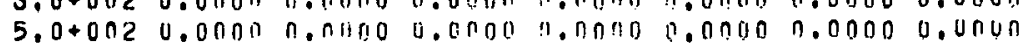

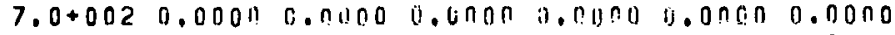

$1,0+0030,00010,0000,0,000,0,00110,00000.0000$

ALPHA 1.000 2.000 3.000 5.000 7.000 $10.00 \quad 20.00 \quad 30.00 \quad 50.00 \quad 70.00 \quad 100.0 \quad 200.0 \quad 300.0 \quad 500.0 \quad 700.0 \quad 1000$ BETA

$1.0-0030.09981 .00001 .00001 .01141 .00001 .00001 .00051 .00001 .000 r 1.00001 .00001 .00001 .00001 .00001 .00001 .0000$ 2.0-003 0.9996 0.0999 1.0100. $3,0-003$. $5,0-0030.0990 .09081 .00001 .0001 .00001 .00001 .000 n 1.00001 .00001 .00001 .00001 .00001 .00001 .00001 .00001 .0000$

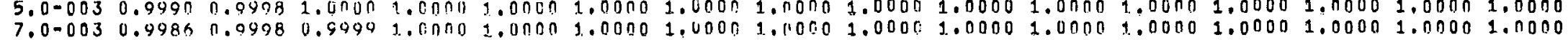
$1.0-0020.998 n \pi .99970 .90991 .00001 .00001 .00001 .00001 .00001 .00061 .00001 .00001 .00001 .00001 .00001 .00001 .0000$

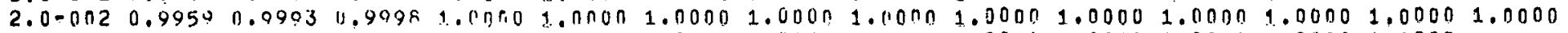

$3.0=0020.993^{\circ} 0.9990 \quad 0.9997 \quad 1.00003 .00001 .00001 .00001 .00001 .00001 .00001 .00001 .00001 .0000$

$5.0-0020.98960 .998310 .9096 \quad 11.09991 .00001 .00001 .00001 .00001 .00001 .00001 .00001 .0000$

$7,0.002 \quad 0.985 ? 0.99760 .9944 \quad 0.09991 .00001 .00001 .00001 .00001 .00061 .00001 .0000$

$1.0=0010.97850 .04 \times 5 \quad 0.49910 .09991 .00001 .00001 .00001 .00009 .00001 .00001 .0000$

$2,0=0010.95470 .99230 .9980,0.09970 .90991 .00001 .00001 .110001 .0006$

$3.0=0010.92760 .06760 .94680 .99050 .99991 .00001 .00001 .0000$

$5.0-001 \quad 0.8096 \quad 0.0764 \quad 0.99360 .0990 \quad 11.99970 .99991 .0000$

$7.0=001 \quad 0.8078 \quad 0.0630 \quad 0.9903 \quad 0.0985 \quad 1.9996 \quad 0.9999$

$1.0+000 \quad 0.7143 \quad 0.0394 \quad 0.91337 \quad 0.9974 \quad 0.9093 \quad 0.9998$

$2.0+000 \quad 0.4545 \quad 0.0378 \quad 0.9528 \quad \pi .09 ? 4$

$3.0+000 \quad 0.2941 \quad 0.7209 \quad 0.91198$

$5.0+000 \quad 0.1429 \quad 0.5082$

$7.0+0000.0821$

$1.0+0010.0435$ 
VALUES OF G FOR $N=4 A N D N H=3$

ALPHA O.001 0.002 Q $0.003=0.00$ BETA

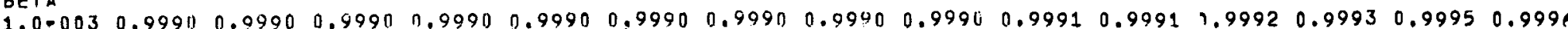

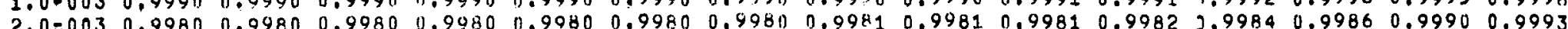

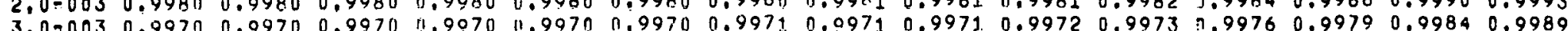

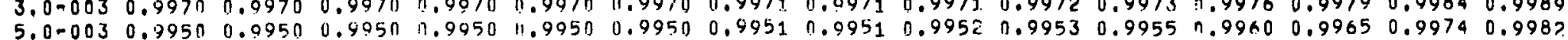
$\begin{array}{llllllllllllllll}7.0=003 & 0.9930 & 0.9930 & 0.9930 & 1.9930 & 11.9930 & 0.9931 & 0.9931 & 0.9932 & 0.9933 & 0.9935 & 0.9937 & 0.9944 & 0.9951 & 0.9964 & 0.9975\end{array}$

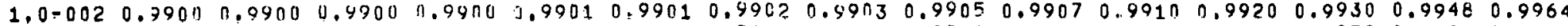

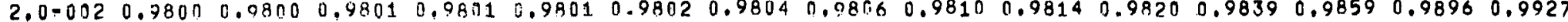
$\begin{array}{llllllllllllllll}3,0=002 & 0.9700 & 0.9701 & 0.9701 & 10.9701 & 0.9702 & 0.9703 & 0.9706 & 0.0709 & 0.9715 & 0.9720 & 0.9729 & 0.9758 & 0.9788 & 0.9843 & 0.9890\end{array}$ $5,0=002 \quad 0.9501$
7 $7.0=0020.93010 .03020 .93020 .93030 .93050 .93070 .93130 .03200 .93330 .93460 .93660 .94320 .94980 .96260 .9736$

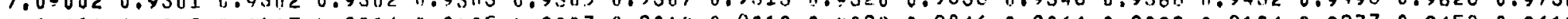
$1.0-0010.90020 .9003$ U.9n04 $2.0=0010.80140 .80160 .00180 .80210 .80240 .80290 .80450 .80610 .80930 .81260 .81750 .83440 .85180 .88650 .9179$

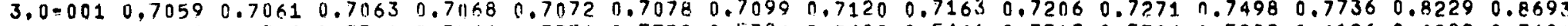
$\begin{array}{lllllllllllllllll}5.0-001 & 0.5336 & 0.5338 & 0.5341 & 0.5346 & 0.5351 & 0.5358 & 0.5384 & 0.54119 & 0.5461 & 0.5513 & 0.5594 & 0.5882 & 0.6196 & 0.6889 & 0.7601\end{array}$

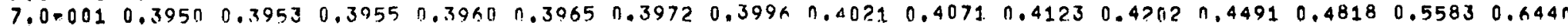

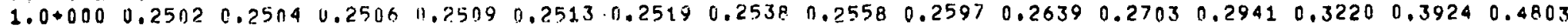

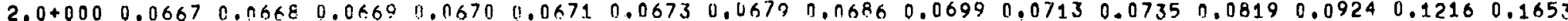
$3.0+0000.0250 \quad 0.0250 \quad 0.02510 .02510 .02520 .02520 .02550 .02580 .02630 .02680 .02770 .03100 .03530 .04730 .0664$ $3.0+0000.025640 .00640 .00640 .00640 .07550 .00650 .00650 .00660 .00670 .00690 .00710 .00800 .00910 .01230 .0176$ $3.0+000.00250 .00250 .00250 .00250 .00250 .00250 .00260 .00260 .00260 .00270 .00280 .00310 .00360 .00480 .0060$

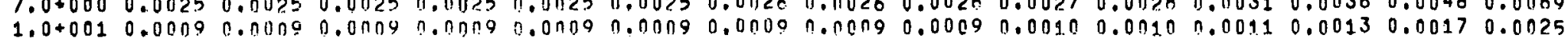

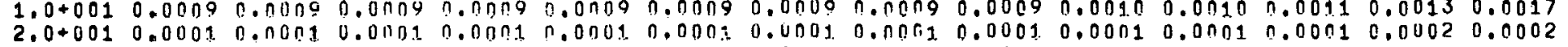
$3.0+0010.00000 .00000 .0 n 000.00000 .00000 .00000 .00000 .00000 .00000 .00000 .00000 .00000 .0001$ $5,0+0010.0000 \quad 0.00000 .011000 .00000 .0000 \quad 0.00000 .00000 .00000 .00000 .00000 .00000 .0000$

$7.0+0010.00000 .00000 .00000 .00000 .00000 .00000 .00007 .00000 .00000 .001100 .0000$

$1.0+0020.00000 .00000 .00000 .00000 .00000 .00000 .00000 .00000 .00000 .00000 .0000$

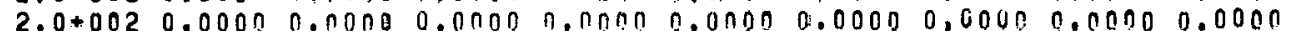

$3.0+0020.0000 \quad 0.00030 .0000,0.00000 .00000 .0000 \quad 0.00000 .0000$

$5.0+0020.00000 .00000 .000 \pi 0.00900 .00000 .00000 .0000$

$7.0+0020.00000 .00000 .00000 .00000 .00000 .0005$

$1.0+0030.00000 .00000 .00000 .00000 .00000 .0000$

ALPHA $2.000 \quad 2.003 \quad 3.000 \quad 5.000 \quad 7.000 \quad 10.00 \quad 20.00 \quad 30.00 \quad 50.00 \quad 70.00 \quad 1.00 .0 \quad 200.0 \quad 300.0 \quad 500.0 \quad 700.0 \quad 1000$ BETA

$1.0=0030.09981 .010091 .00001 .09001 .00001 .00001 .00001 .00001 .00001 .00001 .00001 .00001 .00001 .00001 .00001 .0000$ $2,0=003 \quad 0.9996 \quad 0.99931 .00001 .00001 .00001 .00001 .00001 .00001 .00001 .00001 .00009 .00001 .00001 .00001 .00001 .0000$ $3.0=0030.99940 .09971 .0 n \pi n 1.00 n 1.00001 .00001 .00001 .00001 .00001 .00001 .00001 .00001 .00001 .00001 .00001 .0000$ $5,0-0030.99900 .09931 .00001 .00001 .00001 .00001 .00009 .00001 .00001 .00001 .00001 .00001 .00001 .00001 .00001 .0000$

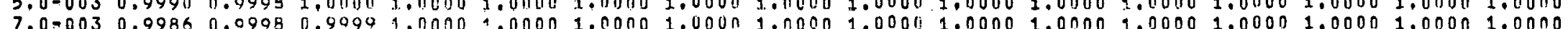

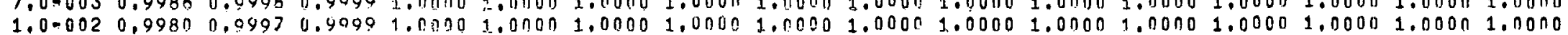

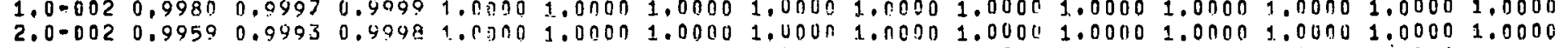
$3,0-0020.99390 .99900 .9997 \quad 1,00001.0000=00001.00009 .00001 .00001 .0000 \div 00001.00001 .0000$ $5,0=0020.9896 \quad 0.99830 .9996 \quad 0.99991 .0000 \div .00001 .00001 .00001 .00001 .0000 \div 00001.0000$

$7.0-0020.985 ? \quad 0.9970 \quad 0.9994 \quad 0.99991 .0000=.00001 .00001 .00001 .00001 .0000=0000$ $1.0=001 \quad 0.9783 \quad 0.09640 .9991 \quad 0.99991 .0000 \leq .00001 .00001 .00001 .00001 .0000=0000$ 2.0.001 $0.95270 .09210 .998[0.9997 \quad 0.99992 .00001 .00001 .00001 .0000$

$3,0.0010 .923 n \quad 0.08670 .9966 \quad 0.99950 .9999 \div .00001 .00001 .0000$

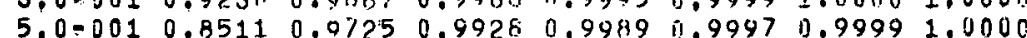

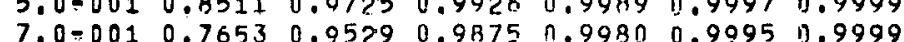

$\begin{array}{lllllll}1.0+000 & 0.6250 & 0.9118 & 0.9758 & 0.9962 & 0.9989 & 0.9997\end{array}$

$2.0+000 \quad 0.2632$. $0.48890 .8963 \quad 1.9824$

$3.0+000 \quad 0.1136 \quad 0.4429 \quad 0.7563$

$5.0+000 \quad 0.0313 \quad 0.1 .667$

$7,0+000 \quad 0.0124$

$1,0 \neq 001 \quad 0.0045$ 


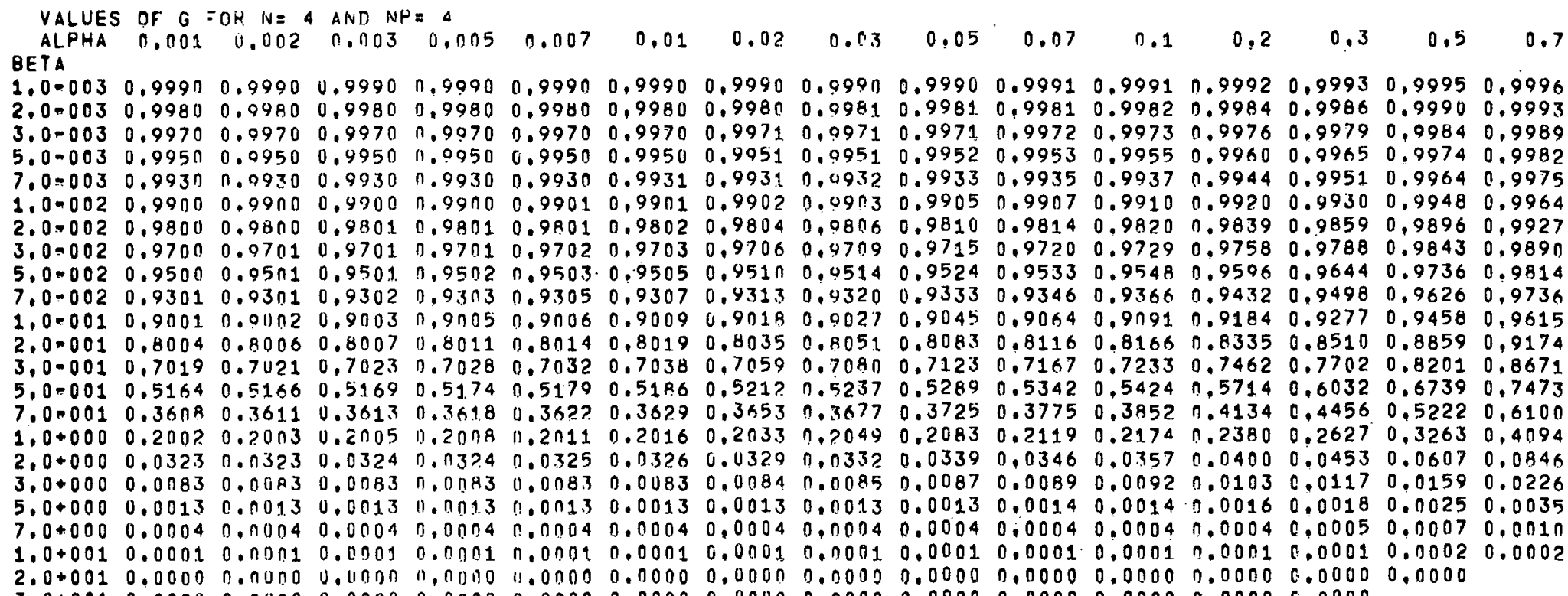


VALUES OF G FOR $N=4$ AND NP= 5 ALPH

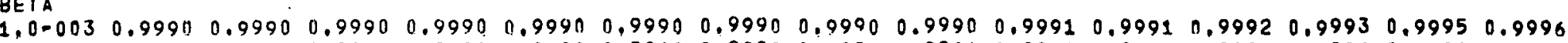

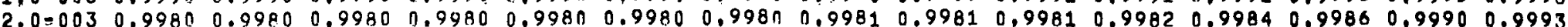

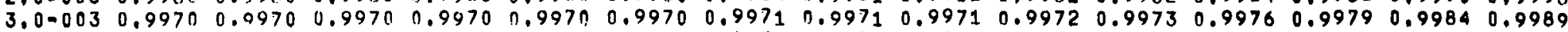

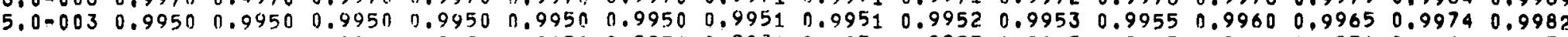
$\begin{array}{llllllllllllllll}7.0=003 & 0.9930 & 0.9930 & 0.9930 & 0.9930 & 0.9930 & 0.9931 & 0.9931 & 0.9932 & 0.9933 & 0.9935 & 0.9937 & 0.9944 & 0.9951 & 0.9964 & 0.9975\end{array}$ $1,0=002 \quad 0.9900 \quad 0.9900 \quad 10.9900 \quad 0.9900 \quad 0.9901 \quad 0.9901 \quad 0.9902 \quad 0.9903 \quad 0.99050 .9907 \quad 0.9910 \quad 0.9920 \quad 0.9930 \quad 0.9948 \quad 0.9964$ $\begin{array}{lllllllllllllllll}2,0.002 & 0.9800 & 0.9800 & 0.9801 & 0.9801 & 0.9801 & 0,9802 & 0.9804 & 0.98166 & 0.9810 & 0.9814 & 0.9820 & 0.9839 & 0.9859 & 0.9896 & 0.9927\end{array}$ $\begin{array}{lllllllllllllllll}3.0 & 002 & 0.9700 & 0.9701 & 0.9701 & 0.9701 & 0.9702 & 0.9703 & 0.9706 & 0.9709 & 0.9715 & 0.9720 & 0.9729 & 0.9758 & 0.9788 & 0.9843 & 0.9890\end{array}$ $5.0-0020.9500 \quad 0.95010 .95010 .95020 .95030 .95050 .95100 .95140 .95240 .95330 .95480 .95960 .96440 .97360 .9814$ 7.0.002 0.93010 .03010 .93020 .9303 .93050 .93070 .93130 .93200 .93330 .93460 .93660 .94320 .94980 .96260 .9814

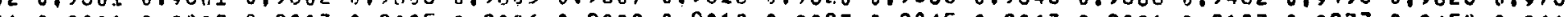
$1.0 \div 0010.90010 .00120 .9003$.

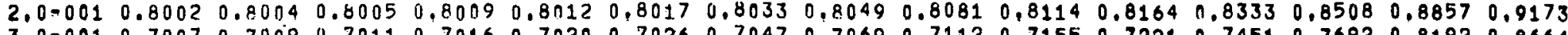
$\begin{array}{llllllllllllllllll}3.0-001 & 0.7007 & 0.7009 & 0.7011 & 0.7016 & 0.702 n & 0.7026 & 0.7047 & 0.7069 & 0.7112 & 0.7155 & 0.7221 & 0.7451 & 0.7692 & 0.8192 & 0.8664\end{array}$ $\begin{array}{lllllllllllllllllll}5,0.001 & 0.5082 & 0.5084 & 0.5087 & 0.5092 & 0.5097 & 0.5104 & 0.5130 & 0.5155 & 0.5207 & 0.5261 & 0.5342 & 0.5633 & 0.5953 & 0.6667 & 0.7411\end{array}$ $\begin{array}{llllllllllllllll}7,0-001 & 0.3402 & 0.3405 & 0.3407 & 0.3411 & 0.3416 & 0.3423 & 0.3445 & 0.3469 & 0.3516 & 0.3565 & 0.3640 & 0.3916 & 0.4233 & 0.4995 & 0.5882\end{array}$

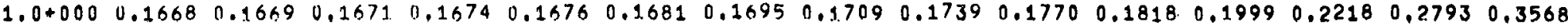

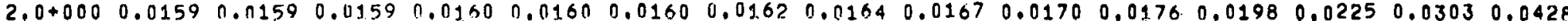
$3.0+0000.00270 .00280 .00280 .00280 .00280 .00280 .00280 .00280 .011290 .00300 .00310 .00340 .00390 .00530 .0076$ $5.0+0000.00030 .00030 .00030 .00030 .00030 .00030 .00030 .00030 .00030 .00030 .00030 .00030 .00040 .00050 .0003$

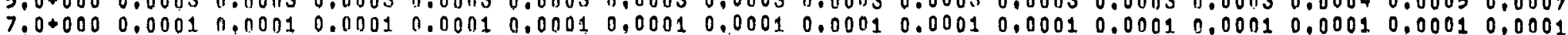

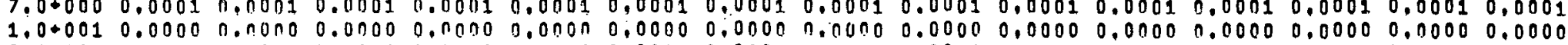
$2.0+0010.00000 .00000 .00000 .00100 .00000 .00000 .00000 .00000 .00000 .00000 .00000 .00000 .00000 .0000$

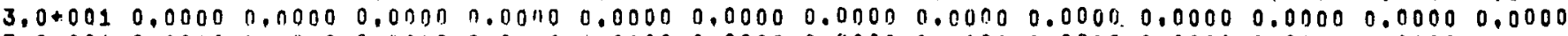
$5,0 \neq 0010,00000.00000 .00000,000110,00000,00000.00000,00000.00000,00000.00000,0000$

$7.0+0010.0000 \quad 0.0000 \quad 0.0000 \quad 0.00000 .0000 \quad 0.0000 \quad 0.00000 .00000 .00000 .00000 .0000$

$1.0+0020,00000.00000 .00000,00000,00000,00000,00000,00000.00000 .00000,0000$

$2.0+0020.00000 .00000 .00000 .00000 .00000 .0000 .000000 .0000 .0 .0000$

$3,0+0020,0000 \quad 1.0000 \quad 0.0000 \quad 0,00000.00000 .0000 \quad 0.00000 .0000$

$5,0+0020,00000.00000,0000,0,110000,00000,00000,0000$

$7,000020.0000 \quad 0.0000 \quad 0.00000 .001100 .0000 \quad 0,0000$

$1.000030 .00000 .00000 .00000 .00000 .0000 .0,0000$

$$
\text { BETA }
$$
BETA

ALPHA $1.000 \quad 2.000 \quad 3,000 \quad 5.000 \quad 7.000 \quad 10.00 \quad 20.00 \quad 30.00 \quad 50.00 \quad 70.00 \quad 100.0 \quad 200,0 \quad 300.0 \quad 500.0 \quad 700.0 \quad 1000$

$1.0-0030.99981 .00001 .00001 .00001 .00001 .00001 .00001 .00001 .00001 .00001 .00001 .00001 .00001 .00001 .00001 .0000$ 2.0-003 $0.99960,09991.00001,00001.00001,00001.00001 .00001 .00001,00001.00001 .00001 .00001 .00001 .00001 .0000$ $3.0-0030.99940 .99991 .00001,00001.00001 .00001 .00001 .00001 .00001 .00001 .00001 .00001 .00001 .00001 .00001 .0000$ $5.0=0030.9990 \quad 0.99981 .00001 .00001 .00001 .00001 .00001 .00001 .00001,00001.00001 .00001,00001.00001 .00001 .0000$

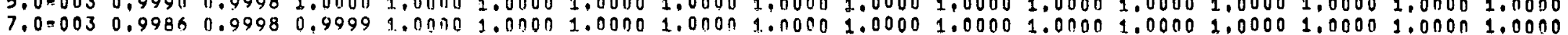

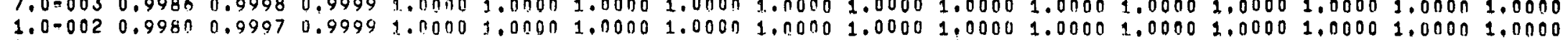

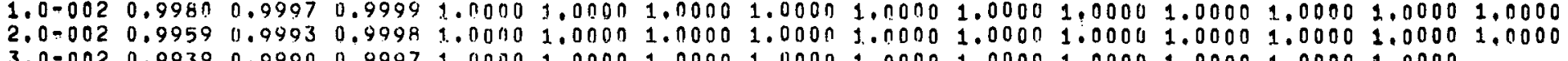

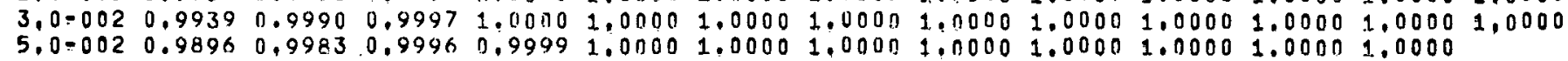
$7,0=0020.9852 \quad 0.09760 .9994 \quad 0.9999 \quad 1.00001 .00001 .00001 .00001 .00001 .00001 .0000$ 1.0=001 $0.97830 .9964 \quad 0.9991 .0 .99991 .00001 .00001 .00001 .00001 .00001 .00001 .0000$ $2.0 .0010 .95240 .9920 \quad 0.99790 .9997 \quad 0.99991 .00001 .00001 .00001 .0000$ $3.0=0010.9212 \quad 0.98640 .9965 \quad 0.9995 \quad 0.9998 \quad 1.00001 .00001 .0000$ $\begin{array}{lllllllll}5.09001 & 0.8377 & 0.9697 & 0.9921 & 0.9988 & 0.9997 & 0.9999 & 1.0000\end{array}$ $\begin{array}{lllllll}5.0 .001 & 0.7203 & 0.9411 & 0.9842 & 0.9975 & 0.9993 & 0.9998\end{array}$ $\begin{array}{lllllll}1,0 \rightarrow 000 & 0.5000 & 0.8611 & 0.9603 & 0.9936 & 0.998 ? & 0.9996\end{array}$ $\begin{array}{llllll}1,0+000 & 0.5000 & 0.8611 & 0.9603 & 0.9936 \\ 2.0+000 & 0.0746 & 0.3333 & 0.6612 & 0.9265\end{array}$ $3.0+000 \quad 0.0136 \quad 0.0787 \quad 0.250$ ?

$5,0+000 \quad 0,00130.0079$

$7.0+000 \quad 0.0003$

$1,0+0010.0000$ 


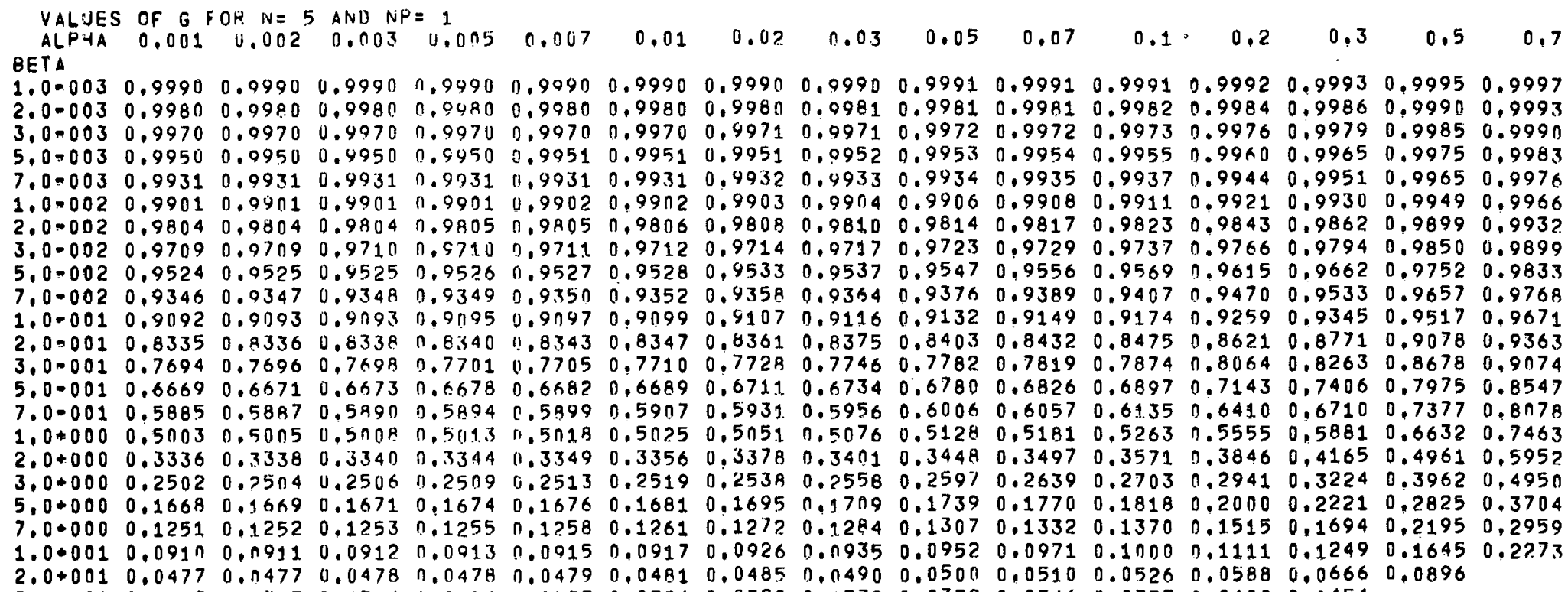

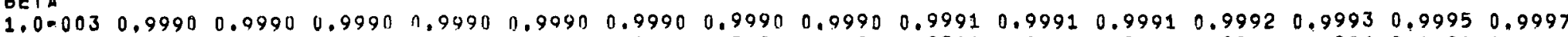
$\begin{array}{llllllllllllllll}2.0-003 & 0.9980 & 0.9980 & 0.9980 & 0.0980 & 0.9980 & 0.9980 & 0.9960 & 0.0981 & 0.9981 & 0.9981 & 0.9982 & 0.9984 & 0.9986 & 0.9990 & 0.9993\end{array}$

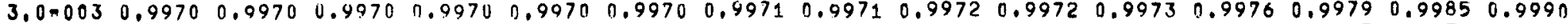

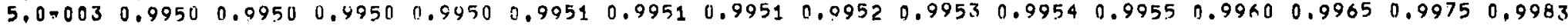

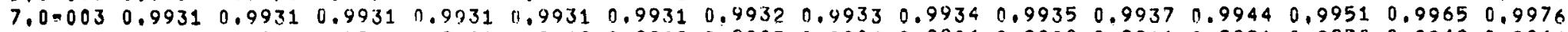

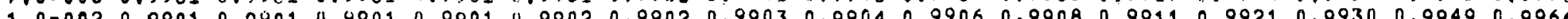

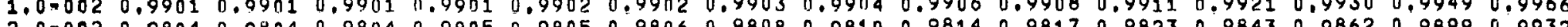

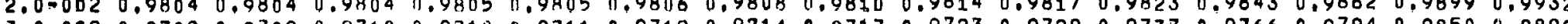
$3.0-0020.97090 .97 n 90.9710 .97109 .971 .0 .97120 .97140 .97970 .97230 .97290 .97370 .97660 .97940 .9850 .9899$

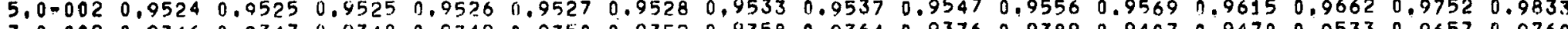

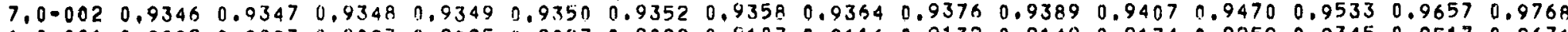
$\begin{array}{lllllllllllllllll}1.0-001 & 0.9092 & 0.9093 & 0.9 n 93 & 0.9195 & 0.9097 & 0.9099 & 0.9107 & 0.9116 & 0.9132 & 0.9149 & 0.9174 & 0.9259 & 0.9345 & 0.9517 & 0.9671\end{array}$ $\begin{array}{llllllllllllllllll}2.0=001 & 0.8335 & 0.8336 & 0.8338 & 0.8340 & 11.8343 & 0.8347 & 0.8361 & 0.8375 & 0.8403 & 0.8432 & 0.8475 & 0.8621 & 0.8771 & 0.9078 & 0.9363\end{array}$ $\begin{array}{lllllllllllllllllll}3.0 .001 & 0.7694 & 0.7696 & 0.7698 & 0.7701 & 0.7705 & 0.7710 & 0.7728 & 0.7746 & 0.7782 & 0.7819 & 0.7874 & 0.8064 & 0.8263 & 0.8678 & 0.9074\end{array}$ $\begin{array}{llllllllllllllll}5.0-001 & 0.6669 & 0.6671 & 0.6673 & 0.6678 & 0.6682 & 0.6689 & 0.6719 & 0.6734 & 0.6780 & 0.6826 & 0.6897 & 0.7143 & 0.7406 & 0.7975 & 0.8547\end{array}$ $\begin{array}{llllllllllllllllll}7.0-001 & 0.5885 & 0.5887 & 0.5990 & 0.5894 & 0.5899 & 0.5907 & 0.5931 & 0.5956 & 0.5006 & 0.6057 & 0.61 .35 & 0.6410 & 0.6710 & 0.7377 & 0.8178\end{array}$

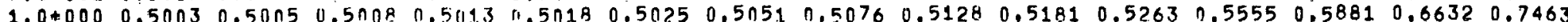
1.040000 .53360 .33300 .33400 .33440 .33490 .33560 .33780 .34010 .34480 .34970 .35710 .38460 .41650 .49610 .5952 2.040000 .33360 .33380 .33400 .33440 .33490 .33560 .33780 .34010 .34480 .34970 .35710 .38460 .41650 .49610 .5952 $3.0+0000.25020 .25040 .25060 .25090 .25130 .25190 .25380 .25580 .2590 .26390 .27030 .29410 .32240 .39620 .4950$ $5,0+0000.16680 .16690 .1671 \quad 0.16740 .16760 .16810 .16950 .171990 .17390 .17700 .18180 .20000 .22210 .28250 .3704$

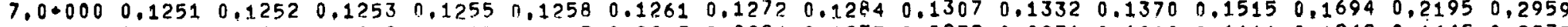

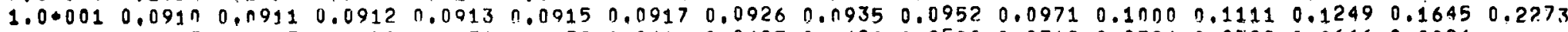

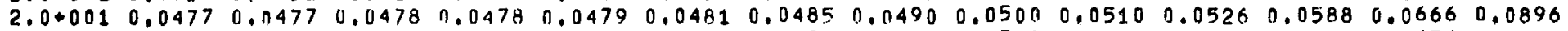

$\begin{array}{lllllllllllllllll}3,0+001 & 0.0323 & 0.0323 & 0.0324 & 0.0324 & 0.0325 & 0.0326 & 0.0329 & 0.0332 & 0.0339 & 0.0346 & 0.0357 & 0.0400 & 0.0454\end{array}$

$\begin{array}{llllllllllllllllllll}5,0+001 & 0.0196 & 0.0196 & 0.0197 & 0.0197 & 0.0197 & 0.0198 & 0.0200 & 0.0202 & 0.0206 & 0,0211 & 0.0217 & 0.0244\end{array}$

$\begin{array}{lllllllllllllllll}7,0+001 & 0.0141 & 0.0141 & 0.0141 & 0.0142 & 0.0142 & 0.0142 & 0.0144 & 0.0145 & 0.0148 & 0.0151 & 0.0156\end{array}$

$1.0+0020.00990 .00990 .00990 .01010 .0100 \quad 0.0100 \quad 0.01010 .01020 .01040 .01060 .0110$

$2,0+0020.00500 .00500 .00500 .00500 .00500 .00500 .00510 .00510 .0052$

$3.0+0020.00330 .00330 .00330 .00330 .00330 .00340 .00340 .0034$

$5,0+0020,00200.00200 .00200 .00200,00200.00200 .0020$

$7,0+002 \quad 0,00140.00140,00140,00140.01140 .0014$

$1.0+0030.00100 .00100 .00100 .001 .0 \quad 0.00100 .0010$

ALPHA $1.000 \quad 2.000 \quad 3.000 \quad 5.000 \quad 7.000 \quad 10.00 \quad 20.00 \quad 30.00 \quad 50.00 \quad 70.00 \quad 100.0 \quad 200.0 \quad 300.0 \quad 500.0 \quad 700.0 \quad 1000$ BETA

$1.0-0030.99981 .00001 .00001 .00001 .00001 .00001 .00001 .00001 .00001 .00001 .00001 .00001 .00001 .00001 .00001 .0000$ $2,0.0030 .99971 .00001 .00001 .00001 .00001 .00001 .00001 .00001 .00001 .00001 .00001 .00001 .00001 .00001 .00001 .0000$

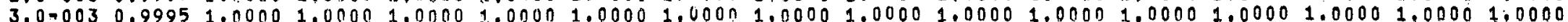
$5,0=0030,99920,99991,00001.00001 .00001,00001.00001 ., 00001.00001 .00001 .00001,00001.00001 .00001 .00001 .0000$ $7,0=0030.99880 .99091 .00001 .00001 .00001 .00001 .00001 .00001 .00001 .00001 .00001 .00001 .00001 .00001 .00001 .0000$ $1.0-0020.99830 .99981 .00001 .00001 .00001 .00001 .00001 .00001 .00001 .00001 .00001 .00001 .00001 .00001 .00001 .0000$ $2.0-0020.99670 .99970 .99991 .00001 .00001 .00001 .00001,00001.00001 .00001 .00001 .00001 .00001 .0000$

$3,0-0020.9950 \quad 0,99950.99991 .00001 .00001 .00001 .00001 .00001 .00001 .00001 .00001 .00001 .0000$

$5,0=0020,99170.99920 .99991,00001.00001,00001.00001 .00001 .00001 .00001 .00001 .0000$

$7,0.002 \quad 0.98850 .99890 .99981 .00001 .00001 .00001 .00001 .00001 .00001 .00001 .0000$

1.0 .0010 .98360 .99840 .99971 .00001 .00001 .00001 .00001 .00001 .00001 .00001 .0000

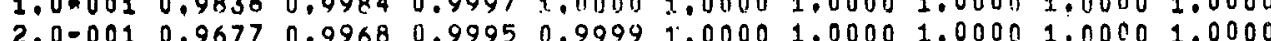

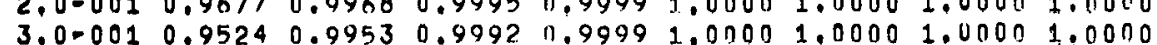

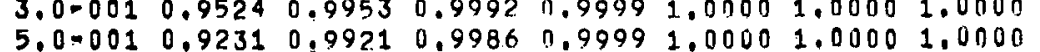

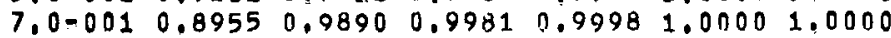

$1.0+000 \quad 0.8571 \quad 0.98440 .9973 \quad 0.9997 \quad 0.99991,0000$

$2.0+000 \quad 0.7500 \quad 0.9692 \quad 0.9945 \quad 0.9995$

$\begin{array}{llllll}3.0+000 & 0.6667 & 0.9545 & 0.9918\end{array}$

$5.0+000 \quad 0.5455 \quad 0.9265$

$7,0+000 \quad 0,4615$

$1.0+0010.3750$ 
VALUES OF G FOR $N=3$ AND $N P=2$ ALPH

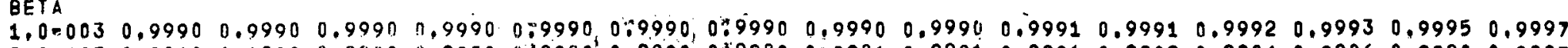

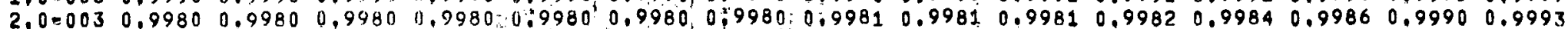
$3.000030 .9970 \quad 0.9970 \quad 0.9970 \quad 0.9970 \quad 0.997 n: 0.9970 \quad 0.9971 \quad 0.9971 \quad 0.997100 .9972 \quad 0.9973 \quad 0.99760 .99790 .99850 .9990$ $5.0=003 \quad 0.9950 \quad 0.9950,0.995$ in $0.9950 \quad 0.9950 \quad 0.9950 \quad 0.9051 \quad 0.9951 \quad 0.9952 \quad 0.9953 .0 .9955 \quad 0.9960 \quad 0.9965 \quad 0.9975 \quad 0.9983$ $7.0=003 \quad 0.9930 \quad 0.9930 \quad 0.9930 \quad 0.9930 \quad 0.9930 \quad 0.99310 .9931 \quad 0.99320 .99330 .99350 .9937 \quad 0.99440 .9951 \quad 0.9964 \quad 0.9976$ $1.0=002 \quad 0.9900 \quad 0.9900 \quad 0.9900 \quad 0.9901,0.9901,0.9901,0.9902 \quad 0.9903 \quad 0.9905 \quad 0.9907 \quad 0,9910 \quad 0.9920 \quad 0.9930 \quad 0.9949 \quad 0.9966$

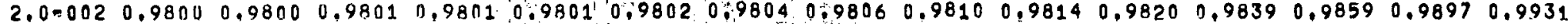

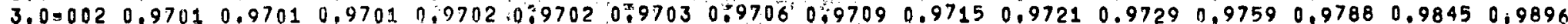
$5.0=0020.95020 .95020 .95030 .9504 i 105050.95060 .951140395150 .95250 .95340 .95490 .95970 .96450 .97400 .9825$ $5.0=0020.9020 .950 .90 .985$

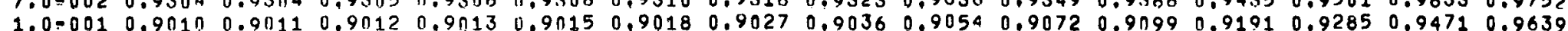

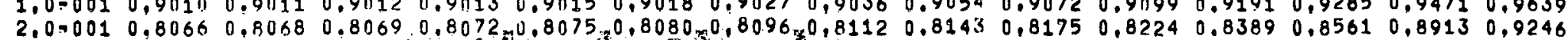

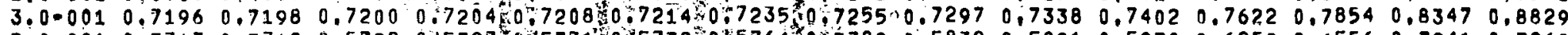

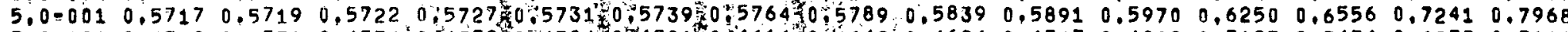

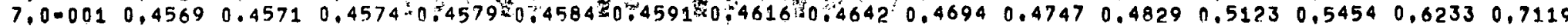

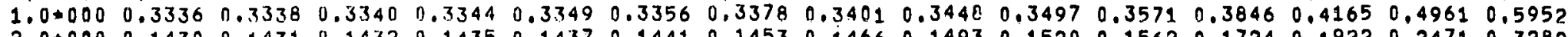

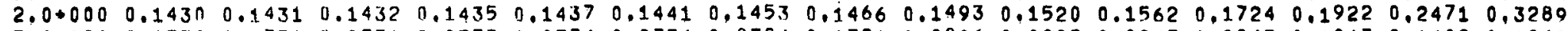
$3,0+000 \quad 0.0770 \quad 0.0771 \quad 0.0771,0.0773,0.0774,0.0776,0.0784,0.0791 .0 .0806 \quad 0,08220,0847.0 .0943 \quad 0.1063 \quad 0.1409 \quad 0.1969$

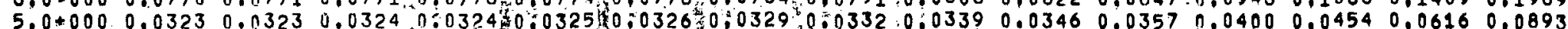

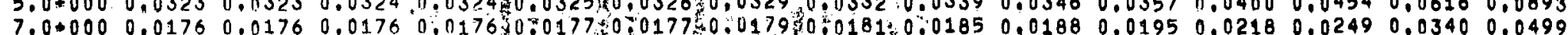

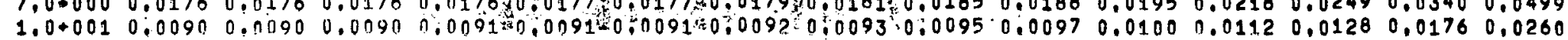

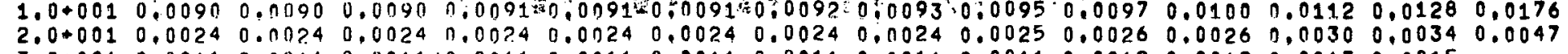
$3.0+0010.00110 .00110 .0011 .0 .00110 .00110 .00110 .00110 .00110 .00110 .00120 .00120 .00130 .0015$ $5,0+0010,00040,00040,0004,0,00040,00040,0004,0,00040,00040,00040,00040,00040,0005$

$7.0+0010.00020 .00020 .00020 .00020000020000020,00020000020000020,00020,0002$

$1,0 \neq 0020,00010,00010,0001,0,00010,00010,0001,0,00010,00010,00010,00010,0001$

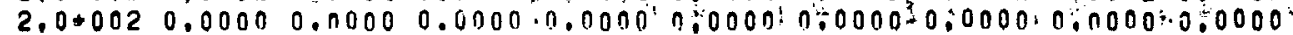

$3.0+002 \quad 0.00000 .00000 .00000 .00000 .00000 .00000 .00000 .0000$

$5.0+002 \quad 0.00000 .00000 .00000 .00000 .00000 .00000 .0000$

$7.0+0020.0000 \quad 0,00000,0000 \quad 0.0000,0.0000,0,0000$

$1.0+0030,00000,00000,0000,0: 00000,000010,0000$ BETA

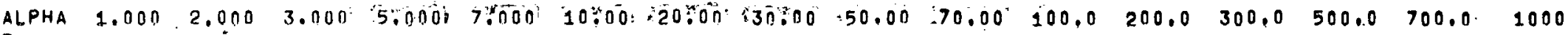

$1.0-0030.99981 .00001 .00001 .00001 .00001 .00001 .00001 .00001 .00001 .00001 .00001 .00001 .00001 .00001 .00001 .0000$ $2.0=0030.99971 .0000 .1 .0000,1.0000 .1 .00001 .00001 .0000 .1 .00001 .00001 .00001 .00001 .00001 .00001 .00001 .00001 .0000$ $3.0=0030.99951 .00001 .00001 .0000,100001100001700001 \% 00001 \% 00001.00001 .00001 .00001 .00001 .00001 .00001 .0000$

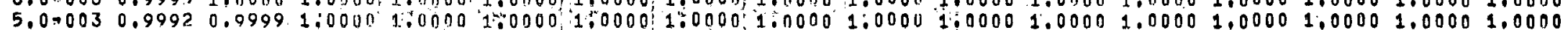

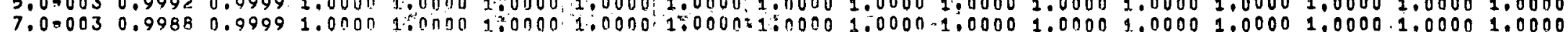
$1.0-0020.99830 .99981 .00001 .00001 .00001 .00001 .00001 .00001 .00001 .00001 .00001 .00001 .00001 .00001 .00001 .0000$ $2.000020 .99660 .99970 .99991 .00001 .00001,00001.00001 .000011 .00001 .00001 .00001 .00001 .00001 .0000$ 3.000020 .99490 .99950 .99991 .0000 .1 .00001 .00001 .0000 .1 .00001 .00001 .00001 .00001 .00001 .0000 $5.0-0020.99130 .99920,9999,100000,1 \% 00001000001,00001.00001 .00001 .00001 .00001 .0000$

$7,0=002.0,98770,998,80,99981,0000: 1,0000 \div 1,0000,1: 00001,00001.00001,00001,0000$ $1,0-0010,98200.9983,0: 9997.1 \% 00001.0000 \cdot 1,00001 \% 00001,00001.00001 .00001 .0000$ $2,0=0010.96150 .99620 .9993 \quad 1.99991 .00001 .00001 .00001 .00001 .0000$ $3.0=0010.9390 \quad 0.99380 .99890 .99991 .00001 .00001 .00001 .0000$

$5,0=001 \quad 0.88890 .9882 \quad 0,997,0,99981.0000,1.00001,0000$

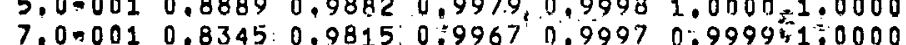

$1.0+000 \quad 0.7500 \quad 0.9692 \quad 0 \% 99450 \% 9995,0 \% 9990100000$

$2.0+000 \quad 0.5000 \quad 0.9130 .0 .9838 \quad 079985$

$3.0+000 \quad 0.3333 \quad 0.8400 \cdot 0.9681$

$5.0 \$ 000 \quad 0.1667 \quad 0.6774$

$7.0+000 \quad 0.0968$

$1,0+0010.051$ ? 


\begin{abstract}
VALUES OF G FOR $N=5$ ANT $N A=3$ BETA

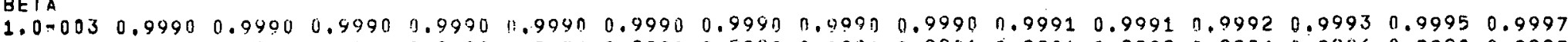

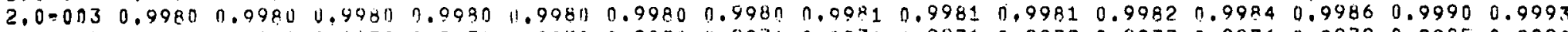
$\begin{array}{lllllllllllllll}3.0-003 & 0.9970 & 0.9970 & 0.9970 & 0.0970 & 0.9970 & 0.9970 & 0.9971 & 0.0971 & 0.9971 & 0.9972 & 0.9973 \quad 0.9976 & 0.9979 & 0.9985 & 0.9990\end{array}$ $\begin{array}{llllllllllllllll}5,0-003 & 0.9950 & 0.0950 & 0.9950 & 1.9950 & 11.9950 & 0.9950 & 0.9951 & 1.0951 & 0.9952 & 0.9953 & 0.9955 & 0.9900 & 0.9965 & 0.9975 & 0.9983\end{array}$ $\begin{array}{llllllllllllllll}7.0-003 & 0.993 n & 0.0930 & 0.9930 & 1.0930 & 10.9930 & 0.9931 & 0.9931 & 0.0032 & 0.9933 & 0.9935 & 0.9937 & 0.9944 & 0.9951 & 0.9964 & 0.9976\end{array}$

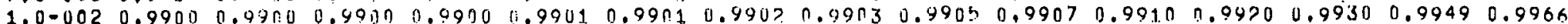

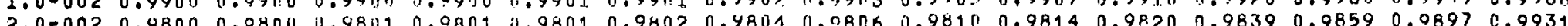

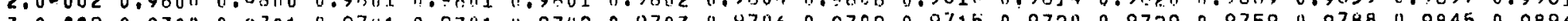
$3.0-0020.97000 .07010 .97110 .0701$. $5.0-0020.95010 .05010 .9501$ n.95 7.0-002 $0.93010 .93 n 20.931120 .9303 \quad n .93050 .93070 .93130 .03200 .93330 .93460 .93660 .94320 .94990 .96320 .9751$

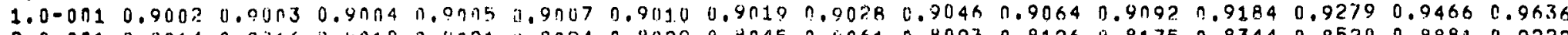
2.0-001 $0.80140 .8116 \quad 0.0018 \quad 0.8 n>1 \quad 11.8 n 24 \quad 0.8029 \quad 0.8045 \quad 0.8061 \quad 0.5093 \quad 0.8126 \quad 0.8175 \quad 0.8344 \quad 0.8520 \quad 0.8881 \quad 0.9222$ $\begin{array}{llllllllllllllllll}3.0001 & 0.7059 & 0.7061 & 0.7063 & 0.7068 & 1: .7 n 72 & 0.7078 & 0.7099 & 0.7120 & 0.7163 & 0.7206 & 0.7271 & 0.7498 & 0.7739 & 0.8252 & 0.8758\end{array}$ $\begin{array}{llllllllllllllll}5.0 .001 & 0.5336 & 0.5338 & 0.5341 & 0.5346 & 0.5351 & 0.5355 & 0.5384 & 0.5409 & 0.5461 & 0.5513 & 0.5594 & 0.5882 & 0.6200 & 0.6923 & 0.7707\end{array}$ $\begin{array}{lllllllllllllllll}7.0-001 & 0.395 n & 0.3953 & 0.3955 & 0.3060 & 1.3905 & 0.3972 & 0.3996 & 0.40 ? 1 & 0.4071 .0 .4123 & 0.4202 & 0.4491 & 0.4822 & 0.5622 & 0.6574\end{array}$

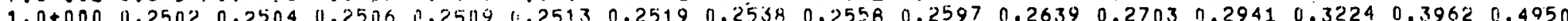
1.0 .0000 .06670 .06680 .06690 .0 .7006710 .06730 .06790 .06460 .06980 .07130 .07350 .08200 .09250 .9330 .1736

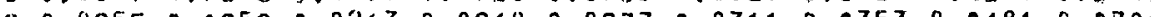

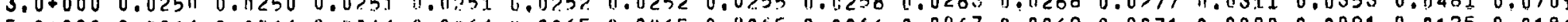

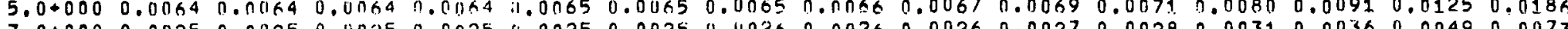

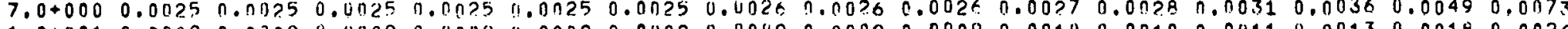
$1.0+0010.00090 .00090 .00090 .011998 .00090 .00090 .00090 .00090 .00090 .00100 .00100 .00110 .00130 .00180 .0026$

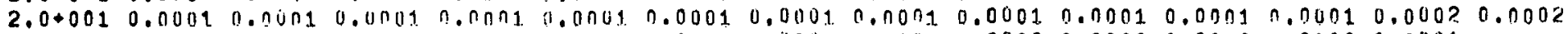

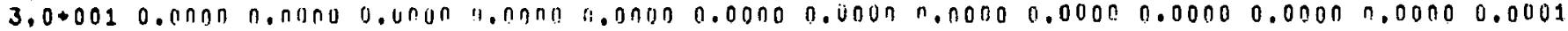

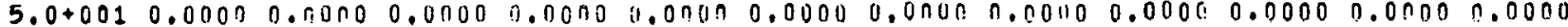

$7.0+001 \quad 0.00000 .010000 .00000 .00000 .00000 .00000 .00000 .00000 .00000 .00000 .0000$

$1.0+0020.00000 .00000 .0000 \quad 0.00000 .00000 .00000 .00000 .00000 .00000 .00000 .0000$

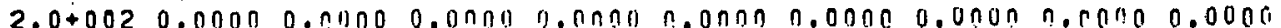

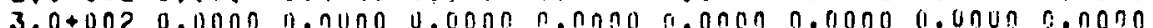

$5.0+0020.0000$ 0.

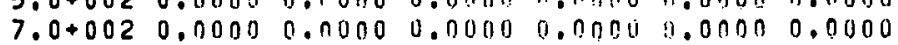

$1,0+0030.00000 .00000 .0000 \quad 0.00000 .00000 .0000$

ALFHA $1.000 \quad 2.000 \quad 3.0005 .000 \quad 7.000 \quad 20.00 \quad 20.0030 .0050 .00 \quad 70.00 \quad 100.0 \quad 200.0 \quad 300.0 \quad 500.0 \quad 700.0 \quad 1000$ BETA

$1.0-0030.99981 .00001 .00001 .00009 .00001 .00001 .00001 .0000 .00001 .00001 .00001 .00001 .00001 .00001 .00001 .0000$ 2.0.003 $0.99971 .01001 .000 n 1.0090,1.00001 .00001 .00001 .00001 .00001 .00001 .00001 .00001 .00001 .00001 .00001 .0000$

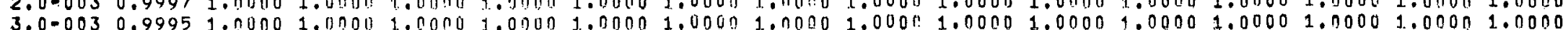

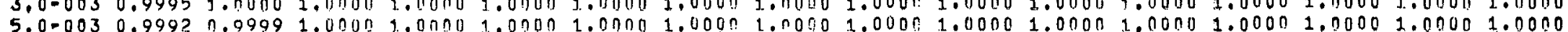
$5,0-003$
$7.0-0.9992$
$1.0-0.9989$

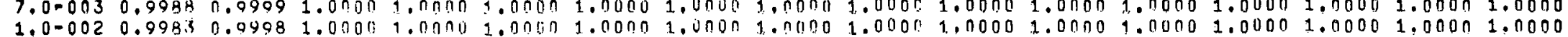
2.0-002 $0.99660 .09970 .99991 .07001 .00091 .00001 .0000 \quad 1.00001 .00001 .00001 .00001 .00001 .00001 .0000$ $3,0-0020.99490 .99950 .99991 .00 n 01.00001 .00001 .00001 .00001 .0008 j_{j} 1.00001 .00001 .00001 .0000$

$5,0-0020.99130 .09920 .99991 .00001 .00001 .00001 .00001 .00001 .00001 .00001 .00001 .0000$

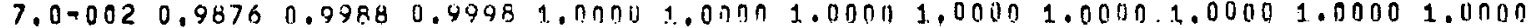

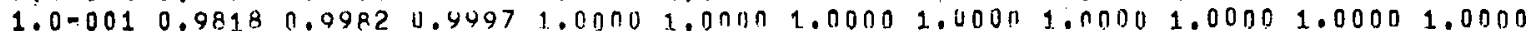

2.0-001 0.96050 .09610 .4993 il. $99091 \% 00001.00001 .000 n 1.000 \pi 1.0000$

$3.090010 .435 n n .09340 .99890 .99491 .00 n n 1.00001 .00001 .0000$

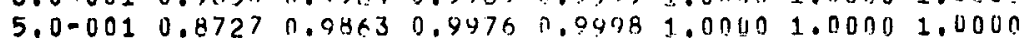

$7.0 .0010 .7965 \quad 0.07620 .4956 \quad 0.9996 \quad 0.99991 .0000$

$1.0+000 \quad 0.6667 \quad 0.0545 \quad 1.9918 \quad 0.9902 \quad 0.9998 \quad 1.0000$

$2.0+0000.30000 .8182 \quad 1.96300 .9064$

$3,0+000 \quad 0.1333 \quad 0.6176 \quad 0.9133$ ?

$5.0+000 \quad 0.0373 \quad 0.2800$

$7.0+000 \quad 0.014 \%$

$1,0+0010.0054$ 
VALUES OF G FOH $N=5$ AIN! $N H=A$ BETA

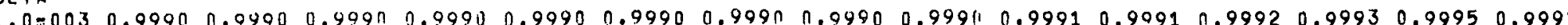
2.0-003 $0.99800 .99800 .998011 .99400 .99400 .99800 .90800 .94810 .99810 .99810 .90820 .99840 .99860 .0990 \quad 0.9993$

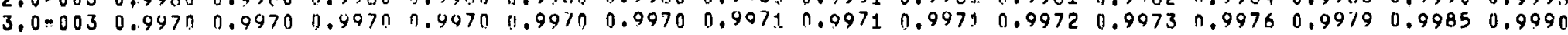

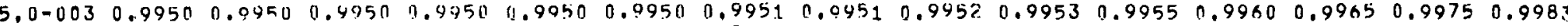
$\begin{array}{llllllllllllllllll}7.0-003 & 0.9930 & 0.9930 & 1.9930 & 11.9931 & 0.9930 & 0.9931 & 0.9931 & 0.9932 & 0.9933 & 0.9935 & 0.9937 & 0.9944 & 0.9951 & 0.9964 & 0.997 \mathrm{~K}\end{array}$ $\begin{array}{llllllllllllllll}1.0-002 & 0.9900 & 0.9900 & 1.9900 & 0.9900 & 0.9901 & 0.9901 & 0.9902 & 0.9903 & 0.9905 & 0.9907 & 0.9910 & 0.9920 & 0.9930 & 0.9949 & 0.9966\end{array}$

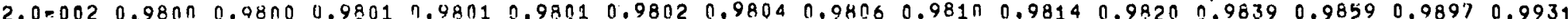

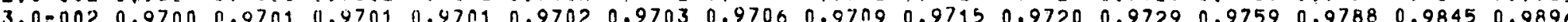

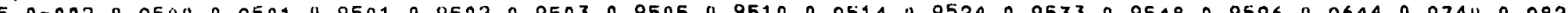

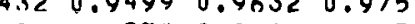
$1.0-0010.9 n 010.01 .2$ v.

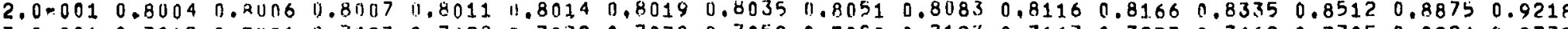
$\begin{array}{llllllllllllllll}3.0=001 & 0.7019 & 0.7021 & 0.7023 & 0.7028 & 0.7032 & 0.7038 & 0.7059 & 0.7080 .0 .7123 & 0.7167 & 0.7233 & 0.7462 & 0.7705 & 0.8224 & 0.8737\end{array}$ $\begin{array}{lllllllllllllllll}5.0-001 & 0.5164 & 0.5166 & 0.5169 & 0.5174 & 0.5179 & 0.5186 & 0.5212 & 0.5237 & 0.5289 & 0.5342 & 0.5424 & 0.571 .4 & 0.6036 & 0.6774 & 0.7583\end{array}$ $\begin{array}{lllllllllllllllll}7,0-001 & 0.3608 & 0.3611 & 0.3613 & 0.3618 & 0.362 ? & 0.3629 & 0.3653 & 0.3677 & 0.3725 & 0.3775 & 0.3852 & 0.4135 & 0.4460 & 0.5261 & 0.6230\end{array}$ $\begin{array}{llllllllllllllll}1.0+000 & 0.2002 & 0.2003 & 0.20105 & 0.2008 & 0.2011 & 0.2016 & 0.2033 & 0.2049 & 0.2083 & 0.2119 & 0.21 .74 & 0.2381 & 0.2630 & 0.3298 & 0.4237\end{array}$ $2.0+000 \quad 0.032 .30 .0323 \quad 0.0324 \quad 0.03240 .03250 .0326 \quad 0.03290 .03320 .03390 .03460 .0357 \quad 0.04 n 0 \quad 0.0454 \quad 0.06160 .0893$ $3.0+0000.00830 .01830 .01830 .00830 .00830 .00830 .00840 .00550 .00870 .00890 .00920 .01030 .01180 .01610 .0239$ $5,0+0000.00130 .00130 .00130 .00130 .00130 .00130 .00130 .00130 .00130 .00140 .00140 .00160 .00180 .00250 .0038$

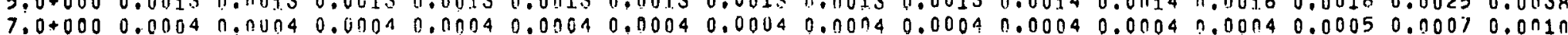

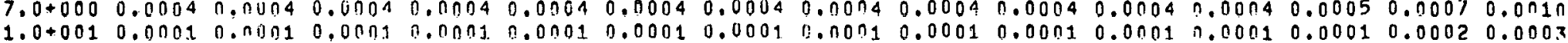

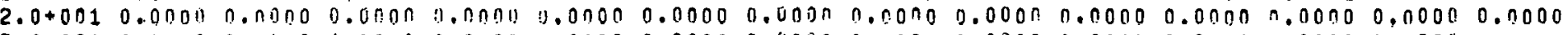

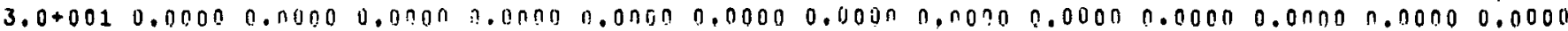

$5.0+0010.00000 .013 n 00.00003 .00000 .00000 .00000 .00000 .00000 .00000 .00000 .00000 .0000$

$7.0+0010.0000 \quad 0.0000 \quad 0.00000 .011000 .00000 .00000 .00000 .00000 .00000 .00000 .0000$

$1.0+0020.0000 \quad 0.0000 \quad 0.00003 .00000 .0000 \quad 0.0000 \quad 0.000 n \quad 0.00000 .00000 .00000 .0000$

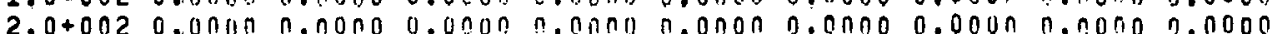

$3.0+0020.000 n$.

$5.0+0020.00000 .00000 .000110 .00 n 00.01000 .00000 .0000$

$7.0+0020.00000 .00000 .110000 .00000 .00000 .0000$

$1,0+0030.0000,0,00000.00000 .0000 \quad 0.00000 .0000$

$$
\text { BETA }
$$

BETA

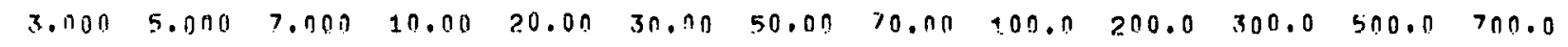

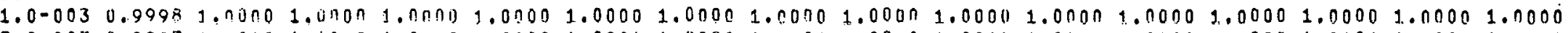
$2,0=0030.99971 .00001 .00001 .00001 .00001 .00001 .00001 .00001 .00001 .00001 .00001 .00001 .00001 .00001 .00001 .0000$ $3,0=0030.99951 .00001 .00001 .00001 .00001 .00001 .00001 .00001 .00001 .00001 .00001 .00001 .00001 .00001 .00001 .0000$

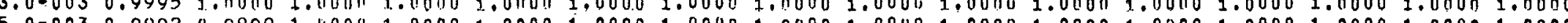
7.0-003 0.998 . 7.0-003 0.998. $1.0=0020.99830 .09931 .00001 .010 n 01.00601 .00001 .00001 .00901 .00001 .00001 .00001 .00001 .00001 .00001 .00001 .0020$ $2,0.0020 .9966 \quad 0.0997 \quad 1.9999 \quad 1.001010 .00001 .00001 .00001 .00001 .00001 .00001 .00001 .00001 .00001 .0000$ $3.0-0020.99490 .09950 .90991 .001103 .00091 .00001 .00001 .00001 .00001 .00001 .00001 .00001 .0000$ $5,0-0020.9913 \quad 0.09920 .99991 .00001 .00001 .00001 .00001 .00001 .00001 .00001 .00001 .0000$

$7.0-0020.987 \mathrm{~K} \quad 11.09880 .99981 .00001 .00001 .00001 .00001 .00001 .00001 .00001 .0000$ $1.0-0010.981 \mathrm{~A} 11.09920 .99971 .00110 \mathrm{~g} 3.00001 .00001 .00001 .00001 .00001 .00001 .0000$ 2.0.001 $0.96010 .0961 \quad 0.9993 \quad 0.9999 \quad 1.00001 .00001 .00001 .00001 .0000$

$3.0-0010.93380 .04330 .9988 \quad 0.9999 \quad 9.00001 .00001 .00001 .0000$

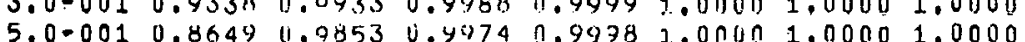

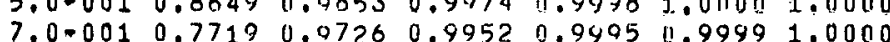

$1.0+0000.600 n 10.04030 .98910 .90900 .99981 .0000$

$2.0+000 \quad 0.1667 \quad 0.6774 \quad 0.4239 \quad 7.0974$

$3.0+000 \quad 0.0476 \quad 11.3443 \quad 0.75 \% 1$

$5.0+000 \quad 0.0076 \quad 0.0747$

$7.0+000 \quad 0.0021$

$1,0+0010,0005$ 


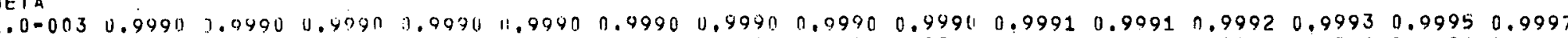
2.0-003 $0.9989 \quad 7.0980 \quad 11.9480 \quad 0.0090 \quad 11.9960 \quad 0.9980 \quad 0.4980 \quad 0.9991 \quad 0.9981 \quad 0.9981 \quad 0.9982 \quad 0.9984 \quad 0.9986 \quad 0.9990 \quad 0.9993$ $\begin{array}{lllllllllllllllll}3.0-003 & 0.9970 & 3.0970 & 1.4971 & 0.9070 & 0.90 \% 10 & 0.9970 & 0.9971 & 0.0971 & 0.9971 & 0.9972 & 0.9973 & 0.9976 & 0.9979 & 0.9985 & 0.9990\end{array}$

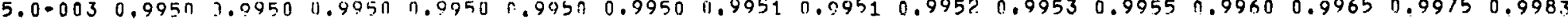
$\begin{array}{llllllllllllllll}7.0-003 & 0.993 i & 3.0930 & 0.9930 & 0.9930 & 11.9030 & 0.9931 & 1.9031 & 0.0932 & 0.9933 & 0.9935 & 0.9937 & 0.9944 & 0.9951 & 0.9964 & 0.9976\end{array}$

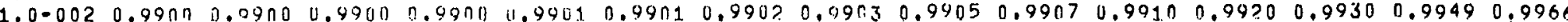

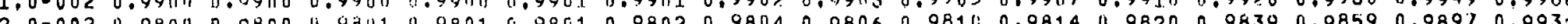

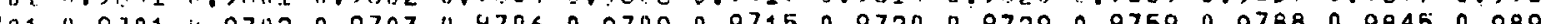

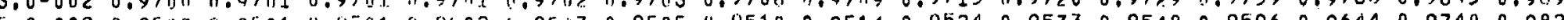
.00-002 0.950n 0.9501 v.

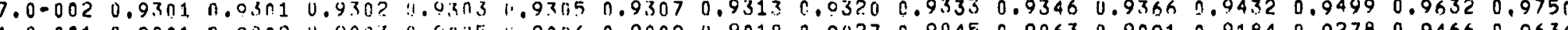

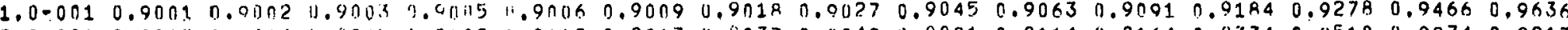

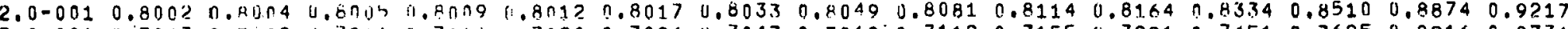

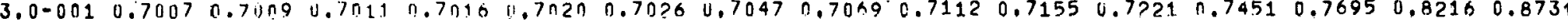
$\begin{array}{llllllllllllllll}5.0-001 & 0.5082 & 0.51) 84 & 0.5 n 47 & 0.509 ? & 0.5 n 97 & 0.5104 & 0.513 n & 0.5155 & 0.5207 & 0.5261 & 0.5342 & 0.5634 & 0.5957 & 0.6702 & 0.752 ?\end{array}$ $\begin{array}{llllllllllllllllll}7.0-001 & 0.34 n ? & 0.34 n 5 & 11.34 n 7 & 11.3411 & 1.3416 & 0.3423 & 0.3445 & 0.3469 & 0.3516 & 0.3565 & 0.364 n & 0.3917 & 0.4238 & 0.5035 & 0.6 n 24\end{array}$

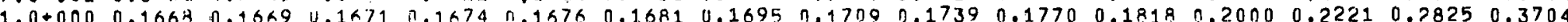
1.0+000 $0.01590 .01590 .01590 .0160 \quad 01000.01600 .01620 .01640 .01670 .01700 .01760 .01980 .02250 .03080 .0453$

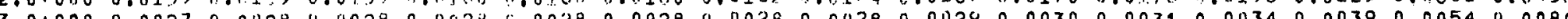
作 $5,0+000$ 0.003 $7.0+000$. 0001 n.n.

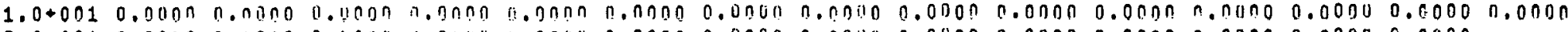

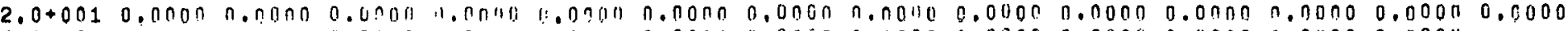

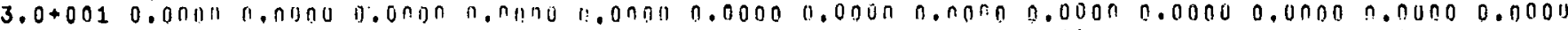

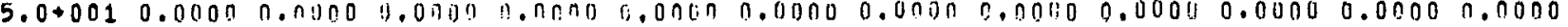

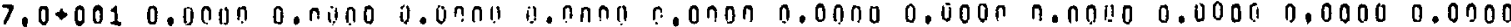

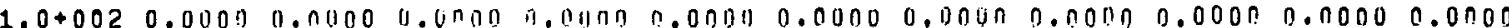

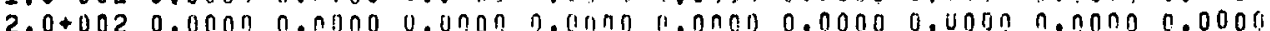

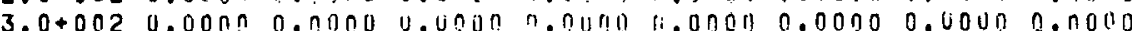

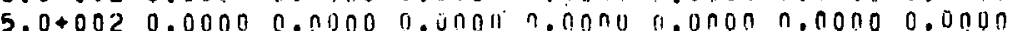

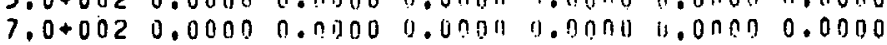

$1,0+0030.00000 .00000,00000,0000$ it.0nण0 0.0000

ALPHA 2.000 2.000 3.100 5.000 7.000 $10.00 \quad 20.0030 .0050 .00 \quad 70.00 \quad 100.0200 .0 \quad 300.0 \quad 500.0 \quad 700.0 \quad 1000$ BETA

$1.0-0030.99981 .00 n 01.00001 .0 n 0 n 1.0 n 001.00001 .000 n 1.00001 .00001 .00001 .00001 .00001 .00001 .00001 .00001 .0000$

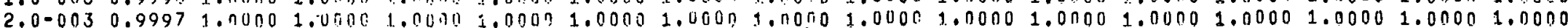

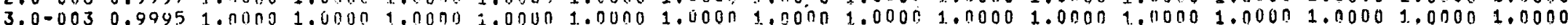

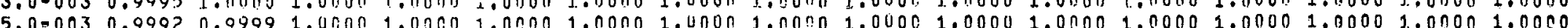

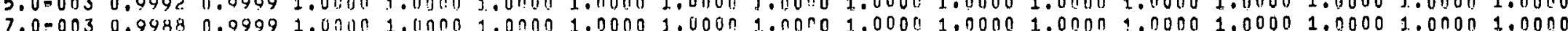

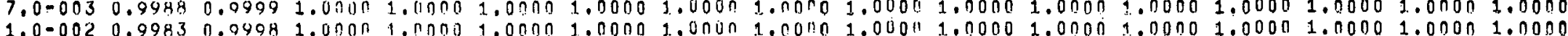

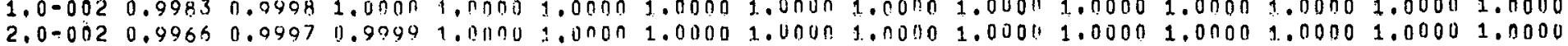
$3.0-0020.90490 .09950 .99991 .01001 .00001 .00001 .00001 .00901 .00001 .00001 .00001 .00001 .0000$

$5.0-0020.99130 .99920 .99991 .00001 .00001 .00001 .00001 .001101 .00001 .00001 .00001 .0000$

$7.0=002 \quad 0.9875 \quad 0.9988 \quad 0.9949$ 1.0030 1.00001 .00001 .00001 .00001 .00001 .00001 .0000

$1.0-0010.98980 .09820 .40971 .01001 .00001 .00001 .000 n 1.00001 .00001 .00001 .0000$

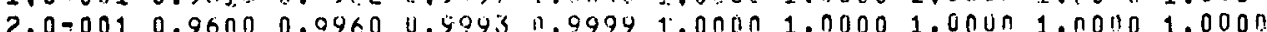

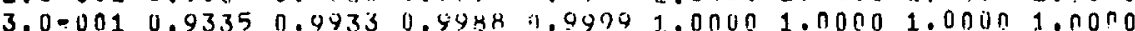

$5.0-0010.86100 .98494 .9973,0.09981 .00001 .00001 .0000$

$7.0=0010.7556 \quad 0.97010 .9947 \quad 1.9995 \quad(1.9999 \quad 1.0000$

$1.0+000 \quad 0.5455 \quad 0.9265 \quad 0.4864 \quad 0.9987 \quad 0.9997 \quad 1.0000$

$2.0+000 \quad 0.0882 \quad 0.51140 \quad 0.6545 \quad 0.9844$

$3.0+000 \quad 0.0163 \quad 0.1479 \quad 0.5007$

$5,0+000 \quad 0.00150 .0159$

$7.0+000 \quad 0.0003$ 
VALUES OF G FOH: $N=6$ AIV N NFF= BET

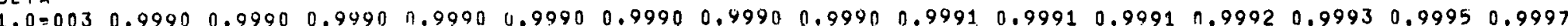

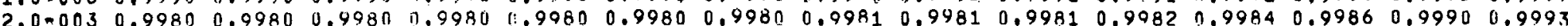
$\begin{array}{lllllllllllllllll}3.0-003 & 0.9970 & 0.9970 & 0.9970 & 7.9970 & 1.997 n & 0.9970 & 0.9971 & 0.9971 & 0.9972 & 0.9972 & 0.9973 & 0.9976 & 0.9979 & 0.9985 & 0.9990\end{array}$ $5.0=003 \quad 0.9950 \quad 0.0950 \quad 0.9950 \quad 0.9950 \quad 0.9059 \quad 0.9951 \quad 0.9951 \quad 0.0952 \quad 0.9953 \quad 0.9954 \quad 0.9955 \quad 0.9960 \quad 0.9965 \quad 0.9973 \quad 0.9984$ $\begin{array}{llllllllllllllll}7.0-003 & 0.9931 & 0.9931 & 0.9931 & 0.9931 & 0.9931 & 0.9931 & 0.9932 & 0.0933 & 0.9934 & 0.9935 & 0.9937 & 0.9944 & 0.9951 & 0.9965 & 0.9977\end{array}$

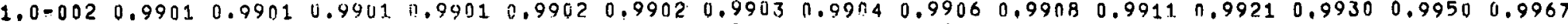

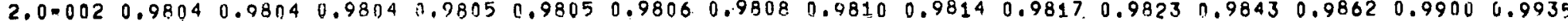

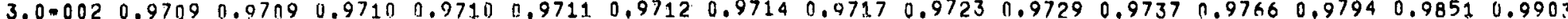
5.0 .0020 .95240 .05250 .95250 .95260 .95270 .95280 .95330 .05370 .95470 .95560 .95690 .96150 .96620 .97540 .9830

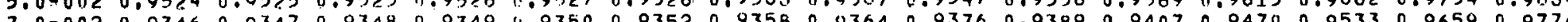

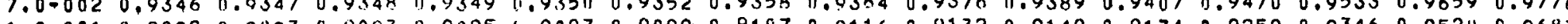

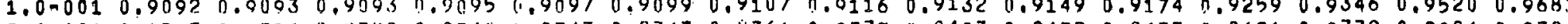

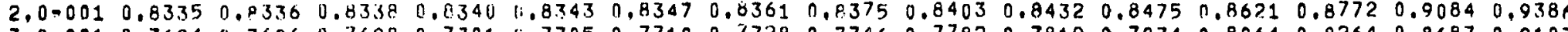
$3.0=0010.76940 .76960 .76980 .7701 \quad 0.7705 \quad 0.77100 .77280 .77460 .77820 .78190 .79740 .80640 .82640 .86870 .9107$ $\begin{array}{llllllllllllllllll}5.0-001 & 0.6669 & 0.6671 & 0.6673 & 0.6678 & 0.6682 & 0.6689 & 0.6711 & 0.6734 & 0.6780 & 0.6826 & 0.6997 & 0.7143 & 0.7407 & 0.7987 & 0.8595\end{array}$ 7.0-001 $0.58850 .58 .7 \quad 0.5890 \quad 0.5894 \quad 0.59990 .5907 \quad 0.5931 \quad 0.5956 \quad 0.6006 \quad 0.6057 \quad 0.6135 \quad 0.6410 \quad 0.6711 \quad 0.7392 \quad 0.8138$

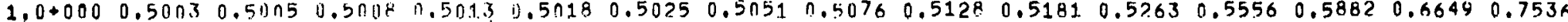

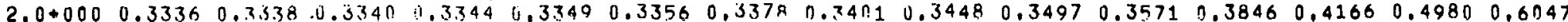
$3.0+0000.250 \% 0.25040 .25060 .25090 .25130 .25190 .25380 .25580 .25970 .26390 .27030 .29410 .32250 .39810 .5040$

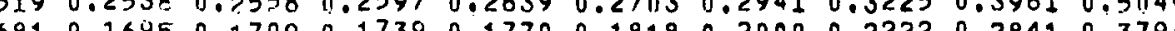

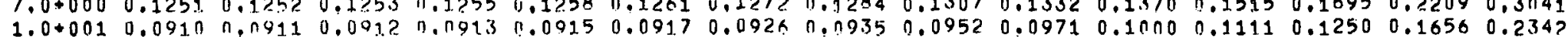

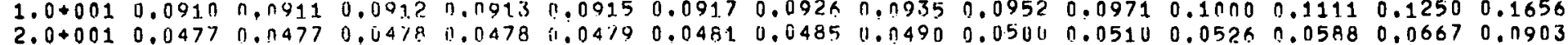

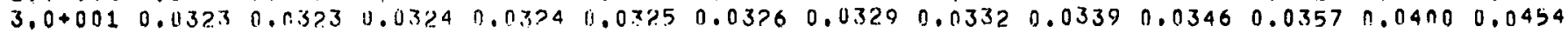

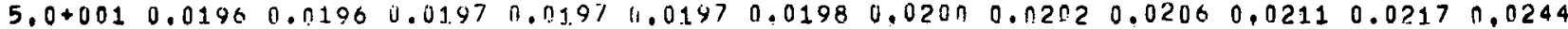

$7.0+0010.01410 .01410 .01410 .01420 .01420 .01420 .01440 .01450 .01480 .01510 .0156$

$1.0 \$ 0020.00990 .00990 .01990 .01000 .01000 .01000 .01010 .01020 .01040 .01060 .0110$

$2.0+002 \quad 0.0050 \quad 0.00500 .00500 .00500 .00500 .0050 \quad 0.0051 \quad 0.00510 .0052$

$3.0+0020.00330 .013330 .4 .3330 .0033 \quad 0.00330 .00340 .00340 .0034$

$5.0+0020.00200 .0020 \quad 0.00200 .002110 .00200 .00200 .0020$

$7.0+0020.00140 .0014$ i. 00140.00140 .00140 .0014

$1.0+0030.0010$ 0.0010 0.0010 0.00100 .00100 .0010

ALPHA $1.000 \quad 2.000 \quad 3.000 \quad 5.000 \quad 7.000 \quad 10.00 \quad 20.00 \quad 30.00 \quad 50.00 \quad 30.00 \quad 100.0 \quad 200.0 \quad 300.0 \quad 500.0 \quad 700.0 \quad 1000$ BETA

1.0-003 $0.99991 .00001 .00001 .00 n 01.00001 .00001 .000 n 1.100001 .00001 .00001 .00001 .00001 .00001 .00001 .00001 .0000$ 2.0-003 0.99971 .00001 .00001 .00001 .00001 .00001 .00001 .00001 .00001 .00001 .00001 .00001 .00001 .00001 .00001 .0000 $3.0-0030.99961 .00001 .000 n 1.00001 .00001 .00001 .00001 .00001 .00001 .00001 .00001 .00001 .00001 .00001 .00001 .0000$ $5,0=0030.99931 .00001 .00001 .00011 .00001 .00001 .00001 .00001 .00001 .00001 .00001 .00001 .00001 .00001 .00001 .0000$

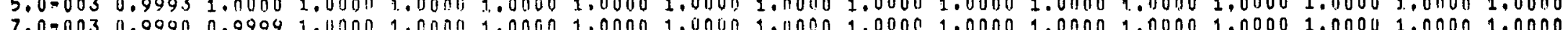
$1.0-0020.99660 .09991 .000191 .00301 .00001 .00001 .00001 .00001 .00001 .00001 .00001 .00001 .00001 .00001 .00001 .0000$ $2.0-0020.99720 .99981 .000 n 1.00301 .00001 .00001 .00001 .00701 .00001 .00001 .00001 .00001 .00001 .0000$ $3.0-0020.99570 .09981 .00001 .00701 .00001 .00001 .00001 .00001 .00001 .00001 .00001 .00001 .0000$ $5,0=002 \quad 0.99290 .99961 .00001 .001001 .00001 .00001 .00001 .00001 .00001 .00001 .00001 .00000$

7.0-002 $0.9901 \quad 0.09940 .9999 \quad 1.001001 .00601 .00001 .00001 .00001 .00001 .00001 .0000$

1.0-001 0.98590 .99920 .9999 1.0001) 1.0000 1.0000 1.00001 .00001 .00001 .00001 .0000

$2.0=0010.97220 .09840 .90981 .00101 .00001 .00001 .00001 .00001 .0000$

$3.0=0010.95890 .09760 .90971 .0001900001 .00001 .00001 .0000$

$5,0.0010 .93330 .9961$ U. 99951.001101 .00001 .00001 .0000

$7.0=0010.90910 .9945$ ij.9094 1.00041 .00001 .0000

$1,0+000 \quad 0.8750 \quad 0.99720 .9991 \quad 0.99091 .00001,0000$

$2,0+000 \quad 0,77780.08450 .99820 .9999$

$3.0+000 \quad 0.70 n 0 \quad 0.0769 \quad 0.9073$

$5.0+000 \quad 0.5833 \quad 0.0621$

$7.0+000 \quad 0.5000$

$1.0+001 \quad 0.4118$ 
1.0-003 0.9940 n.0400 0.9490 0.9990 B.9990 $0.9990 \quad 0.4940 \quad 0.4990 \quad 0.9990 \quad 0.99910 .9991 \quad 0.99920 .99930 .9995 \quad 0.9997$

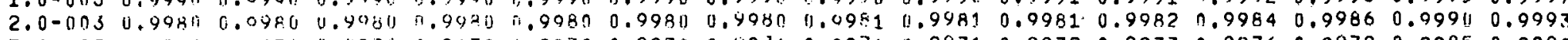
3.0-003 $0.4970 \quad 0.0970 \quad 1.0976 \quad 1.9070 \quad 1.9970 \quad 0.9970 \quad 0.4971 .0 .0971 \quad 0.9971 \quad 0.9972 \quad 0.9973 \quad 0.9976 \quad 0.9979 \quad 0.9985 \quad 0.9990$ $5.0-003 \quad 0.4950 \quad 0.0750 \quad 0.4950 \quad 0.0950 \quad i .9950 \quad 0.9950 \quad 0.4951 \quad 0.0951 \quad 1.9952 \quad 0.9953 \quad 0.9955 \quad 0.9960 \quad 0.9965 \quad 0.9975 \quad 0.9984$ $\begin{array}{llllllllllllllllll}7.0-003 & 0.0931 & 0.0930 & 1.70 .51 & 0.9930 & 0.99 .30 & 0.9931 & 0.9931 & 0.0932 & 0.9933 & 0.9935 & 0.9937 & 0.9944 & 0.9951 & 0.9965 & 0.9977\end{array}$

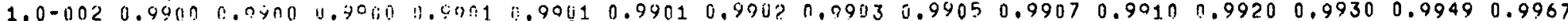

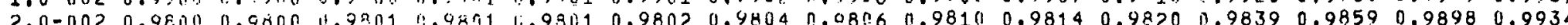

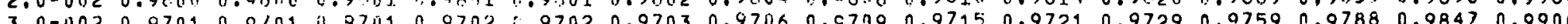

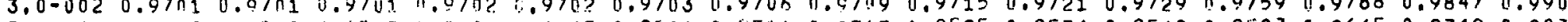

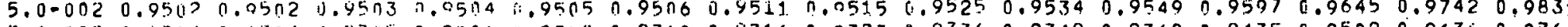

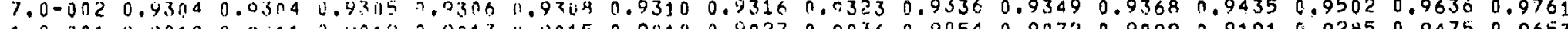

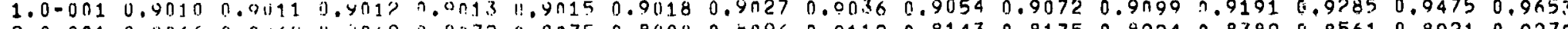

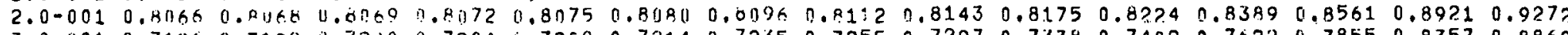
3.0-001 $0.719 \mathrm{~A} \quad 0.7108 \quad 0.720 \mathrm{n} \quad 0.72 n \mathrm{~A} \quad \mathrm{i} .7308 \quad 0.7214 \quad 0.7235 \quad 0.7255 \quad 0.7297 \quad 0.7338 \quad 0.7402 \quad 0.7622 \quad 0.7855 \quad 0.8357 \quad 0.8869$ $\begin{array}{lllllllllllllllllll}5.0-0 & 0.5717 & 0.5799 & 0.572 ? & 1.5727 & 0.5731 & 0.5739 & 0.5764 & 0.5789 & 0.5839 & 0.5891 & 0.5970 & 0.6250 & 0.6557 & 0.7257 & 0.8 n 31\end{array}$

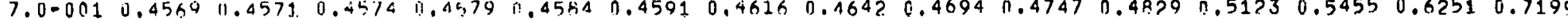

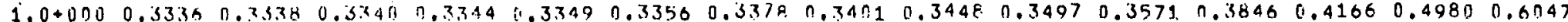

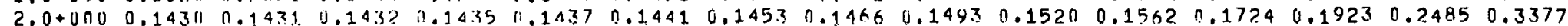
$3,0+0000.0770 .07710 .01790 .07730 .07740 .01760 .47640 .07910 .08060 .08220 .08470 .09430 .10640 .14190 .2031$

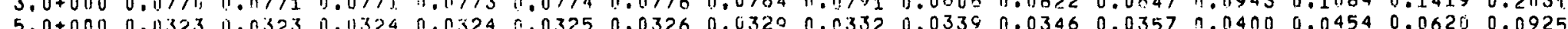

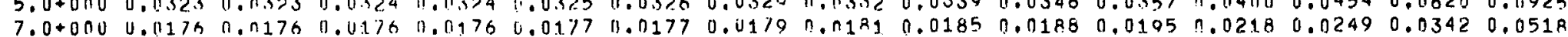

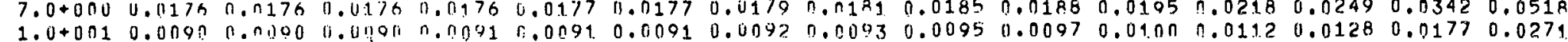
$2.0+0010.0 n 21 \quad 0.01174 \quad 1.017240 .011240 .00240 .00240 .00240 .00240 .00250 .00260 .00260 .0030 \quad 0.00340 .0047$

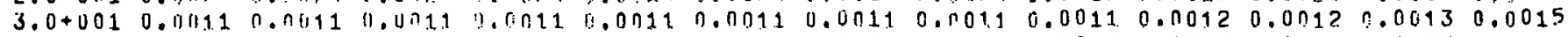

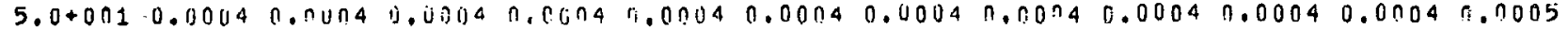

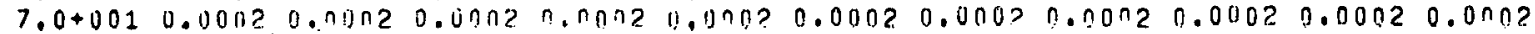

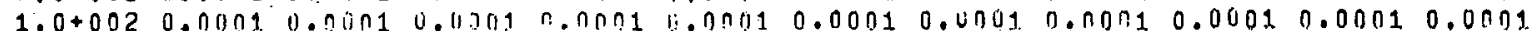

$2,0+0020.0000$. nuno

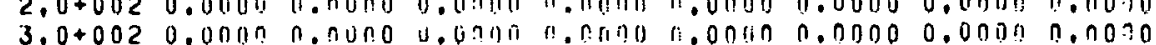

$5.0+0020.0000$ di.n.,

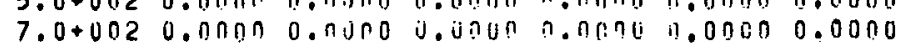

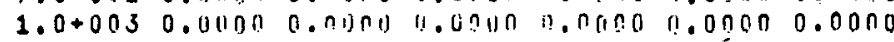

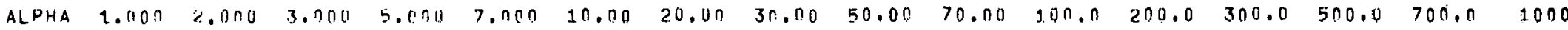
BETA

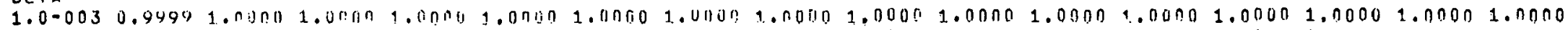

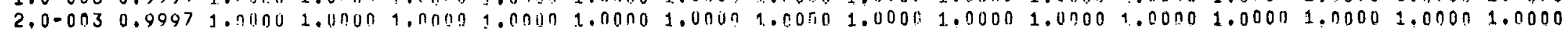
$3.0=0030.99061 .01001 .00001 .00001 .0001 .00001 .00001 .00001 .00001 .00001 .00001 .00001 .00001 .00001 .00001 .0000$

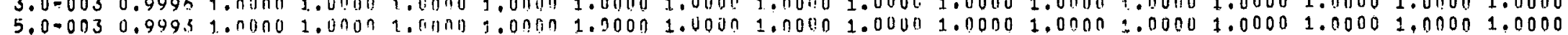

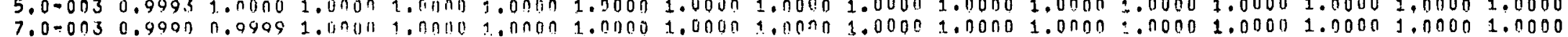

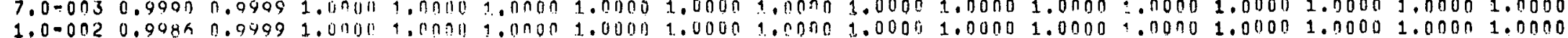
$2.0-0020.99710 .09981 .00001 .00001 .00001 .00001 .00001 .00001 .00001 .00001 .001) 01.00001 .00001 .0000$ $3.0-0020.99560 .09961 .01001 .00009 .00001 .00001 .00001 .00001 .00001 .00001 .00001 .00001 .0000$

5.0-002-0.9926 $0.0996 \quad 1.010001 .00001 .00001,00001.00001 .00001 .00001 .00001 .00009 .0000$

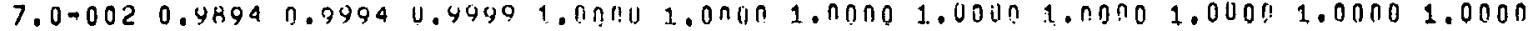

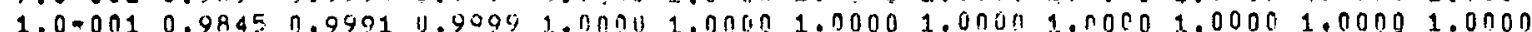

2.0-001 0.90690 .098111 .9498 1.0ntion

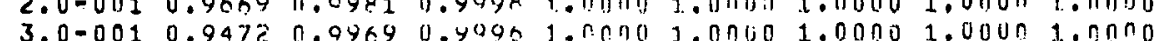

$5.0-0010.90320 .0941$ U.4993 1.000151 .000001 .00001 .0000

$7.0-0010.8547 \quad 0.99 n 7 \quad 0.4949 \quad 0.0999 \quad 1.00001 .0000$

$1.0+000 \quad 0.7778 \quad 0.0845 \quad 0.494 ? \quad 11.99991 .00001 .0000$

$2.0+000 \quad 0.5385 \quad 0.0549 \quad 0.4945 \quad 0.999 \%$

$3.0+000 \quad 0.36840 .0137 \quad 0.9891$

$5.0+000 \quad 0.1892 \quad 0.8489$

$7.0+000 \quad 0.1111$ 
VALUES OF G FOR $N=$ K $A$ NDE NP= ? BETA

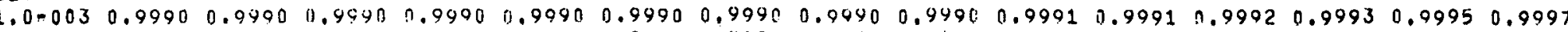

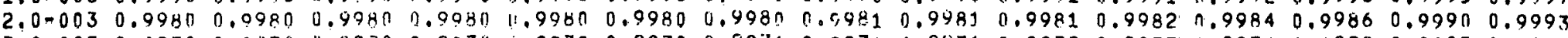

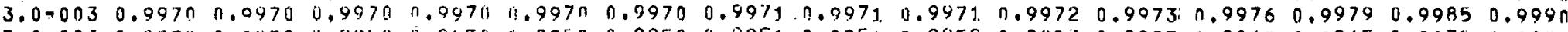

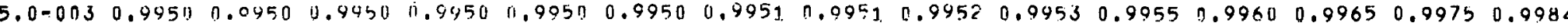
$\begin{array}{llllllllllllllll}7.0-003 & 0.9930 & 0.9930 & 0.9930 & 0.9930 & 0.9730 & 0.9931 & 0.9931 & 0.9932 & 0.9933 & 0.9935 & 0.9937 & 0.9944 & 0.9951 & 0.9965 & 0.9977\end{array}$

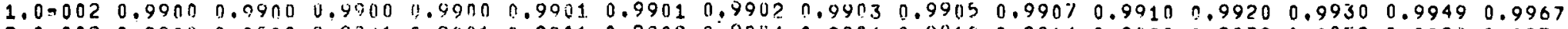

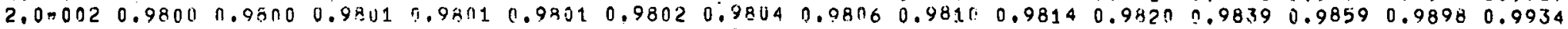
$\begin{array}{llllllllllllllll}3.0-002 & 0.9700 & 0.9701 & 0.9701 & 0.9701 & 0.9702 & 0.9703 & 0.9706 & 0.9709 & 0.9715 & 0.9720 & 0.9729 & 0.9759 & 0.9788 & 0.9847 & 0.9900\end{array}$

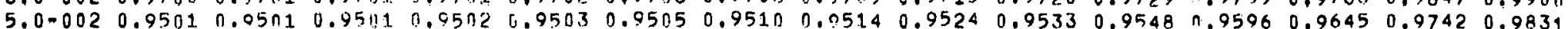

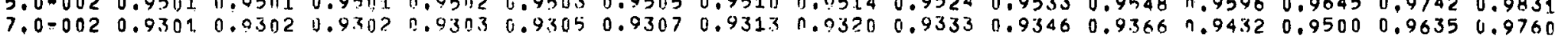

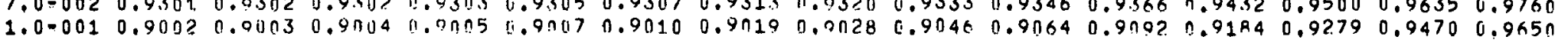

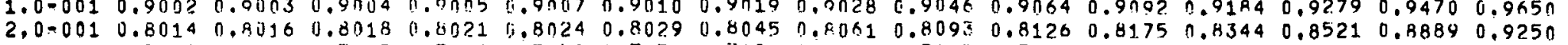
$\begin{array}{llllllllllllllllll}3.0-001 & 0.7059 & 0.7061 & 0.7063 & 0.7068 & 0.70 \% 2 & 0.7078 & 0.7099 & 0.71 .20 & 0.7163 & 0.7206 & 0.7771 & 0.7498 & 0.7740 & 0.8263 & 0.8800\end{array}$ $\begin{array}{lllllllllllllll}5.0-001 & 0.5336 & 0.5338 & 0.5341 & 0.5346 & 0.5351 & 0.5358 & 0.5384 & 0.5409 & 0.546 .0 .5513 & 0.5594 & 0.5882 & 0.6201 & 0.6940 & 0.7776\end{array}$

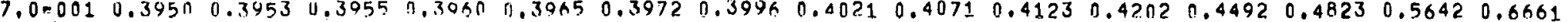

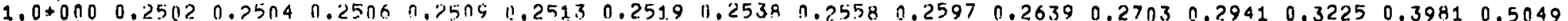

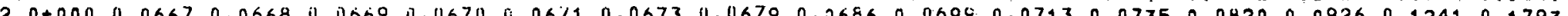
$3.0+0000.02500 .0250 .02510 .02510 .02520 .02520 .02550 .02580 .02630 .02680 .07770 .03110 .03530 .04840 .0727$ (5.0+000 0.025n 0.025 0.0251 .

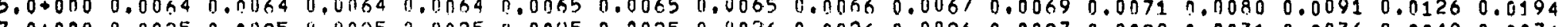

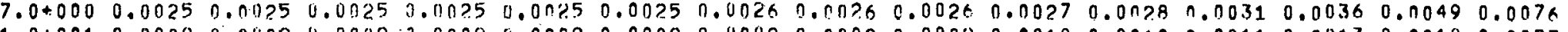

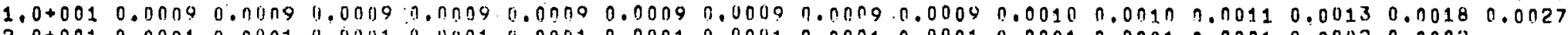

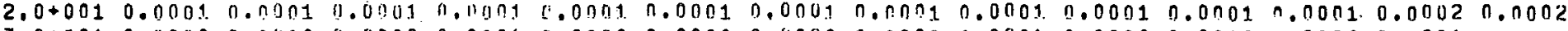
$3.0+0010.00000 .04000 .00000 .0300 .00000 .00000 .00000 .00000 .0001 .0 .00000 .00000 .00000 .0001$

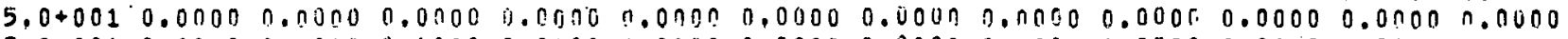

$7.0+0010.00000 .00000 .00000 .00000 .00000 .00000 .00000 .00000 .00000 .00000 .0000$

$1.0+0020.00000 .0000$ j. 00000.00000 .00000 .00000 .00000 .00000 .00000 .00000 .0000

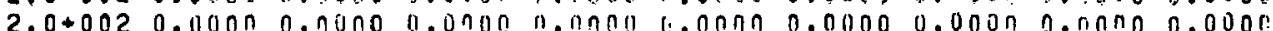

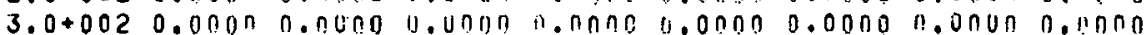

$5,0+0020.000 \pi 0,0000000011,00001.00000,00000.000 n$

$7.0+0020.00000 .00000 .0000 \quad 1.00006 .00000 .0000$

$1.0+0030.00000 .00000 .00000 .0000$ T.00000.0000

$$
A L P H
$$

BETA

$1.0-0030.99991 .00001 .00001 .00001 .00001 .00001 .00001 .00001 .00001 .00001 .00001 .00001 .00001 .00001 .00001 .0000$ $2.0-0030.99971 .00001 .00001 .00001 .00071 .00001 .00001 .00001 .00001 .00001 .00001 .00001 .00001 .00001 .00001 .0000$ $3.0=0030.99961 .00001 .00001 .0 n n 01.00001 .00001 .00001 .00001 .00001 .00001 .00001 .00001 .00001 .00001 .00001 .0000$

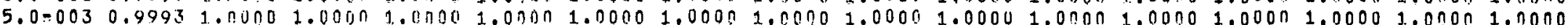

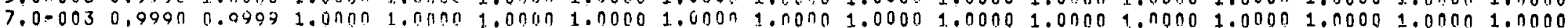
$1,0=0020.998$ h 0.99971 .00001 .091109 .00501 .00001 .00001 .00001 .00001 .00001 .00001 .00001 .00001 .00001 .00001 .0000 $2.0-0020.99710 .99931 .00001 .030119001011 .00001 .00001 .00001 .00001 .00001 .00001 .00001 .00001 .0000$ $3.0-0020.99560 .099 .51 .00001 .03043 .00001 .00001 .00001 .00001 .00001 .00001 .00001 .00701 .0000$

$5.0-0020.99250 .99951 .0110001 .03001 .00001 .00001 .00001 .00001 .00001 .00001 .00001 .0000$

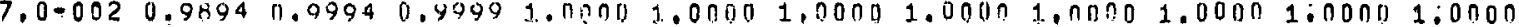

$1.0-0010.98440 .99910 .49991 .010001 .0 n 0011.00001 .000 n 1.00001 .00001 .00001 .0000$

2.0-001 0.96580 .99810 .4948 1.0010.0 j.0non 1.0000 1.00001.0000 1.0000

$3.0-001 \quad 0.94380 .09670 .9046 \quad 1.010011 .00001 .00001 .00001 .0000$

$5.00001 \quad 0.88890 .39320 .9942 \quad 1.04001 .00001 .00031 .000 n$

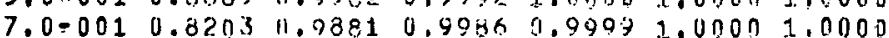

$1.0+000 \quad 0.700 ? 11.07690 .99730 .99931 .00001 .0000$

$2.0+000 \quad 0.3333 \quad 0.9007 \quad 0.94740 .9993$

$3.0+000 \quad 0.1522 \quad 10.76510,9605$

$5.0+000 \quad 0.043211 .4504$

$7,0+000 \quad 0.0172$

$1.0+0010,006$ 


\begin{abstract}
VALUES DF G BOH $N=6$ ANOM $M H=4$ BET BETA

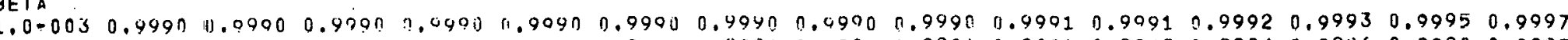

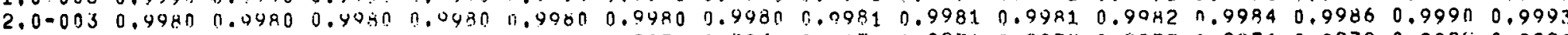

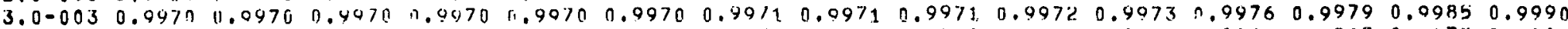

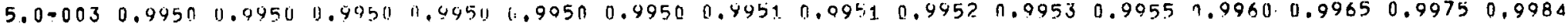
$\begin{array}{llllllllllllllll}7.0 .003 & 0.9930 & 0.0930 & 0.4930 & 11.04311 & 0.903 n & 0.9931 & 0.9931 & 0.9932 & 11.993 .3 & 0.9935 & 0.9937 & 0.9944 & 0.9951 & 0.9965 & 0.9977\end{array}$

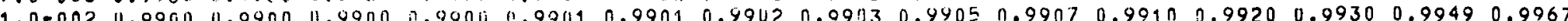

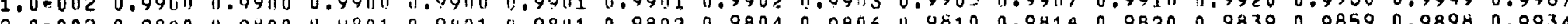
2.0.002 0.9Bח

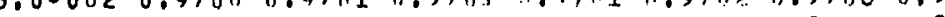

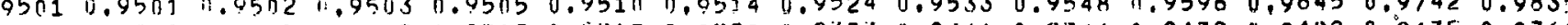

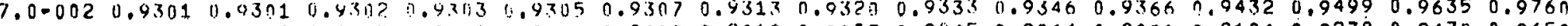

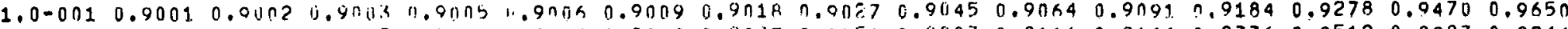

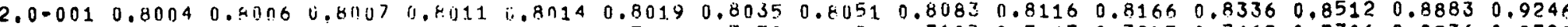
$\begin{array}{lllllllllllllllll}3.0-001 & 0.7019 & 0.7021 & 0.702 .3 & 0.7028 & 1.7 n 32 & 0.7038 & 0.7059 & 0.7080 & 0.7123 & 0.7167 & 0.7233 & 0.7462 & 0.7706 & 0.8236 & 0.878 n\end{array}$ $\begin{array}{llllllllllllllllllll}5,0.001 & 0.5164 & 0.5146 & 0.5169 & 11.5174 & 0.51 \% 9 & 0.5196 & 0.521 ? & 0.5237 & 0.5289 & 0.5342 & 0.5424 & 0.571 .4 & 0.6037 & 0.6791 & 0.7654\end{array}$ $\begin{array}{lllllllllllllllll}7.0-0.1 & 0.3608 & 0.3611 & 15.3613 & 11.361 .2 & 4.3622 & 0.3629 & 0.3653 & 0.3677 & 0.3725 & 0.3775 & 0.3852 & 0.4135 & 0.4461 & 0.5281 & 0.6330\end{array}$ $10+0000.20020 .200311 .201150 .20080 .20110 .20160 .2 n 330.20490 .20830 .21190 .2174 \quad n .23 R 10.26310 .33160 .4333$

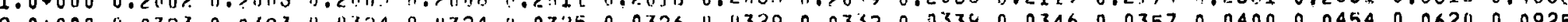
2.0+0 0.

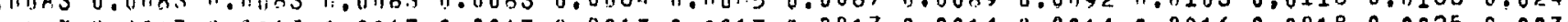

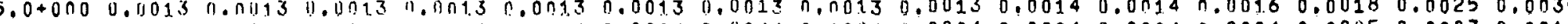

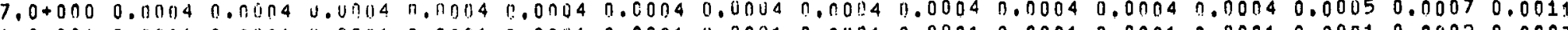

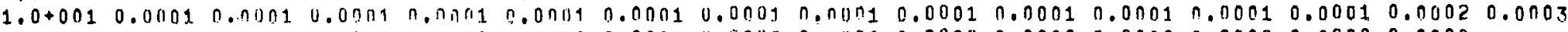

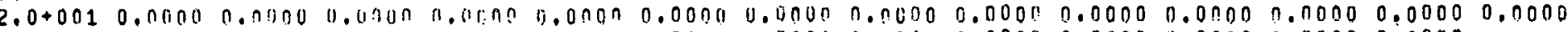

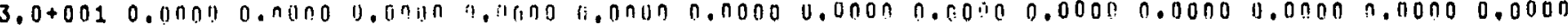

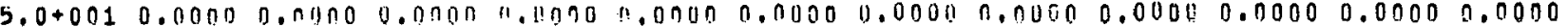

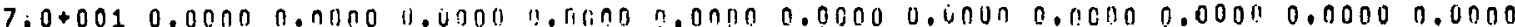

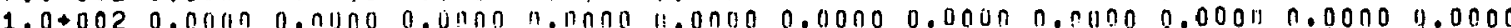

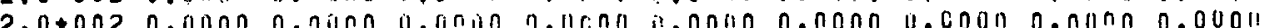

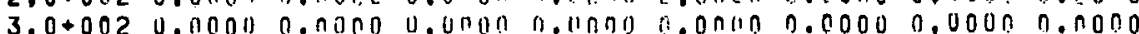

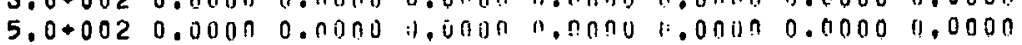

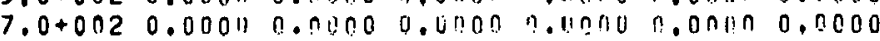

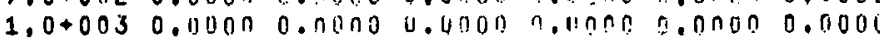

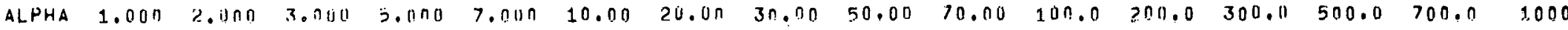
BETA

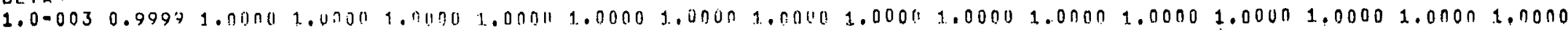
2.0-003 $0.9997,00001.00001 .000 \pi 1.000 n 1.00001 .00001 .00001 .00001 .00001 .00001 .00001 .00001 .00001 .00001 .0000$ 2.0.0.3.

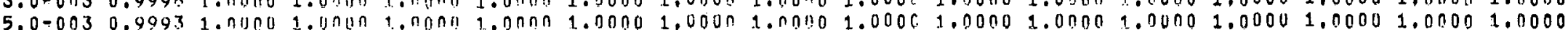

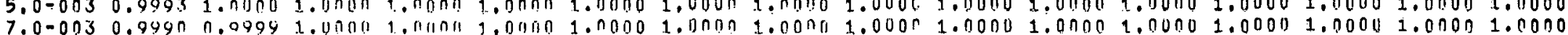

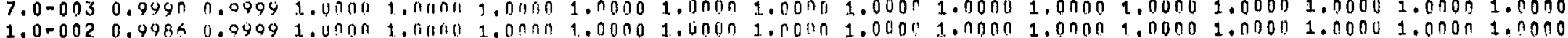
$2.0-0020.9971,0.04081 .00001 .040131 .00001 .00001 .00001 .00001 .00051 .00001 .00001 .00001 .00001 .0000$

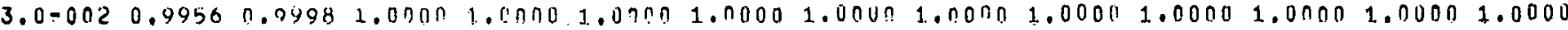

$5.0-0020.99250 .09961 .00001 .00001 .00001 .00001 .00001 .00001 .00001 .00001 .00001 .0000$

$7.0-002 \quad 0.9894 \quad 0.0494$ ij. 40991.010001 .00001 .00001 .00001 .00001 .00001 .00001 .0000

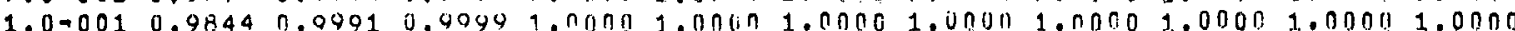

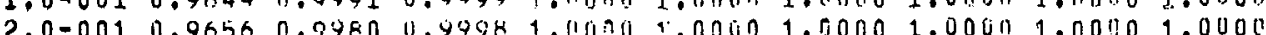

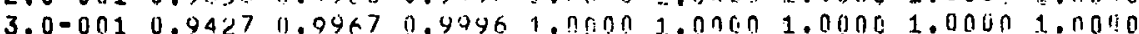

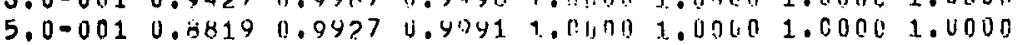

$7.0 .001 \quad 0.7979 \quad 0.9862 \quad 0.9984 \quad 1.9999 \quad 1.001001 .0000$

$1.0+000 \quad 0.63640 .0695 \quad 0.9964 \quad 0.0995 \quad 1.00001 .0000$

$2.0+000 \quad 0.1892 \quad 0.810890 .9733 \quad 1.9985$

$3.0+000 \quad 0.01551 \quad 0.5142 \quad 0.9911$

$5,0+000 \quad 0.0 \pi 89 \quad 0.1400$

$7.0+000 \quad 0.0025$

$1.0+0010.01006$ 
VALUES OF G POH $N=S$ AIV!! $N F=5$

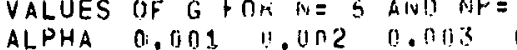

BETA

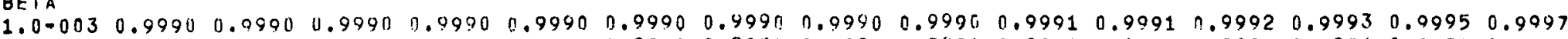

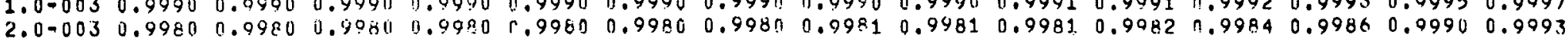

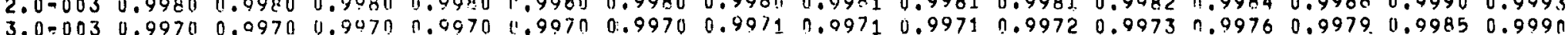

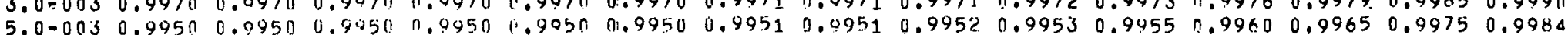

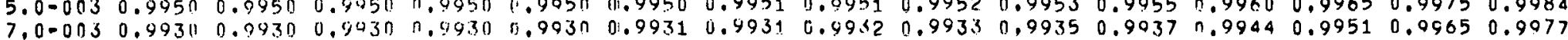

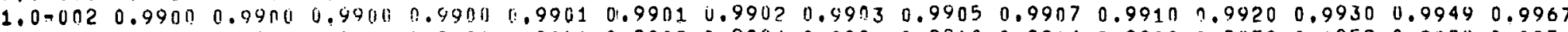

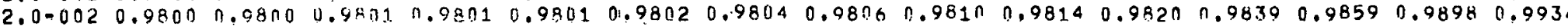
$\begin{array}{llllllllllllllll}3.0=002 & 0.9700 & 0.9701 & 0.9741 & 0.9701 & 0.9702 & 0.9703 & 0.9706 & 0.9709 & 0.9715 & 0.9720 & 0.9729 & 0.9759 & 0.9788 & 0.9847 & 0.9900\end{array}$ $\begin{array}{llllllllllllllll}5.0-002 & 0.9500 & 0.0501 & 0.95(j) & 0.05 n 2 & 1.9503 & 0.9505 & 0.9510 & 0.0514 & 0.9524 & 0.9533 & 0.9548 & 0.9596 & 0.9645 & 0.974 \% & 0.9931\end{array}$

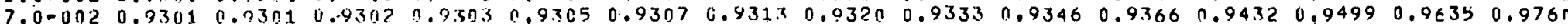

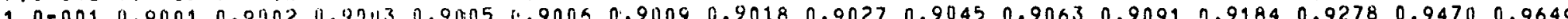

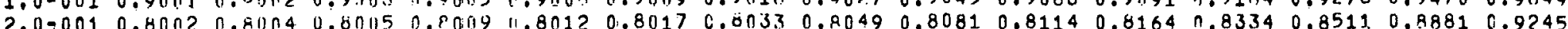

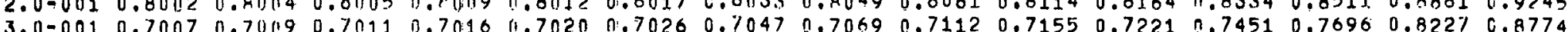

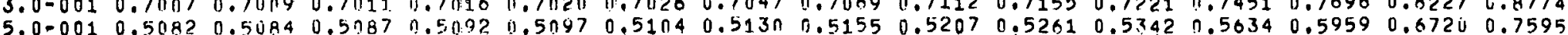

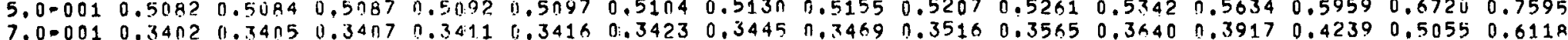

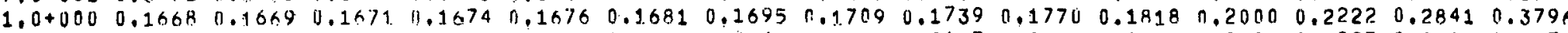
$2.0+000 \quad 0.01590 .01590 .0150 \quad 0.0160 \quad 0.016 n \quad 0.0160 \quad 0.01620 .01640 .01670 .01700 .01760 .01980 .02250 .03100 .047 n$

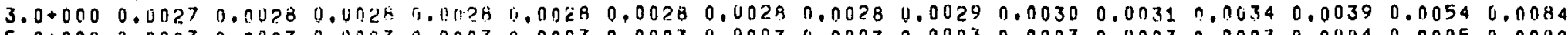
$5,0+000 \quad 0.00030 .00030 .00030 .00030 .00030 .00030 .00030 .00030 .00030 .00030 .00030 .00030 .00040 .00050 .0008$ $7.0+0000.00010 .00010 .01010 .0 n n 10.00010 .0 .0010 .00010 .00010 .00010 .00010 .00010 .00010 .00010 .00010 .0002$

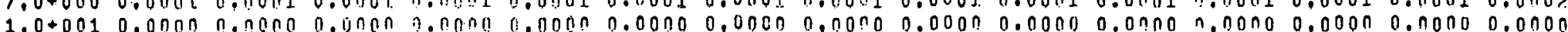

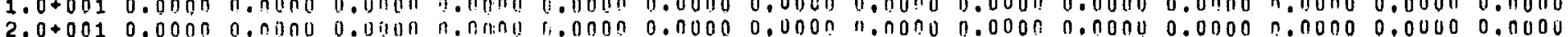

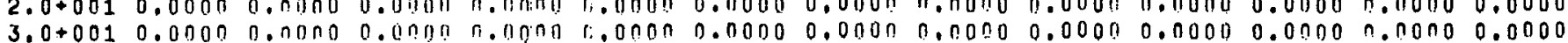

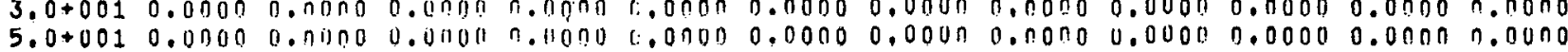

$7.0+0010.00000 .00000 .00190 .00000 .00000 .00000 .00000 .00000 .00000 .00000 .0000$

$1.0+0020.0000 \quad 0.00000 .000180 .00000 .00000 .00000 .00000 .00000 .00000 .00000 .0000$

$2.0+0020.0000 \quad 0.00000 .4 n 000.00000 .0 n \pi n 0.00000 .00000 .0090 \quad 0.0000$

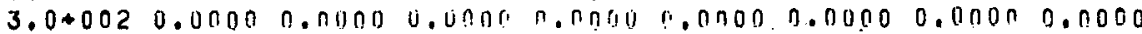

$5.0+0020.00000 .00000 .00000 .0000 \quad 0.00000 .00000 .0000$

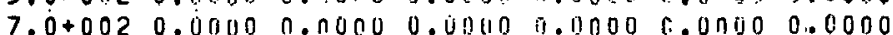

$1.0+003 \quad 0.0000 \quad 0.0000 \quad 0.000 \% 0.00000 .00000 .0000$

ALPHA $1.0002 .000 \quad 3.000500007 .000 \quad 10.00 \quad 20.00 \quad 30.0050 .00 \quad 70.00 \quad 10 n .0 \quad 200.0 \quad 300.0 \quad 500.0 \quad 700.0 \quad 1000$ BETA

$1.0=0030.99991 .00001 .000101 .09001 .0 n 001.00001 .00001 .00001 .00001 .00001 .00001 .00001 .00001 .00001 .00001 .0000$ $2,0=0030.99971 .00001 .000 n 1.00001 .00001 .00001 .000 n 1.00001 .00001 .00001 .00001 .00001 .00001 .00001 .00001 .0000$ $3.0-0030.99961 .00001 .40011 .00001 .0000 .1,00001.00001 .00001 .00001 .00001 .00001 .00001 .00001 .00001 .00001 .0000$ $5.0=0030.99931 .00001 .00 n 01.00001 .000 n 1.00001 .000 n 1.00001 .00001 .00001 .00001 .00001 .00001 .00001 .000 n 1.0000$ $7.0-0030.0990 \quad 0.09991 .00101 .00001 .00001 .00001 .000 n 1.00 n 01.00001 .00001 .00001 .00001 .00001 .00001 .00001 .0000$ $1.0-0020.99460 .09091 .00001 .00001 .00001 .00001 .000 n 1.00001 .00001 .00001 .00001 .00001 .00001 .00001 .00001 .0000$

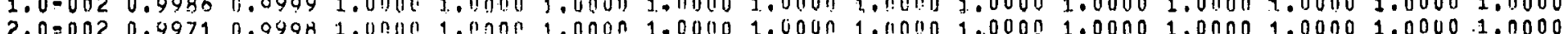

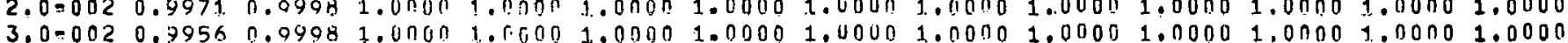

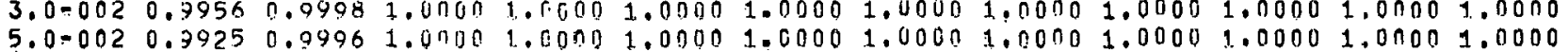

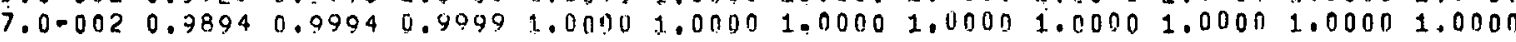

$1.0-0010.79440 .99910 .99991 .00001 .00001 .00001 .00001 .00001 .00001 .00001 .0000$

$2.0=0010.7655 \quad 0.0980 \quad 0.9994 \quad 1.00001 .00001 .00001 .00001 .00001 .0000$

$3.0-001 \quad 0.7424 \quad 0.0946 \quad 0.9996 \quad 1.00001 .000001 .00001 .00001 .0000$

$5.0-001 \quad 0.37840 .99240 .9091$ 1.0000 1.00001 .00001 .0000

$7.0-0010.78290 .98490 .9082 \quad 3.99991 .00001 .0000$

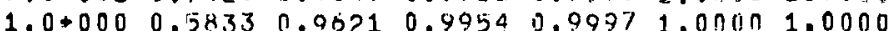

$2.0+000 \quad 0.10140 .07200 .9463 \quad 3.9958$

$3.0+000 \quad 0.31890 .2592 \quad 0.7507$

$5.0+0000.3018 \quad 0.0315$

$7.0+000 \quad 0.0004$

$1,0+0010,1001$ 


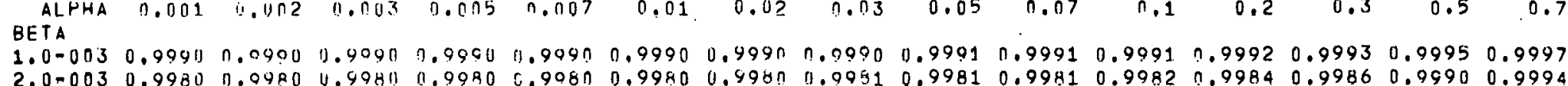
2.0-003 $0.9980 \quad 0.0980 \quad 0.9981 \quad 0.9990 \quad 0.9080 \quad 0.9980 \quad 0.9980 \quad 0.99510 .99810 .99810 .9982 \quad 0.99840 .9986 \quad 0.9990 \quad 0.9994$ $\begin{array}{llllllllllllllll}3.0-003 & 0.9970 & 0.9970 & 0.907 n & 0.9970 & 0.9970 & 0.9970 & 0.9971 & 0.0971 & 0.9972 & 0.9972 & 0.9973 & 0.9976 & 0.9979 & 0.9985 & 0.9990\end{array}$

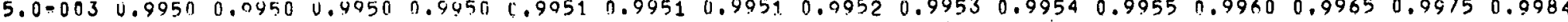
$\begin{array}{llllllllllllllll}7.0-003 & 0.9931 & 0.0931 & 0.9031 & 0.0031 & 0.9931 & 0.9931 & 0.9937 & 0.0933 & 0.9934 & 0.9935 & 0.9937 & 0.9944 & 0.9951 & 0.9965 & 0.9978\end{array}$ $\begin{array}{llllllllllllllll}1.00002 & 0.9901 & 0.0901 & 0.9901 & 0.9901 & 0.9902 & 0.9902 & 0.990 .3 & 0.9904 & 0.9906 & 0.9908 & 0.9911 & 0.9921 & 0.9930 & 0.9950 & 0.9968\end{array}$ $2.0-0020.98040 .00040 .98040 .04050 .98050 .98060 .98020 .08100 .98140 .98170 .98230 .98430 .98620 .09010 .9937$ 3.0-002 0.97090 .07090 .97100 .97100 .97140 .97120 .47140 .97170 .97230 .97290 .97370 .97660 .97940 .98520 .9905

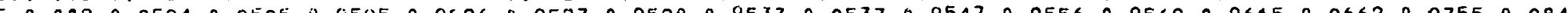

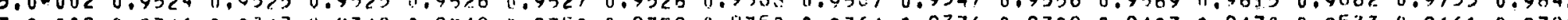

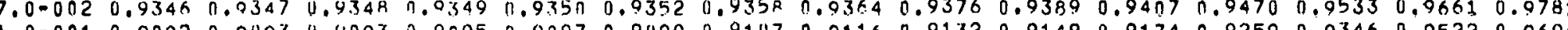
$\begin{array}{lllllllllllllllll}1.0-001 & 0.9092 & 0.0093 & 0.9093 & 0.91995 & 0.9097 & 0.91199 & 0.9107 & 0.0116 & 0.9132 & 0.9149 & 0.9174 & 0.9259 & 0.9346 & 0.9522 & 0.9691\end{array}$

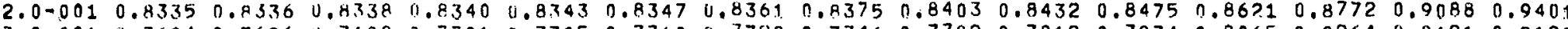

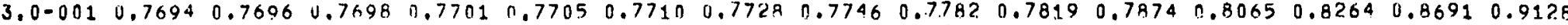

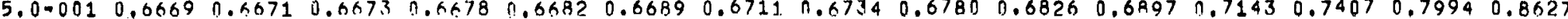
$\begin{array}{lllllllllllllllll}7.0-001 & 0.5885 & 0.5887 & 0.5890 & 0.5494 & 0.5899 & 0.59 n 7 & 0.5931 & 0.5956 & 0.6006 & 0.6057 & 0.61 .35 & 0.641 .0 & 0.6711 & 0.7400 & 0.8178\end{array}$ $\begin{array}{llllllllllllllll}1.0+000 & 0.50 n 3 & 0.511 n 5 & 0.5 n 118 & 0.5 n 13 & 0.5 n 1.8 & 0.5025 & 0.5053 & 0.5076 & 0.5128 & 0.5181 & 0.5263 & 0.5556 & 0.5882 & 0.6658 & 0.7585\end{array}$ $\begin{array}{lllllllllllllllllllll}2.0+000 & 0.3336 & 0.3338 & 0.3340 & 0.3344 & 1.3349 & 0.3356 & 0.3378 & 0.34 n 1 & 0.3448 & 0.3497 & 0.3571 & 0.3846 & 0.4167 & 0.4990 & 0.6110\end{array}$

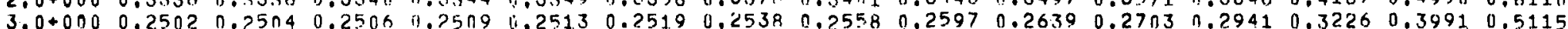

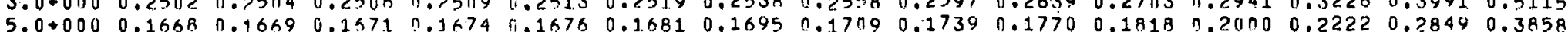

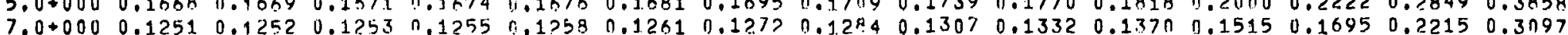

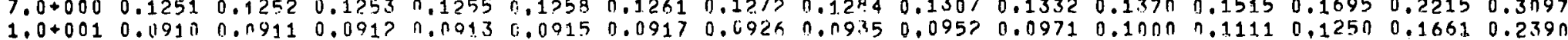

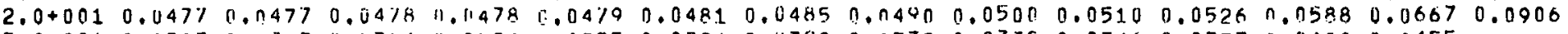

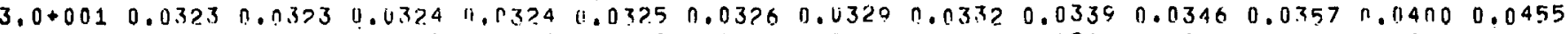

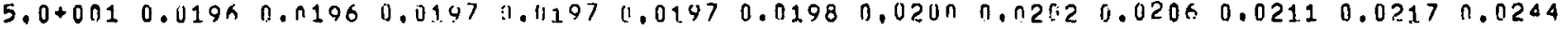

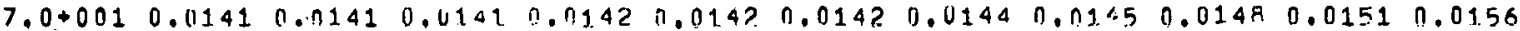

$1.0+0020.00990 .00990 .00990 .01000 .010 n 0.01000 .0102 n .01020 .01040 .01060 .0110$

$2.0+0020.00500 .011500 .005110 .0050 \quad 0.0 n 500.00500 .00510 .00510 .0052$

$3.0+0020.00330 .013330 .00330 .01333 \quad 1.00330 .00340 .00340 .0034$

$5.0+0020.0020 \quad 0.01120 \quad 0.4020 \quad 0.110 ? 0 \quad 0.002 \pi 0.0020 \quad 0.0020$

$7.0+0020.00140 .00140 .01140 .0014 \quad 0.01140 .0014$

$1.0+0030.00110 .00100 .00100 .00100 .00100 .001 .0$

ALPHA $1.000 \quad 2.000 \quad 3.0005 .000 \quad 7.000 \quad 10.00 \quad 20.0030 .00 \quad 50.00 \quad 70.00 \quad 100.0 \quad 200.0 \quad 300.0 \quad 500.0 \quad 700.0 \quad 1000$ BETA

1.0-003 $0.99991 .00001 .4 n 009.00001 .00001 .00001 .00001 .00001 .00001 .00001 .00009 .00001 .00001 .00001 .00001 .0000$

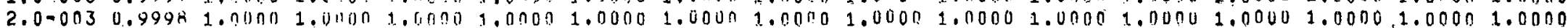

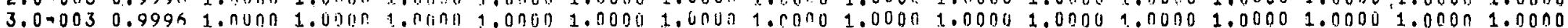
$5.0-0030.99941 .00001 .0001 .0001 .03001 .00001 .00401 .001001 .00001 .00001 .00001 .00001 .00001 .00001 .00001 .0000$

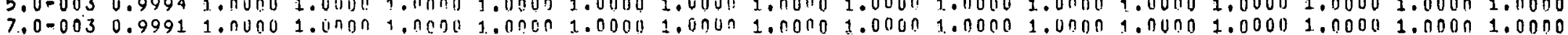

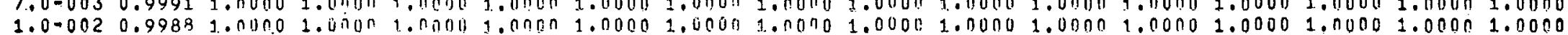

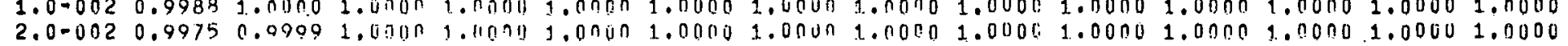
$3.0=0020.49030 .09991 .00001 .90001 .000 n 1.00001 .00001 .00001 .00001 .00001 .00001 .00001 .0000$

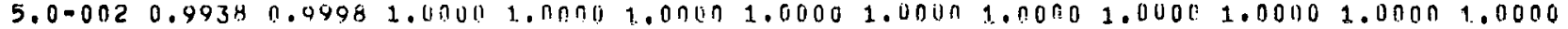

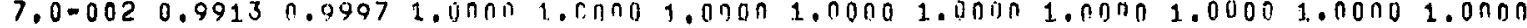

$1.0=0010.98770 .09961 .00001 .00001 .00001 .00001 .010001 .00001 .00001 .00001 .0000$

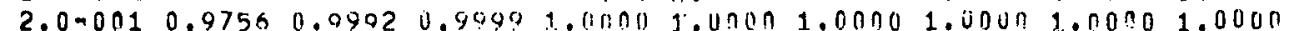

$3.0-0010.46390 .54880 .49041 .010001 .00401 .00001 .00001 .0000$

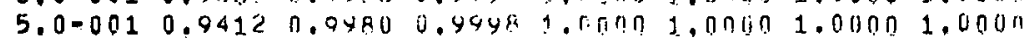

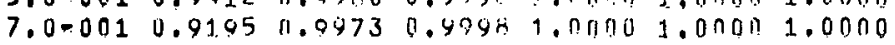

$1.0+000 \quad 0.88890 .0961 \quad 0.4997 \quad 1.110 n 0 \quad 1.00601 .0000$

$2.0+000 \quad 0.801100 .09220 .9094 \quad 1.01000$

$3.0+000 \quad 0.7273 \quad 0.0844 \quad 0.4991$

$5.0+000 \quad 0.6154 \quad 0.0 \mathrm{dn} 8$

$7.0+000 \quad 0.5333$ 


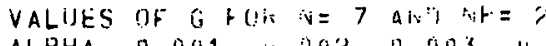
BETA

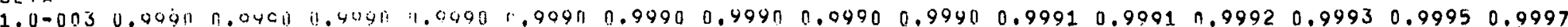

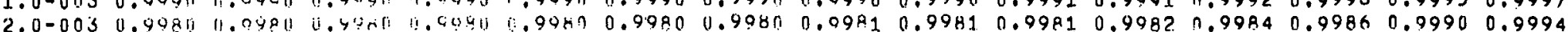

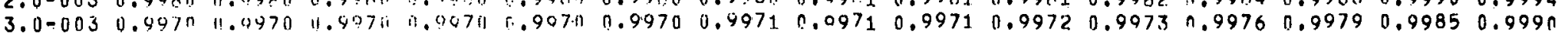

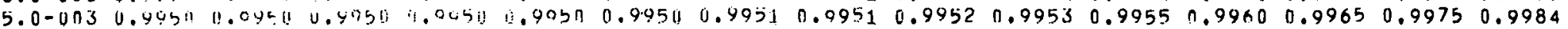

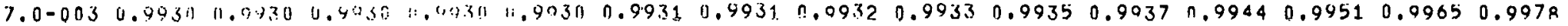

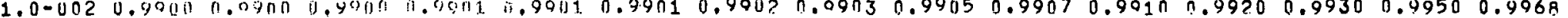

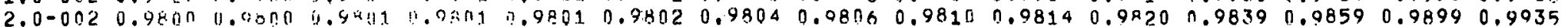
$3.0=0020.97010 .07010 .4711111 .9711218 .97020 .97030 .97050 .07090 .97150 .97210 .97290 .97590 .97880 .98470 .0903$ 3.0-002 0.970

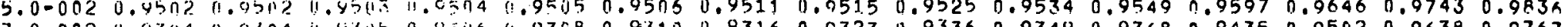

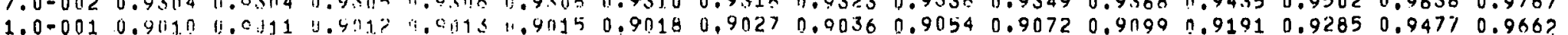

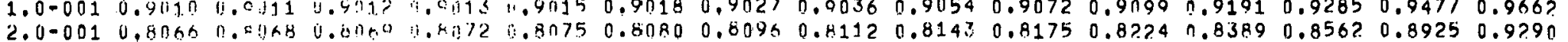

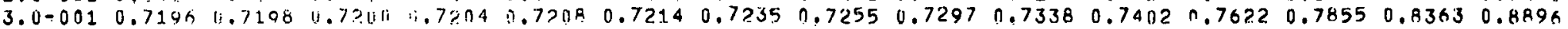
$5.0-0010.5717 \quad 0.5719$ ij.5727 $0.5727 \quad 0.57310 .57390 .57640 .57890 .58390 .58910 .5970 \quad 0.6250 \quad 0.6557 \quad 0.7265 \quad 0.8073$

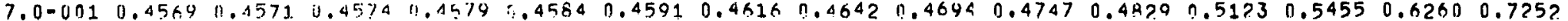

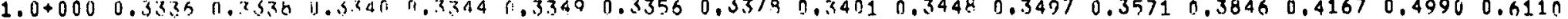

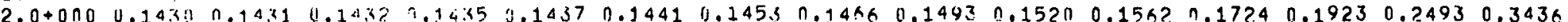
$2.0+00000.07700 .077111 .4710 .07730 .07740 .07760 .07840 .07910 .08060 .08220 .08470 .09430 .10640 .14240 .2075$

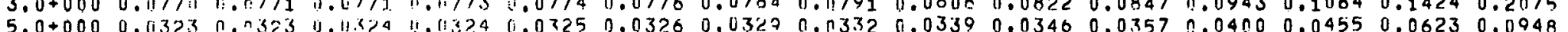

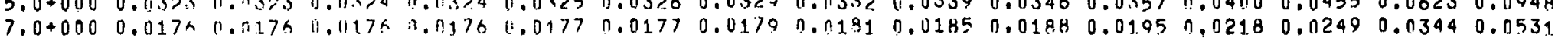

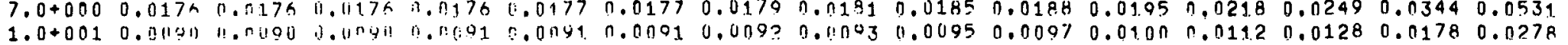

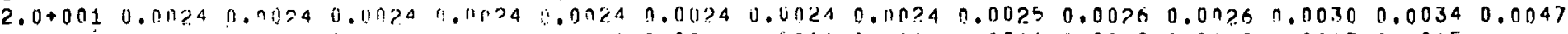

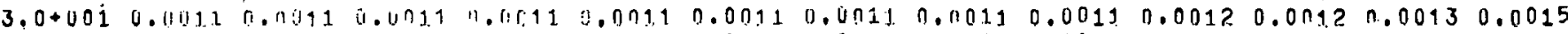

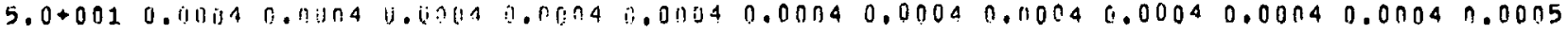

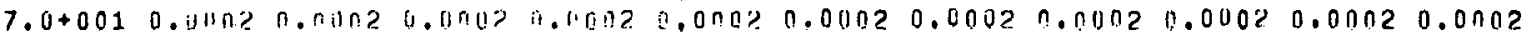

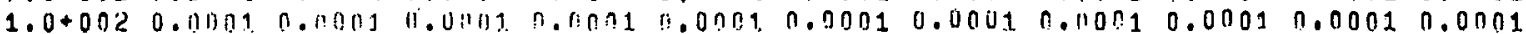

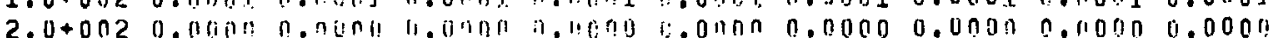

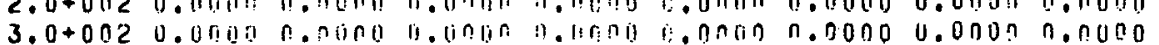

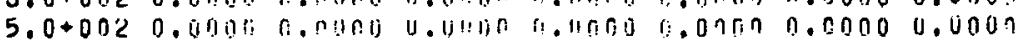

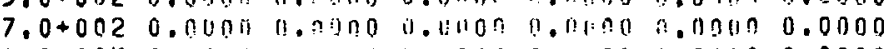

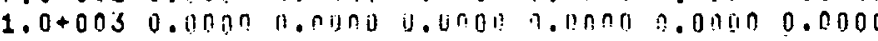

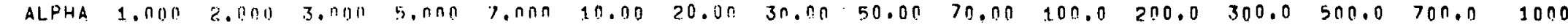
BETA

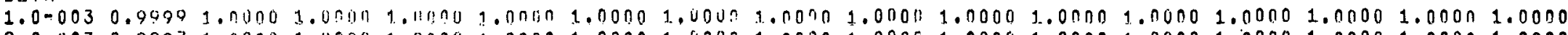

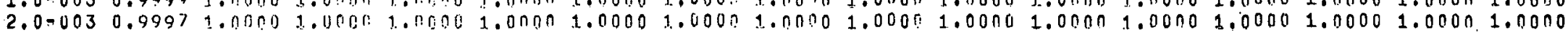

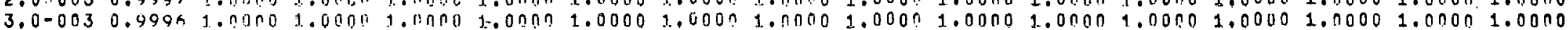

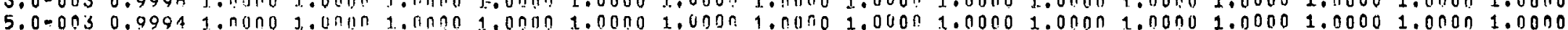

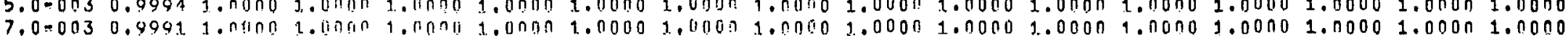

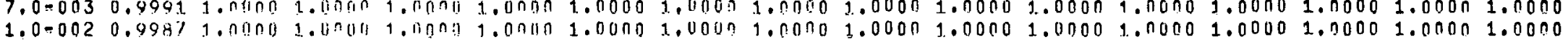
$2,0-0020.99750 .09091 .09011 .000101 .001301 .00001 .00001 .00401 .00001 .00001 .00001 .00001 .001101 .0000$

$3.0-0020.99620 .09091 .00001 .00001 .00001 .00001 .00001 .00001 .00001 .00001 .00001 .00001 .0000$

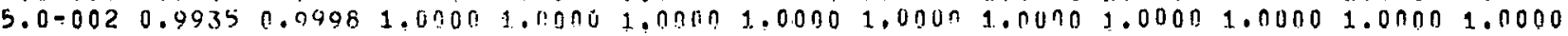

$7.00002 \quad 0.9907 \quad 0.99071 .001) 01.0110001 .00001 .00001 .00001 .00001 .00001 .00001 .0000$

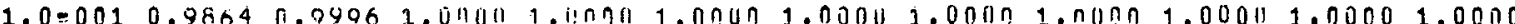

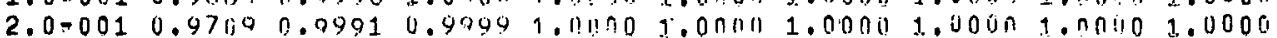

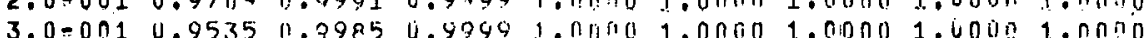

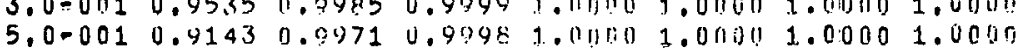

$\begin{aligned} & 7.0=001 \\ & 0.8705\end{aligned} 0.09540 .90961 .00001,00001.0000$

$1.0+000 \quad 0.8070 \quad 0.9972 \quad 0.99941 .00001 .00001 .0000$

$2.0+000 \quad 0.57140 .9770 \quad 10.998 \%, 0.9999$

$3.0+000 \quad 0.4000 \quad 0.4551 \quad 0.4064$

$5.0+000 \quad 0.2105 \quad 0.5947$

$7.0+000 \quad 0.1250$

$1.0+001 \quad 0.0678$ 


\begin{abstract}
VALUES OF G FOH IV $=7$ ANA MPE $=3$ BETA BETA.

$\begin{array}{lllllllllllllll}1.0-003 & 0.9990 & 0.0900 & 0.5990 & 0.9990 & 0.9990 & 0.9990 & 0.9991 & 0.9990 & 0.9996 & 0.9991 & 0.9991 & 0.9992 \quad 0.9993 \quad 0.9995 & 0.9997\end{array}$

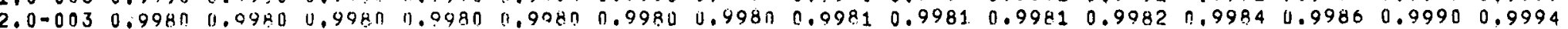
$\begin{array}{llllllllllllllllll}3.0-003 & 0.9970 & 0.0971 & 1.9070 & 0.0970 & 0.9070 & 0.9970 & 0.9971 & 0.9971 & 0.9971 & 0.0972 & 0.9973 & 0.9976 & 0.9979 & 0.0985 & 0.9990\end{array}$ $\begin{array}{llllllllllllllll}5.0=003 & 0.9950 & 11.0950 & 0.9950 & 11.9950 & 10.9051) & 0.9950 & 0.9951 & 0.9951 & 0.9952 & 0.9953 & 0.9955 & 0.9960 & 0.9965 & 0.9975 & 0.9984\end{array}$ $\begin{array}{lllllllllllllllllll}7.0-003 & 0.9930 & 1.0930 & 0.9930 & 0.9930 & {[.9030} & 0.9931 & 0.9931 & 0.0932 & 0.9933 & 0.9935 & 0.9937 & 0.9944 & 0.9951 & 0.9965 & 0.9978\end{array}$

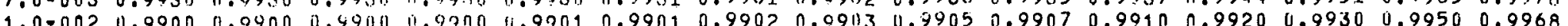

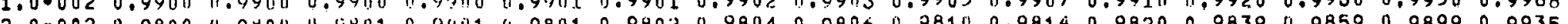
(1)

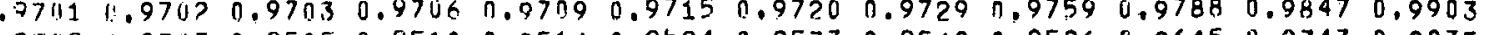

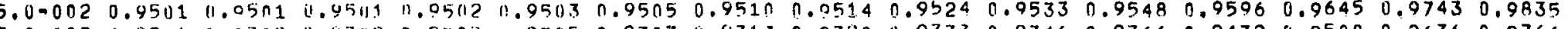

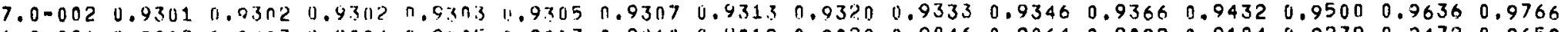
1.0-001 $0.90020 .0103 \quad 0.90 n 4 \quad 0.0105 \quad 0.9007 \quad 0.91110 \quad 0.9010 \quad 0.9028 \quad 0.9046 \quad 0.90640 .9092 \quad 0.9184 \quad 0.9279 \quad 0.9472 \quad 0.9659$

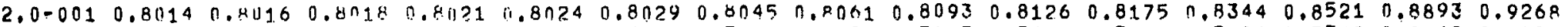
$\begin{array}{llllllllllllllllll}3.0-001 & 0.7059 & 0.71181 & 0.7110 .3 & 11.7168 & 1.7 n 7 ? & 0.71) 78 & 0.7099 & 0.7120 & 0.7163 & 0.721) 6 & 0.7271 & 0.7498 & 0.7740 & 0.8269 & 0.8828\end{array}$ $\begin{array}{llllllllllllllllll}5.0-001 & 0.5336 & 0.5338 & 0.5341 .0 .5346 & 0.5351 & 0.5358 & 0.5384 & 0.54 \Pi 9 & 0.5461 & 0.5513 & 0.5594 & 0.5882 & 0.6201 & 0.6948 & 0.7821\end{array}$ $\begin{array}{lllllllllllllllll}7.0-001 & 0.395 n & 0.3953 & 0.3955 & 0.3940 & 11.3965 & 0.3972 & 0.5996 & 0.4071 & 0.4071 & 0.4123 & 0.4202 & 0.4492 & 0.4824 & 0.5651 & 0.6720\end{array}$ 100000.25020 .25040 .251140 .25190 .25110 .25190 .25380 .25580 .25970 .26390 .27030 .29410 .32260 .39910 .5115

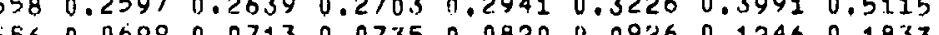
.

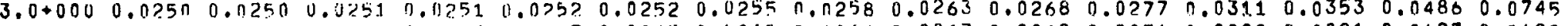

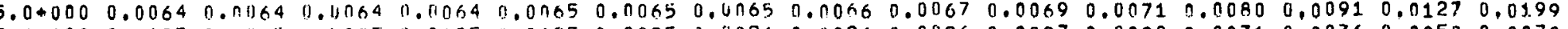
$7.0+000 \quad 0.0025 \quad 0.01325 \quad 10.4025 \quad 0.0025 \quad 0.0 n 25 \quad 0.0025 \quad 0.0026 \quad 0.0026 \quad 0.0026 \quad 0.00270 .00280 .00310 .00360 .0050 \quad 0.0078$ $1.0+0010.00090 .0109$ u.ungs n.00n9 n.0n00 0.0009 0.00000 .00090 .00090 .00100 .00100 .00110 .00130 .00180 .0028 $2.0+0010.00010 .001010 .0001 \quad 1.0 n 010.0 n 010.00 n 10.00010 .00010 .00010 .00010 .00010 .00010 .00020 .0002$

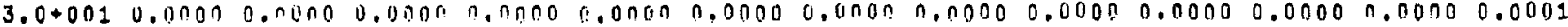

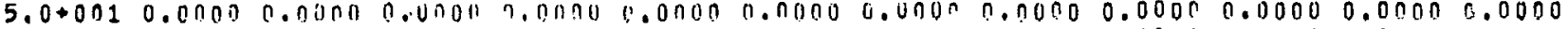

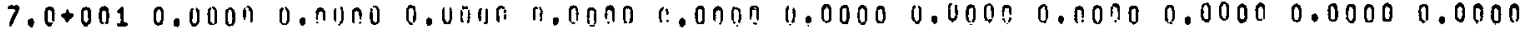

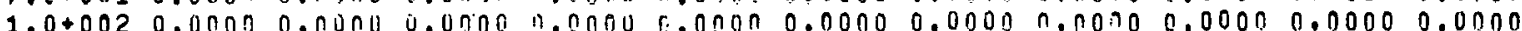

$1.0+002$. 00 (1)

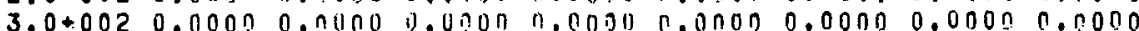

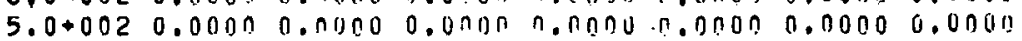

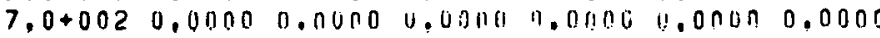

$1.0+003 \quad 0.00000 .00000 .030011 .00000 .00000 .0000$

ALPHA $1.000 \quad 2.000 \quad 3.000 \quad 5.000 \quad 7.000 \quad 10.00 \quad 20.00 \quad 30.00 \quad 50.00 \quad 70.00 \quad 100.0 \quad 200.0 \quad 300.0 \quad 500.0 \quad 700.0 \quad 1000$ BETA

$1.0 .0030 .99901 .00001 .000 n 1.00001 .00001 .00 n n 1.000 n 1.00001 .00001 .00001 .00001 .00001 .00001 .00001 .00001 .0000$

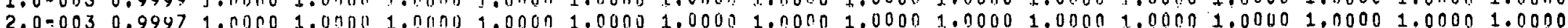

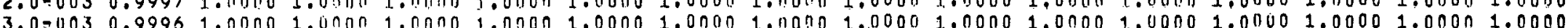

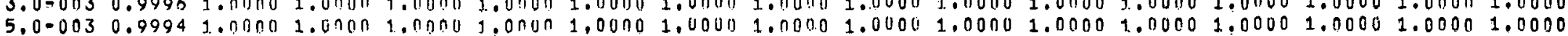

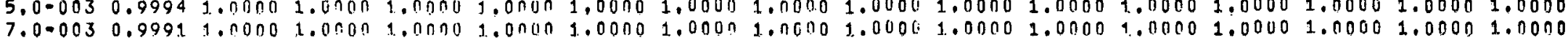

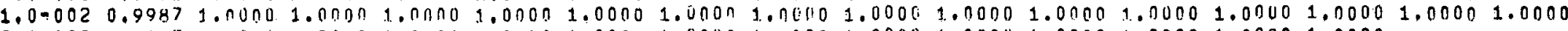
$2.0=0020.99750 .99991 .00001 .00001 .00001 .00001 .00001 .00001 .00001 .00001 .00001 .00001 .00001 .0000$

$3.0-0020.99610 .09991 .00001 .60001 .00001 .00001 .00001 .00001 .00001 .00001 .00001 .00001 .0000$

$5.0-0020.99350 .09981 .00001 .00001 .00001 .00001 .00001 .00001 .00001 .00001 .00001 .0000$

$7.0=002 \quad 0.99070 .99971 .00001 .00001 .00001 .00001 .00001 .00001 .00001 .00001 .0000$

$1.0-0010.98630 .09961 .00001 .00001 .00001 .00001 .00011 .00001 .00001 .00001 .0000$

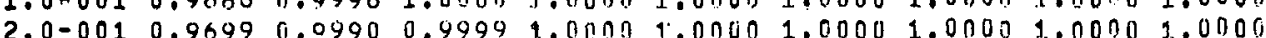

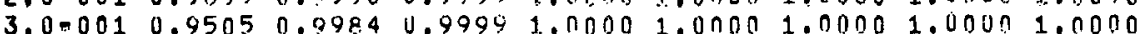

$5.0-0010.90140 .99660 .9097 \quad 1.00901 .00001 .00001 .0000$

$7,0.0010 .83920 .9940 \quad 0.9995 \div .00091 .00001 .0000$

$1.0+000 \quad 0.72730 .98840 .9991$ 1.0000 $1,00001.0000$

$2.0+000 \quad 0.3636 \quad 0.9480 \quad 0.9957 \quad 7.9999$

$3,0+000 \quad 0.1702 \quad 0.8,673 \quad 0,9882$

$5.0+000 \quad 0.04910 .62 ? 0$

$7,0+000 \quad 0.0197$

$1.0 \$ 0010.007 ?$ 
VALUES OF G FON $N=7$ AND NF $=4$

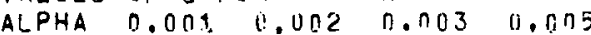
BETA

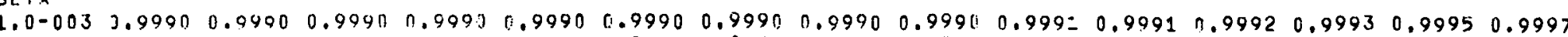

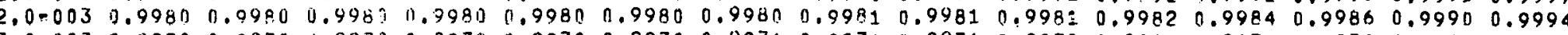
$\begin{array}{lllllllllllllllll}3.0=003 & 0.9970 & 0.9970 & 0.9970 & 0.7970 & 0.9970 & 0.9970 & 0.9971 & 0.0971 & 0.9971 & 0.9972 & 0.9973 & 0.9976 & 0.9979 & 0.9985 & 0.9990\end{array}$ $\begin{array}{llllllllllllllll}5.0-003 & 0.9950 & 0.0950 & 0.9950 & 0.0950 & 0.9950 & 0.9950 & 0.9951 & 0.0951 & 0.9952 & 0.9955 & 0.9055 & 0.9960 & 0.9965 & 0.9975 & 0.9984\end{array}$

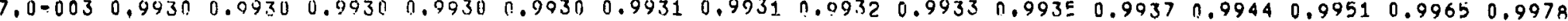

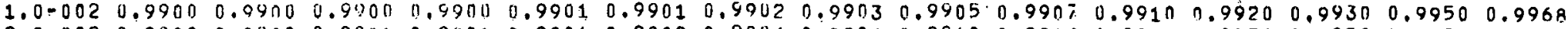
$2.0=0020.9800 \quad 0.0800 \quad 0.980=0.08010 .98010 .98020 .98040 .98060 .98100 .98140 .98200 .98390 .98590 .98990 .9935$ $3.0-0020.97000 .97010 .97010 .97010 .97020 .97030 .97060 .97090 .97150 .97200 .97290 .97590 .97880 .98470 .990 .3$

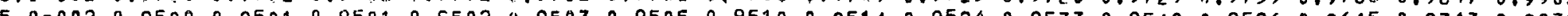

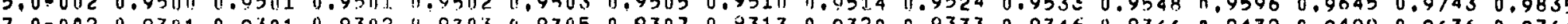

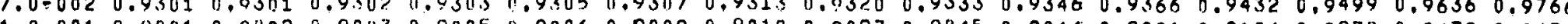

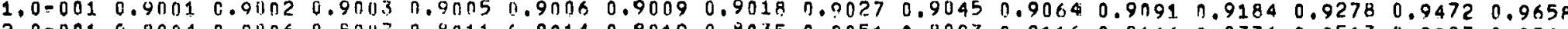
$2.0=0010.8004 \quad 0.8006 \quad 0.60470 .8011 \quad 0.8014 \quad 0.80190 .80350 .8051 \quad 0.8083 \quad 0.81160 .8166 \quad 0.8336 \quad 0.8513 \quad 0.8887 \quad 0.9264$

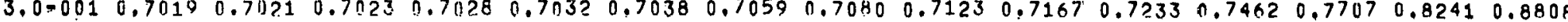
$\begin{array}{lllllllllllllllll}5.0-001 & 0.5164 & 0.5166 & 0.5165 & 0.5174 & 0.5179 & 0.5186 & 0.5212 & 0.5237 & 0.5289 & 0.5342 & 0.5424 & 0.5714 & 0.6038 & 0.6800 & 0.770\end{array}$ $\begin{array}{lllllllllllllllll}7.0-001 & 0.3608 & 0.3611 & 0.3613 & 0.364 .8 & 0.362 ? & 0.36 ? 9 & 0.3653 & 0.3677 & 0.3725 & 0.3775 & 0.3852 & 0.4135 & 0.4462 & 0.5291 & 0.6392\end{array}$

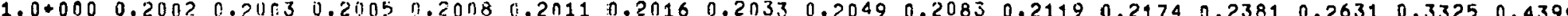
2.04000 0.0323 0.032311 .03240 .03240 .03250 .03260 .03290 .03320 .03300 .03460 .03579 .04000 .04550 .06230 .0948 $3.0+0000.00830 .010330 .00830 .00830 .00830 .00830 .00840 .00850 .00870 .00890 .00920 .01030 .01180 .01630 .0255$

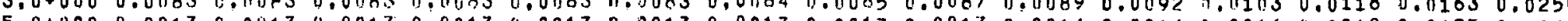
$3.0+0000.00130 .0013$
$7.0+0000.00040$ $7.0+0000.00040 .00040 .00040 .00040 .00040 .00040 .00040 .00040 .00040 .00040 .00040 .00040 .00050 .00070 .0011$

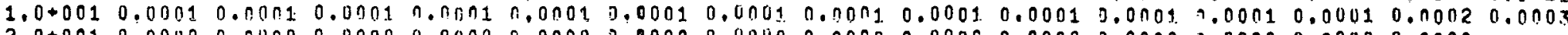

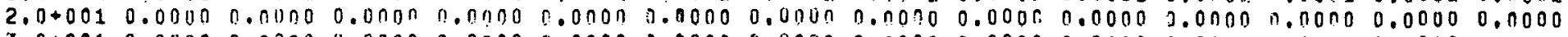

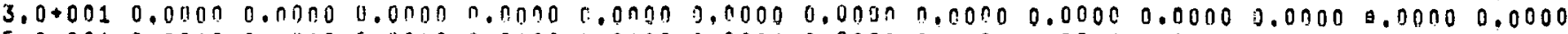

$5.0+0010.00000 .00000 .00000 .00000 .00000 .00000 .00000 .00000 .00000 .00000 .0000 .0000$

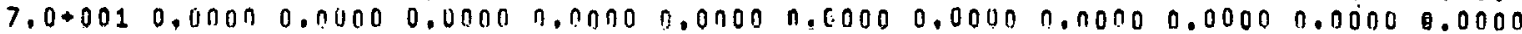

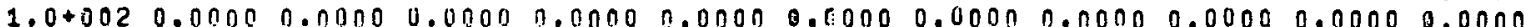

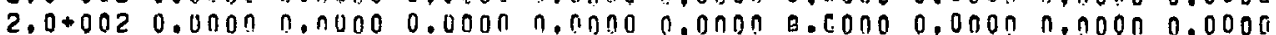

$3.0+002 \quad 0.00000 .0000 \quad 4.0000 \quad 0.0000 \quad 0.0000 \quad 0.0000 \quad 0.40000 .0000$

$5.0+0020.00000 .00000 .00000 .00000,00000,00000.0000$

$7.0+002 \quad 0.00000 .00000 .00000 .0000 \quad 0.0000 \quad 0.0000$

$1.0+0030.00000 .00000 .40000 .00000 .0000 \quad 0.0000$

ALPHA $\therefore 000 \quad 2.000 \quad 3.000 \quad 5.000 \quad 7.000 \quad 10.00 \quad 20.00 \quad 30.00 \quad 50.00 \quad 70.00 \quad 100.0 \quad 200.0 \quad 300.0 \quad 500.0 \quad 700.0 \quad 1000$ BETA

$1.0-0030.99991 .00001 .00001 .00001 .00001 .00001 .00001 .0000 .000001 .00001 .000 n 1.00001 .00001 .00001 .00001 .0000$ $2.0-0030.99971 .00001 .00001 .00001 .00001 .00001 .00001 .00001 .00001 .00001 .00001 .00001 .00001 .00001 .00001 .0000$ $3.0=0030.99961 .00001 .00001 .00001 .00001 .00001 .00001 .00001 .00001 .00001 .00001 .00001 .00001 .00001 .00001 .0000$

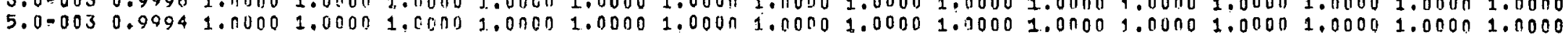

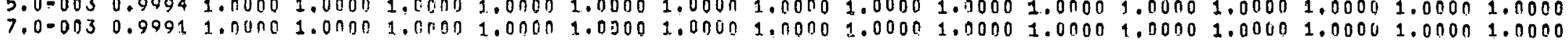

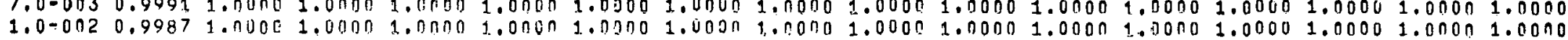

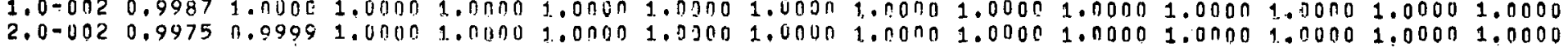
$3.0=0020.99610 .99991 .00001 .00001 .00001 .03001 .00001 .00001 .00001 .00001 .00001 .00001 .0000$

$5.0-0020.99350 .09981 .00001 .00001 .00001 .00001 .00001 .00001 .00001 .00001 .00001,0000$

7.0-002 $0.9907 \quad 0.09971 .00001 .00001 .00001 .00001 .00001 .00001 .00001 .00001 .0000$

$1.0=0010.98630 .09961 .000 n 1.00009 .00001 .00001 .00001 .00001 .00061 .00001 .0000$

$2.0 .0010 .9697 \quad 0.0990 \quad 0.99491 .00001 .00001 .00001 .00001 .00001 .0000$

$3.0=001 \quad 0.9495 \quad 0.9983 \quad 0.9999 \quad 1.00001 .0000 \quad 1.00001 .00001 .0000$

$5.0=0010.39510 .99630 .999 .71 .00001 .00001 .001001 .0000$

.00001 $0.3186 \quad 0.09310 .99951 .00001 .00001 .0000$

$1.0+000 \quad 0.5667 \quad 0.9846 \quad 0.9988 \quad 1.0000 \quad 1.00001 .0600$

$2.0+000 \quad 0.21 n 5 \quad 0.89470 .9909 \quad 3.0997$

$3.0+000 \quad 0.0625 \quad 0.6800 \quad 0.9647$

$5.0+000 \quad 0.0102 \quad 0.2464$

$7.0+000 \quad 0.0028$

$1.0+0010.0007$ 
VALUES OF G FOH $N=7$ ANO NH= BETA

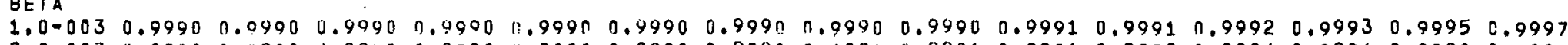

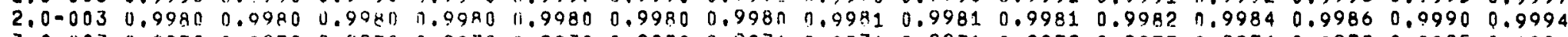
$\begin{array}{llllllllllllllll}3.0-003 & 0.9970 & 0.0970 & 0.9070 & 0.9970 & 0.997 n & 0.9970 & 0.9971 & 0.0971 & 0.9971 & 0.9972 & 0.9973 \quad 0.9976 & 0.9979 & 0.9985 & 0.9990\end{array}$ $5.0-0030.9950 \quad 0.9950 \quad 0.9050 \quad 1.9950 \quad 10.9950 \quad 0.9950 \quad 0.9951 \quad 0.09510 .9952 \quad 0.9953 \quad 0.9955 \quad 0.99600 .99650 .99750 .9984$ $\begin{array}{llllllllllllllll}7.0-003 & 0.9930 & 0.0930 & 0.9030 & 0.9930 & 1.993 n & 0.9931 & 0.9931 & 0.9932 & 0.9933 & 0.9935 & 0.9937 & 0.9944 & 0.9951 & 0.9965 & 0.9978\end{array}$

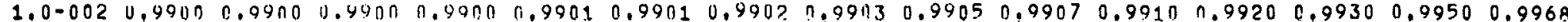
2.0-002 0.9800 0.OBN0 0.9401 0.9801 0.99410 .98020 .98440 .08060 .98100 .98140 .98200 .98390 .98590 .98990 .9935

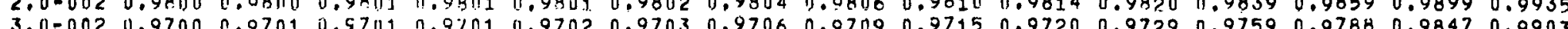
$5.0-0020.95000 .95010 .95010 .90 .903$ 5.0.002 0.95n 0.95n1 0.95110 n.95n2 $7.0-0020.9301 .03 n 10.9310 .93030 .9305 n .93070 .93130 .93200 .93330 .93460 .936 \mathrm{n} n .94320 .94990 .96360 .9766$

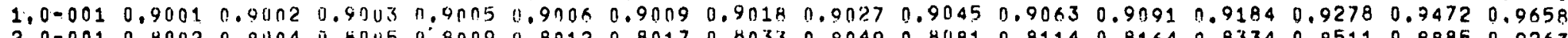

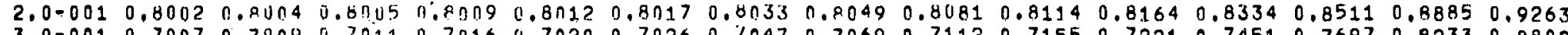
$\begin{array}{llllllllllllllllllll}3.0-001 & 0.7007 & 0.70 n 9 & 0.7 n 11 & 0.7 n 16 & 1.7020 & 0.7026 & 0,7047 & 0.7069 & 0.7112 & 0.7155 & 0.72 .21 & 0.7451 & 0.7697 & 0.8233 & 0.8802\end{array}$ $\begin{array}{llllllllllllllllll}5.00001 & 0.5082 & 0.5094 & 0.5 n \xi 7 & 0.5092 & 0.5 n 97 & 0.5104 & 0.513 n & 0.5155 & 0.5207 & 0.5261 & 0.5342 & 0.5634 & 0.5959 & 0.6728 & 0.7643\end{array}$ $\begin{array}{llllllllllllllll}7.0-001 & 0.3402 & 0.3405 & 0.34117 & 0.3411 & 0.3414 & 0.3423 & 0.3445 & 0.3469 & 0.3516 & 0.3565 & 0.3640 & 0.3917 & 0.4239 & 0.5065 & 0.6181\end{array}$

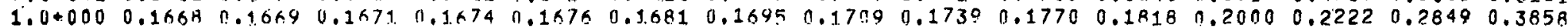
$10+0000.01590 .01590 .01690 .01600 .01600 .01600 .01620 .01640 .01670 .01700 .01760 .01980 .02250 .03110 .0482$ $3.0+0000.01070 .0038$ $3.0+0000.0030 .003$

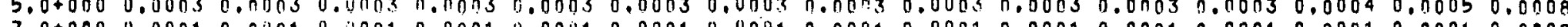
$7.0+000 \quad 0.0001$ n.nun

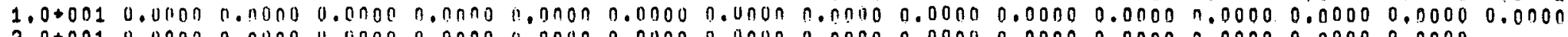

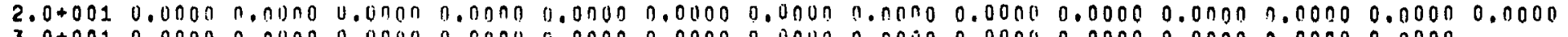

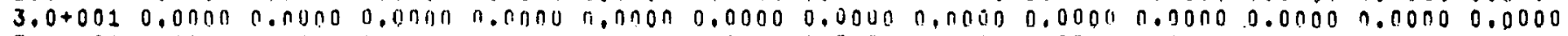

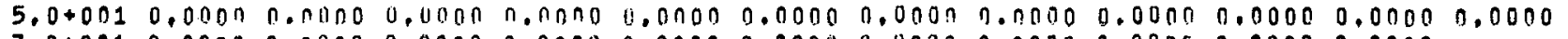

$7.0+0010.00000 .00000 .00000 .00008 .00000 .00000 .00000 .00000 .00000 .00000 .0000$

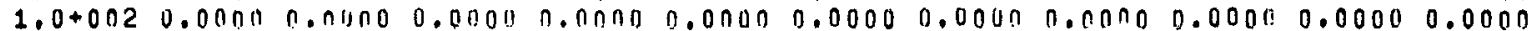

$2.0+0020.00000 .00000 .00000 .00000 .00000 .00000,00000.00000 .0000$

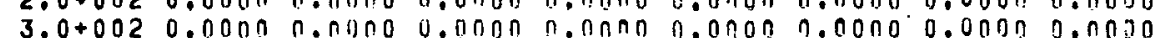

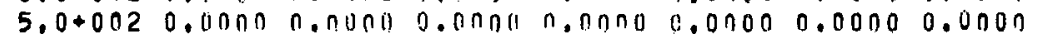

$7.0+0020,00000.00000,00000,0000,0,00000,0000$

$1.0 * 0030.00000 .0000 \quad 0.0000$ 0.0ก00 0.00000 .0000

$$
\text { ALPH }
$$
BETA

1.000030 .99991 .00001 .00001 .00009 .00001 .00001 .000111 .00001 .00001 .00001 .00001 .00001 .00001 .00001 .00001 .0000 2.0.003 $0.99971 .00001 .00001 .000 \mathrm{i} 1.00001,00001.00001 .00001 .00001 .00001 .00001 .00001 .00001 .00001 .00001 .0000$ $3.0 .0030 .99961 .00001 .000 n 1.00001 .00001 .00001 .000 n 1.00001 .00001 .00001 .00001 .00001 .00001 .00001 .00001 .0000$ $5.0-003 \quad 0.99941 .00001 .00101 .00901 .00001 .00001 .00091 .00001 .00001 .00001 .00001 .00001 .00001 .00001 .00001 .0000$ $7.0-0030.9991 .1 .00001 .000 \pi 1.00 n 01.000 n 1.00001 .00001 .00001 .00001 .00001 .00001 .00 n 01.00001 .00001 .00001 .0000$ $1.0-0020.99871 .00001 .00001 .001001 .00001 .00001 .00001 .00001 .00001 .00001 .00001 .00001 .00001 .00001 .00001 .0000$ $2.0-002 \quad 0.99750 .99991 .00001 .00701 .00001 .00001 .40001 .00001 .000111 .00001 .00001 .00001 .00001 .0000$ $3.0-0020.9961 \quad 0.99991 .00001 .001009 .00001,00001.00011 .00001 .00001 .00001 .00001 .00001 .0000$

$5.0=0020.99350 .09981 .00001 .0 n n 01.00001 .00001 .00001 .00001 .00001 .00001 .00001 .0000$

$7.0=0020.99070 .09971 .00001 .00001 .00001 .00001 .00011 .00001 .00001 .00001 .0000$

1.0 .0010 .98630 .99961 .00009 .00001 .00001 .00001 .00001 .00001 .00001 .00001 .0000

$2.00010 .96970 .9990 \quad 0.99991 .0000900001 .00001 .00099000001 .0000$

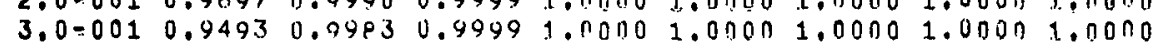

$5.0-001 \quad 0.89200 .9962 \quad 0.9997 \quad 1,00001.00001,00001.0000$

$7.0=001 \quad 0.8047 \quad 0.99240 .9994 \quad 1.00001 .00001 .0000$

$1.0+000 \quad 0.6154 \quad 0.9808 \quad 0.9985 \quad 0.99991 .00001 .0000$

$2.0+000 \quad 0.1143 \quad 0.80440 .9814 \quad 0.9994$

$3.0+000 \quad 0.0216 \quad 0.4126 \quad 0.9004$

$5.0+000 \quad 0.0020 \quad 0.0613$

$7,0+000 \quad 0,0004$ 
VALUES OF G FOH IV: 5 AN! NHE $=1$ BETA

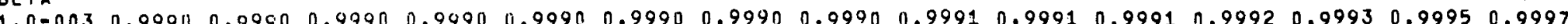

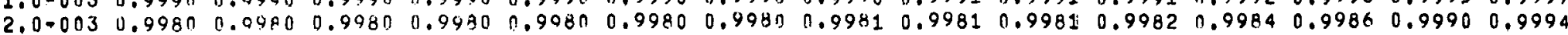
$\begin{array}{llllllllllllllll}3.0-003 & 0.9970 & 0.0970 & 0.9970 & 0.9970 & 10.907 n & 0.9970 & 0.9971 & 0.9971 & 0.9972 & 0.9972 & 0.9973 & 0.9976 & 0.9979 & 0 \% 9985 & 0.9991\end{array}$ $\begin{array}{lllllllllllllllll}5.0-003 & 0.9950 & 0.0950 & 0.9950 & 0.9950 & 1.9951 & 0.9951 & 0.9951 & 0.9952 & 0.9953 & 0.9954 & 0.9955 & 0.9960 & 0.9965 & 0.9975 & 0.9984\end{array}$ $\begin{array}{llllllllllllllllll}7.0-003 & 0.9931 & 0.9931 & 0.9931 & 10.9931 & 0.9931 & 0.9931 & 0.9932 & 0.9933 & 0.9934 & 0.9935 & 0.9937 & 0.9944 & 0.9951 & 0.9965 & 0.9978\end{array}$

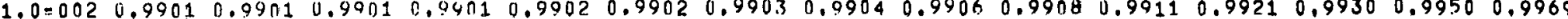

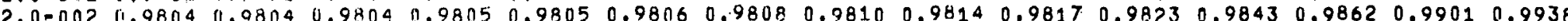
$3,0=0020.97090 .07090 .97100 .971011 .971100 .97120 .97140 .97170 .97230 .97290 .97370 .97660 .97940 .98520 .9907$

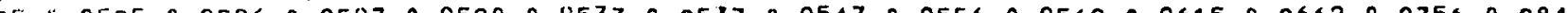
5.0-002 0.9524 0.9525 0.9525 n.9526 n.952 0.95280 .95330 .95370 .95470 .95560 .95690 .96150 .96630 .97560 .9846

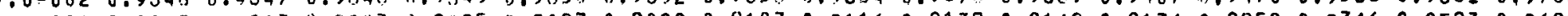
$1.0=0010.90920 .90930 .91930 .91950 .9097$ n.909 0.91 .070 .01160 .91370 .91490 .91 .740 .92590 .93460 .95230 .9697

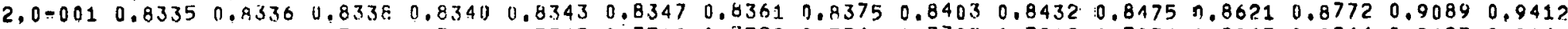
$\begin{array}{llllllllllllllllll}3.0-001 & 0.7694 & 0.7696 & 0.7698 & 0.7701 & 0.7705 & 0.7710 & 0.7728 & 0.7746 & 0.7782 & 0.7819 & 0.7874 & 0.8065 & 0.8264 & 0.8693 & 0.9143\end{array}$ $\begin{array}{lllllllllllllllllll}5.0 .001 & 0.6669 & 0.6671 & 0.6673 & 0.6678 & 0.6682 & 0.5689 & 0.6711 & 0.6734 & 0.6780 & 0.6826 & 0.6897 & 0.7143 & 0.7407 & 0.7997 & 0.8648\end{array}$ $\begin{array}{llllllllllllllllll}7.0 .001 & 0.5885 & 0.5887 & 0.5890 & 0.5894 & 0.5899 & 0.59 n 7 & 0.5931 & 0.5956 & 0.6006 & 0.6057 & 0.6135 & 0.6410 & 0.6711 & 0.7404 & 0.8205\end{array}$

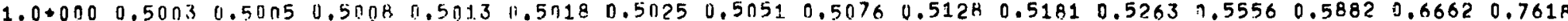

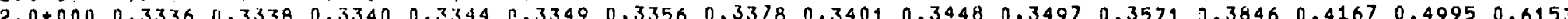
$3.0+0000.25020 .25040 .25060 .25090 .25130 .25190 .05380 .25580 .25070 .26390 .27030 .29410 .32260 .39950 .5160$

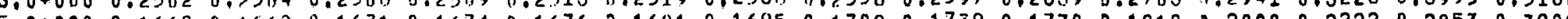

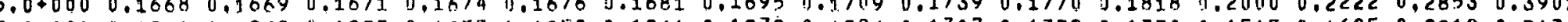

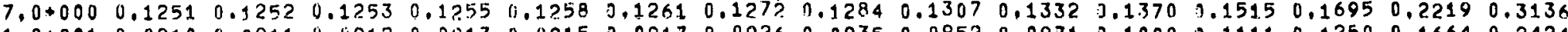

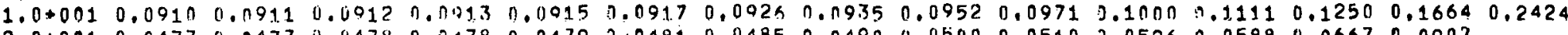

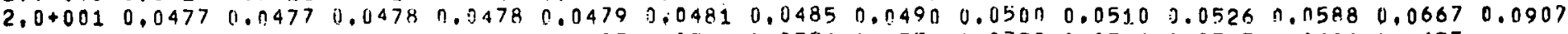

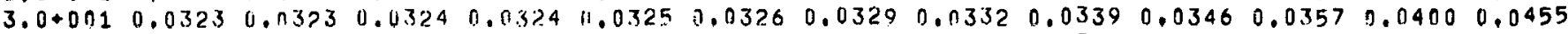

$5.0+0010.0196 \quad 0.0196 \quad 0.0197 \quad 0.0197 \quad 0.0 .297 \quad 0.0198 \quad 0.0200 \quad 0.02020 .0206 \quad 0.02110 .0217 \quad 0.0244$

$\begin{array}{lllllllllllllllll}7.0+001 & 0.0141 & 0.0141 & 0.01 .41 & 0.0142 & 0.0142 & 0.0142 & 0.0144 & 0.0145 & 0.0148 & 0.0151 & 0.0156\end{array}$

$1,0+0020.00990 .00990 .00990 .0100 \quad 0.01000 .0100 \quad 0.01010 .01020 .01040 .01060 .0110$

$2.0+0020.00500 .00500 .417500 .8050,10500.06500 .00540 .00510 .0052$

$3,0+0020,00330.011330,00330.0033 \quad 11.0033 \quad 0.00340 .0034 \quad 0.0034$

$5.0+0020.00200 .00200 .00200 .0020$ i), 0n?0 0.00200,0020

$7.0+0020.00140 .00140 .00140 .0014 \quad 0.0014 \quad 0.0014$

$1,0+0030,00100,00100,00100,0010 \quad 1,00100,0010$

1.00003 0.99991 .011001 .00001 .000001 .00001 .00001 .00001 .00001 .00001 .00001 .00001 .00001 .00001 .00001 .00001 .0000 $2.0-0030.99981 .00001 .00101 .00100 .00001 .00001 .00001 .00001 .00001 .00001 .00001 .00001 .00001 .00001 .00001 .0000$ 2.0.0.0

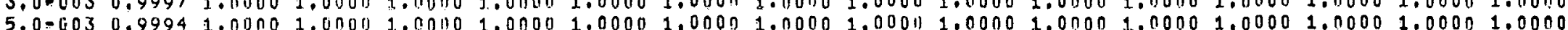

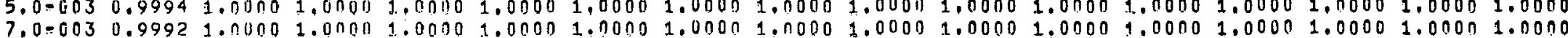

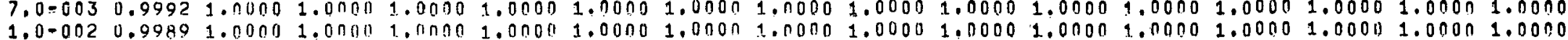

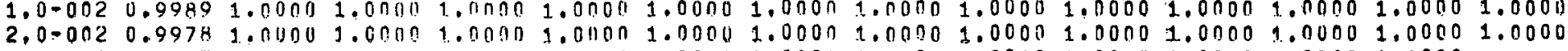

$3.0=0020.99670 .99991 .00001 .00001 .00001 .00001 .00001 .00001 .00001 .00001 .00001 .00001 .0000$

5.0-002 0.99450 .99991 .00001 .00001 .00001 .00001 .00001 .00001 .00001 .00001 .00001 .00000

$7.0-0020.99230 .9 .9991 .00001 .00 .001 .00001 .00001 .00001 .00001 .00001 .00001 .0000$

1.0 .0010 .98900 .09981 .00001 .01001 .00001 .00001 .00001 .00001 .00001 .00001 .0000

$2,0=0010.97830 .99961 .00001 .00001 .00001 .00001,00001.00001 .0000$

$3,0.0010 .96770 .99941,00001.0 n \pi 01.00001 .031001 .00001 .0000$

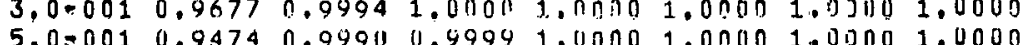

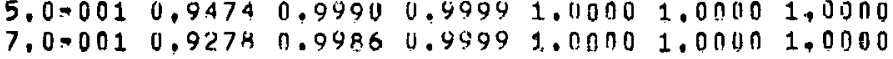

$1.0+000 \quad 0.9000 \quad 0.0980 \quad 0.49991 .00001 .00001 .0000$

$2,0+000 \quad 0,8182 \quad 0.9961 \quad 0,9098 \quad 1.01000$

$3,0+000 \quad 0.75000 .9942 \quad 0.9997$

$5,0+000 \quad 0.6429 \quad 0.0903$

$7.0+000 \quad 0.5625$ 
VALUES OF G FOR $N=B$ ANII NF $=$ Z BETA

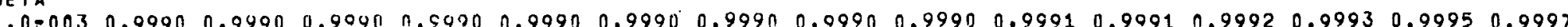

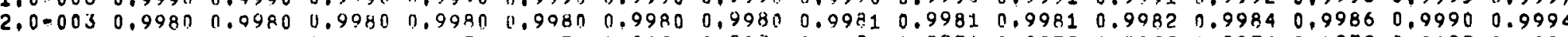
$\begin{array}{llllllllllllllll}3.0-003 & 0.997 n & 0.9970 & 0.9970 .0 .9970 & 0.997 n & 0.9970 & 0.9971 & 0.9971 & 0.9971 & 0.9972 & 0.9973 & 0.9976 & 0.9979 & 0.9985 & 0.9991\end{array}$

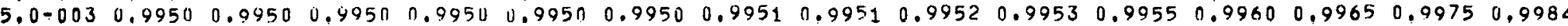
$\begin{array}{lllllllllllllllll}7.0=003 & 0.993 \pi & 0.0930 & 0.9930 & 0.9930 & 0.9030 & 0.9931 & 0.9933 & 0.9932 & 0.9933 & 0.9935 & 0.9937 & 0.9944 & 0.9951 & 0.9965 & 0.9978\end{array}$

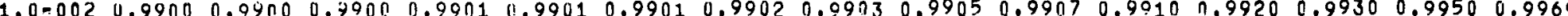

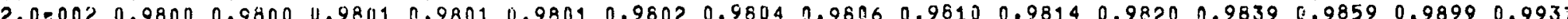
$3.0-0020.97010 .97014 .97010 .97020 .97020 .97030 .97060 .97090 .97150 .97210 .97290 .97590 .97860 .98480 .9904$

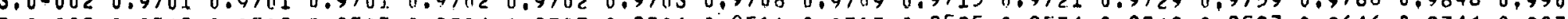

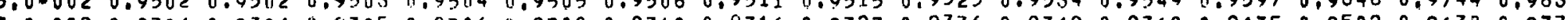

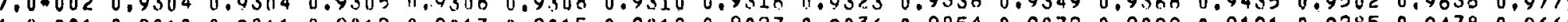

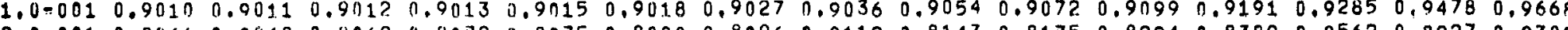

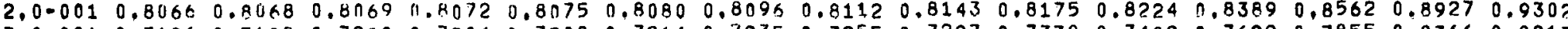
$\begin{array}{llllllllllllllllllll}3.0-001 & 0.7196 & 0.7198 & 0.7200 & 0.7204 & 0.7208 & 0.7214 & 0.7235 & 0.7255 & 0.7297 & 0,7338 & 0.7402 & 0.7622 & 0.7855 & 0.8366 & 0.8913\end{array}$ $\begin{array}{llllllllllllllllll}5.0 .001 & 0.5717 & 0.5719 & 0.5722 & 0.5727 & 0.5731 & 0.5739 & 0.5764 & 0.57 .99 & 0.5839 & 0.5891 & 0.5970 & 0.6250 & 0.6557 & 0,7269 & 0.8101\end{array}$ $\begin{array}{lllllllllllllllll}7,0.001 & 0.4569 & 0.4571 & 0.4574 & 0.4579 & 0.4584 & 0.4591 & 0.4616 & 0.4642 & 0.4694 & 0.4747 & 0.4829 & 0.5123 & 0.5455 & 0.6265 & 0.7289\end{array}$ $\begin{array}{lllllllllllllllllll}1.0+000 & 0.3336 & 0.3338 & 0.3340 & 0.3344 & 0.3349 & 0.3356 & 0.3378 & 0.3401 & 0.3448 & 0.3497 & 0.3571 & 0.3846 & 0.4167 & 0.4995 & 0.6153\end{array}$

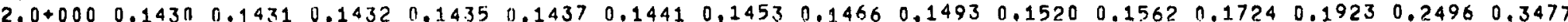
$3.0+0000.07700 .07710 .07710 .07730 .07740 .07760 .07840 .07910 .08050 .082200 .08470 .09430 .10640 .14260 .2105$ $5.0+0000.03250 .03230 .03240 .03240 .03250 .03260 .03290 .03320 .03300 .03460 .03570 .04000 .04550 .06240 .0964$

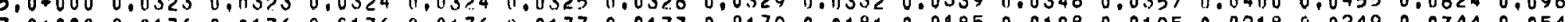
$1.0+0010.00900 .0000 .00900 .00910 .00910 .00910 .00920 .0930 .00950 .00970 .0100 \quad 0.01120 .01280 .01780 .0283$

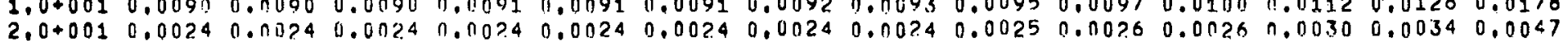

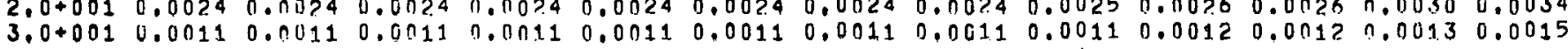

$5.0+0010.00040 .00040 .00040 .00040 .00040 .00040 .00040 .00040 .00040 .00040 .00040 .0005$

$7.0+0010.00020 .00020 .00020 .00020 .00020 .00020 .00020 .00020 .00020 .00020 .0002$

$1.0+0020.00010 .00010 .00010 .00010 .00010 .00010 .00010 .00010 .00010 .00010 .0001$

$2,0+0020,00000,00000,00000,00000,00000,00000,00000.00000 .0000$

$3,0+0020.00000 .0000 \quad 0.0000 \quad 0.00000 .00000 .00000 .00000 .0000$

$5,0+0020.00000 .0000,0,0000,0,00000.0000 \quad 0.0000 \quad 0,0000$

$7.0+0020.00000 .00000 .00000 .00000 .0000 \quad 0,0000$

$1,0+0030,00000.00000 .60000,00000,00000,0000$

ALPHA $1.000 \quad 2.000 \quad 3.000 \quad 5.000 \quad 7.000 \quad 10.00 \quad 20.00 \quad 30.00 \quad 50.00 \quad 70.00 \quad 1.00 .0 \quad 200,0 \quad 300.0 \quad 500.0 \quad 700.0 \quad 1000$ BETA

1.090030 .99991 .00001 .00001 .00001 .00001 .00001 .00001 .00001 .00001 .00001 .00001 .00001 .00002 .00001 .00001 .0000 2.0 .0030 .99981 .00001 .00001 .00001 .00001 .00001 .00001 .00001 .00001 .00001 .00001 .00001 .00001 .00001 .00001 .0000 $3,0=0030.99971 .00001 .00001 .00001 .00001 .00001 .00001 .00001 .00001 .00001 .00001 .00001 .00001 .00001 .00001 .0000$ $5,0=0030.99941 .00001 .00001 .00001 .00001 .00001 .00001 .001011 .00001 .00001 .00001 .00001 .00001 .00001 .00001 .0000$ $7,0.0030 .99921 .00001 .00001 .00 n 01.00001 .00001 .00001 .00001 .00001 .00001 .00001 .00001 .00001 .00001 .00001 .0000$

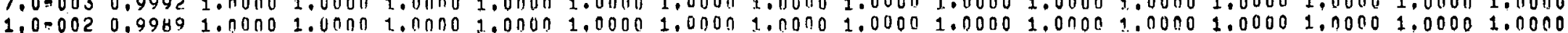
$2,0=0020.99771 .00001 .00009,00001.00001 .00001 .000 n 1.00001 .00001 .00001 .00001 .00001 .00001 .0000$ $3,0.0020 .99660 .99991 .00001 .00001 .00001 .00001 .00041 .00001 .00001 .00001 .00001 .00001 .0000$

$5,0=0020.99420 .99991 .00001 .00001 .00001 .00001 .00001 .00001 .00001 .00001 .00001 .0000$

$7.0-002 \quad 0.9917 \quad 0.99991 .00001 .00001 .00001 .00001 .00001 .00001 .00001 .00001 .0000$

$1.0=0010.98790 .99981 .00001 .00001 .00001 .00001 .00001 .001101 .00001 .00001 .0000$

$2.0-0010.9740 \quad 0.09051 .00001 .000001 .00601 .00001 .00001 .00001 .0000$

$3.0-0010.95850 .09921 .401) \quad 1$, rionn $1.00001 .00001 .000 n 1.0000$

5.000010 .92310 .99450 .99991 .00001 .00001 .00001 .0000

$7.0 .001 \quad 0.8832 \quad 0.9977 \quad 0.9999 \quad 1.00001,00001.0000$

$1.00000 \quad 0.8182 \quad 0.0961 \quad 0.9998 .1 .00001 .00001 .0000$

$2,0+000 \quad 0.6000 \quad 0.9884 \quad 0.9994 \quad 1.0000$

$3,0+000 \quad 0.4286 \quad 0.9771 \quad 0.9984$

$5.0+000 \quad 0.2308 \quad 0.9445$

$7.0+000 \quad 0.1385$ 

BETA

1.0-003 $0.9990 \quad 0.0990 \quad 0.9990 \quad 0.9990 \quad 0.9990 \quad 0.9990 \quad 0.9990 \quad 0.9990 \quad 0.9990 \quad 0.9991 \quad 0.9991 \quad 0.9992 \quad 0.9993 \quad 0.9995 \quad 0.9997$

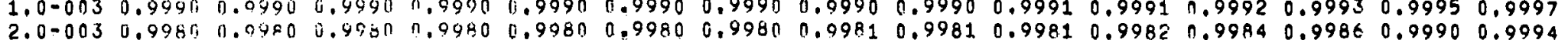

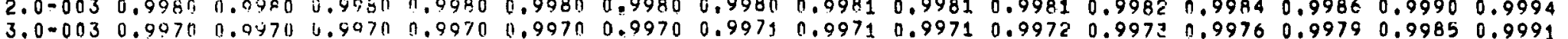

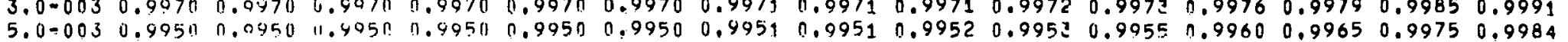

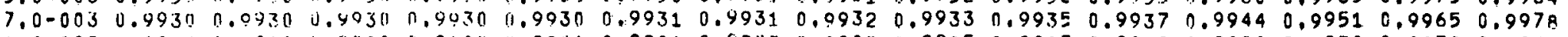

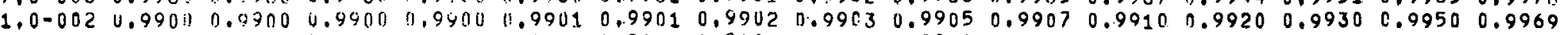

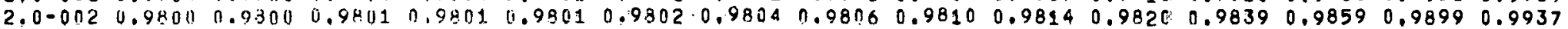
$\begin{array}{lllllllllllllllll}3.07002 & 0.9700 & 0.0701 & 0.9701 & 0.9701 & 0.9702 & 0.9703 & 0.9706 & 0.970 .9 & 0.9715 & 0.9720 & 0.9729 & 0.9759 & 0.9788 & 0.9847 & 0.9904\end{array}$

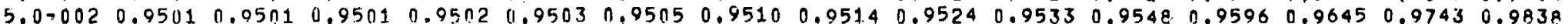
$7.0-0020.93010 .93020 .93020 .930311 .93050 .93070 .93130 .93200 .93330 .93450 .93660 .94320 .95000 .96370 .9370$

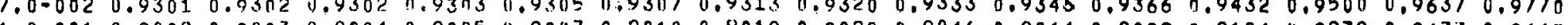
0.9360 .9640 .965

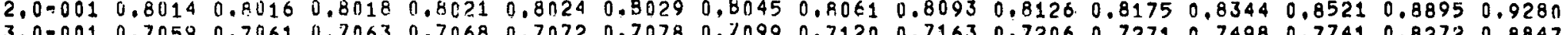
$\begin{array}{llllllllllllllll}3.0-001 & 0.7059 & 0.7061 & 0.7063 & 0.7068 & 0.7072 & 0.7078 & 0.7090 & 0.7120 & 0.7163 & 0.7206 & 0.7271 & 0.7498 & 0.7741 & 0.8272 & 0.8847\end{array}$ $\begin{array}{llllllllllllllllll}5.0=001 & 0.5336 & 0.5338 & 0.5341 & 0.5346 & 0.5351 & 0.5358 & 0.5384 & 0.5409 & 0.5461 & 0.5513 & 0.5594 & 0.5882 & 0.6202 & 0.6952 & 0.7852\end{array}$

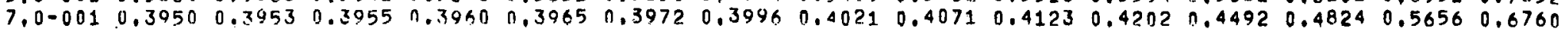

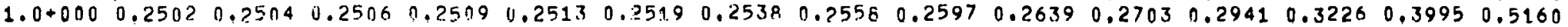

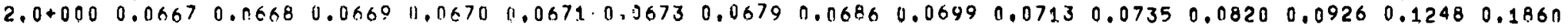
$\begin{array}{llllllllllllllll}3.0+000 & 0.0250 & 0.0250 & 0.0251 & 0.0251 & 0.0252 & 0.0252 & 0.0255 & 0.0258 & 0.0263 & 0.0268 & 0.0277 & 0.0311 & 0.0353 & 0.0487 & 0.0758\end{array}$ $5,0+000 \quad 0.00640 .00640 .00640 .00640 .00650 .00650,00650.00660 .00670 .00690 .00710 .00800 .00910 .01270 .0202$ $3,0+0000.00250 .00250 .00250 .00250 .00250 .0250 .00240 .00260 .00260 .00270 .00280 .00310 .00360 .00500 .0080$

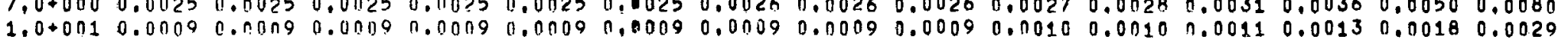

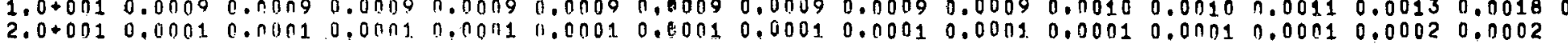

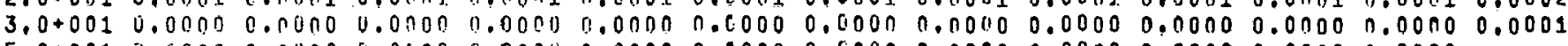

$5.0+0010.00000 .00000 .00000 .00000 .00000 .00000 .00000 .00000 .00000,00000.00000 .0000$

$7.0+0013.00000 .00000 .00000 .00000 .00000,00000.00000 .00000 .00000,00000.0000$

$1.0+0020.0000 \quad 0.00000 .0000 \quad 0.0000 \quad 0.0000 \quad 0.0000 \quad 0.0000 \quad 0.00000 .00000 .00000 .0000$

$2.0+0020.00000 .01000 .00000 .00000 .00000 .00000 .00000 .00000 .0000$

$3.0+0020.00000 .013000 .0000 \quad 0.30000 .00000 .00000 .00000 .0000$

$5.0+002$ 1.0000 0.0000 $0.0000,0.00000 .00000 .00000 .0000$

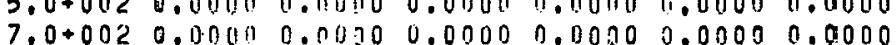

$1,0+0030.00010 .00000 .00000,00000,00000.0000$ BETA

ALPHA $1.000 \quad 2.000 \quad 3.000 \quad 5.000 \quad 7.000 \quad 10.00 \quad 20.00 \quad 30.00 \quad 50.00 \quad 70.00 \quad 100.0 \quad 200.0 \quad 300.0 .500 .0 \quad 700.0 \quad 1000$

$1.090035 .99991 .00001 .001101 .00001 .00001,00001.00001 .00001 .00001 .00001 .00001 .00001 .00001 .00001 .00001 .0000$ 2.0-003 $0.99951 .00001 .00001 .00101 .00001 .0000 \div 00001.00001 .00001 .00001 .00001 .00001 .00001 .00001 .00001 .0000$ $3.0-0030.90971 .00001 .00001 .00001 .00001 .03001 .00001 .00001 .00001 .00001 .0000 .00001 .00001 .00001 .00001 .0000$ $5,0-0030.99941 .00001,00001.00701 .000011,3001,03001,00001.00001,00001.00001900001,00001.00001 .00001 .0000$

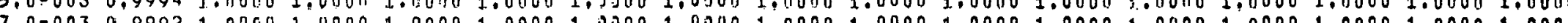
$\begin{array}{llll} & \\ 0\end{array}$ $1.0-0020.99891 .00001 .00001 .00001 .00001 .00001 .00001 .00001 .00001 .00001 .00001 .00001 .00001 .00001 .00001 .0000$ $2,0-0020.99771 .00001 .00001 .00001 .00001,00001.00001 .00001 .00001 .00001 .00001 .00001 .00001 .0000$

$3,0-0020.9966 \quad 0.99991 .00001 .00001 .00001 .00001 .00001 .00001 .00001 .00001 .00001 .00001 .0000$

$5.0>002 \quad 0.99420 .99991 .00001 .00001 .00001,00001.00001 .00001 .00001 .00001,00001.0000$

$7,0.0020 .99170 .09991 .00001 .00001 .00001 .010001 .00001,00001.00001 .00001 .0000$

$1,0.0010 .98780 .9998 \quad 1.00001 .00001 .00001,00001.00001 .00101 .00001 .00001 .0000$

$2.0=001 \quad 0.97320 .99951 .00001 .00009 .00001 .00001 .00001 .00001 .0000$

$3.0=0010.95570 .99921 .00001 .03001 .00001 .0 C 001.00001 .0000$

$5,0=001 \quad 0.9114 \quad 0.9983 \quad 0.999 .91 .00001 .00001,00001.0000$

$7,0.0010 .85450 .9970 \quad 0.99981 .00001 .00001,0600$

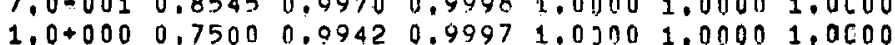

$\begin{array}{llllll}1,0+000 & 0,7500 & 0.9942 & 0.9997 & 1.0300 \\ 2,0+000 & 0.3913 & 0.9733 & 0,9986 & 1.0300\end{array}$

$3,0+000 \quad 0.1875 \quad 0.0291 \quad 13.9961$

$5,0+000 \quad 0.0549 \quad 0.7673$

$7,0+000 \quad 0.0221$

$1,0+0010.008$ 


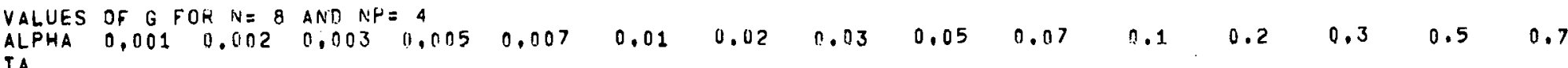
BETA

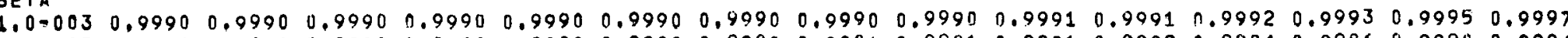
$\begin{array}{llllllllllllllll}2.0 .003 & 0.9980 & 0.9980 & 0.9980 & 0.9981 & 0.9980 & 0.9980 & 0.9980 & 0.9981 & 0.9981 & 0.9981 & 0.9982 & 0.9984 & 0.9986 & 0.9990 & 0.9994\end{array}$ $\begin{array}{llllllllllllllll}3.0 .003 & 0.9970 & 0.9970 & 0.9970 .0 .9973 & 0.9970 & 0.9970 & 0.9971 & 0.9971 & 0.9971 & 0.9972 & 0.9973 & 0.9976 & 0.9979 & 0.9985 & 0.9991\end{array}$

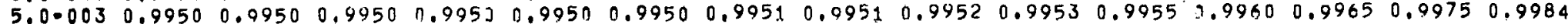
$\begin{array}{lllllllllllllllll}7,0.003 & 0.9930 & 0.9930 & 0.9930 & 0.9930 & 0.9030 & 0.9931 & 0.9931 & 0.9932 & 0.9933 & 0.9935 & 0.9937 & 0.9944 & 0.9951 & 0.9965 & 0.9978\end{array}$ $1.0 .0020 .9900 .909000 .99000 .99000 .99010 .99010 .90020 .99030 .99050 .99070 .99100 .99200 .99300 .9950 \quad 0.0969$

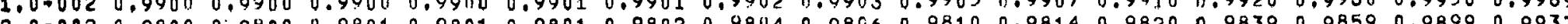

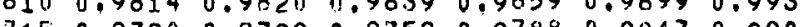

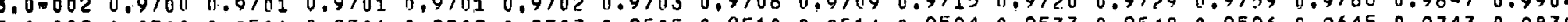
$5.0 .0020 .9500 \quad 0.95010 .95010 .95020 .95030 .95050 .95100 .95140 .95240 .95330 .95480 .95960 .96450 .97430 .9838$ 7.0-002 $0.9301 \quad 0.93 n 1 \quad 0.93020 .93030 .9305 \quad 0.93070 .93130 .93200 .93330 .93460 .93660 .94320 .94990 .96370 .9770$

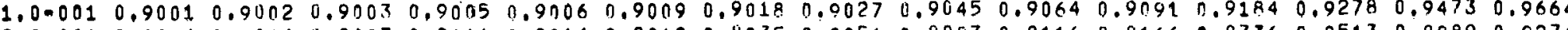
2.0-001 $0.8004 \quad 0.8006 \quad 0.81707 \quad 0.8011 \quad 0.8014 \quad 0.80190 .8035 \quad 0.8051 \quad 0.8083 \quad 0.8116 \quad 0.8166 \quad 0.8336 \quad 0.8513 \quad 0.9889 \quad 0.9276$ $\begin{array}{llllllllllllllllll}3.0-001 & 0.7019 & 0.7021 & 0.7023 & 0.7028 & 0.7032 & 0.7038 & 0.7059 & 0.7080 & 0.7123 & 0.7167 & 0.7233 & 0.7462 & 0.7707 & 0.8244 & 0.8827\end{array}$ $\begin{array}{lllllllllllllllllll}5.0 .001 & 0.5164 & 0.5166 & 0.5169 & 0.5174 & 0.5179 & 0.5186 & 0.5 ? 12 & 0.5237 & 0.5289 & 0.5342 & 0.5424 & 0.5714 & 0.6038 & 0.6804 & 0.7733\end{array}$ $\begin{array}{lllllllllllllllll}7.0 .001 & 0.3608 & 0.3611 & 0.3613 & 0.3618 & 0.362 ? & 0.3629 & 0.365 .3 & 0.3677 & 0.3725 & 0.3775 & 0.3852 & 0.4135 & 0.4462 & 0.5296 & 0.6434\end{array}$ $100000.20020 .00030 .20050 .20080 .20110 .20160 .20330 .20490 .20830 .21190 .21740 .23810 .2632 \quad 0.33290 .4444$ 1.030000 .20020 .20030 .20340 .03240 .03250 .03260 .03290 .03320 .03390 .03460 .03570 .04000 .04550 .06240 .0964

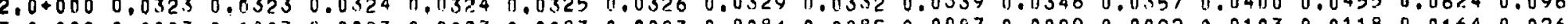

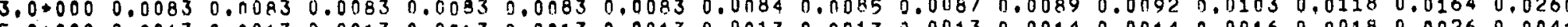

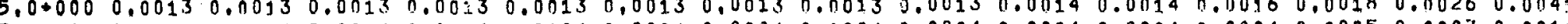
$7.0+000 \quad 0.0004 \quad 0.00040 .00040 .00140 .00040 .00040 .0004 \quad 0.00040 .00040 .00040 .00040 .00040 .00050 .00070 .0011$ $1.0+0010.00010 .00010 .00010 .00 .10 .00010 .00010 .00010 .00010 .00010 .00010 .00010 .00010 .00010 .00020 .0003$ $2.0+0010.00000 .00000 .00000 .00000 .00000 .00000 .00000 .00000 .00000 .00000 .00000 .00000 .00000 .0000$
\end{abstract} $3.0+0010.00000 .00000 .0000,0.00700 .000 n 0.00000 .000 n 0.00000 .00000 .00000 .00000 .00000 .0000$

$5.0+0010.00000 .00000 .00000 .00000 .00000,00000,00000.00000 .00000 .00000 .00000 .0000$

$7,0+0010.00000 .00000 .00000 .00000 .00000 .00000 .00000 .00000 .00000 .00000 .0000$

$1.0+0020.00000 .00000 .00000 .0000,00000.00000 .00000 .00000 .00000 .00000 .0000$

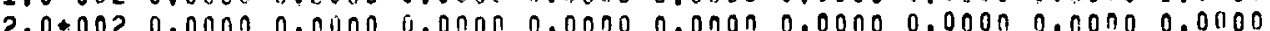

$3,0+0020.000110 .010000 .00000 .00000 .00000 .0000 \quad 0.00000 .0000$

$5.0+0020.00010 .0,000,00000.00000 .00000 .00000 .0000$

$7.0+0020.60000 .00000 .00000 .00000 .00000 .0000$

$1.0+003 \quad 0.0000 \quad 0.0000 \quad 0.00000 .0000 \quad 0.0000 \quad 0.0000$

ALPHA $1.000 \quad 2.000 \quad 3.000 \quad 5.000 \quad 7.000 \quad 10.00 \quad 20.00 \quad 3 n .00 \quad 50.00 \quad 70,00 \quad 100.0 \quad 200.0 \quad 300.0 \quad 500.0 \quad 700.0 \quad 1000$ BETA

$1.0=003 \quad 0.99901 .00001 .00001 .00001 .00001 .00001 .00001 .000001 .00001 .00001 .00001 .00001 .00001 .00001 .00001 .0000$ 2.0.003 0.099 म 1.00001 .00001 .00001 .00001 .00001 .00001 .00001 .00001 .00001 .00001 .00001 .00001 .00001 .00001 .0000

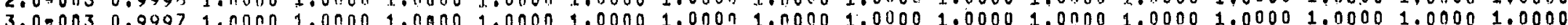

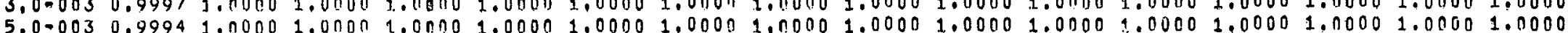

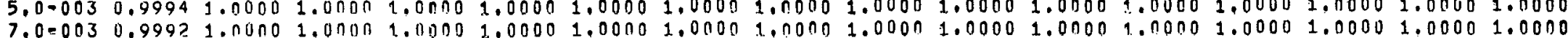
$1,0-0020.99891 .04001 .00001 .00001 .00001 .00001 .00001 .00001 .00001 .00001 .00001 .00001 .00001 .00001 .00001 .0000$ $2.0-0020.99771 .00001 .00001 .03001 .00001 .00001 .00001 .00001 .00001 .00001 .00001 .00001 .00001 .0000$

3.0 .0020 .99650 .99991 .00001 .03001 .00001 .00001 .00071 .00001 .00001 .00001 .00001 .00001 .0000

$5,0=0020,99420.99991 .00001 .00001 .00001 .00001 .00091 .00001 .00001 .00001 .00001 .0000$

$7,0.0020 .99170 .09991 .00001 .00001 .00001 .00001 .00001 .00001 .00001 .00001 .0000$

$1.0-0010.98780 .99981 .00001 .00001 .00001 .00001 .00001 .00001 .00001 .00001 .0000$

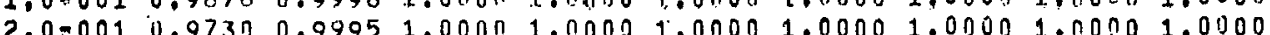

$3,0=0010.95490 .99921 .00001 .00001 .00001 .00001 .00001 .0000$

$5,0=0010,9057 \quad 0.94820 .99991 .00001 .00001 .00001 .0000$

$7,0=001 \quad 0.83540 .9965 \quad 0.9998 \quad 1.00001 .00001 .0000$

$1.0+000 \quad 0.69230 .9922 \quad 0.99961 .00001 .00001 .0000$

$2.0+000 \quad 0.2308 \quad 0.9445 \quad 0.99700 .5999$

$3,0+000 \quad 0.0694 \quad 0.9098 \quad 0.9880$

$5.0+000 \quad 0.0114 \quad 0.3958$

$7.0 .000 \quad 0.0032$

$1,0+0010,000 \mathrm{H}$ 
VALUES OF G FOR N $=8$ AND NP= 5 BETA

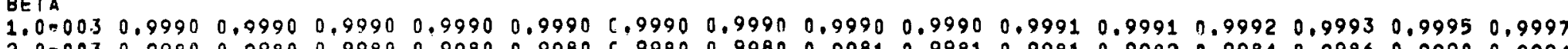

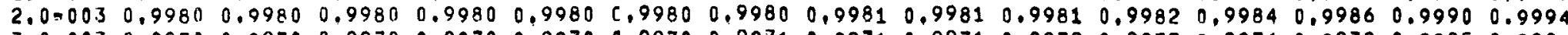
$\begin{array}{llllllllllllllll}3.0=003 & 0.9970 & 0.9970 & 0.9970 & 0.9970 & 0.9970 & C .9970 & 0.9971 & 0.9971 & 0.9971 & 0.9972 & 0.9973 & 0.9976 & 0.9979 & 0.9985 & 0.9991\end{array}$ $\begin{array}{llllllllllllllll}5.0 .003 & 0.9950 & 0.9950 & 0.9950 & 0.9950 & 0.9950 & 0.9950 & 0.9951 & 0.9951 & 0.9952 & 0.9953 & 0.9955 & 0.9960 & 0.9965 & 0.9975 & 0.9980\end{array}$ $\begin{array}{llllllllllllllll}7,0-003 & 0.9930 & 0.7930 & 0.9930 & 0.9930 & 0.9930 & 0.9931 & 0.9931 & 0.9932 & 0.9933 & 0.9935 & 0.9937 & 0.9944 & 0.9951 & 0.9965 & 0.9978\end{array}$

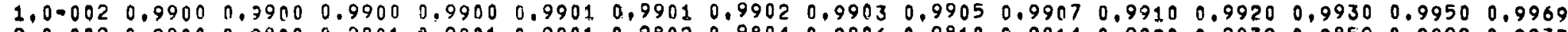
$\begin{array}{lllllllllllllllll}2,0.002 & 0.9800 & 0.9800 & 0.9801 & 0.9801 & 0.9801 & 0.9802 & 0.9804 & 0.9806 & 0.9810 & 0.9814 & 0.9820 & 0.9839 & 0.9859 & 0.9899 & 0.9937\end{array}$

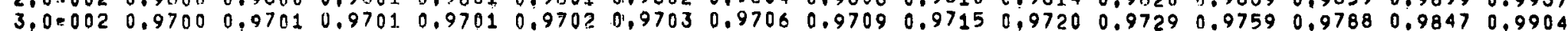
$5,0.0020 .9500 \quad 0.95010 .95010 .95020 .95030 .95050 .95100 .95140 .95240 .95330 .95480 .95960 .96450 .97430 .9838$

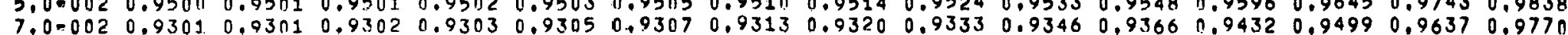

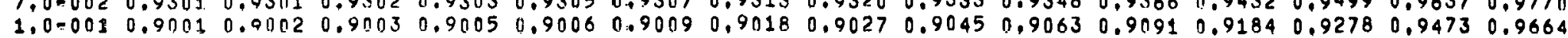

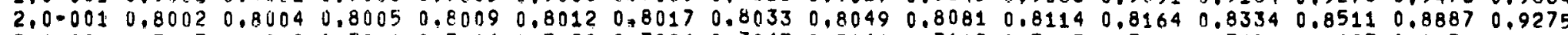

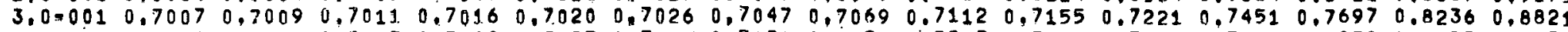
$\begin{array}{lllllllllllllllll}5.0 .001 & 0.5082 & 0.5084 & 0.5087 & 0.5092 & 0.5097 & 0.5104 & 0.5130 & 0.5155 & 0.5207 & 0.5261 & 0.5342 & 0.5634 & 0.5959 & 0.6733 & 0.7676\end{array}$ $\begin{array}{llllllllllllllll}7,0.001 & 0.3402 & 0.2405 & 0.3407 & 0.3411 & 0.3416 & 0.3423 & 0.3445 & 0.3469 & 0.3516 & 0.3565 & 0.3340 & 0,3917 & 0.4239 & 0.5070 & 0.6223\end{array}$

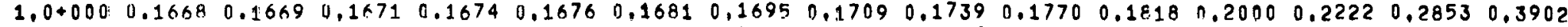
$2.0+000 \quad 0.01590 .01590 .01590 .0160 \quad 0.0160 \quad 0.0160 \quad 0,0162 \quad 0.01640 .0167 \quad 0.0170 \quad 0.0176 \quad 0.0198 \quad 0.02250 .0312 \quad 0.0491$

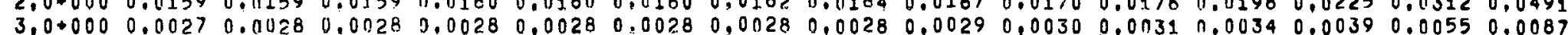

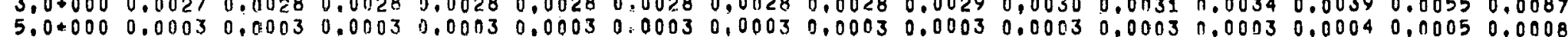

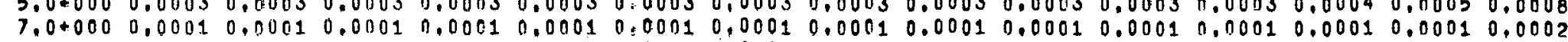
1.000010 .00000 .00000 .00000 .00000 .00000 .00000 .00000 .00000 .00000 .00000 .00000 .00000 .00000 .00000 .0000 $2.000010 .00000 .00000 .00000 .00000 .00000 .00000 .00000 .00000 .00000,00000.00000 .00000 .00000 .0000$

$3.0+0010.00000 .00000 .00000 .00000 .00000 .00000 .00000 .00000 .00000 .00000 .00000 .00000 .0000$

$5,0+0010.00000 .00000 .00000 .00000 .00000 .00000 .00000 .00000 .00000 .00000 .00000 .0000$

$7.0+0010.0000 \quad 0.0000 \quad 0.0000 \quad 0.00000 .0000 \quad 0.0000 \quad 0.0000 \quad 0.00000 .00000 .00000 .0000$

$1.0+0020.00000 .03000 .00000 .00000 .00000 .00000 .00000 .00000 .00000 .00000 .0000$

$2,0+0020,0000,0,0,00,0000,0000,0000,0.00000,00000.00000 .0000$

$3,0+0020,00000,0,000,0000,0,00000,00000,00000,00000,0000$

$5,0+002 \cdot 0,01000.00000 .0000 \quad 0,00000,0000 \quad 0,00000,0000$

$7,0+0020,00000.0000 \quad 0,0000 \quad 0,00000,0000 \quad 0,0000$

$1,0+0030,00000,00000,00000,00000,00000,0000$

ALPHA $1.0002 .000 \quad 3.0005 .0007 .000 \quad 10.00 \quad 20.00 \quad 30.00 \quad 50.00 \quad 70.00 \quad 100.0 \quad 200.0 \quad 300.0 \quad 500.0 \quad 700.0 \quad 1000$ BETA

$1.0=0030.99991 .00001 .00001 .00001 .00001 .30001 .00001 .00001 .00001 .00001 .00001 .00001 .00001 .00001 .00001 .0000$ $2.0=0030.99981 .06001 .00001 .00001 .00001 .30001 .00001 .00001 .0000 \div 00001.00001 .00001 .00001 .00601 .00001 .0000$ $3,0=0030.99971 .06001 .00001 .00001,00001,30001.00001 .00001 .00001,00001.00001 .00001 .00001 .00001 .00001 .0000$

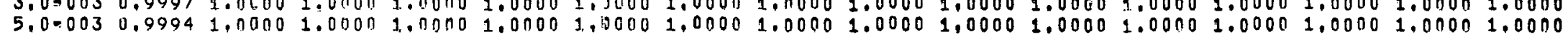

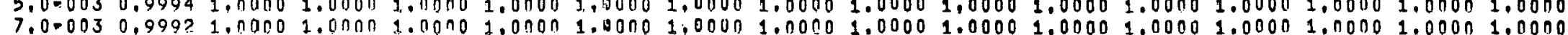

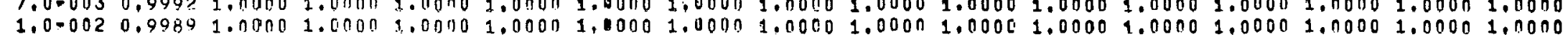

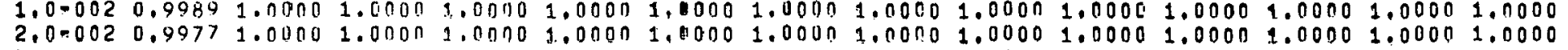
$3,0=0020.99660 .99991 .00001 .00001 .00001,00001.00001 .00001 .00001 .00001 .00001 .00001 .0000$

$5.0-0020.99420 .99991 .00001 .001001 .00001 .00001 .00001 .00001 .00001 .00001 .00001 .0000$

$7,0.0023 .99170 .99991 .00001 .00001,00001,00001.00001,00001.00001 .00001 .0000$

$1.0=001 \mathrm{j}, 9878 \quad 0.9998 \quad 1.00001 .00001 .00001 .00001 .00001 .00001 .00001 .00001 .0000$

$2,0=0010.97300 .99351 .00001 .00001 .00001 .00001 .00001 .00001 .0000$

$3,0.0010 .95470 .9972 \quad 1.00001 .001001,00001,00001,00001.0000$

$5,0.0010 .90280 .99310 .939 .91 .00001 .00001 .00001 .0000$

$7,0=0010.82260 .99620 .99981,00101,00001,0000$

$1,0+000 \quad 0.64290 .9903 \quad 0.9995 \quad 1.00301 .00001,00010$

$2,0+000 \quad[, 1268 \quad 0,89: 8 \quad 0.9037 \quad 0.9999$

$3,0+000 \quad 0.0242 \quad 0,5847 \quad 0.9544$

$5,0+000 \quad 0.0123 \quad 0.1157$

$7.0+000 \quad 0.0005$

$1,0+0010.0001$ 
VALUES OF G FOK $N=9$ AND $N P=1$ BETA

$B E T A$

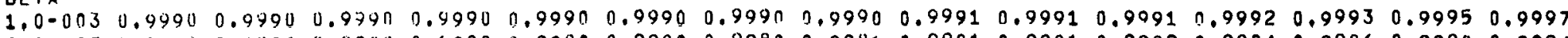

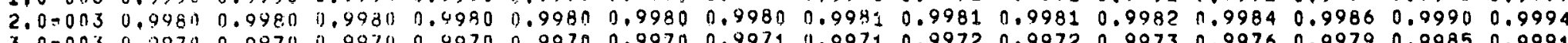

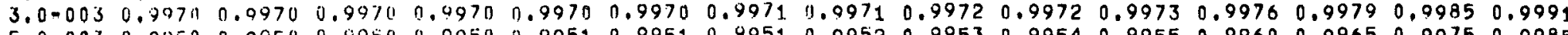

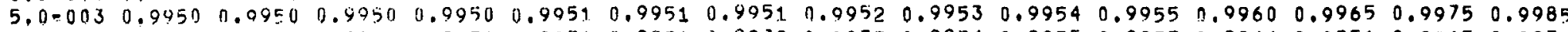
$\begin{array}{lllllllllllllllll}7.0-003 & 0.9931 & 0.9931 & 0.9031 & 0.9931 & 0.9931 & 0.9931 & 0.9932 & 0.9933 & 0.9934 & 0.9935 & 0.9937 & 0.9944 & 0.9951 & 0.9965 & 0.9978\end{array}$ $\begin{array}{llllllllllllllll}1.0-002 & 0.9901 & 0.9901 & u .9301 . & 0.9011 & 0.9902 & 0.9902 & 0.9903 & 0.9904 & 0.7906 & 0.9908 & 0.9911 & 0.9921 & 0.9930 & 0.9950 & 0.9969\end{array}$

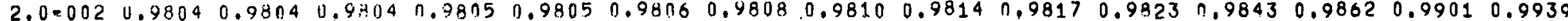

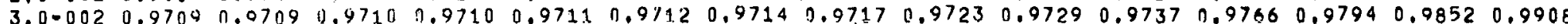
$3.0-002$ 4.9524 0.05250 .95250 .95260 .95270 .95280 .95330 .95370 .95470 .95560 .95690 .96150 .96620 .97560 .9848 (1)

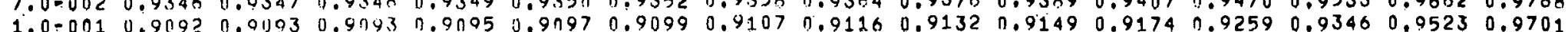

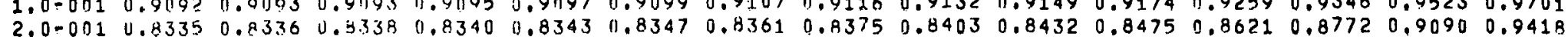

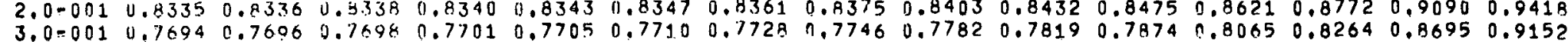
$\begin{array}{lllllllllllllllllll}5.0-001 & 0.6669 & 0.6671 & 0.6673 & 0.6678 & 0.6682 & 0.6689 & 0.6711 & 0.6734 & 0.6780 & 0.6826 & 0.6497 & 0.7143 & 0.7407 & 0.7998 & 0.8663\end{array}$ $\begin{array}{llllllllllllllllll}7.0-001 & 0.5885 & 0.5887 & 0.5890 & 0.5894 & 0.5899 & 0.5907 & 0.5931 & 0.5956 & 0.6006 & 0.6057 & 0.6135 & 0.6410 & 0.6711 & 0.7406 & 0.8223\end{array}$ $\begin{array}{lllllllllllllllll}1.0+000 & 0.5103 & 0.56 n 5 & 0.5 n 08 & 0.501 .3 & 0.5018 & 0.5025 & 0.5051 & 0.5076 & 0.5128 & 0.5181 & 0.5263 & 0.5556 & 0.5882 & 0.6664 & 0.7641\end{array}$

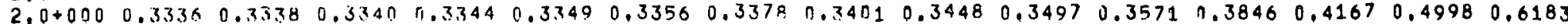

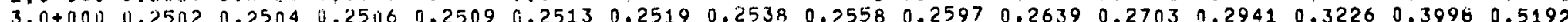
$3.0+0000.16680 .16690 .16710 .16740 .16760 .16810 .16950 .17090 .17390 .17700 .1818 .0 .20000 .22220 .28550 .3031$

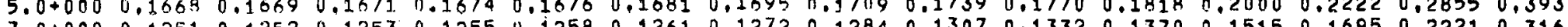

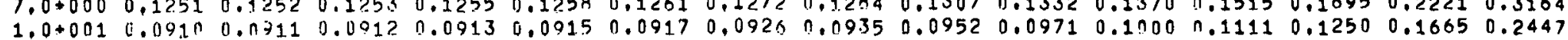

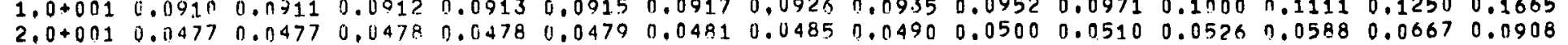

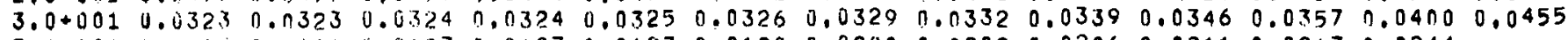
$\begin{array}{llllllllllll}5.0+001 & 0.0196 & 0.0196 & 0.0197 & 0.0197 & 0.0197 & 0.0198 & 0.0200 & 0.0202 & 0.0206 & 0.02110 .02170,0244\end{array}$

$\begin{array}{llllllllllllllll}7,0+001 & 0.0141 & 0.0141 & 0.0141 & 0.0142 & 0.0142 & 0.0142 & 0.0144 & 0.0145 & 0.0148 & 0.0151 & 0.0156\end{array}$

$1.0+002 \quad 1.01099 \quad 0.01) 990.019990 .01000 .01000 .0100 \quad 0.0101 \quad 0.0102 \quad 0.01040 .0106 \quad 0.0110$

$2.0+002 \quad 0.01150 \quad 0.0050 \quad 0.0050 \quad 11.0050 \quad 0.0050 \quad 0.0050 \quad 0.00510 .00510 .0052$

$3.0+0020.00330 .0 .0330 .00330 .00330 .00330 .00340 .00340 .0034$

$5.0+0020.00200 .00200 .00200 .00200 .00200 .00200 .0020$

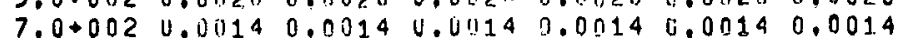

$1,0+0030.0010 \quad 0,0010$ i., $0010 \quad 0.0010 \quad 0.00100,0010$

ALPHA $1.000 \quad 2.000 \quad 3.000 \quad 5.000 \quad 7.000 \quad 10.00 \quad 20.00 \quad 30.00 \quad 50.00 \quad 70.00 \quad 100.0 \quad 200.0 \quad 300.0 \quad 500.0 \quad 700.0 \quad 1000$ BETA

$1,0.0030 .99991 .00001 .00001 .00001 .00001 .00001 .00001 .00001 .00001 .00001 .00001 .00001 .00001 .00001 .00001 .0000$ $2,0.0030 .99981 .00001 .00001 .00001 .00001 .00001 .00001 .00001 .00001 .00001 .00001 .00001 .00001 .00001 .00001 .0000$ $3,0-0030.99971 .00001 .00001 .00001 .00001 .00001 .00001 .00901 .00001 .00001 .00001 .00001 .00001 .00001 .00001 .0000$ 5.000030 .99951 .00001 .00001 .00001 .00001 .00001 .00001 .00001 .00001 .00001 .00001 .00001 .00001 .00001 .00001 .0000 $1.0 .003 .99931 .00001 .00001 .00001 .00001 .00001 .000 n 1.00001 .00001 .00001 .00001 .00001 .00001 .00001 .00001 .0000$

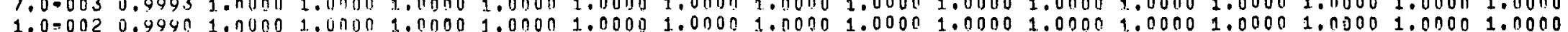

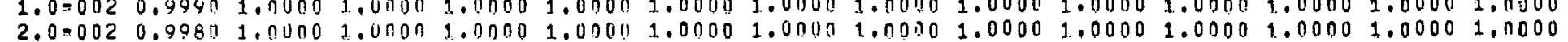

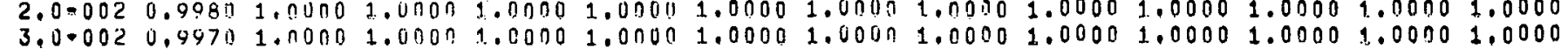
$5,0=0020.99501,00001.00001 .00001 .00001 .00001 .00001 .00001 .00001 .00001 .00001 .0000$ $7,0=0020.99300 .99991 .00001 .00001 .00001 .00001 .00001 .00001 .00001 .00001 .0000$ $1,0=0010.9909 .0 .99991 .00001 .00001 .00001 .00001 .00001 .00001 .00001 .00001 .0000$

$2,0.0010 .9804 \quad 0.9998 \quad 1.00001 .00001 .00001 .00001 .00001 .0000 .1 .0000$

3.0 .0010 .97090 .99971 .011001 .00001 .00001 .00001 .00001 .0000

$5.0=0010.95240 .99951,00001.00001 .00001 .00001 .0001$

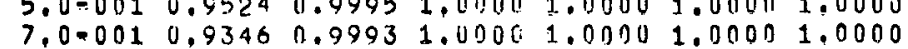

$1,0+0000,9091 \quad 0.99901 .00001 .00001 .00001 .0000$

$2,0+000 \quad 0.8333 \quad 0.9980 \quad 0.9999 \quad 1.0000$

$3.0+000 \quad 0.7692 \quad 0.9971 \quad 0.9999$

$5,0+000 \quad 0.0667 \quad 0.9951$

$7,0+000 \quad 0.5882$

$1,0+001 \quad 0,5000$ 
VALUES OF G FOK $N=9$ ANII $N \dot{r}=2$

BETA

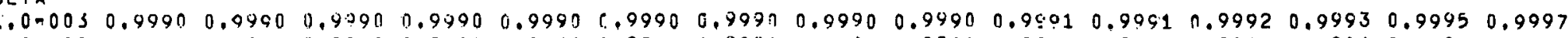

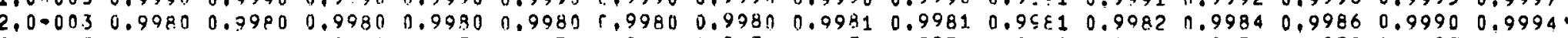
$\begin{array}{lllllllllllllllll}3.0-003 & 0.9970 & 0.7970 & 0.9970 & 0.9970 & 0.997 n & 0.9970 & 0.9971 & 0.9971 & 0.9971 & 0.9572 & 0.9973 & 0.9976 & 0.9979 & 0.9985 & 0.9991\end{array}$ $\begin{array}{llllllllllllllll}5.0 .003 & 0.9950 & 0.9950 & 0.9950 & 3.0950 & 0.9959 & 0.9950 & 0.9951 & 0.0951 & 0.9952 & 0.9953 & 0.9955 & 0.9960 & 0.9965 & 0.9975 & 0.9985\end{array}$ $\begin{array}{lllllllllllllllll}7.0 .003 & 0.9930 & 0.9930 & 0.4030 & 11.9730 & 11.9931 & 0.9931 & 0.9931 & 0.9932 & 0.9933 & 0.9935 & 0.9937 & 0.9944 & 0.9951 & 0.9965 & 0.9978\end{array}$

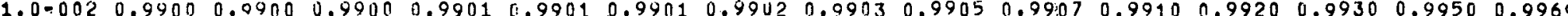
$2.0-002009800009000.95010 .9801099010 .98020 .98340 .98060 .98100 .98140 .98200 .98390 .98590 .08990 .9937$

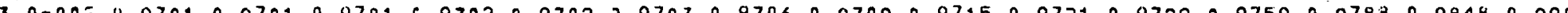

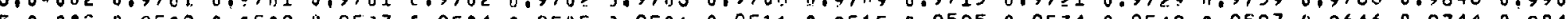

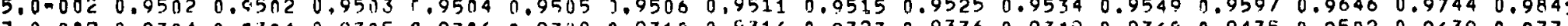
$7.0=0020.93040 .93040 .93050 .93060 .93080 .93100 .9315$ n.9323 0.93360 .93490 .93680 .94350 .95020 .96390 .9774

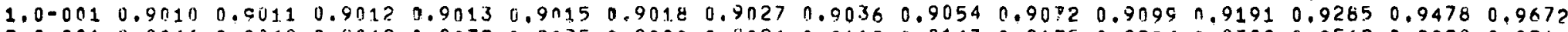
$\begin{array}{llllllllllllllllll}2.0 .001 & 0.8066 & 0.8068 & 0.8069 & 0.8072 & 0.8075 & 0.8080 & 0.8095 & 0.8112 & 0.8143 & 0.8175 & 0.8224 & 0.8389 & 0.8562 & 0.8928 & 0.9310\end{array}$ $\begin{array}{lllllllllllllllll}3.000 & 0.7196 & 0.7198 & 0.7200 & 0.7204 & 0.7208 & 0.7214 & 0.7235 & 0.7255 & 0.7297 & 0.7338 & 0.7402 & 0.7622 & 0.7855 & 0.8367 & 0.8925\end{array}$ $\begin{array}{lllllllllllllllll}5.0001 & 0.5717 & 0.5719 & 0.5722 & 0.5727 & 0.5731 & 0.5739 & 0.5764 & 0.5799 & 0.5839 & 0.5891 & 0.5970 & 0.6250 & 0.6557 & 0.7271 & 0.8120\end{array}$ $\begin{array}{llllllllllllllll}7.0=001 & 0.4569 & 0.4571 & 0.4574 & 0.4579 & 0.4584 & 0.4591 & 0.4616 & 0.4642 & 0.4694 & 0.4747 & 0.4829 & 0.5123 & 0.5456 & 0.6267 & 0.7313\end{array}$ $1.0+000 \quad 0.33360 .73380 .3340 \quad 0.33440 .3340 \quad 0.33560 .33780 .3401034480 .34570 .35710 .38460 .41670 .49980 .6183$ $2.0+0000.14300 .14310 .14320 .14350 .14370 .14410 .14530 .14660 .14930 .15200 .15620 .17240 .19230 .24980 .3506$ $30+0000.07700 .07710 .07790 .07730 .07740 .07760 .07840 .07910 .08060 .08220 .08470 .09430 .10640 .14270 .2126$ ..0.000 0.0770 0.0771 0.0771 0.0773 0.07740 .07760 .07840 .07910 .08060 .08220 .03470 .09430 .10640 .14270 .2126 $5.0+0000.03230 .03230 .03240 .03240 .03250 .03260 .03290 .03320 .03390 .03460 .0357 n .04 n 00.04550 .06240 .0975$ $\begin{array}{llllllllllllllll}7.0+000 & 0.0176 & 0.0176 & 0.0176 & 0.0176 & 0.0177 & 0.0177 & 0.0179 & 0.0181 & 0.0185 & 0.0185 & 0.01,95 & 0.0218 & 0.0249 & 0.0345 & 0.0547\end{array}$ $1.0+0010.0090 \quad 0.0[90 \quad 0.0090 \quad 0.0091 \quad 0.00910 .00910 .3092 \quad 0.0093 \quad 0.0095 \quad 0.0097 \quad 0.0100 \quad 0.011 .20 .0128 \quad 0.0178 \quad 0.0286$

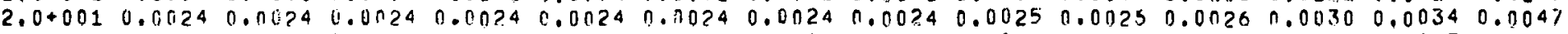

$3.0+0010.00110 .01110 .0011 .0 .00110 .00110 .30110 .00110 .00110 .00110 .00120 .00120 .00130 .0015$

$5.0+0010.00040 .00040 .00040 .00040 .00040 .00040 .00040 .00040 .00040 .00040 .09040 .0005$

$7.0+001 \quad 0.0002 \quad 0.0002 \quad 0.00020 .00020 .00020 .00020 .00020 .00020 .00020 .00020 .0002$

$1.0+002 \quad 0.0001 \quad 0.0010 .00010 .0001 \quad 0.0001 \quad 0.00010 .60010 .00010 .00010 .000=0.0001$

$2,0+002$ j. 0000 0.00no

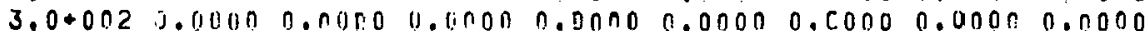

$5.0+002 \quad 1.011000 .00700 .010000 .3000 \quad 1.00000,00000.0000$

$7,0+0020.0000 \quad 0.00 .300 .0 .000 \quad 0.0000 \quad 0.0000 \quad 0.0000$

$1.0+0030.00000 .00000 .00000 .00000 .0000 \quad 0,0000$

ALPHA $1.0002 .000 \quad 3.000 \quad 5.000 \quad 7.000 \quad 20.00 \quad 20.00 \quad 30.00 \quad 50.00 \quad 70.00 \quad 100.0 \quad 200.0 \quad 300.0 \quad 500.0 \quad 700.0 \quad 1000$ BETA

1.070030 .99991 .000 .01 .011001 .00001 .00001 .03001 .00001 .00001 .00001 .00001 .00001 .00001 .00001 .00001 .00001 .0000

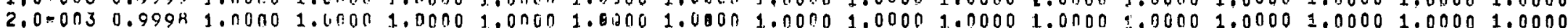

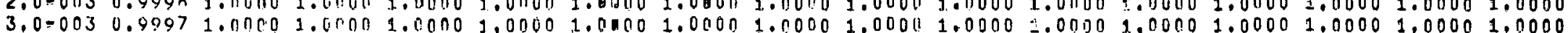

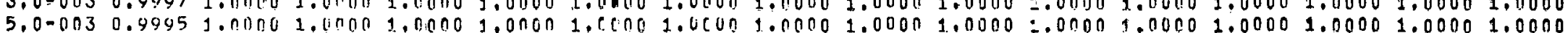

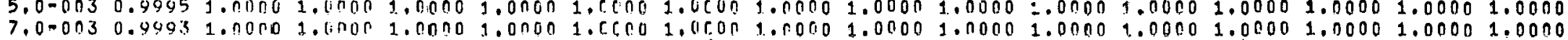
$1.0-0020.99901 .011001 .010001 .00 n 01.00001 .000 \pi 1.00001 .00001 .00001 .00001 .00001 .00001 .00001 .00001 .00001 .0000$ $2.0-0020.99401 .00031 .00001 .07001 .00001 .00001 .00001 .00001 .00001 .00001 .00001 .00001 .00001 .0000$

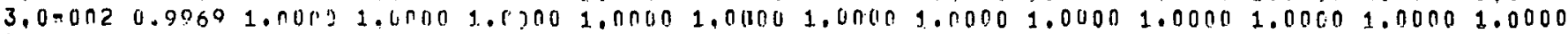

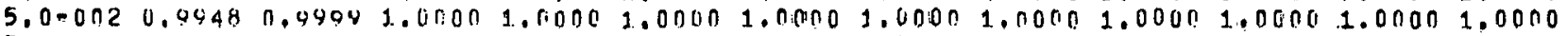

$7,0=0020.99260 .99091 .00001 .00009,00001.00001,00001.00001 .00001,00001.0000$

$1.0-0010.08910 .09901 .601001 .00001 .00001 .00001 .00001 .00001 .00001 .00001 .0000$

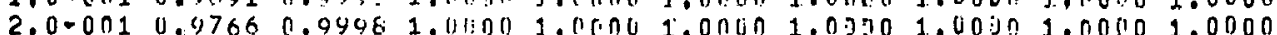

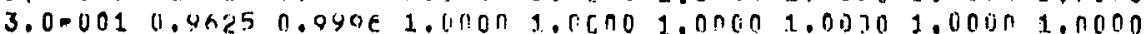

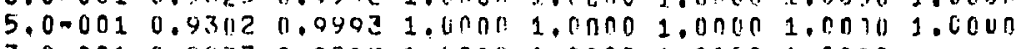

$7,0=0010.89370 .99841 .60001 .00001 .00001 .0000$

$1,0+000 \quad 0.03330 .0980$ b. 409991.00009 .00001 .0000

$2,0+000 \quad 0.52500 .0942 \quad 0.46541 .0000$

$3.0+000 \quad 0.45450 .9884 \quad 0.4096$

$5,0+000 \quad 0.250110 .0715$

$7,0+000 \quad 0.1515$

$1.0+0010.0833$ 


\begin{abstract}
VALUES OF G FOR $N=9$ LINR NP= 3 BETA

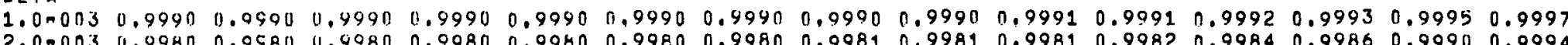

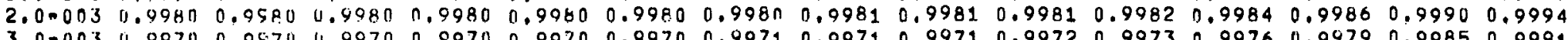
$\begin{array}{llllllllllllllll}3.0-003 & 0.99711 & 0.0570 & 1.9070 & 0.9970 & 0.9970 & 0.9970 & 0.9971 & 0.9971 & 0.9971 & 0.9972 & 0.9973 & 0.9976 & 0.9979 & 0.9985 & 0.9991 .9\end{array}$

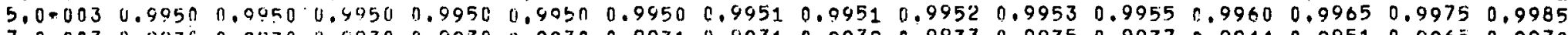

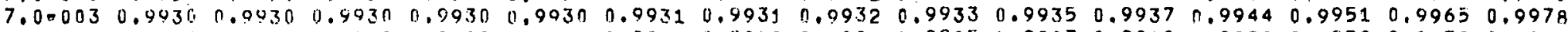
1.0-002 $4.99000 .0900 \quad 0.9900 \quad 0.9900 \quad 0.9061 \quad 0.99010 .99420 .99030 .99050 .99070 .9910 \quad 0.99200 .9930 \quad 0.9950 \quad 0.9969$

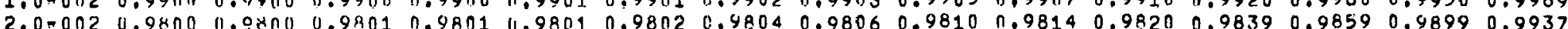
2.070020 .9400
$3.0-002$

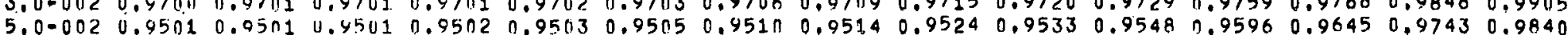

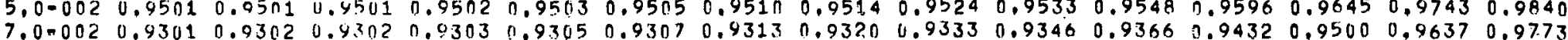
$\begin{array}{lllllllllllllll}1.00001 & 0.9002 & 0.9003 & 0.9004 & 0.9005 & 10.9007 & 0.9010 & 0.9019 & 0.0028 & 0.9046 & 0.9064 & 0.9092 \quad 0.9194 & 0.9279 & 0.9474 & 0.9669\end{array}$

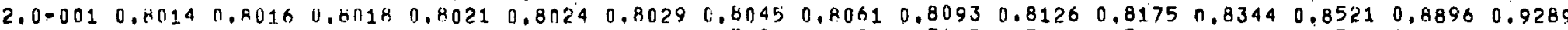
$\begin{array}{lllllllllllllllllll}3,0=001 & 0.7059 & 0.7061 & 0.7063 & 0.7068 & 0.7072 & 0.7078 & 0.7099 & 0.7120 & 0.7163 & 0.7206 & 0.7271 & 0.7499 & 0.7741 & 0.8273 & 0.8859\end{array}$ $\begin{array}{llllllllllllllllll}5.0-001 & 0.5336 & 0.5338 & 0.5341 & 0.5346 & 0.5351 & 0.5358 & 0.5384 & 0.5409 & 0.5461 & 0.5513 & 0.5594 & 0.5882 & 0.6202 & 0.0954 & 0.7873\end{array}$

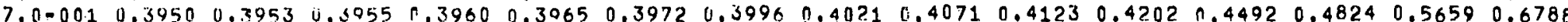
$10+0000.25620 .5040 .25060 .25090 .25130 .25190 .25380 .25580 .25970 .26390 .27030 .29410 .32260 .39980 .5192$

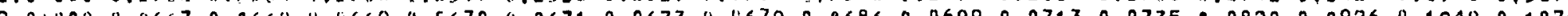
$2.0+0000.06670 . n 648$ 0.

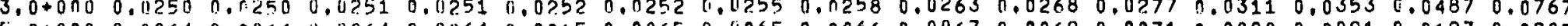
$5.0+000 \quad 0.00640 .006410 .010640 .00640 .00650 .00650 .00650 .00660 .00670 .00690 .00710 .0080 \quad 0.00910 .01270 .0205$ $7.0+000 \quad 0.01125 \quad 0.0025 \quad 11.0025 \quad 0.00250 .0025 \quad 0.0025 \quad 1.0026 \quad 0.0026 \quad 0.00260 .0027 \quad 0.00280 .0031 \quad 0.0036 \quad 0.0050 \quad 0.0081$ $1.0+0010.00090 .00 n 90.0 n 09$ 9.0nng 0.00090 .00090 .00090 .00090 .00090 .00100 .00100 .00110 .00130 .00180 .0029

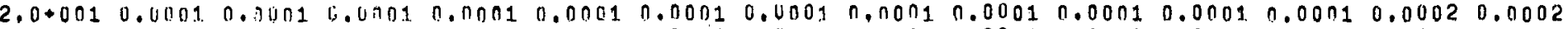

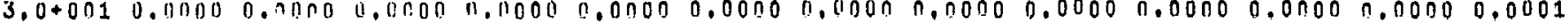

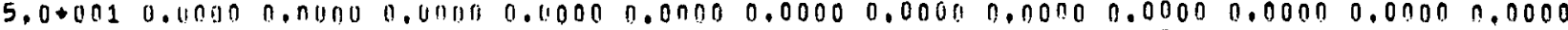

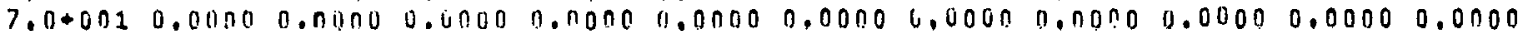

$1,0+0020.00000 .0000 .0000 .00000 .00000 .00000 .00000 .00000 .00000 .00000 .0000$

$1.0+002$.

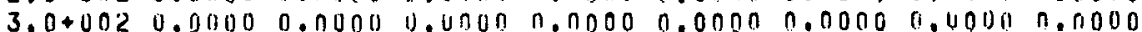

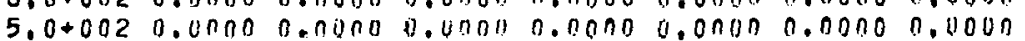

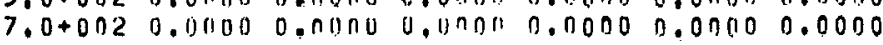

$1.0+0030.00000 .00000 .019000,00000.00000 .0000$

ALPH BETA

$1.0=0030.99991 .00001 .00$ în $1.00001 .000 n 1.00 n 01.001301 .00001 .00001 .00001 .00001 .00001 .00001 .00001 .00001 .0000$

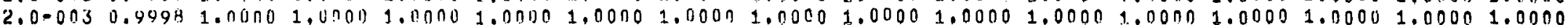
$3.0 .0030 .89971 .00001 .40001,0.0001 .00001 .00001 .00001 .00001 .00001,00001.00001 .00001 .00001 .00001 .00001 .0000$ $5,0=0030.49951 .0,9001.400 n 1.00001 .00001 .00001 .00001 .001001 .00001 .00001 .00091 .00001 .00001 .00001 .00001 .0000$

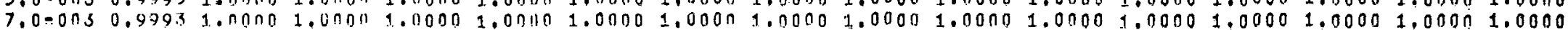

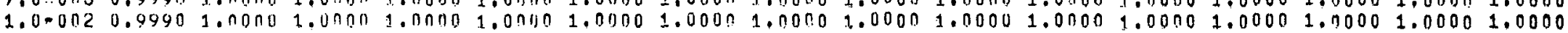

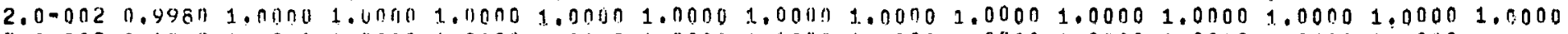
$3,0-0020.99691 .00001 .00001 .00011 .00001 .00001 .00001 .00201 .00001 .00001 .00001 .00001 .0000$

$5.0-002 \quad 0.9448 \quad 0,94091.00001 .00001 .00001 .00001 .00001 .00001 .00001 .00001 .00001 .0000$

$7.0=0020.9925 \Gamma .09991 .00001 .00001 .00001 .00001 .00001 .00001 .00001 .00001 .0000$

$1.000010 .9990 \quad 0.09091 .00001 .00001 .00001 .00001 .00001 .00001 .00001 .00001 .0000$

$2.0 .0010 .47580 .99081 .0110 n 1.00001 .0010 n 1.00001 .400 n 1.00001 .0000$

$3.0=0010.96000 .09061 .4 n n \pi 1.00001 .00001 .00001$. 1000n 1.0000

$5.0-0010.9195 \quad 0.0991 \quad 1.0001) \quad 1.110001 .00001 .00001 .0000$

$7.0=0010.8671 \quad 0.99850 .90091 .000111 .00001 .0000$

$1,0+000 \quad 0.7692 \quad 0.99710 .99991 .00001 .00001 .0000$

$2,0+000 \quad 0,4167 \quad 0.9865 \quad 0.9995 \quad 1.0000$

$3,0+000 \quad 0,2041 \quad 0.9633 \quad 0.9987$

$5,0+000 \quad 0.01606 \quad 0.8684$

$7,0+000 \quad 0,1244$

$1,0+0010.01189$ 
VALUES OF G FOF NV=9 AIN NFE 4 BETA

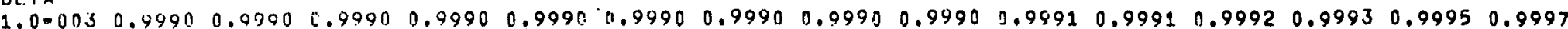

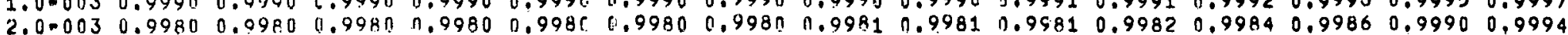
$\begin{array}{llllllllllllllll}3.0-003 & 0.9970 & 0.9970 & 0.9970 & 0.9970 & 0.9970 & 0.9970 & 0.9971 & 0.9971 & 0.9971 & 0.9972 & 0.9973 & 0.9976 & 0.9979 & 0.9985 .0,9991\end{array}$ $\begin{array}{llllllllllllllll}5.0 .003 & 0.9950 & 0.9950 & 0.99511 & 0.9950 & 0.9950 & C .9950 & 0.9951 & 0.9951 & 0.9952 & 0.9953 & 0.9955 & 0.9960 & 0.9965 & 0.9975 & 0.9985\end{array}$ $\begin{array}{llllllllllllllllll}7.0-003 & 0.9930 & 0.0930 & 0.9930 & 3.0930 & 0.9930 & C .9931 & 0.9931 & 0.9932 & 0.9933 & 0.9935 & 0.9937 & 0.9944 & 0.9951 & 0.9965 & 0.9978\end{array}$

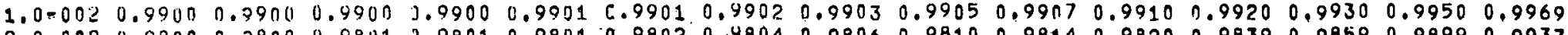

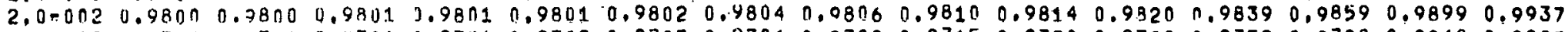
$\begin{array}{llllllllllllllllll}3,0-002 & 0.9700 & 0.7701 & 0.9701 & 9.9701 & 0.9702 & 0.9703 & 0.9706 & 0.9709 & 0.9715 & 0.9720 & 0.9729 & 0.9759 & 0.9788 & 0.9848 & 0.9905\end{array}$ $50-0020.95000 .05010 .9501$ i 95020.95030 .95050 .95100 .95140 .95240 .95330 .95480 .95960 .96450 .97430 .9840

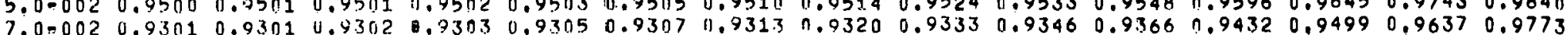

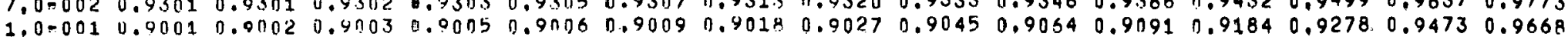

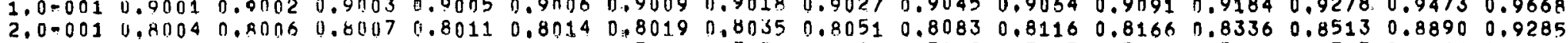
$\begin{array}{lllllllllllllllllll}3.0=001 & 0.7119 & 0.7021 & 0,7023 & 0.7028 & 0.7032 & 0.7038 & 0.7059 & 0.7080 & 0.7123 & 0.7167 & 0.7233 & 0.7462 & 0.7707 & 0.8246 & 0.8840\end{array}$ $\begin{array}{lllllllllllllllll}5.0 .001 & 0.5164 & 0.5166 & 0.5169 & 0.5174 & 0.5179 & 0.5186 & 0.5212 & 0.5237 & 0.5289 & 0.5342 & 0.5424 & 0.5714 & 0.6038 & 0.6806 & 0.7755\end{array}$ $\begin{array}{llllllllllllllll}7.0 .001 & 0.3608 & 0.7611 & 0.3613 & 0.3618 & 0.3622 & 0.3629 & 0.3653 & 0.3677 & 0.3725 & 0.3775 & 0.3852 & 0.4135 & 0.4462 & 0.5298 & 0.6462\end{array}$

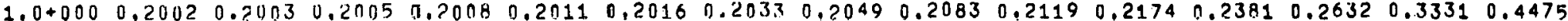
$0.0+0000.03230 .03230 .03240 .03240 .03250 .03260 .03290 .03320 .03390 .03460 .03570 .04000 .04550 .06240 .0975$

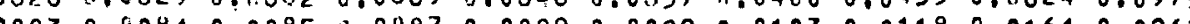

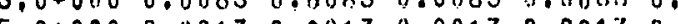
$5,0+0000.00130 .010 .0013$.0013 0.0013 . $7.0+000 \quad 0.00040 .00040 .00040 .00040 .0004 \quad 0.00040 .00040 .00040 .00040 .00040 .00040 .00040 .00050 .00070 .0012$

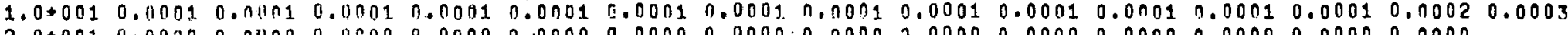
$2,0+0010.00000 .01000 .00000 .00000 .00000 .00000 .0000 .0 .00000 .00000 .00000 .00009 .00000 .00000 .0000$ $3,0+0010.00000 .010000 .00000 .00000 .00000 .00000 .00000 .00000 .00000 .00000 .00000 .00000 .0000$

$5.0+0010.00000 .03000 .00000 .00000 .00000,00000.00000 .00000 .00000 .00000 .00000 .0000$

$7,0+0010.00000,00000.00000 .00000 .00000 .00000 .00000 .00000 .00000 .00000 .0000$

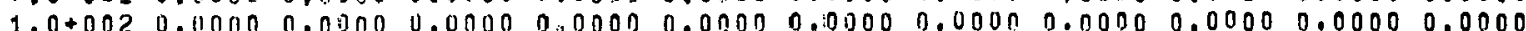

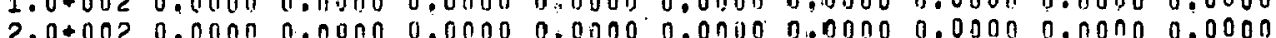

$3,0+0020.00000,0000,0,0000,00000.0000 \quad 0.0000 \quad 0,00000.0000$

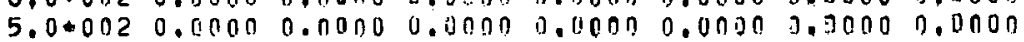

$7.00020 .00000 .00000 .00000 .00000 .0000 \quad 0,0000$

$1.0+0030.00000 .00000 .0000 \quad 0.00000 .00000,0000$ $B E T A$

ALPHA $2.000 \quad 2.000 \quad 3.000 \quad 5.000 \quad 7.000 \quad 30.00 \quad 20.00 \quad 30.00 \quad 50.00 \quad 70.00 \quad 100.0 \quad 200.0 \quad 300.0 \quad 500.0 \quad 700.0 \quad 1000$

1.000030 .99991 .00001 .00001 .00001 .00001 .00001 .00001 .00001 .00001 .00001 .00001 .00001 .00001 .00001 .00001 .0000 $2.0=0030.99981 .00001 .00001 .00001 .00001 .00001 .00001 .00001 .00001 .00001 .00001 .00001 .00001 .00001 .00001 .0000$ 3.0 .0030 .99971 .00001 .00001 .00001 .00001 .00001 .40001 .00001 .00001 .00001 .00001 .00001 .00001 .00001 .00001 .0000 $5,0=003,99951.01) 001.00001 .00001 .00001,0.0001 .00001 .00001 .00001 .00001 .00001 .00001 .00001 .00001 .00001 .0000$

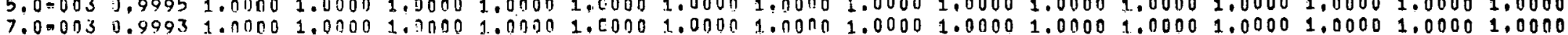

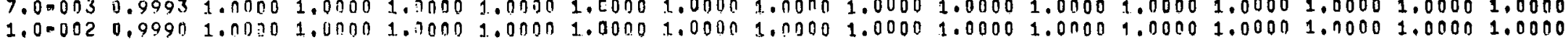
$2.0=0020.99801 .01901 .00001 .70001 .00001 .00001 .00001 .00001 .00001 .00001 .00001 .00001 .00001 .0000$ $3.0-0020.99691 .00001 .00001 .00001 .00001 .00001 .00001 .00001 .00001 .00001 .00001 .00001 .0000$

$5.0=0020.99480 .99091 .00001 .00001 .00001 .00001 .00001 .00001 .00001 .00001 .00001 .0000$

$7,0=002 \quad 0.9925 \quad 0.99091 .0300 \quad 1.00001 .00001 .00001 .00009 .00001 .00001 .00001 .0000$

$1.0=0010.9890 .0 .09091 .00001 .00001 .00001 .39001 .00001 .00001 .00001 .00001 .0000$

$2.0=0010.9 \% 56 \quad 0.0998 \quad 1.00001 .00001 .00001 .33001 .03001 .00001 .0000$

$3.0-0010.05920 .09061 .04001 .0001900001 .03001 .00001 .0000$

$5,0-0010.91430 .9951 \quad 1,000.01 .00001,00001.03001 .0000$

$7,0=0010.84940 .9983 \quad 0.99991 .0000 \quad 1.00001 .0000$

$1.0+000 \quad 0.71430 .99610 .94991 .00001 .00001 .0100$

$2.0+000 \quad 0.2500 \quad 0.9715 \quad 0.4990 \quad 1.0000$

$3.0+000 \quad 0.07690 .6950 \quad 0.9960$

$5.0+000 \quad 0.00127 \quad 0.5674$

$7.0+000 \quad 0.0036$

$1.0 \div 0010.0009$ 


\begin{abstract}
VALUES DF G FOK $N=9$ ANII $N H=5$ BETA

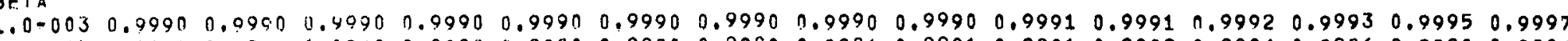

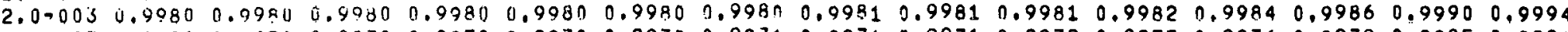
$\begin{array}{llllllllllllllll}3.0003 & 0.9970 & 0.0970 & 0.9970 & 0.9970 & 0.9970 & 0.9970 & 0.9971 & 0.9971 & 0.9971 & 0.9972 & 0.9973 & 0.9976 & 0.9979 & 0.9985 & 0.9991\end{array}$

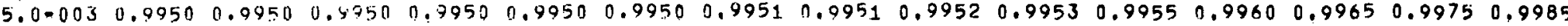
$\begin{array}{llllllllllllllll}7,0-003 & 0.9930 & 0.9930 & 0.9930 & 0.9930 & 0.9930 & 0.9931 & 0.9931 & 0.9932 & 0.9933 & 0.9935 & 0.9037 & 0.9944 & 0.9951 & 0.9965 & 0.9978\end{array}$

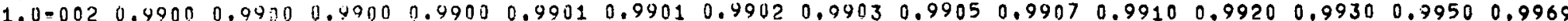

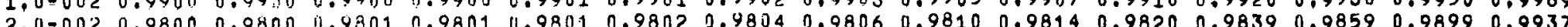
. $0=0020.97000 .97010 .97010 .97010 .97020 .97030 .97060 .97090 .97150097200 .97290 .97590 .97880 .98460 .9905$

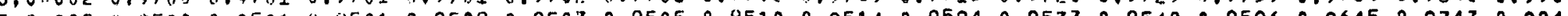

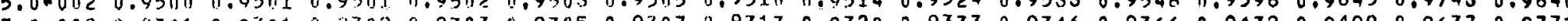

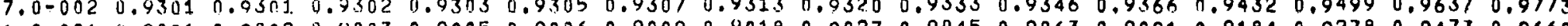

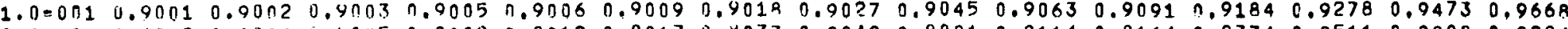

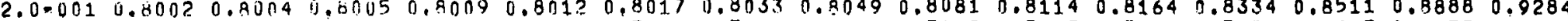

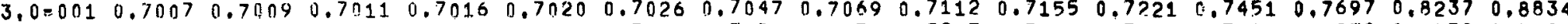
$\begin{array}{lllllllllllllllll}5.0-001 & 0.508 ? .0 .50 R 4 & 0.5087 & 0.5092 & 0.5 n 97 & 0.5104 & 0.5130 & 0.5155 & 0.5207 & 0.5261 & 0.5342 & 0.5634 & 0.5959 & 0.6735 & 0.7698\end{array}$

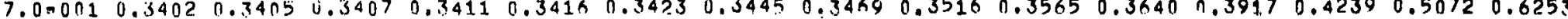

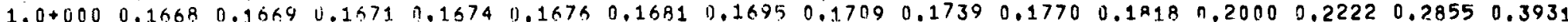
$1.0+0000.01590 .01590 .01590 .01600 .01600 .01600 .01670 .01640 .01670 .01700 .01760 .01980 .02250 .03120 .0497$

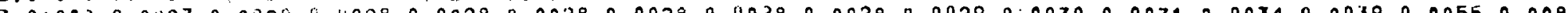

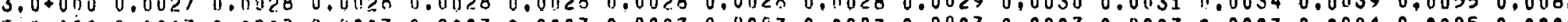

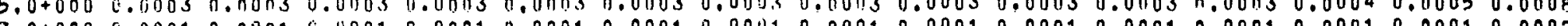

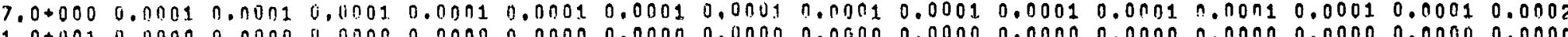

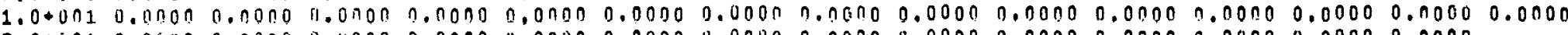
$2.0+0010.000 n 0.00000 .000 \pi 0.000 n 0.00000 .0000 \quad 3.00000 .00000 .00000 .00000 .00000 .00000 .00000 .0000$ $3.0+0010.00000 .0000$ u. $01000,00000.03000 .00000 .00000 .00000 .00000 .00000 .00000 .00000 .0000$
\end{abstract}

$5.0+0010.00000 .0000 \quad 0.00000 .00000 .00000 .00000 .00000 .00000 .00000 .00000 .00000 .0000$

$7.0+0010.00000 .00000 .00000 .00000 .00000 .00000 .00000 .00000 .00000 .00000 .0000$

1.00020 .0000 .0000 .00000 .00000 .00000 .00000 .00000 .00000 .00000 .00000 .0000

$1.0+002$ (1)

$3.0+002$ 0.000 n.000i

$5.0+0020.00000 ., 0000,0000,0.00000,00000,00000.0000$

$7.0+0020.0000 \quad 0.0000 \quad 0.00000 .0000 \quad 0.0000 \quad 0.0000$

$1,0+0030.0000 \quad 0.0000 \quad 0.00000 .0000 \quad 0,00000.0000$

ALPHA $1.000 \quad 2.000 \quad 3.000 \quad 5.000 \quad 7.000 \quad 10.00 \quad 20.00 \quad 30.00 \quad 50.00 \quad 70.00 \quad 100.0 \quad 200.0 \quad 300.0 \quad 500.0 \quad 700.0 \quad 1000$ BETA

$1.0=0030.99991 .000101 .00001 .00001 .00001 .00001 .00001 .00001 .00001 .00001 .00001 .00001 .00001 .00001 .00001 .0000$

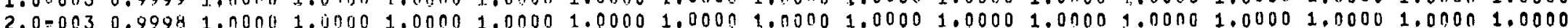

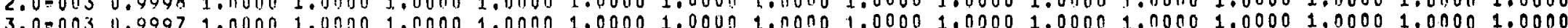

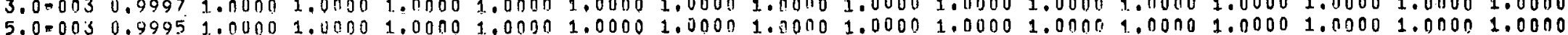

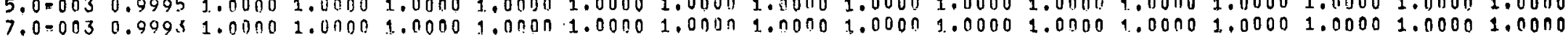
1.090020 .99901 .00001 .00001 .00001 .00001 .00001 .00001 .0000900001 .00001 .00001 .00001 .00001 .00001 .00001 .0000 $2.0=0020.90801 .00001 .00001 .00001 .00001 .00001 .00001 .00001 .00001 .00001 .00001 .00001 .00001 .0000$ $3.0-0020.99691 .00001 .00001 .00001 .00001 .00001 .00001 .00001 .00001 .00001 .00001 .000 B 1.0000$

$5,0=002 \quad 0.99480 .09991 .00001 .00001 .00001 .00001 .00001 .00001 .00001 .00001 .00009 .0000$

$7.0 .002 \quad 0.99250 .9999 \quad 1.00001 .00001 .00001 .00001 .00001 .00001 .00001 .00001 .0000$

$1.0=0010.98900 .99991 .00001 .00001 .00001 .00001 .00001 .00001 .00001 .00001 .0000$

1.0.00: 0 9756 0.0908 1. innn 1.0000 $1.00001 .00001 .000 n 1.00001 .0000$

$3.0-0010.95900 .99001 .40001 .00001 .00001 .00001$, unun 1.0000

5.070010 .91170 .99911 .00001 .00001 .00001 .00001 .0000

$7.0=0010.3374 \quad 0.9981 \quad 0.9999 \quad 1.00001 .00001 .0000$

$1.0+000 \quad 0.6667 \quad 0.9951 \quad 0.9998 \quad 1.00001 .00001 .0000$

$2.0+000 \quad 0.13890 .94290 .9979 \quad 1.0010$

$3,0+000 \quad 0,0268 \quad 0.7381 \quad 0,9879$

$5.0+000 \quad 0.0026 \quad 0.2076$

$7.0+000$ 0.0005

$1.0+0010.0001$ 

BETA

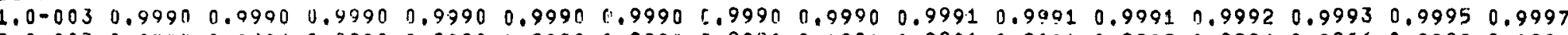

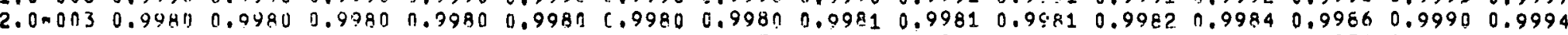
$\begin{array}{llllllllllllllll}3.0=013 & 0.997 n & 0.0970 & 0.9970 & 0.9970 & 0.9970 & 0.9970 & 0.9971 & 0.9971 & 0.9972 & 0.9572 & 0.9973 & 0.9976 & 0.9979 & 0.9985 & 0.9991\end{array}$ $\begin{array}{llllllllllllllll}5.0-003 & 0.9950 & 0.9950 & 0.9950 & 3.9950 & 0.9951 & 0.9951 & 0.9951 & 0.0952 & 0.9953 & 0.9954 & 0.9955 & 0.9960 & 0.9965 & 0.9975 & 0.9985\end{array}$ $\begin{array}{llllllllllllllll}7.0-003 & 0.4931 & 0.9931 & 0.4931 & 3.9031 & 0.9931 & 0.9931 & 0.993 ? & 0.9933 & 0.9934 & 0.9935 & 0.9937 & 0.9944 & 0.9951 & 0.9965 & 0.9979\end{array}$ $\begin{array}{llllllllllllllll}1.0 .002 & 0.9901 & 0.7901 & \text { iJ.9001 } & 0.9901 & 0.9902 & 0.9902 & 0.9903 & 0.9904 & 0.9906 & 0.990 .8 & 0.9911 & 0.9921 & 0.9930 & 0.9950 & 0.9969\end{array}$ $2,0=0020098040.08040 .98040 .98050 .98050 .98060 .98090 .09100098140 .98170 .98230 .98430 .98620 .99010 .9939$ $3.0=0020.97090 .97040 .97100 .97100 .97110 .97120 .97140 .97170 .97230 .97290 .97370 .97660 .97940 .98520 .9909$

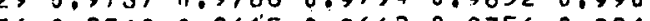

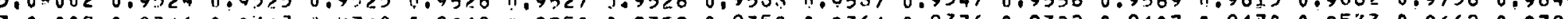

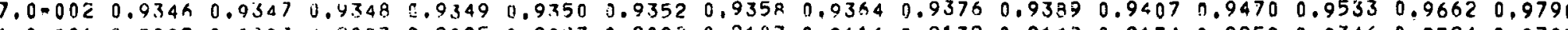

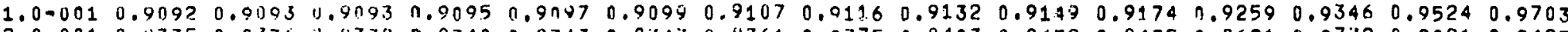
$\begin{array}{llllllllllllllllll}2.00001 & 0.8335 & 0.4336 & 0.8338 & 0.8340 & 0.8343 & 0.834 \% & 0.8361 & 0.8375 & 0.8403 & 0.8432 & 0.8475 & 0.8621 & 0.8772 & 0.9091 & 0.9423\end{array}$ $\begin{array}{llllllllllllllllll}3.0 .001 & 0.7694 & 0.7696 & 1.7498 & 0.7701 & 0.7705 & 0.7710 & 0.7729 & 0.7746 & 0.7782 & 0.78: 9 & 0.7874 & 0.8065 & 0.8264 & 0.8695 & 0.9159\end{array}$ $\begin{array}{lllllllllllllllll}5.0-001 & 0.6669 & 0.6671 & 0.6473 & 0.6678 & 0.6682 & 0.6689 & 0.6711 & 0.6734 & 0.6780 & 0.6826 & 0.6897 & 0.7143 & 0.7407 & 0.7999 & 0.8673\end{array}$ $\begin{array}{llllllllllllllllll}7,00001 & 0.5885 & 0.5887 & 0.5890 & 0.5894 & 0.5299 & 0.5907 & 0.5931 & 0.5956 & 0.6006 & 0.6057 & 0.6135 & 0.6410 & 0.6711 & 0.7406 & 0.8236\end{array}$

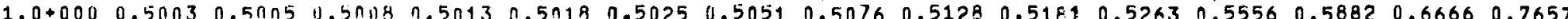
$2.0+000 \quad 0.53360 .31380 .53401133440 .33490 .33560 .33780 .34010 .34480 .34570 .35710 .38460 .41670 .49980 .6203$

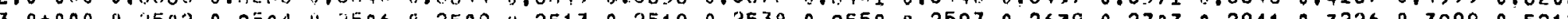
3.0 .0000 .25020 .25040 .259 .0 .25090 .25100 .25190 .25380 .25580 .25970 .26390 .27030 .2910 .32260 .39990 .5213 $5.0+000.16680 .16490 .16710 .16740 .1570 .16810 .16950 .17090 .17390 .17700 .18180 .20000 .22220 .28560 .3952$

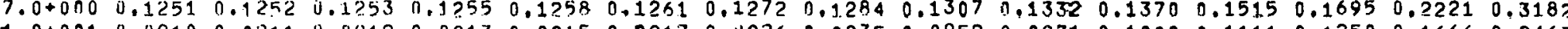

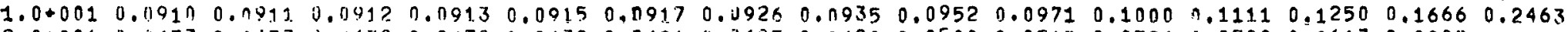

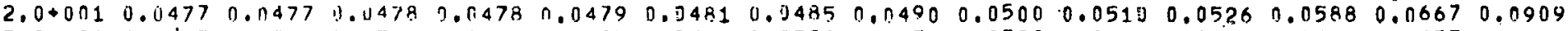

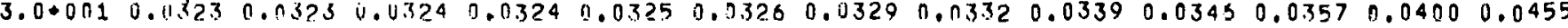

$\begin{array}{lllllllllllllll}5.0+001 & 0.0196 & 0.0176 & j .0197 & 0.0197 & 0.0997 & 0.3198 & 0.0200 & 0.0202 & 0.0206 & 0.0211 & 0.0217 & 0.0244\end{array}$

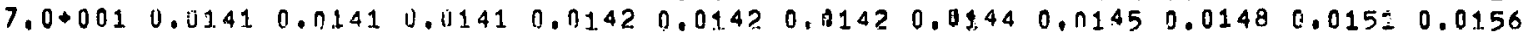

$1,0+0020.010990 .00990 .00990 .01000 .011000 .01000 .09010 .01020 .01040 .01050 .0110$

$2,0+0020.0105 n 0.005110 .07500 .00500 .00500 .00500 .00510 .00510 .0052$

$3,0+002 \quad 3,010330.011330 .07330 .00330 .00330 .0 .0340 .40340 .0034$

$5.0+002 \quad 0.00200 .01020 \quad 10.00200 .00300 .00200 .00200 .0020$

$7.0+002 \quad 4,0014 \quad 0.0014 \quad 0.0014 \quad 0.0014 \quad 0.0014 \quad 0.0014$

$1.0+0030.00100 .00900 .00100 .00100 .00100 .0010$ BETA

$1.0=003 \quad 0.09991 .00001 .00001 .600 \pi 1.00001 .03001 .030 n 1.00001 .00001 .00001 .00001 .00001 .00001 .00001 .00001 .0000$

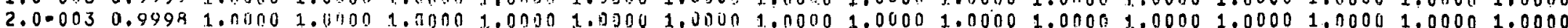

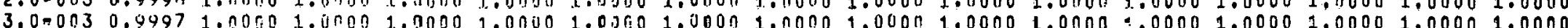

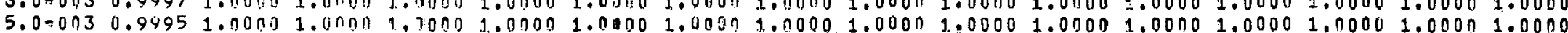

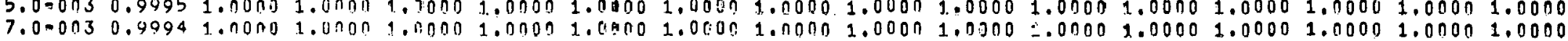

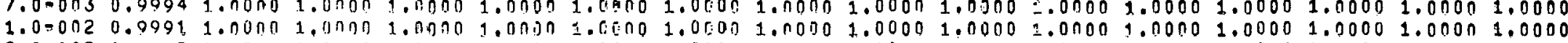
2,0-002 0.9982 1.0000 1.000n 1.00001.00001.00001.000n 1.00001.00001.00001.00001.00001.00001.0000

$3.0-0020.99731 .00001 .00001 .00001 .00001 .06001 .00001 .00001 .00001 .00001 .00001 .00001 .0000$

$5.0=0020.99551 .00001 .01001 .03001 .00001,00001.00001 .00001 .00001 .00001 .00001 .0000$

$7.0=0020.99371 .00031 .00003 .00001 .00301 .09001 .00001 .00001 .00001 .00001 .0000$

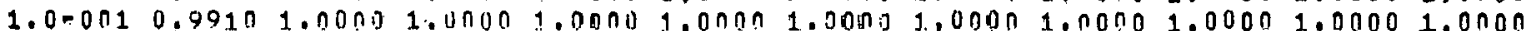

$2.0=0010.98210 .99991 .011001 .00001 .00001 .00001 .00001 .00001 .0000$

$3.0=0010.97350 .99991 .00001 .00001 .00001 .037001 .00101 .0000$

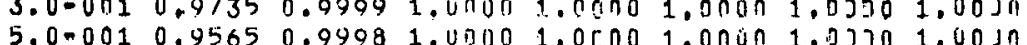

7.0-001 $0.9402 \quad 0.99971 .00001 .00001 .00001 .0030$

$1.0+000 \quad 0.9167 \quad 0.99051 .00001 .00001 .00001 .0000$

$2.0+000 \quad 0.8462 \quad 0.09001 .00001 .0000$

$3.0+000 \quad 0.7857 \quad 0.09851 .0000$

$5.0+000 \quad 0.5875 \quad 0.9976$

$7.0+000 \quad 0.0111$

$1.0+001 \quad 0.5238$ 
VALUES OF G FOR $N=10$ ANI $N P=2$

$$
\text { BETA }
$$

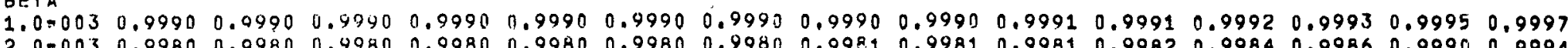

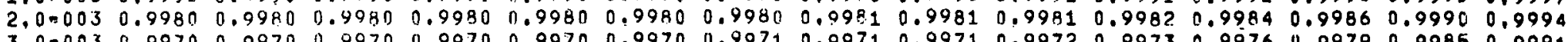

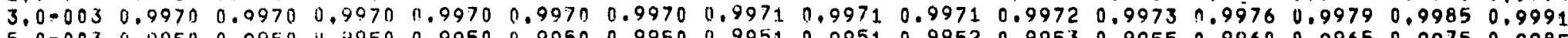

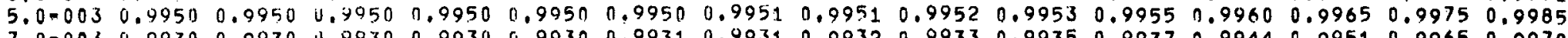

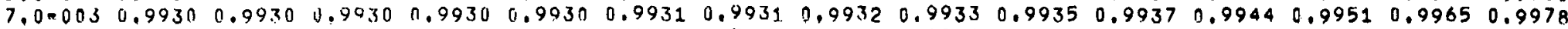
$1.0=0020.9900 \quad 0.9900 \quad 0.90000 .99010 .99090 .99010 .99020 .99030 .99050 .99070 .99100 .99200 .99300 .9950 \quad 0.9969$

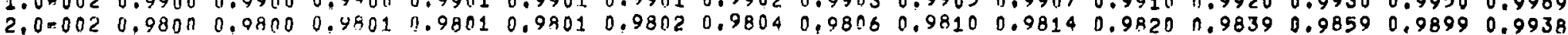

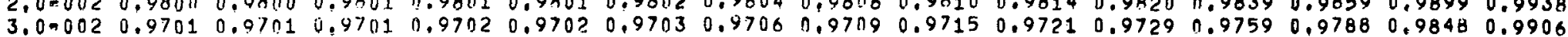

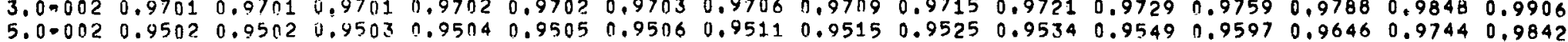

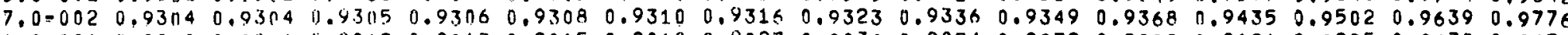
$\begin{array}{lllllllllllllllllll}1.0001 & 0.9010 & 0.9011 & 0.9012 & 0.9013 & 0.9015 & 0.9018 & 0.9027 & 0.9036 & 0.9054 & 0.9072 & 0.9 n 99 & 0.9191 & 0.9285 & 0.9478 & 0.9674\end{array}$

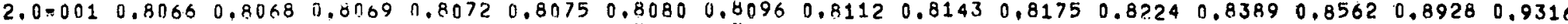
$\begin{array}{llllllllllllllllll}3,0=001 & 0.7196 & 0.7198 & 0.72 .00 & 0.7204 & 0.7208 & 0.7214 & 0.7235 & 0.7255 & 0.7297 & 0.7338 & 0.7402 & 0.7622 & 0.7855 & 0.8368 & 0.8934\end{array}$ $\begin{array}{llllllllllllllllll}5.0 .001 & 0.5717 & 0.5719 & 0.5722 & 0.5727 & 0.5731 & 0.5739 & 0.5764 & 0.5789 & 0.5839 & 0.5891 & 0.5970 & 0.6250 & 0.6557 & 0.7272 & 0.8133\end{array}$ $7.0=0010.45690 .45710 .45740 .45790 .45840 .45910 .46160 .46420 .46940 .47470 .48290 .51230 .54560 .62680 .7330$ 3.0 .000 .0 .33360 .33380 .33400 .33440 .33490 .33560 .33780 .34010 .34480 .34970 .35710 .38460 .51670 .4990 .0 .6203

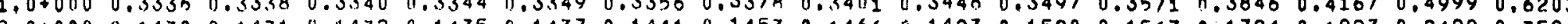
$2.0+000 \quad 0.143010 .14310 .14320 .14350 .14370 .14410 .14530 .14660 .14930 .15200 .15630 .17240 .19230 .24990 .3526$ $3.0+0000.07700 .07710 .0771 \quad 0.07730 .07740 .07760 .07840 .07910 .08060 .08220 .08470 .09430 .10640 .14280 .2140$ $5.0+000 \quad 0.0323 \quad 0.03230 .03240 .0324 \quad 0.03250 .03260 .03290 .03320 .03390 .0346 \quad 0.0357 \quad 0.04 n 0 \quad 0.0455 \quad 6.0625 \quad 0.0982$

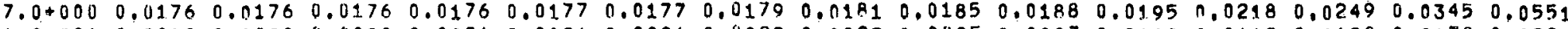
$1.00010 .0090 \quad 0.0090 \quad 1.0090 \quad 0.00910 .0091 .0 .00910 .00920 .00030 .00950 .00970 .0100 \quad 0.01120 .01280 .01780 .0288$ $2.0+0010.00240 .00240 .00240 .00240 .00240 .00240 .00240 .00240 .00250 .00260 .00260 .00300 .00340 .0047$

$3.0+0010.00110 .00110 .00110 .0011 \quad 1.00110 .00110 .00110 .00110 .00110 .00120 .00120 .00130 .0015$

$5.0+0010.00040 .0004 \quad 0.011040 .00040 .00040 .00040 .00040 .00040 .00040 .00040 .00040 .0005$

$7,0.0010 .00020 .0002 \quad 0.000 ? 0.00020 .00020 .00020 .00020 .00020 .00020,00020.0002$

$1.0+0020.00010 .00010 .00010 .00010 .00010 .00010 .00010 .00010 .00010 .00010 .0001$

$1.0+002$
$2,0+0020.0000$
$3.0+002$

$3.0+0020.00000 .00000 .00000 .00000 .00000 .00000 .00000 .0000$

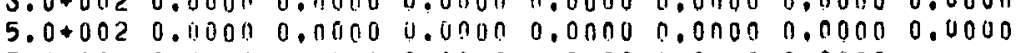

$7.0+002 \quad 0.0000 \quad 0.0000 \quad 0.0000 \quad 0.0000 \quad 0.0000 \quad 0.0000$

$1.0+003 \quad 0.0000 \quad 0.0000 \quad 0.0000 \quad 0.00000 .00000 .0000$

ALPHA $1.000 \quad 2.000 \quad 3.000 \quad 5.000 \quad 7.000 \quad 10.00 \quad 20.00 \quad 30.00 \quad 50.00 \quad 70.00 \quad 100.0 \quad 200.0 \quad 300.0 \quad 500.0 \quad 700.0 \quad 1000$ BETA

1.000030 .09991 .00001 .00001 .00001 .00001 .00001 .00001 .00001 .00001 .00001 .00001 .00031 .00001 .00001 .00001 .0000 $2.0=0030.09981 .00001 .00001 .00001 .00001 .00001 .00001 .00001 .00001 .00001 .00001 .00001 .00001 .00001 .00001 .0000$

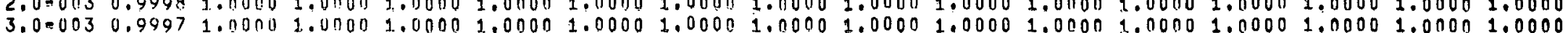

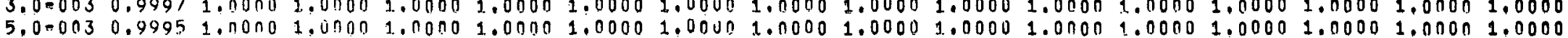

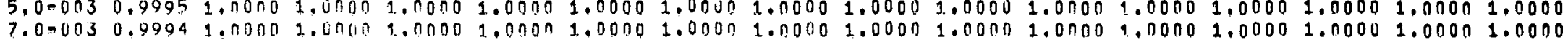
$1.000020 .99911 .010 n 10.00001 .00011 .00001 .00001 .00001 .00001 .00001 .00001 .00001 .00001 .00001 .00001 .00001 .0000$ 2.000020 .99811 .00001 .130001 .00001 .00001 .00001 .00001 .00001 .00001 .00001 .00001 .00001 .00001 .0000

$3,0=0020.99721 .00001 .00001 .00001 .00001 .00001 .000 n 1.00001 .00001 .00001 .00001 .0001 .0000$

$5.0=0020.99521 .00001 .00001 .00011 .00001 .00001 .00001 .00001 .00001 .00001 .00001 .0000$

$7,0=0020.99321 .00001 .00001 .00001 .00001 .00001 .00001 .00001 .00001 .00001 .0000$

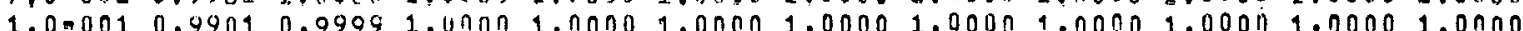

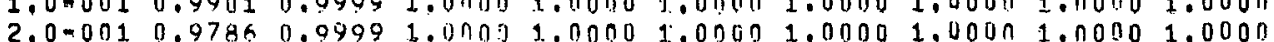

$3,0=0010.96580 .09081 .00001 .00001 .00001 .00001 .00001 .0000$

$5,0=001 \quad 0.9362 \quad 0.9996 \quad 1.0110 .01 .00001 .00001 .00001 .0001$

$7.00001 \quad 0.9024 \quad 0.99041 .011001 .00001 .00001 .0000$

$1,0+0000.84620 .99901 .00001 .00001,00001,0000$

$2.0+000 \quad 0.6471 \quad 0.9971 \quad 0.90991 .0000$

$3.0+000 \quad 0.4783 \quad 0.9942 \quad 0.9099$

$5.0+000 \quad 0.2683 \quad 0.9856$

$7.0+000 \quad 0.164 ?$

1.0 .0010 .0909 


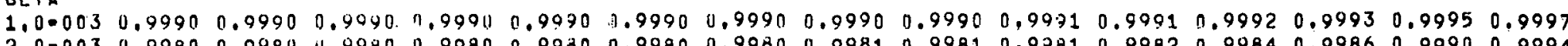

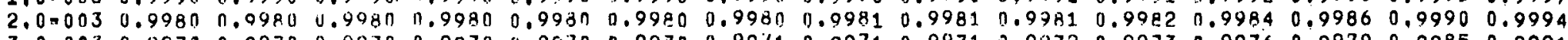

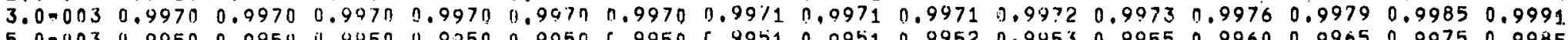

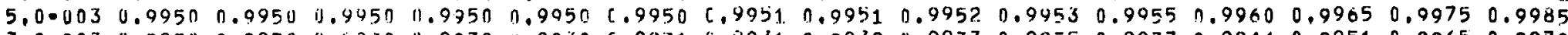

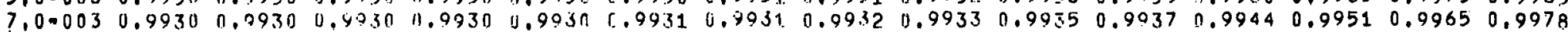

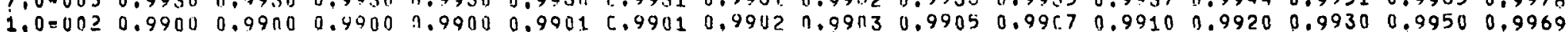

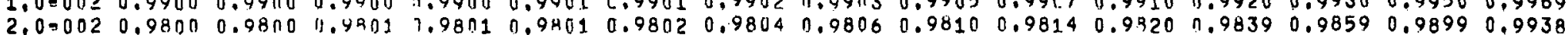

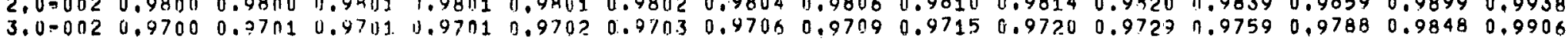

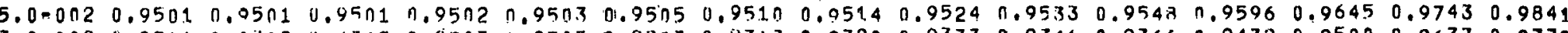
$\begin{array}{llllllllllllllll}7,0-002 & 0.9301 & 0.9302 & 1.9 .902 & 0.0303 & 0.9305 & 0.9307 & 0.9313 & 0.9320 & 0.9333 & 0.9346 & 0.9366 & 0.9432 & 0.9500 & 0.9637 & 0.9775\end{array}$

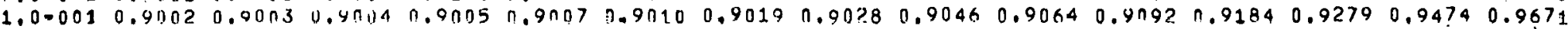

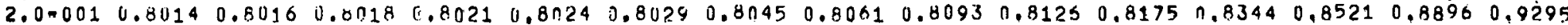
$\begin{array}{llllllllllllllllllll}3.00001 & 0.7159 & 0.7061 & 0.7 n 63 & 0.7068 & 0.7072 & 0.7078 & 0.7099 & 0.7120 & 0.7163 & 0.7205 & 0.7271 & 0.7499 & 0.7741 & 0.8274 & 0.8868\end{array}$

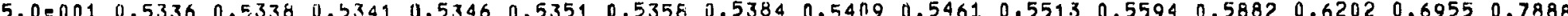
$7.0=0010.39500 .39530 .30550 .39600 .30650 .39700 .39960 .40210 .40710041230 .42020 .44920 .48240 .56600 .6807$

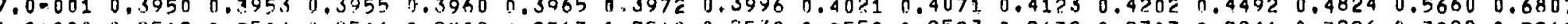

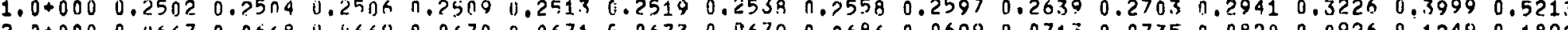

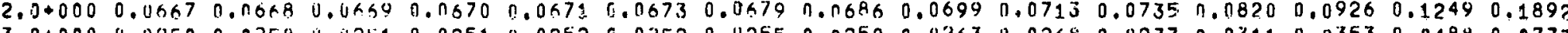

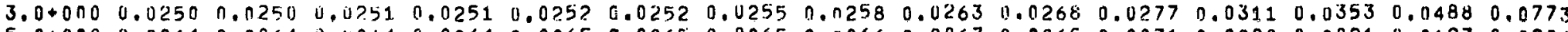
$5.0+0000.00640 .0064 \quad 0.00540 .00640 .00650 .00650 .00650 .00660 .00670 .00650 .00710 .00800 .00910 .01270 .0206$

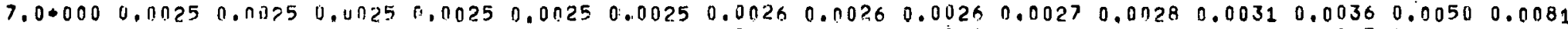
$1.0+0010.00090 .03 n 90.00090 .00090 .00090 .00090 .00090 .00590 .00090 .00900 .00100 .00110 .00130 .00180 .0029$ 2.0+0n1 0.0001 n.n!n1 0.0n01 0.00010 .00010 .00010 .00010 .00010 .0001000010 .00010 .00010 .00020 .0002

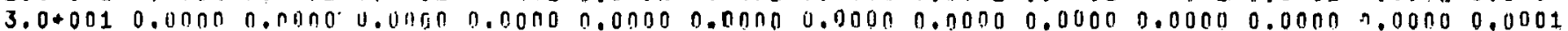

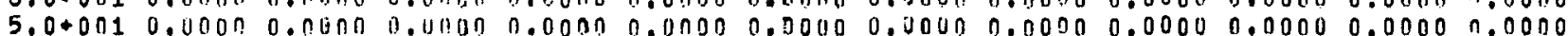

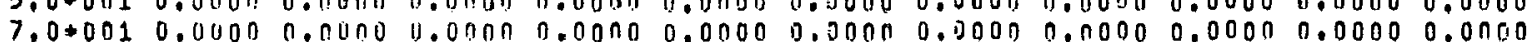

$1,0+0020.00000,00000.00000 .00000 .00000,30000.00000 .00000,00000.00000 .0000$

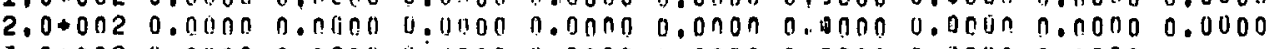

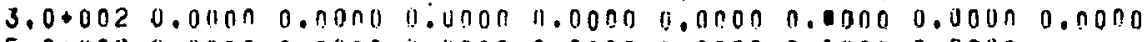

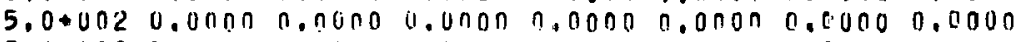

$7,0+0020.00000 .00000 .00000 .0003 .0,0000 \quad 0.0 .000$

$1.000030 .00000 .0000 \quad 0.60000 .00000 .0000 \quad 0.6000$

$A L$ BETA

1.000030 .99991 .00001 .00001 .00001 .00001 .00001 .00301 .00001 .00001 .00001 .00001 .00001 .00001 .00001 .00001 .0000 2.0-003 c.9998 1.0000 1.0000 1.00001.00001.0000 1.00001.00001.00001,00001.00001.00001.00001.00001.00001.0000 $3,0=0030.99971 .00001 .00001 .00001 .00001 .33001 .03001 .00001 .00001 .00001 .00001 .00001 .00001 .00001 .00001 .0000$ $5.0 .0030 .99951 .00001 .00001 .00001 .00001 .03001 .00001 .00001 .00001 .00001 .00001 .00001 .00001,00001.00001 .0000$ $7.0=0030.99941 .00001 .01001 .00001 .00001 .0 .001 .00001 .00001 .00001 .00001 .00009 .00001 .00001 .00001 .00001 .0000$ 1.000020 .99911 .00001 .00001 .00001 .00001 .09001 .00001 .00001 .00001 .00001 .00001 .00001 .00001 .00001 .00001 .0000 $2,000020.99811 .00001 .00001 .00001 .00001 .00001 .00001 .00001 .00001 .0000 \div .00001 .00001 .00001 .0000$

$3.0-0020.99721 .00001 .00001 .00001 .00001 .00001 .00001 .00001 .00001 .00001 .00001 .00001 .0000$

$5.0-0020.99521 .00001 .07001 .00001 .00001 .00001 .00001 .00001 .00001 .00001 .00001 .0000$

$7,0.0020 .99321 .00001 .00001 .00001,00001,00001.00001 .00001 .00001 .00001 .0000$

$1.0-0010.99000 .99991 .00001 .00001 .00001 .00001 .00001 .00001 .00001 .00001 .0000$

$2.0=0010.97800 .99991 .00001 .00001 .00001 .00001 .00001 .00001 .0000$

$3.0=0010.96350 .9998 \cdot 1.00001 .00001 .00001 .00001 .00001 .0000$

$5.0=001 \quad 0.92630 .99961 .00001 .00001 .00001 .00001 .0000$

$7,0.0010 .8777 \quad 0.9993 \quad 1.00001 .00001 .00001,0030$

$1.0+000 \quad 0.7857 \quad 0.99851 .0000 \quad 1.00001 .00001 .0030$

$2,0+000 \quad 0,4400 \quad 0.9932 \quad 0,9998 \quad 1,0000$

$3,0+000 \quad 0.2200 \quad 0.9813 \quad 0.9996$

$5.0+000 \quad 0.0663 \quad 0.9296$

$7.0+000 \quad 0.1208$

1. $0+0010.0098$ 


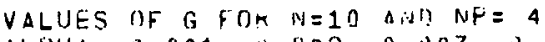

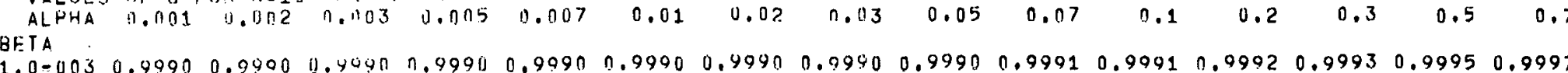

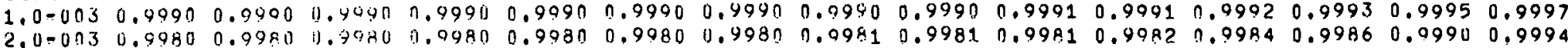
$\begin{array}{llllllllllllllll}3.0-003 & 0.9970 & 0.0970 & 0.4070 & 0.9970 & 0.997 n & 0.9970 & 0.9971 & 0.9971 & 0.9971 & 0.9972 & 0.9973 & 0.9976 & 0.9979 & 0.9985 & 0.9991\end{array}$

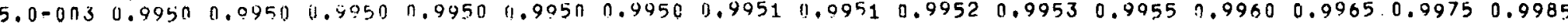

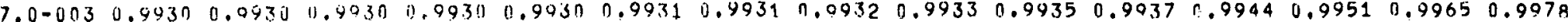

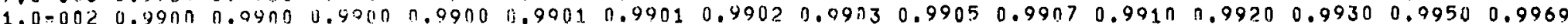

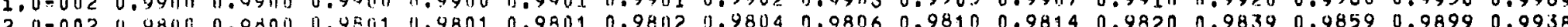
. $3.0 \div 002$ 0.97ח n.07n1 .

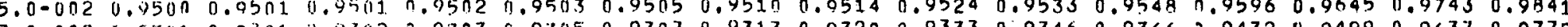

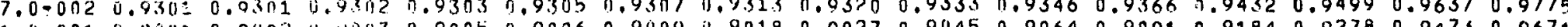

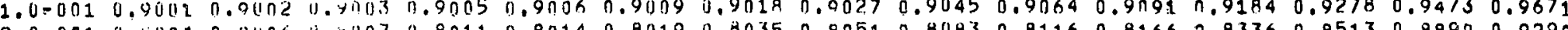

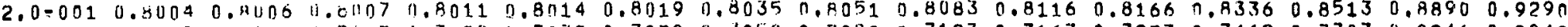
$\begin{array}{lllllllllllllllll}3.0-001 & 0.7019 & 0.70 ? 1 & 0.7023 & 0.7028 & 0.7032 & 0.7038 & 0.1059 & 0.7080 & 0.7123 & 0.7167 & 0.7233 & 0.7462 & 0.7707 & 0.8246 & 0.8849\end{array}$ $\begin{array}{llllllllllllllll}5.0=001 & 0.5164 & 0.5160 & 1.5169 & 1.5174 & 0.51 .79 & 0.5186 & 0.521 ? & 0.5237 & 0.5289 & 0.5342 & 0.5424 & 0.5714 & 0.6038 & 0.6807 & 0.7770\end{array}$ $\begin{array}{lllllllllllllllll}7.0-001 & 0.360 & 0.3611 & 0.3513 & 1.3618 & 0.3622 & 0.36 ? 9 & 0.3653 & 0.3677 & 0.3725 & 0.3775 & 0.3952 & 0.4135 & 0.4462 & 0.5294 & 0.6482\end{array}$

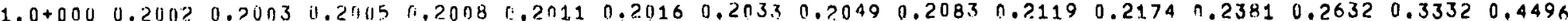

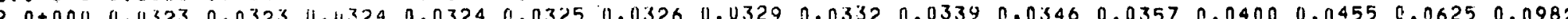

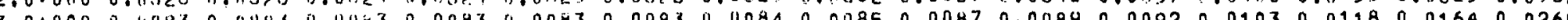

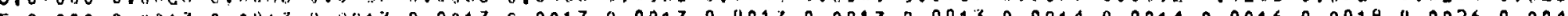

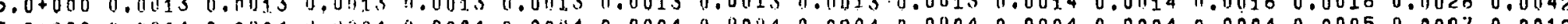

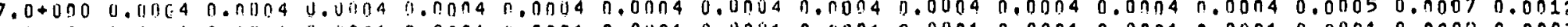

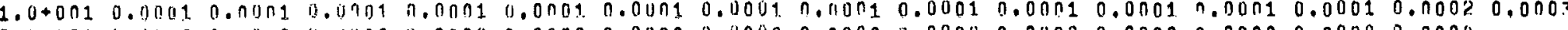

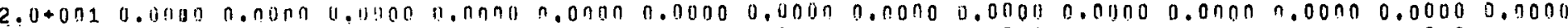

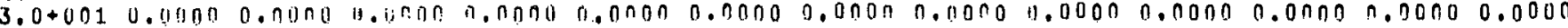

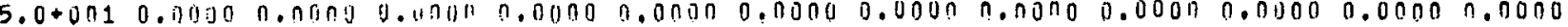

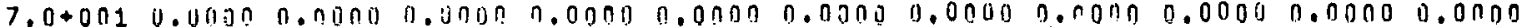

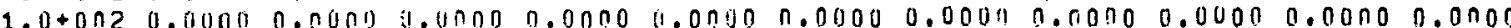

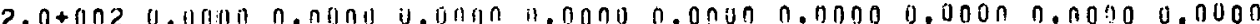

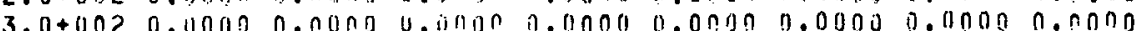

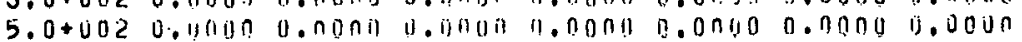

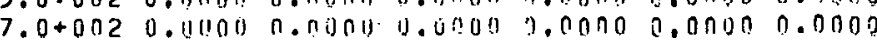

$1.0+0030.01000 .00000 .0000 .00000000000000$

ALPHA $1.00 \pi$ 2.0nO 3.000 $5.000 \quad 7.000 \quad 10.00 \quad 20.0 n \quad 30.00 \quad 50.00 \quad 70.00 \quad 100.0 \quad 200.0 \quad 300.0 \quad 500.0 \quad 700.0 \quad 1000$ UETA

$1.0=0030.09991 .00001 .00001 .00001 .00001 .00001 .00001 .00001 .00001 .00001 .00001 .00001 .00001 .00001 .00001 .0000$ $2.0-0030.49941 .000 j 1.01001 .00001 .00001 .00001 .00101 .00001 .00001 .00001 .00001 .00001 .00001 .100001 .00001 .0000$

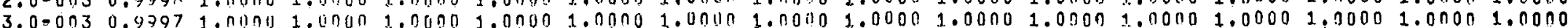

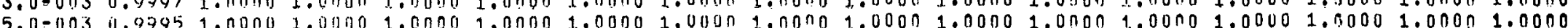

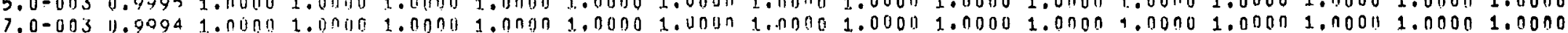

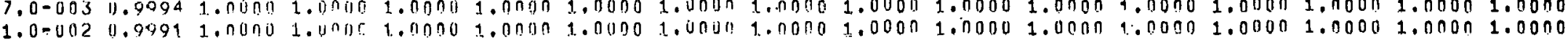

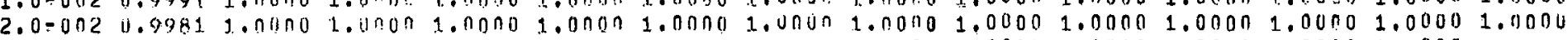
$3.0-0020.99721 .00001 .41001 .00001 .00001 .00001 .00001 .00001 .00001 .00001 .00001 .00001 .0000$

$5.0=0 n 20.59521 .00001 .00001 .00001 .00001 .00 n \pi 1.000 n 1.00001 .00001 .00001 .00001 .0000$

$7.0=002 \quad 4.5 .93 ? .1 .00001 .0 n \pi n 1.00001 .00001 .00001 .00001 .001001 .00001 .00001 .0000$

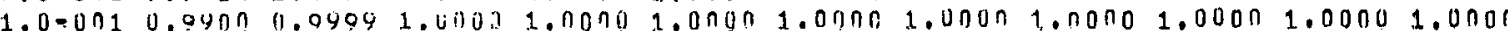

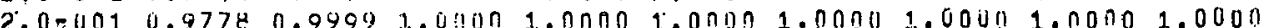

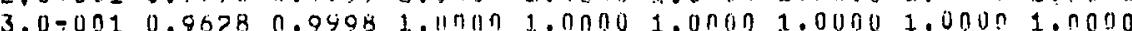

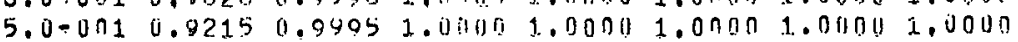

$7.0-0010.0012$ 0.0991 1.0000 1.00000 1.0000 1.0000

$1.0+0000.73330 .99801 .010001 .00001 .00001 .0000$

$2.0+0100 \quad 0.2683 \quad 0.9655 \quad 19.9097 \quad 1.0000$

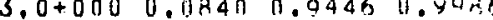

$5.0+000 \quad 0.01390 .7241$

$7,0+000 \quad 0.0039$

$1.0+0 n 10.01010$ 

EETA

1.0-003 $0.99900 .999011 .90900 .9990 \quad 0.9990 \quad 0.9990,9.99900 .09900 .9990 .0 .99910 .99910 .99920 .99930 .99950 .9997$

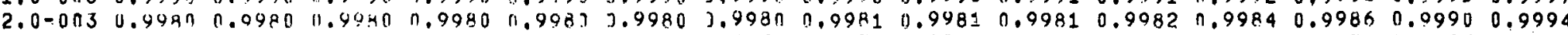
$\begin{array}{llllllllllllllllll}3.0-003 & 0.9970 & 0.0970 & 11.9070 & 0.9970 & 0.9971 & 3.9970 & 1.9971 & 0.0971 & 0.9971 & 0.9972 & 0.9973 & 0.9976 & 0.9979 & 0.9985 & 0.9991\end{array}$

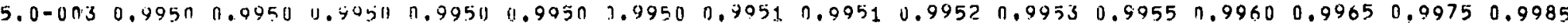

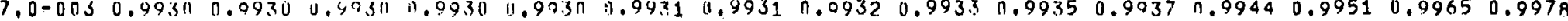

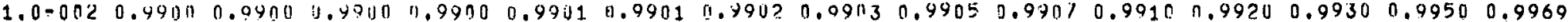

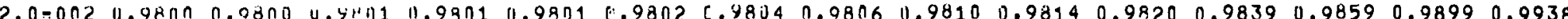
$3,0=0070.97000 .97110 .97010 .97010 .970859703 \quad 1.87060 .97090 .97150 .97200 .97290 .97590 .97880 .98480 .9906$

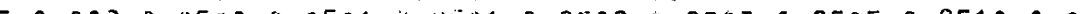

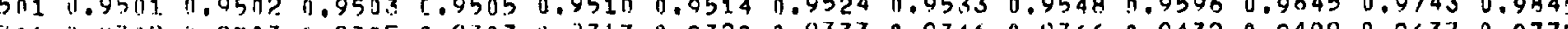

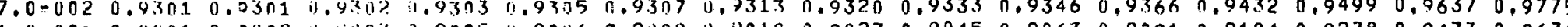

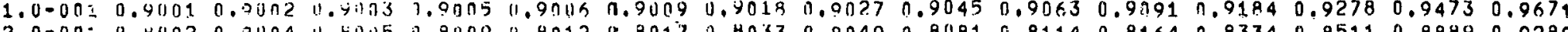

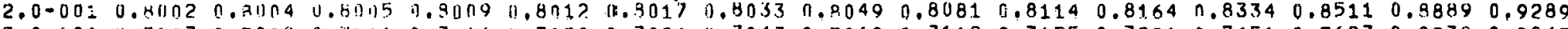

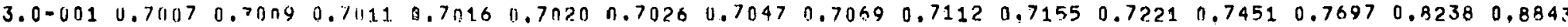

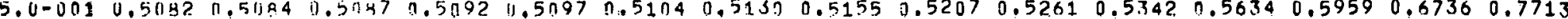

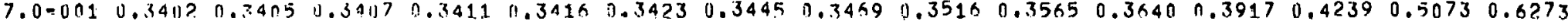
$1.0+000 \quad 0.2664 \quad 0.1669 \quad J .1671=.1674 \quad 0.1474 \quad 0.1681 \quad 0.1695 \quad 0.17390 .17390 .17700 .18180 .2000 \quad 0.2222 \quad 0.2856 \quad 0.3952$

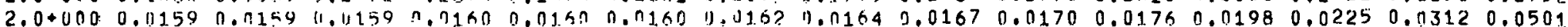

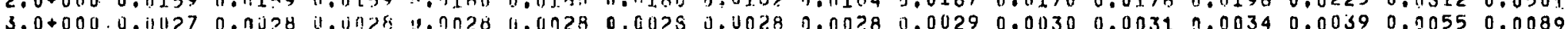

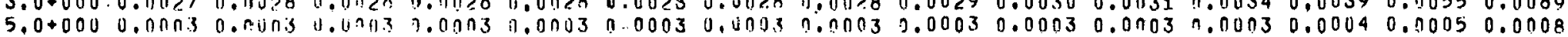

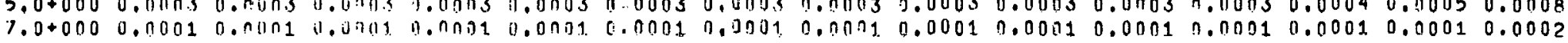

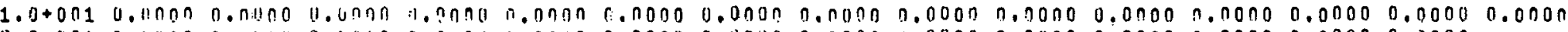

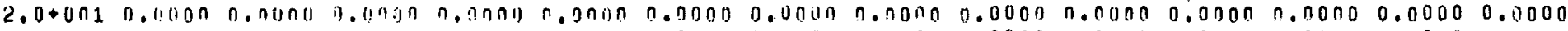

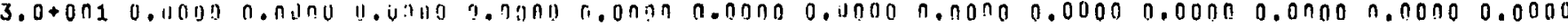

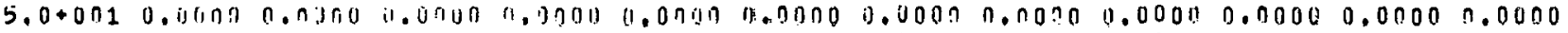

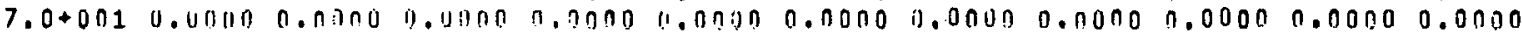

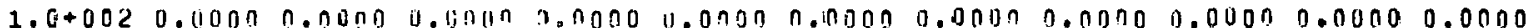

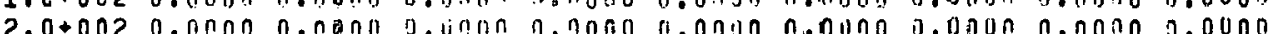

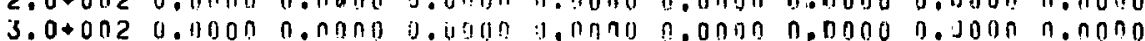

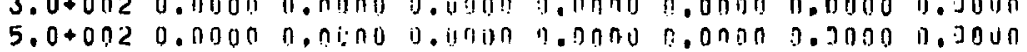

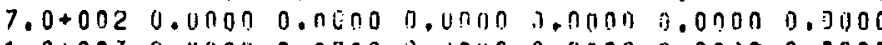

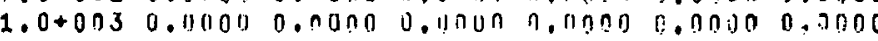

$$
\text { BETA }
$$

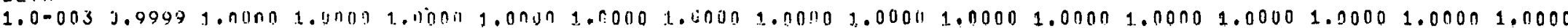

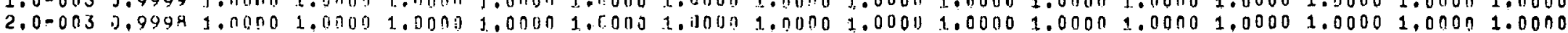

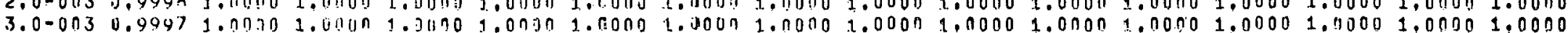

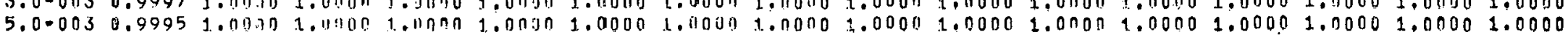

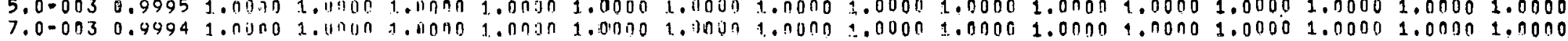

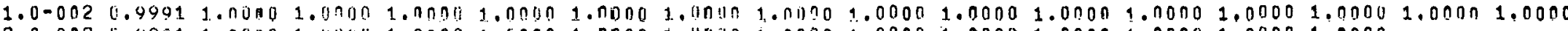

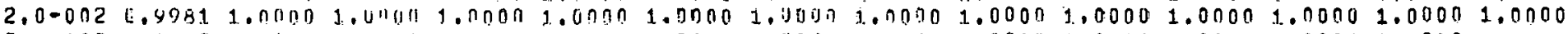

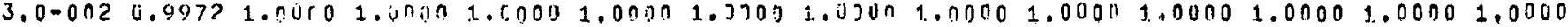

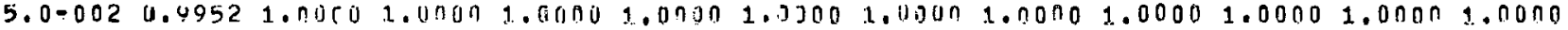

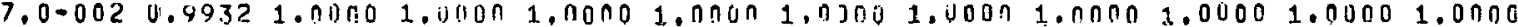

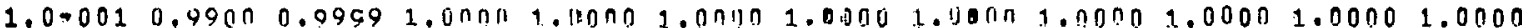

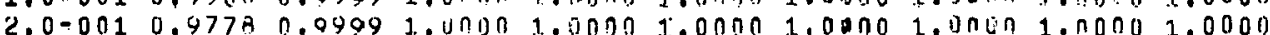

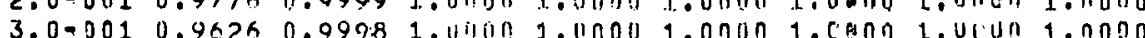

$5,0=001 \quad 0.91910 .9995$ 1.01100 1.0110001 .0000 1.CCDO 1.UCUO

$7.0-0010.85000 .99911 .00001 .00001 .00001 .0600$

$1.0+000 \quad 0.68750 .99750 .99991 .031001 .00101 .0600$

$2.0+000 \quad 0.151170 .97 n .5 \quad 0.8093 \quad 1.00010$

$3.0+0000.0294 \quad 0.4497 \quad 1.9959$

$5,0+000 \quad 0.11028 \quad 0.3430$

$7.0+000 \quad 0.0005$

$1.0+0010.000$ 


\section{APPENDIX D}

FORTRAN Program Statement 
PHOLIRAM CEN R132

B132(CEN)/T, JOHNSON/N, K. CLLARK/FEERUARY 1965

DIMENSION AL = A (31), BETA (31),F(16), FF (31), FOFA(16), ALFAIN( 1 ()

REAIS INPUT TAPE 7.1.(ALFAIN(1.): $I: 1,31)$

1 FURMAT(EIL, U

DU $10 \quad I=1,31$

19230013

10 BETAC(I)=ALFAON(I)

290 REAII INPUT TRPE 7,7\%, IIIII

7 FORMAT (12!E)

If I IIIII) 9000.9999. 295

295 IF. (IIIII-2, 170, 320, 170

170 REAI) INPUI TAPE 7,1 , EMD

DO 2 RS $1=1,3: 1$

285 ALFA(I) =ALFAIN(I)

$10=1$

280 DO $130 \quad N=1,13$

DU 130 NP $=1.5$

GO TO $(1 \% 0, \div 90)$, ID

180 WIITE OUTPUT TAPE $6,2, N, N P$

FURMAT(21H1. VALUES OF.F FOR HEI $2,8 H$ AND NP=12) GO TO 200

190 WRITE OUTPUT TAPE 6, 16, $N$, NAP

16 FURMAT(21H1 VALUES OF $G$ FOR $N=12,8 H$ ANU $N P=12)$

200 WHITE OUTHUT TAPE 6,3 , (ALFA(:) $1=1,15)$

3 FURMAT(GH ALPHA, 5F 7,3,5F7, $2,5 F 7,1,57,0)$

WRITE OUTPIT : TAPE 6,5

5 FURMATC5H BETAD

DO उo $\mathrm{J}=1,31$

B=1.

DU $70 K=1 . N P$

$70 \quad H=B \otimes R E T A(J) * * K$

$I I=0$

DO 4 त $1=1,15$

IF (ALFA(I) $\# B E T A(j)-E M D) 50.50,40$

$50 \quad A=1$.

DO on $K=1, N$

$60 A=A+A L F A(1) \cdots K$

$A=1,1 A$

GO TO $(210,22)), 10$

$210 F(1)=(1,-A) /(1,-A+A+B)$

GO TO 230

$220 F(1)=1.0) /(1,0-A-A+F)$

$230 \quad 11=$

40 CUNTINUE

IF (II) $30,30,80$

80 WRITE OUTPIT TAFE $6,4, B E T A(J),: F(K), K=1,1$ I)

4 FURMAT (1.H,E7.1,16F7.4)

30 CONTI INUE

WHITF OUTHIIT TAPE 6,6

6 FURMAT ( IH )

WRITE OUTPUT TAPE $6,3,($ ALFA $(1), 1=16,31$

WHITE OUTPUI TAPE 6,5

U) $90 \mathrm{~J}=1,31$

$\beta=1$.

D) $110 K=1, N P$

$110 B=B+B E T A(J) * * K$

19230017

19230007

19230018

19230008

19230019

15230010

$1 \$ 230020$

19230029

19.230022

19230023

19230024

19230025

19230027

19230028

19230029

19230030

19230031

19230032

19230033

19230034

19230035

19230009

19230036

19230037

$1923003 \mathrm{~A}$

19230039

19230041

19230041

10230042

19230042 
$11=1.5$

DO 1 in $01=15,31$

IF (ALFA(I)*HFTA(J)-EMD) $150,150,100$

19230044

19230045

$150 \quad A=1$.

Bu 1 ifo $K=1$, is

$100 \quad A=A+A L F A(1) \cdots$

$A=1.1 A$

19230047

19230048

19230049

Gu $\ln (240,250), 10$

$1923005 n$

$240 F(I-15)=(1,-A) /(1,-A+A+B)$

Gu in 260

$250^{\prime} F(I-15)=1.0 /(1,0-A+A+B)$

$26011=1$

10 CUNTINUE

IF $(11-15)>0,90,120$

120 WHITF OUTPUT TAPE 6,4, PFTA $(J),(F(K=15), K=16,11)$

O CONTT INIIE

131 CONTINUE

$2 ? 0$ lic $10,300,310), 10$

$30010=2$

Go. TO 280

S10 IF i IIII-2, $9999,320,320$

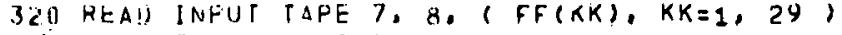

B FURAAT ( 12to.D)

Do $\sin 0$ in $=1$, 1. U

U1) bกU $N P=1,5$

JUMP $=1$

INIT IALL $=1$

LAST $=1^{2} 4$

WRITF OUTPUI TAPE G, $9,^{\circ} N, N P$

FURATI ( $25 H 1$ VALUES OF ALPHA FOK $N=12$, BH AND NPEIA)

WHITE OUTPIJT TAPE 6, 11, ( FF(K1), K1=1, 14 )

11 FORMAT ( 13H GETA/F(A),57.4,3F9.4,F10.4,7F9.4,2F8.4) [iu 10327

325 JUMP $=2$

327 DO $4011 \mathrm{~J}=1,29$

$R=1 . n$

กU $330 K=1$, NP

$33 B \quad B=8+R E T A(J) * K$

$11=0$

חUU $390 \quad I=I N I T I A L$, LAST

ALFA $(1)=U$, U OI) 955

I $i=1$

Q 11 . $A=0.0$

nO OOS $M M=1, N$

$805 A=A+A L F A(1) * 14 M$

$F \cup F A(I)=(A /(A+B))-F F(I)$

(i) $10(8.10,825,840)$, ID

P. $10 \quad 10=2$

IF ( FUFA(1), 8?0, 385, 815

प15 $A L F A(1)=0,0$ GO 10385

8.20 ALFAKEEP $=0.000995$

$A L F A(I)=1: 100.0$

Gu TO

$425 \quad 10=3$

If (FOFA:1), 391, 391, 835 


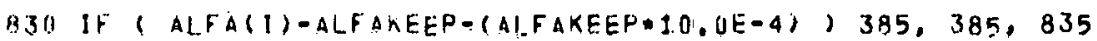

835 ALFAHULD $=A L F A(1)$

$A L F A(I)=(A L F A H O L D+A L F A K E E P) \bullet 0,5$

GU TO 800

340 IF (FOFA(I) $85 \pi, 385,830$

350 IF ( ALFAM()I.10-ALFA(I)-(ALFA(I)+3),0E-4) 1385, 385, 860

860 ALFAKEFP=ALFA(I)

$A L F A(I)=(A L F A H O L D+A L F A K E E P) * 0.5 \quad$

GO TO BOO

$38511=1$

390 CONTTINUE

391 GO Tn ( $392,470 . ;$, JUMP

392 If ( BFTA(J): $3.0,393,398,396$

393 WRITE OUTPUT TAPE 6, 12, BETA(N): ( ALFA(K2), K2=1, 11 )

12 FORMAT ( $1 H, F 7,3,3 X, 4 F 9.5, F 10,5,7 F 9,4,2 F 8,4$ ) GU TO 400

396 If ( BETA(J)-i0.0) 398, 394, 3S4

398 . WRITE OUTPUT TAPE 6, 19, BFTA(I), ( ALFA(K4), K4=1, II)

19 FURMAT $(14$, F $7.3,3 \times, 459,5, F 10,5,2 F 9.4,5 F 9,3,2 F B, 3$ ) GO 70.400

394 WRITE OUTPUT TAPE 6,18 , BETAIJ: ( ALFA(K2), K281, II)

18 FORMAT ( 1H, F7.3, 3X, 4F9.4, F.10,4, TF9.3, 2F8.3)

If ( BETA(J)-500.0, 400, 41., 000

410. WHITE NUTPUT TAPE 6,6

WRITE NUTPUT TAPE 6,13, \& FF(K3:, $K 3=15,29$,

13 FURMAT ( $13 \mathrm{H}$ EETALF (A); F.7, $7,4 F 9.4,10 F 8.4$,

INITIAL $=15$

LAST $=29$

GO TO 400

470 WKITE DUTPIJT TAPE 6,14, BETA(J), ( ALFA(K3), K3=15, Il)

14 FORMAT $(1 H, F 7,3,3 X, 5 F 9,3,13 F 8,2$ )

400 CONTINUE

GO ID ( 325, 500)\% JUMP

500 CONTINUE.

$60 T ? 2.90$

9999 WKITE OUTPUT TAPE 6,17

17 FURMAT (. 1HI)

9000 END 


\section{ACKNOW LEDGMENTS}

The authors wish to acknowledge the helpful criticisms of Leslie Burris, Jr., and R. D. Pierce in preparing this report for publication

\section{REFERENCES}

1. Kremser, A., National Petroleum News 22(21), 42 (May 21, 1930).

2. Smith, B. D., and Brinkley, W. K., AIChE J. 6., 446 (1960).

3. Smith, B. D., Design of Equilibrium Stage Processes, pp. $252-293$, McGraw-Hill Book Company, Inc., New York (1963).

4. Souders, M., and Brown, G. G., Ind. Eng. Chem. 24, 519 (1932).

5. Bartels, C. R., and Kleiman, G., Chem. Eng. Progress 4ㄷ, 589 (1949).

6. Treybal, R. E., Liquid Extraction, pp. 323-331, 2nd ed., McGrawHill Book Company, Inc., New York (1963).

7. Vogel, R. C., et al., Chemical Engineering Division Research Highlights, May 1963-April 1964, ANL-6875, p. 28.

8. Kwauk, M., AIChE J. 2, 240 (1956). 